51

N422

NMAIREF

V.18

II

OF

\author{
by \\ JOHN C. HUDEN
}

MUSEUM OF THE AMERICAN INDIAN

HEYE FOUNDATION

1962 NAMES

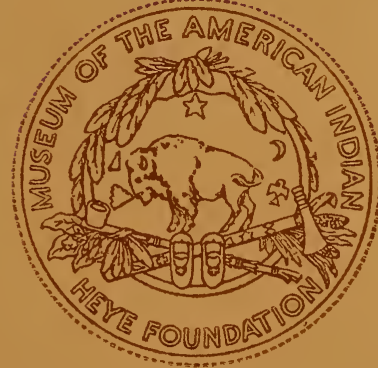

SMITHSONIAN
JUL 202000
LIBRARIES 

CONTRIBUTIONS FROM THE MUSEUM OF THE AMERICAN INDIAN HEYE FOUNDATION VOL. XVIII

\title{
INDIAN PLACE NAMES OF NEW ENGLAND
}

\author{
Compiled by \\ JOHN C.HUDEN
}

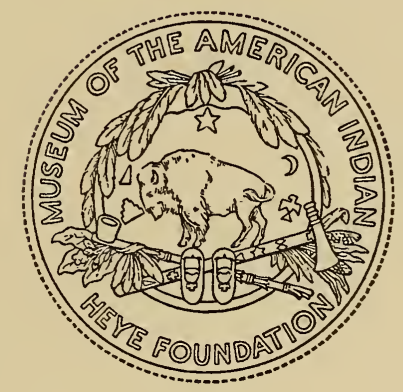

NEW YORK

MUSEUM OF THE AMERICAN INDIAN HEYE FOUNDATION

1962 
Library of Congress catalog card number 62-18399

Printed in Germany at J.J.Augustin, Glückstadt 
Grace Braem Huden with much love 



\section{CONTENTS}

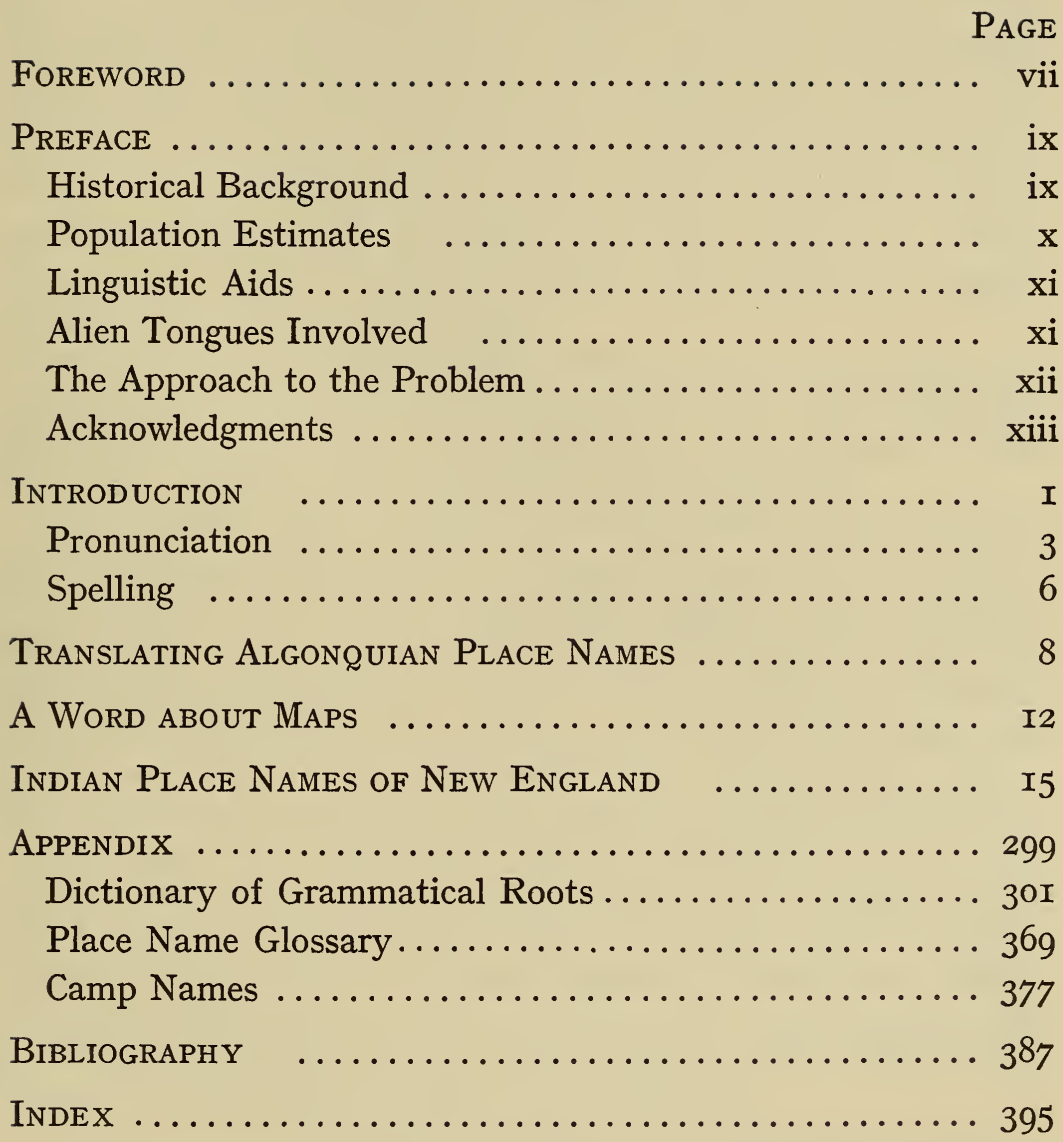





\section{FOREWORD}

The native geographer has come and gone in New England; as is true of so many aboriginal inhabitants, his was a light touch which left little impress upon the land. Because the White emigrant to the region had little understanding of the various local dialects, and even less interest in native cultures, by and large the names the Indian gave to his landmarks have disappeared. Many of these have survived in extremely distorted forms, and only a small proportion of the currently-used place names are clearly defined and readily translatable Indian terms. This is an unfortunate loss to our historic heritage.

As part of a continuing interest in the preservation of information related to all aspects of Amerindian life, this volume is published in an attempt to bring together as large a body of data as possible relating to the toponymy of New England.

The compiler, John C. Huden, has been active in the study of the early Indian history of New England for the past forty years. $\mathrm{He}$ is familiar with several Algonquian dialects, and has spent many years of intensive research into the particular field of place names. From these researches has come an extended record of publications on the subject. Currently on the faculty of the University of Vermont, Dr. Huden has a background of firsthand acquaintance with the region and its aboriginal inhabitants.

It is confidently anticipated that this compilation will prove useful to the reader who is interested in the Indian background of New England. It is also hoped that it will encourage the publication of other studies of a similar nature, so that in due course, the Indian place name structure of the entire United States will be available in published form. While there are many titles in the literature on the subject, much is yet to be done.

The Museum is particularly grateful to Dr. Huden for his untiring efforts in gathering together this information before it is entirely lost, and also to Mr. Lawrence K. Gahan for the generous loan of his notes pertaining to the Massachusetts area.

FRederick J. Dockstader

May, I962

\section{Director}





\section{PREFACE}

Many years ago, J. Hammond Trumbull remarked that, "translating of [Indian] names in Southern New England is far more difficult than in "new" states [Michigan, Minnesota, etc.] where Algonkian is not a dead language. Half the Indian names in Rhode Island are so corrupt as to defy analysis!" (Trumbull, I9I2, p. 68).

This declaration can be applied as arbitrarily to all of New England. Many place names included in this, and other compilations, continue to resist satisfactory translation. Native languages were very difficult for New England colonists, most of whom were not the best of penmen-nor, in many cases, even masters of English usage. Many Indian locatives were first heard from terrified, unwilling lips by prejudiced Puritans-circumstances hardly conducive to linguistic clarity! It is not surprising, then, that a colonist who spelled his own name six or eight different ways would employ a half-dozen variant spellings of a Wampanoag term in a single document.

Even educated English officials had trouble with dialectal differences between such closely-related tongues as Wampanoag, Natick, and Narragansett. Ministers who had studied Latin and Greek found considerable difficulty when they tried to force Algonquian phrases into classical moulds. Some early students attempted to reinforce a then-current belief that the Amerindians were the Ten Lost Tribes of Israel by applying Hebrew grammar to Algonquian constructions; Old Testament cosmology was compared with Indian beliefs, which only compounded the confusion. In addition, many Indian customs and religious ceremonies were suppressed as pagan, and unchristian; the result was an inevitable spotty rendition. It is from just such unsatisfactory bases that the present compilation has arisen.

\section{HISTORICAL BACKGROUND}

It is not the function of this work to set forth anything more than a very brief review of New England Algonquian history. For the purpose of orientation, it must suffice to say that, beginning with the arrival of the earliest Europeans, Algonquianspeaking peoples of the region were continually being mauled, 
expelled, exhorted, acculturated or killed. By the end of the American Revolution, only a pitiful remnant lingered in Connecticut, Rhode Island and Massachusetts, most of whom could speak their aboriginal languages imperfectly, if at all. By I825 the Algonquian languages of Southern New England were practically dead. The last fluent speaker of Wampanoag died around I827, by which time Narragansett had already followed Nauset, Nipmuck and Pennacook into oblivion. By a strange set of circumstances, Mohegan survived until the death of Mrs. Fidelia Fielding in 1908 (Speck, I928, pp. 223-225).

Thus, in three hundred years from the Pilgrim landing at Plymouth Rock, the Wampanoag, Natick, Nipmuck, Narragansett, Pequot, Mohegan, Wappinger, Mahican, Pocumtuck and Pennacook tongues had all disappeared, leaving only an assortment of poorlytranscribed and ill-understood place names as their monuments.

In the North, Abnaki, Malecite and Micmac still survive. Fewer than 2,000 Maine Indians, mostly mixed bloods, today use dialects derived from their ancient language.

\section{POPULATION ESTIMATES}

Swanton (I952) indicates that in I6oo there were from 25,000 to 30,000 Algonquians in New England, speaking upwards of 20 dialects. The I $960 \mathrm{U}$. S. Census presents official figures totaling some 6,084 New England "Indians," most of whom are mixed bloods. It is therefore obvious that from I60o to I960, New England lost about eighty percent of its Indian population, together with ninety percent of their native languages.

Although there are no accurate population statistics availablethe colonists rarely made fine distinctions between the various tribes-the following estimates have been compiled by James Mooney (I928). This classic study, based upon painstaking evaluation of all of the historical material available, remains the most reliable compilation we have to date.

NEW ENGLAND ALGONQUIAN POPULATION IN I600

$\begin{array}{lrlr}\text { Abnaki } & 3,000 & \text { Pennacook } & 2,000 \\ \text { Eastern Niantic } & 400 & \text { Pequot } & 2,200 \\ \text { Mahican } & 3,000 & \text { Pocumtuc } & \text { I,200 } \\ \text { Mohegan } & 2,200 & \text { Wampanoag } & 2,400 \\ \text { Narragansett } & 4,000 & \text { Wappinger } & 1,750 \\ \text { Natick } & 1,200 & \text { Western Niantic } & 600 \\ \text { Nipmuck } & 500 & \text { TOTAL } & 27,450\end{array}$


Authorities agree that this total would represent the minimum, rather than maximum, number. It should also be noted for the purposes of this volume that these figures do not include such non-New England peoples as the Micmac, Malecite, or Delaware, nor the Iroquoian tribes of neighboring New York.

The contemporary population figures of the New England region are of interest, but it must be emphasized that the vast majority are not full-blooded Indians. As a matter of objectivity, it is doubtful whether more than a handful of full-blood Indians remain in New England today, and these latter would be concentrated in upstate Maine.

INDIAN POPULATION OF NEW ENGLAND ACCORDING TO I960 CENSUS

\begin{tabular}{lrlr} 
Connecticut & 923 & New Hampshire & I35 \\
Maine & I,879 & Rhode Island & 932 \\
Massachusetts & 2, I I 8 & Vermont & 97 \\
\cline { 3 - 4 } & & TOTAL & 6,084
\end{tabular}

LINGUISTIC AIDS

Over these three hundred years, satisfactory dictionaries have been compiled for only three of the languages concerned, namely Abnaki, Narragansett and Natick. Although many students have devoted their interest to the languages of the American native, very few have been willing to spend the necessary time to organize that initial tool of linguistic study; rather, they have concerned themselves with grammatical and comparative studies, often failing to establish basic vocabularies for use in continuing research by others.

\section{ALIEN TONGUES INVOLVED}

Most of the non-Algonquian names found in New England are Iroquoian, notably Mohawk. There are several historical and archeological evidences of Mohawk occupation in the extreme northwestern portion of New England, particularly the Champlain Valley of Vermont.

Practically all of the Mohawk place names in Vermont included herein have been taken from French and English maps dated before I790; the rest have been gleaned from old documents of many varieties. These Mohawk names were submitted to Charles A. Cooke, Thawenensere, for examination and comment just prior to his death in I958. His tentative translations were sent to Dr. Floyd G. Lounsbury for checking (see Lounsbury, I96o.) 
Cooke was born on a reservation at Lake of Two Mountains, Québec, on March I2, I870. He spoke only Mohawk until he was twelve, and as a child he made many trips with relatives throughout the Albany-Montréal-Richelieu-Champlain corridor areas. $\mathrm{He}$ recognized all of the names submitted to him, and suggested many more. Lounsbury (I96o, p. 58) makes this interesting observation on Cooke's efforts: "Mr. Cooke may have been translating the European names back into Mohawk in these and in other cases where an original Mohawk name was not historically documented." This may well be-but it would have perhaps been fairer to say that either Huden did not understand Cooke clearly, or else that Cooke, bedridden and able to work only a few hours a day in his last years, did not have the energy to supply anything more than the barest requirements of the task.

\section{THE APPROACH TO THE PROBLEM}

This is not an original work. It represents a compilation of New England place names of Indian origin, assembled from maps, globes, charts, novels, poems, diaries, conversations with informants, and obviously the material in previous compilations. These latter sources are listed in the appended bibliography. It is organized to give the simplest basic information, rather than extended linguistic analyses; to have done the latter would have required another several years preparation, and it was felt better to proceed with the material presently in hand, and assure the publication of those data, which represent the concentrated efforts of the past decade.

The form used throughout this listing presents the Indian place names in boldface type, using the most-commonly-seen spelling; this is followed by any secondary term, in lightface (such as Lake, River, Pond, etc.); then the location is given by county and state for all place names included. When followed by?, this indicates that the place name has been found in early documents, but that its actual location is not known for certain today. The dialect or language is indicated in italics; if doubtful, that doubt is shown by?. Suggested translation of the name, and any comments are then given, followed by variant spellings or cross-references, the latter in boldface. Thus all actual place names are in boldface, linguistic terms are in italics, and the balance is in lightface type throughout the listing. 
Selection of the names to be included has been primarily based on the occurrence of names on historic or quadrangle maps, their appearance in a historical document, or in any generally-accepted work of literature. In several instances, informants have supplied almost-forgotten "undocumented" items. Those which are Indian translations of names invented by whites (e. g., Majihondopemabtunk, "Devil's Foot Prints," a Penobscot translation of such a white-invented term), or pseudo-Indian names (such as "Smile of the Great Spirit," "Indian Lovers' Leap," etc.), have not been included. Some terms which refer simply to Amerindian folklore, but which are not truly place names have been omitted, such as Moshop, Nodumkanwet, and Squant-references to mythological creatures, sometimes confused with locatives.

Many Indian names are preserved in four, five, or, as in the case of Winnepesaukee (see pages 6-7), as many as one hundred forms. In this work, only the major variants are included; extreme cases of linguistic diversity are not used, simply due to lack of space.

\section{ACKNOWLEDGMENTS}

Any compilation of place names depends upon the work of many people; this volume has drawn from the contributions of others in even greater degree. Indian informants have been most helpful, particularly the following Abnaki friends:

Stephen Laurent, Intervale, New Hampshire

Robert-Elie Obumsawin, Odanak (deceased)

Urbain Obumwasin, Odanak

William Simon Obumwasin, Odanak (deceased)

Theofil Panadis, Odanak

John Wadso, Odanak (deceased)

Alice Masta Wawanolett, Odanak

Oliver Wawanolett, Odanak (deceased)

The contributions of Mr. Charles Cooke have been mentioned, as have the appreciated comments of Dr. Floyd Lounsbury, Yale University.

Rev. Thomas M. Charland, O. P., Montréal, and Mr. Herbert E. Putnam, of the University of Vermont, have aided in matters relating to Canadian Affairs. The cartographic knowledge of Mr. Theodore E. Layng, of the Canadian National Archives, Ottawa, has proven of great assistance in locating map details. 
Translations of certain European languages have been patiently provided by Messrs. Paul D. Evans, Malcolm D. Daggett, Brady B. Gilleland, John H. Kent, and James E. Pooley, all of the University of Vermont; and Mrs. Grace B. Huden, of Burlington, Vermont.

The use of documentary material, together with helpful references to many others, has been facilitated by Mr. Harrison A. Brann, Bailey Library, University of Vermont; Miss Virginia A. Knox, Connecticut State Library, Hartford; and Mr. L. Felix Ranlett, Librarian, Bangor, Maine.

Much of the assistance rendered in Massachusetts area place names was unselfishly furnished by Mr. Lawrence K. Gahan, who placed his notes and other material at our disposal. In the early stages of this manuscript, Mr. E. K. Burnett, then Director of the Museum of the American Indian, offered considerable encouragement and wise counsel.

There are many others, too numerous to mention, who have been helpful in this task; but none contributed more in the direct and back-breaking labors of typing, editing and proofreading than Mrs. Nancy S. Abell, and Mrs. Grace B. Huden, both of Burlington. Without these two aides, this volume would truly have never come into being. To all of the foregoing, and to the patience and direction of Dr. Frederick J. Dockstader, I am sincerely grateful.

University of Vermont

JOHN C. HUdEN

Burlington

January, I962 


\section{INTRODUCTION}

In Dunlap and Weslager (I950, p. viii), the late Frank G. Speck commented, "Interpreting some of these Indian place names recorded by early white scribes who knew nothing about Indian tongues is like trying to juggle sand." [Italics mine- JCH]. This volume will amply demonstrate the truth of that observation. While many of the place names herein listed can be readily translated into English, some completely defy analysis, and a few cause the wouldbe interpreter to doubt whether they be truly of Indian origin.

Relatively few aboriginal names have persisted in the northeast. For instance, only two of the six New England states-Connecticut and Massachusetts-have Indian names, as compared with at least twenty-four of the fifty United States at large. This general percentage carries on through all of the toponymy of the region. Most of the names used today are English in origin, although French has left its mark, as have a few other European tongues.

In some instances, original Amerindian names have been replaced by English names suggested by the original, viz.: Mosketuauke, Rhode Island, eventually became Mosquito Hawk, an obvious rendering of the fancied sound. Others, such as Naumkeag, now Salem, and Sicaog, now Hartford, were completely lost and replaced by English names with no references to the original.

Indian names are almost invariably common appellatives. New England Algonquian place names could be, and have been, plain words which appear throughout the region; in fact, Algonquian dialects were (and a few still are) used all along the Atlantic seaboard from Nova Scotia to Virginia. These dialects are sometimes very similar to each other, much as the English use of honour as compared with the American honor-or they may differ completely, as English petrol and American gasoline. The differences are usually noun localisms; grammar and general constructions normally parallel closely, and in all cases, the wider differences indicate long periods long of separation of the two dialects, either in time or in space.

New England Indians did not develop a naming system of the type more familiar today. Rarely did they use terms referring to 
far-away places. Common terms were employed for places in their own neighborhood, as we do for sections within our own frequently-visited areas-farms, cities, towns, or counties. They renamed places and persons almost at will. Lacking writing, they were unable to consolidate these terms, thus no gazetteer developed to firmly establish a given form.

Historically speaking, many Indian place names did not linger very long. Since I600 the Connecticut River has been known variously as Counnitegou, ("Long River"), Manigana tica uoit ("Mahican's River"), Sicojocke ("Mud People"?), and Sokoquois ("South People's River"), etc.; the Mahican, Sicojocke and Sokoki are gone, their names all but forgotten-but Connecticut, the Long River, is still there. Similarly, Lake Champlain has been Petow-pargow or Peton-bowk (the "double pond," or "pond between") to the Algonquian peoples, and Caniadieri Garont ("lake with a bulge") to Iroquoian-speaking Indians. Massachuset, "place of the big hills," (the Blue Hills near Boston), and the name of the major tribe in that region, now officially indicates the whole state area.

The usual references to color, vegetation, flora and fauna, natural phenomena and the like, are to be found throughout the toponymy of New England, and in this category, little difference is to be discerned from place name practices in other parts of the United States-with perhaps only a slightly greater degree of emphasis upon water-usages (pond, lake, river, etc.).

Designations of tribes, mythological characters, and long-discarded processes or implements are to be found. Hundreds of personal names of important personages are recorded; most are at best only approximated in translation, for meanings of a majority of these are lost, while many have been suppressed by early writers as being pagan, ridiculous, or obscene. These proper nouns give great difficulty, for often they are further distorted by attempts to Anglicize not only the form of the name, but often its meaning - a process almost guaranteed to entirely obliterate any resemblance to the original! Furthermore, many Indians were known by more than one name, which only increases the confusion; Massasoit, "the great commander," was also known as Osamequin, "yellow feather." Such dual usages were quite common.

But perhaps the simplest cause for error arose from the basic inability of either side to communicate. When the colonial White asked the Indian the name of a given place, the latter often replied 
by giving his own personal name, thinking this was what was requested. And out of just such simple misunderstandings have come many of the place names of the nation.

\section{PRON UNCIATION}

Completely aside from the matter of translating Indian place names is the question of their actual sound as spoken by natives. It has already been remarked that a majority of the colonists were barely fluent in English, and thus could hardly be expected to handle such alien tongues as Abnaki, Narragansett or Pequot with ease. Moreover, the unfamiliar sounds could give completely different meanings if even slightly mispronounced. Therefore, the first emigrants into the New England region found themselves in linguistic trouble almost immediately upon landing.

The lack of written gazetteers, together with the very few dictionaries or other written records, makes the problem extremely difficult. So it is that the matter of indicating correct pronunciation amounts to a practical impossibility today. Such linguistic studies as have been compiled to date show all too clearly how difficult is this matter of accurate sound. Laurent (I884), for example, gives three pages of extremely detailed directions for pronouncing Abnaki. Even such standard references as The Columbia Lippincott Gazetteer of the World, and The Encyclopedia Americana give varying renderings of similar terms, apparently based primarily upon local usage.

Many of the more commonly-used Indian terms have come to enjoy what may be called a "standard" pronunciation, and this is usually found in the larger dictionaries. Yet it must be emphasized that at this late date there cannot be anything like a "correct" pronunciation of most of these terms. For this reason, this work avoids almost entirely the matter of 'syllabication and pronunciation. Just as in English the sound $b \bar{a} r$ in various contexts wilindicate "to bear," meaning to carry or support; "bare," indicatl ing clear, treeless, or nude; or "bear," referring to an animal, just so are there homonyms in Indian tongues which require context for reasonable interpretations.

Saugatuck has been uttered as Saw'gatuck, Sawgat'uck, Sawgatuck' and Soggertick-all referring to the same place. In the Natick Dictionary (Trumbull, Igor, p. ix), Edward Everett Hale wrote, "with good sense, Eliot used the English [alphabet] letters with the sounds which Englishmen gave them," and, "In Sherman's 
translations, Latin vowel sounds were used, so Eliot's wadchu (mountain) appeared as uijuii."

As a youth, this compiler knew some of the last Montauk and Mohegan speakers. Wyandank Pharaoh, a Montauk, used to say Zzungee! (it's cold!), but Mrs. Nonesuch, a Mohegan, would exclaim, Ssonkee! None of these old people could construct whole sentences, but they had quite a store of nouns, such as torup ("snapping turtle"), pootap ("whale"), nitop ("friend"), etc. They could, and did, use parts of the Lord's Prayer (Speck, I928, p. 25I), and a simple grace before meals; apparently these passages came to them word-of-mouth from great-grandparents who "fit in the Revolution."

We have tried to determine contexts, and the translations in this work reflect contexts wherever found. An attempt has been made to furnish generally-accepted spellings, relying primarily upon that form most commonly used in the region involved. Variants are essentially those which seem to represent secondary usage.

Unfortunately, it is impossible to establish any "guide to pronunciation" which would be consistently helpful for the terms in this volume. Not only have the forms undergone the extreme changes mentioned previously in this section, but there were as many exceptions to the rules of pronunciation in Algonquian as exist in English. One can generalize, for instance, that in Maine, place names have no silent letters; that those letters usually have but one sound, which must be learned by imitation; and that the stress varies in a given word, but normally the strongest accents fall towards the ends of words. Yet an examination of some Maine place names immediately reveals some of the hazards in trying to establish rules:

I. Monosyllables are few, but are usually pronounced as spelled. Example: Quack.

2. Names with two syllables usually stress the first:

$\begin{array}{ll}\text { Apskek } & \text { Ap'skeck } \\ \text { Cobscook } & \text { Cob'skook } \\ \text { Essick } & \text { Ess'ick } \\ \text { Saco } & \text { Say'ko (but also Saw'ko, and Sack'o) } \\ \text { Winskeag } & \text { Wins'keeg }\end{array}$

3. Polysyllabic names usually stress the second or third syllable, but this rule is not rigid: 


$\begin{array}{ll}\text { Metinic } & \text { Me-tin'ick } \\ \text { Pemetic } & \text { Pem-e'tick } \\ \text { Pemskudek } & \text { Pem-skood'eck } \\ \text { Agawam } & \text { Ag'a-wam (but also Aga-wam') } \\ \text { Aquadoctan } & \text { Ak-wa-dock'tan } \\ \text { Matinicus } & \text { Ma-tin'e-kuss } \\ \text { Madahumuck } & \text { Ma-da-humm'uck } \\ \text { Pemjeedgewock } & \text { Pem-jeej'e-wock } \\ \text { Pemtegwatook } & \text { Pem-teg-wa'took }\end{array}$

4. Names formed of many combined syllables break into several forms, and must be learned by practice:

$\begin{array}{ll}\text { Aguahassideck } & \text { A-gwa-hass'ee-deck } \\ \text { Alnambi kwaysah wayk } & \text { Al-nam'bi-kway-sa'wake } \\ \text { Talagodissek } & \text { Ta-la-go-dee'seck }\end{array}$

The Southern New England language groupings follow somewhat similar rules, even to the matter of inconsistency, viz:

I. Words of one syllable are rare, and usually may be readily pronounced as spelled, e.g., Paug.

2. Words of two or three syllables usually stress the first syllable:
Acqueet
Catumb
Mystic
Seipican
Sippican
Wekopee
$\mathrm{Ak}^{\prime}$ weet
Cat'umb
Mis'tick
See'pi-kan
Sip pi-kan
Week'o-pee

3. Polysyllabic words tend to stress the latter ends of words, although this is not rigid:

$\begin{array}{ll}\text { Connecticut } & \text { Co-nek'ti-kut } \\ \text { Wesquadomeset } & \text { Wes-kwa-dom'e-set } \\ \text { Poohookapaug } & \text { Poo-hook'a-pog } \\ \text { Mishimayagat } & \text { Mish'ee-may'a-gat } \\ \text { Nunkatusset } & \text { Nunk-a-tuss'et } \\ \text { Massanagtakwonah } & \text { Mass'a-nag-tak'wo-nah }\end{array}$

In conclusion, this quotation from Speck may be of some pertinence. Speaking of Mohegan-Pequot, he wrote, " ... no doubt the phonetic qualities of the dialect have been somewhat corrupted by a long period of contact with the English..... this dialect is phonetically uniform with other southern New England divisions except for the $\mathbf{y}$ distinction in the transposition of $\mathbf{r}, \mathbf{l}, \mathbf{n}, \mathbf{y} . \ldots$, (Speck, I928, p. 226).

To sum up: In the absence of standard reference works, about the only way to achieve a competent pronunciation for an Indian 
place name in most of New England is to divide it into syllables representing Indian root-meanings and put the stress in the middle or toward the end of the word.

\section{SPELLING}

Reference has been made to the problems involved in the "proper" transcription of Indian terms. Fanny Hardy Eckstorm makes a cogent point concerning this matter in her comments on the lack of a fixed spelling. She points out the confusions between similar words, and asks,

How did Captain John Smith pronounce his Elizabethan English rendition of Indian words ?.... And if, in going further, we today try to explain Smith's Elizabethan-English-Indian forms, or we use the Gallicized Abnaki of Father Rasles, we only compound the difficulty. Our best authority of Modern Abnaki, Father O'Brien, veered inconsistently between English and French notation; unfortunately, he did not live to revise his work. Who shall say today how his words should be transcribed? Yet upon the sound he meant may depend the grammatical root of the term in question." (Eckstorm, I94I, pp. $\mathrm{xx}$, xxi. Italics mine- JCH)

As a simple demonstration of the other side of this coin, the following list is presented, which includes fifty variant spellings, all of the same place name, as found on various official documents. And it should be noted that this is a selected list-there are over fifty more variant spellings for this same body of water! Even so, who is to say which of these many forms is "correct," and which is "wrong"? At this late date, there can be no rule other than the arbitrary decision of most-common-usage, or the dictum of a quasi-official Geographic Board. And both of these authorities can as readily decide in favor of an accepted spelling which might be totally unintelligible to the original propounders of a given place name, were they to return to the scene today.

Wenapasicke

Wenapesioche

Wenepesioca

Wenepesiocho

Wenepesiokee

Winassosawque

Winepisackey

Winepiscocheag

Winepiseoka

Winepisokey

Winipasekek

Winipisaro

Winipisiocke
Winipisioke
Winipisioky
Winipisokee
Winisipisiokie
Winnapissaacka
Winnapissiaukee
Winnapresseakit
Winnapusseakit
Winnapussykat
Winnepesaukee
Winnepeseochee

Winnepisoogee

Winnepissk

Winnepissocay

Winnepossockey

Winnipesse

Winnipiseoca

Winnipiseoce

Winnipiseokee

Winnipisocy

Winnipissacca

Winnipissiaikee

Winnipissioca 


$\begin{array}{lll}\text { Winipiseocee } & \text { Winnepesocket } & \text { Winnipissiocky } \\ \text { Winipishoky } & \text { Winnepiseoge } & \text { Winnipissoake } \\ \text { Winipisiackit } & \text { Winnepiseokee } & \text { Winniposockett } \\ \text { Winipisiakit } & \text { Winnepisioco } & \text { Winnopisseag } \\ \text { Winipisinket } & \text { Winnepisiokee } & \text { Winnopusseag }\end{array}$

To settle this vexing matter, the State of New Hampshire decreed that the "official" spelling shall be Winnepesaukee. Who can argue at this late date which is the most correct form?*

* Yet, even as this volume goes to press, a new "official state map" has just been published by New Hampshire, on which Lake Winnipesaukee appears(!). 


\section{TRANSLATING ALGONQUIAN PLACE NAMES}

Here are some very general rules by which the reader may develop an understanding of the technique followed by the compiler in arriving at a meaning of many of the place names in this volume. While this is by no means a "do-it-yourself kit" for such translating, it is hoped that these suggestions may enable a student to achieve his own analysis of many Algonquian terms.

I. Seek the original or earliest available spelling of the name. This is important, for unless you can establish a fairly early form, your translation may go seriously awry.

a. What dialect does it presumably represent?

b. If the name contains a $\mathbf{v}, \mathbf{f}$, or the ph diphthong, it is probably miscopied; these sounds are absent, but can be easily mistaken in hearing Algonquian speakers.

Thus: Maffachusetts is patently Massachusetts spelled with the old-fashioned $\int$, usually printed in more recent times as f. Another: Foxon, from Poxon.

c. Remembering that $\mathbf{b}$ sometimes changes to $\mathbf{p}$, that $\mathbf{d}$ may change to $\mathbf{t}$, and that $\mathbf{l}, \mathbf{n}, \mathbf{r}$, and $\mathbf{y}$ are interchangeable from one dialect to another, cast the place name into several forms.

Thus: Bash Bish from pashpishau, and Bantam from Peantam. Also, cognates, such as nitap and nidoba, are from Natick and Abnaki, respectively; both mean "friend."

d. Bear in mind the historical shift of $\mathbf{r}$ to $\mathbf{l}$ in Abnaki. Words spelled with $\mathbf{r}$ in Old Abnaki are now customarily found with an $\mathbf{l}$ in Modern Abnaki.

2. Using Algonquian groupings, break the name into syllables. In this, you must seek the basic root, or root-word-a fundamental sound or group of sounds which convey an idea (usually some kind of action).

De Forest (I85I, p. 4I) explains the problem as follows: "[Indian language has] the power of uniting various syllables of different words, into one new word, which should express all of the original terms from which it was compounded. Thus 
a Delaware (kindred dialect of the Mahican) girl in playing with a dog might give utterance to her pleasure by exclaiming Kuligatschis, that is, "thy pretty little paw." This word would be compounded from $K$, "thy," wulit, "pretty," witchgat, "paw," and the diminutive, schis; so that four equally distinct and perfect words would be melted into another equally perfect, which would contain only part of their sounds, but the whole of their meanings." Thus, the parts of those sounds remaining were the roots.

The root word sesek or sisikw (the root is actually $s-s-k$ ) conveys the idea of "rattling," as seeds rattling in a dry gourd; a ceremonial turtle-shell rattle; dry beans or pea pods; or a rattlesnake. Another, -be- or -pe-, is found in many words pertaining to "water," as nebe, or Winnipesaukee. The root -tp- is found in antep, or ontop, meaning "head." Also, - $t n$ - and - $d n$ - are roots for the inseparable - adn-, "mountain," as found in Monadnock.

Sometimes only the root appears in compound words, as Algonquian languages are highly agglutinated-that is, built up from other words, all telescoped into one. In Hampden, Maine, there used to be a high glacial sand bank (long since carried away for gravel), called Tolbuntbessek, "turtle head," because of its shape. The word has this analysis: tolbeh, "turtle," antep, "head," plus the locative -essek. Hence, "turtle head place," or in more grammatical English, "at the place of the (sand bar resembling a) turtle head."

3. See if the syllables are root words similar to those in other analyzed names. A listing of such roots is helpful (for examples, pages $30 \mathrm{I}-368$ of this volume).

To analyze Moskwastooxees: look up a similar term, Moskwaswagamok, the meaning of which is given herein as "muskrat lake." Thus the first part of your word, Moskwas, probably means "muskrat." Your root list gives tuck, or took, as "river, driven by waves"; you also have took-sees, as "brook, or rivulet; diminutive." The name Moskwastooxees, then, probably means, "muskrat's little river," or, more grammatically, "muskrat creek."

4. Then, check this against context: could the meaning indeed refer to the place name? Does it make sense? If your term is attached, say, to a mountain peak, but seems to translate as a waterway (highly unlikely, but it does happen), then there has either been a loss of a syllable, or else the spelling has undergone a major change in the past. There is always the chance that the name was affixed to the locale by a white settler, in error. However, do not require too tight an application of this rule-let it suffice that the name makes 
reasonable sense as an appellative. At this distance from the original source, you are working in a vast sea of uncertainty; it is easy to find yourself swamped!

In order to test the formula suggested above, here are a few examples of Algonquian terms, with their analyses:

Lake Shoonkeek Moonkeek, in Berkshire County, Mass. This was traditionally Mahican territory, but since there are no satisfactory Mahican vocabularies, the related Delaware (Brinton, I888) was used. Confirmation was found in Tooker (I9II), which includes many Delaware terms.

I. The oldest available spelling is Shonkamonk.

2. Dividing this into syllables, we get shonk a monk.

3. This is somewhat similar to Ouschankamaug, a Delaware term derived from ousacheu, "slippery," and -amaug, "fishing place." Also found is schachamek, "slippery things caught with a hook, i. e., "eel."

From this, we decide that Shonkamonk (and therefore Shoonkeek Moonkeek) means "eel fishing place."

4. This makes complete sense, since this lake (now called Pontoosuc) has indeed many eels. The -eek endings of this term are apparently humorous Anglicized additions.

Housatonic River, in Connecticut and Massachusetts:

I. The older available form is Ousatonuc.

2. Divided into syllables: ous-a-tan-uc.

3. Apparently ous derives from Delaware awossi, "beyond, the other side, over;" -atan- is the inseparable form of "mountain;" $-u c$ is a locative ending, "place of."

4. Hence, Housatonic means, "place beyond the mountain(s)." This makes complete sense, since Mohegan people said that they, as well as the river, came from "a place beyond the mountain." A variant form, House of Tunnick, is a poorly modified Anglicization of the original. (See also Aswahdenick, apparently an Abnaki cognate of Housatonic).

But a word of caution should be introduced here: it is usually unsatisfactory to try to unravel, say, Abnaki, by means of Natick root-words. Although there are many similarities shared by the two, (and other cognate tongues), generally speaking such an attempt is as risky as trying to use an Italian dictionary on French words. Your average will be about the same-you may get the general sense of the meaning, but almost never will you 
achieve any precise translation, and you may get a completely wrong interpretation.

To make the problem more difficult, the same form may give considerable confusion unless the root words as originally used by the Indian people can be located. For example:

A. Winnisquam Camp, Chittenden County, Vermont. Abnaki, "at the top of the tree."

B. Winnisquam Lake, Belknap County, New Hampshire. Abnaki, "salmon fishing around here."

Note that these are the same word, same tongue, but have totally different meanings. The first entry derives from the original wanaskiwi, meaning, "end, end-place, top place, or summit." The suffix -quam, or kwam, is peculiarly Abnaki for "tree" in composite words. Hence, wanaskiwi-quam telescope into Winnisquam, meaning "top of the tree." As a final test, Camp Winnisquam is adjacent to a huge apple orchard. In the second form, Lake Winnisquam also has been passed along as an Abnaki or Pennacook term. In this, originally winne- means "hereabouts, or the neighborhood around here," while mskuamagw refers to "red fish," or salmon. Thus, a reasonable translation of winne-mskuamagw would be, "around here, there are salmon."

In like manner, other terms are confusing until the histories as well as the etymologies are considered. Squam, in Essex County, Mass., is often confused with Squam Head, on Nantucket. Squam was originally Wanashquompskqut, from wanasq, "top, summit," plus -ompsk, "rocks," plus -ut, "thither." Taken together, these yield, "at the summit of the rock," and so it is, geographically. In course of time, this has been eroded to Squam, or Squom. But the Nantucket term derives from mi shquam manock, "red fish," or salmon. It, too, has lost syllables over the centuries, leaving only the middle form to contemporary usage.

And, in closing, another example of lost-syllable confusion is Tomheganompskut, which is due course has become Higganum. Without knowledge of the historical changes which some of these terms have undergone, any accurate translation of a term like Higganum would obviously be impossible. 


\section{A WORD ABOUT MAPS}

In preparing this work some 500 maps were consulted, chiefly topographic quadrangles of New England dating from the early I900's. These were painstakingly examined; every Indian-appearing name was noted, then checked against standard vocabularies. Following this, maps of New France, Nova Scotia, New England and adjacent territories were checked with equal care. The more important such sources are listed below.

In northern New England, early French and English map makers wrote Mohawk, Huron, Abnaki, Pennacook, Malecite and Micmac names as they heard them; since there was no written aboriginal language, spellings could not be checked. Likewise, pioneer Dutch, English and German scribes entered names as their European ears registered the two-score dialects used in Massachusetts, Rhode Island and Connecticut. It is not surprising, therefore, to note that Winnepesaukee is found in at least one hundred spellings; that Memphremagog (erstwhile Mehemsabegek, long $\int$, or s miscopied) is now spelled with a ph where none such appears on early maps; or that Montaup became Mount Hope.

Changes within languages can be traced through map entries, as Wambighe to Umbagog; Ouramon into Olamon; etc., as Old Abnaki gradually became Modern Abnaki. Maps also contribute to errors; the continuous process of copying and re-copying of various editions of maps which were published throughout the early days left a synonymy which will probably never be entirely unravelled.

Some old maps yielded many names, as Father Joseph Aubéry's fine I7 I5 chart of New France and New England. Others provided fewer names, and some none at all. The same observation applies to most charts up to about I750, by which time many Indian names had been replaced by English. For example, Naumkeag, Massachusetts, had been renamed Salem; and Sicaog, Connecticut, had become Hartford. Very few "new" Indian names were added to New England places after I750; most of these were imports or transplants due to the influence of Longfellow's Hiawatha or the return of soldiers to New England after the Civil War. 
Occasionally old Indian names were preserved on relatively recent documents, as on the Dorchester map of Lower Canada (I794). On this map alone appears Misquiquaungameck, the Abnaki name for the Clyde River.

The following is a summary of only the more important maps which contributed in a large measure to this study. In addition to these major topographical charts, extensive use was made of a tremendous variety of maps in books, pamphlets and other scattered publications.

I542? Author Unknown. Harleian Mappemonde (Plate VIII). Colored manuscript "Map of the New World." Unsigned, undated; from the original in the British Museum.

I544 JeAn Alfonse, Cosmographie (Plate VI). A manuscript map in the Bibliothèque Nationale, Paris.

I550 Pierre Desceliers, Manuscript Map of the World. (Plate XIV). Original in the British Museum.

I566 Bolognino Zaltieri, Il Desegno del Discoperto della Nova Franza. Venice.

I569 Gerard Mercator, Nova et Aucta Orbis Terrae, etc.

I597 CoRnelius Wytfliet, Nova Francia et Canada.

I6Io Author Unknown. "Simancas" or Velasco Map, sent by Luis de Velasco (junior) to Philip III of Spain in I6I I. Made for James I of England.

I6I3 Sam Uel de Champlain, Map of Canada and New France. Also i6i6.

I624 Captain John Smith, New England; maps to illustrate his Genevall Historie of Virginia.

I627? Author Unknown, Nova Belgii, Niew Niederlandt, Nova Anglica, Almoushicoisen, etc. A Dutch map similar to Visscher, I720, infra.

I650? UnKNown French ARTist, Chemin des Iroquois.

I653 SAmuel DE Champlain, Le Canada. A posthumously published map, similar to his I6I 3 work, supra.

I656 Nicolas SANson, Canada or New France.

I680? Robert Morden, New England and New York.

I688 KARAPINSKI, New France and New England.

I7I5 REv. Joseph AuBÉRy, Carte pour les hauteurs de terre, etc.

I720 CoRElis Visscher, New Map of North America.

I750? The Crown Collection of Photographs of American Maps. Published by the British Museum in $\mathbf{I} 895$.

I755? Thomas Pownall, Grand Pass from New York to Montreal.

I762 William Brassier, Survey of Lake Champlain, etc. A survey made for Sir Jeffry Amherst.

I763 Captain Carver, et al., A New Map of the Province of Canada, (etc). I790? William Hall, Part of the Province of Québec, etc.

I794 The "Dorchester MAP," Plan of Part of the Province of Lower Canada, etc. Made on order of Lord Dorchester.

I8oo- Many local and regional maps made in the Nineteenth Century I900 were examined, but few yielded much additional informat:on. 
I900- U. S. Geological Survey, Quadrangles. Several editions, covering I960 practically all of New England; especially the surveys since I9I4.

I903 Sidney S. Rider, Map of the Colony of Rhode Island; Giving the Indian Names of Locations, etc.

I905 Frank E. Hull and Fred T. Dow, Map of Moosehead Lake, etc. Made for the Kennebec Water Power Co.

I9I I CHARLES EDWARD BANKS, Martha's Vineyard as known to the Indians; Showing its Algonkian Names.

I930 Mathias SpIESS, Map of Connecticut circa I625; Indian Trails Villages, Sachemdoms.

I955- Many road maps, as furnished by oil companies, gasoline stations, 196I chambers of commerce, etc.

I959 Vermont DeVelopment Commission, Vermont Touring Map and Brochure.

i96i Connecticut State Highway Department, Connecticut Map Showing Historic Sites, State Parks, etc.

ig6i Maine State Highway Commission, Official Highway Map 6.

I96I Rhode Island Department of State, Highway Map Showing Historic Sites, etc.

i962 New Hampshire State Planning and Development Council, New Hampshive Tourist Map. 


\section{INDIAN PLACE NAMES OF NEW ENGLAND}

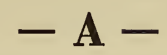

Abacotnetic Bog, Somerset County, Me. Abnaki, "stream opening out from between mountains."

Abagadusset Stream and Point, Sagadahoc County, Me. Micmac, "little parallel river."

Abagamok West Lake, Hancock County, Me. Micmac, "lake parallel to" Nicatous Lake.

Abahguit the sound between Lubec, Washington County, Me., and Campobello Island, New Brunswick. At one time the island was the summer home of Franklin D. Roosevelt. Malecite, "something parallel to the main land."

Abahos Stream, Penobscot County, Me. Micmac, "runs parallel to" [another river].

Abalajackomegus Stream, Piscataquis County, Me. Abnaki, "bare, no trees."

Abaquage Pond, Windham County, Conn. Nipmuck, "place where rushes grow."

Abaquakea Essex County, Mass. Natick, "land of flags or rushes."

Abbouset River, Suffolk County, Mass. Natick, "place of going up stream" (?)

Abenaki Lake and Mountain, Coös County, N. H. Abnaki, "dawn land" or "easterners." Other spellings include Wabanakee, Waubanakie, Wapanakkee, Abnaki, etc.

Aberjona River, Norfolk County, Mass. Natick, "junction or confluence."

Abessah Bar Harbor, Hancock County, Me. Malecite, "clam bake place."

Abnaki Indians, one of the major northeastern Algonquian tribes. Their name, more properly Wabanaki, means "dawn people," or "easterners." They lived in western Maine, Carroll County, N. H., and north into New Brunswick. Today their des- 
cendants mostly live at Odanak (St. Francis), Québec. Also spelled Abenaki, Abenaqui, Waubanakee, etc.

Abocadneticook now Baker River, Grafton County, N. H. Abnaki, "the place of the stream opening out of the mountains." Some translate this "stream narrowed by mountains;" the interpretation seems to depend on which way one is looking.

Abocadneticook the West Branch of Penobscot River, Piscataquis County, Me. Abnaki, see above.

Abocadneticook Pond, Somerset County, Me. Abnaki, see above.

Abojedgewak a stream connecting North and South Twin Lakes, Piscataquis County, Me. Abnaki, "two currents flow, one on each side" [of an island].

Abol Deadwater Pond and Trail, Piscataquis County, Me. Abnaki, "bare, devoid of trees."

Aboljackarmegas Stream, Piscataquis County, Me. Abnaki, "[banks of] swift stream, smooth, bare of trees."

Abonegog the Black River, Lincoln County, Me. Abnaki, "opening wide" as it gets nearer the ocean.

Abonesig River, Lincoln County, Me. Modern Abnaki, "small resting place"(?) or "small baking place."(?) Translation depends upon whether the original was derived from abon, "oven," or "bread," or from abi, "sit down; rest."

Abraquidasset Point, Washington County, Me. See Abagadusset. Abrigada Hill, New Haven County, Conn. Quinnipiac, "hidingplace or shelter." Variant spelling, Abrigador.

Absalona Hill, Providence County, R. I. Probably not Indian, but perhaps named for an Indian named Absalom.(?)

Absalonomiscut Providence County, R. I. Narragansett, "place of the fish trap" or "where fish are caught in a weir."

Acadia Village, Aroostook County, Me. Micmac, "the earth," "the place," or "the land."

Acapasket Barnstable County, Mass. Wampanoag, "at the small cove," or "at the separated branch."(?)

Accomack Plymouth County, Mass. Wampanoag, "on the other side."

Accomonticus Suffolk County, Mass. Natick, "beyond the little river." See Agamenticus. 
Acemy Nantucket County, Mass. Wampanoag, "dry land," [i.e., "ashore"].

Achagomiconset Washington County, R. I. Narragansett, "where there are green meadows," as for pasture. Also Ashagomiconset.

Achagomiconset Meadow, New London County, Conn. Eastern Niantic, "place of green pastures or green meadows."

Achetaqupag see Maruscopag.

Achetonsick see Assapumsic.

Acoaxet Bristol County, Mass. Narrangansett, "at the fishing promontory," or "at the place of pines"(?); or "place of small fields." (?)

Acocisco see Auco Cisco.

Acokesit River. See Acoaxet.

Acomekes or Acomeques New London County, Conn. Mohegan, "land across," or "place on other side." See Accomack.

Acomes Falls, Androscoggin County, Me. Abnaki, "high."

Acontaug Brook. See Aguntaug and Aquntaug.

Acoomemeck Worcester County, Mass. Nipmuck, "at the place across"(?). An ancient village site.

Acoont River, Plymouth County, Mass. Wampanoag, "place across" or "place on the other side."

Acquasimink Fairfield County, Conn. Mahican, "abode of the woodchuck."

Acquebapaug Pond, New London County, Conn. Eastern Niantic, "before the pond" or "alongside the pond." Also Acquibapaug.

Acquebatuck Hill, Windham County, Conn. Nipmuck, "at the end of the river."

Acquedneseth Tract, R. I. See Acquidnesit.

Acqueedenuck or Acquidaneck Windham County, Conn. Mohegan? Nipmuck? "place beyond the hill."

Acqueednuck River, a branch of the Pawtuxet. Washington County, R. I. Nipmuck? Mohegan? "place beyond the hill."

Acqueet River, Plymouth County, Mass. Wampanoag, "boundary or ending place." 
Acquehadongonock Point, Knox County, Me. Abnaki, "place where canoes are slid out of, or into, water." Probably a canoe-harbor at a fishing place.

Acquessuc Franklin County, Me. Abnaki, "place of the slender trout," the oquassa, (Salvelinus oquassa).

Acquettink see Aquidneck.

Acquidnesit Tract, Washington County, R. I. Narrangansett, "place beyond the hill."

Acquiunk Falls and Hill, Windham County, Conn. Nipmuck, "under a tree."

Acqunkoke or Acquunkquoke Windham County, Conn. Nipmuck, "elevated place," or "high land," gunnunkque ohke.

Acrupsak the Hobart Stream, Washington County, Me. Micmac, "rough, rocky bottom."

Acunepequash Brook, Windham County, Conn. Nipmuck, "high level ground."

Acushena Bristol County, Mass. Wampanoag? Nauset? "at the cove," or "swimming place"? or "wet place"? or "fish weir"?

Acushnet City and River, Bristol County, Mass. Narragansett, "at the cove," or "wet place."

Adden Mountain, Essex County, Vt. If Indian, possibly from Abnaki, "a mountain." See Misadene. [But the -adn- root is normally not found isolated; perhaps this is an English name].

Adogenadesk Wolve's Rocks, Washington County, Me. Malecite, "place lashed by storms."

Adonniate Thompson's Point, Chittenden County, Vt. Mohawk, "it is a point."

Adowaukeag Hancock Point, Hancock County, Me. Malecite, "near the knoll where the current runs out."

Adowaukeskeag Falls Point, Hancock County, Me. Malecite, "at the ridge where the strong tide goes out."

Agamenticus Lake, Mountain, and River, York County, Me. Abnaki, "a little river which lies behind an island at its mouth," or perhaps "other side of the little river." Variant, Aggamantukoos. See Sasanow.

Agamuntaëg Pond, Franklin County, Me. Abnaki, "place on the other side of the river." See Agamenticus. 
Agawam Essex County, Mass. Pennacook, "low land," or, "land overflowed by water;" also, "place to unload canoes."

Agawam Hampden County, Mass. (two such places); also in Plymouth County, Mass. Nipmuck, "low land," or "overflowed by water;" also "place to unload canoes."

Agenyateonnia Colchester Point, Chittenden County, Vt. Mohawk, "point at the end."

Aggamoggin Strait, Maine. See Eggemoggin.

Aghenibekki River. See Kennebec.

Agicomook Stony Creek, New Haven County, Conn. Quinnipiac, "sheltered haven." Found also as Aigiocomock, Ajicomick, etc.

Agiochook is Mount Washington, Coös County, N. H. Abnaki, "at the place of the concealed one." Variant, Agiocochook.

Agiscohos see Aziscoös.

Agoncy a mythical (?) Indian place near the mouth of Penobscot River, Waldo County, Me. Huron, "an island." Anciently Aguncia, q. v.

Aguadocta just west of Saco, York County, Me., on I6go map. Abnaki, "at the end of the portage, where we take canoes out of the water."

Aguahadongonock several localities along the Maine Coast in York and Cumberland Counties. Abnaki, "the place where I draw (canoe) out of the water," as at the end of a portage.

Aguahassideck Fort Point, Waldo County, Me. Abnaki, "landing place for canoes." This term was also applied to places where otters slid into the water; "otter-slides."

Aguahega Damariscove Island, Waldo County, Me. Abnaki, "a landing place" for canoes.

Aguncia Waldo County, Me. Probably Huron, "an island." See Agoncy.

Aguntaug Brook, Washington County, R. I. Narragansett, "under a tree," or "big tree place."

Aguspemokick Island, Gould's Island, Newport County, R. I. Narragansett, "short narrow straits." See Aquopimokuk.

Agutteback Pond, Worcester County, Mass. Natick, "kettle pond." This was originally aucuck paug or ohkeek pog; it is near a soapstone (steatite) deposit where Indians made kettles, 
dishes, ladles, etc. Another source translates this as "herb pond," from ohkeit pang.

Ahampatunshauge Pond, Worcester County, Mass. Nipmuck, "beyond the round pond (where rushes grow?)" or "place of small pointed stakes," (some sort of garden fence?)

Ahapaconsett Nantucket County, Mass. Wampanoag, "flags or rushes" to make mats for covering.

Ahbasak and Ahbaysauk Bar Harbor, Hancock County, Me. Abnaki, "where we bake and dry soft shell clams" (steamer clams, Mya arenaria).

Ahionwatha variant of Hiawatha, q. v.

Ahmelagognetercook Cumberland County, Me. Abnaki, "at the place of drying and curing" [fish and game].

Ahquahunwansuh Plymouth County, Mass. Wampanoag, "here the flat land ends," or "the plain ends here."

Ahquanahaganoc Knox County, Me. Abnaki, "a sweat bath." See Pissepunk.

Ahquannis Sowamsoo River, Plymouth County, Mass. Wampanoag, "boundary of Sowam's country."

Ahqueedaukee Belknap County, N. H. Pennacook, "drag-up place [i. e., landing-place] for canoes." Also Ahquedaukenash.

Ahsedakwasic Turner Brook, Aroostook County, Me. Abnaki, "stick pointing to a branch of the stream," a type of directional sign for canoe travelers.

Ahumpatunshaug Worcester County, Mass. Nipmuck, "beyond the round pond," or "little pointed stakes place."

Ahwassoos Aden Orange County, Vt. Abnaki, literally "bear mountain."

Ahyosupsuck Pond, New London County, Conn. Mohegan, "place of wild hemp."

Aigio commuck Stony Creek, New Haven County, Conn. Mohegan, "at the enclosed place," or "sheltered harbor."

Akoaxet See Acoaxet.

Akusenag Bristol County, Mass. Wampanoag, "at the cove." (now Dartmouth).

Akweekek a hill near Eddington, Penobscot County, Me. Abnaki, "at the steep place." 
Alamascook See Alamoosak, Allamoosook.

Alamoosak Lake, Hancock County, Me. Garbled Malecite, "at the fish spawning place."

Alexander see Wamsutta.

Algonkine Point, Grand Isle County, Vt. See below.

Algonquin Mountain, Washington County, Vt. If Micmac, "at the place of spearing fish [as at the bow of a canoe]." If Algonquin, "people of the other shore."

Algonquin State Forest, Litchfield County, Conn. See above.

Alipconk Village, Fairfield County, Conn. Paugussett, [a Wappinger sub-group] "over-flowed place."

Alkarmus Plymouth County, Mass. Wampanoag, "insect which causes oak-galls."

Allagash Lake, falls, and stream, Piscataquis County, Me. Abnaki, "bark cabin," or "birch-bark shelter."

Allagash Falls, lake, river and village, Aroostook County, Me. See above.

Allagask wigamook Churchill Lake and Mountain, Piscataquis County, Me. Abnaki, "at the bark cabin lake."

Allahtwkikamoksis Pond, Aroostook County, Me. Abnaki, "at the little field where much game has been killed."

Allamoosook Pond, Hancock County, Me. Abnaki, "place of the small dog." See Alum.

Almouchicoisen This name, which appears on several old maps, refers to the Indians of northeastern Massachusetts, southeastern New Hampshire and adjacent Maine. Abnaki, "little dog people."

Alnambi-i-menahan Old Town, Penobscot County, Me. Abnaki, "our own [Indian] people's island." cf. Laurent (I884): alnoba, "Indian;" pôgui alnobak, "full-blooded Indians."

Alnambi kwaysah wayk Indian Point, Hancock County, Me. Micmac? "Indian point," or "our own people's point."

Alnobaganock Penobscot County, Me. Abnaki, "place of our [Indian] ceremonial dance." Refers to the Alnobagan ceremonial dance performed at weddings. There were probably many such "dancing places."

Alskachewack the Black River, Windsor County, Vt. Abnaki, "at the great pile of shells." But see Kaskactchawak. 
Alum Lake, Providence County, R. I. Nipmuck, "a dog." Apparently the Pequot people called this same pond A'wumps, q. v. Also Allum, Wallum, etc.

Alum Pond. Several ponds in Worcester County, Mass. See above.

Amaganset Suffolk County, Mass. Natick, "at the well," or perhaps "at the (council) place where we smoke." Smoking was commonly referred to as "drinking weak tobacco." Sometimes translated as "at the fishing place," or "at the point."

Amalingan the regions occupied by the Mahican in western New England, Maine, and in eastern New York. This name is apparently a corruption of Mahican, q. v.

Amanuseag River, same as Ammonoosuc.

Amaseconti Several places of this name in Maine. Abnaki, "plenty of fish."

Amataconet Providence County, R. I. Narragansett, "observation place,"'[?] or "meeting place."'[?]

Ambajack mockamas Falls, Piscataquis County, Me. Abnaki, "slantwise of the regular route."

Ambajejus Lake, Piscataquis County, Me. Abnaki, "two currents, one on either side [of an island]" See Abojedgewak.

Ambajemackomas Pond, Piscataquis County, Me. Abnaki, "a little pond crosswise" of the usual route.

Ameriscoggin Several places of this name in Maine. Probably Old Abnaki, "catching and curing fish." See Androscoggin.

Amessagunticook Farmington Falls, Franklin County, Me. Abnaki, "at the place abounding in alewives," or "plenty of migratory fish here."

Amessecantee now Farmington Falls, Franklin County, Me. Mahican, "plenty of fish." It is believed that Amessecantee was an Indian village established by Mahican refugees from New York and New England around I697.

Amgopilasig Ponds, Penobscot County, Me. Micmac, "little lakes tied together by brooks."

Amiciskeag Kittery Point, York County, Me. Abnaki, "fishing at the point."

Amilcungatiquoke Cumberland County, Me. Abnaki, "at the river where fish are caught and cured." 
Amilesk Big Lake, Washington County, Me. Abnaki, "where provisions are cured and stored."

Amilkan Lisbon Falls, Androscoggin County, Me. Abnaki, "a fish drying place."

Amiskeag same as Amoskeag.

Amitgon pontook Lewiston Falls, Androscoggin County, Me. Abnaki, "at the river-falls where fish are cured."

Ammacongan Falls, St. George River, Waldo County, Me. Abnaki, "fish drying and curing" station. Variant: Ammon-congin.

Ammadamast the Cold Stream, Penobscot County, Me. Abnaki, "fish, or alewives, here."

Ammanatocksuck Hill, Hartford County, Conn. Wangunk (a Wappinger sub-division), "lookout place on a hill near a brook outlet." See Manatuck.

Ammecungan Falls, Presumpscot River, Cumberland County, Me. Abnaki, "where [migratory] fish are caught and cured."

Ammeriscoggin River; same as Androscoggin.

Ammesokantis Lincoln County, Me. Abnaki, "abundance of alewives."

Ammocangen Middlesex County, Mass. Natick, "a fish-curing place."

Ammonoosuc River (Lower), Grafton County, N. H. Abnaki, "small narrow fishing place." Sometimes spelled Omanosek.

Ammonoosuc River (Upper), Coös County, N. H. Abnaki, "small narrow fishing place." Another spelling, Ammoosuc.

Amobscot Muscongus Island, Lincoln County, Me. Abnaki, "fishing near shore ledges."

Amonontuck Hill, Hartford County, Conn. Wangunk, "lookout place"'[?] See Manatuck.

Amoscommun see Androscoggin.

Amoskeag old name for Manchester, Hillsboro County, N. H. Pennacook, "a fishing place." Also spelled Amokeag.

Anackatuseck River. Location unknown, Kent County, R. I.(?) Narragansett, "place where brooks join."? Also Anackatusicke.

Ananepeheren Brook, Fairfield County, Conn.? Mahican, "fish traps." 
Anaquacutt Pond and Stream, Newport County, R. I. Narragansett, "at the end of the river."

Anasagunticook Lake, Oxford County, Me. Abnaki, "at the river with the sandy bottom."

Anchamaunnack kaunack Pond, New London County, Conn. Mohegan, "at the oak (acorn-producing) grove"[?]

Andiarocte the southern end of Lake Champlain, Addison and Rutland Counties, Vt. Mohawk, "where the lake narrows" or "tail of the lake;" also, "dead end of waterway, where lake tapers off to an end."

Androscoggin County, Island, Lake and River, Me. Abnaki, "the place where fish are cured," by drying and smoking.

Androscoggin River, Coös County, N. H. Abnaki, "fish-curing place." Variant, Ameriscoggin.

Aneksassisscuk Island, Penobscot County, Me. Abnaki, "ant's nesting place."

Anequasset Plymouth County, Mass. Wampanoag, "at the abode of the striped ground squirrel."

Angaakwatchwak Coös County, N. H. Abnaki, "place of the White Mountains."

Angualsicook Mahican, "place of barter." See Annisquassicoke.

Aniadarawonte the narrows on Lake Champlain near Ticonderoga, N. Y., and Addison County, Vt. Moharek, "a lake attached to another."

Anitaash Pond, New London County, Conn. Eastern Niantic or Narragansett, "rotten corn." These Indians would bury ears of corn in muck until they decomposed, then eat them.

Ankokemany Nantucket County, Mass. Wampanoag, "path to the end place."

Ankokemaug Dukes County, Mass. Wampanoag, "fishing place at the end."

Anmessok York County, Me. Abnaki, "fish here."

Anmoughcawgen Norfolk County, Mass. Natick, "place for curing fish."

Annabessacook Lake, Kennebec County, Me. Abnaki, "at that particular lake," or "lake where small fish are caught"'[?]. Also given as "at the beautiful body of still water." 
Annahooksett Falls, Merrimack County, N. H. Pennacook, "at the place of beautiful trees."

Annaquassacook Bennington County, Vt. ? Mahican, "at the end of the brook (or marshy place)" or "as far as the brook (or marshy place) extends."

Annaquatucket River and Road, Washington County, R. I. Eastern Niantic, "at the end of the river," or "at the end of the tidal current." Possibly also "overflowing river."

Annasnappet Brook, Plymouth County, Mass. Wampanoag, "at the source [or head] of the stream." Also given as "place of shells," and "gravelly brook"[?].

Annawamscutt Creek, Washington County, R. I. Narragansett, "rock summit" [?], "end of the rocks"[?], or possibly "ruler's hill"'[?]. Also given as Anowanscut, "commander's rock."

Annawomscut Bristol County, Mass. Narragansett, "at the shell rock," probably a shell heap midden. But see Annawon's Rock, below.

Annawoncoate Plymouth County, Mass. Wampanoag, "ruler's house."

Annawon's Rock, Bristol County, Mass. Wampanoag, one of King Philip's most trusted lieutenants, Annawon, was captured in 1675 near this rock in Squannahonk swamp.

Annisnippi Plymouth County, Mass. Wampanoag, "rocks in water." See Annasnappet.

Annisquam Village and Harbor, Essex County, Mass. Natick, "at the rock summit," or "end of the rock."

Annisquam an Abnaki word for "summit" is applied to several White Mountain peaks in northern New Hampshire.

Annisquassicoke now West Arlington, Rutland County, Vt. Mahican, "at the end of the boggy place." Also Angualsicook.

\section{Annocotuckett or Annogotucket R. I. See Annaquatucket.}

Annursnack Hill, Middlesex County, Mass. Nipmuck, "lookout place," or "summit."

Annusautonset River Plymouth County, Mass. Wampanoag, "garden hilled up with hoes," or "hoed garden place."

Annuskumikak Worcester County, Mass. Nipmuck, "broken up land," or "land hoed, ready for planting." 
Anqepenick Worcester County, Mass. Nipmuck, "end of the sloping land."

Anqueet Windham County, Conn. Narragansett?"chipmunk place." Anquepogskit New London County, Conn. Mohegan, "at the end of the small pond."

Anshanduck see Antashantuck.

Antaghantic Neck, Providence County, R. I. Narragansett, "turning backwards river" [oxbows].

Antashantuck Neck and Pond, Providence, R. I. Eastern Niantic, "well forested place." See Meshanticut.

Antassawamock Bristol County, Mass. Narragansett, from an expression meaning "other side of Sowam's land,"[?], or "place of shell heaps." Also given as "backwards-alwaysthings" i. e., lobsters.

Aokeets Pond, Fairfield County, Conn. Siwanoy, "hornet place"? or possibly, "adversary's place"[?].

Apananawapeske River, Hancock County, Me. Abnaki, "place where [the river] opens out [or widens] at the ledges."

Apautuck Rhode Island. A modification of Pawtucket, q. v.

Apehunqunset River, Washington County, R. I. Possibly a corruption of Aponaganset, Apponaug, or Ponaganset? Narragansett? "flags, or rushes." Other roots suggest, "place of traps among the stones," and "waiting place at the ledge."

Apistama Cumberland County, Me. According to DouglasLithgow, the name of the Atlantic seaboard from Casco Bay eastward. Abnaki, "rocky land."

Apmoojene-gamook Chamberlain Lake, Piscataquis County, Me. Abnaki, "lake crosswise" [of the usually-traveled route]. Variants: Appmoojene-quamook, Apmoojenneh-gamook, etc.

Aponahock Brook Kent County, R. I. Narragansett, "oyster place," or "where he roasts oysters;" and possibly, "the waiting place." See Apponaug, and Abonesig.

Aponake or Aponihauk see Apponaug.

Aponeg part of the Sheepscot River, Lincoln County, Me. Abnaki, "where it widens out."

Appalachia Station, Coös County, N. H. See Appalachian Gap.

Appalachian Gap in the Green Mountains, Chittenden County, Vt., derives its name either from a Choctaw expression 
meaning "a helper," or Muskhogean words describing "people on the other side."

Appalachian Trail. See above. This pathway traverses the Appalachian system from Katahdin, Maine to Mount Ogelthorpe in Georgia, a distance of more than 2,000 miles.

Appalachie Pond, Lincoln County, Me. See Appalachian Gap. However, if this name is derived from Old Abnaki, the term might mean "a bare place, devoid of trees," or "treeless land," from abol, "open, smooth, bare," plus auke, "country, land, or place."

Appamapog Fairfield County, Conn. Mahican, "pond where rushes [for mats] grow." Also a brook in Berkshire County, Mass.

Appamatox Cheshire County, N. H. Powhatan (Virginia)?, "a resting place," or "resting under a tree." It may have originated from Abnaki or Pennacook, api-metek, "a bower"[?].

Appaquag River, Windham County, Conn. Windham County, Conn. Nipmuck, "where flags (for making mats) grow." Also found as Appaquaog; and see Abaquage.

Appaum Plymouth County, Mass. Wampanoag, "a trap," or possibly "a waiting place." Also as Appoam, Umpame, etc.

Apponagansett Bay, Bristol County, Mass. Narragansett, "beyond the fishing place." Also given as "oyster bay;" "waiting place" and "jutting reefs."

Apponaug Brook and Village, Kent County, R. I. Narragansett, "where he roasts oysters," (or other shellfish). Also given as "waiting place." Other spellings Aponake, Aponihoak, Aponahock, etc.

Apponecett Plymouth County, Mass. Wampanoag, "waiting place."

Apponequet Lake, Plymouth County, Mass. Wampanoag, "sitting or waiting place."

Appowick part of Damariscotta River, Lincoln County, Me. Abnaki, "widening-out place."

Apskikek on Mopang Lake, Washington County, Me. Abnaki, "at the rock place" or "near the boulders."

Aptuxet Plymouth County, Mass. Wampanoag, "fish weir in the tidal creek," or "waiting place at the small river." 
Apwonnah Fairfield County, Conn. Mahican, "oyster" or "he roasts;" probably where shellfish were baked or roasted.

Aquadocta ancient Abnaki village in York County, Me. Abnaki, "canoe landing place." See Aquadoctan and Aquidnet.

Aquadoctan now The Weirs, Belknap County, N. H. Abnaki, "a landing place" for canoes. Other variants: Aquedoctan, Aquedahtan, Aquedaukenash. See also Aquidny.

Aquahattan where the Merrimack River issues from Lake Winnepesaukee, Belknap County, N. H. Abnaki, "I drag my canoe out."

Aquanahagonog see Ahquanahaganoc.

Aquantaug Brook, Washington County, R. I. Narragansett, "under the trees," or "big trees."

Aquapaukset Plymouth County, Mass. Wampanoag, "end of the small pond, or swamp."

Aquapauksit New London County, Conn. Eastern Niantic, "at the end of the small pond."

Aquaquamset River, Kennebec County, Me. Abnaki, [modified ?] "canoe landing," or perhaps modified Niantic, "rock summit." Apparently this is misspelled; some authorities trace it to M'squamicut, "salmon fishing place."

Aquaquesset Bogs, Plymouth County, Mass. Wampanoag, "end of rocks place."

Aquassik Berkshire County, Mass. Mahican, "large boulder."

Aquebapaug Washington County, R. I. Eastern Niantic, "at the head of the pond." Also Aquabapaug, Acquabapoge, etc.

Aquedaukenash see Aquadoctan.

Aquedenesick Newport County, R. I. Narragansett, "at the small island."

Aquednesset now Dutch Island, Washington County, R. I. Narragansett, "place of the small island."

Aquednessick Newport County, R. I. Narragansett, "at the small island." (Rhode Island).

Aquednet, Aquidnic, Aquidy, Aquiday, R. I. Narragansett, "at the island."

Aquednet Barnstable County, Mass. Wampanoag, "at the island." Aqueedenuck Washington County, R. I. Nipmuck, "at the island," or possibly, "place beyond the hill." 
Aqueednuck Kent County, R. I. Nipmuck[?] or Mohegan[?], "place beyond the hill." See Quidnic, R. I., and Acqueedenuck, R. I.

Aquehedongonock same places as Aguahadongonock, q. v.

Aquehonga Litchfield County, Conn. Mahican, "high place," or "high bluff."

Aquene-ut Dukes County, Mass. Wampanoag, derived from Chief Aquinuh[?]; or perhaps "place of peace." [An Indian named Aquetoquash witnessed Metacom's submission to the English crown in I662.]

Aquibapaug Pond, Windham County, Conn. Nipmuck, "before or alongside of the pond."

Aquidneck Newport County, R. I. Narragansett, "at the island." This translation was supplied by Indians at Rhode Island in 1637 .

Aquidness Nantucket County, Mass. Wampanoag, "at the small island."

Aquidnet Point, Nantucket County, Mass. Wampanoag, "at the island." Also given as Quidnet.

Aquidnic Rhode Island, Narragansett, "the island."

Aquidny Newport County, R. I. Narragansett, "appears as floating, like a ship at anchor, not under way," or "an island." Aquidny might be "a knoll rising out of a flat plain."

Aquidy variant of Aquidnic, q. v.

Aquitamosit or Aquitawosit Washington County, R. I. Narragansett, "at the end of the meadow or plain."

Aquittukasset Pond, Plymouth County, Mass. Wampanoag, "landing place on little stream," or "islands place"[?]

Aqunoonogqutut Nantucket County, Mass. Wampanoag, "at the hollow where a stone stands."

Aquntaug Brook; see Aguntaug

Aquonset Point, Plymouth County, Mass. Wampanoag, "place of fortification."

Aquopimokuk Island, Newport County, R. I. Narragansett, "at the short narrow straits which separate the island from the mainland."

Arambec see Norumbega

Aranbega same as Norumbega, q. v. 
Aransoak The Kennebec River from its source to Norridgewock, Somerset County, Me. Abnaki, "place of rapids."

Araugacutack New Haven County, Conn. Quinnipiac, "place of the furthest cultivated meadow."

Arauscatuck location unknown. Perhaps in New Haven County, Conn. If so, probably Quinnipiac, "furthest river."'[?]

Aresiket River. See Harraseeket

Armonek River; Byram River, between Connecticut and New York. Siwanoy [?], "fishing place," or Delaware [?], "beaver."

Aroosabaug Mirror Lake, Carroll County, N. H. Abnaki, "bright pond."

Aroostook County and River, Me. Micmac, "beautiful, or shining river." See Wallastook.

Arramopskis Falls, on Medomak River, Lincoln County, Me. Abnaki, "falls over small rocks."

Arrockaumecook Aroostook County, Me. Abnaki, "place of working (curing or drying) fish and meat," or "place of working (hoeing?) enclosed land," possibly cornfields. See Rockomeko.

Arrockaumecook Oxford County, Me. See above.

Arrosa-eg see Arrowsic, below.

Arrowamett River, Middlesex County, Conn. Wangunk? Probably from the name of an Indian, Aramet or Arramamett, who deeded land in 1672.

Arrowsic Island and village, Sagadahoc County, Me. Abnaki, "place of the obstruction." This island blocks the channel of the lower Kennebec River. Variant Arrosa-eg.

Arumsunkhungan Island, Penobscot County, Me. Abnaki, "fishing place below the outlet."

Asabeth River, Middlesex County, Mass. Natick, "wild flax place." Also translated as "miry place." See Sebethe.

Asamuck Greenwich Creek, Fairfield County, Conn. Siwanoy, "wild flax"[?].

Asapumsic, Asapumsic, etc. See Assapumsic.

Asawacomuck New Haven County, Conn. Quinnipiac, "plantation or enclosure between" [two streams?]

Ascocompamacke Bristol County, Mass. Wampanoag, "small place closed in by boulders." 
Ascomacut Washington County, R. I. Possibly Eastern Niantic, "trees at this place," but see Misquamicut. Other forms, Ascomicut, Askomackock.

Ascoochames Plymouth County, Mass. Wampanoag "snake-like fish" (eels? pipefish?). Another source translates this as "end of dwarf pines place."

Ascutney Mountain, Windsor County, Vt. Abnaki, "at the end of the river fork."

A shagomisconset see Achagomisconset.

Ashanat see Ashowat.

Ashanteaug Rocks, Washington County, R. I. Narragansett, "lobsters."

Ashappaquonsett Dukes County, Mass. Wampanoag, "where nets are spread."

Ashawaug River, Washington County, R. I. Eastern Niantic, "land in the middle." Spelled also Ashawake, Ashawogue, Ashawog, Ashawa, etc.

Ashaway River and Village, Washington County, R. I. Eastern Niantic, "land between" [river branches].

Ashawog River, New London County, Conn. Eastern Niantic, "place between," or "land in the middle."

Ashcannunsuck or Ashkannunckset Hampden County, Mass. Nipmuck "at the place of narrowing"? or "at the end of the rocks"?

Ashimuet Barnstable County, Mass. Wampanoag, "at the spring."

Ashowat a locality between Amston and Federal, Tolland County, Conn. Mohegan, "place between."

Ashowugh Commock Mystic Harbor, New London County, Conn. Mohegan, "fishing place in harbor, halfway between" large island and mainland.

Ashpaquonsett Dukes County, Mass. Wampanoag, "place where nets are spread" to mend and dry. Variant, Ashappaquonsett.

Ashpatuck River, Litchfield County, Conn. Paugussett, "high place." See Aspanansuck, Aspetuck, Aspinet and Aspotucket.

Ashquoash Worcester County, Mass. Nipmuck, "green garden stuff," such as melons, squash, etc. One authority gives this as part of a word meaning "at the end of" [something].

Ashuelot Bristol County, Mass. Narragansett, "land between place." 
Ashuelot Berkshire County, Mass. Natick, "land between place." Ashuelot Mountain, Pond, River, and Village, Cheshire County, N. H. Pennacook, "to the mountain"? or "place between"? Also Ashwillet, Jossawilok.

Ashumet Pond, Barnstable County, Mass. Wampanoag, "at the spring."

Ashunaiunk River, Washington County, R. I. Narragansett, "rocky point," "stony point," or perhaps "stony stream."

Ashwawott same as Ashowat.

Ashwillet River, Cheshire County, N. H. Pennacook, "place between"? (See Nashua) or, "to the mountain," same as Ashuelot? Also a brook in New London County, Conn.

Askachewack Windsor County, Vt. Abnaki, "near the grassy mountain."

Askaskwigek Wadjo Mount Anthony, Bennington County, Vt. Abnaki, "green grass covered mountain."

Askenonton Grand Isle County, Vt. Mohawk, "a deer." Also see Ohskenonton.

Askomackock see Ascomacut.

Askoonkton Location unknown? Possibly Norfolk County, Mass. Natick, (?) "live upright tree," or "green tree."

Askutegnik the Sugar River, Sullivan County, N. H. Abnaki, "at the end of the river fork." See Ascutney.

Asnacancomic Pond, Worcester County, Mass. Nipmuck, "at the long stone house." Another spelling, Asnacomet.

Asnebumsket Hill, Worcester County, Mass. Nipmuck, "rocks upon rocks," or "boulder cliff."

Asneconick Pond, Worcester County, Mass. Nipmuck, "at the field enclosed by stones." [or "stone house" ?]

Asnela Island. Orson Island, Penobscot County, Me. Abnaki, an Indian rendition of the English "Orson Island" (Asen elan), in the Penobscot River. Orson was an Abnaki chief there before I793.

Asnemscusset Pond, Plymouth County, Mass. Wampanoag, "at the swift flowing rocky brook."

Asnuntuck River, Hampden County, Mass. Pocumtuck, "rocky river." 
Asnuntuck Brook, Hartford County, Conn. Agawam, "stony stream."

Aspanansuck Location uncertain. Washington, County, R. I. Narragansett, "high place," or "brook near the high hill"'[?].

Aspetock River. See Ashpatuck. Variants: Aspetuc, Aspetuck, etc.

Aspetuck Reservoir, Fairfield County, Conn. Paugussett, "at the high place."

Aspetuck River and Hill, Litchfield County, Conn. Probably the same derivation as Aspetuck Reservoir, above; however, Tooker gives "fish net place" for this location.

Aspinet Road, Barnstable County, Mass. Wampanoag, "at the net place."

Aspinook Pond, New London County, Conn. Eastern Niantic, "at the high place."

Aspotucket Providence County, R. I. Narragansett, "at the fishnet cove." But one authority gives, "at the high place." Still another says Aspotucket is a corruption of Pawtucket, "at the falls."

Aspowunk Hampden County, Mass. Nipmuck "place where weirs are made," "summit," or "lookout place"[?].

Asproom an elevation near Ridgefield, Fairfield County, Conn. Mahican, "high, lofty, elevated."

Asquam Lake, Mountain, and River, Carroll and Grafton Counties, N. H. Abnaki. "salmon place."

Asquam Chumakee the Baker River, Grafton County, N. H. Abnaki, "salmon spawning place." Variants, Asquam Chumauke, Asquam Chommeock, etc.

Asqueanunckton Brook, Merrimack County, N. H. Abnaki, "rapid stream which extends as far as mountain."

Asqueebapgamuck Washington County, R. I. Narragansett, "at the end of the double pond."

Asquoach or Asquach Hill, Worcester County, Mass. Probably Natick, "at end of hill," but possibly Nipmuck, "some squashes." Another source gives "boulder hill place," Apsquach.

Assabasset Worcester County, Mass. Nipmuck, "at the place between small brooks." Also, "wild flax place"?

Assabet Brook, Middlesex County, Mass. Nipmuck, "at the miry place." Also a river in Worcester County, Mass. 
Assabumbedock Falls, York County, Me. Abnaki, "sloping, sandy bottom."

Assameekq Bristol County, Mass. Narragansett, "a cave" or "stone roof."

Assanapset Brook, Providence County, R. I. Possibly Nipmuck, "where the net was held down by rocks," or "at the small rocky stream."

Assanotucket Pond, Dukes County, Mass. Wampanoag, "at the tidal stream obstructed by rocks."

Assapumsic Brook, Providence County, R. I. Narragansett, "place where wild hemp is gathered to make cords and nets;" or "great meadow;" or perhaps "stony crossing place." Spelled also Assopumsett, Ossopimsuck, etc.

Assatayag Hampden County, Mass. Pocumtuck, "big trees."

Assawaga Worcester County, Mass. Nipmuck, "place between" or "halfway place." Also Assowaga, Assawaug.

Assawaga "place between." Like Nashua, there are several such places in Southern New England. Also Assawaug.

Assawampsett Plymouth County, Mass. Narragansett, "trading (or barter or exchange) place"? or Wampanoag "place of the large upright rock"?

Assawassuck Hartford County, Conn. Wangunk, "place between" two brooks. (Locally known as Wassuck).

Assawompset Village, Pond, and Neck, Plymouth County, Mass. Wampanoag, "at the place of the large rock," or "stone plain place;" also "trading place" or "place of barter."

Assekonk Brook and Swamp, New London County, Conn. Mohegan, "much green grass at this place."

Assinek or Assunoc "stone place." See Hassunek.

Assinipi Brook, and Village, Plymouth County, Mass. Wampanoag, "rocks in water."

Assonet Bristol County, Mass. Narragansett, "at the rock." This rock is the famous Dighton Rock, bearing aboriginal rock markings.

Assonomock Bristol County, Mass. Narragansett, "place of the rock." Assoowamsoo Plymouth County, Mass. Wampanoag, "the halfway place," or "half way to southwest;" also "other side of Sowam's place." 
Assopamukquod Bristol County, Mass. Wampanoag, "place of giving thanks," or "place of refuge."

\section{Assowetough see Squaw Betty.}

Assunek Worcester County, Mass. Nipmuck, "cave" or "overhang of rocks" or "ledges place." Variant, Hassunek.

Asticou Northeast Harbor, Mt. Desert Island, Hancock County, Me. Probably $A b n a k i$, from the name of a chief who lived there. Some sources claim this is from kaski tegou, Old Abnaki, "deep river."

Astimoost Nantucket County, Mass. Wampanoag, "at the spring," or "at the small stony ford."

Asumsowis Fairfield County, Conn. Probably Paugussett, "a small fish net," or possibly "a small raccoon."

Asupsuck New London County, Conn. Mohegan, "place where wild hemps bounds."

Aswaguscawadic River, Aroostook County, Me. A branch of the Mattawamkeag. Abnaki, "place where a canoe is dragged through a stream" (rather than carried).

Aswaguscawadic Stream. A tributary of Lake Winnipesaukee, Belknap County, N. H. Abnaki, "compelled to drag the canoe through [shallow grassy] stream."

Aswahdenick Chittenden County, Vt. Abnaki, "at the place beyond the hill." See Housatonic.

Atchaubennuck Hill, New London County, Conn. Mohegan, "at the dividing place, or boundary;" "Boundary Hill."

Atgatogwisas Places along the Hoosic River, Bennington County, Vt. Mohawk, "he scoops up fish in a basket."

Atie ompsk a ooe di Mountain (now Moat Mountain), Carroll County, New Hampshire. Old Abnaki, "dogs at rocks, eating meat" ? or "wild animals resembling dogs, among the rocks." ?

Attaquahunchonett Neck, Barnstable County, Mass. Wampanoag, "at the hill where deer abound."

Attawan Beach, New London County, Conn. Roots not clear, nor tribe; possibly Eastern Niantic, "hills or dunes." ?

Attawaugan Village, Windham County, Conn. Possibly Nipmuck, "a knoll, or hill, or height of land." See Adowaukeag, Me.

Attean Lake and Mountain, Somerset County, Me. Abnaki, from name of Attean (Etienne) Orson, I793. 
Attebemeuck Island, Choke-Cherry Island in the Penobscot River. Abnaki, literally "choke-cherry place." Variant Atabeminock.

Atthammonasset or Athemonosseck same as Hammonasset.

Attilah Mountain (Bartlett Peak), Carroll County, N. H. If Indian, this may be a garbled form of attitaash, from the Narragansett, meaning "blueberries." More likely derived from Attila the Hun.

Attitash Lake, Essex County, Mass. Natick (or perhaps Pennacook?) "huckleberries" or "whortleberries."

Auco Cisco Back Bay, Portland, Cumberland County, Me. Micmac, "bay muddy" or "cove muddy."

Aucoot Cove, Plymouth County, Mass. Wampanoag, "a small cove."

Augamtocook Middlesex County, Mass. Natick, "great trees place," or "wading place in the river," or "river cove."

Augomagoit St. Croix Lakes, Washington County, Me. Malecite or Abnaki? "The place where we wade across."

Augutteback Pond, Worcester County, Mass. Nipmuck, "kettlepond." See Agutteback.

Aukumbumsk New London County, Conn. Mohegan, "other side of the rock ledge."

Aumough-cawgin Kennebec County, Me. Abnaki, "places for curing fish."

Auquebatuck Hill Tolland County, Conn. Nipmuck, "top of tree." Another spelling, Ocquebituck.

Aushpook an ancient Mohegan village in New London County, Conn. Mohegan, "place (or brook) where wild flax grows."?

Aussatonag Berkshire County, Mass. See Housatonic.

Austunoog see Housatonic.

Autoposit or Autopscot Nantucket County, Mass. Wampanoag, "at the walled-in well or cistern." L. K. Gahan says, "water drawing place." Also found as "place of wet rocks."

Awanadjo Blue Hill, Hancock County, Me. Abnaki, literally, "the misty mountain."

Awanganis Priestly Lake, Aroostook County, Me. Abnaki, free translation, "little canoe harbor" or "little place where we put canoes in or take them out;" literally "little journey." 
Awannoa Path, Middlesex County, Conn. Wampanoag, "who are you"? Hence: "Englishmen," or "strangers."

Awasaswi Menahan Belknap County, N. H. Abnaki, (literally) "Bear Island." Variant, Awososwi M'naan.

Awashonks Park and Swamp, Newport County, R. I. Awashonks was squaw sachem (suncksqua, "woman who rules") of the Sogkonates. She gave aid to the English in King Philip's War, I675-1676.

Awasoos Island, Penobscot County, Me. Abnaki, "a bear."

Awassawamkeak Sears Island, Waldo County, Me. Abnaki, "the shining sandy beach and beyond."

Awassokik Bearcamp River, Carroll County, N. H. Abnaki, "at the abode of the bear." (Awassos-auke).

A'waumps see A'wumps.

Awcumbuck near Gales Ferry, New London County, Conn. This was the residence of Pequot chiefs before the English came. Possibly Mohegan, "stones used in scraping or drying pelts, or sharpening stones"? or "Occum's place"? Another suggestion is, "across from the ledges."

Awseed local Mohegan name for the Thames River, New London County, Conn. Meaning lost; possibly this is part of a longer name indicating, "it flows down as far as the bay."

A'wumps Pond; now Wallum Lake, Providence County, R. I., and Worcester County, Mass. Pequot, "the Fox," name of a Quinebaug chief, A'waumps, who lived there in I673.

Ayasup Pond, New London County, Conn. Mohegan, "wild hemp, or flags, or rushes."

Azicoös Falls, Lake, Mountain and River, Oxford County, Me. Abnaki, "small pine trees."

Aziscoos Coös County, N. H. Abnaki, "small (young) pine trees."

Azoiquoneset Washington County, R. I. Narragansett, "small island where we get [or use] spruce pitch." Possibly for torches used for spearing fish at night. Another source gives "yellow point place," or "golden flames," suggested by the yellow flames of the burning pitch.

Azzastakak the Missisquoi River, Franklin County, Vt. Abnaki, "contrary to expectations, the stream turns." [i. e., oxbows]. Also Azzasataquake. 
Baamchee nungamook Chamberlain Lake, Piscataquis County, Me. Abnaki, "lake crosswise" of a usually-traveled route.

Baamchenungamis see Baamchee nungamook, below.

Baamchen ungamook Chamberlain Lake, Aroostook County, Me. Abnaki, "extended stretch across the lake," also "lake that is crosswise." Other spellings Boamchenungamo and Boamchenunquamook.

Babaquamshk Windham County, Conn. Nipmuck, "split rock." See Pabaquamsk.

Babbatasset Middlesex County, Mass. Natick, "at the double brook," or "where the brooks unite."

Baboosic Brook and Ponds, Hillsboro County, N. H. Abnaki? Pennacook? "sluggish current." However, this name may come from an Abnaki word babeskw, "a leech or bloodsucker." Other roots give: "middle brook," or "brook between."

Baddacook Pond, Middlesex County, Mass. Natick, "at the round place." See Pataconk.

Bagaduce The Castine Peninsula, Hancock County, Me. Micmac, "large tideway stream."

Bahkah soksik the meadows above Third Machias Lake, Washington County, Me. Malecite, "long straight deadwater."

Bakun Gunahik Crooked Island, Penobscot County, Me. Abnaki, "at the crooked island."

Bamedumpkok Lake, Aroostook County, Me. Abnaki, "place of sand bars."

Bamonewengamok Cross Lake, Aroostook County, Me. Abnaki, "lake athwart or crosswise" of the usually-travelled route.

Banneg Beg Mountain and Pond, York County, Me. See Bonny nague.

Bantam Lake and Village, Litchfield County, Conn. Mahican, "he prays." (Natick form, peantam).

Bapetanshant or Bapetaushaut Washington County, R. I. Narragansett, "hollow place," or perhaps "a cave," or "hiding place"?

Baquag River, Worcester County, Mass. Nipmuck, "clear water," (now Millers River). Also Paquag. 
Bashbish Falls and Mountain, Berkshire County, Mass. Mahican, "it bursts forth," or "it is daybreak." Or Illini, "a waterfall?" (Natick form, pashpisheau).

Bash Bish Falls, Berkshire County, Mass. Mahican, Basha or Mombasha was shot in the Esopus War, hence, "she is shot."

Baskahegan Lake and Stream, Washington County, Me. See below.

Baskahegan River, Aroostook County, Me. Abnaki, "branch downstream," or "branch stream that turns down current." Variants, Bascohegan, Bascanhegan, etc.

Bassoqutogaug Grove Washington County, R. I. Narragansett, "where trees were split"? But, if the correct spelling is Basskutoquoge, then "river branch place."

Bayakosk the Fifth Machias Lake, Washington County, Me. Malecite, "at the end or source of the lakes."

Bedabek Knox County, Me. Malecite, "head of the bay" at Rockland. Variant, Bedabedec.

Beegwatook Pushaw Pond. See Bigwadook.

Beemsquamketook Lake, Penobscot County, Me. Anbaki, "pond in branch of river"? or "extended sandbars in stream"?

Beezelake Pond, Litchfield County, Conn. If Indian, perhaps Mahican? "muddy place" or "sticky place." Another source gives "water place."

Bellamaqueen Bay, Franklin County, Vt. Probably Abnaki, modified by French, "good beaver."

Bemidjiwok Treat's Falls, Penobscot County, Me. Abnaki, "where water runs out swiftly." See Pemigewasset.

Beseck Lake, Middlesex County, Conn. Mohegan, "at the waterplace."

Betcumcasick see Petcumcasick.

Betuckquapock Fairfield County, Conn. Wappinger, "round pond." Variant, Pituquapaug.

Bigaduce Peninsula, Hancock County, Me. See Bagaduce. Variants, Biguaduce, Bigwaduce, Bagyduce, etc.

Bigwadook Pushaw Lake, Penobscot County, Me. Abnaki "bent stream" or "stream turns."

Bimilick Brook, Worcester County, Mass. Said to be from the name of a Narragansett chief, possibly abbreviated from Abimelech [an Old Testament name?]. Also Bumilick, and Bimilikus. 


\section{Boamtuquet see Pentaquiauktook.}

Bog Lake, Washington County, Me. Probably just plain English "bog;" if Indian, Abnaki for "pond."

Boggistow Brook, Middlesex County, Mass. Natick, "turning place."

Boggochaug Hills, Worcester County, Mass. Nipmuck, "at the turning place." See Packachaug.

Bogochico Nantucket County, Mass. Wampanoag, "open, hilly, land."

Bokajenesquis Island; Jug Island, Hancock County, Me. Abnaki, "jug shaped place."

Bombazine Island, Sagadahoc County, Me. Chief Abomazine, or Bomazeen, was an Abnaki leader who was killed at Norridgewock in I724. Abnaki, "keeper of the ceremonial fire." See below.

Bomoseen Lake, Rutland County, Vt. Abnaki, "keeper of ceremonial fire." Other spellings Abomazeen, Bombazine, ObamSaween, etc. William Simon [Obum Sawin], last male Abnaki resident of Vermont, died in I959.

Bonnynague Pond, York County, Me. Abnaki, "spread out lake." Variant Bonnyneag, Banneg Beg.

Bopquam Bay, Franklin County, Vt. Probably modified Abnaki, "a beaver."

Bosebuc Mountain, Oxford County, Me. Abnaki, "at the outlet of the spread-out stream."

Boskquenuguk Island, Broken Island, Penobscot County, Me. Abnaki, "burying ground island."

Bosquenaghook Island, Cemetery Island, Penobscot County, Me. Abnaki, "island where the dead are buried." Other spellings, Bosquenoosick, Boskanosic, etc. See also Bosquenuguk. Probably these all mean "broken land island."

Bosquenoosic Island, Penobscot County, Me. Abnaki, "little broken island."

Boxet Pond, Kent County, R. I. Eastern Niantic, "small pond." See Oxoboxo.

Brassua or Brassway Lake, Aroostook County, Me. EnglishAbnaki? probably from the Abnaki pronunciation of "Frank," a minor chief. 
Buhsuh-gwuntuk-sug Muddy Cove on Thames River, New London County, Conn. Mohegan, "little muddy cove with outlet in river."

Bumbahook Kennebec County, Me. Abnaki, "sandy shoal."

Bungamic Landing, Stream and Reef, Cumberland County, Me. See Bungamug, "boundary-mark."

Bungamug Brook, Cumberland, Me. Abnaki, "fishing place at the boundary."

Bunganock Pond, Oxford County, Me. Abnaki, "at the boundary place."

Bunganut Pond, York County, Me. Abnaki, "at the boundary mark."

Bungay New Haven County, Conn. If Indian, perhaps Quinnipiac, "boundary marker?" Some say this place takes its name from a village in Norfolk County, England.

Bungee Brook, Windham County, Conn. If Indian, possibly Nipmuck, "a boundary." See Bungamug, Me.

Bungy Rock, Washington County, Me. Abnaki, "the boundmark."

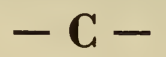

Cabassauk an ancient name for the Merrimack River, N. H. Abnaki, "at the place of the sturgeon."

Cabbadetus Lincoln County, Me. Abnaki, "it is rough, the throat [of the harbor opening] is closed."

Cabbo Lake, Rockingham County, N. H. Abnaki[?], "sturgeon." Perhaps modified from kabbassah?

Cacauwonch Kent County, R. I. Narragansett[?] "the beginning place."

Cacumgunsett Kent County, R. I. Narragansett, "whetstone quarry," or "place of high rocks."

Cahass or Cohass see Coös.

Cajacet see Conanicut.

Cajoot a plumbago mine in Washington County, R. I. This name is not apparently of Indian origin; no Algonkin roots seem to apply. However, one source gives "fir tree plain." Also Cojoot. 
Calumet Worcester County, Mass. Although this word is not Indian, it is included here because of the frequent confusion. This derives from Latin-French calamus, "a reed." Reeds were used in ancient times as ceremonial pipe stems, hence the common name "calumet" for the so-called peace pipe.

Cambolassie Pond, Penobscot County, Me. Micmac, "chain of ponds" connected by streams. Spelled also Cumbolassy.

Canada Falls, Somerset County, Me. Mohawk, "a group of houses" or "village."

Canada Hill, Cumberland County, Me.; see above.

Canangogum Northwestern Providence County, R. I. Nipmuck, "the fence or boundary," or "highland"?

Canapitsit Channel, Dukes County, Mass. Wampanoag, "at the place of the long fish weir."

Canasixet River, Cumberland County, Me. Abnaki, "witch hazel place"?

Canaumet Reservoir, Barnstable County, Mass. Wampanoag, "long fishing place." (eels?)

Candatowa Fairfield County, Conn. Paugusset (greatly modified), "great mountain."

Canestow River, Worcester County, Mass. Nipmuck, "pickerel place."

Caneunsquisset Washington County, R. I. Eastern Niantic, "high place," or possibly "high rocky cliff."

Caniaderi Guarunte or Caniadari Quaront Lake Champlain, north of Shoreham, Addison County, Vt. Mohawk, "lake with a bulge in it," or "wide lake."

Caniaderi-Oit see Yotenyatarokte.

Canibequi River, Kennebec County, Me. Same as Kennebec, q. v.

Canobeola Essex County, Mass. "long lake"[?] This appears to be a constructed term; the dialect or tribe is not recognizable. Perhaps from Seminole?

Canobie Lake, Rockingham County, N. H. Abnaki, "abundant water."

Canonchet Brook, Lake, and Village, Washington County, R. I. Canonchet was a Narragansett chief, the son of Miantunomoh. He was killed by the English in 1675 . 
Canonicut Island, Washington County, R. I. Named for the famous Narragansett chief, Canonicus, who was active in the Pequot War, I637. Also Caunaunacus.

Canopache Nantucket County, Mass. Wampanoag, "long fish weir," or possibly "at the fenced-in water place," or "closed cove place."

Canopaug Brook and Swamp, Providence County, R. I. Narragansett "a long pond."

Canoza Lake Essex County, Mass. Pennacook "pike," or "pickerel."

Canuxsawhory mountains around lower end of Lake Champlain, Vt. and N. Y. Mohawk, "high dwellings."

Capage New Haven County, Conn. Quinnipiac, "closed-in place."

Capanagansitt Providence County, R. I. Narragansett, "place of the enclosed (or plugged-up) well;" or "closed-up meadow"? See Ponaganset.

Capanewagen now Newagen, Lincoln County Me. Abnaki, freely translated as "channel closed up, no choice but to cross here by land." The most acceptable version is "closed route," or "stopped-up trip." Variant, Capenawhagen.

Capaum Pond, Nantucket County, Mass. Wampanoag, "an enclosed place, or harbor."

Capawack Dukes County, Mass. Wampanoag "enclosed harbor, or place of refuge," or just perhaps "overcast, shut in by clouds" [?]. An old name for Martha's Vineyard.

Capawong Franklin County, Mass. Mahican? "enclosed place," or "stopped-up by the bend [oxbows]."

Capawonk Meadow, Hampden County, Mass. Pocumtuck, "enclosed place; protected place; place of refuge; stopped-up by the bend in the river."

Capecorpus York County, Me. Abnaki, "closed to boats,"? i. e., plugged up by sandbars.

Cape Higgon Dukes County, Mass. Just possibly a corruption of Capoag, q. v.

Cape Newagen see Capanewagen.

Cape Poge Pond, Dukes County, Mass. Wampanoag, see below.

Capissic River, Cumberland County, Me. Abnaki, "dammed-up branch." Variants Capisick, Capipissoke, Capesseck, etc. 
Capmanwagan an old spelling (1623) of Kepan-ahwangan, "closed route," now (Cape) Newagen, q. v.

Capoag Dukes County, Mass. Wampanoag, "enclosed pond, or harbor." See Cape Poge and Capawack.

Cappacomuck Swamp, Washington County, R. I. Narragansett, "place of refuge," or "hiding-place,"

Cappiquat Dukes County, Mass. Wampanoag, "at the refugesummit," or "closed-in place." (This is Cuttyhunk Island). See Poocuttahunkanow.

Cappoquidnet Dukes County, Mass. Narragansett? "island of refuge." (No-Man's-Land Island.)

\section{Cappowack see Capoag.}

Cappowongamuck Franklin County, Mass. Mahican, "place enclosed by a bend" in the river.

Capsuptic Lake, Oxford County, Me. Abnaki, "dammed-up spring-fed stream."

Caratunk Lake, SomersetCounty, Me. Abnaki, "forbidden stream," or "crooked stream."

Caribou many place names throughout Maine bear this name. All derive from Abnaki, kalibu, "the shoveler," i.e., "he who gets his food by pawing or shoveling."

Carrabassett River, Franklin County, Me. Abnaki, "small moose place,"? or "sturgeon place"?

Carrartoank Falls. See Curritunk.

Carrituck Somerset County, Me. Abnaki, "crooked swift stream." See Curritunk.

Cascadnac local name for hills around the White River, Windsor County, Vt. Abnaki, "at the steep mountains" or "in the ravine."

Casco Bay, Cumberland County, Me. Micmac, "mud" or "muddy."

Caskak Chesquash Plymouth County, Mass. Wampanoag, "squash gardens"? or "big thicket"? or "deer hide"?

Caskata Beach and Pond, Nantucket County, Mass. Wampanoag, "a sea snail or periwinkle," or possibly "a sturgeon" [?]. Also, "where grass is mowed," kes asketuh.

Casowetchawêge Mountains, Windsor County, Vt. Probably the same as Skitchewaug, q. v. 
Cassacubque ledges near Colchester, New London County, Conn. Mohegan, "at the high rocks."

Cassacubque an ancient village near Mianus, Fairfield County, Conn. Possibly Siwanoy, "high rocks," (Cos Cob).

Cassomacook New London County, Conn. Hammonassett, "rocky place."

Catacoonamug Pond, Worcester County, Mass. Nipmuck, "great long fishing place" (eels?). Another authority gives "thirsty land," kohkuttoonoonk.

Catamawawa North Branch of Marsh River, Waldo County, Me. Abnaki, "very rough"? (k'tamahaga).

Catantaquk Swamp, head of Pachaug River, New London County, Conn. Mohegan, "place of large cedar trees."

Catardin Mountain, Maine. Variant of Katahdin, q. v.

Cataumet Harbor, Neck and Village, Barnstable County, Mass. Wampanoag, "at the ocean," or "landing place near the open sea."

Catawamkeak shores of Knox County, Me., near Rockland. Abnaki, "principal landing place" for canoes.

Cathance River, Washington County, Me. Abnaki, "the principal fork."

Cathaneu River, Washington County, Me. Abnaki, "the main stream."

Cathanisk Washington County, Me. Abnaki, "main branch of the stream," or "main stream."

Catumb Reef, near New London County, Conn. Mohegan-Pequot, "at the place of the great rocks."

Caucaujawatchuk Providence County, R. I. Narragansett, "sharp mountain peak" or "sharp mountain." Found also as Caucan, and Caucaunjawach.

Caucomgomoc Lake, Piscataquis County, Me. Abnaki, "lake abounding with gulls."

Caucumgomock Mountain, Somerset County, Me. Abnaki, "place (or lake) abounding with gulls?" Name transferred to the peak from a lake.

Caucumsquissic Brook, Washington County, R. I. East Niantic, "at the place where there are small sharpening stones." Another sources gives, "sharp stones in a cove;" also, "high cliff"? 
Cawatoquisset River, Bristol County, Mass. Wampanoag, "at the ever-running brook."

Cawcawmsquissick see Caucumsquissic.

Cawkinchawg or Cawgenchaug Middlesex County, Conn. Wangunk, "long swamp."

Cawncawnjawatchuk ProvidenceCounty, R. I. Narragansett, "very long hill." But see Caucaujawatehuk and Quanquanjawatchuck.

Cawsumsett Neck, Bristol County, R. I. Narragansett, "sharp rock place," or "whetstone rock place."

Cawwatoquisset Providence County, Conn. Narragansett, "high place along the brook." But see Cawatoquisset.

Chabanakongkomuk Worcester County, Mass. Nipmuck-Mohegan, "boundary fishing-place," or "place of separation where we fish." Some say "Treaty Pond." This is one of the places rendered as "you fish on your side, I fish on my side, nobody fish in the middle-no trouble." Also Chaubunagungamaug.

Chabatawece Island, Newport County, R. I. Narragansett, "little separated place." Also found as Chibaehuesa, Chibachuweset, Chippacurset, etc. See Chappaquiddick.

Chaboken Pond, Worcester County, Mass. Nipmuck, "hell pond." Literally, "place of separated (spirits)," from tcheppi-ohke.

Chabunnuck between Griswold and Voluntown, New London, County, Conn. Mohegan, "at the place which bounds or divides."

Chachabunk kakowok location unknown; somewhere in Worcester County, Mass. Nipmuck, "at the boundary," or "at the agreement place."

Chachapacasset Kent County, R. I. Narragansett, "near the great widening out place," or "place of the rocks in rushing stream." Other spellings Chackapacauset, Checkapaucasset, etc.

Chachapacassett Bristol County, Mass. Narragansett, "where the stream divides and opens out," or "torrent rocks place."

Chackacust Providence County, R. I. Narragansett, probably same as Chachapacasset, "at the great widening out place." Another source gives "it burns here" or "fire place."

Chackapacauset Neck. See Chachapacasset.

Chagum Pond, Newport County, R. I. Narragansett, "a black bird." (The bobolink? or the redwinged blackbird?) or a 
variation of shakum, (washakum) "surface of the sea, the great provider"? or "pouring out"?

Chamcook River, Aroostook County, Me. Micmac, "spawning place."

Chamquassabamtook Lake, see Chemquasabamticook.

Chanangonum Location unknown; "somewhere in Nipmuck country," possibly Providence County, R. I. Nipmuck, "great reed place"? Another spelling, Chanangongum, "great paint place."

Chapomeset Hill, Providence County, R. I. See Chopmist.

Chapomis Nantucket County, Mass. Wampanoag, "little separated place."

Chapompamiskock Providence County, R. I. Narragansett? Nipmuck? "big fishing place near boundary rock"?

Chappanacoy Nantucket County, Mass. Wampanoag, "(uninhabited) land, separated from rest."

Chappapemeset Nantucket County, Mass. Wampanoag, "at the extended, isolated beach." See also Chopmist.

Chappaquiddick Island, Dukes County, Mass. Wampanoag, "at the separated island," from tseppi-aquidne-auke.

Chappaquoit Barnstable County, Mass. Wampanoag, "at the separated place," or "boundary hill place."

Chappaquonsett Creek and Pond, Dukes County, Mass. Wampanoag, "long place (or thing) of separation." ("long creek separates, etc.").

Chargoggagoggmanchogaggogg Pond, Windham County, Conn. See Chabanakongkomuk.

Chataguay the Swift River, Carroll County, N. H. Pennacook, "the principal stream."

Chaubamaug Windham County, Conn. Nipmuck, "fishing place at boundary."

Chaubanakongkomun or Chabunakongkomun. See Chabanakongkomuk. Mass.

Chaubaqueduck Dukes County, Mass. Same as Chappaquiddick.

Chaubatick Providence County, R. I. An ancient village of the Narragansett tribe; "at the forked river," or "the river which bounds." 
Chaubongum Pond Windham County, Conn. Nipmuck, "the boundary mark," or "the limit."

Chawonk Neck, Lincoln County, Me. Abnaki, "great neck" or "big peninsula."

Cheapschaddock New London County, Conn. Nipmuck, "big rocks at boundary place."

Chebacco Lake, Essex County, Mass. Probably Pennacook, "big pond;" but possibly Natick, "crossed over." Another source gives Old Abnaki or Pennacook roots, tsibai-ki, "place of departed spirits."

Chebatigosuk Penobscot County, Me. Malecite, "short way from river" or "short cut across."

Chebeague Village, Strafford County, N. H. Abnaki, "almost separated." See below. Variant, Chebeaque.

Chebeague Island, Cumberland County, Me. Abnaki, "almost separated." At low tide a connecting beach is exposed; at high water the sea separates the two high ends. Literally, the name means "separated place."

Chebeguadose a large bay in Hancock County, Me. Malecite, "principal cove" or "great bay."

Chebogardinac Knox County, Me. Penobscot, "at the high hill."

Checapscaddock an ancient Mohegan village, New London County, Conn. Mohegan, "big rocky hill."

Checkechnusset Brook, Washington County, R. I. Eastern Niantic, "at the boundary" or "brook at the place of separation." Another spelling, Chechechnessett.

Checkecknessett Brook, New London County, Conn. and Washington County, R. I. Eastern Niantic, "at the boundary" or "at the separation brook."

Cheebeeantups a bald hill near Colchester, New London County, Conn. Mohegan, "a skull." Literally, "a separated head," "a head cut off the body."

Cheebee Atque Seep Monument Brook, the boundary between Aroostook County, Me., and New Brunswick, Canada. Passamaquoddy, "separated by marking (or boundary) brook."

Chee Coke the falls in the Connecticut River near Bellows Falls, Windham County, Vt. Pennacook, "the great kettle." 
Cheekheek Windham County, Conn. Nipmuck? "fire place." Cheekwakat see Chequaquet Lake.

Cheemahn Island, Long Island, Penobscot County, Me. Abnaki, "a large island."

Cheepauke Location unknown; probably Providence County, R. I. Narragansett? "a place apart" or "an isolated place."

Cheesechankamuck a branch of the Farmington River, Hartford County, Conn. Tunxis, "great enclosed place for fishing," or "big fish weir pool."

Cheetoskeunke Kent County, R. I. Nipmuck or Narragansett? "at the principal wading-place (ford, or bridge?)."

Chegewanussuck Falls, Lincoln County, Me. Malecite, "large rapids and falls."

Chegony Island, Sagadahoc County, Me. Probably Micmac, "knee shaped."

Chehockset Merrimack County, N. H. Pennacook, "at the principal forest grove."

Chemangase Pond, Washington County, R. I. Eastern Niantic, "small canoe"? or "small waterway" ? or "big brant goose"? Other spellings Chemagaze, Chemaunguz, etc.

Chemaun Nayaug Chimney Point, Addison County, Vt. This name is a strange mixture of Chippewa and Natick, "canoe point."

Chemo Lake, Penobscot County, Me. Abnaki, "a large bog."

Chemquam Sabamticook Lake, Piscataquis County, Me. Abnaki, "where there is a large lake together with a river."

Chemquassabamticook Stream, Aroostook County, Me. Same as above.

Chemung Road, Norfolk County, Mass. Delaware, "the great horn"? or (modified) Natick, "a canoe"?

Chemung Strafford County, N. H. If Delaware, "big horn"; if modified Natick, "a canoe."

Chemunganock Hill and Pond, Washington County, R. I. East Niantic? This name is badly distorted. Probably "at the abode of the brant goose"; possibly "big stink place," (rotting vegetation) or "place where we put down paddles," or "big ash tree place."

Chemunganset Pond. See Chemunganock.

Chemunkanuck Pond. See Chemunganock. 
Chenayok Carroll County, N. H. Abnaki, "principal neck of land," the Moultonboro penninsula in Lake Winnepesaukee.

Cheôuanasäg Falls, Knox County, Me. Abnaki, "at the big portage."

Chepachague Washington County, R. I. Eastern Niantic, "principal turning place." Also Chepachuach.

Chepachet River and Village, Providence County, R. I. Narragansett, "place of separation," that is, where rivers divide. Also, "boundary place."

Chepachewag River, Washington County, R. I. East Niantic, "principal turning place." Other spellings Chepacheway, Chepachuach, etc.

Chepacheweset Plymouth County, Mass. Wampanoag, "boundary place."

Chepados Hill, New London County, Conn. Mohegan? "principal hunting place"? Perhaps, "hill where the big nuts grow." Another spelling, Chepadas.

Chepatset Nipmuck territory in the Northwestern part, Providence County, R. I. Nipmuck, "boundary place" or "place of separation."

Chepinoxet Island, Kent County, R. I. Narragansett, "little separated place," or "Devil's Island," or "Hell Island," i. e., "place of the departed spirits."

Chepiwanoxet Island and Village, Kent County, R. I. Narragansett, "at the end of the small separated place." (Narrow neck covered at high water).

Chepontuc Ottauqueechee Falls, Windsor County, Vt. Abnaki, "principal fall in river."

Cheppipogut Bristol County, Mass. Wampanoag, "at the separated pond" or "place of the out-of-the-way, isolated pond." Also given as "spirit's-place pond." Variant, Chuppipoggut.

Cheputneticook Grand Lake, the Schoodic system, Washington County, Me. Abnaki, "at the place of the big hill stream."

Chequapee Hampden County, Mass. Nipmuck, "rushing water," (or "cedar tree?") See Chicopee.

Chequaquet Lake, Barnstable County, Mass. Wampanoag, "at the fire place"? or "it is daybreak," ? or "big swamp place."? Also "big hill place." 
Chequesset Barnstable County, Mass. Wampanoag, "violent waters." (Possibly "great waves because of north wind?" or "big rock place"?)

Chesewane Island, Bristol County, R. I. Narragansett, "big shells," or "great scattered shells," or "large shell heaps." Also given as Chesewanne and Chesewannock, but see Chisawamicke, Mass.

Chesquonopog Worcester County, Mass. Nipmuck, "great long pond."

Chesuncook Lake and Village, Piscataquis County, Me. Abnaki, "at the place of the principal outlet."

Chewonki Neck, Lincoln County, Me. Abnaki, "a great bend," or "a large ridge."

Chibachuesa Island (Prudence Island) Newport County, R. I. Narragansett, "separated at high tide" (from Patience Island)? Variants: Chibachuweset, Chebbachuesset, etc.

Chibacoweda Patience Island, Newport County, R. I. Narragansett, "separated by a passage" i. e., from Prudence Island.

Chibanook Seponac Lake, Penobscot County, Me. Abnaki, "the big opening."

Chiboctous Hancock County, Me. Malecite, "the big bay."

Chickabi River; see Chicopee.

Chickamug near Stonington, New London County, Conn. Eastern Niantic, "big fishing place," or "fish-weir place."

Chickamug Washington County, R. I. Eastern Niantic, "fish trap," or "fish weir," or perhaps "a fishing place; the principal fishing place."

Chickasheen Brook, Washington County, R. I. This name might be Eastern Niantic, Narragansett, or even Nipmuck. It has several possible meanings: "fish weir," "high water," or "cedars." Most likely, however, is "big spring."

Chickatawbut Road, Norfolk County, Mass. Natick, "his house is on fire," or, "his house is burning violently." The name of a chief at Weymouth, c. I62I. One source suggests "place of raging water"'[?]

Chickawaukee Lake, Knox County, Me. This is not a genuine Indian name, but a composite attempt at "good, sweet, fresh, or drinkable." 
Chickemmoo Dukes County, Mass. Wampanoag, "fish weir."

Chickons Hampden County, Mass. Nipmuck, "burned place, burned so as to be clear, ready for planting."

Chickons Cattones Akees Hampden County, Mass. Natick?, "small plantation." Also Cottinyakies, Cottinackeesh, Kitikanakish. (But a Chickens lived at Fairfield, Conn., in I725.)

Chickwolnepy Stream, Coös County, N. H. Abnaki, "frog pond." Also Chickwalnipy, Chigglneepa. See Jigwallick.

Chicomico Creek, New London County, Conn. Nipmuck, "large enclosed place," or "big house."

Chicopee Hampden County, Mass. Nipmuck, "violent water," 嫼 (or "cedar tree"?)

Chicopee York County, Me. See above.

Chikabi see Chicopee.

Chikkabi Hills, Hartford County, Conn. Nipmuck, "cedar?" or "birch bark"? See also Chicopee.

Chilnucook Grand Lake, Washington County, Me. Abnaki, "great open place."

Chimenasanganum Big Lake, Washington County, Me. Abnaki, "at the place where boards are heaped up near the big lake."

Chimkazaooktook Aroostook County, Me. Abnaki, "big black stream."

Chimmenticook Lake and Stream, Aroostook County, Me. Abnaki, "principal islands in river."

Chimon Island, Fairfield County, Conn. If Indian, possibly Pangussett, "he paddles" a canoe? But see Mamachimins.

Chinquist Barnstable County, Mass. Wampanoag, "big rocks place."

Chipchug Pond, New London County, Conn. Nipmuck, "place apart," or "boundary place."

Chipchug Pond, Washington County, R. I. See above.

Chipohke see Cheepauke.

Chiponaug Point, Kent County, R. I. Narragansett, "separated or isolated point"? "place of the large oysters" ? or "principal resting place"?

Chippachaug Mason's Island, New London County, Conn. Mohegan, "place separated," apart from main land. Also Chippichuock. 
Chippachooag Washington County, R. I. East Niantic, "where it [stream] divides," or "place of separation."

Chippachuachack R. I. See Chippachooag and Chippuachack. Chippacurset Island, R. I. See Chibachuesa. Another spelling, Chippecurset.

Chippaquiddick see Chappaquidick.

Chippascutt Plymouth County, Mass. Wampanoag, "at the place of separation."

Chippenhook Village, Rutland County, Vt. If Indian, perhaps Abnaki, "big extended run of water." If not Indian, perhaps Dutch, as Shippen's Hoek[?] One source gives Natick, "separated place." See Chibanook.

Chippewa Indians, also known as Ojibwa, were the largest Algonquian-speaking tribe. They lived in the region of the Great Lakes, and Longfellow's Hiawatha includes many of their legends, although Hiawatha was actually an Iroquoian hero. Their name is a reference to their puckered-seam moccasin.

Chippopoquet Plymouth County, Mass. Wampanoag, "at the place of the separated pond." Also spelled Chuppipoggut.

Chippuachack Washington County, R. I. East Niantic, "at the boundary hill place." See Quowatchaug.

\section{Chiputneticook see Cheputneticook.}

Chipuxet River, Washington County, R. I. Narragansett, "little separated place," or "isolated small pond."? Spelled also Cheppuxet, Chepuckset, etc.

Chisapeak Bay, Merrymeeting Bay, Sagadahoc County, Me. Abnaki, "at the large part of the river," or "big salt bay."

Chisawamicke Plymouth County, Mass. Wampanoag, "at the old field." Spelled also Chessawanacke.

Chisawannock Bristol County, R.I. Narragansett, "principal fishing place"? or "muddy bottom"? This is Bristol Harbor. Other spellings Chesewane, Chesewanock, Chisweanocke, etc.

Chochichok Falls of the Cochecho River, Strafford County, N. H. Abnaki, "at the rapid current." (Perhaps "big kettle" ?)

Chockalaug River, Providence County, R. I. Nipmuck, "fox place." See Chockalog, above.

Chockalog Pond, Worcester County, Mass. Nipmuck, "fox place"? or "burned land"? See also Chickwolnepy. 
Chockseit Worcester County, Mass. Nipmuck, "fox place."

Chocorua Lake, Mountain and Village, Carroll County, N. H. Pennacook, from the great chief whose name may have meant "sharp knife." (Or perhaps from tsikweres, "a frog." One source gives "fox.")

Chomowauke Washington County, R. I. East Niantic, misspelling of Ohomowauke, meaning "owl place," i. e., a place of refuge or concealment.

Chopequonset Providence County, R. I. Narragansett, "isolated plantation," or "separated fields" or "fields at boundary place."

Chopmist Hill and Village, Providence County, R. I. Narragansett? "boundary or dividing place." (Given also as "principal crossroads").

Chouacoet part of the Saco River, York County, Me. Abnaki, "at the outlet." (This name used by Champlain and Lescarbot.)

Chuppipogut Plymouth County, Mass. Wampanoag, "at the boundary pond," or "at the closed-up pond" (kuppi poget)?

Chuquisak probably a creek outlet near Yarmouth, Cumberland County, Me. Abnaki, "at the muddy place."

Chusick Brook, Hampshire County, Mass. Pocumtuck, "mountain place."

\section{Cinebaque see Kennebec.}

Coaksett Newport County, R. I. Narragansett, "pine place," or "at the place of pines." See Cokesit and Coaxet.

Coassatuck Hill, New London County, Conn. Mohegan? or Niantic? "pine tree place," or "pine tree river." Also Cowissatuck.

Coassit Franklin County, Mass. Pocumtuck, "at the pine tree place." (Probably connected with Koessek, q. v.)

Coasuck Brook, Franklin County, Mass. Pennacook, "pine-tree place."

Coaticook River, Essex County, Vt. Abnaki, "the river near the pines."

Coatue Beach, Nantucket County, Mass. Wampanoag, "at the pine tree place." (Also Coatuit, Cotuit, Coituate, etc.)

Coaxet Newport County, R. I. Narragansett, "pine place." See also Cowekesit. 
Cobosse Kennebec County, Me. Abnaki, "a sturgeon."

Cobossecontee Lake, Kennebec County, Me. Abnaki, "plenty of sturgeon." Variants: Cobeskonte and Cobbasseeconteag.

Cobscook Bay, Washington County, Me. Malecite, "rocks under water," or "underwater boulders." In several Maine place names, cook means "turbulent, eddying currents."

Cocacocks New Haven County, Conn. Quinnipiac, "drinking place."

Cocasset Pond, Norfolk County, Mass. Natick, "at the small kettle." (Glacial kettle hole?) Also translated "boundary place" and "pine-hill place."

Cocheco Marsh, Point, Pond and River, Strafford County, N. H. Abnaki, "place of the rapid current." Variants, Cochecha, Cochchechoe, etc.

Cochessett Plymouth County, Mass. Wampanoag, "place of small pine trees."

Cochichewick Lake, Essex County, Mass. Pennacook, "at the place of the swift current."

Cochikuack Brook, New London County, Conn. Mohegan, "fast, strong, dashing current."

Cochituate Lake and Village, Middlesex County, Mass. Natick, "place of swift water."

Cochnewagon Lake, Kennebec County, Me. Abnaki, "closed up route." See Capanewagen.

Cochpinnecote Barnstable County, Mass. Wampanoag, "at the green bank" (grass covered rim?) Also given as "owl's nest place."

Cockamong River, Byram River, between Connecticut and New York. See Armonek and Comonck.

Cockampoag Washington County, R. I. Narragansett, "long pond." Also Cocumpaug.

Cockaponset State Forest, Middlesex County, Mass. Wangunk, "the boundary is at this falls."

Cockenoe Island and Harbor, Fairfield County, Conn. Montauk, "he interprets and sets things down." From name of the Indian who arranged Norwalk deeds in I652. Also Checkinoo.

Cocumcussuc Washington County, R. I. Eastern Niantic? "high place" or "place of high rock." 
Cocumscusset Brook, Washington County, R. I. Eastern Niantic? "place of high rock." (Given also as "whetstones-place," "stones in brook," and "outlet of cove.")

Coddank New London County, Conn. Mohegan-Pequot, "cleared land."

Coddude Nantucket County, Mass. Wampanoag, "the end," or "the summit"[?]

Codtaumut Barnstable County, Mass. Wampanoag, "desired place"[?], "place of trading" [?] or "where they sing" [?]

Coes Reservoir and Village, Worcester County, Mass. Nipmuck, "pine tree."

Coeset Kent County, R. I. Narragansett, "pine place." See Cowekesit.

Coginchaug Brook, Middlesex County, Conn. Wangunk, "at the place where they cure fish" by drying and smoking. Similar to Androscoggin. Another source gives "long swamp."

Cohanit Bristol County, Mass. Narragansett, "at the long place," or "at the pine place"?

Cohannet Road, Plymouth County, Mass. Wampanoag, "at the long place," or "at the pine place."

Cohasset Village, Norfolk County, Mass. Natick, "high place (where we fish)" or "at the promontory." See Quonahassit.

Coheassuck Kent County, R. I. Narragansett, "pine tree place"? or "brook near the pines"?

Cohoes Brook, Hillsboro County, N. H. Abnaki, "a pine tree."

Cohoos Pond, Cheshire County, N. H. Abnaki, "a pine tree."

Coicus see Nonacoicus.

Cokesit see Coaksett.

Colicum Pond, Worcester County, Mass. Nipmuck, "whistling duck."

Collegewidgwock see Kollegewidgewook. Abnaki, "mixed rapids."

Collicut Brook, Aroostook County, Me. Abnaki, "at the place of flames."

Comet Hollow, Essex County, Mass. Natick, "at the house." Also given as "long hollow" or "pine hollow." See Asnacancomic. 
Commquessakumkanet (Herring Pond) Plymouth County, Mass. Wampanoag, "at the rock which stands erect." Variant, Commassakumkanit.

Comonck River; Byram River, between Connecticut and New York. Siwanoy [?], "fishing place."

Comphegan see Quamphegan, "scoop net."

Compo Neck, Fairfield County, Conn. Paugussett, "long pond"? or "scoop net"?

Compounce Pond, Hartford County, Conn. Named after an old Tunxis Indian, John Compound; hence Compound's Pond, etc.

Conamicut Island; same as Conanicut, Quonanicut, etc.

Conampsquenooncoat River, Essex County, Mass. Natick, "at the long stone ledge." Sometimes given as "long rat meadow field."

Conanicut Island, Park and Point, Washington County, R. I. Narragansett, "the especially long place." Named for chief Canonicus, who died in I647. Variants Conanticut, Canonicut, Quonaniquot, Quononicut, Quononiquit, etc.

Conaquetoque Island, Washington County, R. I. Narragansett, "place of the long stream."

Conaquotoag see Quanacontaug or Quonacontaug.

Conaytuck Brook, New London County, Conn. Nipmuck, "long stream," or "tall tree"? Also Connoughtug.

Conconchewachet see Caucaujawatchuk and Cawncawnjawatchuk.

Condeskeag Me. See Kenduskeag, "eel weir place."

Congamond Lakes, Ponds and Village, Hampden County, Mass. Nipmuck, "long fishing place." Another source gives "parched land place."

Congamuck Lakes, Hartford County, Conn. Nipmuck, "long fishing place."

Congamund Pond, Hartford County, Conn. Nipmuck, "long enclosed fishing-lake." See Congamuck.

Conic Lake and Stream, Washington County, Me. If this should be spelled Gonic, it is probably Abnaki, "spearing place."

Conimicut Point and Village, Kent County, R. I. Narragansett; probably from the name of Canonicus's granddaughter, Quenimiquet (or Quinimikit). 
Connaug Pond, Providence County, R. I. Narragansett, "long place."

Connecticut Lakes, River, and State. Rising in Coös County, N. H., the Connecticut flows south to separate New Hampshire and Vermont, then divides Massachusetts and Connecticut. Hence in several New England dialects, "the place of the long river."

\section{Connitic see Connecticut.}

Conob Pond, Washington County, R. I. Eastern Niantic, "long rock".

Conockonquit Rose Island, Newport County, R. I. Narragansett, "place at the long point."

Conohasset see Cohasset, and Quonahassit.

Conomo Point and Village, Essex County, Mass. Natick, "plenty of fish," or possibly, "long fish (eels, or lampreys)."

Consamasset Washington County, R. I. Eastern Niantic, "place of sharp rocks" ? or "place of long fish (eels)" ?

Consamasset Land, Providence County, R. I. Narragansett, "sharp rocks place." Also Consamset, Consmassic, Cawsumsett.

Conskuet Island, Newport County, R. I. Narragansett, "at the long rock or reef," "at the long outlet," or "the long pouringout place."

Consue Nantucket County, Mass. Wampanoag, "the long soft, miry place" or "extensive bog."

Consumpsit Rock, Bristol County, R. I. Narragansett, "sharpening rock," "whetstones," or "sharp rock."

Conticoog Branch. See below.

Contocook Merrimack County, New Hampshire. Originally pakunteku, possibly from Abnaki, "nut trees river," or Natick, "small plantation at river."

Contoocook River, Essex County, Mass. Pennacook, "place of the river near the pines." Given also as "at the river of the crows," but see Coaticook.

Coocatoonemaug Brook and Pond, Worcester County, Mass. Nipmuck, "eel fishing place." See also Catacoonamug.

Cooksacky Rutland County, Vt. Mahican, "snake-place," or "owl place," or "rocky point."

Coojoot see Cajoot. 
Coonamesset Pond, Barnstable County, Mass. Wampanoag, "long fish place," also "pine place."

Coonempus Road, Block Island, Newport County, R. I. Narragansett, "long reef" or "long gravelly place"?

Coös Intervales, along both sides of the Connecticut River from Newbury, Orange County, Vt., and Haverhill, Grafton County, N. H., northward through Essex County, Vt., and Coös County, N. H. Coösuc [a Pennacook subdivision], "the place of the pine trees."

Coös County, N. H. Pennacook, "a pine tree."

Coös Junction, Coös County, N. H. Pennacook, "a pine tree."

Coösauk Falls, Coös County, N. H. Pennacook, "at the place of the pines."

Coösuck now Newbury, Orange County, Vt. Pennacook, "at the place of the pines." Variant, Koes-seck.

Coöxissett Plymouth County, Mass. Wampanoag, "at the grove of small pines."

Copassanatuxet Kent County, R. I. Narragansett, a modification of Occupessatuxit, "cove on small tidewater river or inlet." Other spellings Copassnetuxit, Copessnatuxit, etc.

Copecut Bristol County, Mass. Wampanoag, "thicket place, place of refuge; closed-in place."

Copicut Neck, Dukes County, Mass. Wampanoag, "at the closedup place."

Copicut River and Hill, Bristol County, Mass. Narragansett, "at the refuge place."

Coppoanissett Plymouth County, Mass. Wampanoag, "little harbor" or "small landing place (for canoes)." Also Coppanissett.

Coquitt Bristol County, Mass. Narragansett, "an arrow point," or possibly, "at the high point."

Corum Fairfield County, Conn. Paugussett, "a valley," or "low country." Perhaps originally Moonouhkoigeum, "low land," later corrupted into Moncorum.

Cosattuck see Coassatuck.

Coschow Path, Middlesex County, Conn. Natick, "high" or "summit," or from a Wangunk family of that name.

Cos Cob Village, Fairfield County, Conn. Siwanoy, "high rock." See Cassacubque. 
Coskata Beach and Pond, Nantucket County, Mass. Wampanoag, "grove of trees"? or "broad woods"?

Cossonowock Middlesex County, Conn. Hammonasset, "long stone place," or "pestles-place." Also Cassomacook and Coussounacock.

Cotackta Nantucket County, Mass. Wampanoag, "hill top," or "at the summit."

Cottinackeesh Hampden County, Mass. Nipmuck, "little farms place," or "he digs and plants a field." Also translated as "ground planted now, little heaps" i. e., hills of corn and beans.

Cottonchusett Neck, Barnstable County, Mass. Wampanoag, "piled up hills place." A reference to sand dunes, or to hills of corn and beans?

Cottoyowsekeesitt Plymouth County, Mass. Wampanoag, "miry brook meadow."

Cotuit Barnstable County, Wampanoag, "at the long planting fields."

\section{Coucomgomoc Lakes, etc. See Caucomgomoc.}

Counnitegou The Connecticut River. Abnaki, "the long river." So spelled on a I7I3 map published in France.

\section{Couxsachrage see Koghserage.}

Cowamps a locality in Hartford County, Conn. Tunxis, "sharpening rock," or "whetstone rock." Also Cowomsq, Cowomsk, Cowomsque; these last possibly "sharp, jagged rocks."

Cowasset River, Worcester County, Mass. Nipmuck, "at the place of pines."

Cowassit New London County, Conn. Mohegan, "at the pine woods." Given also as Cowissick, Koessek, etc.

Cowate Middlesex County, Mass. Natick, "pine tree place." An ancient village site.

Cowaude Kent County, R. I. Narragansett, "pine place."

Cowautacuck Litchfield County, Conn. Mahican, "pine woods place."

Cowekesit Kent County, R. I. Eastern Niantic, "pine tree place" or "place of young pines." Spelled Coweset, Cowesit and Cowesuck. 
Cowesawaskoög Peak, Hillsboro County, N. H. Pennacook, "sharppointed mountain place." Possibly the same as Kearsarge, q. v.

Cowesiseck River, Mill River, Lincoln County, Me. Abnaki, "pine tree place."

Cowissewaschook Kearsearge Mountain, Merrimack County, N. H. Abnaki, "at the pointed mountain."

Cowsigan Narrows, Sheepscot River, Kennebec County, Me. Malecite, "rough rocks." Also Cowsegan and Cowseagan.

Cowsumpsit Bristol County, R. I. Wampanoag, "place of sharp rocks."

Cowsumsett Neck, Plymouth County, Mass. Wampanoag, "place of sharp rocks."

Cowwaus New London County, Conn. Mohegan, "pine grove."

Cowwautatuck River, New London County, Conn. Mohegan, "pine woods river." (If Corwrautacuck, "pine woods land.")

Crackatuxet Cove, Dukes County, Mass. Wampanoag, "swampy little stream, or creek."

Crockemago "corn-hoeing?" See Rockomeko.

Cromesit Point, Plymouth County, Mass. Wampanoag, "at the fishing place; deserted place," or "deserted fort."

Cummaquid Harbor and Village, Barnstable County, Mass. Wampanoag, "enclosed place" or "harbor." Also given as "shore of the island."

Cumnuck Island, Washington County, R. I. Narragansett, "shutin place." (As this island is in a shallow pond, perhaps the meaning includes "we can walk over to it.") Also Cumnoc.

Cunney Mountain, Grafton County, N. H. (Probably Sawyer's Mountains?) If this is Indian, it is possibly the same as Kineo, q. v.

Cupheag a cove near Stratford, Fairfield County, Conn. Paugussett, "at the cove," or "at the enclosed place," or "place of shelter."

Cuppacomuck Swamp, Ledyard Pine Swamp, New London County, Conn. Mohegan, "place of refuge," or "enclosed grove" where we hide from our enemies.

Cuppan augunit an ancient place of refuge north of Stonington, New London County, Conn. Pequot or Eastern Niantic, "enclosed hollow place," probably a geologic kettle hole. 
Cupsuptic Brook, Mountain, Pond and River, Oxford County, Me. Abnaki, "a closed-up stream." Some Indians say the closure was a wooden fish trap.

Curritunk Falls, Township and Village, Somerset County, Me. Abnaki, "crooked swift stream."

Cushankamaug Hartford County, Conn. Tunxis? "eel-fishing place." Also, Onshankamaug.

Cushena Newport County, R. I. Narragansett, "wet land?" "rough country"? or possibly "near where it (the tide) runs out."

Cushenag Pond, Plymouth County, Mass. Wampanoag, "wet place." See Acushnet.

Cushnoc near Augusta, Kennebec County, Me. Abnaki, "head of the tide." Also found as Cusinock, Cushnoog, Kussinak, etc.

Cusumpe Pond, now Asquam Lake, Grafton County, N. H. Abnaki, "choked-up pond." Also possibly, "large rocks."

Cutchegun Rock, New London County, Conn. Mohegan, from the name of Caleb Cutchegun, an Indian who lived near this rock. Said to be the largest detached boulder in New England. The famous Uncas lived near here, circa I637. Also found as Corchegan.

Cuttoquat Plymouth County, Mass. Wampanoag, "at the great tidal river." See Titicut.

Cuttyhunk Harbor and Island, Dukes County, Mass. Wampanoag, from poo-cutahunk-anow, "a thing that lies out in the great water." Another authority gives papaquantuck as a possible source, meaning "broken land."

Cuxabesis Lake, Piscataquis County, Me. Malecite, "little swift water." Also Cussabexis.

\section{$-\mathbf{D}-$}

Daaquam River, Aroostook County, Me. Abnaki, "thy beaver." Damariscotta Lake, and Village, Lincoln County, Me. Abnaki, "plenty of alewives."

Damariscove Island, Lincoln County, Me. Not Indian? More likely English, "Damerell's Cove"[?] See Aguahega. 
Damisokantic An old name for Megantic Pond, Franklin County, Me. Abnaki, modified from Namesokantsik, "place of many fish."

Darongowa Apple Tree Point, Chittenden County, Vt. Mohawk, "a large duck."

Dawinehneh Big and Little Otter Creeks, Addison County, Vt. Mohawk "at the abode of otters" (The Mohawks made a pun on this name and that of a French missionary, the Abbé Thavenet, whom they called Père Dawinet.)

Debsconeag Deadwater and Lakes, Piscataquis County, Me. Abnaki, "ponds at the high place" or "ponds at the head of the waterway."

Deekeewenskek upper Dobsy Lake, St. Croix System, Washington County, Me. Abnaki, "at the head or the source of the river."

Dekaswenkarorens near Swanton, Franklin County, Vt. Moharek "a sawmill." The French had a sawmill here in the late I740's and early I750's, on land leased from Missisquoi Indians, chiefly Abnaki, and Mahican.

Delaware Indians inhabited New Jersey, eastern Pennsylvania, and much of lower New York. Calling themselves Lenni Lenape, "real men," they were a confederation of three subgroups, the Unami, Unalachtigo and Munsee. Many of the smaller Algonquian tribes looked upon them as their ancestors.

Deowcook Rattlesnake Peak, Berkshire County, Mass. Mahican, "that hill," or "great hill," kdj-watchuk.

Deyehonwakwatha now the Carrying Place, Grand Isle County, Vt. Mohawk, "with which one picks up a canoe."

Donwagayon meadows in Addison County, Vt. Mohawk, "an old muskrat lodge."

Douaquec Hancock County, Me. Ancient name (I688) of territory between Penobscot and Union Rivers. See Douaquet.

Douaquet ancient name for Falls Point, Hancock County, Me. Malecite, "a ridge;" a glacial kame. See also Adowaukeag.

Dyonondakren probably Mt. Mansfield and Camel's Hump, Chittenden County, Vt. Mohawk, "mountains near to each other." 
Eackhonk River, Washington County, R. I. Narragansett, "this is the end of the fishing place," or "as far up as the migratory fish go." Another authority suggests megunk, "a dry tree," or "a large tree.") See Egunk.

\section{Eascoheag see Escoheag.}

Easkissey Hill, Bristol County, Mass. Wampanoag, "old traps," "little green things," or perhaps "melon vines" [?]

Ebeeme Mountain, Piscataquis County, Me. If Indian, perhaps Abnaki, "extended." Also given as Ebeemin; in which case the translation would be "berries," perhaps huckleberries.

Ebenecook Harbor, Lincoln County, Me. Abnaki, "opens out behind the entrance-place."

Edali andalach sime mook Castine Neck, Hancock County, Me. Abnaki, "place where they waited and rested."

Edali chichi quasik Cape Rosier, Hancock County, Me. Abnaki, "the place where it is very narrow."

Edali qu saguh holdemook Prospect Ferry, Hancock County, Me. Abnaki, "where one goes across."

Edali seback lemook Castine Neck, Hancock County, Me. Abnaki, "where they waited for the tide."

Edali teh wakeel amook Bald Hill Cove, Waldo County, Me. Abnaki, "the place where they run up hill" as a trial of strength and endurance.

Edali weekek hadimook Hampden Narrows, Penobscot County, Me. Abnaki, "place where they (dwarfs, like Irish leprechauns) made marks on the cliff."

Edal skowasimook Castine Neck, Hancock County, Me. Abnaki, "where you would have to wait and keep watch."

Edawi maniwik inlet of Hermon Pond, Penobscot County, Me. Abnaki, "where you can go either way." (Here one has a choice of two routes downstream.)

Edjida Waskodek cleared place near Orono, Penobscot County, Me. Abnaki, "grassy place cleared by fire."

Eggemoggin Reach, Hancock County, Me. Malecite, "fish weirplace."

Egol bayik the Third Machias Lake, Washington County, Me. Malecite, "long, narrow lake." 
Egunk Hill, New London County, Conn. Mohegan, "at the turn or bend" in a river or trail.

Egunk Sonkapaug New London County, Conn. Mohegan, "at the turn, a cool spring."

Ehkapsak Little Falls Stream, Washington County, Me. Malecite, "rocky bottom."

Ekonk Windham County, Conn. Nipmuck? "a bend or turn." See Eackhonk, and Egunk.

Ekwanok Mountain, Bennington County, Vt. A fake? Many Vermont students have labelled this as an Indianized form of "equinox." If it were Indian, its Abnaki form would be, roughly, "place of the fog;" if Delaware, "where clothing is distributed;" if Chippewa, "place of the woman." See Naidni, and Retaw-erif.

Elandam ook ganop skitschwak the East Branch of the Penobscot River, Penobscot County, Me. Abnaki, "where the falls go down like steps." See Skitchewaug.

Elat Windham County, Conn. Nipmuck, "toward the hills." [?]

Ellala gwaga waysek Islands, Penobscot County, Me. Abnaki, "where freshets overflow."

Elligo Pond, Orleans County, Vt. If Indian, possibly from $A b$ naki, "a birch bark dish" or "bowl-shaped, or scooped-out." Some suggest "it is a good" pond, but Day effectively argues against this on grammatical ground. Also Eligo Scootloon.

Elligo-sigo now Black River, Orleans County, Vt. Abnaki, "good river"? This name, like Elligo above, defies translation. Perhaps Elligo-sigo means "a good place to plant (or return to) in the springtime."

Ellitegway gamek a stream connecting Lewey's Lake and Big Lake, Washington County, Me. Abnaki, "where two lakes are connected by a river," or "waterway which enters into another."

Emikus wassissek Ant Hills Island, Penobscot County, Me. Penobscot, "at the ants' nests."

Emmetinic Matinicus Island, Knox County, Me. This old form appears in the Jesuit Relations, I6Ir. See Matinicus.

Ennenstyacks same as Yennenstyaks, q. v.

Epawames Fairfield County, Conn. Mahican, "covering tree," or "bark-covering of a tree"[?] Variant, Apawamis. 
Epituse probably Fisherman's Island, Lincoln County, Me. Malecite, "sticking up, you have to go around."

Eptchedgewak Penobscot County, Me. Abnaki, "up and downstream currents (or opposing currents) meet here."

Epuk unikek Harrison's Island, Washington County, Me. Malecite, "island you have to go around."

Equies Brook and Swamp, Bristol County, Mass. Said to be from Mohegan, an abbreviation of "Tantoquieson's." See Tantaquidgeon.

Erascohegan Parker's Island, Sagadahoc County, Me. Abnaki, "a watching place."

Escoheag Village, Kent County, R. I. Quinebang (a Nipmuck subdivision), "this is as far as the fish-spearing goes," or "fork in the river where we spear fish." This is given as "three forks in the river," or "source of three rivers." Also, possibly from muskoheag, "red land," or from mickuckaskheek," a meadow." Variants, Escoheague and Eastrig. See Neastoquaheaganuck.

Escumbuit Island, Rockingham County, N. H. Micmac, "at the watching place."

Escutassis Brook and Pond, Penobscot County, Me. Abnaki, "brook trout," or "small trout."

Escutnagen Mopang Lake, Washington County, Me. Abnaki, "trout lake."

Eskweskwewadjo Bald Mountain, Katahdin Range, Piscataquis County, Me. Abnaki, "green tree covered mountain," or "evergreen hill."

Espowet Creek, Newport County, R. I. Narragansett? or Wampanoag? "at the large cove." See also Sapowet.

Esqui wamigook Spruce Island, Washington County, Me. Abnaki, "greens right down to the shore," or "place of green boughs."

Essick Stockton, Waldo County, Me. Abnaki, "clam place." These were soft clams, Mya arenaria, used for baking and steaming.

Eyamquittoo wau connuck Mohegan Village, New London County, Conn. Mohegan, "town or plantation of equals or brothers." This name was also given to Brothers Town, a New England Christian Indian settlement at Deansboro, N. Y. 
Fennapoo Bowditch Ledge, Baker's Island, Barnstable County, Mass. If this is Indian, it may be a corruption of a Wampanoag word, pennapu, meaning "sloping seat," or "lobster trap." However, since F is absent in Algonkian dialects, it may be from sennapu, "rock seat."

Foxon New Haven County, Conn. Mohegan? Pequot? Foxon, alias Poxon, witnessed deeds in I640. A possible derivation may be puck sun, "flat stone," or "level rock."$$
\text { - G - }
$$

Gaentake now Beaver Brook, Rockingham County, N. H. Pennacook, "red river."

Gallowa Several places along the Maine coast; exact locations now lost. Derived from the mythical Malecite bird (either a giant eagle or osprey) which could seize and carry away pigs, lambs, or infants. The Micmac had a similar roc-like bird, Kulloo-ok, which has traces in Malecite legends surviving in Washington County, Maine. Also Gallawass.

Ganawes Wood Creek, Rutland County, Vt. Mohawk, "the river."

Ganonwarohare Isle aux Têtes, north of Franklin County, Vt. Mohawk, "head is hoisted up." See Wdepsek.

Gansett Barnstable County, Mass. Wampanaog, "at the drinking place," or "at the well."

Gashee Pond, Plymouth County, Mass. Wampanoag, "warm pond." Also Gushee.

Gashekwaneh Lake Champlain near West Haven, in Rutland County, Vt. Mohawe, "big fish spear." Also Kohshahquahna.

Gassabias Lake, Hancock County, Me. Abnaki, "small clearwater lake."

Gawaysik see Pokey.

Gawenidakhe Isle la Motte, Grand Isle County, Vt. Moharek, "island alongside" (another island).

Gawenio Grand Isle County, Vt. Mohawk, "the large or beautiful island."

Gaywaysick Crawford Lake, Washington County, Me. Malecite, "large body of water." 
Gebeag Islands, Casco Bay, Cumberland County, Me. See Chebeague.

Geeyahoo wudchi oose taughannick New London County, Conn. Mohegan, "we come from beyond the distant (western) mountains." Said to be the origin of Taconic, q. v.

Genesag arumsis Lake, Washington County, Me. Malecite or Abnaki, Said to be from gan "bone" and arumsis (modern Abnaki, alamoos) "little dog;" hence "bones for the little dog"? Probably this is an Indian guide's joke; other roots give "clay as white as bleached bones."

Genesee Brook, Washington County, R. I. Onondaga, "beautiful valley," or Seneca, "there it has fine banks."

Gesquoquasset Barnstable County, Mass. Wampanoag, "sturgeon stream," or possibly, "at the wide rocks."

Godmorrocke Marsh, York County, Me. Probably an English word; but if Indian, possibly Natick, kuhpoke munnohke, "protected landing place" at island? or kuhpoke munnocks, "haven of the brant goose"?

Gonic Strafford County, N. H. Abnaki, "salmon spearing place."

Gotomska Bristol County, Mass. Mohegan, "big rocks" or "top of rocky cliff."

Grand Manan Channel, Washington County, Me. A composite French and Micmac term, "Big Island" Channel.

Greylock Mountain, Berkshire County, Mass. Greylock was the Waranoke chief of the Missisquoi refugees from Massachussetts and Connecticut, plus a few from New York, circa I723. Possibly his Indian name was Wabantep, meaning "White Head."

Guagas Stream, Hancock County, Me. Micmac, "rough stream" or "low flooded ground."

Guna Brook, Litchfield County, Conn. If Indian, perhaps a corrupted form of a Mahican word, gunnunk, "a tall standing tree."

Gunasquamecook ancient name for Indian village at Passamaquoddy, Washington County, Me. Probably Malecite, "long point harbor or beach."

Gungy Wamps an elevation near Groton, New London County, Conn. Mohegan, "high rocks." 
Guonitogou Connecticut River. Pennacook? Abnaki? "the Long River."

Gyobscot Point, Sagadahoc County, Me. Abnaki, "standing-up rocks place."$$
-\mathbf{H}-
$$

Hahkik Watpuk Seal Head, Fox Islands, Washington County, Me. Malecite, "seal's head."

Hakewamepinke Washington County, R. I. Narragansett "end of the dry field"? or "edge of the bank"?

Hammonassett Beach, Point and River, New Haven County, Conn. Hammonassett, "at the place of small islands or sandbars." Some say, "where we dig holes in the ground" for planting or for water. Variants, Hamonascitt, Homonasuk, Ammonassuk, etc.

Hanol menahanol Five Island Rapids, Penobscot County, Me. Abnaki, "five islands." See Nanenol Mehanol.

Haquessuk Kuppamuke Barnstable County, Mass. Wampanoag, "enclosure with stones around it" or "place of refuge at boundary brook" or "thicket at boundary stream."

Harco monco Pond, Worcester County, Mass. Nipmuck, "hook shaped fishing place"?

Harraseeket River, Cumberland County, Me. No satisfactory explanation available; perhaps not Indian. If Abnaki, perhaps "full of obstacles, small islands."

Hash kinnet Chaopket Nantucket County, Mass. Wampanoag, "at the great long cove" or "stone field hill summit place"?

Hasnebumskeag see Asnebumsket.

Hassacky Fairfield County, Conn. Siwanoy, "swamp, meadow." Variants, Hessekee, Hossockie, etc.

Hassamanisco Location unknown. Possibly Hartford County, Conn. Tunxis? "gravelly place" or "place of small stones."

Hassanamesit Worcester County, Mass. Nipmuck, "place where there is (much) gravel" or "at the place of small stones."

Hassawassuck Hartford County, Conn. Wangunk, "between two brooks." See Assawassuc.

Hassumps Road Middlesex County, Conn. Wangunk, "rock ledge," or "overhanging rocks." 
Hassunek Hill, Worcester County, Mass. Nipmuck, "at the stony place" (Perhaps a ledge; a rock-shelter in an overhang; a cave?) Eliot gives "a ledge of rocks."

Heagan Mountain, Waldo County, Me. Pennacook? "a dwelling." Possibly named for chief Sampson Hegan, I698.

Hesseke Meadow New Haven County, Conn. Quinnipiac, "meadow place" or "swampy land."

Hiawatha Lake, Norfolk County, Mass. Onondaga or Mohawk, "maker of rivers," circa I550.

Higganum Village and Reservoir, Middlesex County, Conn. Mohegan; this is the middle part of Tom heganom psk "quarry where we get stone for axes (tomahawks)."

Higganum ompos or Higgan ompsk Middlesex County, Conn. Mohegan? "tomahawk rocks." See Higganum.

Hinkum Pond, Rutland County, Vt. If Indian, possibly from Wampanoag or Narragansett, honck, "gray goose," the Canada goose.

Hoanantum Hill. See Nonantum.

Hobbomoc Pond, Worcester County, Mass. Natick, "evil spirit." Named for a Wampanoag sagamore who helped the Pilgrims in I620. Other spellings Hobomoco, Hobbamocke, Hoboomook.

Hobbomocka Hockomock Point, Arrowsic Island, Sagadahoc County, Me. Abnaki, "hellish place" or "hell gate."

Hoccanum Hampshire County, Mass. Mahican, "shaped like a hook" See Hockanum.

Hochelaga Camp, Grand Isle County, Vt. Moharek, "at the beaver dam." This is the ancient name of Montréal, Province of Québec, Canada.

Hockamock Swamp, Bristol County, Mass. Narragansett, "hookshaped place" or "land formation like a hook."

Hockananum Fairfield County, Conn. Paugussett, "place very much hook-shaped."

Hockanum Hill, Franklin County, Mass. Natick, "hook-shaped."

Hockanum River, Tolland County, Conn. Podunk, "a hook." Other spellings Hoccanum, Hoccanam, etc.

Hockanum Village, Hartford County, Conn. Podunk, "a hook." Other places and streams of same name in Fairfield and New Haven Counties. 
Hocquan Barnstable and Hampshire Counties, Mass. Natick, "a hook."

Hogamockock Point, Lincoln County, Me. No satisfactory translation of this old name. Perhaps Abnaki, "place of the palisade made of sharpened logs driven into the ground."

Homoganset Shore, Washington County, R. I. Narragansett, "at the fishing place." (This word may be the remainder of a phrase meaning in addition, "at low tide there are fresh water springs.") Also translated as "hunting grounds."

Honkamonk Pond, Litchfield County, Conn. Mahican, "hookshaped lake" or possibly "wild goose place" (?) See also, Oushankamaug "eel fishing place."

Hooksett Merrimack County, N. H. See Annahooksett and Onnahooksett.

Hoosac Tunnel, Berkshire County, Mass. Mahican, "rock place"? or "mountain rock"?

Hoosac Hill, Rockingham County, N. H. Mahican? "stone place"?

Hoosac Mountains, Bennington County, Vt. Mahican, "rim of the earthen kettle," or "stone place"[?]. Or perhaps Natick, abbreviated from nahoosic, "a pinnacle."

Hoosic River, Bennington County, Vt. Mahican, "rim of the kettle," or from the Natick name roussockhosick, "writinghouse." [?]

Hoosichisic Lake, Norfolk County, Mass. Natick, "place of the writings." Given also as Hoosicwhisic.

Hoosicwhisic Pond, Norfolk County, Mass. Natick, "at the place of the small kettle"? or "kettle runs almost dry"? or "place of the writings"?

Hosokey Meadow, Worcester County, Mass. Nipmuck, "marsh land" Spelled also Hosokie.

Housatonic River and Village, Berkshire County, Mass. Mahican, "beyond the mountain" Other spellings Hoestunnuc, Housetunnack, House of Tunnick, etc.

Housatonic River, Litchfield and New Haven Counties, Conn. and Berkshire County, Mass. Mahican, "at the place beyond the mountain."

Humarock Plymouth County, Mass.? Wampanoag? "shell place"? or "rock carving"? 
Humhaw Brook, Middlesex County, Mass. Natick, "filled up, brimming."

Hummock Pond, Nantucket County, Mass. From the name of a Nantucket chief, Nana Humacke.

Humolatski hegon Roque Bluffs, Washington County, Me. Abnaki, "many carvings on rocks."

Huson Tract, Strafford County, N. H. Modified Natick, "a stone." Also Husow, Whisow, Hussun and Husone.

Hyannis Village, Barnstable County, Mass. Wampanoag, from Anayanough's Place. Anayanough (or Iyanogh, "He who wages war") was a chief who lived here in the early I60o's.

Hyponeco Brook, Cheshire County, N. H. Pocumtuck[?] "on both sides of the falls."

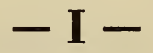

Iagoo Pond, Washington County, R. I. Chippewa, "boaster, or story teller." A corruption by way of Longfellow's Hiawatha.

Iannough's see Hyannis, "The Warrior."

Igowam see Agawam.

Indian Joe see Sozap Nebees.

Indian Molly see Mali Bowk.

Irocoisia on ancient maps this name was affixed to the Champlain Valley, embracing the western parts of Rutland, Addison, Chittenden, Franklin Counties and all of Grand Isle County, Vermont. See Iroquois.

Iroquois Lake, Chittenden County, Vt. Algonquin, from Iriakhoire, plus French suffix -ois, "real adders." This term of hatred was applied by the Algonquians to the Iroquois people.$$
-\mathbf{J}-
$$

Jabish River, Hampshire County, Mass. If Indian, perhaps from Mahican, "swarms of flies." Also given as "boundary place," or "separated hill," cheppi-ach.

Jamaica Plain and Pond, Suffolk County, Mass. Natick, "beaver." Jamaica Windham County, Vt. Natick, "beaver."

Jebucto Strafford County, N. H. Abnaki [?] from the name of an Indian who lived at Chebeague in 1680. 
Jeebege Island. See Chebeague.

Jigwallick Marsh along Lewis Creek, Addison County, Vt. Modified Abnaki, "frogs." See Chickwolnepy.

Jimskitikuk Stillwater Branch, Penobscot County, Me. Abnaki, "big, still water."

Jossawilock see Ashuelot.

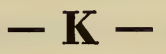

Kabaumkeag Washington County, Me. Malecite or Abnaki? "stopped up by sandbars."

Kachewalunk Pond. See Uncache walunk, "acorn place."

Kachkesset Nantucket County, Mass. Wampanoag, "where grass is cut or mowed" or "green place."

Kadesquitt see Kenduskeag and Condeskeag.

Kaghsk-i-binday a camp ground near Brewer, Penobscot County, Me. Abnaki, "cedars."

Kahgognamock (Black River, West tributary of the Penobscot) Penobscot County, Me. Abnaki, "big bony fish place."

Kahkoguamook Lake. Same as Caucomgomoc Lake.

Kahnonahjik White Squaw Island, Penobscot County, Me. Abnaki, "long island."

Kahus weskeete heenouk Washington County? or Aroostook County? Me. Micmac, "muskrat eaters," or "muskrat people," a term of derison applied to the Malecite.

Kahwehniyo same as Gawenio, q. v.

Kahyonhes the Otter Creek, Rutland and Addison Counties, Vt. Mohawk, "long stream."

Kamankeag Pond, Franklin County, Me. Abnaki? or Malecite? "near the weir," or perhaps "near the submerged weir."

Kamesset Point, Dukes County, Mass. Wampanoag, "at the place of great fish;" also given as "pine place."

Kamouraska County, Province of Québec, Canada. This name is applied to several places in northern New England, notably Windham County, Vt. Abnaki, "birch bark here."

Kanakolus Bay, Waldo County, Me. Abnaki, "long flame"?

Kananghetne a point near Damariscotta, Lincoln County, Me. Abnaki, "a sharp turn." 
Kancamagus Mountain, Grafton County, N. H. Named for a Pennacook chief, alias John Hodgkins, I648. Possible meaning, "plenty of small fish."

Kangatsigwi Kearsarge Mountain, Merrimack County, N. H. Abnaki, "very steep."

Kanionra Chimney Point, Addison County, Vt. Mohawk, "smokehole at the top" or "chimney-hole."

Kanondoro The Narrows at Thompson's Point, Chittenden County, Vt. Mohawk, "narrow portal."

Kansas Village, Bennington County, Vt. Kaw, "the wind," or "the south wind."

Kanyagwaronton ridges of hard rock, Franklin County, Vt. Mohawk, "flint mounds."

Kanyatatakwaronte Lake Champlain (north of the narrows), Chittenden County, Vt. Mohawk, "the bulge in the waterway." Variant, Ganyadaragwa-ronde.

Kaoosi Menahan Cow Island, Penobscot County, Me. EnglishAbnaki, "cow's island."

Kaouasaki Grand Lake, Schoodic System, Washington County, Me. Penobscot-Abnaki, "place of gulls." This name is found on Father Aubéry's map of I7I5.

Kappowongamick Hampden County, Mass. Mahican, "place shut in by the bend in the river." Also Kuppowankomuck.

Karsaootuk Stream, Aroostook County, Me. Abnaki, "pine river."

Kaskaashadi an ancient name for the Merrimack River, N. H. Pennacook or Old Abnaki for "deep obstructed, broken water."

Kaskactchawack River, now the Black River, Windsor County, Vt. Abnaki, "at or near the mountains with steep sides."

Kaskekouke now the Hoosac River, near Pownal, Bennington County, Vt. Abnaki, modified by French, "steep place."

Kaskoi Menahan Grand Isle County, Vt. Abnaki, "heron island."

Kaskoo naguk Mark Island, Knox County, Me. Abnaki, "crane island."

Kassanum ganumkeag a "rips" in a stream near Lincoln, Penobscot County, Me. Abnaki, "rapid current over coarse gravel."

Katachinoc Penobscot County, Me. Abnaki, "big mountain place"? 
Katahdin Mountain, Piscataquis County, Me. Abnaki, "the principal mountain." Other spellings include Ktaahden, Ktahdin, Taddon, etc.

Katama Bay and Point, Dukes County, Mass. Wampanoag, "the great fishing place," or perhaps "crab place," Katawamacke?

Katamaug Dukes County, Mass. Wampanoag, "the great fishing place."

Katananuck Dukes County, Mass. Wampanoag, "crab fishing place"?

Katawamkeag the Great Landing Place, Lermond's Cove, Knox County, Me. Abnaki, "the great landing place," or "much sand exposed when the tide is out."

Katawamskiway see Katawamkeag and Kawap skitchwak.

Kathahnis River. See Cathance.

Katomuckett Island, Dukes County, Mass. Wampanoag, "great fish place," or "great spring place."

Katopskonegan Falls and Deadwater, the West Branch of the Penobscot River, Piscataquis County, Me. Abnaki, "long portage around rocks" or "portage around large rocks."

Kattenanit Suffolk County, Mass. Named in honor of Job Kattenanit, a Natick preacher at Hopkinton circa I676. Probable meaning, "great expanse of sea" or "ocean."

Kawakussaki Lake, same as Kaouasaki, q. v.

Kawamasohkakannit Hill, Plymouth County, Mass. Wampanoag, "at the field with the pines and the brook."

Kawap skitchwak West Branch of Machias River, Washington County, Me. Abnaki, "rough, rocky rapids and falls," (like steps?)

Kawassentekwa bare, rough places along the Connecticut River, Windham County, Vt. Abnaki, "it falls into the river because of the high wind."

K'chenamenahan Grand Isle County, Vt. Abnaki, "the principal (or large) island." Also found as Kitchee Menan, Gitshee Menan, etc.

K'chi mugwock i menahan Orson Island, Penobscot County, Me. Abnaki, "big bog island."

K'chi Muskegw Windham County, Vt. Abnaki or Pennacook? "great (or principal) meadow." 
K'chi Nayok same as Chenayok, q. v.

K'chi penabsq' mananook Treat Island, Penobscot County, Me. Abnaki, "big sloping ledges at the islands."

K'chi Pontegok Strafford County, N. H. Abnaki, "place of great river falls."

K'chi Ponteguh Cheshire County, N. H. Abnaki, "great falls in the river," the Connecticut River at Walpole, opposite Bellows Falls, Vermont.

K'chi p'saganum the stream connecting Big Lake and Lewey's Lake, Washington County, Me. Abnaki, "where they split boards to make skids for canoes." (so as to protect the bottoms from gravel in the streams).

K'chi punahaquot a brook near Sipp Bay, Washington County, Me. Malecite, "principal place where tomcods come to spawn."

Kearsarge Mountain, Merrimack County, N. H. Abnaki or Pennacook, "pointed mountain"' ?]. This may not be an Indian name; if it is, translations vary from "proud, selfish," or "high place," to ki saage, "land that is harsh, rough, difficult."

Kearsarge Village, Carroll County, N. H. See Cowissewaschook, also Kearsarge, above.

Kebec the Narrows or Chops on Merrymeeting Bay, Sagadahoc County, Me. Abnaki and Micmac, "closed, or plugged." Same root-meaning as Québec, and Capawack, q. v.

Kebo Mountain, Hancock County, Me. If Indian, perhaps Abnaki, from kiba, "I fall"' ?].

Kebumkewis Second Lake, Washington County, Me. Malecite, "little mud bar lake."

Kecheachy same as Cocheco, q. v.

Kedinket Island, Washington County, R. I. If Indian, perhaps Narragansett, "a ship," or "on the ship," or "(it resembles) a little ship."

Kedumcook The Hook, Hallowell, Kennebec County, Me. Abnaki, "shallow, gravelly ford."

Keecamewett Bristol County, Conn. See Kickamuit.

Keeck Pond Providence County, R. I. Narragansett, "kettle pond" from ohkuk, "a kettle."[?]

Keekamanset Spring, Bristol County, Mass. Narragansett, "at the great valley." 
Keekamoochaug see Kekamoochaug.

Keekamuit Neck, Bristol County, Mass. Narragansett, "great spring."

Keekomkwak places along Barton River, Orleans County, Vt. Abnaki, "abode of sucker fish."

Keektawhank Berkshire County, Mass. Mahican, "rapid stream," or "dashing current."

Keenaht Nassick now Treat Webster Island, or French Island, Penobscot County, Me. Penobscot-Abnaki, "steep island."

Keesaug Brook, Carroll County, N. H. Abnaki, "principal outflow."

Keesequechan Bristol County, Mass. Narragansett, "swift water." Also Quequechan.

Keetadoganapskwa Seebis Whetstone Brook, Windham County, Vt. Abnaki, "brook where we get whetstone."

Keetahden Mount Mansfield, Chittenden County, Vt. Abnaki, "the principal mountain."

Keetutenny Litchfield County, Conn. Mahican, "principal town."

Keewaydin Trail, Addison County, Vt. Chippewa "north," "people of the north," or "north wind."

Keght Niganish Whiting River, Washington County, Me. Malecite, "the principal fork."

Keheketooksook Pond, Litchfield County, Conn. Mahican? Paugussett? possibly "outlet of large stream."

Kehtah Hanit or Kehtuh Hannupog the Atlantic Ocean and large bays along the coast. Natick, "principal salt bays." Kehtanit or Keihtannit was anciently the title of "The Lord God."

Kehtitanunk perhaps present-day Cuttyhunk, q. v.

Kejigigilhasis a camp site near Lake Fairlee, Orange County, Vt. Abnaki, "a chickadee."

Kekamoochaug Worcester County, Mass. Nipmuck, "where the earth trembled."

Kekamowadchaug Worcester County, Mass. Nipmuck, "mountain where the earth trembles."

Ke Kepan Agliesek the ancient name for Arrowsic Island, Sagadahoc County, Me. Abnaki, "(island) which almost closes the channel." The French fought the English here in I723. 
Keketticut see Titicut. Also spelled Keticut.

Kekuttokunta Hill Plymouth County, Mass. Wampanoag, "conference place," or "parley place."

Kemboes Kisek ancient name for Dyers Neck, Lincoln County, Me. Probably Abnaki, "at the grassy place, near a network of streams."

Kenduskeag Village and Stream, Penobscot County, Me. Malecite "eel weir place." Before being modified by the English, the name was pronounced Katesquit.

Keneba-eg River. See Kennebec.

Kenemicut see Conimicut.

Kennebago Lake and Mountains, Franklin County, Me. Abnaki, "long pond," or "large lake."

Kennebec County, Maine. Abnaki, "long level water without rapids," or "long quiet water." Some students have wrongly traced this to the Chippewa word kenabeek, "serpent."

Kennebec River, Maine. This stream anciently was called Aransoack or Orantsoack, "rapids up-river," from Moosehead Lake to Norridgewock. Below Skowhegan it was known as Canebas or Kenebas, "long water," as far as Merrymeeting Bay. From there to the sea, Sagadahoc, "where the river flows out."

Kennebunk Pond, River and Village, York County, Me. If Abnaki, perhaps, "long sandbar;" if Micmac, "long cut (ditch) banks."

Kenosha Lake, Fairfield County, Conn. Mahican, "pickerel" or "pike."

Kenoza Lake, Essex County, Mass. Pennacook, "a pike” or "a pickerel."

Kenunck Pacooke Brook, Litchfield County, Conn. Mahican, "where the body of water bends or turns."

Keoka Lake, Oxford County, Me. Abnaki, "where they get (red) earth for pots" ? or if a transfer from Keuka, N.Y., Iroquoian, "canoes pulled out of water."

Kepamkiak near the Campobello Ferry at Lubec, Washington County, Me. Malecite, "stopped-up by a gravel bar."

Kequassagansett Pond, now Gates Pond, Worcester County, Mass. Natick, "at the place of the principal wells," or "open fields place." 
Kermes Plymouth County, Mass. See Alkarmus.

Kescayogansett Pond, Barnstable County, Mass. Wampanoag, "return to fishing place"? or "high, rough place"?

Kesebem Lake, Aroostook County, Me. Old Abnaki, "chief, or principal, lake."

Kesickamuck Washington County, R. I. Narragansett, "stony fishing place" or "stone we stand on when fishing." Variant Kesikomuck, possibly "wide beach."

Kesiog Pond, Hampden County, Mass. If Nipmuck, "place of briars or thorns." If Pennacook, "difficult place." See Kearsarge. Also commonly Siog Pond.

Kessalogesso Modik Five Island Rapids, Penobscot County, Me. Abnaki, "where there is a swift water current."

Kestaubeunk Fairfield County, Conn. Mahican, modified by Dutch, "principal camping place." (Probably the same as Kestaubauck in Westchester County, N. Y.).

Kestokas Field, Nantucket County, Mass. Wampanoag, "at the great tidal creek."

Keta Kouan the great portage at Waterville, Kennebec County, Me. Abnaki, "principal crossing."

Ketaumet see Cataumet.

Ket h'nik Denny's River, Washington County, Me. Malecite, "principal fork."

Keticut Plymouth County, Mass. Wampanoag, "on the great river."

Ketsi M'weskw several places in Maine. Old Abnaki, "Great Good Spirit; God." Modern Abnaki, K'chi Niwaskw.

Ket takone adchu Berkshire County, Mass., and Bennington County, Vt. Mahican, "great woody mountains," the Taconic range.

Kettigwewick West Branch of Penobscot River, Penobscot County, Me. Abnaki, "place of the great stream."

Ketumscut off New London County, Conn. Mohegan, "place of the great rocks." See Catumb.

Kewaydin Lake, Oxford County, Me. Chippewa, "north" or "northpeople."

Kewoutaquak see Cowwautatuck, "land of pine woods." 
Kiasobeak now Nicatowis Lake, Hancock County, Me. Abnaki, "clear water lake."

Kickamuit River, Bristol County, R. I. Narragansett, "at the large spring." (This is the most common of sixteen listed spellings).

Kickemus Path, Middlesex County, Conn. Pennacook? "great moose." From an Indian's name; the father of Mamooson.

Kikon a locality north of Bradford, Orange County, Vt. Abnaki, "a field."

Kinabsk atnek now Mount Waldo, Waldo County, Me. Abnaki, "steep rocky mountain."

Kineo Mountain, Piscataquis County, Me. Abnaki, "sharp peak." (This mountain is said by Indians to be Moose Rock, the petrified body of a moose slain by Glooskap, a wonderworker). There is another Kineo in Grafton County, N.H.

King Philip Mountain, Hartford County, Conn. King Philip was the English name for Metacom, Metacomet, or Pometacom, son of the great Massasoit. Metacom led an Indian insurrection in New England, I674-I676.

King Philip's House; see Pometacomet.

King Philip's Rock, Norfolk County, Mass.; see Metacomet.

Kinkajou on the Allagash River, Aroostook County, Me. Micmac, "wolverine." from gigwadju (literally, "beast of the mountain.") This animal was believed to have supernatural powers. See Lunksoos.

Kinnicum Pond, Rockingham County, N. H. Pennacook, "the long one,"? or Natick, "a mixture"? Various plant leaves, plus red osier bark, mixed with tobacco, for smoking.

Kishkituckock Litchfield County, Conn. Mahican, "place or land near the bank of the stream," or "by the river side." Variants, Keshkiscotuk and Kiskotock.

Kisnop Brook, Litchfield County, Conn. Mahican, "principal outlet"? or "big rock"? See Sconnoups.

Kissacook Hill, Middlesex County, Mass. Pennacook, "stony place," ? or "principal stream"?

Kissenaug New Haven County, Conn. Paugussett, "place of big stones."

Kitachanniqut Kent County, R. I. Narragansett, "principal long place," or "principal long beach." 
Kitamuckqut Washington County, R. I. Narragansett "on the mainland opposite." Variants Kitacka muck nut, Kittack quamuck opelle, Kittacka mucket, Kittackquam uckquiet.

Kitchee Ponteguh rapids on the Connecticut River, Windham County, Vt. Abnaki, "the principal rapids (or falls) in the river."

Kitemaug west bank of Thames River, New London County, Conn. Mohegan, "the principal fishing place." Also Kittemaug.

Kittansett Point, Plymouth County, Mass. Wampanoag, "the great expanse of water; the ocean," also, "at the great point in the sea."

Kitteaumut Plymouth County, Mass. Wampanoag, "principal fishing place"? or "large spring"?

Kittituck Stream, now Blackstone River, Worcester County, Mass. Nipmuck, "at the large (or principal) tidal river."

Klaganissecook Falls, Mattawamkeag River, Penobscot County, Me. Old Abnaki, "place of the noisy little stream." Other roots indicate, "little opening, like a door."

Klondike Essex County, Mass., and Brook, Aroostook County, Me. Athapaskan, "hammer-water," referring to driving stakes into river bed so as to make a salmon trap.

K'mokadich Moosabek Reach, Washington County, Me. Malecite? Abnaki? "plenty of vile insects." Porpoises were tried out here for their oil; perhaps the stench and the decaying flesh attracted flies.

K'noonaghek Argyle Boom Island, Penobscot County, Me. Abnaki, "long island."

Koaktekauk Essex County, Vt. Abnaki, "river at the place of the pine trees." See Coaticook.

Koategw Carroll County, N. H. Abnaki (literally), "Pine River."

Kobossee Island, Penobscot County, Me. Abnaki, "a sturgeon."

Kobpakommocket Plymouth County, Mass. Wampanoag, "refuge place, or hiding place." Probably in a swamp. Also Kowbissowonket.

Kochekok River, Essex County, Mass. Pennacook, "place of the rapid current," "big eddies," or "great kettle."

Kochisuk exact locality unknown; probably several such along Passadumkeag Stream, Penobscot County, Me. Abnaki, "eel-weir place." 
Kodaak Wadjo Mount Washington, Coös County, N. H. Abnaki, "summit of the highest mountain."

Kodttukoet Plymouth County, Mass. Wampanoag, "at the summit of a hill."

Koessek now Vernon, Windham County, Vt. Pennacook, "at the place of the pine trees." White captives from Massachusetts were brought here by Indians in King Philip's War,I675-1676. (Koessek was part of Squakheag). See also Coassit.

Koessinock Essex County, Vt. Pennacook, "place of the pines."

Koghserage northern Vermont and New York. Mohawk, "it is winter" (An old Choreographic Map of this country is marked "Koghserage Ancient Beaver Hunting Grounds of Six Nations.") Variant, Cochserage.

Kogwees a camp site on Lake Fairlee, Orange County, Vt. Abnaki, "a little porcupine."

Kohanza Lakes, Fairfield County, Conn. If Indian, perhaps the name is garbled Mahican, "pike fish."

Kohshahquahna see Gashekwaneh.

Kok adjeweem gwasebem Roach Pond, Piscataquis County, Me. Abnaki, "kettle (shaped) mountain lake."

Kok adjeweem gwasebemsis Spencer Pond, Piscataquis County, Me. Abnaki, "little kettle-shaped mountain lake."

Kokadjo Mountain and Village, Little Spencer Mountain, Piscataquis County, Me. Abnaki, "kettle mountain." At Moosehead Lake, Glooscap killed a large moose, which became Mount Kineo, then pursued a calf. To lighten his burden he threw down his kettle, which became Kettle Mountain.

Kokohas wantepek a promontory at the western entrance to Penobscot Bay, Lincoln County, Me. Abnaki, "at the owl's head;" hence the English name Owl's Head.

Kolelemook Lake, Sullivan County, N. H. Abnaki, "shining pond."

Kollegewidgewock Hancock County, Me. Abnaki, "mixed salt and fresh water rapids." See Kuladam itchwan.

Konesanouskek St. George Peninsula, Knox County, Me. Abnaki, "at the long rocky place."

Kongscut Mountain, Hartford County, Conn. Wangunk, "at the high place" or "high, rocky place." 
Konikey Cliff, Plymouth County, Mass. Wampanoag, "long rock" or "high rock." But see Kodttukoet.

Konkapot River, Litchfield County, Conn. Named for a Stockbridge Mahican chief; possibly his name meant "long spout."

Konkapot Village, Berkshire County, Mass. Mahican, from the name of a Stockbridge chief, Capt. John Konkapot, I775.

Konomoc Lake, New London County, Conn. Perhaps Pequot, "(plenty of) lamprey-eels (long fish) here." See Taubakonommok.

Koomasabunkawitt Plymouth County, Mass. Wampanoag, "great or principal herring, alewive, or mossbunkers fishing-place." (Mossbunkers are menhaden, used for fertilizer).

Kotget Nantucket County, Mass. Wampanoag? "a piece of land"? or "a heap"? An old name for Muskeget Island.

Kowawesuck-pumotaash Plymouth County, Mass. Wampanoag, "long line, or field of small pines."

Kozoapskwa Thompson's Point, Chittenden County, Vt. Abnaki, "long stony place."

K'seusk-i-naghassik Little Hemlock Island, Penobscot County, Me. Abnaki, "black growth on small island." Lumbermen call hemlocks and another evergreens "black growth."

K't-ahguantek the Grand Landing Place, Knox County, Me. Abnaki, "the principal landing place."

K'tchi-peskwahonda Fort Knox, Waldo County, Me. Abnaki, "big guns."

K'tolbeh-i-ahmikenaqu-naghek Namokanok Island,Lincoln County, Me. Abnaki, "snapping turtle shell (shaped) island."

K'tolbewik Fourth Lake Machias, Washington County, Me. Abnaki, "snapping turtle lake."

Kuhtuhquetnet Dukes County, Mass. Wampanoag, "at the great place on the island."

Kuladam-itchwan Blue Hill Tide Falls, Hancock County, Me. Abnaki, "mixed salt-and-fresh water rapids." When the tide is running out, the stream is less salt than the ocean; as the tide rises, the stream becomes almost as salty as the Atlantic.

Kuncanowet Hills, Merrimack County, N. H. Natick, "near the long sharp places (ridge of hills)." 
Kunckiunkqualluck Hampshire County, Mass. Pocumtuck, "rolling hills land," or "high round hills place."

Kunckquachu now Mount Toby, Hampshire County, Mass. Mahican, "very high mountain."

Kuppi komuk Swamp, New London County, Conn. MoheganPequot, "closed place; enclosure; place of refuge."

Kuppowonkunok Hampshire County, Mass. Pocumtuck, "at the enclosed place near the bend."

Kushequa see Gashekwaneh.

Kuskaidzowak the Black River, Windsor County, Vt. Abnaki, "broad current."

Kussompskauk New London County, Conn. Mohegan? or Narragansett? "place of hot stones." Possibly a clam-baking or oyster-roasting site. See Pissepunk.

Kussus Kook Lake, Penobscot County, Me. Abnaki, "stony falls"?

Kusumpe Pond; see Cusumpe, and Kussompskauk.

Kutshamakin Hill, Norfolk County, Mass. Natick, "big plume, "or great feather." Also Kuttis-amequin, "cormorant's plume." Named for the sachem of Dorchester, I643.

Kuttanoo New Haven County, Conn. Quinnipiac, "the sea." Long Island Sound.

Kuttis-amequin see Kutshamakin.

Kuttisog New London County, Conn. Narragansett? "cormorants."

Kuttoowaug New London County, Conn. Mohegan? "speaking place." If Kuttoowang, "bend in the sea; a bay" [?]

Kuttutuck the Blackstone River, Windham River, Conn. Nipmuck, "the principal river," or "the great river." Also Kuttatuck, Kittituck, Kuttuck, Quttuc, etc.

Kwagustchusk see Quagachusque.

Kwanok sagamik Loon Lake, Penobscot County, Me. Abnaki, "lake that comes to a point."

Kwaykway-nahmak Long Reach, Penobscot County, Me. Abnaki, "big long fish place," possibly "sucker fish place."

Kwedawi-manwik Hermon Pond Inlet, Penobscot County, Me. Abnaki, "downstream, rivers not far apart."

Kwel-bedjwanosik on Stillwater Branch, Penobscot County, Me. Abnaki, "where currents turn as they meet." 
Kwenosakek mouth of Lamoille River, Chittenden County, Vt. Abnaki, "at the place of pike-fish."

Kwesahkamegus see Quisquamego.

Kwetahwamkitik near Pea Cove, Penobscot County, Me. Abnaki, "long stream, sandy bottom."

Kweu-euk tonoonk hegan the Moose River, Aroostook County, Me. Abnaki, "snowshoes."

Kwikwimes-witicook Marsh River, Waldo County, Me. Abnaki, "black duck stream."

Kwini teguh the Connecticut River. Abnaki, "the long river."$$
-\mathbf{L}-
$$

Lacasse come cook Aroostook County, Me. Abnaki, "place of bark cabin." Same place as Allagask-wigamook, Allagash, etc.

Lakeutta Nantucket County, Mass. Lenape? "sandy place," from lekau, "sand," and modified locative $u t$.

Lampopeag Branch, Aroostook River, Aroostook County, Me. Abnaki, "(crooked) like-a-rope stream."

Lapomique Branch (of Aroostook River), Aroostook County, Me. Abnaki, "crooked, like a rope."

Lashaway River, Worcester County, Mass. Nipmuck, "between." See Nashua.

Liswoosuckit Nipmuck modification of Niswosaket, now Woonsocket?

Loshtock the Long River, St. John's River, Aroostook County, Me. Abnaki? Malecite? "wide, shallow, shining."

Louisquisset Pike, Providence County, R. I. Nipmuck, "at the meeting place." Other spellings (more than 20) include Locasquisset, Loquassuck, Loquasquocit, etc.

Lunksoos Mountain, Pond and Stream, Penobscot County, Me. Abnaki, "a catamount."

\section{$-\mathbf{M}-$}

Ma-adameg Falls, Knox County, Me. Abnaki, "alewives place." Ma-ada-ouamkeag same as Mattawamkeag, q. v.

Ma-adom-cog Island, Sagadahoc County, Me. Abnaki, "alewives place," or perhaps "shad place"[?] 
Maanepes Brook, Fairfield County, Conn. Mahican, "sluggish stream," or "slow water."

Maanexit Windham County, Conn. Nipmuck, "path," or "gathering;" also "place of meekness." Also a river in Worcester County, Mass.

Maasbaak Mossback Meadow, Orange County, Vt. Abnaki, "much water place," or "overflowed."

Mabautuantucksuck Hill, Hartford County, Conn. Wangunk, "outlet of rushing river"? or "waters rushing past wooded hill"?

Machaquamaganset Washington County, R. I. Eastern Niantic? or Narragansett? "place of big beach wells," i. e., hollow logs sunk in sand so that they fill up with fresh water at low tide.

Machegony ancient name for Portland, Cumberland County, Me. Micmac, "shaped like a large knee." Also Machigonne.

Machemoodus East Haddam, Middlesex County, Conn. Wangunk, "there is a bad noise." Variants Machamadoset, Machmadonset, and Machamoodus.

Machemux Fairfield County, Conn. Paugussett? possibly a corruption of machequox, "a belt of wampum," or perhaps from the name of Mahackemo, Sachem of Norwalk?

Machepaconapunsuck Washington County, R. I. Eastern Niantic? "Big enclosure near falls in the brook"? or "big bank near brook falls"?

Machewisis Falls, Penobscot County, Me. Abnaki, "bad little falls."

Machias several place names in Maine, particularly the Lake System in Aroostook County, are known by this name, from Abnaki, meaning "bad little falls."

Machimucket Brook, Windham County, Conn. Nipmuck, "bad fish here," or Narragansett, "it stinks" or perhaps "great fishing place" ? See Mashamoquet.

Machipiscat New London County, Conn. Eastern Niantic, "stony path" or "rocky trail."

Machipscat Kent County, R. I. Narragansett, "a stony path" or "rough place."

Machlich-Nagook Hardwood Island, Penobscot County, Me. Abnaki, "growth of hardwoods on this island." 
Machuset Brook, Plymouth County, Mass. Wampanoag, "at the hill."

Mackinackock a small cove near Thompson's Point, Chittenden County, Vt. Abnaki, "the abode of (small) turtles."

Macomet Nantucket County, Mass. Wampanoag, "at the meeting place," or "at the little house."

Maconessett Neck, Plymouth County, Mass. Wampanoag, "barren place."

Macwahoc Lake, Stream and Village, Aroostook County, Me. Abnaki, "wet ground, bog." Also Macwakook.

Macwahock Brook, Piscataquis County, Me. Abnaki, "wet ground, bog."

Madagam Lake, Penobscot County, Me. Abnaki, "(soon at) the end of the lake." But see Madagamus.

Madagamus Hill, Waldo County, Me. Abnaki, "snowshoe tracks" left by Glooskap when he jumped across Penobscot Bay, in pursuit of a moose calf.

Madagascal Pond and Stream, Penobscot County, Me. Abnaki, "meadows at mouth." But see Madagascar.

Madagascar the Brushwood section of Bradford, Orange County, Vt. Abnaki, "poor game, bad meat," that is, rabbits in summer. But see Madagascal.

Madahondo apskwa Devil's Den, Wright's Mountain, Orange County, Vt. Abnaki, "the devil's rocks."

Madahumuck Bay, Falls and Point, Lincoln County, Me. Abnaki, "alewives caught around here."

Mada-Kamigosek modern Indian name for Camden, Knox County, Me. Penobscot-Abnaki, "big ridge place."

Madaket Nantucket County, Mass. Wampanoag, "at the end of the land," or Narragansett, "cloudy, overcast." Also Madaquet.

Madamascontee modern Indian name for Damariscotta, Lincoln County, Me. Abnaki, "abundance of alewives."

Madamaswok Cold Stream, Penobscot County, Me. Abnaki, "alewives."

Madam Bettox Mountain, Knox County, Me. Abnaki? perhaps "alewives' pond," name transferred to nearby hill ? The pond is now known as Chickawaukee. Other spellings Medambettox, Medambetek, etc. 
Madamiscomtis River, Blackman Stream, Penobscot County, Me. Abnaki, "plenty of alewives."

Mada-waniganook Carry, French Island, Penobscot County, Me. Abnaki, "(where we put our canoes into the water) at the end of the portage."

Madawaska Lake, River and Village, Aroostook County, Me. Micmac, "where one river runs into another," or Abnaki, "where there is much hay"?

Maddequecham Pond, Nantucket County, Mass. Wampanoag, "foothill place."

Madi tegou now the White River, Windsor and Orange Counties, Vt. Abnaki, "bad useless river," as on Father Aubéry's map, I7I5. The name survives in Mad River (Washington County, Vt.), reached by portage from the White River.

Madnaguk Island, Lincoln County, Penobscot County, Me. Abnaki, "big island."

Madombedeag Bay, same as Medomac, q. v.

Mad River see Madi tegou.

Madunke-hunk Webster Stream, Penobscot County, Me. Abnaki, "far away, at the height of land, stream."

Magaenak Middlesex County, Mass. Natick, "where the Mohawks came," or "wolf-place." An ancient village site.

Magallo River, Rockingham County, N. H. Malecite, "caribou," "the shoveler," see below.

Magalloway, Mountain in Coös County, N. H. and river in Oxford County, Me. Malecite, "the shoveler," i. e., the caribou, which gets its food by shoveling snow to one side with its hoofs.

Magasewanussuck Falls, Lincoln County, Me. Abnaki, "great fresh water falls."

Magawok Bay, Cumberland County, Me. Abnaki, "a bog swamp, or lowland." Also Magwaak.

Magegunuck Neck, Cumberland County, Me. Micmac? "a big knee or elbow;" that is, shaped like a bending joint. See Machegony.

Magesemanussick Falls, York County, Me. Abnaki, "great fresh water falls."

Magnus see Quaiapen. 
Magomiscock Hill, Worcester County, Mass. Nipmuck, "place of the large rock," or possibly "place of waste land."

Magonck Great Neck, New London County, Conn. Mohegan, "grove of large trees." Also Magunk.

Maguaguavic the Little St. Croix River, Aroostook County, Me. Malecite, "abode of big eels." Also Mekkatewek.

Magumkaquog Middlesex County, Conn. Natick, "place of the gift or offering"? or "place of great trees"?

Maguncaquog Middlesex County, Mass. Natick, "place of the gift."

Magunco Hill, Middlesex County, Mass. Natick, "gift or grant."

Magunhukquok Middlesex County, Mass. Natick, "place of the offering."

Magunk see Magonck.

Magunkahquog Middlesex County, Mass. Natick, "at the place of the gift or grant."

Magunkook Hartford County, Conn. Wangunk, "place of large trees."

Magunquaquog Middlesex County, Mass. Natick, "place of the gift or grant;" but some sources give "place of great trees."

Magunticoog same as Medumcook, q. v.

Magurrewock Lakes, Washington County, Me. Malecite, "at the place of the shoveler;" i. e., the caribou.

Magus Hill, Norfolk County, Mass. Nipmuck, for John Magus, a Nipmuck chief, who deeded Hardwick land in I686.

Magwak see Mohawk.

Magwin tegwak Lincolnville Beach, Knox County, Me. Abnaki, "choppy seas."

Magwonkkomuk Middlesex County, Mass. Natick, "at the enclosed place of the grant," or "gift of a plantation."

Mahackemo Fairfield County, ${ }^{-}$Conn. Pangussett, "fire consumes him"? Named for a Sachem of Norwalk.

Mahantic a swamp near Newton, Fairfield County, Conn. Paugussett, "cedar or spruce trees."

Maharnes Hill, Fairfield County, Conn. Mahican, "the one who gathers them together." Possibly the same as Mianus, Mayannoes, etc. 
Mahican Indians, often confused with the Mohegan, q. v. Their home extended from the upper Hudson Valley in New York into Vermont and sections of Connecticut. Their descendants are known as Stockbridge Indians, a group with whom they allied in I736. Their name means "wolf;" they figure prominently in The Last of the Mohicans, by Cooper.

Mahkahkahssen Cliff, Fairfield County, Conn. Mahican? Delaware? "the (very) large rock."

Mahkeenac Lake, Berkshire County, Mass. Mahican (Stockbridge), "the abode of the Mahicans." Also Maheekanac.

Mahkonlahgok "The Gulf," near Katahdin, Piscataquis County, Me. Abnaki, "gap in the stream," or "hole in the river."

Mahmansuck at Billings Lake, New London County, Conn. Mohegan, "the brook connecting the ponds."

Mahmunsqueag Windham County, Conn. Nipmuck, "gravelly place."

Mahnagwanegwa Sebem Rainbow Lake, Penobscot County, Me. Abnaki? translation of the English name, "Rainbow Lake"? (manawakon, "small rainbow").

Mahnekebahntic Caribou Lake, Piscataquis County, Me. Abnaki, "big cedar swamp near the falls in the river"? or, "where they got cedar bark for packs."

Mahoosuc Range, Coös County, N. H., and Oxford County, Me. Abnaki, "abode of hungry animals," possibly bears or wolves (or both). But a Natick word nahoosic means "a pinnacle," i. e., mountain peak. It has been suggested that the Abnaki term possibly refers to Mohegan-Pequot refugees who fled from Connecticut to Maine following the Pequot War of 1637.

Mahsunquammasec the Waits River, Orange County, Vermont. Abnaki, "big river at outlet," an apt description of the Waits as it flows into the Connecticut.

Mahtawesweskeetehenoouk A name given to the Micmac of Nova Scotia, who briefly conquered and occupied the coast of Maine. Malecite, "porcupine eaters."

Mahutchet Plymouth County, Mass. Wampanoag, "on the path or trail."

Mais Tchuseäg The Piedmont, probably in Essex County, Mass. Natick? Pennacook? "large mountains place." Pownall 
suggests "country on this side of the hills." See also Massachusetts.

Majabigwaduce The Bagaduce River, Hancock County, Me. Abnaki, "big tideway river."

Maji Neowaska was a demon, supposed to dwell on the highest peak of Mount Washington, Coös County, N. H. Abnaki, "Bad Spirit." See Ketsi M'weskw.

Majomansuck Pond, see Mayomansuck.

Majum-quassebem Pond, Knox County, Me. Abnaki, "worthless (or bad) pond."

Makamacheckamucks Hill, Worcester County, Mass. Nipmuck, from the Catacoonamaug chief Mahmachekomock. The name may mean "where we get small bait fish," mamachoog; or "big mountain strewn with rocks," or "big, useless plantation."

Makewaumaqueset Plymouth County, Mass. Wampanoag, "at the end of the worthless field," or "barren meadow ending place."

Maklic-ongomoc Pleasant Lake, Aroostook County, Me. Abnaki, "a lake (surrounded by) hardwoods."

Makoron Swamp, Fairfield County, Conn. Quinnipiac, "a gift," "waste land," or perhaps "turtles."[?]

Maktepos see Mashpee.

Makwamcusk Washington County, Me. Abnaki? Malecite? "red beach."

Malamake River, same as Merrimac, q. v.

Malecite an Algonquian tribe, part of whom live at Passamaquoddy, Maine. Their name comes from Micmac, "poor garbled, barely understandable speech," applied in derison. Actually, both languages are mutually understandable. See Mahtawesweskeeteheenonk.

Mali bowk Washington County, Vt. Abnaki, literally, "Molly's Pond." Indian Molly was the wife of Indian Joe, of Revolutionary War fame.

Mali panjahlok Washington County, Vt. Abnaki, "Molly's Falls."

Maliseet an old-fashioned spelling of Malecite, q. v.

Mallego Brook, Strafford County, N. H. Abnaki, "deep, ravinelike." 
Mallicooniganus Pocumpus Lake, Washington County, Me. Malecite, "short, quick carry." This is a short portage where it is not necessary to unload canoes for the carry; a "lazy portage."

Malmanick New Haven County, Conn. Possibly a corruption of manantick, "cedar swamp"? or "island creek"?

Mamachimins possibly Chimon Island, Fairfield County, Conn. Paugussett, possibly "Mamechon's little island" or "barren, unoccupied little island." Another spelling, Mamachimons.

Mamachoag Brook, New London, New London County, Conn. Narragansett, "where mummies, small fish, abound" (Another Mamachoag Brook is in Lyme, Conn.)

Mamacock Fort Trumbull Point, New London County, Conn. Western Niantic or Mohegan, "the great hook." (There is a Mamacock Creek in East Lyme.)

Mamanasco Lake, Fairfield County, Conn. Mahican, "united outlets," or "two sharing same outlet." Another spelling, Mammasco.

Mamaniskak Washington County, R. I. Eastern Niantic, "near the joined rocks."

Mamantapett River, Bristol County, Mass. Narragansett, "at the island camp." Also given as "wading river," or "ford."

Mamantapit Providence County, R. I. Narragansett, "customarily he walks in the water" (here); or "wading-place."

Mamaquag Brook, Washington County, R. I. Eastern Niantic, "small fish," called even now by children, "mummies." Variants, Mammaquog, Mummaquog, etc. ("Smelt" are moamitteang.)

Mamaquog part of Natchaug River, Windham County, Conn. Mohegan, "small bait fish abound here."

Mamasack New London County, Conn. Mohegan, "thistles," or "big nettles"?

Mamasunquobscook River, Piscataquis County, Me. Abnaki, "stream with rough stones," hence, poor traveling by canoe.

Mamattaquesset Norfolk County, Mass. Natick, "where they cross the brook," or "at the wading place." These were stepping-stones, "grouped-together-rocks place." 
Mameeg an early name for New London, New London County, Conn. Probably a corruption of Nameeg, "fishing place."

Maminketesuck Fairfield County, Conn. Wappinger, "swift, strong flowing brook, where there are alewives." Variant, Manuhketsuck.

Mamooson Avenue, Middlesex County, Conn. Natick, "smooth stone," or "bare rocks." Mamooson was a son of Kickemus.

Mamusqunke land on east side of Naugatuck River, New Haven County, Conn. Quinnipiac, "gravelly place,"? or "place of smooth stones"?

Mamwhauge Bristol County, Mass. Narragansett, "place of the ravine;" also, "moving ground."

Manahnekook the Penobscot River. Abnaki, "river full of islands."

Manahnook Grand Manan Island, Washington County, Me. Abnaki, "the island."

Manakaway Fairfield County, Conn. Wappinger, "the great meadows"?

Manamoaskeagug Plymouth County, Mass. Natick, "place abounding in beaver."

Manamoiett see Monomoy, and Manamoyik.

Manamookeagin Plymouth County, Mass. Wampanoag, "many beavers."?

Manamoquack Bay, Barnstable County, Mass. Wampanoag, "at the place of many fish." (Or "many clams"? )

Manamoyik Barnstable County, Mass. Wampanoag, "carrying place" or "burden-place," a portage. Also Maunamoitt.

Manan Islands, off Falmouth, in Casco Bay, Cumberland County, Me. Abnaki, "island." See Menanouze.

Mananduk Suffolk County, Mass. Natick, "lookout place." Also given as "cedar swamp."

Mananexit see Mayanexit, or Maanexit.

Mananis the small island which guards the harbor at Monhegan, Lincoln County, Me. Abnaki, "little island."

Mananosick Berkshire County, Mass. Mahican, "the high peak."

Mananscussett Plymouth County, Mass. Wampanoag, "place of the lookout hill." 
Mananticut River, Norfolk County, Mass. Natick, "at the lookout place," or "at the island in the river." Variants, Manatacat, Manatiquot.

Manaskek Arrowsic Island, Sagadahoc County, Me. Abnaki, "island with grassy covering."

Manaskoos Green Island, Penobscot County, Me. Abnaki, "green, grassy."

Manatohqua Essex County, Mass. Natick, after a chief also known as Black William. Ruler of an ancient Sachemdom comprising Nahant and Swampscott; "wooded island"?

Manatuck Mountain, New London County, Conn. Eastern Niantic, "at the lookout place."

Manatuck Hill, Hartford County, Conn. Massacoe, (a Wappinger sub-group), "look-out place."

Manaumet Worcester County, Mass. Nipmuck, "lookout place."

Manchage Worcester County, Mass. Nipmuck, "ye shall be strengthened."

Manchage Worcester County, Mass. Nipmuck? Natick? "place of departure," "place of marveling." ?

Manchaug Pond and Village, Worcester County, Mass. Nipmuck, "island of rushes," or "island where flags grow."

Manchuk River, Providence County, R. I. Narragansett, "lookout hill." But see Manchaug, Mass.

Mandawessoe Island, Penobscot County, Me. Abnaki, "a porcupine" (not "hedgehog").

Maneikshan Plymouth County, Mass. Wampanoag, "appearance of blackness," or "dark earth." Also "brant goose." Variant, Monechkan.

Manesaydik an Indian name for Bar Harbor, Hancock County, Me. Abnaki, "place of collecting clams."

Manet Beach, Norfolk County, Mass. Natick, "near the island."

Manetic Reservoir, Hartford County, Conn. Tunxis, "the high place," or "observation place."

Manexit River, Worcester County, Mass., and Windham County, Conn. Nipmuck, "near the path," or "he gathers them together." See Mayanexit River and Maanexit.

Mangunk akuk on Thames River, near Mohegan Village, New London County, Conn. Probably Mohegan-Pequot, "at the 
grove of large trees," but possibly, "where we made big wooden pots."

Manhan New Haven County, Conn. Quinnipiac, "an island." Also Munhan.

Manhan River, Hampshire County, Mass. Nipmuck, "island."

Manhannock Wright's Island, Hartford County, Conn. Wangunk, "at the place of the island," or possibly, "sheltered island."

Manhanock Pond, Piscataquis County, Me. Abnaki, "at the place of the island," possibly also "shelter island," a canoe harbor.

Manheigin Island, mouth of Penobscot River, Hancock County, Me. Abnaki? Micmac? "a passage"? or "the island"? See Monhegan, Moratiggon.

Manhumsqueeg Windham County, Conn. Mohegan, "whetstone place." Also Mahmunsqueag and Munhunsqueeg.

Manickmung Mountain, Windham County, Vt. Possibly Mahican, from monachnung, "place where the mountain heaps up," as in a monadnock. In other contexts, monachnung means "place on an island."

Manigana-tica-uoit name used by Champlain on his I629 map for the Connecticut River. Probably Abnaki, modified by French, "Mahican's river place," or "river of the Mahicans."

Manipsconasset Rock, Providence County, R. I. Narragansett, "place of the split rock island."

Manisses Block Island, Newport County, R. I. Eastern Niantic, "little island." Also given as Narragansett, "little god." See also Montowese.

Manitoese Avenue, Middlesex County, Conn. Paugussett, "Little God." Manitoese was Sowheag's nephew. See also Montowese.

Manitoo assenihah Plymouth County, Mass. Wampanoag, "the Great Spirit's rocks."

Manitoo-auke a term used by some southwestern New England Indians for "God's Country," i.e., "Heaven." (But on Long Island, Mantowacks meant "place of the periwinkle shell.")

Manitook Lake, Hartford County, Conn. Wangunk, probably "observation place," but possibly "cornfields, we guard them." Some suggest this means "Place of the Gods," or "God's Land."[?] See Manitoo-auke. 
Manitwa tootan Dukes County, Mass. Wampanoag, "God's town." A Christian Indian village on Martha's Vineyard.

Mankantosit River, Middlesex County, Conn. Hammonassett, same as Menenketesuck, q. v.

Mannahanose see Massaco.

Mannamoiset Bristol County, Mass. Narragansett, "point place," "place at end of portage," or "resting place," etc.

Mannanpenokean Brook, Berkshire County, Mass. Mahican, "heaps of fallen rocks."

Mannapensecon Berkshire County, Mass. Mahican, "heap of fallen rocks."

Manomet Beach, Bluffs, Reef and Village, Plymouth County, Mass. Wampanoag, "where they carry burdens on their backs," hence, "a portage."

Manonscusset Worcester County, Mass. Nipmuck, "lookout hill place."

Manoonckasuck see Menunketesuck.

Manoosnock Essex County, Mass. Natick, "at the summit," or, "at the lookout place."

Manset Hancock County, Me. Abnaki, "at the island."

Manshacket Cove, Dukes County, Mass. Wampanoag, "at the sheltering island."

Manshuck see Manchuck.

Manskussehoank Mountain, now Rattlesnake Mountain, Berkshire County, Mass. Mahican, "lookout summit place," or "nest at summit brook"? See Manskussichank.

Manskussichank now Rattlesnake Mountain, Berkshire County, Mass. Mahican, "stream emerging from and flowing through rock heaps."

Mantawassuk Johnson Brook, Penobscot County, Me. Abnaki, "at the mouth of," or "inlet."

Manunkateset Brook, New London County, Conn. Western Niantic, "place of alewives, or menhaden." Also Manunketesuck, Manuncketesseck, and Manaquatesset.

Manunkatuck New Haven County, Conn. Quinnipiac, "place of alewives," or alewive's tidal stream."

Manunketesuck Stream, Fairfield County, Conn. Siwanoy, "strong, flowing brook." 
Manunkquag Point, New London County, Conn. Eastern Niantic, "end of the land."

Manwhage Swamp, Bristol County, Mass. Narragansett, "at the refuge or shelter place."

Maquam Pond, Plymouth County, Mass. Wampanoag, "a beaver." Also a village and beach in Franklin County, Vt.

Maquan Lake, Plymouth County, Mass. Wampanoag, "a beaver."

Maquoit Bay, Cumberland County, Me. Abnaki, "a wet place."

Maranacook Lake, Kennebec County, Me. Abnaki, "plugged-up lake," "deep lake" or "black bass here" [?] Also Marancook.

Marandus Brook, Litchfield County, Conn. If Indian, possibly Natick, "cedar swamp," from mananduk.

Maranocook see Maranacook.

Maraspin Creek, Barnstable County, Mass. Probably a Dutch modification of the Wampanoag term Massapeague, "the land at the great cove."

Marchin Bay, now Casco Bay, Cumberland County, Me. Old Abnaki, "a wolf." Indicated as Baie de Marchen on Champlain's map, I607; this was as close as the French could get to manrhesem, "bad animal," or wolf.

Mardad poquehy Pond, Nantucket County, Mass. Wampanoag, "bare place, shallow, clear."

Margalloway see Magalloway.

Maromas Middlesex County, Conn. Natick? "bare."

Maroonscook Pond, now Lake Cobbosecontee, Kennebec County, Me. Abnaki, "plugged-up pond"? or "black bass pond"?

Marshpee Barnstable County, Mass. Wampanoag, "big still water," great pool," or "large pond."

Maruscopag New Haven County, Conn. Quinnipiac, "at the narrows." See also Achetaqupag and Warunscopage.

Masacksicke Hampden County, Mass. Nipmuck, "big meadow."

Masardis Village, Aroostook County, Me. Abnaki, "place of white clay."

Mascachaug Brook and Cove, Providence County, R. I. Narragansett, "place of rushes."

Mascachusett Location uncertain, probably near Greenwich, Kent County, R. I. Narragansett, "near place of flags or rushes." 
Mascakonage Brook, Providence County, R. I. Narragansett, "place of long rushes" (cat tails?). Other spellings, Mascacowage, Mascachowage, etc.

Mascamp York County, Me. Natick? Pennacook? "big rock." Variant, Maskomp.

Mascanomo Essex County, Mass. Pennacook, "big eel," "great long fish." If Natick, perhaps "big plantation." See below. Variant, Masconomet.

Mascoma Lake, Pond, River and Village, Grafton County, N. H. If Abnaki, "salmon fishing," "much grass," or "red rocks." If Natick, perhaps "big plantation," "big beach," or "grassy swamp."

Masconks York County, Me. Abnaki, "big goose" or "big rocks."

Mascopic Lake, Essex County, Mass. Natick, "at the place of the large rocks." Also given as "a large, enclosed place, a large cove," and "cedar."

Masenunckquock location unknown, probably New London County, Conn. Mohegan, "place of nettles," massonog-ock? or "place of lobsters," muschundang?

Mashacket Plymouth County, Mass. Wampanoag, "at the great palisaded house," or "at the castle."

Mashakamockett Pond, Dukes County, Mass. Wampanoag, "place of the big springs."

Mashamee River, Grafton County, N. H. Abnaki? Pennacook? perhaps remnants of words meaning, "big fish," or "place between"?

Mashamoquet Brook and State Park, Windham County, Conn. Nipmuck, "at the important fishing place." Also Mashamugget, Mashamugket and Massamugget.

Mashamugget Hill, Worcester County, Mass. Nipmuck, "spring of water," or "great water spring." Translated also as "great fishing place."

Mashamugget Meadow, Worcester County, Mass. Nipmuck, "great meadow-grass country." But see Mashamugget Hill and Mashamoquet, Conn. [Narragansett, "big stink" ?]

Mashantackack an ancient village site near Montville, New London County, Conn. Mohegan, "this place is well forested." 
Mashanticut Brook, Providence County, R. I. Narragansett, "small river or brook running through grove of trees."

Mashantucket New London County, Conn. Mohegan, "place of big trees."

Mashantuxet Brook, New London County, Conn. Pequot, "at the little forest," or "place of small trees" ? See Mushantuxet.

Mashapaug Pond and Village, Tolland County, Conn. Nipmuck, "a large pond." See also Massapaug and Mashipaug.

Mashapaug Pond, Kent County, R. I. Narragansett, "a large pond or cove." Also Maushapog, Mashepok, etc.

Mashapauge Worcester County, Mass. Nipmuck, "large pond" or possibly "worthless pond." (Swampy land, unfit for planting ?)

Mashapequottuck River, the Thames River, New London County, Conn. Mohegan, "great Pequot River," or "river of the great destroyers of men."

Mashaquamaganset Plymouth County, Mass. Wampanoag, "place where there are big rocks in the pool."

Mashashinnet Plymouth County, Mass. Wampanoag, "at the place of large stones," or perhaps "place of great springs."

Mashatatack Brook, Providence County, R. I. Narragansett, "well forested place," or "place of big trees." Also Mashantatuck, Mishauntatuk, etc.

Mashattaneeseck Hill, Washington County, R. I. Narragansett, "brook near great hill," or "great hill near brook."

Mashawmut Suffolk County, Mass. Natick, "at the great canoe landing," or "at the great neck of land."

Mashawshimet Plymouth County, Mass. Wampanoag, "Big spring place." Variant, Mashinock.

Masheet Island, Barnstable County, Mass. Wampanoag, "bad place."

Mashentuck Brook and Mountain, Windham County, Conn. Mohegan, "many trees." Also a town in Providence County, R. I.

Mashenups Pond, Windham County, Conn. Mohegan, "big rock." See also Moshenupsuck.

Mashepagocke Middlesex County, Mass. Natick, "land near the great pond." See also Mashepagonoke. 
Mashepagonoke Pond, Middlesex County, Mass. Natick, "at the place of the wicked destroyer," or "place of bad pestilence." Also translated, "place of big bank."

Masheshattuck Hill Hillsboro County, N. H. Natick? "big wooded mountain."

Mashipaug Alexander's Lake, Windham County, Conn., and Gardiner's Lake, New London County, Conn. See Mashapaug.

Mashipawog see Mashpaug.

Mashnee Island, Barnstable County, Mass. Wampanoag, "big house."

Mashomuck perhaps two places by this name in Windham County, Conn., and Worcester County, Mass. If as spelled, the term is Nipmuck, "where they go by boat or canoe." However, if Massomuck, q. v., is meant, the term is Nipmuck, "great fishing place."

Mashonaug Island, Washington County, R. I. Eastern Niantic, "nettles"? or "dug-out canoe place"? or "place reached by boat"?

Mashoquen mentioned by Captain John Smith, is somewhere along the coast of Cumberland (or York) County, Me. Perhaps it is Abnaki, "big hook," or Micmac, "big knee."

Mashpaug Ponds, Washington County, R. I. Eastern Niantic, "great ponds."

Mashpeag New London County, Conn. Mohegan, "land at great cove."

Mashpee Pond and Village, Barnstable County, Mass. Wampanoag, "land near the great cove or pond."

Mashpoag Norfolk County, Mass. Natick, "a great pond."

Mashquaponitib Nantucket County, Mass. Wampanoag, "where the big nets are spread."

Mashquomuoh or Massquamack Plymouth County, Mass. Wampanoag, "marshy, grassy haven or hiding-place."

Mashucket Plymouth County, Mass. Wampanoag, "grass land," or, "grassy place near large outlet."

Maskataquatt Providence County, R. I. Narragansett, "place of rushes," or "grassy place."

Maskechusett Brook and Hill, Kent County, R. I. Narragansett, "at the grassy place," or "place of flags." 
Maskeekohwogam near Lake Bomoseen, Rutland County, Vt. Abnaki, "much grass, hay curing."

Maskerchugg River, Kent County, R. I. Narragansett, "rushes here." Also Maskachuag, Masquachug, Musquechuge.

Maskituash Bristol County, R. I. Narragansett, "hay-marsh" or "grassy place."

Maskwenozakek mouth of the Missisquoi River, Franklin County, Vt. Abnaki, "where muskellunge abound."

Masocksicke Hampden County, Mass. Nipmuck, "big meadow."

Maspenock Pond, Worcester County, Mass. Nipmuck, "overflowed land," or "boggy place"; also given as "choice fishing place" and "great narrow path."

Masquabamisk Meadow, Franklin County, Mass. Mahican, "at the place of the red rocks." Possibly "wet rocks," a boundary place.

Masquachowawaug Washington County, R. I. Eastern Niantic, "place where rushes grow"? or "red fish (salmon) fishing place"? See Misquamicut.

Masquaseecook Lake, Penobscot County, Me. Abnaki, "birch stream."

Masquetuck Bristol County, Mass. Narragansett, "river of reeds," "fish weir river," or "wide river."

Masquomcussick Hampden County, Mass. Nipmuck, "grassy enclosed place," or "grassy haven." Or perhaps "big sharp stones place"?

Masquomoh Swamp, Bristol County, Mass. Wampanoag, "grassy place."

Masquomp Hampden County, Mass. Nipmuck, "red rock."

Masquopeck Nantucket County, Mass. Wampanoag, "land near the reedy cove" or "wide pond."

Massabequash Essex County, Mass. Natick, "lands near great body of water." (But another source gives "rats".)

Massabeseck York County, Me. Abnaki, "large pond."

Massabeset see Mattabesic.

Massabesic Lake, Hillsboro County, N.H. Abnaki, "near the great brook."

Massachaug Pond, Watch Hill, Westerly, R. I.? Eastern Niantic, "land near the great hill." Given also as "land where rushes grow." 
Massachuset an ancient Indian Village; exact location not known, region of Blue Hills, Mass. ? Natick, "at the great hills" or "at the range of hills," i. e., the Milton hills. This tribe, for whom the state was named, occupied lands from Salem, Essex County, southward to Brockton, in Plymouth County, Mass. Also Natick.

Massachusetts Bog and Gore, Oxford County, Me. Natick, "at the place of the large mountain."

Massachusetts State, Anglicized Natick, "at the place of large hills;" also Messatsoosec, "hill in the form of an arrowhead."?

Massacksic Hampden County, Mass. Nipmuck, "at the great marsh." Also Massaksicke.

Massaco Territory, Hartford County, Conn. Tunxis, "great lowland or outlet." Mannahannose was "Grand Massaco," sachem of Simsbury, Granby and Barkhamstead in 1656 . Variants, Massaqua, Mussawco, Mossocowe, Mushko.

Massagika York County, Me.? Abnaki, "big outlet."

Massamoskeht Worcester County, Mass. Nipmuck, "place of much grass" or "great hay country."

Massanagtakwonah Bristol County, Mass. Narragansett, "dry trees hill" or "canoe wood hill"? Also translated as "flax fields along the river"?

Massanegtocaneh Providence County, R. I. Nipmuck? "place of source of the great stream" ? or "union of great streams."? But see Massanagtakwonah, above.

Massapanoch Plymouth County, Mass. Wampanoag, "great miry place," or "large swamp."

Massapaug Rockingham County, N. H. Abnaki, literally "great pond." See Mashpaug.

Massapeag Village, New London County, Conn. Mohegan, "place at the large cove."

Massapee same as Marshpee.

Massapoag Pond, Middlesex County, Mass. Natick, "large pond." Ponds of this name also in Norfolk and Plymouth Counties, Variant, Mashapoog.

Massaquockumnie Brook, Worcester County, Mass. Nipmuck, "big shaking meadow." 
Massasaugatukut Plymouth County, Mass. Wampanoag, "at the mouth of the large tidal stream." An old name for Marshfield.

Massasecum .Lake, Hillsboro County, N. H. Named for a Pennacook warrior; possibly "tall, slender, erect."

Massasoit Avenue, Bristol County, R. I. Also a road in Barnstable County, Mass. Wampanoag, "great commander." This chief befriended English colonists at Plymouth, 1620. See Osamequin.

Massassomineuk Plymouth County, Mass. Wampanoag, "where big sour berries abound," i. e., large cranberries, or grapes.

Massatucksett see Mastuxet.

Massaucunnock New Haven County, Conn. Quinnipiac, "place of the great (longtails) fish hawks," probably ospreys.

Massaugatucket Washington County, R. I. Narragansett, "at the great outlet of the tidal river."

Massaukatucket Plymouth County, Mass. Wampanoag, "at the outlet of the large tidal stream."

Massawamsog Middlesex County, Conn. Wangunk, "place of the great declivity." Sometimes as Meshomasic.

Massawipi now Holland Pond, Orleans County, Vt. Abnaki, "much good clear water."

Massekump Path, Middlesex County, Conn. Natick? Wangunk? "Big Boulder." Massekump was a landholder Indian and witness to several deeds.

Masshapauge Pond, Worcester County, Mass. Nipmuck, "big pond."

Masshattaneesec Hill, Washington County, R. I. Narragansett, "brook at the great hill."

Massiby Plymouth County, Mass. Wampanoag, "big water."

Massick Island, Middlesex County, Mass. Natick, "large brook," or "large outlet."

Massomuck Stream, Windham County, Conn. Nipmuck, "great fishing place." But see Mashomuck.

Massomuck Worcester County, Mass. Nipmuck, "great fishing place." This was in the neighborhood of Wabaquasset Village, I700. But see Mashomuck.

Masstucksett Brook, Bristol County, Mass. Narragansett, "at the place of the large brook." 
Mastomquoog Island, Knox County, Me. Abnaki, "the place of big rocks"?

Mastuxet Brook and Cove, Washington County, R. I. East Niantic, "at the big brook." Also Massatucksett.

Maswachi Berkshire County, Mass. Mahican, "great mountain."

Maswasehi Mountain, Berkshire County, Mass. Mahican, "bird's nest standing up," or "big bird's nest"?

Matachuest Barnstable County, Mass. Wampanoag, "the place without hills."

Matacomacok Swamp, Hartford County, Conn. Sicaog, "bad plantation" or "bad going, where paths or trails are difficult" or "ineffective refuge."

Matacompemiscok Washington County, R. I. Eastern Niantic, "place (far away) up country."

Matagamon Village, Penobscot County, Me. Abnaki, "far on the other side."

Matagamook Grand Lake, Penobscot County, Me. Abnaki, "old, exhausted lake," fished out, grown up with weeds; or "far away lake."

Matagamooksis Second Lake, Penobscot County, Abnaki, "little far away lake," etc., diminutive of Matagamook.

Matagoodus Stream, Penobscot County, Me. Abnaki, "bad landing place (for canoes)."

Matagwadis Penobscot County, Me. Abnaki, "landing place at the confluence, usually overflowing, or covered with water."

Matanawcook Islands, Lake and Stream, Penobscot County, Me. While these now bear the same name, their old names are dissimilar, and have different meanings. Their original names and meanings follow:

Matanawcook Islands, originally Metinahguk. Abnaki, "small, broken water."

Matanawcook Lake, originally Madagamok. Modern Abnaki, "lake that ends almost at the river."

Matanawcook Stream, originally Matanaghe-seebo. Abnaki, "at the end of an island."

Matatucket River, Washington County, R. I. Eastern Niantic, "poorly forested," or possibly "at the worthless river."? 
Mataubaun Fairfield County, Conn. Siwanoy, "it is day," or, "dawn has come."

Matchapoxet Pond, Barnstable County, Mass. Wampanoag, "near the useless little pond." Possibly, "bad swamp place." ?

Matchapquake Plymouth County, Mass. Wampanoag, "place of the bad bog."

Matchecompomiskok Bristol County, Mass. Narragansett, "place of the bad little rocks."'? See Matacompemiscok.

Matchepukgunnupponset Bristol County, Mass. Wampanoag, "at the impassable falls."

Matchuk Meadows, Worcester County, Mass. Nipmuck, "bad, or useless land."

Mateguasaden Mount Philo, Chittenden County, Vt. Abnaki, "rabbit mountain." See Madagascar.

Matetacoke see Mattatuck.

Matianock see Mattaneaug and Matianuck.

Matianuck Hartford County, Conn. Pocasset, "the lookout place."

Matincook Mountain, Essex County, Vt. Natick, "the high land observation place," or "he searches from there."

Matinicus Island and Rock, Knox County, Me. The largest of a group of islands well out to sea. Abnaki, "far-out island."

Matitacooke see Mattatuck.

Matomy Hill, Providence County, R. I. Narragansett, "a lookout place," or "observation height."

Matowepesack see Mattapoisett.

Mattabaget Hampden County, Mass. Nipmuck, "at the edge of the pond," or "at the far distant pond."

Mattabeesick Pond, Rockingham County, N. H. Natick, "place of the great body of still water." But see Mattabesec.

Mattabesec near Middletown, Middlesex County, Conn. Wangunk, "a resting place." See also Mattapoisett, Mass. (Some sources give translation as "place of large body of water," or "place of large brook.") Other spellings, Mattabesic, Mattabezeke, Mattapeaset, Matowepesack, etc.

Mattabesett Middlesex County, Conn. Wangunk, "(at the end of carrying place or portage), a resting place." See also Mattapoisett. 
Mattacheese Barnstable County, Mass. Wampanoag, "old fields."

Mattacomacok Hartford County, Conn. Poquonock, "at the furthest enclosure."

Mattagodus Stream, Penobscot County, Me. Abnaki, "bad sliding;" that is, rocky bottom, bad for canoe landings.

Mattakeesee Barnstable County, Mass. Wampanoag, "big meadow." Possibly "little trees," such as beach plum bushes.

Mattakesett Creek, Dukes County, Mass. Wampanoag, "at the place of black mud." (If the mud was thin and deep, the sense of bad mud, quicksand-like mud is carried.)

Mattakeunk Pond and Stream, Penobscot County, Me. Abnaki, "at end of swift stream."

Mattamiscontis Lake and Mountain, Penobscot County, Me. Abnaki, "plenty of alewives."

Mattanawcook Pond, Penobscot County, Me. Abnaki, "at the end of the gravel bar."

Mattaneaug Hartford County, Conn. Sicaog, "abandoned fields," or "remains of encampment," or "furthest country"?

Mattaoolanic Hampshire County, Mass. Nipmuck, "where waters meet and mingle."

Mattapan Suffolk County, Mass. Natick, "he sits down," hence "a resting place," or "end of portage," also "edge of bank."

Mattapoisett Plymouth County, Mass. Wampanoag, "a resting place," or "edge of the cove," also "resting place on portage."

Mattapoysett River, Bristol County, R. I. Pocasset, "resting place." See Mattapoiset.

Mattaquitcham Pond, Nantucket County, Mass. Wampanoag, "edge of the rocks."

Mattaseunk Stream, Aroostook County, Me. Abnaki, "furthest rapid stream."

Mattatig New Haven County, Conn. Quinnipiac or Paugusset, "bad tree," or "poorly wooded." Variant Mattatuck.

Mattato Hill, Providence County, R. I. Nipmuck? "bad hill"? Probably an abbreviation, with some syllables lost.

Mattatuck State Forest and Trail, Litchfield County, and River, New Haven County, Conn. Paugussett, "without trees," or "badly wooded." 
Mattatuxot River, Washington County, R. I. Eastern Niantic? Narragansett? "at the worthless little river"? or "poorly wooded place"?

Mattawa Lake, Franklin County, Mass. Pocumtuck, "bad place," or "bad going."

Mattawamkeag Lake, Penobscot County, Me. If Abnaki, "fishing place beyond gravel bar." If Malecite, "rapids at mouth;" if Micmac, "on a sand bar."

Mattawehausk Path, Middlesex County, Conn. Wangunk? "bad rocks"? or "black rocks"? or "furthest rocks"? Mattawehausk was Sowheag's granddaughter.

Mattetakonitt Meadows, Providence County, R. I. Narragansett, "at the great spring," or "distant spring."

Mattimockamus Lake, Washington County, Me: Abnaki, "far-off small lake."

Mattonkeeset Bay, Dukes County, Mass. Wampanoag, "at the place of black mud."

Mattoonuc Neck, now Point Judith, Washington County, R. I. Eastern Niantic, "place of the lookout hill."

Mattuckhott New Haven County, Conn. Quinnipiac, "in the woods," or "at the tree."? See Mattatuck.

Matumpseck Island, Hartford County, Conn. Tunxis, "place of the bad rock," or "bad ledges."

Matunuck Village, Washington County, R. I. Narragansett, "high place" or "observation place."

Matush Windham County, Conn. Nipmuck? "leggings"?

Mauchetan Middlesex County, Conn. ? Hammonassett? "ebb tide."

Maumansuck Windham County, Conn. Nipmuck? "where two streams meet"? See Mahmansuck.

Maumsuck Pond, New London County, Conn. Mohegan, "stream connects two ponds," or "two streams meet."

Maunamushog Barnstable County, Mass. Wampanoag, "deep fishing place"?

Maunipensing Brook, Plymouth County, Mass. Wampanoag, "gently descending brook," or "gently sloping field." Also Maunpenseconet.

Mauntunsq West Rock, New Haven County, Conn. Quinnipiac, "difficult rock" (to climb). 
Maushantuxet now Ledyard, New London County, Conn. Mohegan, "at the great woods."

Maushapogue Providence County, R. I. Narragansett, "land at the great cove," the ancient name for Cranston. Also Mashapaug, Maushapoque, etc.

Mausup see Moosup and Pessicus.

Mauswaseekhi Monument Mountain, Berkshire County, Mass. Mahican, "at the smooth, bare mountain shaped like a bird's nest." Sometimes translated as "fisher's nest," or "osprey's nest."

Mauwee Brook, Lake and Peak, Litchfield County, Conn. Mahican, "hungry animal," perhaps a bear.

Mawiagwaug see Mamaquog.

Mawnantuck Hill, Hartford County, Conn. Shortened form of Wangunk, mabautau antucksuck, "hill near swift running brook."

Maximus see Machemux.

Mayanexit Windham County, Conn. Nipmuck, "where the road lies," and "where we gather."

Mayannoes Neck and Creek, Fairfield County, Conn. Paugussett, from the name of Chief Mayano, who was killed in I683. Also, Mehawnoes, Mianus, Maharnes, etc.

Mayawaug Hartford County, Conn. Tunxis, "at the meetingplace," or "at the junction of routes and paths." Also seen as Mayyoawang, Mayowack, etc.

Mayomansuck Pond, New London County, Conn. Pequot? Mohegan? "common outlet of two ponds," or "meeting of two outlets."

\section{Medambetek or Medambetox see Madam Bettox.}

Meddybemps Lake and Village, Washington County, Me. Passamaquoddy-Abnaki, "plenty of alewives."

Medomac Bay, River, Point and Village, Lincoln County, Me. Abnaki, "place of many alewives."

Medumcook River, Aroostook County, Me. Malecite, "blocked by sandbars."

Medum Keunk Lake, Penobscot County, Me. Abnaki, "blocked up by sand." Also Maddunkeunk, Medunkeunk, etc. 
Meduxnekeag Lake and River, Aroostook County, Me. Malecite, "falls or rapids at the mouth."

Meeseecontee Farmington Falls, Franklin County, Me. Abnaki, "plenty of alewives," or other migratory fish?

Meeshawn Barnstable County, Mass. Wampanoag, "a landing place," or "ferry," or "great neck of land."

Meesocdowhok Burnt Land Island, Penobscot County, Me. Abnaki, "big outlet," or "big confluence."

Megansett Harbor, Barnstable County, Mass. Wampanoag, "at the fish weir."

Megantic Lake, Franklin County, Mass. Abnaki, "lake trout place," derived from Old Abnaki expression, namagwottik. See Damisokantic.

Megantig Pond, Franklin County, Mass. Pocumtuck? Pennacook? "a pipe stem." But see Megantic, above.

Megkwahlagas Penobscot County, Me. Abnaki, "red pool"? or "red rock pool"?

Megkwakangamosis Harrow Pond, Aroostook County, Me. Abnaki, "little marshy (or muddy) lake."

Megunhuttake New Haven County, Conn. Quinnipiac "place of the gift, or grant." Spelled also Mequenhuttocke.

Megunticook Lake, Knox County, Me. Micmac or Malecite, "big mountain harbor."

Megurrewock Lakes, Washington County, Me. Abnaki, "the shoveler," the caribou which paws or shovels snow away so that it can reach its food.

Megwak-angamik Mud Pond, Piscataquis County, Me. Abnaki, "wet ground, bogs around lake."

Mehtukquaaumsett Plymouth County, Mass. Wampanoag, "fishing place near trees," or "fish trap made of saplings" or "place marked out by line of trees."

Mekwamkesk a beach near Robbinston, Washington County, Me. Micmac, "at the red rocks" or Abnaki, "at the small red beach."

Melanpswangamoc or Melaxswangarmo Joe-Mary Lakes, Penobscot County, Me. Abnaki, "many rocks at the portages near this lake," or Micmac, "abounding in rocks of all shapes and sizes." 
Memachogue Hampden County, Mass. Natick, "fish sprats," or "mummies," from mummichoog.

Memada-Kamioguk said to be an old name for Princeton, Washington County, Me. Penobscot-Abnaki, "a point of land."

Memeeneesitt Creek, Sagadahoc County, Me. Abnaki, "little islands everywhere."

Memkeeswe Stream, Washington County, Me. Malecite, "good landing places."?

Memphremagog Lake, Orleans County, Vt. Abnaki, "where there is a great expanse of water." Variants Mamlawbagak, Memeraoubeke, Memeranbegak, Memsahabegeck.

Mempticook Stream, Washington County, Me. Abnaki, "overflowing river."

Menada Beach, Dukes County, Mass. If Indian, perhaps from the Wampanoag word munnawhateaug, "fertilizer fish," or "menhaden." Possibly also "out-of-the-way place."

Menameset Hampshire County, Mass. Nipmuck, "at the place where fish abound."

Menanbawk Essex County, Vt. Abnaki, literally "island pond."

Menanhant Barnstable County, Mass. Wampanoag, "on the island."

Menannah Bay and Island, Knox County, Me. Abnaki, "the island, out at sea."

Menanouze Island, Petit Manan, Washington County, Me. Abnaki, "small island," far out at sea.

Menasquassicook Matinicus Island, Knox County, Me. Abnaki, "at the grassy islands."

Menauhant Barnstable County, Mass. Wampanoag, "deep water at point."

Menchoiset Plymouth County, Mass. Wampanoag, "much food here"?

Menehanis-cook Boom Islands, Penobscot County, Me. Abnaki, "at the very small islands."

Menemesseck Worcester County, Mass. Nipmuck, "at the great fish-weir," or "at the important fishing-place."

Menemsha Pond, Dukes County, Mass. Wampanoag, "middle island," or "lookout cliff." Also suggested as "sour berries," i. e., cranberries. 
Menes-saganaganis Big Lake, Washington County, Me. Abnaki, (free translation) "collected boards split and fastened to bottom of canoes" for protection while being dragged through low water.

Menhaneekek Ragged Lake, Piscataquis County, Me. Abnaki, "small islands at this place."

Meniekec St. George Island, Knox County, Me. Abnaki, "at the place of the sheltering island." Such islands as these afforded ample windbreaks or shelter for canoes. Also Meniquet.

Menikoe Point, Cumberland County, Me. Abnaki, "shelter islands."

Menikpodik Pokomoonshine Lake, Washington County, Me. Abnaki, "collecting tying-bark." At this place Indians obtained cedar or basswood fiber to make string, twine, braid, etc.

Menikuk Cape Harbor, Lincoln County, Me. Malecite-Micmac, "at the island," "or shelter for canoes."

Menomee Pond, Plymouth County, Mass. Wampanoag, "seed corn" or "grain for seeding."

Menotonomy Rocks, Park, Middlesex County, Mass. Narragansett, from the name of the sachem Wannamenotonomy, or Waunamatanemet.

Mensecommook River, Aroostook County, Me. Malecite, "come and fetch." Probably a specific place on the stream where provisions were stored.

Menucketuck Reservoir, New Haven County, Conn. Western Niantic, "strong-flowing stream." Name transplanted from Menunketesuck River. Also Menunketuck, and Menunquatucke.

Menuhkikook Worcester County, Mass. Nipmuck, "place where (you shall be) strengthened."

Menunketesuck Island and River, Middlesex County, Conn. Western Niantic, "strong-flowing stream."

Menunquatucke Gilford, Belknap County, N. H. Probably Western Niantic transplanted from Guilford, Conn., "strong flowing stream." This name and the two preceeding are often given as "place of menhaden," which would be munnoquoteauauke; or "menhaden river," munnahtotook. 
Mcrriconeag Peninsula, near Harpswell, Cumberland County, Me. Abnaki? Malecite? "a lazy carry," where canoes could be dragged or lifted over, without unloading.

Merriconeag Sound, Cumberland County, Me. Malecite, "lazy portage."

Merrimac River and Port, Essex County, Mass. Pennacook, "deep place." One authority translates this as "noise of the falls." Also Merrimaege.

Merrimack County, Town and River, N. H. Abnaki, "at the deep place." Also a Village in Hillsboro County, N. H. Variant, Merramacke.

Mesacket Cove, Dukes County, Mass. Wampanoag, "at the place of two brooks."

Mesakketesagewick Socatean Stream, Aroostook County, Me. Abnaki, "stream separating burned timber and burned fields"?

Mesebigou Sebec Lake, Piscataquis County, Me. Abnaki, "the big lake." This name appears on Father Aubéry's I7I5 maps.

Meselipemapskek near Pemaquid Point, Lincoln County, Me. Penobscot-Abnaki, "very extended ledges."

Meshantic Brook, Providence County, R. I. Narragansett, "woody place," or "canoe-tree"?

Meshanticut City, Brook and State Park, Providence County, R. I. Mohegan, "at the place of (many) big trees," or "well forested." Also Meshantacuck, Meshantituck, etc.

Meshapock Brook; see Mashapaug.

Meshenups Pond; see Moshenupsuck and Snipsic.

Meshmusketucktecutt Plymouth County, Mass. Wampanoag, "the great meadow at the tidal creek."

Meshomasic Mountain, State Forest and Park, Middlesex County, Conn. Wangunk, "at the place of great springs," "great declivity." Also Meshomassuc, Mesomussuck, Mesomersic.

Meskaskeeseehunk Brook, Penobscot County, Me. Abnaki, "little spruce brook."

Meskeekwagamasic Black Pond, Knox County, Me. Abnaki, "grassy pond," or "pond with grassy banks."

Messalonskee Lake, Kennebec County, Me. Abnaki, "white clay here." 
Messatoocus Smith Brook, Penobscot County, Me. Abnaki, "big brook."

Messatsoosec Hill, Suffolk County, Mass. Natick, "great hills at the mouth" of the Charles River; or "dry trees place;" or "hill in form of an arrowhead." See Massachusetts; Massachuset.

Messenegtaquaneh Bristol County, Mass. Narragansett, "at the place of the dry tree hill."

Messubisuk see Massabeseck.

Metacom Avenue, Bristol County, R. I. Wampanoag, the name of King Philip, son of Massasoit. Meaning is unclear, possibly "at a faraway place." See Pometacomet.

Metacomet Lake, Hampshire County, Mass., and Metacomet Trail, Hartford County, Conn. A variant of Metacom, above.

Metallak Brook and Mountain, Coös County, N. H. Also a Pond in Oxford County, Me. Pennacook. This was the name of the last Coösuck man, who died at Stewartstown, Coös County, around I850. His name possibly meant "The Last Man."

Metatoxet River, Washington County, R. I. Narragansett, "well wooded stream place." Another spelling, Metatuxet.

Metaubscot an ancient Indian village site on Coweset shore, Kent County, R. I. Narragansett, "place of black rocks" or "black cliff place." Variant, Mettobscot.

Metecompemiscock see Matacompemiscok.

Metewemesick Worcester County, Mass. Nipmuck, "at the place of dark earth."

Metichawon Falls, Litchfield County, Conn. Mahican, "because of big rapids or the swift current" here the shad stopped in their migration.

Metinic Island, Knox County, Me. Abnaki, "far out island." Variant, Metineäg.

Mettawee River and Valley, Rutland County, Vt. Origin doubtful; possibly Narragansett, "black earth," or Natick, "poplar trees."

Mettuckquashapock New London County, Conn. Narragansett? Eastern Niantic?" place of the eel traps."

Metukpogkottomis Bristol County, Mass. Wampanoag, "grove of white oak trees." 
Miacomet Pond, Nantucket County, Mass. Wampanoag, probably "where we meet to fish," or "enclosure where we meet." Also suggested as "dry field." Variant, Myacomet.

Miamogue west side of Bridgeport Harbor, Fairfield County, Conn. Paugusset, "where we come together to fish."

Miantanomi Hill and Avenue, Newport County, R. I. Narragansett, "he wages war"'[?]. From the name of a chief murdered (or executed?) by a brother of Uncas in I643.

Miantonomo New London County, Conn. See Miantanomi.

Mianus River, Fairfield County, Conn. Paugussett, "Mayanno's River." Mayanno was a chief who was killed in I683; his name means "he who gathers together." He was also called Pessicus, "a sturgeon." See Mayanno's, Mayannoes.

Michigan Camp, Windsor County, Vt. Chippewa, "big lake."

Michiscouy see Missisquoi.

Micmac Indians, although primarily a Canadian tribe, invaded the Maine coast in the late I500's. Their name means "allies." They were closely related to the Malecite and Abnaki. These may have been the first Indians encountered by whites, when the Vikings sailed into their territory circa I0oo A.D.

Mikumwessak "woods fairies." These "wee people" had very narrow Indian faces. They were reticent and rather solitary, but very friendly to Indians. They wore red caps with pointed earpieces, like those the Indians wore in winter. It is said that a certain Mikumwes warned the Abnaki of approaching Mohawk war parties. The Mikumwessak dwelt in the woods; they were larger than the rock fairies, Wanagameweswak.

Millimagassett Lake, Penobscot County, Me. Abnaki, "where duckhawks abound."

Millinocket Lakes and Village, Penobscot County, Me. Authorities disagree on this name. Preferred is Abnaki, "this place is admirable." Other interpretations: Abnaki, "many coves;" and Malecite, "broken by outjutting rocks and islands."

Minacomuc Island, Washington County, R. I. Eastern Niantic, "field in low lands," "berry farm," or "enclosed plantation in a deep place." Or possibly Narragansett, "berry fields." Variant, Minnacommuck, Munnacommuck.

Minamok River, Fairfield County, Conn. Wappinger, "islands place," or perhaps "berries place." 
Mincomonk Meadow, Hampshire County, Mass. Nipmuck, "overacross place," possibly "land across the brook." Also Mincommuck.

Mingo Beach, Essex County, Mass., and Mingo Rock in York County, Me. Mingwe was a name applied by the Delaware to the Iroquois, particularly the Mohawk; Mingo is a corruption of this. A term of hatred and fear, it meant "treacherous, stealthy, or sneaky," and was commonly used during the colonial period to refer to those Iroquois who had settled in Pennsylvania.

Minhan Island, Hampshire County, Mass. Nipmuck, "the island."

Minhannock New London County, Conn. Narragansett, "island place," or "at the island."

Minnabaug Washington County, R. I. Eastern Niantic, "berry pond," probably cranberries; perhaps also "deep pond." One source suggests "island in the pond." Also Minebauge.

Minnacommuck see Minacomuc.

Minnechaug Mountain, Hartford County, Conn. Wangunk, "berry mountain."

Minnechoag Hampden County, Mass. Nipmuck, "berry mountain."

Minnecopscook Seal Rock, Washington County, Me. Malecite, "plenty of rocks here."

Minnehonk Lake, Kennebec County, Me. Abnaki, "berriesstream," or more likely, "many geese."

\section{Minnesquam see Winnisquam.}

Minnewokun Hancock County, Me. Malecite, "many bends," also Abnaki, "very crooked route."

Minnie Island, State Park, New London County, Conn. Mohegan, "small island."

Minniwawa River, Cheshire County, N. H. Malecite, "many waters."

Minotoquid River, Norfolk County, Mass. Natick, perhaps, "lookout place," or possibly "berry island."

Minusing Island, Fairfield County, Conn. Mahican, "small island."

Mioonkh-tuk now East Haven, New Haven County, Conn. Quinnipiac, "where tidal streams come together," or "meeting of tidal rivers." 
Mioxe's Pond, Nantucket County, Mass. Wampanoag, from the name of a Chief Mioxeo, circa I640.

Misadene the White Mountains in Coös and Grafton Counties, N. H. Abnaki, "great mountains." This name appears on Father Aubéry's map of I7I5.

Miscoe Hill, Worcester County, Mass. Nipmuck, "great hill." But see Misquoc.

Mishanegitaconnet Bristol County, Mass. Narragansett, "squirrel hunting ground." Also translated as, "field of slaughter."

Mishanneke Hill, Kent County, R. I. Narragansett, "a squirrel." Spelled also Mishanoke, Mishannok, etc.

Mishanups see Moshenupsuck.

Mishaum Point, Bristol County, Mass. Narragansett, "great neck," also "great landing place for canoes" or "big ferry." Other places named Mishaum are in Plymouth, Norfolk, Middlesex and Suffolk Counties., Mass.

Mishawamut Neck, Middlesex County, Mass. Natick, "at the great spring."

Mishawomut Kent County, R. I. Narragansett, "big landing place" or "big neck of land." See Shawmut.

Mishawum Lake and Suburb, Middlesex County, Mass. Natick, "great landing place," etc. See Mishaum and Shawmut.

Mishawum Suffolk County, Mass. Natick, "great landing place." An ancient village at Charlestown.

Mishimayagat "the great trail" from New York to Boston, along the shore, except from Guilford to New Haven, Conn. Quinnipiac, "the great trail."

Mishnepettuut Bristol County, Mass. Wampanoag, "at the place where the large stream twists."

Mishnock Pond, Road and Swamp, Kent County, R. I. Narragansett, "they go and come by water, by canoe." Other roots suggest "squirrel." Variant, Michnic.

Mishoasket Pond, Providence County, R. I. Narragansett, "muskrat place, "or possibly "wide yellow meadow" (cowslips?).

Miskianza Brook, Washington County, R. I. If Narragansett, this name is so modified that any translation is guesswork. Possibly, "trout" or "salmon" or "grass"? More likely: in Italian, mischianza means "a mixing;" in Colonial times it 
denoted a very elaborate entertainment. A mischianza was given by British army officers at Philadelphia on May I8, I778, in honor of Sir William Howe.

Miskwa-aubik Redrock Point, Chittenden County, Vt. Chipperwa, "it is a red rock."

Mispecky Washington County, Me. Abnaki? "overflowed." See Maasbaak.

Misquamicut Hill and Village, Washington County, R. I. Narragansett, "red salmon at this place." Variants, Mishquomacuk, Mishquamicuk.

Misquatucket Brook, Plymouth County, Mass. Wampanoag, "at the red river."

Misquiquaungameck now the Clyde River, Orleans County, Vt. Abnaki, "meadows at the end of the lake" or "much grass at the end of the lake." Also, "salmon fishing at end of lake."

Misquitanxit Washington County, R. I. Eastern Niantic, "at the place of the meadows." Also, Misquitanset.

Misquitucket Plymouth County, Mass. Wampanoag, "at the red tidal stream," or "red creek" also "wide stream."

Misquoc Hill, Worcester County, Mass. Nipmuck, possibly part of ussunamis-co ("small rock" or "pebble")? Also Miscoe, Misko, etc.

Missatchawag Windham County, Conn.? Nipmuck, "place at the great hill." Also Miscetchawog.

Missaugatucket River, Plymouth County, Mass. Wampanoag, "at the great outlet of the tidal river."

Missiassick Penobscot County, Me. Abnaki, "important flint place"? See Missisquoi and Missisiasuk.

Missisiasuk on Lake Champlain at the mouth of the Missisquoi River, Franklin County, Vt.; this was an important Abnaki village, I700-I758. Abnaki, "people of the great grassy meadows."

Missisquoi Bay and River, Franklin County, Vt. Abnaki, translations include "big woman," "big rattlesnake," "much flint," and "great grassy meadows." The last-named is most favored. Also Michiscouy, Petagoubky, q. v. See Greylock.

Missogkonnog Worcester County, Mass.? Nipmuck, "plantation near big outlet"? or "big miry tract"? 
Mistasenee Rock Point, Chittenden County, Vt. Abnaki, "big stone."

Mistic Pond, Middlesex County, Mass. Natick, "the great tidal river." More commonly Mystic, q. v.

Mistic or Mystic River New London County, and Suffolk County, Conn. Mohegan, "great tidal river."

Mistick Middlesex County, Mass. Natick, "great tidal river."

Mistuckset Brook, New London County, Conn. Narragansett? "Little Mystic" or "Mystic brook place." Also given as Mustucksuck.

Mitaganessuk Falls, Eddington Bend, Penobscot County, Me. Abnaki, "at the end of the high tide." This falls marked the place beyond which flood tides from the Atlantic did not go upstream. Variant Mitangowessuck.

Mittaubscot Providence County, R. I. Narragansett, "place of black rocks." See Metaubscot.

Mittineag Hampden County, Mass. Nipmuck, "abandoned fields" or "remains of encampment."

M'kaga-ook took the Little Black River, Aroostook County, Me. Abnaki, "black river." Another spelling, M'kaazaooktook.

M'kazanikut-menahan Black Island, Penobscot County, Me. Abnaki, "black growth (hemlock) island."

M'kazawitego the Black River, Windsor County, Vt. Abnaki, literally "Black River." Found also as M'kazawi sebo. See Kaskactchawack.

M'kuigen Red Hill, Bennington County, Vt. Abnaki, "it is red."

M'nadagahimis the Cold Stream, Penobscot County, Me. Abnaki, "migrating fish," possibly shad or alewives.

Moantukcake Hill, Worcester County, Mass. Nipmuck, "at the tree where they assemble" ? or "at the bend in the river" ?

Moccasin Brook, Worcester County, Mass. Nipmuck, "a shoe."

Moccasin Hill, Essex County, Vt. Abnaki, "a shoe."

Moges Islands, Cumberland County, Me. Abnaki? "wet"? or "black"?

Mogewetu Tolland County, Conn. Podunk? "large dwelling" or "big house."

Mohanhegumewog New London County, Conn. Mohegan, "place of the Mohegan people," or "wolf people." 
Moharmet's Plantation, Dover County, New Hampshire Abnaki or Pennacook, (manrihi) "lavish, wonderful," "a soothsayerhe whom others distrust."

Moharmot's Hill, Rockingham, N. H.; see above.

Moharnes' River; see Mianus.

Mohawk this name is found in a variety of topographic applications throughout New England. It derives from the name of the easternmost Iroquois tribe, applied in derision, fear and contempt by the New England Algonquian peoples. The term means "cowards," "cannibals," "hungry animals," and "wolves."

Mohegan Bluffs and Village, Providence County, R. I. Mohegan, "a hungry animal; (especially) a wolf."

Mohegan Village, New London County, Conn: Mohegan, "wolf people." This was the home of the famous Indian preacher, Samson Occum. See Occum; Mohanhegumewog. The Mohegan tribe is not to be confused with the Mahican, though their name also means "wolf." They lived in the upper Thames Valley, Connecticut. Before I637 they were a part of the Pequot tribe.

Moheganick New London County, Conn. Mohegan, "place of the wolf people," i. e., the Mohegans.

Mohican see Mahican.

Mohootset Pond, Plymouth County, Mass. Wampanoag, "at the abode of the owl."

Moiegans Loups, New London County, Conn. Abnaki and French, "Mohegan Indians," at Mohegan Indian Village. French Colonial officials in Canada called all Indians Loups, "wolves."

Moissehassaky Fairfield County, Conn. Paugussett, "black mire bog."

Molasses Pond, Hancock County, Me. Some Indians have said that this came from an Old Abnaki root, meaning "deep," but this is doubtful. Some say it means "a small island," from monasses, which is equally questionable. See Molunkes.

Molechunkemunk Lake, Oxford County, Me. Abnaki, "stream in a deep ravine"?

Molligwasset Providence County, R. I. Narragansett? or Nipmuck? "valley place." 
Molly's Falls and Molly's Pond. See Mali bowk, Mali panjahlok.

Mollywooket Brook, Coös County, N. H. Abnaki, "at the deep place."

Molnichwock Brook, Coös County, N. H. Abnaki, "very deep place" or "ravine"?

Molockett Mountain, Oxford County, Me. Abnaki, "at the sheer cliffs." But local history says, "place of Mary Agatha, last of the Pequaketts" who died in I8I6. See Mali bowk.

Molunkes or Molunkus Stream, Aroostook County, Me. Abnaki, "a ravine," or "high banks on both sides."

Molynuchgamog Lake, Franklin County, Me? Abnaki, "steep banks lake."

Momagegwetuck Windham County, Conn. Nipmuck? Mohegan? "river abounding in small fish." See Mamaquog.

Momauguin Beach, New Haven County, Conn. Named for a chief of the Quinnipiac tribe. Also called Momauquin, perhaps "Black Feather," sachem circa I638.

Mommock Essex County, Mass. Pennacook? Natick? "place of noises" - the falls of the Merrimack.

Momoronock River, Fairfield County, Conn.? Siwanoy? Paugussett? Delaware? from the name of Chief Momoronock, "he assembles the people." Variants, Mamarack, Momohanock, Moworronoke, Mamaroneck, etc.

Mona Nantucket County, Mass. Wampanoag, "it is deep." Also Moona.

Monadnock Mountains, in Cheshire County, N. H., and Essex County, Vt. In Abnaki, this means, "at the mountain which sticks up like an island;" while in Natick, "at the most prominent island." The name is applied to peaks of resistant rock surrounded by a peneplain. These were used as lookout stations. Tooker (I904) says, "at the most prominent mountain." Also Monadnaëg.

Monadoc see Monadnock.

Monahigan "The Grand Isle" of John Smith, I6I4? See Monhegan.

Monakewego Fairfield County, Conn. Paugussett? Siwanoy? perhaps "plenty of meat," or "deep at the end," or possibly, "deep at the point's end." 
Monamesset Neck, Barnstable County, Mass. Wampanoag, "at the deep fishing place," or "lookout place."

Monamsha Dukes County, Mass. Wampanoag, "cranberries." Also Minamsha.

Monanis Bay, Pemaquid, Lincoln County, Me. Abnaki, "the small island."

Monasses Block Island, R. I. Narragansett, "little island." See Manisses.

Monatiquot Norfolk County, Mass. Natick, "at the deep tidal stream," or "lookout place."

Monchauset Plymouth County, Mass. Wampanoag, "place of dark earth."

Monchusett Bristol County, Mass. Narragansett, "lookout place."

Moncouadi Washington County, Me. Malecite or Micmac, modified by French. Same place, and same meaning as Passamaquoddy, q. v. Variant, Moncouacadie.

Monhantick New Haven County, Conn. Paugussett, "a spruce or cedar swamp."

Monheage Middlesex County, Conn. Natick? "heaped-up place," or "place of mounds"?

Monhegan Island, Knox County, Me. Malecite? Micmac? "out to sea island." Variant, Monhagon.

Monhiggin Plymouth County, Mass. Wampanoag, "place of islands."

Monhiggon River, Plymouth County, Mass. Wampanoag, "place of islands." Also given as "it is deep here"[?]

Monhungunuck now Voluntown, New London County, Conn. Mohegan, "the Mohegan's place."

Moniack ancient Natick (and Pennacook?) name for mouth of the Merrimack river, "place of the island."

Moniecook Barter Island, Knox County, Me. Micmac, "at the island."

Monnebassa Pond, Humphrey's Pond, York County, Me. Abnaki, "deep water."

Monohassett Island, Dukes County, Mass. Wampanoag, "at the small island."

Monomonac Lake, Worcester County, Mass. Nipmuck, "at the deep place;" or "deep black mire." 
Monomonock Lake, Cheshire County, N. H. Abnaki, "at the very deep place." (Perhaps Natick, "place where there is grain for seed")? Also as Monomak.

Monomoy Island and Point, Barnstable County, Mass. Wampanoag, probably "lookout or observation place," but possibly "deep water." See also Monomoy Marshes, and Manamoyik.

Monomoy Marshes, Nantucket County, Mass. Wampanoag, "deep, black" (mire). Other spellings Monomout, Monomoiett, Monomoyet, etc.

Mononkatesuck see Menunketesuck.

Mononowuttock Middlesex County, Conn. Hammonassett, perhaps "island dwellers"?

Monoosmoc Brook, Worcester County, Mass. Nipmuck, "deep, miry place," or possibly "deep stream."

Monoosuck Worcester County, Mass. Nipmuck, "deep brook."

Monoposhke see Mooapske.

Monponset Pond, Plymouth County, Mass. Wampanoag, "at the deep clear place," or "at the deep inlet."

Monsapec Village, Washington County, Me. Malecite, "island far at sea."

Monson Hampden County, Mass. If Indian, perhaps Pocumtuck, "moose people." But more likely, from an English name.

Montauk Point, south of Stonington, Conn. Now part of New York State, Montauk was before I625 and as late as I790 a favorite fishing place for Eastern Connecticut Indians. Montauk, "at the fort," or "fort place high land."

Montaup Neck, Bristol County, R. I. Narragansett, "sufficiently fortified"? or "lookout place"? See Montop.

Montinicus Island, off Rockingham County, N. H. Abnaki, "little island far off, separated from the mainland." See Matinicus.

Montononesuck Brook, New London County, Conn. Eastern Niantic? "wade across the brook to the island"?

Montop Hill, Bristol County, R. I. Narragansett, "this island is sufficiently fortified." This is the original name for Mount Hope. Possibly from M'ontep, "the head." See Montaup.

Montowese Village, New Haven County, Conn. Quinnipiac, a chief named, "Little God," who sold land in I638. 
Montsweag Bay, Brook and Village, Sagadahoc County, Me. Modified Abnaki, "narrow, dug-out channel."

Monuhchogog Worcester County, Mass. Nipmuck, "near the deep pond"? or "ye shall be strengthened"? See also Manchaug and Manchage.

\section{Monuhchug see Manchaug.}

Mooanum see Wamsutta.

Mooapske New London County, Conn. Eastern Niantic, "black stones." Also Monoposhke, "black, muddy."

Moodus Reservoir, River and Village, Middlesex County, Conn. Wangunk, "bad noise," from mache moodus. The "Moodus Noises," probably caused by subterranean shiftings, are still a cause for concern.

Moonassachuet River, Washington County, R. I. Narragansett, "deep backward (reversing?) current."

Moosabek Reach, Washington County, Me. Abnaki, "moosehead rock."

Moosalamoo Mountain, Addison County, Vt. Abnaki, "he trails the moose," or "the moose departs."

Moosamuttuck River, New Haven County, Conn. Quinnipiac, "big springs in the river," or "big rocks in the stream," just possibly, "Big Shoulders," the name of a chief?

Mooscoog Mountain, Grafton County, N. H. Pennacook, "meadow place," or "grassy place," or "abode of the moose." ? This is probably the same mountain as Moosilauke.

Moosehausic Providence County, R. I. Narragansett, "at the great marsh." Variants, Mooshassuck, Mooshawset, Mowshawsuck, Moshosick, etc.

Mooseleuk Mountain, Aroostook County, Me., and Stream, in Piscataquis County, Me. Abnaki, "moose place."

Mooselookmeguntic Lake, Franklin County, Me. Abnaki, "portage to the moose feeding place," or "moose feeding among big trees"?

Moosepayechick an island at Jonesport, Washington County, Me. Abnaki, "moose's head." See Moos-i-katchick.

Moosetookmeguntic Oxford County, Me. Abnaki, "moose's river (running through or out of) swampy place." 
Moos-i-katchick Cape Rosier, Hancock County, Me. Malecite, "a moose's rump." Indians imagined this to be the moosecalf killed by Glooscap.

Moosilauke Brook and Mountain, Grafton County, N. H. Abnaki, three defensible translations: "at the place of the ferns," and "good moose place" along the brook; also, "at the smooth place" on the summit.

Moosompsquetuh Bennington County, Vt. Natick, "near (or upon) smooth (weathered) rocks."

Moosup Pond, River and Villages in Windham County, Conn., and Providence County, R. I. Narragansett, named for Chief Mausup, a brother of Miantanomi, q. v. He was also called Pessicus, q. v.

Mootinoo Island, said to be off the Isles of Shoals, Rockingham County, N. H. Abnaki, somewhat modified, for "far off island."

Mopang Stream, Washington County, Me. Malecite, "solitary place."

Moratiggon Island, Knox County, Me. Probably Old Abnaki, "passage, or strait" between the island and the mainland. This island, now known as Monhegan, was a domain of Samoset. Variants. Moratiggan, Morratiggin.

Moscachuck see Musquechuge.

Moscatage see Musquechuge.

Mosemadage Castine, Waldo County, Me. Abnaki, "snow shoe marks." Spelled also Mosemadega.

Moshantatuck Brook; see Mashanticut.

Moshassuck River, Providence County, R. I. Narragansett, "great brook in the marshy meadow."

Moshenupsuck Tolland County, Conn. Nipmuck, "great brook," or "great outlet;" perhaps "great pond."

Moshowunganuck Washington County, R. I. East Niantic, "at the place of the great bend," in the Pawcatuck River.

Moshtatuck Creek, Nantucket County, Mass. Wampanoag, "grass river."

Moshwansicutt River and Pond; see Moswansicut.

Moskituash Creek, Bristol County, R. I. Narragansett, "meadow."

Moskituauke Washington County, R. I. Eastern Niantic, "grassy land." Sometimes Mosquito Hawk. 
Moskwaswagamocsis Daggett Pond, Aroostook County, Me. Abnaki, "little muskrat pond."

Moskwaswagamok Shallow Lake, Aroostook County, Me. Abnaki, "muskrat lake."

Mossonachud Hill, Worcester County, Mass. Nipmuck, "hill covered with dried trees," or perhaps "canoe-wood hill."

Moswamcut Pond; see Moswansicut.

Moswansicut Pond and River, Providence County, R. I. Narragansett, "place of mist and fog," or "red hill," or "at the great bend."?

Mount Hope see Montaup, Montop.

Mountquies Neck, York County, Me. If Indian, perhaps Abnaki, "salt-water (or tidal) ditch."

Mountsweag Bay, Lincoln County, Me. Abnaki, "dug-out narrows."

Mousam River, formerly Cape Porpoise River, York County, Me. If Indian, perhaps Abnaki, "grandfather," or Old Abnaki, "a snare."

Mouscochuck Creek; see Musquechuge.

Moxisset Essex County, Mass. Natick, "place of small grasses"? or "small shoes"?

Moz Mushkeg Grafton County, N. H. Abnaki, "moose meadow."

Mozias a locality in Essex County, Vt. May be Abnaki, "moose meat," or perhaps from the name of a White man named Mozier or Mosier.

Mozodepowadso Mount Mansfield, Chittenden County, Vt. Abnaki, "moose head mountain" from its fancied resemblance to the horned animal's head.

Mozodup Nebes Moosehead Lake, Piscataquis County, Me. Abnaki, literally, "moosehead lake."

M'skootuk Lake, Washington County, Me. Abnaki, "trout place." (But this root gives trouble; the translation might be "at a point of land," or "fireplace.") See M'skutook and Schoodic.

M'skutook an abbreviation for Pemskudek, Marsh's Farm, Old Town Island, Penobscot County, Me. Abnaki, "extended burned-over place," near the river. See M'skootuk.

M'squamagweseebo Hale Brook, Penobscot County, Me. Abnaki, "salmon river" or "salmon brook." 
M'squam Chumaki Belknap County, N. H. Abnaki, "salmon spawning place."

M'squam Nebis now Squam Lake, Grafton County, N. H. Abnaki, "salmon lake." This title noted on Aubéry's map of I7I5.

M'squamscook now Exeter, Rockingham County, N. H. Abnaki, "at the abode of salmon."

M'teoulin, Madeluno, and Madewahun, (modern Abnaki and Malecite); Manrigwa in Old Abnaki; and Magermette or Majalmit in Modern Abnaki all mean "one who drums," that is, "soothsayer," "witch or sorcerer," etc. These terms figured in unofficial or local names, most of which were suppressed after 1630 by White religious authorities. Some of these have been re-located or discovered by linguistic students, e. g., Kinkajou.

Muchquachema Swamp, Plymouth County, Mass. Wampanoag, "red fox."

Muckcheese Middlesex County, Conn. Natick? "small animal," such as a kit beaver, or bear cub.

Muckhaneek probably the same place as Mohegan Village, New London County, Conn. Mohegan-Pequot, "wolf people."

Mucqut Cove; see Kitamuckqut.

Mugaleep-ahwangan Pond, Penobscot County, Me. Malecite, "the caribou trail."

Mugget Hill, Worcester County, Mass. Nipmuck, "beaver"? Natick, "grass"?

Muggs Holes, New London County, Conn. Mohegan, "storage bins"? These may be old, dry spring basins; the holes were indeed used for storing potatoes, turnips, etc., but see Mukyaweesug. (Were these hiding places for "wee people"?)

Mugunkwaquog Worcester County, Mass. Nipmuck, "place of the gift."

Mugurrewock Lakes, Washington County, Me. Malecite, "the shoveler," or caribou.

Muhhekaneew Litchfield County, Conn. etc. Mahican, "people of the flowing river." Other sources give "wolf people." These were Mahican, not Mohawk, nor Mohegan.

Mukquata Neck, Washington County, R. I. Eastern Niantic, "place of rushes." 
Mukyaweesug New London County, Conn. Mohegan, "little boys" or "little people;" leprechaun-like dwarfs. This name has been translated also as "whippoorwills." The Abnakis have similar "wee people;" see Wanagamesswak.

Mulpus Brook, Worcester County, Mass. Nipmuck, "little heaps" probably muskrat or beaver lodges. But some say this is from the name of a French settler, de Mulpuis.

Mundoo-uscoo took the Eastern River, Kennebec County, Me. Abnaki, "Devil's-rush river." These rushes were probably cat-tails, supposed by some Indians to have magical powers.

Munhan New Haven County, Conn. Quinnipiac, "an island." Spelled also Manhan.

Munhumsqueeg see Manhumsqueeg.

Munnacommuck Windham County, Conn. Nipmuck, "island place," or "island plantation," or "berry plantation." See Minnacommuck, Mincommuck, etc.

Munnatawkit Island, either Fisher's Island or Plum Island, south of New London and Niantic respectively; politically now part of New York, but formerly important to Eastern Connecticut Indians. Western Niantic? "menhaden country," where (said Roger Williams) the Pequots went to fish and to make new fields of corn, fertilized by the menhaden.

Mun-olammon ungan West Branch of Pleasant River, Piscataquis County, Me. Abnaki, "heaps of red paint in making."

Munponset Plymouth County, Mass.? Natick, "at the deep clear place."

Munsungan Lake and River, Piscataquis County, Me. Abnaki, "humped-up island."

Munt Hill, Rockingham County, N. H. Pennacook? or Natick? Said to be the name of an Indian who lived near there. Sometimes translated as "a basket."

Murranook Monhegan Island, Knox County, Me. Malecite, "the island." See Monhegan.

Musabek Grafton County, N. H. Abnaki, "moose head rock."

Muscatanupus Pond, Hillsboro County, N. H. Pennacook? Natick? "small red tortoise."

Muscatuapus Merrimack County, N. H. Pennacook, "the large pickerel." 
Muschaug Ponds, Washington County, R. I. Eastern Niantic, "place of rushes." Variant, Mushaug.

Muschopaug Pond, Worcester County, Mass. Nipmuck, "muskrat pond," or "wide pond." Also Muskopog, Muscopauk, etc.

Muscongus Island and Sound, Lincoln County, Me. Abnaki, "many rock ledges," or "rock," or "large rock ledges."

Muscoota Litchfield County, Conn. Mahican? "meadow," or "grassy land," or "burned pasture."

Muselenk an island near Eastport, Washington County, Me. Malecite and modified English, "moose island."

Mushantuxet New London County, Conn. Pequot, "well forested," or "place of large trees." Also Mushantucksett. See Mashantuxet.

Mushattchuckapeake Providence County, R. I. Narragansett, "pond at the great mountain," or "big hill near the edge or bank of the pond."

Mushawwomuk Norfolk County, Mass. Natick, "at the great canoe landing," or "great ferry," depending upon the occasion; mishoonhomwock means "they go or come by water."

Mushuagusset Pond, Washington County, R. I. Eastern Niantic, "muskrat place." Also Mushuagannie.

Musicatchick Cape Rosier, Hancock County, Me. Malecite? Abnaki? "moose's rump," the rump of the moose-calf killed by Glooscap.

Muskagwah marshes or meadows along the Connecticut River, Essex County, Vt. Abnaki, "a marsh."

Muskapasset Plymouth County, Mass. Wampanoag, "wide cove"? or "grassy, open land."?

Muskechuge see Musquechuge.

Muskeget Channel and Island, Nantucket County, Mass. Wampanoag, "great green place," or "grassy place."

Muskehtu-auk Plain, Hartford County, Conn. Tunxis, "green grass place;" also called Mosquito Hawk Plain.

Musketaquid Middlesex County, Mass. Massachuset, "place of rushes." Sometimes given as "rushy creek," or "rushy island." Also Musquetequid.

Musquacook River, Aroostook County, Me. Abnaki, "muskrat place," or "birch bark place." 
Musquash a bog, lakes, mountain and stream in Washington County, Me., a road in Hillsboro County, N. H., and a meadow in Orange County, Vt. All are Abnaki, meaning "reddish-brown animal," the muskrat.

Musquashcut Pond, Plymouth County, Mass. Wampanoag, "abode of muskrats."

Musquauke New Haven County, Conn. Quinnipiac, "red earth." Musquebaug Worcester County, Mass. Nipmuck, "red pond."

Musquechuge Kent County, R. I. Narragansett, "place where rushes grow." Variants, Musquachug, Mascachuge, Muskachaug, etc.

Musquetaug Washington County, R. I. Narragansett, "place of rushes."

Musquetohauke Brook, Providence County, R.I. Narragansett, "grassy place." Variants, Musquetohaug, Mosquito Hawk, etc.

Musquetuxet Providence County, R. I. Narragansett, "red brook"? or "brook in grassy place," or "grassy meadow brook land." Given also as "place of herbs."

Musquinepash Plymouth County, Mass. Wampanoag, "trout" (plural).

Mussachuck Creek, Bristol County, R. I. Pocasset, "at the place of flags or rushes."

Mussaco Hartford County, Conn. Tunxis, "great lowland" or "big outlet." Also Mussauco; see Massaco.

Musshauge Swamp, Worcester County, Mass. Nipmuck, "grassy pond," or "grassy place."

Mutanagwes Dochet Island, Washington County, Me. Passamaquoddy-Abnaki, "place for storage." Also Muttonegwenish, and Muttoneguis.

Mutighticoss see Titicus, Tittituck.

Muttock see Muttught Keese.

Muttoneguis Dochet Island, Washington County, Me. Abnaki? Malecite? "little island near us," used for storage?

Muttonegwenish Little Dochet Island, Washington County, Me. Abnaki? Malecite? "tiny island near us," used for storage?

Muttught Keese Plymouth County, Mass. Wampanoag, "big shoulders." From the name of a famous chief at Namasket. Also Muttock. 
Muxquatah Neck, Washington County, R. I. Narragansett, "place of rushes."

Muxquataug Pond, Washington County, R. I. Narragansett, "place of rushes." Also Muxquata, Muxqua, etc.

Muyquataug Washington County, R. I. Eastern Niantic? "path to the pond place" ? or "place of rushes." Variant Muyquatage.

Myacomet Pond; see Miacomet.

Mystic River and Village, New London County, Conn. PequotMohegan "the great tidal river." Also Mistick.

Mystic Lakes and Pond, Middlesex County, Mass. Natick, "great tidal stream."

Mystic River, Suffolk County, Mass. See Mistic.

\section{$-\mathbf{N}-$}

Naacook, Naamhok, Naimkeak, Naumkeag etc. Pennacook? Abnaki? Natick? "at the fishing place." Several such scattered over eastern New Hampshire and northern Massachusetts.

Naamcoyicke Creek, Barnstable County, Mass. Wampanoag, "heaps of fish," possibly drying for fertilizer? But see Nanacoicus.

Naamkeak Middlesex County, Mass. Pennacook, "at the abode of eels."

Naaomuck Neck, Washington County, R. I. Narragansett, "narrows fishing place." Variant, Naomuck.

Nabnasset Pond, Hampden County, Mass. Nipmuck, "near the dry land" ? or pond "number twelve"? or "chestnut tree place"?

Nachecot Providence County, R. I. Narragansett, "middle place."

Nacheek location unknown, but see Nachick below.

Nachick Hill, Kent County, R. I. Narragansett, "my house."

Nacommuck Brook, Worcester County, Mass. Nipmuck, "enclosed point of land," or "garden at the point," or "sandy field"?

Nadhuset Stream, Plymouth County, Mass. Narragansett, "procure meat or game," or "hunting place."

Nagatossett location unknown, probably New London County, Conn. Eastern Niantic, "sandy brook."

Nagatuck see Naugatuck.

Naggawoomcom Pond, Worcester County, Mass. Nipmuck, probably a variant of Agawam in the sense of "a canoe unloading place," "basin at the sandy place." 
Nagog Pond and Village, Middlesex County, Mass. Wampanoag, "at the sandy place," or "at the corner" or "near the path."

Nagusset Abagadusset Point, Sagadahoc County, Me. Abnaki, "at the small point."

Nagwadog Lone Tree Hill, Orleans County, Vt. Mahican? "one tree."

Nagwamqueeg Mallison Falls, Presumpscot River, Cumberland County, Me. Abnaki, "a single sand bar"?

Nahant Essex County, Mass. Natick, "the point." Also translated as "almost an island;" an older version makes it "two things (islands) surrounded," probably surrounded at high tide. (Nahanton, or Nahantum, was a chief in this region.)

Nahapassumkeck Plymouth County, Mass. Natick, "right there at the roasting place." Also given as "place of posts or stakes."

Nahatan Street, Middlesex County, Mass. Natick, from William Nahetan, a Christian Indian circa 1650.

Nahett Peninsula or Neck, Bristol County, R. I. Narragansett, "at the point."

Nahigonset Island, Washington County, R. I. Narragansett, "at the small point." Variant, Nahiganset.

Nahma jimski congomoc Haymock Lake, Aroostook County, Me. Abnaki, "fishing place at dead water lake," on high land.

Nahmajimskitegwek Penobscot County, Me. Abnaki, "dead water fishing place meets stream" (tumbling out of high place)? See Nahma jimski congomoc.

Nahmakanta Lake, Piscataquis County, Me. Abnaki, "plenty of fish."

Nahnek-bahntik Caribou Lake, Piscataquis County, Me. Abnaki, "big island falls." This place was an important source of wycobee, or leatherwood, so important in manufacturing pack-goods.

Nahoosik see Hoosic, and Mahoosuc.

Nahteawanet Plymouth County, Mass. Wampanoag, "four cornered gap," or "square shaped hole."

Nahumkeag Falls, Island, Pond and Stream, Kennebec County, Me. Abnaki, "eels run out."

Nahwesetuck anciently a Mohegan village on Fenton River, Windham County, Conn. Mohegan, "small fish stream." 
Naiag Middlesex County, Conn. Hammonassett, "a point." See Nayaug.

Naidni Rutland County, Vt. This is a jape - just the word 'Indian' spelled backwards.

Naiog Hartford County, Conn. Wangunk, "at the point."

Naiwayouk Point, New London County, Conn. Western Niantic? "crooked point." See Noank.

Nalabongan Sebasticook Lake, Penobscot County, Me. Abnaki, "above the lake," or "the lake above." Nala, or nara seem to indicate a resting-place at a pond above a stretch of rapids.

Nalagamoik Pond, Penobscot County, Me. Abnaki, "above the lake."

Nalagwem Menahan Verona Island, Hancock County, Me. Abnaki, "the island upstream from bogs."

Nalaseemagamobsis Shad Pond, Penobscot County, Me. Abnaki, "below the sluggish place near the small rock," where guides and hunters rested after poling up the stream.

Nallahamcongan alias Natanis Franklin County, Mass. Abnaki? Pennacook? "fishing place downstream?" See Nalumsunkhungan, Natanas.

Nallahoodus Mountain, Penobscot County, Me. Abnaki, "below the rapids"? or "fall on either side" ? See Nulhedus.

Nallawagwis Washington County, Me. Malecite? Abnaki? "below the whirlpool, or great eddy"? But see Narraguagus.

Naltaug Brook, Worcester County, Mass. Nipmuck, "narrow place." Alternate spelling Nautaug.

Nalumsunk-hungan Rips, Ayers Rips, Penobscot County, Me. Abnaki, "fish curing place below the outlet."

Namaaskotic Lake Wallis, Essex County, Vt. Abnaki, "salmontrout place."

Namacock Neck, Barnstable County, Mass. Wampanoag, "at the fishing place."

Namadunkeeunk see Nemadunkehunk.

Namagonic Seymour Lake, Orleans County, Vt. Abnaki, "salmon trout spearing place." See Nammygoe.

Namaquaog exact location not known, Windham County, Conn. Probably Nipmuck, "fish-place." (Namacush? see Togue.) 
Namareck Hartford County, Conn. Podunk? or Poquonnock? "fishing place." Other spellings Namelake, Nameroc, Nameroake.

Namaskeese Plymouth County, Mass. Wampanoag, "heaps of fish," perhaps for fertilizer?

Namasket Plymouth County, Mass. Wampanoag, "at the abode of fish."

Namaskik Hillsboro County, N. H. Abnaki, "at the fishing place." See Amoskeag. Another spelling Namaoskeag.

Namassakeeset Plymouth County, Mass. Wampanoag, "heaps of fish."

Namdamassuagum Knox County, Me. Abnaki, "sucker lake." See Nemdamassuagum.

Nameaug New London County, Conn. Mohegan-Pequot, "the fishing place." Other spellings Nameek, Nameeg, Nameug, etc.

Nameaug Essex County, Mass. Natick, "fishing place" possibly "place where eels are caught."

Namequoit Point, Barnstable County, Mass. Wampanoag, "at the fishing place."

Nameroke formerly an Indian village at Warehouse Point, Hartford County, Conn. Podunk, "fishing place." See Namareck.

Nameunkquasset Plymouth County, Mass. Wampanoag, "at the end of the heaps of fish."

Namkeag Essex County, Mass. Natick, "fishing place," or "eeling place."

Namkecke River, Rockingham County, N. H. Abnaki, "fishing place."

Namkook Boston Neck, now Salem. Washington County, R. I. Narragansett, "at the fishing place." Also Namacoke and Nameoke.

Nammygoe Pond, Piscataquis County, Me. Penobscot, "salmon trout." Same as the Togue or Nammacush. See Namagonic.

Namokanok Island, Penobscot County, Me. Abnaki, "turtle shell island."

Nampscoscoske an ancient name for Wells, York County, Me. Abnaki, possibly, "comes out of sandy place," a stream? or "sandy, rocky land"? 
Nampshaket Barnstable County, Mass. Wampanoag, "temporary sojourning place"?

Nampsic Pond Providence County, R. I. Narragansett, "fishing place."

Namquit Pond, and Point, Kent County, R. I. Narragansett, "fishing place." Another source gives this as a contraction of Quinnemquit, "high spring."

Namquoxet Shore, Providence County, R. I. Narragansett, "at the little beach," or "at the small fishing stand."

Namshaket Creek, Barnstable County, Mass. Wampanoag, "a fishing place." But see Nampshaket, above.

Namucksuck New London County, Conn. Western Niantic? "fishing place at outlet."

Namyak probably this country was Pequot or Mohegan territory, around New London, Conn. The name means "fishing place" in those tongues. Also Namyoke.

Nanachestawack Fairfield County, Conn. Mahican, "place of safety," or "fort."

Nanacoicus Pond, Worcester County, Mass. Nipmuck, "an earthen pot," or "an earthern kettle."

Nanakumas Nantucket County, Mass. Wampanoag, "the shore," literally, "dry land." Variant, Nunna Koma.

Nanantomqua Worcester County, Mass. Nipmuck, "rocky point"? But see Nonantum.

Nana penahekan Berkshire County, Mass. Mahican, "stone heaps; piles of fallen rocks." See Mannan penokean.

Nanapenhakan Brook, Berkshire County, Mass. Mahican, "stream coming out of stone heaps." Variants, Nanipenihekan, Nanahpenahekan, Mannanpenokean.

Nanaquacket Pond, Bristol County, Mass. Wampanoag, "swamp dries up." Also Namaquaket, Nunnacket, Nunnaquacket.

Nanaquonset Island, Fox Island, Washington County, R. I. Narragansett, "above the confluence of two streams"? "narrow strait," or "long beach; long dry shore."?

Nanenol Mehanol off Burlington, Chittenden County, Vt. Abnaki, "five islands."

Nanepashemet Village, Essex County, Mass. Nipmuck, "he who walks at night," i. e., The Moon. Nanepashemet was the 
great Moon Chief of the Naumkeag at Salem in I6r6. A "Queen Nanepashemet" was killed in I62I. Another possible translation is "dry fountain."

Nanequoxet Fox Island, Washington County, R. I. Narragansett, "above the confluence of two rivers," or "narrow strait." See Nonnequasset.

Nanhoc location unknown, perhaps York County, Me.? Abnaki, "downstream place," or perhaps a modification of Manhoc, "island place"?

Nanipsick Pond, Providence County, Conn. Narragansett, "place of the narrow body of water"? or "dried-up pool"? See Nipsic.

Nannaquaket Pond, Newport County, R. I. Narragansett, "it dries up."

Nannaquokset Neck; see Nonequasset.

Nannihiggonsick R. I. This is the name of a small island described by Roger Williams. Rough translations include "good ferry," "narrow strait," etc. Some references state that this name may be the original of the term Narragansett.

Nannonicky Dukes County, Mass. Wampanoag, "dry land"?

Nanomesset Island, Dukes County, Mass. Wampanoag, "at the narrow place abounding in fish." Now Gosnold Island.

Nanrantsoak the Old Abnaki name for Norridgewock, q. v.

Nanshigganset see Nahigonset.

Nansquatog Windham County, Conn. Nipmuck, "above the confluence of two rivers."

Nantascot Nantasket, Plymouth County, Mass. Wampanoag, "at the place of two points" (which appear at low tide). Also "narrow bridge place" (at low tide), Nantoyusk-ut? See Nahant.

Nantasket Brook, Windham County, Conn. See below.

Nantasket Norfolk County, Mass. Natick, "at the strait," or "place of the ebb tide." See Nantascot.

Nantiganset Bay, Little Narragansett Bay, Washington County, R. I. Narragansett, "at the small point," or "at the place where the river is no longer narrow."

Nantucket County, Island, Mass. Wampanoag, either "in the midst of waters" or "at the far-off (at sea) place" or "where 
it is, the sea gets broader;" also Narragansett, "far off, among the waves," and "point of land in the stream." Perhaps "narrow river," the strait between the island of Nantucket and Tuckernuck Island.

Nantusiunk Newport County, R. I. Goat Island, in Newport Harbor. Narragansett, "narrow ford or strait." Also Nomsusmuck, and Nantusununk or Nantuzenunk. See Nantascot.

Napackamach Fairfield County, Conn. Mahican? "fish weir," or "fish trap place."

Napchecoy Nantucket County, Mass. Wampanoag, "at the hill near the shore."

Naperhan Creek, Fairfield County, Conn. Wappinger, "a snare or trap."

Napucke Fairfield County, Conn. Paugussett, "at the pool" of water.

Naquag Worcester County, Mass. Nipmuck, "a point or angle."

Naquamke Falls, Kennebec County, Me. Abnaki, "place of eels." See Nequamwicke and Nahumkeag.

Narantsoak Somerset County, Me. Abnaki, na-itswak, "downcurrent," or "rapids." See Norridgewock.

Narmacungawak Brook, Coös County, N. H. Abnaki, "a deep fishing place."

Naromiyock nowhusun katankshunk Brook, Litchfield County, Conn. Mahican, "fishing place in the gravelly stream near the big hill." (Or possibly a name built up by Whites).

Narragansett many places; parks, trails, villages, and names in New England. All are Narragansett, meaning "at the small narrow point." This was an important Rhode Island tribe which occupied most of Rhode Island west of Narragansett Bay. Until defeated during King Philip's War they were the most powerful tribe in southern New England. Also Narraganset. See Nahigonset, and Nannihiggonsick.

Narragooe an ancient Abnaki village, location uncertain; translation perhaps, "above the marsh," or "upstream from canoe landing." See Narraguagus below.

Narraguagus River, Hancock County, Me. Abnaki, "above the boggy place."

Narshapogge Hartford County, Conn. Probably Wangunk, "two ponds near together." Also Neeshapaug. 
Nasauket Kent County, R. I. Narragansett, "at the neck of land," "land between rivers."

Nasawi the land between the Waits River and the Connecticut River, Orange County, Vt. Abnaki, "midway," or "land between."

Nasawtuck Hill, Middlesex County, Mass. Natick, "the middle river," or "river between."

Nasbaug Strafford County, N. H. Abnaki, "three ponds."

Nashamoiess Dukes County, Mass. Wampanoag, "in the middle of the dark land;" or Natick, "small path between."

Nashanticut Providence County, R. I. See Mashanticut.

Nashaquits Cliffs, Nantucket County, Mass. Wampanoag, "between islands," or "middle of islands."

Nashawag Windham County, Conn. Nipmuck, "between two river branches." Variants, Nashaway, Nashua, Nashawog. See Ashawog.

Nashawake Worcester County, Mass. Nipmuck "place between river branches."

Nashawannuck Hampden County, Mass. Nipmuck, "the meadow between."

Nashawena Island, Dukes County, Mass. Wampanoag, "it lies between."

Nashawena Road, Barnstable County, Mass. Wampanoag, "half way," or "between."

Nashawomauk Nantucket County, Mass. Wampanoag, "in the middle of the country." But see Shawmut.

Nashayte Nantucket County, Mass. Wampanoag, "place between two tidal streams," or "two branches" of the harbor.

Nashoba Brook, Middlesex County, Mass. Nipmuck, "between waters." See Neshobe, etc.

Nashua City and River, Hillsboro County, N. H. Pennacook, "between" streams.

Nashua Reservoir and River, Worcester, Mass. Nipmuck, "between" streams. Variant Nashawa-eg.

Nashuae Komuck Dukes County, Mass. Wampanoag, "the half-way house."

Nashuatukqut New London County, Conn. Nipmuck? "between two rivers," the Yantic and the Quinebaug. Also found as Nashuatukut. See Shetucket. 
Naskeag Point, Harbor and Village, Hancock County, Me. Abnaki, "place at the end."

Nasket Point, Sagadahoc County, Me. Abnaki, "at the end."

Nasketucket Bay and Village, Bristol County, Mass. Narragansett, "at the end of the tidal stream."

Nasnocomacock Plymouth County, Mass. Wampanoag, "at the halfway place," or "at the place between." Another source gives "end of plantation" or "as far as the plantation."

Naspatuckett River; see Woonasquatuckett.

Nassahegan State Forest, Hartford County, Conn. Poquonock or Tunxis, "he has two houses" or "he lives at halfway place." ? Also spelled Nosohogon, "second dwelling place." An Indian by this name sold lands in Connecticut in I673.

Nassaque mentioned by Capt. John Smith. Possibly Nasket, q. v.

Nassawket Shore; see Nasauket.

Nasso-emek Dyer's Neck, Kennebec County, Me. Abnaki, "land between."

Nassouac the ancient name for Merrymeeting Bay, Sagadahoc County, Me. Abnaki, "land between." Variant, Naxoat.

Natanas Brook, Franklin County, Mass. Probably Abnaki, from Natanas (or Natanis), a brother of Sabbatus; both were helpful to the Americans in the Revolution. It is suggested that the name comes from Natick, nootimis, "oak tree," but other evidence traces it to Nathan-is, "little Nathan." See Nallahamcongan, Sabbatus.

Natanis Brook, Cheshire County, N. H. Abnaki, "little Nathan." Natardin Mountain, Me. See Katahdin.

Natchaug State Forest, Windham County, Conn. Nipmuck, "between rivers."

Natchick Hill; see Nachick.

Natick Village and Falls, Kent County, R. I. Natick, "the place I seek," that is, "my home, my house." But another source gives "a plain," or "a clearing." Also a village in Middlesex County, Mass. See Massachuset.

Naticook now Litchfield, Hillsboro County, N. H. Abnaki, "at the place where the river flows downward."

Natuah possibly in Kennebec County, Me. Abnaki, "a meadow or intervale"? 
Naubuc Village, Hartford County, Conn. Wangunk, "overflowed, flooded."

Naugatuck River and Village, New Haven County, Conn. Quinnipiac, "a single tree." (Naugatuck Reservoir is in Fairfield County.) Another spelling, Nawcotuck. See Nagwadog.

Naukeag Lakes and Village, Worcester County, Mass. Nipmuck, "fishing place," or "sandy place," or "soft earth."

Nauma Nantucket County, Mass. Wampanoag, "(long) point?" or "(long) sand spit," "(long) fish"? Quon-nauma?

Naumkeag or Nahum Keke Essex County, Mass. Natick, "eel place;" another source gives "sandy land."

Nausauket Village, Kent County, R. I. Narragansett, "at the second outlet," or "between outlets." Another spelling, Nausaucat.

Nauseag Hancock County, Me. Abnaki, "the point of land." See Nasket.

Nauset Barnstable County, Mass. Nauset, "at the place between" (Cape Cod Bay and the Atlantic Ocean ?), or "on the point."

Naushon Island, Dukes County, Mass. Wampanoag, "angular"? "middle"? "Spirit of God"? "big spring"? "first"?

Nau-wot Fairfield County, Conn. Wappinger? "a great way" or "far distant." See Nawottok.

Nawaas the general Connecticut region. Also Nowaas.

Nawbesetuc the site of an ancient Indian village, Tolland County, Conn. Mohegan, "land at the pond," or "overflowed river country," bottom land.

Naweag see Noank.

Nawlombages Etna Pond, Penobscot County, Me. Abnaki, "a little pond upstream."

Nawottok Hampden County, Mass., and Hampshire County, Mass. Possibly a transplant by White settlers from Connecticut; Wangunk, "land far away?" Otherwise given as "middle of the river," that is, on or at a small island in the stream.

Naxoat the St. John's River, the boundary between parts of northern Maine and Canada. Malecite? Abnaki? perhaps "far away" or, "at the point of land"'[?]. See Nassouac.

Nayacke see Nayaug.

Nayage see Nayaug. 
Nayantacawnick a small island off shore from New London County, mentioned by Roger Williams; the precise spot is not known. Narragansett, "at the place across from the Niantic." Probably Gull Island or Plum Island, N. Y.

Nayantaquit Eastern New London County, between Thames and Pawcatuck rivers. Narragansett? "points of land on tidal stream."

Nayanticot (Point Judith), also Nayanticut. See Nianticut.

Nayas Hampden County, Mass. Nipmuck, "a point, or angle."

Nayasset Hampden County, Mass. Nipmuck, "at the small point."

Nayatt Point and Road, Bristol County, R. I. Narragansett, "at the point." Also Nayot, Nayott.

Nayaug the point at the mouth of Roaring Brook, Glastonbury, Hartford County, Conn. Probably Wangunk, although this word which means "point or angle" is used in several major dialects.

Nayaug Colchester Point, Chittenden County, Vt. Mahican, "a point."

Nayump River, Fairfield County, Conn. Mahican, "point, rocky." See also Nayumps, below.

Nayumps Rock Point, Fairfield County, Conn. Mahican, "rocks at the point." (Where the Nayump river runs into Housatonic).

Nayumpsk New London County, Conn. Western Niantic? "rocky point." Spelled also Nyumph.

Nayyag Hampden County, Mass. Nipmuck, "at the point."

Nayyocossuck Hampden County, Mass. Nipmuck, "brook near the point of land."

Neaguamkot Kennebec County, Me. Abnaki, "the first place," hence, "old village"? See Negan odanak.

Neanscott River, Androscoggin County, Me. Abnaki, probably "at the place where they cure fish," but possibly "trembling, boggy banks."

Neanticoet and Neanticot see Nianticut.

Neastoquoheaganuck see Escoheag.

Nebisonbik mineral springs in Essex County, Vt. Abnaki, "mineral water place."

Nebissee-woldam-wogan Weeping Rocks, near Pownal, Bennington County, Vt. Abnaki, "waters of sorrow," according to Rowland Robinson, Vermont's famous blind author who 
collected and preserved many Indian names. (Also in Mohawk, Kanyatare Keahea, "lake of death"). This landmark was destroyed in a road improvement program in I960.

Nebraska Notch, Chittenden County, Vt. Osage, "the flat water," or "wide, flat expanse of river."

Necadoram the main stream of the Sebasteguk River. Penobscot County, Me. Abnaki, possibly "better route up this fork."

Nechawonak see Newichawannock; also Nechawonek, Nechewannick, Nechowanuck, etc.

Neconaugamook Round Pond, Third Machias Lake, Washington County, Me. Abnaki? Malecite?" third long lake."[?]

Necotok location unknown, perhaps in Knox County, Me?. Abnaki, "fork in river." See Nicatow.

Neddick Cape, York County, Me. Micmac, "heaving in sight of a solitary upstanding island." Variants, Nedick, Neddock.

Neekequaw Pond, Washington County, R. I. Narragansett, "home place, or house"? or "double pond"? Also Neekeequoweere and Neekeequawese.

Neekounegan the right bank carry, past Old Town Falls, Penobscot County, Me. Abnaki, "the better, or preferred carry."

Neesepegesuck Brook and Ponds, Middlesex County, Mass. Nipmuck, "two narrow outlets." Also found as "two pond brook."

Neeseponsonet Worcester County, Mass. Nipmuck, "in the neighborhood of two clear ponds," or "at the second waterfall."

Neetmock River, the Blackstone River, New London County, Conn., and Kent County, R. I. A modern form of Nipmuck, "fresh water place."

Negan odanak Old Town, Penobscot County, Me. Abnaki, "the first, or original town;" that is, "the old town."

Negas now Fort Point, Penobscot County, Me. Abnaki, perhaps part of their word for "portage," perhaps part of a longer word meaning "short cut." Also Negew. See Negunisis.

Neghechewanck a small island at the confluence of Cocheco and Salmon Falls rivers, York County, Me. Abnaki, "island in two currents" or "island at the great bend."

Neguasseag River Sagadahoc County, Me. Abnaki, "a pond" or "place of the pond," also "first widening' as we go up-river." (See Nequasset.) 
Negunisis Knox County, Me. Abnaki, contraction and modification of Ouinigansees, "small (or short) portage." Also Negunsis.

Negunket River; see Neguntequit and Ogunquit.

Neguntequit ancient name for Wells, York County, Me. Abnaki, "old, abandoned fields."

Negunticook Camden Harbor, Knox County, Me. Micmac, "big mountain harbor."'

Neguttaquid River, Berwick, York County, Me. Abnaki, "old island," or "first island." See also Neguntequit.

Negwamkeag near Augusta, Kennebec County, Me. Probably Malecite, "a single sand bar."

Negwasseg see Nequasset.

Negweesen the great boulder at Rock Point, Chittenden County, Vt. Mahican? Abnaki? "single rock."

Nehaiwe Berkshire County, Mass. Mahican, "place down stream."

Nehantic see Niantic.

Nehantic State Forest, New London County, Conn. In memory of the Niantic Indians who formerly lived here. This name means practically the same as Nehantuck, q. v.

Nehantuck Niantic Point, New London County, Conn. Western Niantic, "point of land on a tidal river or estuary." See Niantic.

Nehumkeag or Nahumkeag see Nahumkeag.

Nehumkee or Nehumkike Several such places in York, Cumberland, Knox, Penobscot and Kennebec Counties, Me. Abnaki, "eel place."

Nekatatacomet Plymouth County, Mass. Wampanoag, "divided field" or "divided plantation."

\section{Neketow see Nicatow.}

Nekrangen the mouth of the Sheepscott River, Lincoln County, Me. Abnaki, perhaps "the opening." See Klanganissecook.

Nemadunkehunk, Webster Stream, Penobscot County, Me. Malecite? Micmac? "straight-up-the-hill stream."

Nemasket Plymouth County, Mass. Wampanoag, "at the fishing place," or possibly "grassy land." (There was a Nemasket Village in Boston Harbor to circa I79o.)

Nemdamassuagum Gardiner's Lake, Washington County, Me. Abnaki, "sucker lake." 
Nemonunck or Nominick Windham County, Conn. Nipmuck, "land to be seen far off," or "elevated land."

Nemoset Mountain and Pond, Middlesex County, Mass. Nipmuck, "at the fishing place."

Neotaconkonitt Hill, Providence County, R. I. Narragansett, "at the short (or scant) boundary mark," the hill surmounted by Cononicus' Boulder. There are at least 60 variants of this name.

Neotuquet River, York County, Me. Abnaki, "at the solitary river."

Nepash Stream, Hartford County, Conn. Sicaog, "upright stake" or possibly from nepaus, "the sun"(?)

Nepasooenegg Brook, Mohawk Brook, Hampden County, Mass. Nipmuck, "at the upright stone."

Nepaug Reservoir and River, Hartford County, Conn. Wangunk, "fresh pond."

Neponset Reservoir and River, Suffolk County, Mass. Natick, perhaps an abbreviation of rinneponset, "a good fall," that is, easily negotiated by canoe parties. (Perhaps this has something to do with nepun, "early summer." Another set of roots give a hint of "sleeping," or "dreaming.") Variants, Naponsitt and Naponset.

Nepp Point, Washington County, Me. If Indian, perhaps nepe, "water" in several dialects.

Nepsucknet Plymouth County, Mass. From Wampanoag, "at the place where the brook runs all summer," or "at the brook which has its outlet in the pond."

Nequamwicke Falls, Kennebec County, Me. Abnaki, "eel-place."

Nequaseag River, Kennebec County, Me. Abnaki, "place of clear (or shallow) water."

Nequassabemasis Knox County, Me. Abnaki, "small pond."

Nequassebem Knox County, Me. Abnaki, "a pond or lake."

Nequasset Pond and Village, Sagadahoc County, Me. Abnaki, "at the pond."

Nequechoke Lake, Bristol County, Mass. Narragansett, "near the hilly place."

Nerigwocke, Neriwocke see Norridgewock. 
Nesayik now Boyden's Lake, Washington County, Me. Malecite, "the muddy lake." Spelled also Nesseik, "roily water." Indians say the water is roily because of the death-throes of "a huge water monster," the Wiwiliamecq.

Nesenkeag Eel Brook, Hillsboro County, N. H. Abnaki, "they come here two-by-two," probably the so-called silver eels.

Nesepack Ponds, Addison County, Vt. Abnaki? "two ponds"?

Neshaw Holmes Hole, Dukes County, Mass. Wampanoag, "two of a kind," or "they go two-by-two," the silver eel, Muraena argentes.

Neshobe the Otter Creek Valley near Brandon, Rutland County, Vt. Natick, "very full of water." Also a river in Addison County, Vt.

Neshunganset Brook, Washington County, R. I. Narragansett, "in the middle of the fishing place." Also Neshunganes.

Neskett Maine Coast, between Penobscot Harbor and Mt. Desert Island. Abnaki? "rough places"?

Nesopack Pond, Berkshire County, Mass. Mahican, "eels pond," "they come two by two," i. e., silver eels. But see Nesepack.

Nesowadnehunk Stream, Piscataquis County, Me. Abnaki, "swift stream between mountains," or "swift stream in the mountain ravine."

Nesquabinanset Plymouth County, Mass. Wampanoag, "at the first water pool."

Nesuntabunt Mountain, Piscataquis County, Me. Abnaki, "three heads." From a certain angle, the height seems to be three headed; that is, with three peaks.

Netahme Puntook falls on Otter Creek near Vergennes, Addison County, Vt. Abnaki, "first falls on river," above river mouth.

Netakamikus Knox County, Me. Penobscot-Abnaki, "big ridge." Variant, Netukhamakoos.

Netop Mountain, Bennington County, Vt. Natick, "my friend."

Neutaconkanut Hill, Providence County, R. I. Narragansett, "at the short or scant boundary mark." Also Natakonkanet, Neotacankonitt, Neutaqunkanet, etc.

Newagen Cape and Village, Lincoln County, Me. Abnaki, part of a longer word meaning "route across the land," or "route stopped and turned across land," kepan-ahwangen. 
Newashe Hartford County, Conn. Podunk, "a point of land"?

New Canada Township, Aroostook County, Me. Mohawk, "the houses."

Newdick Point, Sagadahoc County, Me. Malecite, "at the solitary place."

Newichawannock Rapids, York County, Me. Abnaki, "at the place between two strong currents and rapids," or "between swift river forks." Variants, Nechawonak, Nechawonek.

Newichawannuk Hill, Windham County, Conn. Nipmuck, "extended rapids, at the fork in the river." Spelled also Newichawannak.

Newichwanimak Lake, (Great East Lake) Carroll County, N. H. Pennacook, "the place of extended rapids, at the fork."

Newichwannock River and Village, Strafford County, N. H. Pennacook, "where rapids extend a considerable distance, at a fork."

Nexaongermek now Stetson Pond, Penobscot County, Me. From Abnaki, "the lake between" Etna Pond and Newport Lake, but not connected with either.

Nezinscot River, Oxford County, Me. Abnaki, "place of descent, going down-river by canoe."

Niagara Falls, Piscataquis County, Me. Seneca, "the collarshaped object," or "the neck-piece."

Niantic Indians who lived between the Connecticut River and the Rhode Island boundary. Invading Pequots split the tribe in two; some remained in Connecticut between the Connecticut River and Niantic Harbor, others went east to live in western Rhode Island. Thus we have the terms Western Niantic and Eastern Niantic; there is actually almost no difference between the two dialects. The term Niantic translates as "those who live at the point." Variants, Nehantic, Nyantic.

Niantic R. I., and various points in Connecticut. West Niantic, "point of land on the tidal estuary." See Nianticut and Nyantaquit.

Nianticut Point Judith, Washington County, R. I. Eastern Niantic, "at the tidal creek near the point." Also Nayanticut.

Niben a meadow south of Bellows Falls, Windham County, Vt. Abnaki, "summer." 
Nicatous Lake, Hancock County, Me. Abnaki, "the little fork" in the stream. See also Nicatowis.

Nicatow old name for Medway, Penobscot County, Me. Abnaki, "the river fork."

Nicatowis Fork, of Passadumkeag River, Hancock County, Me. Abnaki, "the small fork."

Nichewaug Village, Worcester County, Mass. Nipmuck, "at the midway place," or "place between."

Nichiquiwanick N. H. Sometimes incorrectly [?] translated as "place of my wigwam." See Newichwannock.

Nichmug River, Grafton County, N.H. Abnaki, "fishing place at the fork."

Nickwacket Mountain, Rutland County, Vt. Abnaki, "at the fork," or possibly "abode of squirrels" ?

Nigakws-wakeag Skiff Pond (or North Lake) tributary of the Spednick River, Washington County, Me. Abnaki, "the forktailed terns' nesting place."

Nikaagamok Lake, Penobscot County, Me. Abnaki, "forked lake." See also Nikanagamak, below.

Nikanagamak Ragged Lake, Piscataquis County, Me. Abnaki, "forked lake." Spelled also Nigidawagamek.

Nikisipik a locality near Franklin, Merrimack County, N. H. Abnaki, "fork in the stream."

Nimpanick Hickanuh Dukes County, Mass. Wampanoag, "the place where a tree was split during a thunder storm," or "place of thunder clefts."

Ninigret Pond, Washington County, R. I. East Niantic, "a war chief." Ninigret was a chief of the Niantic, I654.

Ninipoket Plymouth County, Mass. Wampanoag, "at the fresh pond." Nipchossuck see Nippsatchuk. Variants, Nipsachoke, Nipsachook.

Nipmuck many place names in southern New England. All derive from Nipmuck, "fresh water place," or, "fresh water fishing place," the name of a tribe living in Connecticut, Rhode Island and Massachusetts.

Nippahonset Pond, Bristol County, Mass. Narragansett, "at the fresh water pool."

Nippaniquet Pond, Bristol County, Mass. Narragansett, "at the small fresh water pond." 
Nippeake New London County, Conn. Eastern Niantic, "water country," probably meaning "well watered, plenty of fresh water brooks."

Nippenick Lake, Plymouth County, Mass. Wampanoag, probably "fresh water pool," but possibly "at the summer place."

Nippoo Pond and Hill, Strafford County, N. H. Abnaki or Pennacook, "fresh water pond." Variants, Nepo, Nebo, Nippo, etc.

Nippowance Fairfield County, Conn. Paugussett form, same as Rippowams, q. v.

Nippowin Fairfield County, Conn. Siwanoy, "good water."

Nippsatchuk Hill and Swamp, Providence County, R. I. Narragansett, "water near the hill."

Nipsic Hartford County, Conn. Wangunk, "fresh pool," or "water brook." Another spelling Nipsuck, "at the pool" or "where water flows out."

Nipsquanauge New London County, Conn. Wangunk? "land at the long pond."

Nisopack Ponds, Fairfield County, Conn. Mahican, "double pond" or "two ponds."

Nisquitianxset Washington County, R. I. Narragansett, "defiled or unclean place"?

Nissitissett Hillsboro County, N. H. Abnaki, "two brooks" or "between brooks."

Nissitisset Hill and River, Middlesex County, Mass. Natick, "near the two small rivers."

Niswosaket Providence County, R. I. This is an ancient name for Woonsocket, R. I. Probably Narragansett, the name means approximately "water broken up as it goes rapidly downward"? or "place of two brooks"?

Nitchewog Worcester County, Mass. Nipmuck, "land or place between."

Niupp aquashneag Brook, New Haven County, Conn. Quinnipiac? "water at place of flags or rushes" or "rushy pool."

Noag see Nayaug. Variant, Noyaug.

Noanet Peak and Brook, Norfolk County, Mass. Natick, "at the far place," or "at the dry place?" or "place of dry trees"? Spelled also Noanat. 
Noank Bay, Point and Village, New London County, Conn. West Niantic, "it is a point."

Noautuk see Norwottuck.

Nobscot Hill, Middlesex County, Mass. Natick, "at the rocky place."

Nobscusset Harbor and Point, Barnstable County, Mass. Wampanoag, "at the place of little reefs, or small rocks." Variant, Nobsquassit.

Nobska Point, Barnstable County, Mass. Wampanoag, "rocks."

Nobsquassit Barnstable County, Mass. Wampanoag, "at the rock ledge cliff."

Nockum Hill, Bristol County, R. I. Narragansett, "land can be seen far off," or possibly "sandy."

Nodawangak Lincoln County, Me.? Abnaki, "dancing place," or "where we dance." There were several such places.

Noepe Dukes County, Mass. Wampanoag? an old name for Martha's Vineyard. See Nope, and Noepi.

Noepi Nantucket County, Mass. Wampanoag? "in the midst of waters." An old name for Nantucket. See also Nope.

Noeutuck Hampshire County, Mass. Natick? Mahican? "middle of the river." See also Noautuck, Norwottuck.

Noguncoth Perhaps a I649 spelling of Ogunquit, q. v.

Nohlkai Menahan in Moosehead Lake, Piscataquis County, Me. Abnaki, literally "deer island."

Nohono Dukes County, Mass. Wampanoag, possibly an abbreviation of the terms for "dry land" or "beach;" or "middle place," or "far away."

Nokatay Island, Bristol County, Mass. Narragansett, "one alone," or "first (island) come to;" possibly, "where the channel splits."

Nokhigan a locality near Goshen School, Orange County, Vt. Abnaki, "flour, or meal-mortar" (A typical Mohegan-type log mortar was there until I94I. Local legend had Coösucks grinding fine corn meal there). The words "no-cake," nokeag, rokeag, yokheag have the same root-meaning: "fine-ground meal."

Nokomis Pond, Somerset County, Me. Abnaki, "my grandmother."

Nolan gamoik Ripogenous Lake, Piscataquis County, Me. Abnaki, "resting place after a long hard carry above a long stretch of falls or rapids." 
Nolat keeheemungan Freese Island, Penobscot County, Me. Abnaki, "between the hunting grounds."

Nolkaianak Essex County, Vt. Abnaki, "deer here."

Nollesemic Lake, Penobscot County, Me. Abnaki, "resting place at falls above the long stretch."

Nollidgewanticook River, Knox County, Me. Abnaki, "place above the falls in the river"? See also Norridgewock.

Nolumbeke see Norumbega.

Nolwottog Hampshire County, Mass. Nipmuck, "far-away place."

No Man's Land (Island), Dukes County, Mass. Wampanoag, modified by Colonists from the name of Chief Tequenoman of Nantucket, I664.

Nomascom Grafton County, N. H. Abnaki, "long fish"? More likely a modification of Mascoma, q. v.

Nomsussmuc Island, Newport County, R. I. Narragansett, "white beach place"? Possibly also "infertile mud"? or "place of little heaps"?

Nonacoicus Brook and Pond Worcester County, Mass. Nipmuck, "dry earth"? "dry pines"? or "earthen pot"?

Nonantum Middlesex County, Mass. Natick, "I am well minded, I rejoice," or Narragansett, "I am glad."

Noncook Pond, Litchfield County, Conn. Mahican, "sandy place," or "at the shore."

Nonequacket Island and Shore, Washington County, R. I. Narragansett, "dry land place," i.e., the shore.

Nonequasset see Nonequacket. Variants, Nonequausut, Nonequksett.

Nonequit Cove, Pond and Point, Newport County, R. I. Narragansett, "dry land," the shore. Also Nonnequid, Nonquid, etc.

Nonganeck see Nonequit.

Noni's Spring, New London County, Conn. Mohegan, from the name of an Indian who lived near the spring before 1800 .

Nonnecoicus Norfolk County, Mass. Natick, "dried soft stuff," perhaps dried mud, possibly baked clay, as earthen pots. See Nonacoicus.

Nonnequasset Washington County, R. I. Narragansett, "narrow swamp place"? or "above the confluence of two rivers." 
Nonnewaug River, Litchfield County, Conn. Mahican, "dry land" referring to stream drying up in summer.

\section{Nonniquatuc see Nonequit.}

Nonnowigil Mehanol Chittenden County, Vt. Abnaki, literally "five islands."

Nononomesset Island; see Nanomesset.

Nonotuck Hampshire County, Mass. Natick, "in the middle of the river," or "narrow river," or Paugussett, "far-away place."

Nonquitt Bristol County, Mass. Narragansett, "dry place," or "place of harvest," or perhaps "landing place."

Nookagee Brook, Worcester County, Mass. Nipmuck, "the sandy place," or possibly "the clay place."

Noomuck see Namkook.

Nooseneck Hill and Village, Kent County, R. I. Narragansett, "at the place of the beaver." See Noozapoge.

Noosnippi Pond, Plymouth County, Mass. Narragansett, "beaver pond."

Nootash Hill, Newport County, R. I. Narragansett, "carry loads in them, on your back;" that is, "baskets."

Nootimishash Tolland County, Conn. Podunk? "oak trees."

No-ottut Merrimack County, N. H. Natick, "far-away place."

No-ottut Hampden County, Mass. Paugussett, "a great, long journey."

Noozapoge Washington County, R. I. Narragansett, "beaverpond." See Nooseneck.

Nope Dukes County, Mass. Wampanoag? abbreviation of p'nops quessaugamaug, "menhaden fishing place." This is an ancient name for Martha's Vineyard. Variant, Nopi.

Nopque Nantucket County, Mass. Wampanoag, "menhadenplace"? or "utmost, furthest place."?

Noquochoke Lake, Bristol County, Mass. Narragansett, "soft earth place." See also Nequechoke.

Noronoke Lake, Fairfield County, Conn. Perhaps Mahican, "dry land" or Paugussett, "far-off country"?

Norridgewock Village, Somerset County, Me. Probably Abnaki, "where swift river descends". Possibly Malecite, nah-wisewauk, "two torrents at this place." Variant, Noridgewa-ëg. 
Norsaton now Noroton Village, Fairfield County, Conn. Siwanoy, (free translation) "creek almost dry at low tide."

Norumbega Point and Village, Hancock County, Me. Also a Park and Reservoir, Middlesex County, Mass. This name appeared on maps as early as 1550 , with a very large territory so labelled. Ganong (IgI2) wrote that this word is a European transplant, and that the following Abnaki name, however similar, is coincidental: Nolumbeke, "still water between falls." Variants, Aranbega, Nanrhbegwa, etc.

Norwalk see Norwauke.

Norwauke Fairfield County, Conn. Siwanoy, "at the point of land." Also given as Norwaack. But see Norwottock.

Norwottock Rockingham County, N. H. Natick? "far away place." See also No-ottut.

Norwottock Mountain, Hampden County, Mass. Natick? or Paugussett? "far from us."

Nosahick Lake, Washington County, Me. Abnaki? Malecite? "place of roiled water," or "muddy lake." See Nesayik.

Notaconkanut Hill; same as Neutaconkanut.

Nowaas Hartford County, Conn. Podunk, "between rivers"? or "at the point"?

Nowashe Hartford County, Conn. Podunk, "between" rivers.

Nowatunamon Fairfield County, Conn. Siwanoy, "dried up place" also "dry, ready for harvest."

Nowedonah Fairfield County, Conn. A Long Island chief who helped the Dutch in their Connecticut struggles, circa I645. His name is Montauk, "I go seek;" presumably the enemy.

Nowesit Neck, Newport County, R. I. Narragansett, "middle place."

Nowpaug Washington County, R. I.? New London County, Conn? Niantic, "dry pond"? "beaver"?

Noycoy Hampden County, Mass. Nipmuck, "soft stuff," such as mud, or clay.

Nubanusit Brook and Lake, Hillsboro County, N. H. Pennacook, "at the place of gently sloping banks"? or, "small summer place"?

Nucksisset Plymouth County, Mass. Wampanoag, "at the small landing place." 
Nuequiauke location uncertain. New London County, Conn.? Western Niantic? Paugussett? "broad land"?

Nuhkehkummees Plymouth County, Mass. Wampanoag, "a small shelter."

Nukacongamoc Pond, Piscataquis County, Me. Abnaki, "lake which is beginning" or "headwater pond," or "pond beyond which boat and burden have to be carried."

Nukkekummees Newport County, R. I. Narragansett, "sought for place" or "desired home." But see Nuhkekummees.

Nulhedus Mountain, Somerset County, Me. Abnaki, "(water?) fall on each side."

Nulhegan River, Essex County, Vt. Abnaki, "my log trap;" a deadfall.

Nullie Kunjewa Brook, Coös County, N. H. Abnaki, "fishing place downstream."

Numachinagamook Elbow Lake, Piscataquis County, Me. Abnaki, "lake that opens on one side."

Numchenugmawis Second Lake, Washington County, Me. Malecite, "lake one-sided;" inlet and outlet on the same side of the lake, so one may go straight up in a canoe.

Numdemosees Stream, Washington County, Me. Malecite, "little sucker-fish swim up" (to spawn?). Possibly also, some other small migratory fish.

Numtsceenaganawis Elbow Lake, Piscataquis County, Me. $A b$ naki, "little crossways pond."

Nungee Brook and Swamp, Hampshire County, Mass. Nipmuck, "trembling, quaking."

Nunkatusset River, Plymouth County, Mass. Wampanoag, "narrow meadow," "winding brook." Also, Nunketest.

Nunkertuck New London County, Conn. Mohegan, "crooked stream."

Nunnakomac Middlesex County, Conn. Hammonassett, "dry land," or "at the shore."

Nunnaquahgat Neck, Newport County, R. I. Narragansett, "dry meadow." Variant, Nummastaquyt.

Nunnatucket River, Washington County, R. I. Narragansett, "a creek that dries up."

Nunnawauk Road, Fairfield County, Conn. Siwanoy, "dry land." 
Nunnipaug Dukes County, Mass. Wampanoag, "fresh pond," or "narrow pond." Variant Nunpoak, "dry pond?"

Nupskonaug New London County, Conn. Mohegan, "land at long pond." See Nipsquanauge.

Nusalk Chunangan possibly on Penobscot Bay, Knox County, Me. Perhaps Abnaki, "village near the big fish weir." See Nutskamongan.

Nuscongus see Muscongus.

Nusquash Pond, Sagadahoc County, Me. See Musquash.

Nutskamongan probably Ayer's Rips, Penobscot County, Me. Abnaki, perhaps "good cornfield" or "fishing place." It was here that the Mikumwessak, the dwarfs or "wee people" came to warn the Abnaki that the Mohawk were enroute east, on the warpath. The Mikumwessak were wood-dwellers, larger and more friendly than the rock-dwelling Wanagameswak.

Nyac New Haven County, Conn. Quinnipiac, "a point."

\section{$-\mathbf{O}-$}

Oantenocke or Ouantinuck; see Weantinock.

Oawascoage River; see Owascoag.

Obscob New London County, Conn. Mohegan, "white rock." See also Webomskat.

Obumkeag River, York County, Me.? Abnaki, "sucker-fish place" from abodumquen-keag.

Obwebetuck Hill. See Ocquebituck.

Occawa Nantucket County, Mass. Wampanoag, "on the other side,"? or, "over against (the fishing place)"?

Occooch Pond, Dukes County, Mass. Wampanoag, "small." (But possibly "flies.")

Occopaug New Haven County, Conn. Quinnipiac, "head of the pond." See Aquibapaug. Another spelling, Aquebogue.

Occum Village New London County, Conn. Mohegan, named for Samson Occum, I723-I792, a famous Indian preacher who worked for Indian education; he was also a prime mover in the exodus of New England Indians to Brothertown, N. Y. 
Occupaspatucket Cove, Washington County, R. I. Narragansett, "near the cove on the shallow tidal creek." Variant Occupaspawtuxet.

Occupasstuxet Road, Kent County, R. I. Narragansett, "small cove on tidal creek," or "cove on small tidal creek."

Occupessuatuxet Kent County, R. I. Narragansett, "small cove on tidewater."

Ocinamunt Pond, Barnstable County, Mass. Wampanoag? Perhaps originally Oshamamucks, "eel fishing place."[?]

Ockoogangansett Hill, Middlesex County, Mass. Nipmuck, "at the plantation," or "plowed fields place."

Ockseattkontack New Haven County, Conn. Quinnipiac, "wading place (or fording-place) at the creek," possibly where the Mishimayagat, "principal trail," turned eastward.

Ocquebituck Hill, Tolland County, Conn. Nipmuck, meaning doubtful. Perhaps "separated from river," or else "top of a tree;" probably the latter.

Ocquiunk Hill, Windham County, Conn. Nipmuck, "under the tree." See Acquiunk.

Ocsechoxit Worcester County, Mass. Nipmuck, "fox country." See Woonksechocksett.

Odana site of ancient Koessuk, Windham County, Vt. Abnaki, "dwellings."

Odonwagayon meadows near Swanton, Franklin County, Vt. Moharek, "old beaver or muskrat lodges."

Ogesh Kuppi Dukes County, Mass. Wampanoag, "small enclosure."

Oggawame see Agawam.

Oggunikonqquamesut Middlesex County, Mass. Nipmuck, "at the plowed fields, or plantation."

Ogoncog River; see Ogunquit.

Ogunquit Village, York County, Me. Micmac, "lagoons within dunes"? Also Abnaki, "place of waves," from antegw-quit.

Ohio Brook, Washington County, Me. Mohawk, "the large or beautiful river."

Ohio Hill, Windsor County, Vt. Mohawk, "big or beautiful river."

Ohkakquiset Bristol County, Mass. Wampanoag, "at the small hollow" (or kettle-hole)? or Wonqui quaset, "crooked rocks 
place" ? or Oke-aquesset, "field ending place" (earth, dirt, field ?)

Ohkonkumme Dukes County, Mass. Wampanoag, "village on the other side" (across the island).

Ohnowarake a small cove in Lake Champlain, Chittenden County, Vt. Mohawk, "turtle place."

Ohomowauke a pine swamp near Ledyard, New London County, Conn. Eastern Niantic, "owl's place." This was a favorite refuge of Indians when attacked. Also Ohomauke.

Ohomowauke Swamp, Washington County, R. I. Eastern Niantic, "at the abode of owls." This was a hiding-place for Indians during colonial wars, hence "place of refuge, or concealment."

Ohskenonton Grand Isle County, Vt. Mohawk, "a deer."

Oiocommuck see Aigio commuck.

Okemo Mountain, Windsor County, Vt. Chippewa, "a chieftain" or Abnaki, "a louse."

Okenmakomesut Middlesex County, Mass. Natick, "at the field (enclosure) on the other side." This was a farming village regularly visited by John Eliot, I604-I69o.

Okommakemsit see Okenmakomesut.

Okpaak an ancient Abnaki village, perhaps in Cumberland County, Me.? "at the bend"? or "cleared land"?

Olamon Island and Village, Penobscot County, Me. Abnaki, "vermilion, red paint."

Oloostook River; see Aroostook, Wallastook, Oolastook, and Walastegw, all Abnaki or Malecite words meaning "beautiful river."

Omeconset Meadow, New London County, Conn. Eastern Niantic? Narragansett? "drinking place," or "well place" (wuttahhommunk), or possibly "herring fishing place"?

Ompompanoosuc River and Village, Windsor County, Vt. Abnaki, "mushy, quaky land."

Omquemenikeag Lake, North Schoodic Lake, Washington County, Me. Abnaki, "place of cranberries."

Onagunset see Wunnegunset.

Onawa Lake, Piscataquis County, Me. Chippewa, "Awaken!" (In Longfellow's Hiawatha, "Awaken, O Beloved!') 
Ondawa Stream, branch of Batten Kill, Bennington County, Vt. Mohawk? "white stream."?

Onderiguegon Lake Champlain, Drowned Lands south of Rutland County, Vt. Mohawk, "lake stopped up without outlet."

Oneadalote Lake Champlain, Vt. Oneida, "a lake."

Oneco Village, Windham County, Conn. Mohegan, named for Oneco, the son of Uncas in The Last of the Mohicans.

Ongeachonta Mountain, Kennebec County, Me. Abnaki? "top (or summit) of mountain"?

Onkatomka Island, Dukes County, Mass. Wampanoag, "top of the rock."

Onkawoom Nantucket County, Mass. Wampanoag, "land on the other side"? or "landing-place," similar to Agawam?

Onkoskopsug New London County, Conn. Mohegan, "Uncas' spring.'

Onkowam Plymouth County, Mass. See Agawam.

Onnahookset Cheshire County, N. H. Pennacook, "at the place of beautiful trees."

Onota Lake, Berkshire County, Mass. Mahican, "blue"? "deep"? or possibly modified from Oyatuck, "he dwells at a waterstream."

Onset Plymouth County, Mass. If Indian, perhaps Wampanoag, "place at the point," or "sand place," or "shellfish place," or "plain, sandy place."

Onshaukamaug Hartford County, Conn. Wangunk? a "fishing place for eels."

Onyatalot Lake Champlain, north of Shoreham, Addison County, Vt. Oneida, "standing lake," or "(wide lake) standing in a stream" or "(possessed) standing lake."

Ooalakguemook the Allagash River, Aroostook County, Me. Abnaki? Malecite? "birch river," or "birch place," or "black camp river"?

Oolaghesee Cape Rosier, Hancock County, Me. Malecite, "the entrails." This is a vein of white quartz under water, which resembles water-soaked moose entrails. Glooscap, the legendary Malecite hero, killed a moose calf and threw the insides to his dog. There they remain. 
Oolagweskwigamicook the Allagash River, Aroostook County, Me. Abnaki, "bark cabin stream," or "bark-house place." Also Allagaskwigamook.

Oolamonogamook Silver Lake, Piscataquis County, Me. Abnaki, "vermilion paint lake."

Oolamonoosuk Olamon Island, Penobscot County, Me. Abnaki, "place of red paint."

Oolastook see Wallastook.

Ooniganissek Cape Jellison Neck, Waldo County, Me. Abnaki, "at the short carry." Also Ouinigansek.

Oosoola or Ussoola Kennebec County, Me. Abnaki, "flooded." Opechee Bay, Belknap County, N. H.; and Stream in Waldo County, Me. Chippewa, "a robin."

Opponaugue see Apponaug and Opponenaubock.

Opponegansett see Apponagansett and Ponaganset.

Opponenaubock see Apponaug.

Opuitowaxet Washington County, R. I. Narragansett, "fording place at the end of a portage," or "ford at the waiting place."

Oquassanock Brook, Orange County, Vt. Abnaki, "at the place of the slender trout."

Oquasskikonaquam Lake Sunapee, Merrimack County, N. H. Abnaki, "slender trout at the end of the field." Price (I958) says: "place abounding with slender trout."

Oquossoc Village, Franklin County, Me. Abnaki, "a blue slender trout," or "place of the oquassa trout."

\section{Oramphegan see Quamphegan.}

Orantsoak parts of the Kennebec River, Kennebec County, Me. Abnaki, "rapids" or "swift water." Also Aransoak.

Orcawa Nantucket County, Mass. See Occawa.

Ordonakis said to be near Lyme, Grafton County, N. H. Pennacook? "at the place of small dwellings"?

Oregon Barnstable County, Mass. Possibly a modification of Wauregan, q. v. If not, perhaps from French ouragan, "hurricane," or Latin origamum, "wild sage," or even Spanish orejón, "big ear."[?]

Orenaug Hill, Litchfield County, Conn. Quinnipiac? Paugussett? "pleasant place." Also see Waurenaug. 
Original Moosehead Lake, Piscataquis County, Me. This is not an Indian name but a French word meaning "elk," adapted by certain French-speaking Indian guides, who use this name for "moose." Variants, Orignal, Orignac.

Orkatucket see Annaquatucket.

Oronauke Fairfield County, Conn. Quinnipiac, "curved place" or "land at the bend." Other spellings Oronooke, Oronoke, Oronoque, Orronack. See Woronoco and Wauregan.

Orono the site of the University of Maine, Penobscot County, Me. Abnaki, from the name of Chief Joseph Orono, who may have been a white man adopted into the tribe in his boyhood. This name is not translatable; it may not be Indian.

Ortocoag River, Cumberland County, Me. Abnaki, "where we mend canoes." Also Oriocog.

Osabeg Hills, York County, Me. Abnaki, "the other side of the stream or pond." See Ossipee.

Osamequin another name for Massasoit; from Wampanoag, "yellow feather."

Osceola Island, Plymouth County, and Mountain, Berkshire County, Mass.; and Mountain in Grafton County, N. H. Derives from Asi-yaholo, "black drink," the Seminole term applied to the famous leader of that tribe. The word refers to a ceremonial potion.

Oselaga Camp Hochelaga, Grand Isle County, Vt. Mohawk, "at the beaver dam," from Hochelaga, q. v.

Osotonac Creek, York County, Me. Pennacook? "land beyond the mountain." See Housatonic.

Osquepaug River, Washington County, R. I. Narragansett, "at the end of the pond," not "whiskey pond."

Osquoon Cape Rosier, Hancock County, Me. Malecite, "the liver." This is a large reddish brown rock, supposed by Indians to be the liver of a moose calf killed by the legendary hero, Glooscap.

Ossaghrage an ancient Indian village, York County, Me. Perhaps Abnaki, "place near salt water." But see Koghserage; the Mohawk ranged as far east as Gaspé.

Ossapimsuck Brook. See Assapumsic. 
Ossipee Lake, Mountain and Village, Carroll County, N. H. and Pond, York County, Me. Abnaki, "water on other side," or "beyond the water."

Oswegachie River, Essex County, Vt. Mohawk, "a long bay or estuary."

Oswegatchie Hill, New London County, Conn. Oneida or Mohawk? "long bay or estuary." But if not Iroquoian, perhaps Mohegan, "outlet near the mountain"?

Otstenrowanen Rock Point, Chittenden County, Vt. Mohawk, "the large rock."

Ottauquechee River, Windsor County, Vt. Natick? "swift mountain stream," or "cat tails, rushes, near a swift current"?

Otternic Pond, Hillsboro County, N.H. If Indian, possibly Abnaki, "at the dwellings."

Ouätchuset see Wachusett.

Ouchamanunkanet Meadow, Providence County, R. I. Narragansett, "cultivated plantation at the halfway place."

Oûi-ina-oûasset see Pemigwasset.

Ouiniganisis-ikuk Stillwater Branch, Penobscot County, Me. Abnaki, "stream with the very short carry."

Ouinous-tic River; same as Winooski, q. v.

Ouisnouski Bay, now Malletts Bay, Chittenden County, Vt. Abnaki (modified by French influence), "at the place of wild onions." Another spelling, Ouynouske.

Oukote Norfolk County, Mass. Natick, "a kettle"? or "hills."?

Oulakese-Awangan, a route along the Penobscot River between Abol and Chesuncook, Piscataquis County, Me. Abnaki, "route of entrails," or "route of sausage casings" because ponds "on this trail are strung along like links of sausage."

Oumgomkos Franklin County, Mass. Pocumtuck, "place over across."

Ouneganisis a portage at Stillwater, Penobscot County, Me. Abnaki, "the very short carry." This was just a few yards, around a tricky waterfall; thus, "a small portage."

Ouramana Lake, Penobscot County, Me. Old Abnaki, "vermilion," "shining," or beautiful." See Olamon.

Ousatunick see Housatonic. 
Ouschankamaug Hartford County, Conn., and Berkshire County, Mass. Mahican, "eel-fishing place." Also Ouschanpamaug, Washakamaug, etc.

Ouwerage an ancient Abnaki village. Possibly the same place as Ossaghrage?

Ouy goody St. John's River, Aroostook County, Me. Malecite, "canoe place"? Spelled also Oxgoudy.

Owanux Cumberland County, Me. Old Abnaki, "strangers (here)" from a8eni, "strangers," i.e., colonists. Variant, Awanoots.

Owascoag River, York County, Me. Abnaki, "at the grassy place."

Owassoosen Bear Notch, Orange County, Vt. Abnaki, "bears' rocks," or "bear's den."

Oweantonnuc see Weantinock. Spelled also Ouwanatonnuc in which version it may mean "at the cloudy, misty hill"?

Owenamchock a locality in Fairfield County, Conn. Mohegan, "beyond the fishing place."

Owenoke Fairfield County, Conn. Mahican, "lowland" or "valley," or "country beyond."

Owtanic Coös County, N. H. Pennacook, "at the village."

Owunnegunset Hill, Windham County, Conn. Mohegan, "at the place of the portage," or "place near the kettle-hole"?

Owweonhungganuck Tolland County, Conn. Nipmuck, "where fish come to lay eggs"? or "place where we gather eggs," (gull, plover, or turtle eggs). Another source gives "beyond the drinking place," and "beyond the fishing place." Also Oweeonhonganock, Owwaenungganuck.

Oxecoset Creek, New London County, Conn. Eastern Niantic, "fox country," also "place of small pines." See Woonsechockset and Wonksacoxet.

Oxoboxo Brook and Lake, New London County, Conn. Mohegan, "a small pond."

Oxopaugsuck Brook, New London County, Conn. Mohegan, "outlet of the small pond."

Ozwazogehsuck Penobscot Brook, Penobscot County, Me. Abnaki, "waded across by 'quartering' (because of the swift current)." One Indian called it "yellowish brown brook," but Nudénans gives answegan, vadum trajicio, "to cross a ford." 
Paamtegwitook the Penobscot River below Bangor, Penobscot County, Me. Abnaki, "extended rivers united in one stream."

Pabachismusk Hartford County, Conn. Tunxis, "fishing place at the bend of the stream," the Farmington River.

Pabaquamske The Split Rock on Quinebaug River, Windham County, Conn. Mohegan, "split rock."

Pacamkik Dukes County, Mass. Wampanoag, "at the abode of codfish," (or haddock?) or "dark land" (well fertilized). Also "open land."

Pacanaset Providence County, R. I. Narragansett, "little cleared place."

\section{Pacanaukett see Pokanoket.}

Pacanoket all the land in Bristol, Warren and Barrington, part of Swansea, and Seekonk, eastern Rhode Island. This territory was called Sowams by the Narragansett. See Pokanoket.

Pacatuck Brook, Hampden County, Mass. Nipmuck, "open, clear river." See also Pawcatuck.

Pachade Plymouth County, Mass. Wampanoag, "the turn" (as in a road or trail).

Pachamaquast Plymouth County, Mass. Wampanoag, "at the cleft rock."

Pachassett River, Bristol County, Mass. Wampanoag, "at the small branch," or "boundary."

Pachasuck Hampden County, Mass. Nipmuck, "split, or divided, brook."

Pachatanage Washington County, R. I. Narragansett, "dividing, or boundary place."

Pachaug Neck, Bristol County, Mass. Narragansett, "the turning place."

Pachaug State Forest, Windham County, Conn. Village and Lake, New London County, Conn. Mohegan, "the fork, or turning aside place." (Some say "divided river.")

Pachawesit same as Pocasset?

Pachest Plymouth County, Mass. Wampanoag, "turning place." 
Pachet Brook, Newport County, R. I. Narragansett, "at the boundary," or "dividing place," or "turning place."

Pachet Brook, Barnstable County, Mass. Wampanoag, "at the turning place." See Pochet.

Pachgatgotch Hill, Litchfield County, Conn. Mahican, "where the river divides." See Pishgachtigok.

Pachquadnach Litchfield County, Conn. Mahican, "at the mountain with bare, cleared sides."

Pachquinacook Fairfield County, Conn. Wappinger, "elevated land," a plateau.

Pachuach Hampden County, Mass. Nipmuck, "bends," or "turning-off place."

Pachusett Plymouth County, Mass. Wampanoag, "dividing hill place," or "place of the boundary hill," or "hill of quails"?

Packachaug Hills, Worcester County, Mass. Nipmuck, "at the turning place." Also Packachoag, Packachoog, etc.

Packachoog Worcester County, Mass. Nipmuck, "bare mountain place," or "treeless mountain."

Packamohquah Neck, Nantucket County, Mass. Wampanoag, "open country," If spelled Pacomoquash, perhaps "place of fallen rock"? See also Poncammooncoe.

Packwacke Gorge, Berkshire County, Mass. Mahican, "open place." Probably a shortened form of a word or phrase meaning "open place below the gorge."

Packwock Stream, Aroostook County, Me. Abnaki? Malecite? "clear, transparent, shallow place."

Pacomsuck Island, Windham County, Conn. Nipwuck, "swift narrow brook," or "cleared lands." See Peagscomsuck.

Pacomtock possibly Hartford County, Conn., the Farmington River in its upper reaches? See Pocumtuck and Pacomsuck.

Pacoquarocke New Haven County, Conn. Quinnipiac, "a long stretch of cleared land." Ancient name for the east bank of Naugatuck River near Ansonia and Derby. Also Pacoqurocke.

Pacoussett see Pocasset, Paugasset and Paugusset.

Pacowachuck New Haven County, Conn. Quinnipiac, "place of the bare hill."

Padaquonch or Padaquonk. See Pataconk. 
Pagassett Fairfield County, Conn. Paugussett, "place where the river widens." Variants include Pawgasuck, Paugasset, Pawgasset, etc.

Pageacoag Providence County, R. I. Narragansett, "muddy place."

Paghmaigah New London County, Conn. Mohegan, "at the divided path," or "where the trail splits."

Paghuntanuck an ancient Abnaki village, location possibly in York County, Me.? Abnaki, "land broken up for cultivation."

\section{Pagiscott see Pejipscot.}

Pagolgwansek Penobscot County, Me.? Abnaki, "shallow place, clear."

Pagonaumischaug possibly Hartford County, Conn. Sicaog, "fishing place near the bare hill."

Pagwonk New London County, Conn. Narragansett or Eastern Niantic, "cleared land." Also Paugwonk.

Pahahauk Franklin County, Vt. Mahican, "he waits for him (or them?)" a rendezvous at Sciaticook or Schaghticoke on Missisquoi Bay.

Pahcupog see Pauquapaug.

Pahegansuc Hill, Hartford County, Conn. Wangunk, "bare hill place." Spelled also Pegansic.

P'ahnmoiwadjo Squaw Mountain, Piscataquis County, Maine. Abnaki, "woman's mountain." This is a Modern Abnaki term introduced by Indian guides. (The word for "woman" is p'hanem, perhaps from French femme? There is no f-sound in Abnaki; p'hanem is pronounced puh-hanem).

Pahquioke Fairfield County, Conn. Mahican, "cleared land," broken up for planting. Also, Siwanoy, "open country," or "cleared land." Variants, Paquiag, Poquuag, Poquaug, Poquuaug, Pyquag, Poquiogh, etc.

Pahquioque Worcester County, Mass. Nipmuck, "cleared lands."

Pahquopog Pond, Washington County, R. I. Eastern Niantic, "clear pond," or "shallow pond."

Pais-unk Hancock County, Me. Malecite, "clams place." (the $M y$ a arenaria species).

Pakanoket Bristol County, R. I. Narragansett, "at the small plantation." 
Pakemit Norfolk County, Mass. Natick, "at the clear place." See also Punkapoag.

Pakonokick see Pakanoket, below.

Pakwakek Pickpocket Falls, Rockingham County, N. H. Abnaki, "at the place of arrows"?

Paliten Chittenden County, Vt. Modern Abnaki pronunciation of "Burlington."

Palmasicket Hill, York County, Me. Meaning obscure; perhaps Abnaki, "at the place you walk by." (If Panmasicket, "where the brook opens out."

Pamanset River, Bristol County, Mass. Wampanoag, "at the inlet or cove."

Pamaquasset New London County, Conn. Western Niantic, "watered or flooded meadow"? or "across the meadow"?

Pamaquesicke River, Hampshire County, Mass. Nipmuck, "at the ledges." But see Pamechipsk.

Pameacha Pond, Middlesex County, Conn. Wangunk? "crooked mountain," or "mountain near the small river"?

Pamechage see Pomachaug.

Pamechipsk Hills, Providence County, R. I. Narragansett, "crosswise rocks," or "transverse rocks;" that is, rocks across the path or trail.

Pamedomcook or Pemidumcook Lake, Penobscot County, Me. Abnaki, "shallow sandy place or bar extending across the lake," or "shallow place between two lakes."

Pamet River, Barnstable County, Mass. Wampanoag, "wading place," or "at the shallow cove."

Pametoopauksett Swamp, Plymouth County, Mass. Wampanoag, "cove in a little pond," "running water swamp," or "small crosswise pond"'[?]

Pamgockamock Lake, now Mud Pond, in Aroostook County, Me. Abnaki, "extended shallow (and mucky?) still water."

Pampaskeshanke Brook, Fairfield County, Conn. Siwanoy? "double stream." Also Pampaskehauke.

Pamuet Barnstable County, Mass. Wampanoag, "going-over place," or "ford."

Panahemsequet same as Penobscot, q. v.

Panamske probably in Penobscot County, Me. Abnaki, "extended rocks," or "sloping rocks." 
Panatucket Essex County, Mass. Pennacook, "(noisy) falls in the river."

Panawansot Hill, Piscataquis County, Me. Abnaki, "the place of spread-out, extended fog," i. e., Katahdin Mountain.

Panhanet Plymouth County, Mass. Wampanoag, "at the small creek, or inlet."

Panhomuk Bristol County, Mass. Wampanoag, "strange unfrequented place," or "out-of-the-way meadow;" also, "out-of-the-way plain."

Pannaheconnok Litchfield County, Conn. Mahican, "extended country"? or "spread out lakes"? The Twin Lakes near Salisbury. Also spelled Paunahecannuck.

Pannawambskek Penobscot River above Old Town, Penobscot County, Me. Abnaki, "where the channel opens out of the rocks." Also given as Pannawanskek, Pannowauke, etc.

Pannaway salt marshes near tidal creeks, Rockingham County, N. H. Abnaki, "where (rising tide) water spreads out."

Pannukog now Concord, Merrimack County, N. H. Pennacook, the "downhill place."

Panoket Island, Plymouth County, Mass. Wampanoag, "far-off place," "small thing away off," or "sloping place."

Panomescett Neck, Bristol County, Mass. Narragansett, "at the small fishing place."

Panootan New Haven County, Conn. Quinnipiac, "extended village," or "distant out-of-the-way village."

Panoumke Old Town, Penobscot County, Me. Abnaki, "opening between rocks."

Pansacaco Pond, Washington County, R. I. Narragansett, "crooked outlet," or "crooked current." See Pascachuto.

Pantacunset Providence County, R. I. Narragansett, "at the round place."

Pantigo Hill, Windsor County, Vt. Abnaki, "rapids or falls in river."

Pantoocoög Falls, Essex County, Mass. Pennacook, "place of the falls in the river."

Pantook Reservoir, Coös County, N. H. Abnaki, "falls in the river."

Pantookaëg the falls at Lowell, Middlesex County, Mass. Same as Pawtucket, q. v. 
Pantoosuck Mountain, Hartford County, Conn. Wangunk, "at the falls in the brook." This may be an abbreviation of Ammonantacksuck, which includes a "lookout-place" root perhaps meaning "hill lookout-place near the brook with the falls." See Mawnantuck.

Pantukket Falls, Essex County, Mass. Nipmuck, "at the falls in the river."

Panway Strafford County, N. H. Abnaki, "the rising tide spreads out (here)."

Papanomscutt Washington County, R. I. Narragansett, "place (where we get) winterfish," that is, frostfish or tomcods paponaumsuog. Also translated as "lookout place," and "broken rocks." Variants, Popanomscut, Poppanomscut, Poppanompscut, etc.

Papaquinapaug Pond and River, Providence County, R. I. Narragansett, "shallow long pond," or "double long pond"?

Papasgush Washington County, R. I. ? Narragansett, from the name of one of King Philip's counselors.

Papasquash Neck, Bristol County, R. I. Narragansett, "broken rocks"? or "double hill"? or "partridges"? See Paupasquachuke.

Papoose Rock, New London County, Conn. Western Niantic? "an infant." (But perhaps a corruption of Papasquanch, "double hills"?

Papootic York County, Me. See Poodik.

Pappacontucksquash River, Hampden County, Mass. Nipmuck, "cleft bank rocks," or "gorge."

Pappoose Ponds, Oxford County, Me. Narragansett, "my child."

Pappoose Squaw Island, see Poppasquash.

Papuduc and Papuding see Poodik.

Paqua Pond, Dukes County, Mass. Wampanoag, "open or clear" pond.

Paquaback Providence County, R. I. Narragansett, "clear or shallow pond." Also Paquabuck.

Paquabaug Island, Litchfield County, Conn. Paugussett? "clear, open pond" or "shallow pond." See Pauquapaug. 
Paquanaug Hartford County, Conn. Wangunk, "cleared lands." Spelled also Paquanauke, Paquaanocke, Paquanick, Pakwaunic, Paquanunke, Paquanage, Paquanauke.

Paquantuck River, Windham County, Conn. Nipmuck, "clear, open or shallow river." Also Poquannotuck, Poquantuc, etc.

Paquantuck Stream, Providence County, R. I. Nipmuck? "clear or shallow river."

Paquatanee Waldo County, Me. Abnaki, "far from us," off the beaten track, out of the way.

Paquatnach see Pachquadnach.

Paquattuk the Pawcatuck River, between Connecticut and Rhode Island. Eastern Niantic, "the clear, shallow tidal stream."

Paquinapaguoque Meadows, Providence County, R. I. Narragansett, "place of shallow (or clear) long pond." Given also as Paquinapaquoge.

Paquoag Franklin County, Mass. Nipmuck, "an open or clear place."

Paquonk quamaug Hampshire County, Mass. Nipmuck, "at the shallow fishing place."

Parmachenee Lake and Stream, Oxford County, Me. Abnaki, "across the usual path," aslant, deviating from the mosttravelled route. But see Pomersimbeke.

Pasacomuck Hampshire County, Mass. Nipmuck, "where it (the trail ?) turns or branches."

Pasakasock Rockingham County, N. H. Abnaki, "place of division or of branching."

Pasam-Aquâda or Possam-Accâda same as Passamaquoddy, q.v.

Pasaôumkeag same as Passadumkeag, q. v.

Pascachute Pond, Washington County, R. I. Narragansett, "dividing place." Variant, Pascachuto.

Pascatiguage Worcester County, Mass. Nipmuck, "place clear of trees," or "open place near the stream."

Paschuchammuck Cove, Kent County, R. I. Narragansett, "divided fishing place," or perhaps "turning place."

Pascoag Village, Reservoir and River, Providence County, R. I. Nipmuck, "the dividing place," two branches of the Blackstone River. Also spelled Pascoage, Paskhoage, etc. 
Pascodumoquonteag see Passamaquoddy.

Pascomanset Neck and River, Bristol County, Mass. Narragansett, "at the fork in the path"? or "meadow boundary"? (If Passemansit, "miry meadow place.")

Pascomattas Washington County, R. I. Eastern Niantic, "muddy place"?

Pascommuck School, Hampshire County, Mass. Nipmuck, "where it turns or branches," or "plantation where road forks."

Pascongamoc Holeb Pond, Moose River. See Pescongamoc.

Pasconuquis Providence County, R. I. Narragansett, "muddy cove."

Pascunas Path, Middlesex County, Conn. Natick? "steep."? Pascunas was a landholder and witness to deeds.

Pasharanack an Abnaki village, location uncertain; "near the jutting-in bay."

Pashebesauke Middlesex County, Conn. Western Niantic, "near the outlet or river mouth." Also Pashpeshank.

Pashesauke Middlesex County, Conn. Western Niantic, "split outlet," the mouth of the Connecticut at Saybrook. See also Passquishhunk.

Pashipscot Sheepscott, Kennebec County, Me. Abnaki, "channels split by rocks;" also Micmac, "where tide flows splitwise."

Pashquishhook see Pashesauke and Passquishunk.

Pasipuchammuck Kent County, R. I. Narragansett, "divided fishing place." See Paschuchammuck.

Paskeegh Windham County, Conn. Nipmuck, "at the branch of the stream." See Pascoag.

Paskesickquopoh Pond, Hampshire County, Mass. Nipmuck, "pond that branches."

Paskhoage Providence County, R. I. Nipmuck? Narragansett? "land at the branch." See Pascoag.

Paskhommuck Hampshire County, Mass. Nipmuck, "turning place," or "dividing place," a boundary-mark.

Paskuisset Pond and Brook, Washington County, R. I. Narragansett, "miry place."

Pasmocadie Washington County, Me. Same as Passamaquoddy. 
Pasocha Valley, Nantucket County, Mass. Wampanoag, "miry land," or mucky meadow?" or "bare hill" valley, a valley with sparsely wooded sides? Also found as "boundary hill."

Pasomkasik Passumpsic River, Caledonia County, Vt. Abnaki, or Pennacook, "stream with the clear sandy bottom" See also Pesammes.

Paspatanage Windham County, Conn. Nipmuck, "at the branch in the stream." But see Paspatonage.

Paspatonage Brook and Neck, Washington County, R. I. Narragansett, "small inlet place," or "boundary at small inlet."

Pasque Island, Dukes County, Mass. If Indian, perhaps Wampanoag, "split." Another spelling, Pasquenese, "little split." Perhaps, "barren place" and "small barren place." See Penikese.

Pasqueset Pond, Washington County, R. I. Eastern Niantic, "at the miry place," or "muddy place at the end."

Passaconaway River and Village, Carroll County, N. H. Pennacook, "papoose bear," or "bear cub," from papisse-conwa. Named for a famous leader of that tribe, father of Wannalancet, who was important in colonial history. He figures prominently in Whittier's Bridal of Pennacook.

Passadumkeag River and Village, Penobscot County, Me. Abnaki, "rapids over the gravel beds."

Passagas-sa-waukeag at head of Belfast Bay, Waldo County, Me. Malecite, "place for spearing sturgeon by torch light."

Passaguanik shore of New Found Lake. Grafton County, N. H. Abnaki, "sandy bottom at the fork, landing place."

Passaiaco Washington County, R. I. Narragansett, "muddy place."

Passamagamet Lake, Piscataquis County, Me. Abnaki? Malecite? "at the place of many fish."

Passamaquoddy Bay, Washington County, Me. Malecite, "place of abundance of pollack."

Passanoke Washington County, R. I. Narragansett, "muddy land." Also Passanoquke.

Passaquatch Plymouth County, Mass. Wampanoag, "at the divided hill."

Passataquack same as Piscataqua?

Passatuthonsee River, Kent County, R. I. Narragansett, "muddy shallow place," or "muddy ford." 
Passawaukeag Lake, Waldo County, Me. Malecite, "place of the sturgeon."

Passcogue see Pascoag.

Passenchaug Hill, Middlesex County, Conn. Natick? "steep (bluffs) mountain."

Passenchaug Meadow, Middlesex County, Conn. Wangunk, "miry land," or "mucky place."

Passeonkquis Cove and Pond, Kent County, R. I. Narragansett, "the miry place." Also Passconuquis, Paskonucquish, etc.

Passonagesit Norfolk County, Mass. Natick, "where bluffs rise up," ? or "slippery descent place,"? or "miry place"?

Passpatonage Brook; see Paspatonage.

Passquessit south of New London County, Conn. Narragansett, "at the place where the bluffs stand up."

Passquishunk Middlesex County, Conn. Western Niantic? "land at the outlet," the mouth of the Connecticut River. Also given as Pashquishhook, perhaps "muddy, split outlet"?

Passuatuxet see Pawtuxet.

Passumpsic River and Village, Caledonia County, Vt. Abnaki, "flowing over clear sandy bottom." (Pesammes on Father Aubéry's map, I715).

Passuntquanucke Neck, Barnstable County, Mass. Wampanoag, "at the end of the sandy place," ? or "slippery rock landing place"?

Paston the Modern Abnaki pronounciation of Boston, Mass. (All New Englanders are Pastonaki, similar to Colonial French Baston and Bastonnais).

Paswampisic Caledonia County, Vt. Abnaki, "great clear place." See Passumpsic.

Paswonquitte location uncertain, Kent County, R. I. Narragansett, "at the muddy bend."

Patackhouse see Potock, Potucko's and Quaiapen.

Patackosi Brook, Town Brook, Plymouth County, Mass. Wampanoag, "narrow," or "short and narrow," also "round (rock place)."

Patacomumscott Windham County, Conn. Nipmuck, "place of the round rock." See Puttacawmaumshcuck. 
Pataconk or Pattequonk Middlesex County, Conn. Wangunk, "at the round place," possibly Cedar Lake.

Pataconkset Kent County, R. I. Narragansett, "at the round place."

Pataguanset or Pattaquonset Lake, New London County, Conn. East Niantic, "at the round, shallow place." Another spelling Patagunset.

Patagumkis or Pattagumkus Stream, Penobscot County, Me. Malecite, "sandy round cove" or "gravelly bend."

Patagumskocte Washington County, R. I. Narragansett, "place of the round rock."

Patant atonet Plymouth County, Mass. Wampanoag, "windy hill place" or "thunder hill place."

Patapso possibly Sagadahoc County, Me. Abnaki? "round rock."?

Pataquasek or Pataquasak see Pattaquasset.

Pataquongamis Telosimis Lake, Piscataquis County, Me. Abnaki, "little round lake."

Pataug Plain, Worcester County, Mass. Nipmuck, "a bog”? or "small trees."?

Pataweekongomoc Telos Lake, Piscataquis County, Me. Abnaki, "lake near burned-over land."

Pataweektook Ragmuff Stream, Penobscot County, Me. Abnaki, "stream (running through) burned-over land." Variants, Paytayweektook, P'tawektook, etc.

Patawomut River and Rocks, Washington County, R. I. Narragansett, "miry meadow place." But see Potowomut.

Patchogue River, Middlesex County, Conn. Western Niantic, "at the place of turning."

Patcongomic Lake and Portage, Piscataquis County, Me. Abnaki, "round lake," possibly the lake now called Telos?

Pateook Litchfield County, Conn. Mahican, "place at the falls."

Patequonque see Pattaconk.

Pat-higget Plymouth County, Mass. Wampanoag (modified by English), possibly from Patusuc, "falls at the mouth," or "falls in the brook." An ancient name for Plymouth, sometimes called Patuxet, q. v.

Patnisset Barnstable County, Mass. Wampanoag, "at the small hill." 
Patopacassit Pond, Plymouth County, Mass. Wampanoag, "where strait widens." Also given as "foamy place where narrows open into a small pond."

Patowomuck see Potowomut.

Pattaconk Hill; see Pattaquonk.

Pattagawonset see Pattaquonset.

Pattagussis Aroostook County, Me. Abnaki? Malecite? "little oxbow," or "little back turn." See Petagoubky.

Pattaquasset Middlesex County, Conn. Hammonassett, "at the round place."

Pattaquattic Hill and Ponds, Hampden County, Mass. Nipmuck, "at the round place," or "at the round hill."

Pattaquodtuck Windham County, Conn. Nipmuck, "round hill near the river." Spelled also Pattaquottuck.

Pattaquonk Hill, New London County, Conn. Western Niantic, "round hill." Found also as Padaquonch and Padaquonk. See Pataconk.

Pattaquonquomis Lake, Allagash River, Aroostook County, Me. Abnaki? Malecite? "long double lake?" or "long lake joined to a round pond?" or "long fishing place in the round lake." ?

Pattaquonset Pond, New London County, Conn. Western Niantic, "at the small round place." This may have been a small rock, or hill, or a sweat-lodge; see Pissepunk.

Pattaquottuck see Pattaquodtuck.

Pattukett the Providence River. See Pawtucket.

Pattyquonck Hill, Middlesex County, Conn. Wangunk, "round place."

Patucket Falls, Hampshire County, Mass. Nipmuck, "at the falls in the river."

Patuisset Barnstable County, Mass. Wampanoag, "at the small falls, or rapids"?

Patuxet Plymouth County, Mass. Wampanoag, "at or near the little falls."

Patuxett River, Providence County, R. I. Narragansett, "at the little falls." Also Patuxit.

Patuxit see above.

Paucahak or Paukahak see Pocasset. 
Paucamack Pond, Washington County, R. I. Narragansett, "open or clear pond," or "shallow pond."

Paucatuck Brook, Hampden County, Mass. Nipmuck, "shallow river," or Natick, "falls in the river."

Paucatunnuc see Poquetanuck,

Pauchasset River; see Pocasset.

Pauchaug Brook and Meadow, Franklin County, Mass. Narragansett, "at the turning place."

Pauchauog Washington County, R. I. Narragansett, "where they play games and dance," or "they are playing"? but more likely, "the turning place." See Pachaug.

Paucomptucke River, Franklin County, Mass. Pocumtuc, "narrow swift river." This is the Pocumtuc, now the Deerfield River.

Paudowaumset New London County, Conn. Mohegan-Pequot, "trading or barter place."

Paug Hartford County, Conn. Sicaog, "a pond." (This is a generic name for "pond." Variants occur as poque, baug, etc.)

Paugachauge Newport County, R. I. Narragansett? Wampanoag? "at the clear, open place"? or "pond near the hill"? or "bare hill"?

Paugamaug Pond, Washington County, R. I. Narragansett, "shallow or clear fishing place."

Pauganuck Fairfield County, Conn. Paugussett, "cleared land." Paugasset New Haven County, Conn. Quinnipiac, "where river widens." See Paugussett.

Paugatuck River; see Pawcatuck.

Paugeamapauge Pond, Providence County, R. I. Narragansett, "divided pond" or "shallow fishing place at pond."

Paugunt New London County, Conn. Mohegan, "place of the ponds,"? or "cleared land."?

Paugus Bay, Belknap County, N. H. Abnaki, "small pond." Paugus was an Indian killed at Fryeburg, Me., in I725.

Paugus Middlesex County, Mass. Natick, "small pond."

Paugussett Trail, Fairfield County, Conn. Paugussett, "a swift current in the divided river," or "the river widens out where the forks join"? also "place of the small pond." This was a small tribe occupying parts of eastern Fairfield County and New Haven County, Conn. 
Paugwonk New London County, Conn. Mohegan, "pond at the cleared place," or "crooked pond." Also Pogwonk.

Pauhunganuck Brook, Hampden County, Mass. Nipmuck, "at the place where millstones are quarried;" (or perhaps stone mortars made ?); "within the bend;" also "little field brook;" also "small enclosed field."

Paukatuck see Pawcatuck.

Paukopunnakuk Hill, Plymouth County, Mass. Wampanoag, "where you turn aside from the narrowed path"? or "refugeplace"? or "bear place"?

Paukunawawock Hill, Kent County, R. I. Narragansett, "bear's place."

Paukyowohhog New London County, Conn. Nipmuck, possibly "purifying place," a sweat-lodge? (See Pissepunk). Perhaps, "cleared land."?

Paumpagussit Bristol County, R. I. Narragansett, "God of the Sea."

Paupakquamcook Pond, Plymouth County, Mass. Wampanoag, "at the double ponds," or possibly "quail country."

Paupasquachuke Worcester County, Mass. Nipmuck, "at the double hill."

Paupasquachuke Kent County, R. I. Narragansett, "double hill place," papiske wadchu auke.

Paupasquachuke New London County, Conn. Nipmuck? "place of the double hill."

Paupasquatch see Paupasquachuke, above.

Paupattokshick New London County, Conn. Mohegan-Pequot, "round bare hills near the river."

Paupock (singular) Paupoksuog (plural) Poopoohquotog (plural) This name or cognates appear in several places in Southern New England. Its translation is "quail" or "quails."

Pauquanauge Hartford County, Conn. Wangunk, "land cleared and prepared for planting." See Poquetanuck.

Pauquapaug Brook, Litchfield County, Conn. Mahican, "clear water pond." Also, Pahquepaug, Pequabuck, Poquaback, etc.

Pauqununch Fairfield County, Conn. Paugussett? "clear creek"? "divided creek"? "long split rock reef"? This seems to be a remnant of a longer word. 
Pausatuck Plymouth County, Mass. Wampanoag, "miry, or mucky, river." (From pusseog, "mud.")

Pautage Worcester County, Mass. Nipmuck, "a neck, where the land juts out."

Pautapaug Hill, Windham County, Conn. Nipmuck, "the jutting cove or pond." This name fits a pond nearby. Other spellings Poattapogue, Potabauge, Potapogue.

Pautipaug Windham County, Conn. Nipmuck, "miry land." Given also as Pootapaug, Pautiboag, etc.

Pautonk Hartford County, Conn. An alluvial island or bar at the mouth of the Farmington River. Possibly from Mahican, paudtau-ank, "he brings it hither;" that is, someone brings his fishing equipment. Pautunk might refer to building up of lowlands or deltas by alluvial soil. See also Podunk.

Pautuckquitt Fields, or Pautuckqut Field and River. See Pawtucket.

Pautuxet New London County, Conn. Eastern Niantic? Narragansett? "at the little falls." Also Pawtucksit.

Pauwaget Pond, Washington County, R. I. Eastern Niantic, "small clear meadow." Other spellings Pawwawget, Pawamack, Pawwanget and Pauwanganset.

Pawcatuck River and Village, New London County, Conn. Eastern Niantic? Pequot? "the clear divided tidal stream;" or "open divided stream." Given sometimes as Pawcatucket, Pawkeatucket, Paquatuck, Paucatuck, etc.

Pawchauquet Washington County, R. I. Eastern Niantic? "at the dividing place;" more likely "at the boundary."

Pawcomet (now Beach Pond) Washington County, R. I. and New London County, Conn. Narragansett? Eastern Niantic? "at the small beach."

Pawconakik, Pawkeesett, Pawkunnawkutt; see Pokanoket.

Pawhikehatt River, Plymouth County, Mass. Wampanoag, "at the shallow branch,"? or "at the winding river."?

Pawkamauket Bristol County, Mass. Wampanoag, possibly "at the cleared land" (pokanoket, or pokonoket), or "fort-place" or "place of refuge." (Pawkamauket was the spelling used by King Philip's secretary in I670.)

Pawkhungernock see Puckhunkonnuck. 
Pawmechaug see Pomachaug.

Pawmet Barnstable County, Mass. Wampanoag, "at the sea shore," or "at the swimming place."

Pawnook Lake, White Oak Pond, Penobscot County, Me. Abnaki, "large opening," or "the opening." Also found as Ponoke.

Pawonbowk a cove in Lake Champlain, Thompson's Point, Chittenden County, Vt. Abnaki, "dish-shaped pond."

Pawpoesit Barnstable County, Mass. Wampanoag, "snipe (or partridge) country,"? or "at the little swamp." ?

Pawquabunke Providence County, R. I. Narragansett? "shallow pond."

Pawqutaboque see Pocotopaug.

Pawsacacow see Pascachute.

Pawtuckaway Mountains and Pond, Rockingham County, N. H. Abnaki, "falls in river"? or "clear, open, shallow river"?

Pawtucket City and Reservoir, Providence County, R. I., Windham County, Conn. and Bristol County, Mass.; Narragansett, "at the falls in the tidal stream."

Pawtucket Falls, and Village, Middlesex County, Mass. Natick, "at the falls in the river."

Pawtuckquachooge New London County, Conn. Eastern Niantic, "at the hill near the river falls."

Pawtuxent Falls, Washington County, R. I. Eastern Niantic, "at the small falls."

Pawtuxet Plymouth County, Mass. Narragansett, "at the little falls." Others in Middlesex County, and Hampden County.

Pawtuxet City and River, Providence County, R. I. Narragansett, "at the little falls." Also a cove of this name in Kent County.

Pawwating Village, Plymouth County, Mass. Chipperwa, "at the falls or rapids."

Paw Woh Pond, Barnstable County, Mass. Wampanoag, "a conjurer or medicine-man; a ceremonialist." See Pow wow.

Paynatuck see Pawcatuck.

Payquage see Poquiag.

Peacepunk see Pissepunk and Pesuponck.

Peagscomsuck Island, Windham County, Conn. Nipmuck, "cleared lands." 
Peagwompsh Windham County, Conn. Nipmuck? Mohegan? "bare rocks."

Peamecha Middlesex County, Conn. Natick? possibly, "small river near the hill;" or "land by the stream." See Sebethe.

Peantam (Bantam) Litchfield County, Conn. Natick, "he is praying."

Peboamauk Falls, Coös County, N. H. Abnaki, "wintry place," or "country of the winter."

Pecausset Pond, Middlesex County, Conn. Wangunk, "a small clearing."

Pecheneganook Rapids, St. Francis River, Aroostook County, Me. Micmac, "long portage because of rapids."

Pecowsic Hampden County, Mass. Nipmuck, "where the river opens out." But some derive this from Indian names of "red or gray fox." (Another spelling Peccawoosuck may be translated "clear, open brook.")

Pedaugbiouk headwaters of Damariscotta River, Lincoln County, Me. Abnaki, "double cove" or "round bay."? One source gives "place where spreading out waters turn about." See Pedcoke gowake, below.

Pedcoke gowake Carry, Lincoln County, Me. Micmac, "reverse bends," S-bends in the stream.

Pedee Nantucket County, Mass. Wampanoag, "pitch or gum," from a tree such as pine, spruce, wild cherry.

Peech-oonegonik Village, Aroostook County, Me. Malecite, "at the long portage."

Peegwon Place, Middlesex County, Conn. Natick? "tear drop" (lachrymal fluid).

Pegan Middlesex County, Mass. Natick, "bare." But perhaps an abbreviation of a longer word such as Quamphegan?

Pegansic Hill, Hartford County, Conn. Wangunk, "bare, unwooded place," or some say Eastern Niantic, "bear's abode," pegunapar, but this is unlikely. See Pahegansuc.

Pegusset Middlesex County, Mass. Natick, "where waters widen out."

Pegu usset New Haven County, Conn. Quinnipiac? Pangusset? See Paugussett. Found sometimes as Pequ usset.

Pegwacket or Pegwakik. See Pigwacket. 
Pejipscot near Brunswick, Cumberland County, Me. Abnaki, "extended long rapids." Also Pejebscot, Pejepscot, Pegypscott, Pejepschaëg, etc.

Pekdabowk possibly Shadow Lake, Orleans County, Vt. Abnaki, "smoke pond."

Pekenut Norfolk County, Mass. Natick, "bare place."

Pekketegw Rockingham County, N. H. Abnaki, "branched river." The Piscataqua river.

Pekontuk Otter Creek, Rutland and Addison Counties, Vt. Abnaki, "crooked river"? or "clear open stream"?

Pemadinisikek Clifton-Dedham-Oldham Hills, Penobscot County, Abnaki, "extended little mountains," or "range of small hills."

Pemadnek Mount Desert Island, Hancock County, Me. Abnaki, "extended mountains," or "mountain range." See also Pemetic.

Pemadumcook Lake, Piscataquis County, Me. Malecite, "extended sand bar place."

Pemaquid Point and Village, Lincoln County, Me. Micmac, "extended land," long peninsula running into the sea. Other spellings include Pemaquida, Pemaquideag, Pemakwid, etc.

\section{Pematagoet see Pentagoet.}

Pematchuwatunch Mountain, Franklin County, Mass. Pocumtuck? "twisted or winding ridge of mountains,"? or "village in twisted mountain range,"? or "joined crossways."?

Pemategu Mt. Desert Island, Hancock County, Me. Abnaki, pem "extended," plus antegre "a wave;" translated as "long rows of breakers."

Pematinek (Routes), Penobscot County, Me. Abnaki, "route (to) the distant mountain range." See Matanawcook.

Pemenos Windham County, Conn. Nipmuck, "small path," or "narrow trail."

Pemetic Hills, Mt. Desert Island, Hancock County, Me. Abnaki, "range of mountains." A more correct spelling is Pem-etnic.

Pemidjuanosick Falls, Penobscot County, Me. Abnaki, "place of the extended falls, rapids or swift current."

Pemigewasset Mountain and River, Grafton County, N. H. Abnaki, "swift extended current," or "successive rapids." 
Pemigewasset Pond, Belknap County, N. H. Abnaki, "extensive rapids."

Pemjeedgewock Falls, Treat's Falls, Penobscot County, Me. Abnaki, "extended, rapidly descending current."

Pemmaquan River, Penobscot County, Me. Abnaki, "long ridge covered with maple trees."

Pemmaquan Lake and Stream, Washington County, Me. Abnaki, "slope or ridge covered with maple trees."

Pemmoquittaquomut Hillsboro County, N. H. Pennacook, "at the place of two ponds joined by a straight stream or ditch."

Pemskudek see M'skutook.

Pemsquamkutook Birch Stream, Penobscot County, Me. Abnaki, "hardwoods near river." Sometimes translated "birch river."

Pemtaquamcook the Penobscot River. See Pemtegwatook.

Pemtaquiauktook mouth of the Pembroke River, Washington County, Me. Malecite, "extended waters joined" or "extension of stream into larger body." Sometimes found as Boamtuquet, "broad stream place."

Pemtegwatook the main stream of the Penobscot River, below Bangor, Penobscot County, Me. Abnaki, "extended descending river current."

Pemunneaht Fairfield County, Conn. Paugusset, "a string or cord," especially a fish line. Variants, Pemenante and Pem munneaht-om, "line for fishing."

Penacook Village, Merrimack County, N. H., and River, Worcester County, Mass. Pennacook, "downhill," or "at the foothills."

Penagou so named on Aubéry's I7I5 map; probably in Merrimack County, N. H. Pennacook, modified by French, "sloping land," or "foothills."

Penapsquacook Penobscot County, Me. Abnaki, "steep rocky banks."

Penecoög Ridge, Merrimack County, N. H. Pennacook, "foothills" or "sloping land." The Pawtuckaway Range.

Penetahpoh Nantucket County, Mass. Wampanoag, "crooked wading place," or "crooked pond." Also suggested, "whales here," but unlikely.

Penhongansett see Ponaganset. 
Penikese Island, Dukes County, Mass. Wampanoag, meaning now lost. Perhaps "small waves thunder down," or "small, sloping land." It may refer to Chief Penakeson, related to one of Metacom's chieftains. Also Pasquenese.

Penjejawock Stream, Penobscot County, Me. Abnaki, "current falling down raggedly." See Pemjeedgewock; also Bemidjwok.

Penkese Island, Worcester County, Mass. Nipmuck, "at the shallow place"? May refer to Penakeson; see Penikese, above.

Pennacook Lake, Merrimack County, N. H. Pennacook, "at the foothills" or "sloping down place." Pennacook Indians occupied upper Connecticut Valley, south-central New Hampshire, northeastern Massachusetts and southern York County, Maine, in early times. After the King Philips War ended in I676, they migrated north to Canada, New Hampshire and Maine.

Pennamaquan River, Penobscot County, Me. Abnaki, "extensive area covered by maple trees."

Pennechuck York County (?), Me. Abnaki, "slope of mountain"? or "bottom of the mountain"? or "at the rapids."?

Pennecook Falls, Rumford Falls, Oxford County, Me. Pennacook, "at the foot hills" or "down hill," or "at the bottom of the hill." Variants, Pennecoo, Penacuk, Pennycook, etc.

Pennessewassee Lake, Oxford County, Me. Natick, "a strange, shining-then-fading light."

Pennichuck Brook, Hillsboro County, N. H., and Merrimack County, N. H. Pennacook, "at the rapids."

Penobscot County, River, Lake in Penobscot County; Village in Hancock County; Pond and River in Piscataquis County, Me. Malecite? Abnaki? "at the descending rocks" or "at the extended ledges." The Penobscot Indians, closely related to the Abnaki, live chiefly at Old Town, Maine, and the adjacent Penobscot Valley.

Penobscot Middlesex County, Mass. Pennacook? "place of sloping ledges." Variant, Penobsceäg.

Penobseese Stream, Lincoln County, Me. Abnaki, "little Penobscot."

\section{Penobskeag see Penobscot.}

Penobsquisumquisebou the Sandy River, Kennebec County, Me. Abnaki, "sloping rocks (fragments?) river." 
Penoomskeeook the Penobscot River. Abnaki, "place of rocky falls."

Penopeauke the Penobscot River. Abnaki, "place of sloping rocks."

Penopsquacook Waldo County, Me. Abnaki, "steep, rocky place."

Pentagoet former name of Castine and vicinity, Hancock County, Me. Abnaki, modified by French, "at the falls."

Pentucket Lake, Pond and River, Essex County, Mass. Natick, "at the twisting river."

Peormug seeboog New London County, Conn. Mohegan, "fish in the brook." An old name for Poquetanack Cove.

Pepemightughk on the boundary between New York and Connecticut, (Fairfield County). Delaware? Mahican? "selected tree," possibly a surveyor's mark.

Pequabuck River, Hartford County, Conn. Wangunk, "clear, open pond." Also a village in Litchfield County, Conn.

Pequag or Pequiog Worcester County, Mass. Nipmuck, "cleared or cultivated land."

Pequatit New London County, Conn. Narragansett, "at the Pequot's place."

Pequawket Pond, Carroll County, N. H. Abnaki, "broken land."

Pequawkett Jockey Cap Mountain, Oxford County, Me. Malecite, "punched-up-through place."

Pequid Brook, Norfolk County, Mass., Pequot? or Natick, "waterplace"? or "cleared, plowed land." ?

Pequimmet Norfolk County, Mass. Natick, "at the shallow place," or "place of open springs."

Pequiog Worcester County, Mass. Nipmuck, "cleared land."

Pequod Middlesex County, Mass. Pequot, "destroyers of men."

Pequonnock Fairfield County, Conn. Paugussett, "a small plantation" near Bridgeport. Some sources say the correct form is Paquanan auke, from Narragansett "battlefield," or "slaughter place." Variant, Pequannuc.

Pequot an important Connecticut tribe who were almost annihilated during the Pequot War of I637. Their name means "the destroyers," and has been applied to a large number of sites and places in Connecticut, Rhode Island, and Massachusetts. It is also found as Pequod. 
Pequot Tract, Franklin County, Mass. Pequot-Mohegan, "destroyers."

Pequotauk New London County, Conn. Mohegan? Narragansett? "territory of the Pequots," part of New London County.

Pequot sepos New London County, Conn. Mohegan, "little river of the Pequots." The Mystic River?

Pequt a term used to distinguish between the Narragansetts of Rhode Island and the Connecticut Pequot Indians. "Destroyers."

Pequt toog "The Pequots," a name given by Roger Williams.

Perquanapaqua Berkshire County, Mass. Mahican, "clear long pond," or "clear still water."

Pesammes now the Passumpsic River, Caledonia County, Vt. Abnaki, "clear, sandy bottom."

Pesaumkamesquesit Pond, now Blackmore Pond, in Providence County, R. I. Narragansett, "very small plain or meadow, at its end."

Pescedona now the Androscoggin River, Cumberland County, Me. This ancient name is Abnaki, and probably means "a branch."

Peschameeset Island, Dukes County, Mass. Wampanoag, something to do with small fish; perhaps, "where we catch and split small fish"? Also, "blue place"? Variant, Peshamesset.

Pescongamoc Pond, Piscataquis County, Me. Abnaki, "branched pond."

Peskadamioukkanti the St. Croix River, the boundary between Washington County, Me., and New Brunswick. Malecite, "plenty of pollack." See Passamaquoddy.

Peskebegat Lobster Lake, Picataquis County, Me. Abnaki, "split lake."

Peskebskitegwek Soper Brook, Eagle Lake, Aroostook County, Me. Abnaki, "branch of the deadwater stream."

Peskedopikek Alder Brook, Penobscot County, Me. Abnaki, "branch at the place of alder bushes."

Peskeompscut Franklin County, Mass. Nipmuck, "at the split rocks."

Pesketuk the Piscataquis River, York County, Me. Abnaki, "the split river," or "river fork." 
Peskutum-akadi Passamaquoddy Bay, Washington County, Me. Micmac, "place of the pollack."

Pespataug Pond, Washington County, R. I. Eastern Niantic, "the land at the bursting-out place," or "at the small inlet."

Pespataug New London County, Conn. Narragansett, "where the stream flows out," possibly the mouth of the Pawcatuck River, or that of the Mystic River, the eastern boundary of the Pequot country.

Pesquamscot River and Pond, Washington County, R. I. Narragansett, "at the cleft rock," or "split boulder place."

Pessakenew Agenek West Quoddy Light, Washington County, Me. Abnaki, "at the lighthouse." Also Pessaken-i-higanek, "at the lighting apparatus."

Pessicus from the name of a Narragansett chief who was killed fighting Mohawk invaders in Maine. Malecite, "sturgeon." (In Natick, the word psukses means "little bird.") Also, Pessacus; see Passagas sa waukeag; Moosup.

Pesuckapaug location probably in Middlesex County, Conn.? Wangunk, "mucky or miry pond." See Pishatipaug.

Pesuponck Washington County, R. I. Narragansett, "a hot house." Roger Williams tells of such sweathouses where the men went "first to cleanse their skin, secondly to purge their bodies. ... I have seen them run (summer and winter) into brooks to cool them without the least hurt." Several such place names are found throughout Connectitut, Rhode Island and elsewhere in New England.

Pesut amesset an old camp ground at Brewer, Penobscot County, Me. Abnaki, "seen only when near it."

Petagoubky now the lower reaches of the Missisquoi River, Franklin County, Vt. Old Abnaki, "river full of (unexpected) bends and eddies," that is, back turns or oxbows.

Petapauket Middlesex County, Mass. Natick, "swampy place."

Petapawag River, Middlesex County, Mass. Natick, "boggy place where our feet sink in."

Petaquapen Fairfield County, Conn. A Paugussett chief? See Petuckquapaug.

Petch-kajisk Washington County, Me. Malecite? Abnaki? "sharp bend," or "sharp bend at ledge."? 
Petcongomac Aroostook County, Me. Abnaki, "crooked pond" or "lake that turns."

Petcumcasick Penobscot County, Me. Abnaki, "round bend with a gravelly bottom." Also Betcumcasick.

Peteconset Meadow, Washington County, R. I. Narragansett, "small plantation." Variant Petequonset.

Petegwamgamek Round Pond, Aroostook County, Me. Abnaki, "round lake."

Petekamkes Penobscot County, Me. Abnaki, "the gravelly bend." (The old form of Patagumkis.)

Petekesisk Aroostook County, Me. Abnaki, "at the little oxbow turns."

Petekwangamessis probably Telosinis Pond, Piscataquis County, Me. Abnaki, "little back turns."

Petit Manan Point, Washington County, Me. French and Abnaki, small "island."

Petonbowk now Lake Champlain. Abnaki, "waters that lie between" the Green Mountains and the Adirondacks. (A Micmac term petoobook means "a long dish of (salt) water.")

Petowamacha Hills, Hampden County, Mass. Nipmuck, "jutting(up) mountains," or "bulging mountains."

Petow Bowk Lake Champlain. Abnaki, "lake that lies between" New York and Vermont.

Petow Pargow now Lake Champlain. Mahican, "double pond," or "two great ponds joined;" or Nipmuck, "two bulges"[?]

Petowtucke River, New Haven County, Conn. Quinnipiac, "country around the falls."

Petowwag Hampden County, Mass. Nipmuck, "bulging place." One authority gives "land from whence water flows to us." See Petowamacha.

Pettaconsett Kent County, R. I. Narragansett, "at the small enclosure, or plantation"? or "place of the round rocks"?

Pettaquamscutt Cove, River and Rock. Washington County, R. I. Narragansett, "at the round rock." Also Pettiquamscut, Pettycomscok, etc.

Pettopaugsett Plymouth County, Mass. Wampanoag, "place of the small round pond." 
Pettukaway Mountains, Rockingham County, N. H. Pennacook, "round"? But see Pawtuckaway.

Petuckquapaug Fairfield County, Conn. Siwanoy, "round pond." Also given as Betuckquapock and Petuquapen.

Petukunok River, the Chester River, Middlesex County, Conn. Hammonassett, "round rock place."

Pewagon West Branch of the Pemmaquan River, Washington County, Me. Malecite, "small portage."

Pewampskine Path, Middlesex County, Conn. Wampanoag? Natick? "little blond girl" or "small whitish child," from the name of a female chief or Sunksqua. Possibly a white captive adopted into the tribe? A man named Powampskin deeded land here in 1673 .

Pewonganuck River, Hampden County, Mass. Nipmuck, "at the place of whetstones," or "place of small long stones." Also "country of small bends or turns."

Piamikin Fairfield County, Conn. Paugussett, "where the path is narrow."

Pichet Mountain, Aroostook County, Me. If Indian, perhaps Abnaki, "split."?

Pico Peak, Rutland County, Vt. Abnaki, "the pass or opening." Or perhaps from Spanish term pico, "peak." Pico overshadows Sherburne Pass in the Green Mountains.

Picosick Hampden County, Mass. Nipmuck, "river opens out"? or "red fox."? See Pecowsic.

Piggscut River, Cumberland County, Me. Abnaki, "at the branch."

Pigscomsuck Island, New London and Windham Counties, Conn. Mohegan, "clear land." Found also as Paukyowohhog and Pesquamscot, "where stream divides into two currents." See also Pacomsuck.

Pigsgussett Middlesex County, Mass. Natick, "where stream widens." See Pegusset.

Pigwacket now Fryeburg, Oxford County, Me. Malecite? "land naturally clear or open;" also given as "broken, punched-up land." See Pequawket.

Pigwaduk gamok Pushaw Lake, Penobscot County, Me. Penobscot-Abnaki, "bent stream lake." Variants, Pigwatook and Bigwadook. 
Pikaghenahik Crooked Island, Penobscot County, Me. Abnaki, "curved or crooked island."

Pimsepoese River, Plymouth County, Mass. Wampanoag, "creek which supplies food." Also given as "crooked creek," or "extended little river," etc.

Piscasset the Lamper Eel Stream in Rockingham County, N. H. Abnaki, "at the branch" (of the river).

Piscatacook Litchfield County, Conn. Mahican, "at the river branch." Variants, Scatacook, Schachtigoke, etc.

Piscataqua Point, York County, Me.; the River is the boundary between Strafford County, N. H., and York County, Me. Pennacook, "the place where the river divides." Also Piscatua.

Piscataquis County, Me., and Stream in Penobscot County, Me. Abnaki, "at the river branch," or "at the little divided stream."

Piscataquis awangen Piscataquis County, Me. Abnaki, "a route via the forked (Piscataquis) river."

Piscataquog River, Hillsboro County, N. H. Abnaki, "where the river divides."

Pisgah many locations by this name are found in New England; most refer to mountains. If Abnaki, the meaning is, "dark;" if Mahican, "muddy." However, the nearby presence in Vermont of Mount Hor suggests Old Testament Hebrere, "a mountain." (see Numbers 20:22 and 2I:20).

Pisgatoek River, Strafford County, N. H. Abnaki, "at the place of the river branch."

Pishatipaug Middlesex County, Conn. Wangunk, "muddy or miry pond."

Pishgachtigok Litchfield County, Conn. Mahican, "where the stream branches."

Piskaquoag River, Merrimack County, N. H. Pennacook, "place of the branch or tributary."

Pispogutt Plymouth County, Mass. Wampanoag, "at the miry pond."

Pisquasent Washington County, R. I. Narragansett, "muddy rocks place," or "slippery rocks place."

Pisqueheege Fairfield County, Conn. Paugussett, "at the river branch." 
Pissak Plymouth County, Mass. Wampanoag, "swampy place."

Pissapogue Washington County, R. I. Narragansett, "a hothouse," a ceremonial hot air or steam bath place. Probably this gives the name to Hothouse Pond.

\section{Pissatapaug see Pishatipaug.}

Pissepunk "Ceremonial Hothouses," Hartford, Middlesex, and New London Counties, Conn. Narragansett, pesuponck, an especially tight hut or cave where men built hot fires and took ceremonial sweat-baths. (From pesuppau-og, "they are sweating.")

Pissepunk Hill, Fairfield County, Conn. Paugussett, "a sweatlodge." See Pesuponck.

Pissoups Windham County, Conn.? Nipmuck, "mucky place."?

Pistapaug Mountain and Pond, New Haven County, Conn. Quinnipiac, "muddy pond." Also Pistepaugh.

Pitawi teguk the Stillwater Branch, Penobscot County, Me. Abnaki, "at the roundabout river" or "meandering river;" (locally, "the back way.")

Pitchawamache Swamp, Hampshire County, Mass. Nipmuck, "at the place of low, miry land." Also, Pitchawam.

Pitchgussett Middlesex County, Mass. Natick, "muddy or slippery ledge place."

Pitchoohutt Plymouth County, Mass. Wampanoag, "muddy pool."

Pitow baygook Long Island, Waldo County, Me. Abnaki, "between two channels;" also given as Pitau-begwi-menahanuk, "the island between two channels."

Piwan gamosis Field's Pond, Penobscot County, Me. Abnaki, "little round pond."

Plausawa Mountain, Merrimack County, N. H. Named for an Abnaki warrior; meaning obscure, but possibly "wild pigeon" or "short yell." He was killed near here in I753.

Poakyowohog Windham County, Conn. Nipmuck, "land cleared and prepared for planting." See Paukyowohog.

Poataskehung Brook, Fairfield County, Conn. Mahican, "green cleared land," or "green pasture"?

Poattapoge see Pauquapaug.

Pocahantas Road, Fairfield County, Conn. Pocahontas (I595-I6I7) was the daughter of Powhatan, an important Virginia chief; 
her real name was Matoaka. Pocahontas is translated as "she is playful," although Strachey gives "a sharp pointed instrument, such as an awl."

Pocamsus Lake Washington County, Me. Abnaki, "little shallow" or "little clear."

Pocapawmet Plymouth County, Mass. Natick, "at the shallow swimming (or wading) place," or "at the cleared trail." Also given as "at the stopped-up inlet or cove."

Pocasset several Rivers, Lakes and Streams in New England. All are Natick and cognate tongues, meaning, "where the stream widens." Other spellings, Pochasset, Pohkasset, etc.

Pochassic Hills, Hampden County, Mass. Nipmuck, "at the narrow outlet."

Pochasuck Hampden County, Mass. Nipmuck, "narrow outlet," or "narrow brook."

Pochaug River, Middlesex County, Conn. Western Niantic, "where they divide." Also Patchogue, Pooachoage, Pochawg, etc.

Pochet Island and Neck, Barnstable County, Mass. Wampanoag, "at the narrow place." Or possibly "at the turning place." See Pachet.

Pochick Bluff and Rip, Nantucket County, Mass. Wampanoag, "divided reef," or "bulging-out place." Also given as "turnaside place."

Pochoboquett Plymouth County, Mass. Wampanoag, "small pond opens up," or "opening of small pond." Also translated as "boundary pond place."

Pochoke location unknown. Natick? "turning place."

Pochuppunnukaak Plymouth County, Mass. Wampanoag, "the divided path." See Paukopunnakuk.

Pockapockhunk Bristol County, Mass. Narragansett, "creek between two hills"?

Pocketapaces Neck, Dukes County, Mass. Wampanoag, "divided cove, on tidal river."

Pockhanoket Pokanoket, Bristol County, Mass. Narragansett, "wood or land at the other side of the water"?

Pockquamscutt Plymouth County, Mass. Wampanoag, "place of the split rock." 
Pocksha Pond, Plymouth County, Mass. Wampanoag, "where he turns aside."

Pockwasook Penobscot County, Me. Abnaki, "low water," or "shallow water."

Pockwockamus Pond, Piscataquis County, Me. Abnaki, "little muddy pond."

Pocomo Head, Nantucket County, Mass. Wampanoag, "round fishing place," or "clear fishing place."?

Pocopassum an ancient Abnaki village, possibly in Cumberland County, Me. Abnaki, "cleared land"? or "small round cove"?

Pocopawmet Barnstable County, Mass.? or Plymouth County, Mass.? Wampanoag? Natick? "at the closed cove," or "at the plugged-up inlet."

Pocomtakuke probably in the Deerfield Valley, Franklin County, Mass.? A variant of Pocomtuck, "narrow, swift river" or "clear, open stream"?

Pocotopaug Creek and Lake, Middlesex County, Conn. Wangunk, "divided pond," or "two ponds." Also Pagetupaug, Pocatopaug, Paw qut a boque, Poacatoobuck, etc.

Pocowset Middlesex County, Conn. Wangunk, "stream widens here."

Pocoy Shore, Nantucket County, Mass. Wampanoag, "open."

Pocumpcus Lake, Washington County, Me. Micmac, "at the gravelly place."

Pocumtuck Mountain, River, and Valley, Franklin County, Mass. Also, the Deerfield Valley, Windham County, Vt., Pocumtuck, "narrow swift river," or possibly "clear, open stream." These Indians occupied the Deerfield and Connecticut Valleys in Vermont and Massachusetts. Mohawk warriors destroyed their Deerfield, Mass., fort in I666.

Podpis Harbor, Nantucket County, Mass. Wampanoag, "divided harbor," or possibly "bay place."

Podunk Hartford County, Conn. Nipmuck, "where you sink in mire," a boggy place. Podunk, or Pautunke, is the name of a tribe or sachemdom and the territory they inhabited. Their lands are marked Nowaas on maps of the early I6oo's.

Podunk Pond and Village, Worcester County, Mass. Natick, "at the place where the foot sinks," or "at the miry place." 
Poekquamscutt Plymouth County, Mass. Wampanoag, "at the place of broken rocks," or "split rocks."

Pogamqua River, Cumberland County, Me. Abnaki, "shallow, sandy."

Pogatanack Brook; see Poquetanuck.

Poggaticut Washington County, R. I. Eastern Niantic, "at the clear tidal creek." There was a chief so named.

Poggatossur Brook, Hartford County, Conn. Agawam, "clear pool." But see Pogotossuc.

Pogopskekok Stream, Kennebec County, Me. Abnaki, "a shallow gravelly place."

Pogotossuc Hampden County, Mass. Nipmuck, "at the small hollow," or "outlet of the small hollow." Also translated as "steep falls brook."

Poguassek Knox County, Me. Abnaki, "moonlight," or "moonlit place." See also Pok-o-moonshine.

Pogue Windsor County, Vt. If Indian, this may be a word taken from pog or paug, meaning "pond" in several dialects. See Capawack.

Pogumkik the Ogunquit River, in York County, Me. Micmac, "lagoon formed by sand dunes."

Pogwonk see Paugwonk.

Pohenagamook Lake, Penobscot County, Me. Abnaki? "spreadout lake."?

Pohgassek Franklin County, Mass. Pennacook? Pocumtuck? "place of the small pond." But see Poguassek.

Pohio New London County, Conn. Western Niantic, "cleared land." See Poquiogh.

Pohoganse Pond, Washington County, Me. Abnaki? Malecite? "shallow, sandy pond."?

Pohoganut Dukes County, Mass. Wampanoag, "at the place of the pipe."

Pohomoosh Stream, Washington County, Me. Micmac? "shallow creek."?

Pohquantuck River; see Paquantuck.

Pohqui Plymouth County, Mass. Wampanoag, "open, clear, shallow." 
Pohtaiyomsek New London County, Conn. Mohegan, "at the place of the prominent (projecting) rock," the southwest boundary of Mohegan territory.

Pohtatuck Nèw Haven County, Conn. Paugussett, "land near the falls."

Pojac Point, Washington County, R. I. See Potock.

Pojassick Franklin County, Mass. Pocumtuck, "narrow brook," or "narrow outlet."

Pokahganeh Pond, Penobscot County, Me. Abnaki, "white perch."

Pokamquoh Neck, Nantucket County, Mass. Wampanoag, "narrow place."

Pokanoket Bristol County, Mass. Wampanoag, "at the cleared land," or "fort," or "refuge." This was a favorite haunt of King Philip.

Pokanoket Plymouth County, Mass. Wampanoag, "at or near the cleared lands." Also Pokanokik.

Poke-o-moonshine Lake, Washington County, Me. Probably an English corruption of pok-wajan-i-tagook, Abnaki, "stumps in the brook." But note the Micmac term pocomoosh, "(salt) water extending inwards."

Pokey Lake, Washington County, Me. Abnaki, "clear, shallow, open." Another name, Gawaysik.

Pok-o-moonshine Brook, Strafford County, N. H. Possibly mixed Abnaki and English, "pond clear as moonlight." See Poke-omoonshine.

Pokonoket see Pokanoket and Pawkamauket.

Pokumkesawangamoksis Harrington Lake, Piscataquis County, Me. Abnaki, "shallow sandy pond on a canoe route."

Pokutcheco Nantucket County, Mass. Wampanoag, "at the harbor," or "enclosed place."

Poland Androscoggin County, Me. Possibly named after Polan, chief of the Sokoki, killed in I756.

Polpis Harbor, Nantucket County, Mass. Wampanoag, "branching harbor, or cove."?

Polyganset see Apponagansett and Ponaganset.

Pomachaug New London County, Conn. Mohegan, "across the path," or "over the hill."? Variants Pamechage, Pomechoag, Pummechog, etc. 
Pomacuck New London County, Conn. Mohegan, "narrow path" or possibly "place of the grant," or "land of tribute." Also Pomoacooke and Pomatuck.

Pomagusset Worcester County, Mass. Nipmuck, "at the stream which is crossways of the path or trail," or possibly "at the dancing place."

Pomamgansett Pond, Kent County, R. I. Narragansett, "at the small bend"? or "athwart the path"? or "place of tribute"?

Pomauknet Dukes County, Mass. Wampanoag, "he gets there by water," or "country reached by water travel."

Pomecanset an ancient Indian village near Pawtuxet Falls. See Pomamgansett.

Pomecansett Neck, Kent County, R. I. Narragansett, "athwart the path"? or "at the small bend"? or "at the place of tribute."? Also Pumgansett.

Pomegobset Hammond Pond, Penobscot County, Me. Abnaki, "slanting ledges."

Pomersimbeke now Parmacheene Lake, q. v. Oxford County, Me. Old Abnaki, perhaps "(extended) little crosswise pond," the headwaters of the Androscoggin River. This name is so indicated on Aubéry's I7I5 map.

Pometacomet Bristol County, Mass. Wampanoag, "chief's house," one of the titles of Metacom, or King Philip.

Pomham Rock and Shore, Providence County, R. I. Narragansett, possibly "he travels by sea." Pomham was a Narragansett sachem, killed in I676.

Pomkeag Stream, now La Pomkeag, Penobscot County, Me. Abnaki, "the place of rocks."

Pomkikin Worcester County, Mass. Nipmuck, "shallow place," or "fording place." Also Pommakin.

Pompanoosuc see Ompompanoosuc.

Pompanuck Creek, Bennington County, Vt. Wangunk? "place for playing games"?

Pompashpissett Barnstable County, Mass. Wampanoag, the same place as Pispogutt, q. v., but the translation may be "rock or boulder at the miry place."

Pomperaug River, New Haven County, and Trail, Fairfield County, Conn. Paugussett, from the name of the sachem of Woodbury, 
Conn., around I725; meaning obscure, but possibly, "place to walk, or to play," or "rocky place."? Variants, Pomperage, Pomperague, Pompawraug, etc.

Pompositicut Middlesex County, Mass. Natick, "at the clear river," or possibly "cliff at or near tidal stream."

Pompwanganug Hill, Windham County, Conn. Nipmuck, "where the trail turns or bends." Also Pomponagaug.

Ponaganset Pond and River, Providence County, R. I, and town in Bristol County, Mass. Narragansett, "oyster processing place," or "waiting place at the cove"?

Poncammooncoe Neck, Nantucket County, Mass. Wampanoag, "open or clear fishing place." See Packamohquah.

Pondock River, Kent County, R. I. Narragansett, "at the falls."

Ponemah Village, Hillsboro County, and Cemetery, Middlesex County, N. H. Chippewa, "the blessed hereafter," or "the sweet bye-and-bye."

Pongokwahemook Eagle Lake, Piscataquis County, Me. Abnaki, "place of the woodpeckers."

Pongokwayhaymock Lake, Aroostook County, Me.Abnaki, "woodpecker lake," from pogakmokqueh, "pounding on wood."

Pongonquamook Lake, Aroostook County, Me. Abnaki, "muddy lake," source of the Allagash River.

Poniken Village, Worcester County, Mass. Nipmuck? "put down your burden" (at end of portage?). But if Quassaponiken, perhaps "edge of bank" or "fording-place."

Ponikin Hill, Worcester County, Mass. Nipmuck, "put down your burden." Spelled also Ponnakin.

Ponkapog Norfolk County, Mass. Natick, "clear, open pond, "or "shallow pond." Also Punkapog and Punkapoag.

Ponsamsick Mountain, Litchfield County, Conn. Mahican, "the place of fallen rocks."

Ponscachuto Pond; see Pansacaco and Pascachute.

Ponset Hampden County, Mass., and Middlesex County, Conn. Western Niantic, "at the falls."

Pontiac Highway, Kent County, R. I. Ottawa, "the falls in the river;" the name of the famous Ottawa chief, circa I765.

Pontoocook Cove, Coös County, N. H. Abnaki, "near the falls in the river." 
Pontoosuc several sites in Mass., all Mahican or Nipmuck, "falls on the brook."

Pontoosuc Hartford County, Conn. Wangunk, "falls on the brook," at Glastonbury. Also Poontook suck.

Pontpetsicke Suffolk County, Mass. Wampanoag "falls in small brook."

Pontugwotchaug New London County, Conn.? Mohegan, "place of the falls in the river, near the mountain."

Ponus Fairfield County, Conn. Siwanoy? Possibly from ponam mohsketu ussenat, "he applies herbs as part of his work." Hence, "medicine-man" or Pow Wow. Ponus was a sagamore of Stamford, r64I. But another source says Pon-ees, "little falls."

Poo Cutahunk Anow see Cuttyhunk.

Poodatuck Franklin County, Mass. Mahican, "land near the falls."

Poodhumsk New London County, Conn. Mohegan, "projecting rocks," or "reefs sticking out of water." Also Poodhumseck. See Paudowaumset and Pohtaiyomsek.

Poodik now Cape Elizabeth, Cumberland County, Me. Malecite, "sticking out into the water," a prominent rock formation. Also Porpooduck, Purpoodik.

Poohookapaug Pond, Worcester County, Mass. Nipmuck, "cats' pond." But if (Pookhookapaug, "pond where we smoked tobacco.")

Poohpoohsaug location unknown; possibly from Natick, "cats," or "wild cats."

Poohunck New London County, Conn? Mohegan, "wildcat place,"? or "miry place"?

Pooksha Pond, Plymouth County, Mass. Wampanoag, possibly "it smokes," or, "it is misty."

Poontoosuc see Pontoosuc.

Poosumsuck River, Windsor County, Vt. Abnaki, "mushy, quaky, boggy outlet." (It has been suggested that this name is a modified form of a Virginia dialect word aposom, but "the opossum" is rare this far north.)

\section{Pootapaug see Pautipaug.}

Pootatuck River and State Forest, Fairfield County, Conn. Paugussett? "falls in river," or "county around the falls." Also Potatuc, perhaps "miry stream." 
Pootatugock Providence County? R. I. Narragansett? "at the place of the cove in the river," or possibly "at the shallow cove in the river."

Pootowoomet Neck; see Potowomut.

Popanompscut Bristol County, R. I. Wampanoag, "lookout hill, place." But possibly "winter-fish (frost fish) place"? or "place of the double boulder."? See Papanomscutt.

Popasquash Island, Franklin County, Vt. Natick, "partridges"? or Narragansett, "double thing," or "double island," or "double hill."

Popokomuk wodchussu Whetstone Falls, Penobscot County, Me. Abnaki, "near the mountain with many cranberries."

Popoquash Neck, Bristol County, Mass. Pequot, "quail."

Popossego quohock egge Brook, Essex County, Mass. No satisfactory translation available; perhaps Natick, "place where quahog clams abound" [?] Also Popossesgos quock egg.

Poppanompscut same as Papanomscutt, q. v.

Poppaquinnapog see Papaquinapaug.

Poppasquash Island, Bristol County, R. I. Narragansett, "partridges," or "broken rocks"?

Popponesset Bay and Beach, Barnstable County, Mass. Wampanoag, "at the place of the frost fish or tomcod;" or, "place of the obstructed inlet," or, "lookout place."

Poppotonuck Mountain, Hartford County, Conn. Tunxis, "at the double mountain."

Popsquatchet Hills, Nantucket County, Mass. Wampanoag, "rocky hills," or "double hill"?

Popumossett Norfolk County, Mass. Natick, "place where they go to and fro," or "the trail."

\section{Poquaback see Pauquapaug.}

Poquahaug Island, New Haven County, Conn. Quinnipiac, "the round clam," Venus mercenaria, the quahog clam, also called co-hog, or pookaw. These shells were used to make wampum beads.

Poquanatuck Providence County, R. I. Narragansett, "shallow river," or "stream."

Poquannatuck River, New London County, Conn.? Nipmuck? Pequot? "shallow river"? or "the plain near the river"? 
Poquannoc and variants, Paquanaug, Paquanick, Pequonnock, Poqonnock, etc. In several dialects, "cleared lands."

Poquanticut School, Bristol County, Mass. Narragansett, "at the clear or shallow stream."

\section{Poquatocke see Pawtucket.}

Poquaug see Paquoag.

Poquechanneeg New London County, Conn. Mohegan? "creek between two hills," or "valley between the hills"?

Poquetanuck Village, New London, Conn. Mohegan, "land broken up as plowed for crops." (Local persons say "broken, as cracks in mud during dry spells or low water.")

Poquiag Worcester County, Mass. Nipmuck, "cleared land."

Poquian see Uncas.

Poquiant Brook, Washington County, R. I. Eastern Niantic? This seems to be part of Poquiantep, "bald head," but possibly this is just "clear place," or "cleared land." Another possibility is Poquian, "defender."

Poquiogh New London County, Conn. Eastern Niantic, "open land," or "open meadows, cleared for planting."

Poquiunk Brook, Washington County, R. I. Narragansett, "clear or shallow stream." Variants, Poquiant, Poquinunk, and Poquyent.

Poquomock Neck, Nantucket County, Mass. Wampanoag, "open country." See Packamohquah.

Poquonock there are several places bearing this name in Connecticut, deriving from cognate Algonquian roots; all mean, "cleared land (for cultivation)."

Porchcommock Pond, Barnstable County, Mass. Wampanoag, "divided enclosure."

Porpooduck see Poodik.

Posneganset see Punhanganset.

Posseps Cangamock Cathance Lake, Washington County, Me. Abnaki, "pebble bottom lake."

Postatugock see Pootatugock.

Potabaug see Pautapaug.

Potagansett Pond, Middlesex County, Conn. Mohegan, "at the jutting rock," or "at the reef." Also Potaguansett. 
Potanamaqut Harbor and Village, Barnstable County, Mass. Wampanoag, "foaming island place," etc. See Potenumacut.

Potapaug see Pautapaug.

Potateuk New Haven County, Conn. Quinnipiac, "land near the falls."

Potatuck New Haven County, Conn. Quinnipiac, "land near the falls." Also Powtatuck.

Potaywadjo Ridge, Piscataquis County, Me. Abnaki? See Potowadjo, "wind blows over mountain."

Potcomet Nantucket County, Mass. Wampanoag, "at the muddy beach." Also spelled Pottacohannet, "at the long miry beach." (If Pottacomet or Pettacomet, "round (or curved) beach place.") One informant says "round house."

\section{Potebaug see Potepaug.}

Potenumacut Harbor, Barnstable County, Mass. Wampanoag, "foaming island place," or "jutting out dry land place."

Potepaug Worcester County, Mass. Nipmuck, "miry pond," or "damp, marshy land."

Potobek Lilly Bay, Piscataquis County, Me. Abnaki, "bulge in the lake," or "cove in the lake."

Potock Point and Rock, Bristol County, R. I. Potock was a Narragansett counselor of Queen Quaiapen, before 1675 .

Potonapa Pond, Hillsboro County, N. H. Old Abnaki, "cove in the pond."

Potoowoomuck Neck and River, Kent County, R. I. Narragansett, "muddy country," or "where rushes grow" and possibly "trading place"?

Potowadjo Hill, Piscataquis County, Me. Abnaki, "wind blows over the mountain." The Abnaki word for "whale" is podebe, "the blower." Thus the mountain is sometimes called Whale Mountain. See Potaywadjo.

Potowhommet Franklin County, Mass. Pennacook? "damp' field place."

Potowomuck Neck and River, Kent County, R. I. Narragansett, "at the place where miry land sinks." (Some give also "where rushes grow.")

Potowomut Village, Kent County, R. I. Narragansett, "low meadow land." (Another set of roots gives "where there is a 
going-to-bring-again," that is, "a trading-place" or "mart." These two latter may be descriptions of activities at the place rather than translations of the name.)

Potowomut Hartford County, Conn. Wangunk, "boggy damp field." Potowoomuck, Pootowoomuck, Potowomut, etc.

Potowoome see Potowomut, above.

Potquient see Poquiunk.

Pottacohannet Nantucket County, Mass. Wampanoag, "curved long fishing promontory"? See Potcomet.

Pottanumacutt Harbor and Road, Barnstable County, Mass. Wampanoag, "at the whale fishing place." Also given as Portanimicut, or Portnameqout, "at the place of the foaming," probably ocean breakers.

Pottapaug Hill and Pond, Worcester County, Mass. Nipmuck, "marshy, damp bog," or possibly "a bulging cove or pond." Also Pottapogue, Pottapoug, etc.

Potuckco's Ring, New Haven County, Conn. Quinnipiac, "round"? This name may have come from that of Patackhouse, a suncksqua or "queen," the sister of Chief Nassahegon.

Potumska Bristol County, Mass. Narragansett, "round rock," or "jutting reef."

Poucha Pond, Dukes County, Mass. Wampanoag, "it opens out," or perhaps "small hill."

Poughkeeste now Buzzard's Bay, between Barnstable and Dukes Counties to eastward, with Plymouth and Bristol Counties, Mass., to westward. Wampanoag, "bay with coves," or perhaps same as Pocasset [?].

Powachaug New London County, Conn. Mohegan, "mountain near falls," or "steep mountain."

Powaget Pond, Washington County, R. I. Narragansett? Eastern Niantic? "small clear meadow."

Powahag Fairfield County, Conn. Pangussett, "customarily he dips and immerses himself there."

Powakasik Newport County, R. I. Narragansett, "at the widening out place."

Powaw River, Rockingham County, N. H. See Pow wow.

Powisset Norwalk County, Mass. Natick, "at the small, low field"?

Powntuck Windham County, Conn. Nipmuck, "river-falls." 
Powntucket see Pawtucket.

Powntuxet see Pautuxet.

Pow Wow River and Pond, Rockingham County, N. H. Abnaki, Natick, and other New England Algonquian Indians had similiar words, freely translated as "sorcerer" or "medicine man."

Powwow Hill and River, Essex County, Mass. See Pow Wow. (If Natick, perhaps "small plain place.")

Poxon same as Foxon.

Poyasuck Hampden County, Mass. Nipmuck, "small brook".

Pquakis Pond, Oxford County, Me. Old Abnaki, a diminutive form of Pequawket, "little humped-up." ?

Presumpscot River, Cumberland County, Me. Abnaki, "ledges in channel." Variant Presumskeag.

Prosewamenos Fairfield County, Conn. Paugussett, from name of the sachem Prosewamenos, who deeded land in I640.

P'sahn Bar Harbor, Hancock County, Me. Malecite, "clam bake place."

Psazeske Stream, Sagadahoc County, Me. Abnaki, "muddy branch."

Psinkskihigan-i-ontop one of the Nesowadnehunk Mountains, near Katahdin, Piscataquis County, Me. Abnaki, "notched head;" it resembles the notch in the blunt end of an arrowhead.

Psinkwandissek a location on Passadaumkeag Stream. Penobscot County, Me. Abnaki, "place of scalping" or "scalping rock," from roots psik, "split" and antep "head."

Psiscontic Brassua Lake, Moosehead, Piscataquis County, Me. Abnaki, "place of splitting branches (and bark?) (to make canoes)."

Puccatannock River New London County, Conn. Mohegan, "shallow river." Local name for the Thames at Poquetanuck.

\section{Puckanokick see Pokanoket.}

Puckcommeagon River Hampden County, Mass. Nipmuck, "white oak tree."

Puckenokick see Pokanoket.

Puckhunk Hill, Washington County, R. I. Eastern Niantic, "clear stream," "smoke place," or "bear" [?]. 
Puckhunkonnuck New London County, Conn. Mohegan, "bear hill"? or "place of the bear's den."?

Puckhussunaug Pond, New London County, Conn. Mohegan, "place of broken stones," or possibly "place cleared of stones."

Puckquahawks New Haven County, Conn. Quinnipiac, "open plains" or "cleared land." Also Pukquahaks.

Pudjquenssis-ak Knox County, Me. Abnaki, "abode of the powerful sorceress." ? Variant, Pukdjinskwes.

Pug Lake in Washington County, Me., and Pond and Lake in Piscataquis County, Me. Doubtful origin; if this is Indian, it is perhaps Abnaki, meaning a "pond," or "a shallow place."

Puggamugga River, Cumberland County, Me. Abnaki, "shallow, sandy."

Pughquonnack see Poquannoc.

Pujejewock see Penjejawock.

Pukanauket Barnstable County, Mass. Wampanoag, "at the small plantation," or "cleared land."

Pukwannusett Washington County, R. I. Narragansett, "bear's abode."

Pumgansett see Pomecansett.

Pumgustuck Falls, Cumberland County, Me. Abnaki, "falls near the mouth of the river."

Pumham see Pomham.

Pummachog see Pomachaug.

Pumpissett River, Barnstable County, Mass. Wampanoag, "at the crooked place," or perhaps "it runs down to the sea." Also "shallow place."

Pumpumbashunk New London County, Conn. Mohegan, "rocky reefs at the river mouth." Also Pumpinbashoonk.

\section{Pumpsoquattick see Paupasquatch.}

Puncatest Neck Road, Newport County, R. I. Narragansett, "low meadow," or "shallow when overflown." Variants, Puncoteast, Punkatees, Ponquatist, etc.

\section{Punhanganset see Ponaganset.}

Punkapoag Pond, Norfolk County, Mass. Natick, "shallow fresh-water pond." 
Punkatesset Hill, Middlesex County, Mass. Natick, "shallow brook."

Punkups New Haven County, Conn. Quinnipiac, from name of an Indian called Puckahomp, "bare rock."

Punonakanit Barnstable County, Mass. Wampanoag, "out of the way beach," or distant enclosure."

Punset see Cockaponset and Ponset.

Purchaed Plymouth County, Mass. Wampanoag "turning place." Variants Purchade, Pochead, Pocheag, etc.

Purpoodik see Poodik.

Puscommatas Pond, now Burden's Pond. Washington County, R. I. Eastern Niantic, "muddy pond" or "miry stream."

Pushaw Stream, Penobscot County, Me. Included only because of a frequent confusion. It is not Indian, but was named for an early English settler. The Indian name was Pigwaduk.

Putchaug Brook, Cheshire County, N. H. Nipmuck, a "turning place," or "division place."

Puttacawmaumshcuck Tolland County, Conn. Nipmuck, "at the round rock," or "at the fishing place near the round rock."

Puttawuamscut River, Providence County, R. I. Narragansett, "place of the round rock." (Another source says, "where we snare birds in a net;" but this appears to be a declaration of activity at the place rather than a translation of the name.)

Puttuckqupmscut Washington County, R. I. Narragansett? "at or near the round rock." Other spellings, Petaquamscot, Puttaquomcuts, etc.

Pyquiag Fairfield County, Conn. Paugussett, "open land."

Pyqyag Hartford County, Conn. Wangunk, "open land," for cultivation; "open meadows."

\section{$-\mathbf{Q}-$}

Quabacook near Merrymeeting Bay, Sagadahoc County, Me. Abnaki, "sunken land, swamp."

Quabaconk Worcester County, Mass. Nipmuck, "red pond," or "swampy land." Also Quabacutt, Quabakonk, Quabauk, etc.

Quabbin Mountain, Hampshire County, Mass. Also Reservoir and Park, Hampden, Franklin and Worcester Counties, Mass. 
Nipmuck, "it twists and turns about"? "crooked streams"? Named for a chief?

Quabeag Bay, Cumberland County, Me. Abnaki, "swampy cove, or bay."

Quaboag Pond and River, Worcester County, Mass. Nipmuck, "before the pond," or "pond-before"? More likely an abbreviation of $m$ 'squ'boag, "bloody pond," or "red pond."

Quacataug Washington County, R. I. Eastern Niantic, "swampy place," or "where the land trembles."

Quacataug Hill, New London County, Conn. Eastern Niantic, "swampy land." Also Quahquetough and Quaukataugh.

Quack near Cape Elizabeth, Cumberland County, Me. Abnaki, "meadows," or "marshes."

Quaco rocks in the sea near Cape Neddick, York County, Me. Micmac, "hooded seals" (now extinct).

Quacumquasit Pond, Worcester County, Mass. Nipmuck, possibly from the name of Chief Quacuunquasit of Quaboag. Other possible derivations include Pequot words for "black ducks."

Quacumquasset Pond, Hampden County, Mass. Nipmuck, "black ducks marsh," or "end of the marsh." But see below.

Quaddick Reservoir and State Park, Windham County, Conn. If Nipmuck, perhaps "bend or oxbow in river." If Narragansett, "miry place." See Pattaquattic, Pattaquodtuck and Pawtuckquachooge. Also found as Anadic and Anaddic.

Quadochqoik River, location uncertain; perhaps in Oxford County, Me.? Abnaki? "place of the mountain range"? or "at the mountain between the waters"? or "place between whirl pools"?

Quag Pond, Worcester County, Mass. Nipmuck, "where land shakes and trembles;" a shaking marsh.

Quagachusque D'Orville's Head, Washington County, Me. Abnaki? Malecite? properly spelled Kwagustchus'k, "dirty mountain." So called because it appeared black after being burned over.

Quagana Hill, Middlesex County, Mass. Natick, "swamp" near a hill.

Quaganapoxet Salt Marsh, New London County, Conn. Mohegan, "quagmire at the small pond."

Quahmsit Barnstable County, Mass. Wampanoag, "at the rock." 
Quahog Point, Dukes County, Mass. Natick, Narragansett, etc., "round clam," the Venus mercenaria, probably from kuppogki hogki, "thick shell." Also poquahock; called pookaw on Nantucket, it was commonly known as co-hog elsewhere. This is the shell from which most wampum beads were made.

Quahtannet Plymouth County, Mass. Wampanoag, "at the great village." Also Sachtananet.

Quaiapen Bristol County, Mass. Quaiapen was the squaw-sachem or "Queen" of the Narragansett people in the early I670's.

Quaiombog Cove New London County, Conn. Eastern Niantic, "scoop up fish in cove," using scoop nets?

Quaise Nantucket County, Mass. Wampanoag, "at the extreme point." Another source gives "reeds, or flags-place."? Also Quayze.

Quakansick Bennington County, Vt. Mahican, "shaking or trembling land," boggy places along the Hoosic River.

Quaket Neck and Pond, Newport County, R. I. Narragansett, "narrow swampy place," abbreviation of Nonnequaket or Nanniquacut. Another spelling, Quacut.

Quakish Lake, Penobscot County, Me. Abnaki, "boggy, flooded; low places near water." See Guagas.

Quamaskechett-Tookepessett Plymouth County, Mass. Wampanoag, "high grass wilderness," or "high grass in an abandoned place."

Quamatucumpic Washington County, R. I. Narragansett, "rocks in the long stream," or "extended deserted land." Variant, Quanatumpic.

Quambaug New London County, Conn. Pequot, "end of pond," or "long pond."

Quampakasset Pond, Bristol County, Mass. Wampanoag, "little long pond," or "small red pond."

Quampaug River, Bristol County, Mass. Wampanoag, "long pond."

Quamphegan Falls, York County, Me., and Strafford County, N. H. Abnaki, "dip net." Here the fish were so abundant that they could be "dipped out" in a net. See Wussquamhegonset, and Atgatogwisas.

Quampiasan Landing, York County, Me. From Abnaki, a canoe launching place, "enter vessel, immersed in water up to a certain mark." 
Quamquit Cove, Newport County, R. I. Narragansett, "wide place." But see Nonquitt.

Quamscook Kennebec County, Me. Abnaki, "salmon place."

Quana Hampden County, Mass. Nipmuck, "long." Probably this is just part of original longer name.

Quanabog Cove and Neck, New London County, Conn. Eastern Niantic, "long pond."

Quanacontaug Pond, Washington County, R. I. Narragansett, "extended deserted place," or "at the long beach"? or "two long ponds in succession"?

Quanaconwampith Plymouth County, Mass. Wampanoag, "trees near the meadow."

Quanaduck Meadow, New London County, Conn. Eastern Niantic, "long tidal stream," or "long tidal estuary." See Quonatuck.

Quanapaug Pond, Fairfield County, Conn. and Stream in New Haven County, Conn. Paugussett, "long pond." Also Queannepauge and Quenopooke.

Quanascomcook Washington County, Me. Abnaki? Malecite? "rock summit"? or "plenty of long rocks in the bay, or pond." One Indian said, "plenty of long fish place;" these might be eels, pike, or pickerel. Usually the particular fish which appeared in quantity would be named, as in "plenty of sturgeon," e. g., Cobossecontee.

Quanata Hill, Worcester County, Mass. Nipmuck, "boundary place," or "long hill," or "tall tree."[?]

Quanatock Brook, Hampden County, Mass. Nipmuck, "long stream," or "tall trees."

Quanatumpic Grove, Washington County, R. I. Narragansett, "long ford, or wading place."

Quanatusset Windham County, Conn. Nipmuck, "at the long brook." Also Quanutusset, Quatissik, Quatiske, etc.

Quanduck Brook, Windham County, Conn. Wangunk, "long stream."

Quanesusett Norfolk County, Mass. Natick, "long brook."

Quanhiggin River, Plymouth County, Mass. Wampanoag, "long pointed stick," or "long spear," or "long house."[?]

Quanitick Worcester County, Mass. Natick, "tall trees," or "long tree." Or possibly, "long river." 
Quannapowitt Lake, Middlesex County, Mass. Natick, "at the long pond," or, less likely, "long falls."

Quannipi Alton Bay, Belknap County, N. H. Abnaki, "the long lake."

Quannuntowock see Quassaconkanuck.

Quanotock Harbor, New London County, Conn. Eastern Niantic, "long tidal stream."

Quanpaukoessut Plymouth County, Mass. Wampanoag, "at the little long pond," or "place at the end of the swamp."

Quanquanjawatchuck Providence County, R. I.? Narragansett, "at the hill with two long ridges." See Cawcawnjawatchuck.

Quanset Cove and Pond, Barnstable County, Mass. Wampanoag, "long place." But if an abbreviation of Aqounset, this may possibly mean "boundary place," or "at the fort."

Quansigomog Middlesex County, Mass. Natick, "pickerel fishing place," or "long brook basin." See Quinsigamond.

Quansoo Dukes County, Mass. Wampanoag, "long outlet."

Quantabacook Lake, Waldo County, Me. Abnaki, "plenty of game (fur-bearing) animals."?

Quantisset Windham County, Conn. Nipmuck, "long brook."

Quantituck Hill, Plymouth County, Mass. Wampanoag, "tall tree" or "long river."

Quantuckoyog see Yantuckkoyog.

Quanumpacke Swamp, Plymouth County, Mass. Wampanoag, "at the long pond," or "swampy place."

Quapaukuk Berkshire County, Mass. Mahican, "at the place before the pond," or perhaps "at the pond before." Also translated, "as far as the pond."

Quaquadne Hill, Middlesex County, Mass. Pennacook, "high hill."

Quaquananawich Worcester County, Mass. Nipmuck, "high observation (lookout) place."

Quaquoountuck Hampden County, Mass. Nipmuck, "shaking marsh creek."

Quarasksucks New Haven County, Conn. Mohegan, "long green lands," or "long green place near the brook."

Quasapaug Worcester County, Mass. Nipmuck, "pickerel pond"? (But see Oquassa, "slender blue trout.") 
Quascacunquen Falls, Essex County, Mass. If Abnaki, "the long ridge;" if Natick, "the long bank."

Quashnet Road, Barnstable County, Mass. Wampanoag, "at the small cove." See Acushnet.

Quassaconkanuck Pond, Washington County, R. I. Eastern Niantic, "stone fence boundary mark," or "turning place at the stone wall."

Quassapaug Pond, New Haven County, Conn. Quinnipiac, "stones in the pond," or "gravelly pond." But if an abbreviation of Kehtequasset, "the largest."

Quassaponikin Hill, Worcester County, Mass. Nipmuck, "at the largest fording place," or "greatest shallow section."

Quassink Pond, Worcester County, Mass. Nipmuck, "at the stony place."

Quassuck Pond, Worcester County, Mass. Nipmuck, "at the very large outlet."

Quatenus Dutch Island, Newport County, R. I. Another frequently mistaken term; it is not Indian, but derives from early Latin, meaning "as far as." Also Quetanis or Quotenis.

Quatoncanit Providence County, R. I. Narragansett, "large plantation," or "large enclosure."

Quatuck River, Washington County, R. I. Eastern Niantic, "the large (tidal ?) stream."

Quawawehunk Washington County, R. I. Eastern Niantic, "where the land shakes and trembles." This was the location of the Great Swamp Fight, December, I675.

Quaweejoos D'Orville Head, Washington County, Me. Malecite, "dirty mountain," dirty appearing because it had been burned over.

Quawquinnippau Pond, Washington County, R. I. Eastern Niantic, "stream with a wide turn."

Quayachick Essex County, Mass. Pennacook, "high hill."

Quayz Nantucket County, Mass. Wampanoag "place of growing reeds"? or "the extreme end." ? See Quaise.

Queachick Essex County, Mass. Pennacook, "swift current," See Cochichewick.

Quebaog see Quaboag. 
Quebek Hartford County, Conn. Tunxis, "stopped up place," or "enclosed place."

Queghommateh Windham County, Conn. Nipmuck, "shaking or trembling mountain."

Quenaumett Barnstable County, Mass. Wampanoag, "look out place." See Cataumet.

Quenibeck Worcester County, Mass. Natick, "long pond."

Quentabacook see Quantabacook.

Quequachanoke Washington County, R. I. Eastern Niantic, "place of a strong rapid current."

Quequataug see Quacataug.

Quequecham Bristol County, R. I. Narragansett, "very swift current." Also Quequechan.

Quequeteant Bristol County, Mass. Narragansett, "swift water" or "torrent."

Quesquitcumegek Ridge Knox County, Me. Abnaki, "long carry over high land," or "long ridge." Also Quesquitcumgee.

Quetequash Hills, Island and River, Plymouth County, Mass. Wampanoag, "red rocks." See Quittacus.

Quibiquesson River, Lincoln County, Me. Abnaki, "little long river," or "long brook."

Quidnesset Road, Washington County, R. I. Narragansett, "at the small island." Variants, Quidnissit and Aquidnesit.

Quidnet Nantucket County, Mass. Wampanoag, "at the island."

Quidnic River and Pond, Windham County, Conn., and Pond, Providence County, R. I. Nipmuck, from Acqueedenuck, "place at the end of the hill."

Quidnick City and Reservoir, Kent County, R. I. Narragansett, "at the end of a hill."

Quillicksq Hampden County, Mass. Nipmuck, "mixed water and earth," i. e., mud, or mire.

Quillipiac New Haven County, Conn. Quinnipiac, "where we change our route." See Quinnipiac.

Quinabaag River, Worcester County, Mass. Nipmuck, "long pond." See Quinnebaug.

Quinacquck Hampden County, Mass. Nipmuck, "high land." 
Quinamoge Meadow, Washington County, R. I. Eastern Niantic, "long fish," i. e., "eels" (probably lampreys).

Quinapaug Suffolk County, Mass. Natick, "long pond." Also Quinibaug, Quineboag, Qunnubage, etc.

Quinapoxet Worcester County, Mass. Nipmuck, "at the place of the little long pond," or perhaps "the long swamp."

Quinebaug Pond, Windham County, Conn., and River, New London County, Conn. Nipmuck, "long pond."

Quinetusset Windham County, Conn. Nipmuck, "long brook."

Quinibaak Cheshire County, N. H. Abnaki, "at the long pond."

Quinibeck Camp, Orange County, Vt. Abnaki, "a long pond."

Quinicuntauge see Quonocontaug.

Quinnamuck Dukes County, Mass. Wampanoag, "at the long fishing place."

Quinnatisset Brook and Pond, Windham County, Conn. Nipmuck, "long brook," or "little long river."

Quinneaska Island, Chittenden County, Vt. Abnaki, "long joint," or "long elbow."

Quinnebaug River, Hampden County, Mass. Nipmuck, "long pond."

Quinnebequi ancient name for the Kennebec River. Abnaki, "long still water."

Quinnehtukqut The Connecticut River. In several Algonquian dialects, "country at the long river." Also Quinetucquet, Quinnihticut, etc.

Quinnepoxet Worcester County, Mass. Nipmuck, "place of the little long pond."

Quinnibeque the Charles River, Suffolk County, Mass. Abnaki, transplanted from Maine, "long still water;" the Kennebec. Also Quinobeque, Quinobequin, Quinnebequon.

Quinnipaugh Pond, New Haven County, Conn. Quinnipiac, "long pond." Other spellings Quillipoke, Quillipog, etc.

Quinnipiac River and Village, New Haven County; Conn. Quinnipiac, from quinnuppin-uk, "where we change our route" and go inland north of New Haven harbor, along the Great Path Mishimayagat from New York to Boston.

Quinnipiac Territory, much of New Haven County, Conn., and part of Hartford County. Variants Quillipeak, Quillipeage, Quillipiac, Quinopiock, Quinnypiock, and Quinnepyooghq. 
Quinnisk-wouk-ook near Robbinston, Washington County, Me. Malecite, "at the long gravel bar."

Quinshepaug Worcester County, Mass. Nipmuck, "pike pond," or "pickerel pond."

Quinsibis Island, Aroostook County, Me. Abnaki, "long little stream," or "long brook."

Quinsigamond Lake and River, Worcester County, Mass. Nipmuck, "pickerel fishing place." Another translation, "enclosed place at the long brook."

\section{Quinsnaket see Quinsnikit.}

Quinsnikit Hill, Providence County, R. I. Narragansett, "at my stone house."

Quintikoock according to Roger Williams, the Narragansett applied this name to Indians dwelling in the Connecticut valley.

\section{Quinunicut see Conanicut.}

Quinuqui Komuk Plymouth County, Mass. Natick, "high enclosure or tower."

Quisquamego Knox County, Me. Abnaki, "long ridge," or "long peninsula." Also Kwesahkamegus.

Quisset Hill, Worcester County, Mass. Nipmuck, "at the place of the small pines." Also spelled Quissit; see Coeset.

Quitemaug Hill, Worcester County, Mass. Nipmuck, "the great fishing place." Named for John Quittemug, a Nipmuck counselor in I630.

Quito Hill, Cumberland County, Me. If Indian, perhaps Abnaki, "long flow," but more likely Spanish, from the capital of Ecuador.

Quittacus Ponds, Plymouth County, Mass. Wampanoag, "red rocks?" or "long brook." ? Other spellings Quitiquos, Quittiquash, Quiticus, etc.

Quittapeage Rock, New London County, Conn. Mohegan, "sunk in bay," or "reef in harbor." ?

Quittaub Plymouth County, Mass. Wampanoag, "he sinks," as into water, or mire.

Quittiquash Ponds, Plymouth County, Mass. See Quittacus.

Quittuwashett Pond, Plymouth County, Mass. Wampanoag, "at the great hill." 
Quitquasset Plymouth County, Mass. Wampanoag, "at the islands."

Quksett Neck, Kent County, R. I. Narragansett, "miry place," "muddy place," or "rocky place"[?]

Qumatumpick Washington County, R. I. Narragansett, "at the long ford," or "long wading place." ? Also given as "sinking swamp."

\section{Qummunagat see Canonicut.}

Qunnoskwamkook near Robbinston, Washington County, Me. Malecite? Micmac? "the long gravel bar."

Qunnubage Worcester County, Mass. Nipmuck, "long pond."

Qununkwattchu Hampden County, Mass. Nipmuck, "the high mountain." Variant, Qununque wachu.

Quoaug Rock, Point Judith, Washington County, R. I. Eastern Niantic, "round clam" (Venus mercenaria).

Quodduck sunckset Brook, New London County, Conn. Mohegan, "turning place near the outlet."

Quoddy see Passamaquoddy.

Quohoag Bay, Cumberland County, Me. Abnaki? Natick? "hard clam," or "chowder clam" (Venus mercenaria).

Quonackquk Hampden County, Mass. Nipmuck, "high place."

Quonacontaug a salt-pond partly in New London County, Conn. and partly in Washington County, R. I. See Quonocontaug.

Quonahassit Norfolk County, Mass. Natick, "long rock place," or "a fishing promontory."

Quonatuck Meadow New London County, Conn. See Quanaduck.

Quonektacut Hampden County, Mass. Nipmuck, "at the long river," the Connecticut.

Quonepaug Hill, New Haven County, Conn. Quinnipiac, "long pond."

Quonnipaug Mountain, Middlesex County, Conn. Quinnipiac, "long pond."

Quonochontaug Ponds, Washington County, R. I. Eastern Niantic, "the long, long pond."

Quonocontaug Pond, Washington County, R. I. Eastern Niantic? "at the extended pond," or "at the long beach."

Quononicut see Conanicut. 
Quonopataug see Quanacontaug. Variants, Quanaquataug, Quonaquatog, Quonaquontaug, etc.

Quonopaug Brook, Kent County, R. I. Narragansett, "long pond."

Quonset Point and Village, Washington County, R. I. Narragansett, "long place," (or abbreviation of petequonset, "a round shallow cove.")

Quonshapage Worcester County, Mass. Nipmuck, "long fish pond," that is, "pickerel pond."

Quontabacook Lake, Waldo County, Me. Abnaki, "plenty of (muskrat or beaver) at this pond."

Quoquinnakeesapassananagnog Hillsboro County, N. H. Pennacook, "at the place of the long falls in many hills and meadows." But see Quoquinnapasskeesanahnog, below.

Quoquinnapasskeesanahnog Hillsboro County, N. H. Pennacook, may mean "where the panther hunts for small birds," or "where the broad-tailed hawk hunts for small birds."

Quoquiquasoug Brook, New London County, Conn. Mohegan, "the long muddy outlet."

Quosopanagon Meadow, Middlesex County, Mass. Natick, "plain near the river."

Quotonset Beach, Middlesex County, Conn. Hammonassett, "at the place of dunes," or "gravelly place."

Quowatchaug Washington County, R. I. Narragansett, "high hill." Also Quowchauk.

Qussuknashunk Rock, Plymouth County, Mass. Wampanoag, "at the place of the upright, elevated rock," in the water; or "stream near the rocky hill."

Quttonckanitnuing Providence County, R. I. Narragansett, "wide planted place," or "wide garden." ?

\section{$-\mathbf{R}-$}

Raggertask Island, Ragged Island, Knox County, Me. Abnaki? Micmac? possibly from naghetobsk, "island rocks." ?

Rahonaness Plain, Fairfield County, Conn. Siwanoy? Mahican? Possibly an Indian's name? Any translation would be guesswork. "Sandy."? Mohawk: roaneh, "lady." 
Ramapoge Hampden County, Mass. Natick? "fish pond," from namaaspoag.

Ramapoo Fairfield County, Conn. Delaware or Mahican, "they are en route," or "temporary dwellers." (Other sources give "stream formed by round ponds," and "river which empties into round ponds.")

Ramassoc ancient Penobscot village (I628), Penobscot County, Me. Old Abnaki, "fish-place," or "alewife place."

Ramaug Lake, Litchfield County, Conn., abbreviation of the $M a$ hican word wauremang, "good fishing," or quonkemang, "fishing place at the river-bend."?

Rameson Island, Penobscot County, Me. Abnaki, "below the alewife place;" in this case, below Alamoosuk Lake.

Rampopeag see Lampopeag.

Raskohegan Island, Sagadahoc County, Me. Abnaki, "watching place;" same as Erascohegan, Skowhegan, etc. Other spellings Rasthegon, Lastiggin, and Reskêgon.

Regiochne Rock Rogeo, in Lake Champlain, near Burlington, Chittenden County, Vt. Mohawk, "place of the Cowardly Spirit."

Retacumuckut Washington County, R. I. Narragansett? "on the mainland opposite," west of Canonicut Island. See also Neutaconkonut.

Retaw-erif a joke; spell this backwards.

Rippogenus Pond, Piscataquis County, Me. Abnaki? "small rocks, gravel."?

Rippowams Fairfield County, Conn. Siwanoy, "standing rocks," or "rocky cliff." Also Rippowance. See Nippowance.

Roatan Fairfield County, Conn. Siwanoy? "creek almost dry at low tide." Variant, Noroton.

Rockabema Lake, Aroostook County, Me. Abnaki, "a woodpecker."? See Rockomeko.

Rockomeko Mountains, Oxford County, Me. Abnaki? "hoed-up corn land." ? See Romomeko.

Rodsio Canyatare Lake Champlain. Mohawk, "Cowardly Spirit's Lake."

Rogeo Point, Chittenden County, Vt., and Rock, in Grand Isle County, Vt. Mohawk, "at the place of the cowardly spirit." 
Romomeko Androscoggin County, Me. Abnaki, "good corn country."

Roswic same as Arrowsic, q. v.

Rottsiichni Rock Rogeo in Lake Champlain; Mohawk, "The Cowardly Spirit," or "The Weakling."

Rowayton Fairfield County, Conn. Siwanoy? "creek almost dry at low tide."

Rumfeekungus, properly Rumsickhungas Penobscot County, Me. Abnaki, "alewive fishery below outlet." (There is no $\mathrm{f}$-sound in Abnaki; this f-letter was really a long s, mis-copied.)

Runckinheage Fairfield County, Conn. Siwanoy?" at the boundary place."

Runksoos see Lunksoos.

Rutawoo River, New Haven County, Conn. Quinnipiac, "flowing out of a pond."

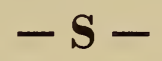

Sabada Pond,Cumberland County, Me.Abnaki, "provision cache"? or "thoroughfare."?

Sabagwagum Hadley's Lake, Washington County, Me. Abnaki, "lake near salt water."

Sabagwilha Hathaway Point, Franklin County, Vt. Abnaki, "a sea duck."

Sabao Mountain, Hancock County, and the West Branch of Machias River, Washington County, Me. Malecite, "passage," or "almost through." This river word, transferred to the mountain, may suggest how the travelers felt "almost through" their journey when this landmark was sighted.

Sabbatia Lake, Mass. Exact location unknown. Probably from Sabbatus, q. v.

Sabbatus Pond, River and Village in Androscoggin County, Me., and Heights in Merrimack County, N. H. An Abnaki rendition of the French St. Jean Baptiste, an Abnaki who aided the Continentals in Arnold's I775 invasion of Québec. Another Sabbatus was killed at Canterbury, N. H., in I753.

Sabino an ancient village site near the mouth of the Sagadahoc River in Maine.(?) Said to be the name of an Abnaki chief.

Sabonac Pond. See Saponac. 
Sabotowan Big Spencer Mountain, Piscataquis County, Me. Abnaki, "end of pack or bundle, where strap is pulled together." This was Glooscap's pack, which he threw down along with his kettle Kokadjo, so that he could pursue a moose calf.

Sacadiock same as Sagadahoc, q. v.

Sacarabig Cumberland County, Me. Abnaki? "outlet of falls or rapids near a pond?" See Saccarappa.

\section{Sacarappa see Saccarappa.}

Sacasawaki River, Waldo County, Me. Abnaki, "place near a small outlet," or "land near a brook"?

Sacatyhock see Sagadahoc.

Saccanossett Hill, Providence County, R. I. Narragansett, "along the little seashore trail," or "black earth place."

Saccarappa Falls, Cumberland County, Me. Abnaki, (free translation) "where we stop to rest and tie up loose ends or knots on our packs." Also given as "pond outlet, then rapids, then falls." Also Sacarappa.

Sachacha Pond, Nantucket County, Mass. Wampanoag, "place of the boulder-stream hill." See also Sasagacha.

Sachem Peak, Grafton County, N. H., Head in New Haven County, Conn., and Pond, in Newport County, R. I. This is a common New England Indian title for "chief." It is literally, "a chief," or "he has the mastery." See Sagamore.

Sachimma Comock Kent County, R. I. Narragansett, "the prince's house," or "ruler's plantation."

Sachimo Comaco Norfolk County, Mass. Natick, "sachem's house," or "ruler's plantation."

Sachtalen Sock's Island, Penobscot County, Me. An Abnaki rendition of the French name, Jacques' Island.

Sachuck Hill, Providence County, R. I. Nipmuck, "at the mountain."

Sachuest Bay, Beach, Point and River, Newport County, R. I. Narragansett, "at or near the great hill." Also Sachueeset, Sachues, Sachuset.

Sackatehock see Sagadahoc.

Sackatucket Barnstable County, Mass. Wampanoag, "at the mouth of the tidal river." 
Saco River, Carroll County, N. H. Abnaki, "flowing out," or "outlet." Also a city and river in York County, Me.

Saconaset see Sockanosset.

Saconesset Hills, Barnstable County, Mass. Wampanoag, "dark earth." See Sockanosset.

Sacunyte Fairfield County, Conn. Mahican, "path along the sea shore." Another source gives Sacunyte Napocke, "at the outlet of a pond." (Also Delaware, shajahikananey.)

Sadawga Lake, Windham County, Vt. If Mohawk, possibly "swiftly flowing water," "side hill," or "a house burst open." This lake, in Pocumtuck country, was named for an old Indian who remained there. A I640 map shows a Mohawk outpost near here.

Sag Pond Aroostook County, Me. Abnaki, "outlet."

Sagadahoc County and River, Me. Abnaki, "the outflowing of a swift stream as it nears the sea," particulary the lower Kennebec River.

Sagamore Beach and Village, Barnstable County, Mass., and Hill in Plymouth County, Mass. Wampanoag, "chief;" literally, "he has mastery of."

Sagamore Creek, Rockingham County, N. H. Abnaki, "chieftain." (Abnaki spelling sôgmô.)

\section{Sagamore Sam see Shawsheen.}

Sagaquash Island, Plymouth County, Mass. Wampanoag, "hard rocks." Given also as Sagaquish, Saquish, etc.

Sagassett Sagadahoc County, Me. Abnaki, "near the outlet," a local name for the mouth of the Kennebec River.

Sagatobscot Hill, Worcester County, Mass. Nipmuck, "at the place of hard rock."

Saghibpatook Falls, Piscataquis County, Me. Abnaki, "falls in a rough, difficult river."

Sagiask Windham County, Conn. Nipmuck, "hard rocks." Also Sagioshk.

Sagistonac Falls, Berkshire County, Mass. Mahican, "at the place of hard rocks."

Sagon-dagon Lake, Newport Pond, Penobscot County, Me. Abnaki, "level place." (Some Indians say, "old portage.")

Sagoquas Plymouth County, Mass. Natick, "hard rocks." 
Sagosset Island, Sagadahoc County, Me. Abnaki, "near the outlet."

Sagumskuffe Coös County, N. H. Probably Sagoniskusse, Abnaki or Pennacook, "place of the small hard rocks," or "difficult passage because of rocks."

Sagumumpsketuck the Hop River, Tolland County, Conn. Nipmuck, "river that runs through hard rock."

Sagus same as Saugus.

Sahbahesset Fox Island Thoroughfare (or Narrows), Washington County, Me. Abnaki, "thoroughfare."

Sahbimskitegwek Thoroughfare Brook, Piscataquis County, Me. Abnaki, "rocks at outlet of stream;" but many Indians say, "stream connecting two lakes." Another source gives, "a stream that empties (flows) between two large bodies of water."

Sahkabehaluk Moose River, Piscataquis County, Me. Abnaki, (a free translation), "flat place, most volume tributary," or "outlet of water body discharging most volume."

Sahkahegan Telos Lake, Aroostook County, Me. Abnaki, "artificial outlet connecting" with another water system.

Sahnchecontucket Dukes County, Mass. Wampanoag, "at the outlet of the principal long tidal stream," or "place of the principal cold water stream." Also Sanchecantacket.

Sahnghib pahntook Falls, Piscataquis County, Me. Abnaki, "rough falls in the river," at outlet.

Sakadamkiak outlet of Saco River, York County, Me. Malecite, "sandbar at the outlet."

Sakessett Pond, Bristol County, Mass. Narragansett, "at the small outlet."

Sakonnet City, Height, Point and River, Bristol County, R. I., and Bristol County, Mass. Narragansett, "abode of the black goose," or "rocky outlet," or "at an outlet." Also Saconnet, Sakonet, Seaconnett, etc.

Salko Hill, Washington County, Me. Abnaki? Micmac? possibly from words meaning "the outlet," or "humped up like a turtle's back."'(?)

Salquin Island, off mouth of Kennebec River, Sagadahoc County, Me. Abnaki? Micmac? possibly "high, humped up like a 
turtle or horseshoe crab, far off sea."? Found also as Sutquin, Sequin, Satquin, Sodkin, Zedquin on maps dated I607 to I647.

Sammauchamoi Pond, Plymouth County, Mass. Wampanoag, "place where we feed, or get provisions."

Samoset Barnstable County, Mass. Wampanoag? Samoset, the famous Sachem of Monhegan, greeted the Pilgrims in 1620. Also called Osamoset, "he walks over much"?

Samp Mortar Reservoir, Fairfield County, Conn. Natick, "corn bruised in a mortar and boiled." Samp was a favorite New England meal, with sugar and milk, until at least I925.

Sampopeag see Lampopeag.

Sanchekantacket Dukes County, Mass. Wampanoag, "place of the long cold river." Also, Sanchecantacket.

Sancoik Bennington County, Vt. Probably French, "St. Croix," but if Indian, possibly Mahican, "I emerge from hiding."

Sankaty Head, Nantucket County, Mass. Wampanoag, "cold stream,"? or "cold hill."?

Sankrohonk Hampden County, Mass. Nipmuck, "land at the outlet." Also, Sankrohoncum.

Sanqutagnappipanquash Plymouth County, Mass. Wampanoag, "fording place at the outlet of the cold water pond;" also given as "it pours out from under contorted rocks."

Santuit Pond, Barnstable County, Mass. Wampanoag, "cool water place."

Sapokonist Brook, Barnstable County, Mass. Wampanoag, "miry field."

Saponac at Chibanook Lake, Penobscot County, Me. Abnaki, "the great outlet," or "big opening."

Sapowet Marsh, Newport County, R. I. Narragansett, "by the river," or "wet, miry place."

Saquaische Penobscot County, Me. Abnaki, "going out" or "outlet." See Sunkaze.

Saquassis diggin Island; see Sebascodegan.

Saquatucket River, Barnstable County, Mass. Wampanoag, "at the mouth of the tidal stream."

Saquid mouth of St. George River, Knox County, Me. Abnaki, "the outlet." 
Saquish Neck, Plymouth County, Mass. Wampanoag, possibly "hard rocks," or "plenty of clams;" the soft shell clam Mya arenaria, used in steaming (hence sohqussog, "they spit or squirt.")

Saracasks New Haven County, Conn. Quinnipiac, "green banks," or "grassy banks."

Saratogue Rivière de Saratogue, now the Batten Kill, Bennington County, Vt. Mohawk, "the side hills;" or perhaps "where heel prints may be seen (in soft ground)."

Sasagacha Pond, Nantucket County, Mass. Wampanoag, "sour, black berries" (alder berries, blackberries, over-ripe cranberries or unripe grapes? One source suggests "black slippery fish, eels.")

Sasagook apaug Worcester County, Mass. Nipmuck, "alder pond"? Some give "black snake pond." (Possibly eels, sassamanquock, "shiny black and slippery ?") Another source translates this as "rattlesnake pond," but a "rattlesnake" would be sesek, or sesegk in Massachusetts; further north, sisikw. See Sisikwa menahan.

Sasaketasick Worcester County, Mass. Nipmuck, "dark flowing, muddy stream."

Sasanet Island, Dukes County, Mass. Wampanoag, "at the place of black slippery rocks."

Sasanoa the lower Kennebec River, now the Black River. Named for Chief Sasanou, who met Samuel de Champlain. See Sasanow, below.

Sasanow now Mt. Agamenticus, York County, Me. Named by John Smith "Sasanow's Mount," after Chief Sasanou, an Abnaki sachem, I607.

Sasaquash Plymouth County, Mass. Wampanoag, "rattlesnakes"?

Sasco Swamp, Fairfield County, Conn. Wappinger? Delaware? "mud." Also Sasquenaugh.

Sasonkususett Pond, Bristol County, Mass. Wampanoag, "place of small sassafrass bushes."

Sasquenaugh Sasco Swamp, Fairfield County, Conn. Probably Wappinger, "muddy river," same roots as Susquehanna, q. v. But see Sassaquin.

Sassamon's Cove, Bristol County, Mass. Wampanoag, named for John Sassamon, Metacom's secretary. His murder at this 
place in 1675 triggered King Philip's War. Possible meaning, "cranberry."

Sassaquin Pond, Bristol County, Mass. Narragansett, perhaps "cranberries," or "he is slow." Possibly the name of a chief, "Black Feather."

Sassawich Beach, Newport County, R. I. Narragansett, "eel trap."

Sasson Kussett Pond, Plymouth County, Mass. Wampanoag, "muddy, warm."?

Sassucksuck Brook, New Haven County, Conn.Quinnipiac, "black earth brook," or "muddy brook." See Saukiog, Sioascauk and Sicaog.

Sataylan Penobscot County, Me. English, "shad island," as pronounced by Abnaki.

Satucket Barnstable County, Mass. Wampanoag, "at the mouth of the tidal creek."

Satucket Plymouth County, Wampanoag, "at the mouth of the tidal stream."

Satuit Plymouth County, Mass. Wampanoag, "cold brook." (Salt, cold stream?)

Saucauoca Nantucket County, Mass. Wampanoag, "dark land." (Possibly magnetic iron on the beach? or black mud?).

Sauga Point, Washington County, R. I. Narragansett, "at the outlet."

Saugatuck Reservoir, River and Village, Fairfield County, Conn. Pangussett, "outlet of the tidal river."

Saugatucket Pond and River, Washington County, R. I. Eastern Niantic, "at the outlet of the tidal stream."

Saughtuckquett Plymouth County, Mass. Wampanoag, "at the mouth of the tidal creek."

Saugus River and Village, Essex County, Mass. Natick, "the outlet," or "small outlet."

Saugus Island, Penobscot River, Penobscot County, Me. Natick, "small outlet?" or Abnaki, "rough, difficult."?

Saugutagnappiepanquash see Sanqutagnappipanquash.

Saukiog Hartford County, Conn. Wangunk, "the ground is dark," sometimes found as Sicaog or Suckiaug. 
Sauks Island, Washington County, R. I., If Indian, perhaps Eastern Niantic, "an outlet," or an abbreviation for "black mud." Possibly, however, it derives from osakiwog, "yellow earth people," i.e., the Sauk tribe.

Saukwonk River Hampshire County, Mass. Natick, "outlet place," or "bend at the outlet."

Sauquish see Saquish.

Sauquonckackok New London County, Conn. Mohegan, "at the ledge of hard rocks." Also translated as "land in a high place." See Sagumumpsketuck.

Sauseunk Avenue, Middlesex County, Conn. Wangunk, from the name of one of Sachem Sowheag's sons, Sause-Unk, "Uncas of the South." He sold lands in 1672.

Sawaams see Sowams.

Sawacook Sagadahoc County, Me. Probably Abnaki, "sloping land," but possibly a Narragansett import, "shell beads," sawhosachick. See Sawgogue.

Sawacotuck an old name for the Saco river at its mouth, hence Abnaki, "mouth of the tidal stream." See Saugatuck, etc.

Sawad Apskek the Sawadabscook River, Penobscot County, Me. Abnaki, "at the place of sloping ledges." See Saw watap skechuwas.

Sawaquatock see Sawkatucket.

Sawcatucket River; see Saugatucket and Massaugatucket.

Sawcomst Plymouth County, Mass. Wampanoag, "flowing out over rocks." Also Saukompsk.

Sawcook Foothills, Hillsboro County, N. H. Abnaki, "sloping land."

Sawgogue Washington County, R. I. Narragansett? Eastern Niantic? "loose shell beads, unstrung wampum" (or, "at the outlet"?) Also Squakheag, Sawgoge, Sawgoog, etc.

Sawhegan Falls, Coös County, N. H. Pennacook? "south country," or "waiting place."?

Sawish Lake, Aroostook County, Me. Abnaki, "sluggish."

Sawkatucket Barnstable County, Mass. Narragansett, "at the outlet of the tidal creek." Given also as Sawahquatock.

Sawkhead see Sawquid.

Sawquid Lincoln County, Me. Abnaki, "outlet place." 
Saw watep skechuwas Sawmill Brook, Hampden County, Mass. Nipmuck, "pouring forth alone it comes out," also translated as "continuous outflowing current runs over sloping ledges." See Sawad Apskek.

Saxafrax (Sassafrass) Point and Cove, R. I. Narragansett, "eels"? or "place of the upright rocks."?

Sayquish see Saquish.

Scaggrock River, Aroostook County, Me. Old Abnaki, "green place," or "grassy place."

Scammon Pond, Hancock County, Me. If Indian, perhaps Abnaki, "maize," or "Indian corn."

Scamscammuck Spring, Bristol County, R. I. Narragansett, "rock enclosure," referring to a walled-up spring. Another spelling, Scamscamnet.

Scantic Hampden County, Mass. Nipmuck, "where the river branches."

Scantic River and Village, Hartford County, Conn. Nipmuck, "branch of the river." Originally Peskantuk. Other spellings Scantuck, Skeantocke, Scuntock.

Scanticook Hartford County, Conn. A village and fort anciently on the north bank of Scantic River; Nipmuck, "at the river fork."

Scantuk River, Hampden County, Mass. Nipmuck, "river branch."

Scargo Lake, Barnstable County, Mass. Wampanoag, "it flows out." Possibly part of an ancient word for "bass."?

Scargo Mountain, York County, Me. Old Abnaki, "skunk"? or "outlet"?

Scatacook Kent County, R. I. Nipmuck, "fork in river." Also Scatacoke, Scatacosh, etc.

Scataway Hill, Cumberland County, Me. Old Abnaki, "burnedover place"? or "little mountain"? (If this were a tidewater location, Scatuate would fit).

Scaticook Litchfield County, Conn. Mahican (Stockbridge), "confluence of two rivers," or "where rivers divide." Variants Scatacook; in New York, Schaghticoke, etc.

Scatuate near Dover, Strafford County, N. H. Abnaki, "slack water," or "ebb tide."

Scatuk see Schoodic River. 
Schachticoke an ancient village on Missisquoi Bay, Franklin County, Vt. Mahican, "where the river branches."

Schaghaticauke Litchfield County, Conn. Mahican, "where the rivers fork." Also Pishquachticook.

Schaticook now Hoosac River, Bennington County, Vt. Mahican, "bend in the stream," or "fork in the stream."

Schenob (Schnob) Brook, Berkshire County, Mass. Mahican, "great pond outlet"? Also found as ashim ops "water spring at the rocky ledge." See Moshenupsuck.

Schodac Brook, Merrimack County, N. H. Abnaki, "trout place."

Schodack Litchfield County, Conn. Mahican? "where there was a fire," or "burned-over land," or "fireplace." Possibly also "trout place," or "point of land."

Schohomogomoc Hill, Strafford County, N. H. Abnaki, "lake with fire markings near it."

Schoodic many waterways in Maine bear this name, deriving from Abnaki or Malecite, "trout place," or perhaps, "point of land."

Schooset Plymouth County, Mass. Wampanoag, "fire place."

Scitico Village, Hartford County, Conn. Nipmuck, "land at the river branch." These are also found: Skitico, Squitikko.

Scittery gusset Creek, Cumberland County, Me. Perhaps another spelling of Squidrayset, a chief of territory near Lynn, Mass. Some say Abnaki-English, "flows rapidly, continuous current."

Scituate Plymouth County, Mass., and Providence County, R. I. Wampanoag, "at the cold spring, or cold brook." But possibly slack water, between tides?

\section{Scoakequanocksett see Sockanosset.}

Scodoqua River, Franklin County, Vt. Abnaki, "trout stream."

Sconnoups Brook, Litchfield County, Conn. Mahican, "dark rocks." Also Succonups.

Sconokoskes Pond, Middlesex County, Conn. Wangunk? "small stones at outlet," or "outlet with small stones."?

Sconticut Neck and Point, Bristol County, Mass. Narragansett, "at (the end of) the cold brook," or possibly "at the end of the crooked stream."

Sconunganuc Hampden County, Mass. Nipmuck, "plenty of corn here," or "green stuff place." 
Scoodeag River, Washington County, Me. Malecite? Abnaki? "fire-place," or "burned-over land."

Scoodik the upper St. Croix River, Washington County, Me. Malecite, "the point or end."

Scook Pond, Plymouth County, Mass. Wampanoag, "snake," or possibly a contraction of Quassacook, "stony place."?

Scotomak Caledonia County, Vt. Abnaki, "trout place."

Scouhegan Falls; see Skowhegan.

Scucurra Snake Hill, New Haven County, Conn. Mohegan, "snakes here."

Scusset Beach, Harbor and River, Plymouth County, Mass. Wampanoag, "at the wading place," or possibly "at the outlet." One reference said, "something about sparks of fire here." Another gave "small fires, possibly torches for luring fish."

Scutcuk see Scaticook.

Seanna Avenue, Middlesex County, Conn. Wangunk, from the name of one of Sachem Sowheag's sons.

Seapuit River, Barnstable County, Mass. Wampanoag, "in the current," or possibly "in the undertow."

Seaukum Hartford County, Conn. Wangunk, "muddy place," or perhaps, "hard going." ?

Sebacook see Sebec.

Sebago Lake, Cumberland Conuty, Me. Abnaki, "big lake," or "big still water." See Sobagwa.

Sebahticook Indian Pond on Kennebec River, Kennebec County, Me. Abnaki, "passage, or thoroughfare, or narrows."

Sebaik see Sebayck.

Sebaim Pond, Piscataquis County, Me. Abnaki, "much water." Same place as Sebec Lake.

Sebamook Moosehead Lake, Piscataquis County, Me. Abnaki, "large lake."

Sebasco Estates and Harbor, Cumberland County, Me. Abnaki, "almost through passage," or "portage almost completed."

Sebascodegan Island, Cumberland County, Me. Abnaki, "carry or passage almost completed." Also Sebascodiggin, Saquasisdiggin, etc. 
Sebascohegan River, Penobscot County, Me. Abnaki, "a portage or carrying place."

Sebaskiak Neck, Washington County, Me. Abnaki, "narrow place in or near a stream."

Sebasticook Lake, Penobscot County, Me. Penobscot-Abnaki, free translation, "the shortest route;" literally, "almostthrough-place." Also Sebastoocoög.

Sebayek narrows at Quoddy Village, Washington County, Me. Abnaki? "narrow stream, or narrow current;" but Seebyik (Pleasant Point) is Micmac for "peninsula." Also Sebayik.

Sebec Lake and Village, Piscataquis County, Me. Abnaki, "much water."

Sebesteguk River, Kennebec County, Me. Abnaki, "short passage river."

Sebethe River, Middlesex County, Conn. Mohegan? Wangunk? "a brook." Sometimes given as "small river." A cognate of Seboeis or Sebes; the substitution of $t h$ for $s$ is rare.

Seboeis Lake, Piscataquis County, Me. Abnaki, "small lake," or "small waterway."

Seboois Village, Penobscot County, Me. Abnaki, "little stream."

Seboomook Elm Stream, Penobscot County, Me. Abnaki, "at or near the large stream."

Sebosenec Hillsboro County, N. H. Abnaki, "at the place of the stony stream."

Seboy cook Washington County, Me. Same as Sebayik, q. v.

Secarabigg Falls, Amancongon River, Cumberland County, Me. Abnaki, "pool, then rapids, then falls." See Saccarappa.

Seccasaw Plymouth County, Mass.? Natick, "black earth," or possibly "squirting clams"? or "hard rocks."?

Secesakut Hill, Providence County, R. I. Narragansett, "black rocks place." Variants, Secesakutt, Sekescute, etc.

Seche nayaug Hartford County, Conn. Wangunk, "spring at the corner," or "pool at the point," sohken-nayaug? .

Seconchet Village; see Seconchqut Village, and Seekonk.

Seconchqut Village, Dukes County, Mass. Wampanoag, "at the summer place," or "late-spring place."

Seconnet Bristol County, Mass. Narragansett, "early summer, or late spring, place"? (Where we catch sequanamauquock, the bream?) 
Sedunkehunk Stream, Penobscot County, Me. Abnaki, "rapids at mouth." Sometimes (incorrectly) spelled Segeunkedunk. Perhaps the earlier and more complete name was Matasededunkehunk, "enters the river with rapids."

Seebaticook Indian Pond, Piscataquis County, Me. Abnaki, "lake-stream place."

Seeboomook Lake, River and Village, Piscataquis County, Me. Abnaki, "at the place of the large river or lake."

Seeboomook Village, Somerset County, Me. Abnaki, "large lake, or stream."

Seebyik Pleasant Point, Washington County, Me. Micmac, "a peninsula."

Seeconnesset Barnstable County, Mass. Wampanoag, "small late-spring, or early-summer, place." ? But see Sockanosset.

Seekonk River, Bristol County, R. I. Wampanoag, "the mouth of the stream," or "outlet;" possibly "wild goose." Also found as Seaconke, Seaconk, Seakunk, and Sikunke.

Seekonk Bristol County, Mass. Narragansett, "wild black goose," or "pouring-out place," or "rocky beach."

Seeogamook Stillwater Lake, Washington County, Me. Malecite, "passing without rapids into a stream." Also Shogomock.

Seepoocke Washington County, R. I. Eastern Niantic, "salt pond" or "salt water." Also found as Seepooke, Seepoke, Seippog. "Sour water"? See Sebago and Sobagwa.

Seewamuck Point, Bristol County, R. I. Narragansett, "at the place where we catch bream," (porgies, mishouppaog) literally, "at the place of early summer fish." (See Sowams.) One source translates this as "big plain (or meadow) place;" another, "place of sewan."

Segocket mouth of St. George River, Knox County, Me. Abnaki, "at the outlet." Variants Segoquet, Segocket, Segohquet.

Segotago an ancient Indian village, possibly in Cumberland County, Me. Abnaki, "outlet of the river."

Segreganset Bristol County, Mass. Narragansett, "place of hard rocks," or "where it pours out."

Seguin Island and Passage, Washington County, Me. Abnaki, "humped up" like a turtle, or horseshoe crab (limulus polyphemus). 
Segumkendunk River, Penobscot County, Me. Abnaki, "rapids at the confluence." See Sedunkehunk.

Segunesit Windham County, Conn. Possibly Nipmuck, "where we go in the spring or early summer." But see Sockanosset.

Seguski-menahanikuk an island in the Penobscot River, Penobscot County, Me. Abnaki, "broken island place," or "plowed-up island."

Seipican River, Plymouth County, Mass. Wampanoag, "a long stream." See Sippican.

Seketegansett Barnstable County, Mass. Wampanoag, "darkcolored spring place."

Sekidawe Franklin County, Vt. Abnaki? "beaver dam." However, a Mohawk word for "beaver" is dawinet.

Sekled Obscus Lakes, Penobscot and Washington Counties, Me. Malecite, "shark-shaped rock."

Seminenal River, Salmon Falls River, Strafford County, N. H. Abnaki, "pebbles," or "coarse gravel."

Senaglecouna early northern boundary of Maine, Aroostook County. Malecite? "long rocky banks."

Seneca Mountain, Essex County, Vt. Natick? "place of stones"? Perhaps named after the Seneca Indians, or may even derive from Lucius Annæus Seneca, the Roman philosopher.

Senechataconet Providence County, R. I. Narragansett, "stony angle (or corner) of the plantation,"? or "stepping-stones ford." ?

Seneptuit Pond, Plymouth County, Mass. Wampanoag, "at the gravelly (or rocky) place."

Seneteconnet Plymouth County, Mass. Wampanoag, "at the rock-strewn fields," or "gravelly place."

Senexet Valley and Meadow, Windham County, Conn. Nipmuck, "place of small stones."

Sengekontacket Pond, Dukes County, Mass. Wampanoag, "at the cold, long creek," or "rocky, long creek." Found sometimes as "lookout tree place." Also Sengehontakit.

Sennebec or Sennibec Pond, Knox County, Me. Abnaki, "rocks in the pond."

Sennemahgesso Eagle Rocks, Orange County, Vt. Abnaki, literally, "rocks of the eagle." 
Seogogguanegabo Cumberland County, Me. Probably Abnaki, "big canoe landing place," but possibly "extended dunes."

Sepaconit or Sepaconnet River, Plymouth County, Mass. Wampanoag, "at the long stream." Also, "pouring out at beach."

Sepasonnet Plymouth County, Mass. Wampanoag, "at the gravelly stream," or "stones in the river."

Sepos Tamesuck or Sepaw Tamesuck Brook and Cove, New London County, Conn. Mixed Mohegan (sepos) and English (T(h)ames), "outlet of the River Thames."

Sepsis-edal-apskit Hancock County, Me. Abnaki "where a bird is carved or punched into the rock." (This picture near Sedgwick has been practically obliterated; the weather, and many souvenir-hunting vandals have taken their toll).

Sepunamus Avenue, Middlesex County, Conn. Wangunk, "younger sister." Sepunnemoe is a better spelling; she was a Wangunk squaw of some means who deeded land in I662. Sepunamus might be translated as "little river-fish."

Sequankit New Haven County, Conn. Quinnipiac "place of summer (dwellings),"? or "what is left, what remains." Also Sequanaukit.

Sequassen Pond, Litchfield County, Conn. Tunxis, Sequassen was a sachem of the Tunxis Indians, circa I635.

\section{Sequin see Sowheage.}

Seremobscus Muscongus Island, Lincoln County, Me. Abnaki, "place of extended ledges."

Sesachacha Pond, Nantucket County, Mass. From Wampanoag, "boulder hill here." But see Sasagacha.

Sesuit Creek and Neck, Barnstable County, Mass. Wampanoag, "cold water" or "cold creek;" also translated as "big spring."

Setamachut Hill, Providence County, R. I. Narragansett, "at the great stony hill"? or perhaps "place of strong currents." ? Variants Settemeechut, Sissamachute, Schichemachute.

Setnesset location unknown. Natick? "stretched out, or extended, tract."

Setuat Providence County, R. I. See Satuit.

Setucket Road, Barnstable County, Mass. Wampanoag, "at the mouth of the tidal creek." 
Sewadapskak Stream, Penobscot County, Me. Abnaki, "place of the sloping ledge."

Shabokin Pond, Worcester County, Mass. Natick, "place of the departed" (chepiohkomuk) or "hell." Variants, Shabikin, Shabbukin, Chabikkin.

Shadogee see Chataguay.

Shaganiscathoke Washington County, R. I. Eastern Niantic, "land at the side of the hills," or possibly," land of the green hills," or "land between hills." Variant, Sheganishkachoke.

Shakameeko Litchfield County, Conn. Mahican? "slippery fish" or "straight fish;" that is, "eels." See Shonkamonke.

Shakum Pond, Middlesex County, Mass. Natick, probably a contraction of Washacum; or "enclosed between"?

Shamcook Shore, Washington County, R. I. Eastern Niantic, abbreviation of "salmon fishing place," or "great fishing place."?

Shamuet Barnstable County, Mass. Wampanoag, "at the spring."

Shamunganuck see Chemunganock.

Shanaks Fort, Cheshire County, N. H. If Indian, perhaps Pennacook, "halfway place" or "place between."

Shankhassick near Exeter, Rockingham County, N. H. Abnaki, "at the hidden outlet of a stream."

Shannock River, New London County, Conn. Eastern Niantic? Mohegan? "place between" (streams)? or "stony place"? Also Shannuck and Shawnuck.

Shannock Hill, River and Village, Washington County, R. I. Mohegan, "where two streams meet." People more poetically inclined look to Niantic, "big squirrel," or Natick, "morning star." Variant, Shannuck.

Shantituck Brook, Providence County, R. I. Narragansett, "where two large tidal streams unite," or (more likely) "large trees near the river," Mishantig-tuck.

Shantok New London County, Conn. Mohegan, "midway up river." This was an old Indian fort almost halfway from New London to Norwich; it is now a park.

Shaomet Franklin County, Mass. See Shawmut.

Shatterack Mountain, Windham County, Vermont. Pocumtuck? "big mountain,"? "fireplace,"? or "between mountains."? 
Shatterack Brook and Mountain, Hampden County, Mass. Nipmuck? Abnaki? possibly "where two streams meet," or "foaming place"?

Shaum Bristol County, Mass. Wampanoag, "the neck" (or as in Shawmut, "landing place.") (Also found as Shaume, Shum, etc.; often confused with Shimuet, "at the spring.") Cape Cod was sometimes labeled Shaume or Mishaum, "big neck."

Shaume Neck (Cape Cod Peninsula), Barnstable County, Mass. Nauset, "the neck." See Shaum, above.

Shawacotoc an ancient name for Saco, York County, Me. Old Abnaki, "(country around) the mouth of the river." A tribe of that name lived there; variants of the name include Saco, Sawco, Shawakotock, Shawocotuck, etc.

Shawamug New London County, Conn. Mohegan, "fishing place at the fork in the stream (where two streams meet)."

Shawkemo Creek and Hills Nantucket County, Mass. Wampanoag, "place of springs," or "spring field," but possibly "gnats." See Okemo and Shekomeko.

Shawme State Forest, Barnstable County, Mass. Nauset, same as Shawmut, "the neck."

Shawmut Suffolk County, Mass., and Village, Somerset County, Me. Natick, (I) "he goes there by water;" (2) "ferry;" (3) "at the neck" (where we draw up our canoes). This is an ancient name for Charlestown, Boston, and Dorchester.

Shawnuck see Shannock.

Shawomet Kent County, R. I. Narragansett, "at the peninsula, the land between waters," or "at the neck." There is more than a hint of "canoe landing place" in this word. See also Shaomet, Shawmut, etc.

Shawomet Bristol County, and Plymouth County, Mass. Wampanoag, "at the neck," or possibly "at the canoelanding-place." This was anciently a Wampanoag village.

Shawsheen River and Village, Middlesex County, Mass. Probably Nipmuck, from the name of Sagamore Sam, alias Shoshanim.

Shawunkhassick River, Rockingham County, N. H. Abnaki, "at the hidden outlet of a stream."

Shawwunk New London County, Conn. Mohegan, "where the streams join," literally, "place between." 
Shebeag see Chebeague.

Shecoway River, Cumberland County, Me. Abnaki, "sheldrake," or "American merganser." This bird is also called Shecorway, Skeecoway and Ussikawai, "rough shaggy crest."

Sheehauge Fairfield County, Conn. Paugussett, "land between," or perhaps, "eels here." Also, with another inflection, possibly "loose unstrung beads, or shell money." See Sawgogue.

Sheepscot Pond, River and Village, Waldo County, Me. Abnaki, "many rocky channels." Also, Shepscooke, Shippscutt, etc.

Sheganishkachoke see Shaganiscathoke.

Shekomeko Brook, Lichtfield County, Conn., and Dutchess County, N. Y. Mahican, "principal house," or "headquarters" (of Moravian missions). See Chicomico.

Shemunkanuck see Chemun ganock. Also Shemunkanug.

Shenecossett New London County, Conn. Pequot, "level land."

Shenewemedy Essex County, Mass. Natick, "spring meadow place."

Shenipsit Lake, Tolland County, Conn. Mohegan, "at the great pool."

Shenskonet Brook and Hill, Providence County, R. I. Narragansett, "strong field"? or "wholly enclosed place"? or "level land"?

Shepaug Reservoir and River, Litchfield County, Conn. Tunxis, "great pond." Variants, Shippoack, Shippang, Sheppog, etc.

Shequocket Barnstable County, Mass. Wampanoag, "at the fork"? or "big swamp."?

Shetucket River, New London County, Conn. Mohegan, "land between rivers." Some authorities give Mashetucket, "at the great river." Variants Shawtucket, Showwatuckhet, Showtucket.

Shewamet Neck Bristol County, Mass. Narragansett, "at the neck." See Shawmut, Shawomet.

Shewatucket Stream, Washington County, R. I. Narragansett, "at the place between tidal streams." Variants, Shewatuck, Shewtuck, Shewtuk, etc.

Shewatucquese Stream, Washington County, R. I. Narragansett, "place between small streams," or "small place between streams." Also given as Showatucquese. 
Shewunk see Shannock.

Shickasheen Brook, Washington County, R. I. Narragansett, "great spring."

Shimmoah Nantucket County, Mass. Wampanoag, "a spring," (of good water). Also Shimo, Shimmoo, Shimuet, etc.

Shimmunk Hampden County, Mass. Nipmuck, "at the springs." Also spelled Shipmuck.

Shinskatuck Brook and Hill, Providence County, R. I. Narragansett, "spring-fed river."

Shipmuck Hampden County, Mass. Nipmuck, "springs," "big watery place," or "big bog." ?

Shippan Fairfield County, Conn. Paugussett, "the shore, where the sea begins."

Shippaquonset Washington County, R. I. Eastern Niantic, "place apart from big point, or from long point"?

Shockolog Pond, Worcester County, Mass. See Chockalog.

Shogonaug Providence County, R. I. Narragansett, "land on the side of the hill."

Shohomagock's Hill, Piscataquis County, Me. Abnaki, from an Indian so named. But see Schohomogomoc.

Shoneeto now Beaver Pond, Rockingham County, N. H. Pennacook? Natick? "big outlet"? or "rocky place."?

Shonkamonke Pond, Berkshire County, Mass. From Mahican? or Delaware? "abode of eels." Also given as "cultivated land." See Shoonkeek Moonkeek, below.

Shoonkeek Moonkeek Lake, Berkshire County, Mass. Mahican, "abode of straight fish; eels."

Showaluckqut Worcester County, Mass. Nipmuck, "where the river forks."

Showatucket River, New London County, Conn. Mohegan, "land between the rivers."

Shuckquam Plymouth County, Mass. Wampanoag, "grassy meadows."

Shumack Stream, Washington County, R. I. Possibly Narragansett, sumhup, "a beaver," but more likely just the name of the "sumac bush," which is an Arabic word.

Shuman Kanuc Hill, Washington County, R. I. Narragansett, "high enclosed place," or "place of refuge high up." 
Shumuit Worcester County, Mass. Nipmuck, "at the spring" of good water.

Shunock River, New London County, Conn. Mohegan? "stone place," or possibly a corruption of Shawnuck, "where streams join." See also Shawwunk.

Siasconset Nantucket County, Mass. Narragansett, "at the place of many bones." or "great bones place." Possibly because skeletons of whales, etc., were strewn about.

Sibegehanuck Cross Island, Washington County, Me. Malecite, "passage by water," or "sea-avoiding passage."

Sicaog Hartford County, Conn. Sicaog, "dark earth" or "muddy place." The Sicaog Indians lived in and around Hartford.

Sickcompsqu Hampden County, Mass. Nipmuck, "black rocks," or "hard rocks"?

Sickenames River, New London County, Conn. Mohegan? "black fish," probably the tautog (plural).

Sicojocke an early name for the Connecticut River, probably from Wampanoag, "dark colored earth," or "dark land;" i. e., mucky land. Also found as Sioasock.

Siguenoc Washington County, Me. Abnaki, "at the humped-up place." See Seguin Island.

Sigwanawock Stream, Coös County, N. H. Pennacook, "springtime place."

Sigwooganock River, now Israel's River, Coös County, N. H. Abnaki? Pennacook? "toward the south," or more likely, "place where we return in the springtime."

Sikunke see Seekonk.

Simquish Lake, Washington County, Me. Abnaki, "dip up a drink."

Singrawac River, Coös County, N. H. Abnaki, "springtime place."

Sinuessutt New London County, Conn. The eastern boundary of Mohegan territory. Possibly Mohegan, "place of small stones." But see Sneeksuck.

Sioascauk Fairfield County, Conn. Paugussett, "black or dark colored earth," or "covered at high tide." Also Sioascock.

Sioug Pond, Hampden County, Mass. Nipmuck, "thorns or briars," or Natick, "sour or bitter." 
Sip Pond, Cheshire County, N. H. Perhaps this is the English word "sip." Possibly Old Abnaki, "a bird;" or Malecite, "a duck."

Sipp Bay, Washington County, Me. Perhaps an English name; if Abnaki, "a bird." (Or from -sebe, cognate with the -sippi in Mississippi ?)

Sippenak Brook, Hancock County, Me. Abnaki, "edible lily roots." Sippewisset Harbor, Barnstable County, Mass. Wampanoag, "at the little river." See Seboois.

Sippican River, Neck and Harbor, Plymouth County, Mass. Wampanoag, "long stream," or "gravelly stream."

Sippigunnet River, Plymouth County, Mass. Wampanoag, "at the long river."

Sippiquonet Plymouth County, Mass. Wampanoag, "at the long stream."

Sipsaconta Lincoln County, Me. Abnaki, "plenty of birds," perhaps ducks. Also Sipsisconta.

Sisikwa Menahan Belknap County, N. H. Abnaki, literally, "Rattlesnake Island" in Lake Winnepesaukee.

Sisladobsis Lake (Lower), Lower Dobsy Lake, Washington County, Me. Malecite, "rock shaped like a shark or dogfish." Also Sysladobsis.

Sisladobsis Lake (Upper) Penobscot County, Me. See Lower Sisladobsis.

Sisquisic Cousin's River, Cumberland County, Me. Abnaki, "muddy place." See also Sysquissett.

Siwanoy were Indians living in Fairfield County, Conn. and westward into New York. Probable meaning, "south people." Also Siwanog.

Skahogan a locality in Essex County, Vt. Abnaki, "a sharp stick." (As a pole for the center of a haystack.)

Skaket Barnstable County, Mass. Wampanoag, from Namskeket, "at the fishing place."

Skaki or Skwasi Cumberland County, Me. Old Abnaki, "a way or place or method of standing or waiting." See Skowhegan, Squakheag, etc.

Skamonikoos Windham County, Vt. Abnaki, "corn harvest moon." 
Skamscommuck Providence County, R. I. Narragansett, "rock enclosed spring place." See Scamscammuck.

Skanentgraksenge the shore of Lake Champlain near Burlington, Chittenden County, Vt. Mohawk, "a notably bad landing place."

Skanetoghrowa Lake Champlain. Moharek? "largest lake."

Skatehook Berkshire County, Mass. Mahican, "at the branch of the stream"? or "fire place."?

Skatutakee Hill and Lake, Cheshire County, N. H. Abnaki, "a fire swept by here."

Skauton Barnstable County, Mass. Wampanoag, "edge of the sea."

"Skeag Island, Cumberland County, Me. Abnaki, part of Naamaskeag, "a fishing place."

Skeecoway Creek, Cumberland County, Me. Abnaki, the American merganser, "rough shaggy crest," or "sheldrake."

Skenunganock Hampden County, Mass. Nipmuck, "green field."

Skeset Meadow, Plymouth County, Mass. Wampanoag, "at the outlet," or perhaps "overflowed."

Skiga-Nason or Skeega-Nassun-Edowa? Washington County, Me. Malecite, "April-moon place." (Where this band returned in spring to their cleared lands near a stream, to prepare for summer crops and fishing.?)

Skinequit Pond, Barnstable County, Mass. Wampanoag, "at the salmon fishing place," or perhaps "first blood place."

Skipmaug Hampden County, Mass. Nipmuck, "chief fishing place."

Skitchewaug Mountain and Trail, Windsor County, Vt. Probably Abnaki, "big mountain," if applied to the mountain. But if transplanted from nearby Black River, perhaps Micmac or Malecite, Kawap skitchwak "rough rocky rapid current," or "falls go down like steps."

Skitticook Branch, Piscataquis County, Me. Abnaki, "dead water," "sluggish stream," "slack water," "standing water."

Skogogwaganock Penobscot County, Me.? Abnaki, "place of the snake-dance."?

Skokorat New Haven County, Conn. Quinnipiac? "snake hill," Skug-adchu.

Skookum Chuck Brook, Grafton County, N. H. Chinook Jargon, "dashing water" or "rapids." 
Skoonkeekmoonkeek Lake; see Shonkamonke, Shoonkeek Moonkeek.

Skowhegan Village, Somerset County, Me. Abnaki, "place of waiting and watching," a fish-spearing place.

Skuakheag Franklin County, Mass. Abnaki or Pennacook, "sharp instrument," probably a fish-spear; but see Squakheag.

Skuamkeag Windham County, Vt. Abnaki, "salmon place" or "red place." Probably Skuakheag or Squakheag, miscopied or misspelled. See also Skowhegan.

Skudek Washington County, Me. Probably Abnaki, "fire place," or "burned-over place."

Skug River, Essex County, Mass. Pennacook, "a snake."

Skukoal Island, Penobscot County, Me. Abnaki, "meadow grass."

Skunkamug River, Barnstable County, Mass. Wampanaog, "green field"? But see Skunkamug, Conn. Variant, Scunganuyk.

Skunkamug the Hop River, Tolland County, Conn. Nipmuck, "eel fishing here." Also Skungemaug and Shonkamonk; and Narragansett, neshuongok, "eels." (Literally, "they go in twos.")

Skunkscut see Kongscut.

Skutarza or Skutarzy Abnaki, "trout-place." See Escutassis or Skuthazis. Several places so named.

Skuthazis or Escutassis Stream, Penobscot County, Me. Abnaki, "brook trout."

Sneech Pond, Providence County, R. I. Nipmuck, "rocks at the outlet"? See Sneechteconnet.

Sneechteconnet River, Bristol County, Mass. Nipmuck? "rock in or along the river." This is a local name for the Blackstone River. See Tittituck.

Sneeksuck New London County, Conn. Mohegan, "stone shelter (overhang) near the' brook outlet;" or "at the cave," or "at the stone house."?

Snipatuit Pond and Village, Plymouth County, Mass. Wampanoag, "at the rocky river." Other spellings Snippatuit, Snipatuett, etc., sometimes translated as "log palisade," or "big stakes place."

Snipsic Pond, Tolland County, Conn. Mohegan, abbreviation of moshenupsuc "the great outlet." 
Soadabscook Penobscot County, Me. Abnaki, "place of large, water-smoothed rocks." See Sowadabscook.

Soakatuck see Saugatuck.

Soansacut see Moswansacut. Also spelled Soansarut.

Sobagwa the Atlantic Ocean. Abnaki, "salt water." See also Sebago.

Sobscook Nichols Rock, Penobscot County, Me. Abnaki? Micmac? "rock around which the tide current appears to boil."

Socatean Stream, Somerset County, Me. Abnaki, "divided into two parts."

Sockanosset Barnstable County, Mass. Wampanoag, "place of dark-colored earth."

Sockanosset Cross Road and Hill, Providence County, R. I. Narragansett, Sockanoco was a Narragansett chief. Possible meaning, "dark colored land."

Sockhigones An old name for the lower Saco River and its valley, in York County, Me.

Soggahannego Lincoln County, Me. Abnaki, "where the river enters the ocean sea." Said to be the home of Samoset, q. v.

Soghali Menahan Piscataquis County, Me. English-Abnaki, "sugar island." Modern Abnaki substitute L for R, hence soghal or soogle for sugar; Mali for Mary, etc.

Sogkunate Point, River and Village, Newport County, R. I. Narragansett, "haunt of the black goose," or "land at outlet," or a cognate of sackahickneyah (Delaware), "a path along the seashore."?

Sokones Barnstable County, Mass. Wampanoag, "dark earth." See Sockanosset.

Sokoquois Rivière des; the Connecticut River. Abnaki? Pennacook? modified by French, "south people's river." So named on Aubéry's I7I5 map.

Solikuk Isle au Haut, Hancock County, Me. Micmac or Malecite, "place of shells." This seems questionable; Captain John Smith called this Sorico, perhaps Sonico, from hassun-ohke, "stony place."'[?]

Sologismoodik Five-Island Falls, Penobscot County, Me. Old Abnaki? "depression-place," or "ravine-place."? 
Somersic Rattlesnake Hill, Hartford County, Conn. This is really a water-name, not a hill-name; probably Wangunk, "at the large outlet." Also Somasick and Meshomasick.

Sonanoxet Island, Fox Island Washington County, R. I. Narragansett, "crushed by heavy stones," as in a deadfall trap? More likely, "place too stony to dig with hoes," or "little stony place."

Songo Pond, Oxford County, Me. Mohegan, "cold water."

Sonkipaugsuck Hartford County, Conn. Wangunk, "outlet of cold brook."

Sonkpog New London County, Conn. Western Niantic, "cold pond."

Sonoogawanock River, Coös County, N. H. See Sigwooganock.

Sooneybeag Pond, Knox County, Me. Abnaki, "stones in pond." Also Sooneybek.

Soongapog New London County, Conn. Mohegan, "cold spring."

Soucook River, now Israel River, Coös County, N. H. Abnaki, "rocky place" (Another stream of same name in Merrimack County.) But see Sigwooganock.

Souhegan River, Worcester County, Mass. Nipmuck, "southwest river," also "watching place."

Souhegan River, Hillsboro County, N. H. Pennacook, "watching place; still-water fishing." Also Souheganock.

Souneunk Stream, Piscataquis County, Me. Abnaki, "(extensive?) rapid stream."? See Sourdnahunk.

Sourdnahunk Lake, Piscataquis County, Me. Abnaki, "extensive rapid stream."

Sowadabscook River, Penobscot County, Me. Abnaki, "at the place of sloping ledges," or "smooth sloping rocky banks."

Sowames see Sowams.

Sowampsett Pond and River, Bristol County, R. I. Wampanoag, "at the place of red rocks." Also Soewompsit, etc.

Sowams Bristol County, R. I. Wampanoag, "stony plain path"? or "southwest place." But see Sowamsett. Also Sohomes.

Sowamsett River, Plymouth County, Mass. Narragansett, "at or in the south country," or "stony plain," or "beach trees." ? This is possibly Metacom's "Southwest Place." 
Sowangan Island, Penobscot County, Me. Abnaki, "the bald eagle." Another spelling, Sowungun.

Sowanoxet Island, Fox Island, Washington County, R. I. Narragansett, "place of small shells?" See Sonanoxet.

Sowcook Cheshire County, N. H. Abnaki, "sloping ground," or "descending land." Also translated as "outlet place;" see Saco. Variant Sowcoög.

Sowheage Avenue, Middlesex County, Conn. Wangunk, "Southland" or "South Sachemdom." Sowheag was a sachem of the Wangunks, known also as Sequin, which may signify that he was the last of a large group; he sold land in I635.

Sowhigginock same as Saco. See also Skowhegan.

Sowhomes see Sowams.

Sowniganock Coös County, N. H. Abnaki, "to the south going" (trail).

Sowockatuck a Sokoki (Abnaki) village on Saco River, noted by John Smith, I6I6. Abnaki, "country around the river outlet."

Sozap Nebees Joe's Pond, Caledonia County, Vt. Abnaki, “Joseph's Pond." "Indian Joe" was a Revolutionary character who aided the Continentals.

Spednic Falls, Washington County, Me. Abnaki, "visible, but shut in by mountains."

Spoart Newport County, R. I. Narragansett, "large cove." See Espowet.

Squabage Worcester County, Mass. Nipmuck, "red pond."

Squakheag Windham County, Vt.; Franklin County, Mass.; and Cheshire County, N. H. Abnaki, "watching place," similar to Skowhegan, Me. This name has been the subject of much research, but "watching place" seems to be the best translation. Other possibilities include roots indicating "red rocks," "black swamp-earth," "quaking bogs," "forearm," "tears," etc.; but the history, territory and etymology all point to "waiting, watching," and "spearing fish." This was one of King Philip's last rallying places, I675-I676.

Squakheague Washington County, R. I. See Squakheag.

Squam River, Essex County, Mass. Natick, "at the end (or top) of the rock." 
Squam Head, Nantucket County, Mass. Wampanoag, either "at the summit, or end of rock," or (less likely) an abbreviation of mishquammanock, "salmon."

Squam Lakes and Mountain, Carroll and Grafton Counties, N. H. Abnaki, "salmon."

Squamagonic (also Gonic) Strafford County, N. H. Abnaki, "salmon spearing place."

Squamcut Providence County, R. I. Narragansett, "red earth," or "salmon place."

Squamicuck New London County, Conn. Eastern Niantic, "salmon fishing place." Variants M'squamicook, Squammacut.

Squamicut see Squammicost and Misquamicuck.

Squammicott Washington County, R. I. Eastern Niantic, "salmon fishing place."

Squamokwisseeboo Stream, Penobscot County, Me. Abnaki, "salmon in little stream."

Squamscott Bloody Point, Rockingham County, N. H. Pennacook, "at the end of the rocks," or "red rocks."

Squamscut Bog, Hillsboro County, N. H. Abnaki, "place at the end of the rocks," or "red rocks."

Squannacook River, Middlesex County, Mass. Natick, "green place," or possibly "place for taking salmon, or for catching bream?"

Squannacook River, Worcester County, Mass. Possibly a corruption of Natick, Squamacook, "salmon fishing place,"? or "in the season of gardens," or "green place"?

Squannahonk Swamps, Bristol County, Mass. Narragansett, "green place." Other spellings Squannakonk, Skwannakonk, etc.

Squannequeest Plymouth County, Mass. Wampanoag, "place where mud ends."

Squanticook Brook, Franklin County, Mass. Pennacook, "place of the green brook." See Squenatock.

Squantuck New Haven County, Conn. Quinnipiac, "at the end or branch of the river." See Wesquantook.

Squantum anciently a village in Cheshire County, N. H. Natick, "angry god;" but if the spelling is correctly Squontam the translation is "door, or gateway." 
Squantum Suffolk County, Mass. Natick, "a door or gate," also Narragansett, "angry god." A Wampanoag named Squantum, or Squanto, was the interpreter for Massasoit in 1620 .

Squapan Lake, Mountain and Village, Aroostook County, Me. Abnaki, "bear's den."

Squapnassutte New Haven County, Conn. Quinnipiac, "place at the top rocks," or "between rocks"? Possibly "red rocks." See also Squibnocket.

Squatesit Nantucket County, Mass. Wampanoag, "red place," or "red brook."?

Squattock Lake, Aroostook County, Me. Abnaki, "trout river."

Squaw a multitude of topographical features, such as Squaw Peak, Squaw Pond, Squaw Rock, etc., are scattered throughout New England. They all stem from a cognate NarragansettNatick-Nipmuck term, squaw, esqua, eskwaw, meaning "a female, one of womankind." In common usage, this refers to an Indian woman, or an Indian's wife. The word is a feminine suffix for nouns, as "Kinjames," and "Kinjamesqua."

Squaw Bally Bristol County, Mass. Narragansett, mixed with English? "One of womankind; a female," but Bally may be a modification of Betty. See Squaw Betty.

Squaw Betty Bristol County, Mass. Wampanoag; Squaw Betty was the name of a Wampanoag woman, Sausaman, the wife of Nanooswanum. She inherited a large tract of land from her father, Paumatuck Quasuk, in I673.

Squazodek Washington County, Me. Abnaki, "a lookout place" or "waiting-place."

Squenatock Brook, Franklin County Mass. Pocumtuck, "long stream"? See Squanticook, "red stream," from m'squenatock?

Squepaug Washington County, R. I. Eastern Niantic, "red pond" if from misque pang; "end of pond" if from usque paug.

Squeteague Harbor, Barnstable County, Mass. Wampanoag, "the weakfish," or "the gluey one." This fish is also called chequit.

Squibnocket Point and Pond, Dukes County, Mass. Wampanoag, "at the place of red (or black) rocks." Possibly "sticky earth, (or clay) cliff." 
Squidrayset Creek, Cumberland County, Me. Named for Chief Squidrayset, of Salem, Mass. Variants, Squiddera Gusset, Squitheragusset, Squintregust, etc.

Squimonk Pond, York County, Me. Abnaki, "red fish place," probably salmon. This was Bonnabeg Pond.

Squitcomegek Falls, Knox County, Me. Abnaki, "at the long ridge."

Squog Hillsboro County, N. H. Pennacook, an abbreviation of Piscataquog, "at the river branch."

Squog Kane Kaneek Berkshire County, Mass. Mahican, "cold water spring," "spring at big rock," or "big fishing place at rock." Perhaps from Musquaquag Konet, "red, or wide swamp enclosed place"? or "big refuge place."?

Squontonk Brook, New Haven County, Conn. Quinnipiac, "red river"? or "place of red earth." ?

Squotuck Hartford County, Conn. Tunxis? "red river"? or "place of red earth."?

Statuckquett Plymouth County, Mass. Wampanoag, "at the source of the creek;" or perhaps "place of no trees."

Stockbridge Indians; see Mahican.

Subecwangamook Hadley's Lake, Washington County, Me. Old Abnaki? "portage or trail toward the salt water," and "portage or trail toward the large body of water."

Succanesset Barnstable County, Mass. Wampanoag, "place of black shells," (for wampum)? or "bass-fishing place?" or "early summer place"? See Sockanosset.

Succotash Point, in Washington County, R. I. Narragansett, "shelled corn kernels separated from the cob and beaten to a pulp." (Modern succotash has beans in it.)

Suchow Hampshire County, Mass. Perhaps from Chinese, but if Indian, possibly Nipmuck, "between the hills," or "dark colored lands," or "big hill" or "rocky hill."

Suckatunkanuck Hill, Providence County, R. I. Narragansett, "dark colored earth (or rocks) at the summit."

Suckiaug Hartford County, Conn. Sicaog, "muddy land" or "dark colored land" or "black earth." Variants Sicaiock, Suckiagge, Sicaogg.

Suckquackheag Franklin County, Mass. Pennacook? "black swamp, or dark, miry place." See Squakheag. 
Sucktequesite Plymouth County, Mass. Wampanoag, "at or near the small dark river," or "at the outlet of the small river."

Sugalmanahan Sugar Island, Penobscot County, Me. Abnaki, "sugar island." See Soghali Menahan.

Suker Pond, Providence County, R. I. If Indian, probably from Narragansett, "it pours forth."

Sumpauge Pond, Worcester County, Mass. Narragansett, or Nipmuck, "male beaver," or "pond with small stones; a gravelly pond."

Sunapee Hill, Hampden County, Mass. Pennacook, "rocks in the water," or "stony pond."

Sunapee Lakes, Merrimack and Sullivan Counties, N. H. Pennacook, "rocky pond."

Suncook Mountains, Belknap County, and Pond, Rockingham County, N. H. Pennacook, "rock place."

Suncook Rivers, Merrimack County, N. H. and Oxford County, Me. Abnaki, "at the rocky place." Also Suncoog.

Sungahnee took Lewis Creek, Addison County, Vt. Abnaki, "fish weir river" or Mahican, "wolf trap river."

Sunkataradunk mouth of Kennebec River, Sagadahoc County, Me. Abnaki, "concealed outlet of large swift currents." See Sunkaze.

Sunkatunkarunk same as Sunkataradunk, Sunkaradunk, etc.

Sunkaze Rips and Stream, Penobscot County, Me. Abnaki, "concealed outlet."

Sunkeath see Sawkhead.

Sunkipaug New London County, Conn. Western Niantic, "cold pond."

Sunmuckquommuck Hampden County, Mass. Nipmuck, "extended plantation."

Sunsicke Hills, Franklin County, Mass. Pocumtuck, "at the stony place," or Pennacook, "boulder brook."

Suntaug Lake, Essex County, Mass. Natick, "stony ground," or "place of boulders."

Suponic Pond, Penobscot County, Me. Abnaki, same as Chibanook and Saponac, "big opening." 
Susquesong Cousin's Island, Cumberland County, Me. Abnaki, "muddy place."

Susquetomscut Brook, New London County, Conn. Mohegan, "at the place of mixed rocks and mud," or possibly "muddy stream flowing near (or over) rocks." Also Suskotomscot and Susquoetomscutt; possibly "place of the red ledges."

Sussgussugg see Susquesong, Sisquissic and Sysquissett.

Sutamachute see Setamachut.

Sutquin same as Seguin.

Swackadock Cumberland County, Me. Abnaki, "swift stream outlet place." Similar to Sagadahoc.

Swagadahock this name apparently was applied by early explorers to the Saco, the Kennebec and other Maine rivers at their mouths. The general meaning is "pouring forth at outlet place."

Swamcot see Misquamicut, R. I.

Swammicott Valley, Providence County, R. I. Narragansett, "south plantation."

Swampscott Essex County, Mass. and River in Rockingham County, N. H. Natick, "at the place of the red rocks."

Swango Island, Sagadahoc County, Me. Abnaki, "an eagle"?

Swank wahiganus Washington County, Me. Abnaki, "little bell, or chimes," so named for a small English early settler named Bell; Indians would say, "Let's stop at Little Bell's place."

Sweektaconet see Sneechteconnet.

Swegotchy see Oswegatchie.

Swegustagoe River, Royall's River, Cumberland County, Me. Abnaki, "mouth of the river."

Swichichog New London County, Conn. Mohegan, "place to get clams"? or "dark earth ?"

Sybaik see Sebayek.

Syenpauge see Sympaug.

Sympaug Brook and Pond, Fairfield County, Conn. Possibly Paugussett or Western Niantic, from sumhupaog, "beavers."

Sysladobsis Lake, see Sisladobsis.

Sysquissett Cousin's River, Cumberland County, Me. Abnaki, "muddy place," or "muddy stream." 
Taascamessick Bennington County, Vt. Mahican, "small crossing place," or "small ford" (across the Walloomsac).

Tabamapaug Pond, Providence County, R. I. Narragansett, "this pond is sufficient," ? or, more likely, "clear or shallow pond." See Paugamaug and Paugeamapauge; also Taubakonommock.

Tabusintac Stream, Washington County, Me. Micmac, "two by two," or "they go in pairs;" possibly silver eels.

Tabuttantam Fairfield County, Conn. Paugussett, "he gives thanks."

Taccomhuit Path, Middlesex County, Conn. Wangunk, "he who strikes" (repeated blows) See also Takemmy.

Tachamugus Middlesex County, Conn. Wangunk, "forest bear," or "digging bear"? From the name of an Indian. Possibly the same as Terramuggus, q. v.

Tachannike Berkshire County, Mass. Delaware? This is Zeisberger's name for the Taconic Mountains, translated as "full of timber."

Tacoma Lake, Kennebec County, Me. Nisqually [of Washington state], "snow covered, or great white breast." By that state's legislative decree, "Mountain That Was a God." Possibly applied locally by a returning early traveler[?].

Taconic this name is applied to many features of the topography of New England, particularly mountains (e. g., Mt. Washington, in Mass.). The derivation is unclear; it may be from Mahican or Natick, meaning "steep ascent," "small field or plantation in the forest," or "forest." Variants, Tachannike, Taghkannuc, Taughkanghnick, etc.

Taconic Mountains, Bennington County, Vt. Natick, "ladder," or "steep ascent." (But Tooker says, "field in the woods.") Variant, Tachonaëg.

Taconic Mountains and Trail, Berkshire County, Mass. Mahican, see above.

Taconnet River, Kennebec County, Me. Abnaki, "at the wading place, or ford."

Tacook Penobscot County, Me. Abnaki, "waves."

Tadmuck Brook and Meadow, Middlesex County, Mass. Natick, "wading-place." L. K. Gahan says, "blueberry place." 
Taghkanick Berkshire County, Mass. Mahican, "forest" or "wilderness;" ? if Tohkonick, "enough water."

Taghkannuc Mountain, Litchfield County, Conn. Mahican, "wild lands," ? "forest"? Spelled also Taughkanghnick.

Taghommay Middlesex County, Conn. Western Niantic, "corn grinding path." See also Takemmy.

Tagwahogan York County, Me. Modern Abnaki, "a corn mill" or "grinding instrument, for grains." See Nokhigan.

Tagwesi Menahol Penobscot County, Me. Abnaki, literally "twin islands."

Tagwonk New London County, Conn. Eastern Niantic, "a stone mortar" for grinding grain and acorns. Also Tagwouncke, Tagunk, Tangwonk, Togguhwhonk, etc; see Takemmy.

Tahanock Knox County, Me. Micmac, "toward the open sea;" this is on Simanca's Map, I6Io.

Tahanto Point, Worcester County, Mass. Nipmuck, possible meaning "principal point or neck of land." George Tahanto was chief of the Nashaway in I700.

Tahonwenta Grand Isle County, Vt. Mohawk? "canoe launching," "where one drops a canoe into it."

Tahwomp Place, Middlesex County, Conn. Wangunk, "white dugthings (white jack-in-the-pulpit roots)" ? or "white digger"?

Takebi-suk Penobscot County, Me. Abnaki, "at the place of the cold water (spring)." This one is at Cold Stream, Enfield.

Takekomuit Bristol County, R. I. Narragansett, "place of the spring," or perhaps "fountain."

Takemmy Trail, Dukes County, Mass. Wampanoag, probably, "trail to the corn-meal mill," or "the corn grinding path" (togguhum-mai); or possibly "camp field" (tetant-komet, or tuppuksinnoonk).

Takwangan York County, Me. Old Abnaki, "crush by repeated strokes," that is, using a rock "pestle to crush corn. Also Taggwangun.

Talagodissek Treat-Webster Island. Penobscot County, Me. Abnaki, "painting place for squaws." Also spelled Tallagodisik.

Talaquya Bristol County, Mass. No suitable New England roots seem to apply. Perhaps this name is a transplant from Tahlequah, the Cherokee capital in Oklahoma? 
Talhanio Dukes County, Mass. Wampanoag, "low meadow."

Tallawanda Worcester County, Mass. Nipmuck, "hoarse voiced ones;" that is, "bitterns or cranes."

Tamarack Road, Litchfield County, Conn. and Pond in Coös County, N. H. Possibly of Indian origin; the name is in common use for certain larches and pines.

Tamescot Lincoln County, Me. Abnaki, "alewife place."

Tamesuck the Thames outlet, New London County, Conn. English-Mohegan, "Thames outlet." See Sepos-Tamesuck.

Tamett Brook, Plymouth County, Mass. Wampanoag, "flooded place," or "low place."

Tammesham Hampden County, Mass. Narragansett, "my wife," (my wife's place?). Also Nummetawamus.

Tamonquas Brook, Windham County, Conn. Mohegan, "a beaver."

Tamtashua Fairfield County, Conn. Paugussett, "summit of the mountain."

Tanipus Pond, Hillsboro County, N. H. Natick, "a tortoise"? or "herring"?

Tankeroosen Brook; see Tunkahoosen.

Tankiteke Fairfield County, Conn. Mahican? "forest dwellers."

Tantaquidgeon Lodge, New London County, Conn. Mohegan, meaning lost. The Tantaquidgeons were prominent in Mohegan tribal affairs. One man of the name still survives (I960).

Tantiusque Worcester County, Mass. Mohegan, from the name of Chief Tantaquieson, or Tantaquidgeon, q. v.

Taphamshashack River, New Haven County, Conn. Quinnipiac, "at the place of cold springs." Also found as Tappanshashick, Tappunshasic, etc.

Taphance Fairfield County, Conn. If Pangussett, possibly "he buys" or "he is satisfied." But more probably Siwanoy or Wappinger, "cold spring."

Taquahunga Falls, Franklin County, Vt. Moharek, "haunt of bitterns."

Tarratine Village, Somerset County, Me. The Tarratines were Micmacs from Nova Scotia, who invaded and controlled parts of Maine around I6oo. It is doubtful that the word is of Indian origin, although southern New England Indians in 
the I60o's were said to have called the Abnaki Tarratines, or Taretyns. The Puritans used this term also in referring to the Abnaki, and the contemporary Malecite call the Penobscot by the same name.

Tashima Nantucket County, Mass. Wampanoag, "the great" (or principal) spring."

Tashmoo Pond, Dukes County, Mass. Wampanoag, "big spring," or perhaps "deserted pond."

Tashmuit Barnstable County, Mass. Wampanoag, "at the great spring."

Tashnuc Spring, Dukes County, Mass. Wampanoag, "deserted place."

\section{Tashua see Tamtashua.}

Tassacaumet Bristol County, Mass. Wampanoag, "at the deserted beach."

Tassacauset Neck, Bristol County, Mass. Narragansett, "at the abandoned carrying place (or portage)." But see Tassacaumet.

Tataesset Hill, Worcester County, Mass. Natick, "near the thing that rolls on an axis;" the "rocking-stone place."

Tatamuckatakis Fairfield County, Conn. Paugussett, "little meadow trembles (when you walk on it)."

Tatetuck Fairfield County, Conn. Paugussett, "principal stream."

Tatnic Brook and Hill, Windham County, Conn. Nipmuck, "at the great hill."

Tatnit Hill, Worcester County, Mass. Nipmuck, "at the great hill." Another spelling, Tatnuck.

Tatnock Marshes, York County, Me. Abnaki, "at the shaking place," or "quivering meadows."

Tatomuck Brook, Fairfield County, Conn. Paugussett, "trembling meadow." Variants, Tatomok, Tomuk, etc.

Tattahassun Worcester County, Mass. Nipmuck, "at the top of the shaking or rocking boulder."

Tattamacuntaway River, New London County, Conn. Mohegan, "place of trembling bogs."

Tattamasket Plymouth County, Mass. Wampanoag, "winding around place," or perhaps "berry place." Also Tatumasket.

Tattaquamock-pangok Pond, Litchfield County, Conn. Paugussett? Mahican? "pond in the boggy trembling meadow." 
Tatuppequaog New London County, Conn. Pequot, "level, open land that trembles" when walked on. Boggy land along the Thames River, near Mohegan Village. Probably abbreviated from Tattaquannockpancook.

Taubakonommock Hill, New London County, Conn. Western Niantic, "place where there are many lamprey eels." Given also as Tabaconomock, Konomok, etc.

Tauchaug Meadow, Hartford County, Conn. Tunxis, "land at the brook."

Taughkannuc see Taconic.

Taukonnock see Taconic.

Taupagoh River, now Williams River, Berkshire County, Mass. Mahican, "an isolated or solitary pond," or Natick, "satisfactory pond," or possibly "wise speaking."

Taupoowaumsett Pond, Plymouth County, Mass. Wampanoag, "at the place of wise counsel," or "here they preached;" possibly "deserted meadow."

Tausakaust see Tassacauset.

Tauseguse Oxford County?, Me. Old Abnaki? "upon the back," i.e., carrying goods over a portage.

Tauskounk Meadow, Kent County, R. I. Narragansett, "a ford or wading-place;" possibly the site of a bridge. Variants, Toskiounke, Toskeunke, etc.

Tautemco Nantucket County, Mass. Wampanoag, "camp field." Variant, Tawtemko. See also Takemmy.

Ta wak be dee eeso Wadso Camel's Hump, Washington County, Vt. Abnaki, "prudently, we make a campfire in a circle near water (and rest) at this mountain." Given by Rowland Robinson as "resting place, or sit down place, mountain."

Tawawag New London County, Conn. Mohegan-Pequot, "useless or abandoned lands." Also found as Tawawog. (The Indians near New London moved away from these fields sometime before 1650 ).

Tchebatigosak Penobscot County, Me. Abnaki, "crossing at the separated place;" or freely, "crossing which does not take so long." Sometimes given as "crossing at the boundary."

Tchobacco Lake; see Chebacco. 
Teapanock Pond, Washington County, R. I. Eastern Niantic, "at the great clearing," or "at the great cove." Found also as Teapanocke, Teapannock, Tepanock, etc.

Teaticket Barnstable County, Mass. Wampanoag, "at the principal tidal stream." Also, Tecticut, Tehticut, etc.

Teckyadoughinyariga the Narrows at Chimney Point, Addison County, Vt., and Crown Point, N. Y. Mohawk, "two points of land standing opposite each other."

Teckyadough Nigarige The Narrows on Lake Champlain near Charlotte, Vt., and Essex, N. Y. Mohawk, "two points of land which have come close together." Also Teckyadough Nigarêge.

Teconnet same as Taconnet, q. $v$.

Tecumseh Mountain, Grafton County, N. H. Sharenee, "I cross somebody's path." The great warrior here commemorated was also called Meteor, and Crouching Panther: he lived I768-I8I3.

Teddon, Tadden, Toddin, etc., Piscataquis County, Me. These are various English slurrings of the Abnaki Katahdin, "principal mountain."

Tegoak Rapids, Penobscot County, Me. Abnaki, "waves." This is "the cook" or "cauldron" at the Old Town. Modifications of this word are used as "river-water driven by waves."

Teightaquid Middlesex County, Mass. Natick, "principal land, or island."

Teikiming Dukes County, Mass. Wampanoag, "a fountain," or "cool springs place." Given also as Tockiming.

Tekebisek anciently a village near Cold River, Cheshire County, N. H. Abnaki, "at the place of the cold water spring." Also given as Ticopeesok, the Abnaki spring.

Telastinis Lake, "Little Telos;" see Pataquongamis.

Telos Lake, Piscataquis County, Me. Now called Pataweekongomoc, q. v. Probably not Indian; some guides say it is Tea-los, "without tea," since there is a Coffee-los lake nearby. A most likely suggestion is that Telos is from Greek, meaning "far," "ultimate," or "the end."

Telosinis Lake; see Pataquongamis.

Temahkwecook Aroostook County, Me. Abnaki, "beaver place." But see Macwahoc. 
Temiscouata Lake, Aroostook County, Me. Abnaki, "bottomless," or "exceedingly deep all around." See Tommy Squatter.

Tepenegine near Sheepscot River, Lincoln County, Me. Abnaki, "head of the island."

Tepikamicut Plymouth County, Mass. Wampanoag, "at the great clearing." Also Tippecummicut.

Tequenoman see No Man's Land.

Terramuggus Lake, Hartford County, Conn. This is the name of a Wangunk chief. Another form, Tachamuggus, suggests "forest bear," or "digging bear." He sold land here in I662.

Tetaukimmo Nantucket County, Mass. Wampanoag, "principal cool spring." But one authority says, "boggy place around a big spring;" another, "camp field spring."

\section{Tetiquet see Titicut.}

Tewissicke same as Capisic, q. v.

Texas Falls, Addison County, Vt. Hasinai (a Southwestern tribe), "a friend, an ally."

Tgawistaniyonteh St. Anne's Mission, Grand Isle County, Vt. Mohawk, "there a bell is suspended." There are legends of similar places at Lake St. Catherine, Rutland County, and at Ferrisburg, Addison County, Vt.

Tiasquam River, Dukes County, Mass. Wampanoag, from the name of Metacom's great warrior, Tiashq, or Tuspaquin. Sometimes translated as "big salmon," or "red feather." See Tisquaquin.

Ticklenaked Pond, Caledonia County, Vt. It is very doubtful that this hilarious name is of Indian origin; if so, it is possibly a much modified Delaware word meaning "beaver kittens here."'[?]

Ticonderoga on Lake Champlain opposite Shoreham, Addison County, Vt., where Lake George spills into Lake Champlain. Mohawk, "where two currents or rivers join."

Ticonic Falls; see Taconnet.

Tieronderaquegon Lake Champlain near West Haven, Rutland County, Vt. Moharek, "lake closed in, where two rivers are stopped up," or more grammatically, "two rivers without an outlet;" South River of Lake Champlain and Wood Creek. 
Tihonet Pond, Plymouth County, Mass. Wampanoag, "at the abode of crane or bittern." Also Tionet.

Tioga Hill and River, Belknap County, N. H. Oneida, "at the river forks."

Tiogue Lake, Providence County, R. I. Narragansett? "low place" or "low land," or if Tiohquonque, "it is low." (If borrowed from New York, see Tioga.)

Tiowawaye Third Lake, Penobscot County, Me. Abnaki? "empty your canoes,"? or "sit in the stern of your canoes."?

Tippecansett Pond, Kent County, R. I. Narragansett, "at the great clearing." Variants Tipecanset, Tippecan, Tippecanaunit, etc.

Tirsick perhaps York County, Me. ? Old Abnaki, "at the bend or curve in the stream"?

Tiscatuck Swamp, Washington County, R. I. Eastern Niantic, "at the ford, or wading-place." Variant, Tiscatuk.

Tishcottic Swamp, Washington County, R. I. Narragansett, "at the wading place, or ford." Also Tishcottie.

Tismattuck Washington County, R. I., and New London County, Conn. Eastern Niantic? "wading place," or "crude bridge over the river," or "river over which we cross on a driftwood bridge." Also Tismatuck.

Tisquaquin Pond, Plymouth County, Mass. Wampanoag, a chief called also Watuspaquin. The English called him "The Black Sachem," but the name means "big dark feather," or "black plume." He was killed in King Philip's War, I676.

Tist Norfolk County, Mass. Natick, "a bridge," or "a fording place." Sometimes Tisk.

Titicus Village, Fairfield County, Conn. Mahican, "place without trees."

Titicut Bristol County, Mass. Narragansett, "the principal river."

Titicut Village, Plymouth County, Mass. Wampanoag, "at the great tidal river."

Titticut Road, Newport County, R. I. Narragansett, "place of the principal (or great) river."

Tittituck the Blackstone River, Bristol and Plymouth Counties, Mass. Wampanoag, "the great, or principal river." 
T'Kopesuck the large spring at Indian Point, Hancock County, Me. Abnaki, "large cold spring place."

T'makwa Sibo Cheshire County, N. H. Abnaki, literally "beaver brook."

Toantik Pond, Middlesex County, Conn. Wangunk, "wading place."

Toaskeunck Kent County, R. I. Narragansett, "a bridge (made of wood)"?

Tobago Pond. A misspelling of Sebago, q. v.

Tobaskick Falls, York County, Me. Abnaki, "cold water springs."

Tobegewock Pond, York County, Me. Abnaki, "rapid current at the outlet."

Tobyan Swamp, Washington County, R. I. Eastern Niantic, "camp"? Perhaps this was a refuge camp?

Tockiming Dukes County, Mass. Wampanoag, "place of gushing springs, or fountain."

Tockwotton Hill, Providence County, R. I. Narragansett, "steep ascent to be climbed"? or "shaped like a pounding mortar?"

Togue Ponds and Ledges, Piscataquis County, Me. Abnaki? The togue is the same as the namaycush, or nammycush (meaning "long fish") - the large deepwater lake trout, Cristivomer namaycush. See Tuladi.

Togus Lakes, River, Stream and Village, Kennebec County, Me. Old Abnaki, "brook (entering cove)" or "little stream." See Wallamatogus and Togue.

Tohke commumwachak Suffolk County, Mass. Natick, "springs at the lookout place," or "fountain near the mountain."

Toikiming Nantucket County, Mass. Wampanoag, "at the mill," or "at the gushing spring"?

Tokanock see Taconnet.

Tokekommunwadchuck Worcester County, Mass. Nipmuck, "gushing spring at the lookout mountain."

Tokenake Fairfield County, Conn. Mahican, "the forest," literally, "tree-land." Chief Tokenake sold land here in I640.

Tokopissett Plymouth County, Mass. Wampanoag, "little wilderness place."

Tolam Cumberland County, Me. Old Abnaki, "canoes on sand"? or "a canoe landing place"? 
Tolbabawk Orange County, Vt. Abnaki, "snapping turtle pond."

Tolbuntbessek Penobscot County, Me. Abnaki, "turtle head," a rock at Hampden.

Tomah Stream, Washington County, Me. Either Micmac, "pipe rock;" Abnaki, "deep;" or Natick, "it is flooded." Any of these could apply. See Tomahegan.

Tomah Island, Penobscot County, Me. Abnaki, probably from the name of the minor Chief Tomah. See Tomah Stream.

Tomahegan Pond, now Moosehead Lake, Piscataquis County, Me. Old Abnaki, "deep drag," from temi-isquattan, "deep all around," or possibly an import from Connecticut. See Higganum, Takwangan and Tomhegan.

Tomakergo Fairfield County, Conn. Paugussett, "flood tide."

Tomaquag Brook, Washington County, R. I. Narragansett, "they who cut" (beavers). Variants, Tommaquag, Tommoeweague, Tummunkque, etc.

Tombegewoc Pond; Dering Pond, York County, Me. Abnaki, "place of the rocky reef in the pond."

Tomhannock Creek, Bennington County, Vt. Natick? "full up" or "brimming;" or, "it is flooded."

Tomhaummucke Hampden County, Mass. Nipmuck, "it is flooded," or "at the overflowed place." Also translated as "place of grinding," or "mortar place."

Tomhegan Pond, Somerset County, Me. Abnaki, "an axe." See Higganum and Tomahegan.

Tomheganompsket Middlesex County, Conn. Wangunk, "at the axe (or tomahawk) stone quarry." See Higganum.

Tomhollisick Brook, Hampden County, Mass. Nipmuck, "samp mortar brook," or "grinding place."

Tommy Squatter fishing spots in deep water near Thompson's Point, Chittenden County, Vt. Abnaki, "deep water all around here," temi isquattam.

Tomock River, Fairfield County, Conn. Siwanoy, "flooded."

Tonemy Hill or Tonnemony Hill, York County, Me. Abnaki (abbreviated), "red paint source." See Wannametoname.

Tonisset Neck, Bristol County, R. I. Narragansett, "pine place." Variants, Tonissit, Tuisset, etc. 
Tonomy Hill, Newport County, R. I. Narragansett, from the name of a sachem of Acquidneck; see Wannemetonomy.

Tonquewack a summit in the Russell Mountains, Somerset County, Me. Abnaki, "great top place."

Tonset Barnstable County, Mass. Wampanoag, "hill-place." Another source makes this an abbreviation of Annusautonset, "hoed garden place."

Took-sunk Plymouth County, Mass. Wampanoag, "wading place" or "ford." (Or perhaps "wading place where they trod out clams.")

Topabskwa Split Rock (in Lake Champlain) between Chittenden County, Vt., and Essex, N. Y. Abnaki, "the split or cloven rock." This was an important boundmark between Mohawk and Abnaki territories.

Topamisspauge (now Randall's Pond) Washington County, R. I. Narragansett, "little camp at a pond"? or if Topansissipaug, "little twisting river pond"?

Topeent an ancient village near Boston? Natick? "he encamps," or "abandoned, deserted"? ?

Toquams Fairfield County, Conn. Paugussett, "round rock."

Toquamske Middlesex County, Conn. Wangunk, "round rock," or "heavy rock."

Torube Pond, Knox County, Me. Old Abnaki, "snapping-turtle," Chelydra serpentina, or (along the coast) a large sea-turtle, a terrapin. Also any of the edible Emydidae inhabiting fresh or brackish water. In Montauk dialect, torup; in Delaware, tulpe.

Torup Pond, New Haven County, Conn. Quinnipiac? Mohegan? "snapping-turtle," or "terrapin." See Tolbabawk, Toulbah, Torube.

Toskaunk Meadow, Kent County, R. I. Narragansett, "a bridge," or "a ford." Also Toskeunke, Toskiounke, etc.

Toskibunke Providence County, R. I. Narragansett, "a wooden bridge."

Totant An ancient village near Boston, Suffolk County, Mass. Same as Topeent? or possibly from Natick, "(the stream) winds about."?

Totapoag Norfolk County, Mass. An ancient Massachusetts village. Natick, "twisted pond." 
Totawamscut Kent County, R. I. Narragansett, "at the crossing, by means of (stepping) stones."

Totheet Suffolk County, Mass. An ancient village near Boston. Probably same as Topeent, but possibly Natick, "beating place," where wild flax was beaten, or corn pounded?

Totoket Mountain, New Haven County, Conn. Western Niantic, "on the great tidal stream." Variant, Totocott, Titicut, q. v.

Totomak Brook, Fairfield County, Conn. Mahican, "a meadow that trembles" when walked on.

Totonnock Somerset County, Me. Abnaki, "shaking or quaking place," or "a quaky place, a bog."

Touissett Neck, Bristol County, R. I. Narragansett, "fording place." Another source gives "worn-out fields." ?

Touisset Bristol County, Mass. Wampanoag, "fording place."

Toulbah Mountains, Penobscot County, Me. Abnaki, "snapping turtle." See Torup.

Touskounkanet Providence County, R. I. Narragansett, "an enclosure near the (stone) fording place." See Totawamscut.

Towansett Neck, Bristol County, Mass. Narragansett, "at the wading place (or ford)." Same as Touisset and Toweset.

Towantic Village, New Haven County, Conn. Quinnipiac, "wading place in the river," or "ford across the river."

Towantuck Pond, Middlesex County, Conn. Wangunk, "fording place in the river," or "at the wading place."

Towcocks Windham County, Conn. Nipmuck, "cold place" or "cold weather;" or possibly "at the wading place."

Towequensuck New London County, Conn. Mohegan, "at the long wading-place," or "at the long ford."

Toweset Neck, Mount Hope Bay, Bristol County, R. I. Narragansett, "near the old fields"? or "at the place of pines"? See Touisset.

Towessek Sagadahoc County, Me. Abnaki, "broken passage." Also Tussek.

Towtaid Worcester County, Mass. Nipmuck, "open, solitary, deserted field."

Towtenhemon Hartford County, Conn. Tunxis, named after the Sachem of Poquonnock, Towtenhemon, circa I650. 
Towunucksett River, Hampden County, Mass. Nipmuck, "at the ford." Another meaning, "bridge of stepping stones," or possibly "mortar place;" where corn was pounded.

Towwoh York County, Me. Abnaki? "corn grinding place"? or "damp place?" But see Towessek. Variant, Towow.

Toyaskquit River, Providence County, R. I. Narragansett, "place of the bridge."

Toyusk New London County, Conn. Narragansett, "a bridge." Formerly a crude stepping-stones arrangement near Groton.

Tsabakwa Knox County, Me. Abnaki, "I hide my provisions," or, "I cache my food."

Tuck Mountain, Waldo County, Me. Doubtful; if Indian, probably Abnaki, "a tree." But more likely from English.

Tuckachawan Windham County, Conn. Nipmuck, "mist over the wooded hill."

Tuckernuck Island, Nantucket County, Mass. Wampanoag, "a round loaf of bread," because of its appearance. (It also resembles togguwhonk, "a mortar;" and it lies among ketah tugooak, "great waves.") Also, Tuckanuck, Tuckonoc.

Tueskennckinck R. I., location uncertain. Probably Narragansett, "a fording place."

Tug several mountains by this name in New England. All of them derive from the Natick word m'tughk, "a tree." A Maine locale may be from the cognate Abnaki, meaning "a tree," or possibly, "river."

Tugemaug Norfolk County, Mass. Natick, "place of grinding."

Tuladi Brook, Aroostook County, Me. Malecite? "the nammycush?" See Togue.

Tulamdie River, Aroostook County, Me. Abnaki? Malecite? "canoe on the sandbar."

Tulanic Stream, Aroostook County, Me. Abnaki, "place where they make canoes."

Tumpum Pond, Plymouth County, Mass. Wampanoag, "carrying strap"? (possibly English "hillocks" or "heaps"?)

Tunipus Beach and Pond, Newport County, R. I. Narragansett, "tortoise, or turtle." Another source says "small herring." Any of these would fit. 
Tunk Lake, Mountain and Stream, Hancock County, Me. Abnaki, "principal or large swift stream," abbreviated from k't-hunk; possibly English.

Tunkahoosen Brook, Tolland County, Conn. Mohegan, "fast flowing brook"? or "fast flowing gravelly stream"?

Tunxis Hill, Fairfield County, Conn. Wangunk, little river." Perhaps abbreviation of Tunckseasapose, "fast flowing and winding stream."

Tunxis River, Hartford County, Conn. See Tunxis-sepos.

Tunxishoag Tunxis Island, New Haven County, Conn. Paugussett, "at the place of the small stream." Another account of this place indicates the name was tuckahoe (Indian turnip or Jack-in-the-pulpit), roots which could be roasted and eaten like bread.

Tunxis-sepos Hartford County, Conn. Wangunk, "fast flowing little stream."

Tuppatuett Plymouth County, Mass. Wampanoag, "twisting river," or possibly "twisted outlet"?

Turramuggus see Terramuggus.

Tuscomanset Plymouth County, Mass. Wampanoag, "fording place at the point."

Tuskatucket River, Kent County, R. I. Narragansett, "at the ford (or wading place) in the tidal stream."

Tuskheganuck New London County, Conn. Mohegan, "where we cross the river by wading." Also Tusheganuck.

Tuttomnest Neck, Barnstable County, Mass. Wampanoag, "twisted place." Another source gives "blueberry place."

Tuxis or Tooksees New Haven County, Conn. Quinnipiac, "little stream," or "little river."

Tuxisshoag Pond New Haven County, Conn. Quinnipiac, "land at the little river." Variants, Tuxishocke, Tuxishog, Tuchshishhoag.

Tyetilegogtakook the Batten Kill, Bennington County, Vt. Mahican? "country around the river of toads."?

Tyontkathotha Mount Philo, Chittenden County, Vt. Moharek, "lookout place."

Tzawapa Knox County, Me. Old Abnaki, "a crossing place in a river or stream," that is, "a ford." Also Tzawipi. 
Uckatimes Island, Dukes County, Mass. Wampanoag, "small neck at the end."

Ueques Worcester County, Mass. Nipmuck? Mohegan? "this is the end, or boundary."

Uhquanchaug Windham County, Conn. Nipmuck, "at the end of the hill," or possibly "hook-shaped hill."

Uhuio New London County, Conn. Mohegan, "it curves and winds about." Also Uhuhyock.

Uhuponckash New London County, Conn.? Western Niantic, "pipes for tobacco (ceremonial pipes?)." See Calumet.

Ukquiessa Neck, Dukes County, Mass. Wampanoag, "at the end."

Ulmsaket Ponds, Aroostook County, Me. Malecite, "where the points meet each other." See Umsaskis.

Umbagog Lake, Oxford County, Me., and Coös County, N. H. Abnaki, "clear lake." Also Umbagoög. See Wambighe.

Umbawaug Pond, Fairfield County, Conn. Paugussett; Umpewag was an Indian chief who signed a deed for Stamford lands in I66o.Perhaps the name meant "conqueror, to whom tribute is brought." Also Umpawag, Umpawaug, Umpewaug.

Umbazookskus Stream, Piscataquis County, Me. Abnaki, "clear, gravelly outlet."

Umcolcus Deadwater Lake and Stream, Penobscot and Aroostook Counties, Maine. Abnaki, "a whistling duck." Variants, Umcolquis, Umcoleus, Umkolkwees, etc.

Umpachene River, Berkshire County, Mass. Mahican, said to be derived from the name of a chief. Sometimes translated as "top man," sometimes as "conqueror."

Umpame Plymouth County, Mass. Wampanoag, "resting place," or perhaps "turn around."

Umpammonoosuc Brook, now Oliverian Stream, Grafton County, N. H. Abnaki, "at the bare (treeless) extended deep outlet."

Umpanawang Hill and Pond, Fairfield County, Conn. Paugussett, "beyond the bend," or if Umpanamaug, "beyond the fishing place."

Umpewage Fairfield County, Conn. Pangussett, "beyond the bend." 
Umpog Creek, Fairfield County, Conn. Paugussett, "a fishing place"? or "beyond the bend"?

Umsaskis Lake, Aroostook County, Me. Malecite, "points which run out to meet each other," or in reference to the chain of ponds there, "linked together like sausages." Variants, Umasaskis and Ulmsaket.

Umsquaquospem Washington County, Me. Malecite, "first lake" we come to, going upstream; Big Machias Lake, or Lower Sabao.

Umsquattanack Hampden County, Mass. Nipmuck, "at the beaver or muskrat domes," or perhaps "rocks sloping down place," also "place at the end of the village or mountain."

Unaganek Washington County, Me. Abnaki, "at the portage," near Lubec.

Uncachewalunk Pond, Worcester County, Mass. Nipmuck, "brook at the end of the hill," (where we make dishes?), or "place of acorns," ? or "place beyond the good hill." Also spelled Uncachewhalomaug and Uncachewalumock.

Uncanoomuc Mountain, Hillsboro County, N. H. Pennacook, "at the end of the fishing place," or "beyond the fishing place."

Uncanoonuc Hill, Middlesex County, Mass. Pennacook, "breast," or "nipple."

Uncanoonucks Mountain, Hillsborough County, N. H. Pennacook? "a breast," or "nipple."

Uncas ville, New London County, Conn. Mohegan. Uncas was the family name of a powerful chief of that tribe; his name is a corruption of Wonkus, "fox" (literally, "the circler.") Another name for this same person was Poquiam, "The Defender," I588?-I683?

Uncataquisset Norfolk County, Mass. Natick, "at the end of the small stream," or "place of overturned rocks."

Uncatena Island, Dukes County, Mass. Wampanoag, "like a hill," or "end of the hill," or "shows from afar."

Uncawannuck Dukes County, Mass. Wampanoag, "fishing place at the furthest place."

Unchechewhaton Pond, Worcester County, Mass. Nipmuck, "at the end of the wooded hills."

Uncheckcathaton Pond, Worcester County, Mass. Nipmuck, "cedar mountain." 
Unchemamuck Hartford County, Conn. Wangunk, "fishing place for eels." See Onshaukamaug.

Uncoa Fairfield County, Conn. Paugussett, "beyond," or "over yonder." Variants, Uncowa, Uncoway, Uncowah, etc.

Uncushnet River, New London County, Conn. Mohegan, "at the end of the rapid stream." Variants, Ungoshet, Unquoshnet.

Ungowa-umsock Middlesex County, Conn. Mohegan, "abode of old squaws, long tailed ducks."

Ungwonshackook New London County, Conn. Mohegan, "place at the bend or fork of the stream." Also Unggwongskakooke, Unggwonsuckcook.

Unjaware possibly in York County, Me. Abnaki, "sheltered place"? or "end of the portage."?

Unkapaukook Litchfield County, Conn. Mahican, "land at the end of the pond," or "place at the bend in the pond."

Unkawa Fairfield County, Conn. Probably Paugussett, the short form of Uncawamuck, "beyond the fishing-place." Variant, Unquowa, Uncoa, etc.

Unkety Brook, Middlesex County, Mass. Natick, "at the end, or boundary place."

Unquamonk Hill, Hampshire County, Mass. Nipmuck, "at the end place" or "at the boundary."

Unquawomuck Kent County, R. I. Narragansett, "beyond the fishing place" or "the fishing place farther away."

Unquety Hills, Norfolk County, Mass. Natick, "boundary," or "(hills which are) the boundary."

Unsatuit Plymouth County, Mass. Wampanoag, "the place of flooding."

Unshemamuck Pond, Hartford County, Conn. Tunxis? "eelfishing place." See Onshaukamaug.

Upaquoag Woods, Hartford County, Conn. Wangunk, "land where flags (or rushes) grow." See Abaquage.

Uppanash Konameset Dukes County, Mass. Wampanoag, "covering mats at the long fishing place," or "flags (or rushes) used for making mats, at the large fishing place."

Uppooquantup New Haven County, Conn. Western Niantic? "eat (bass) head." Sea-bass heads and fine corn meal make a sort of "head-cheese." This name may be comparatively recent; 
it is near Momauguin. There was another such place near Niantic.

Upquedopscook River, Aroostook County, Me. Abnaki, "top of the rocks place."

Usgah River, Maine. Abnaki? "bitter water."?

Usquaiok River, Hampden Coounty, Mass. Nipmuck,"at the end of this land," or "boundary place."

Usquepaug Village, Washington County, R. I. Narragansett, "at the end of the pond." (In the early I80o's attempts were made to link this with the Gaelic word for whiskey, usquebeotha; there is no connection). Variants, Usquebaug and Usquepaugh.

Ussowwack Franklin County, Mass. Nipmuck, "at the end-place," or "at the boundary." Also translated as "seething pot."

Usuntabunt Piscataquis County, Me. Abnaki, "wet head"? (now Rainbow Lake). But if a corruption of Nesuntabunt, the meaning is "three heads."

Utlooskes Washington County, Me. Malecite, "cast a small net there,"? or "soak small hides there."? This is the Little River, near Perry; perhaps the name is just "small stream in that direction," or "go to the small cove."?

\section{$-\mathbb{W}-$}

Waashacum Ponds, Worcester County, Mass. Natick, "surface of the sea." Also translated as "springs bubbling forth."

Wabacosoos Lake, York County, Me. Abnaki, "white, shining."

Wabademsolduwak Wajowuk Coös County, N. H. Penobscot, "The White Mountains."

Waban Middlesex County, Mass. Natick, "the wind," especially "the east wind."

Waban Lake, Norfolk County, Mass. Natick, "east wind."

Wabaquasset Tolland County, Conn. Nipmuck? "a place covered with rush matting," possibly a house. One source gives "tabernacle covered with rush thatching," perhaps one of Eliot's meeting houses; he had spelled it Wabuhquohish.

Wabaquasset Providence County, R. I. Narragansett, "flags or rushes for making mats." 
Wabaquassick Windham County, Conn. Nipmuck, "place of the white stones,"? or "mats for covering a lodge"? Also Wabasquassuck.

Wabaquassit Worcester County, Mass. and Tolland County, Conn. Nipmuck, (at the place where we make) "mats for house coverings." Variant, Wabaquassuck.

Wabaquassuck see Wabaquasset.

Wabasgach Washington County, Me. Abnaki, "light colored boards," or "white planks" (now Robbinston Mills).

Wabash Pond, Worcester County, Mass. Natick, "the white things" (white stones?); or Narragansett, "the winds?"

Wabassus Lake, Washington County, Me. Malecite, "shining," or "torchlight." Variant, Wabasses.

Wabeno bahntuck Penobscot County, Me. Abnaki, "white water falls," or "the foamy white cataract."

Wabenock Sagadahoc County, Me.? Abnaki, "the white beach place."

Wabigenek at Kendall Head, Washington County, Me. Abnaki, "white bone." This is a quartz ledge which resembles a large white bone, at a distance.

Wabiggamus Abnaki village, Penobscot County, Me. Abnaki, "the little clear lake." Also Warbeggamus.

Wabissick Avenue, Middlesex County, Conn. Wangunk, "at the little white place," or "at the small place eastward." Wabissick was a female chief or Suncksqua.

Wabossagock Liberty Point, Robbinston, Washington County, Me. Abnaki, "white planks place."

Wabquisset an ancient village, "west of the Quinebaug River," Windham County, Conn.; or the same place as Wabaquasset?

Wabuhquosish same as Wabaquasset, q. v.

Wacagasaneps Plymouth County, Mass. Wampanoag, "house and adjoining fields."

Wacatuc Worcester County, Mass. Natick, "bend in the river," or "at the end of the river." Also Wacantuck, Wacuntug.

Waccago ancient Abnaki village, possibly Cumberland County, Me.? Abnaki, "head of the bay."

Wachamaucutt Plymouth County, Mass. Wampanoag, "hilly land." 
Wachamotusset Plymouth County, Mass. Wampanoag, "at the barren brooklet," or "at the end of the brooklet."

Wachaqueage New London County, Conn. Western Niantic, "country near the mountain."

Wachemscusset Brook, Plymouth County, Mass. Wampanoag, "corn fields place."

Wachipauke Pond, Grafton County, N. H. Abnaki, "mountain pond."

Wachocastinook Creek, Litchfield County, Conn. Mahican, "at the place where walnut trees grow on the hill," or possibly "land at the stony hill."

Wachpusk Plymouth County, Mass. Wampanoag, "hilly ridge" or "hills back."

Wachquatnach Litchfield County, Conn. Mahican, "extending to the mountain," or "the end of the mountain." Also, Wechquadnach.

Wachu New Haven County, Conn. Quinnipiac, "a hill, or mountain."

Wachusett Mountain, Reservoir and State Park, Worcester County, Mass. Natick, "near the mountain."

Wackquigut Back Bay, Cumberland County, Me. Abnaki, "at the end of the bay." See Wakogumaak.

Wacobske Cliff, Dukes County, Mass. Wampanoag, "a shining rock."

Wacuntug Worcester County, Mass. Nipmuck, "a bend in the river." A Nipmuck village, Wacuntuc, was located here in the late I6oo's. See Wacatuc.

Wadadeag see Watatick.

Wadatatuk Middlesex County, Conn. Hammonasett, "treeboughs"? or "river from uttermost distant land"? See Woodtick and Mattatuck.

Wadawannuc Point, New London County, Conn. Eastern Niantic? "a place destitute of trees."? Other spellings Wadawanak, Wattawanett, Woddowonuck, etc.

Wadchusett see Wachusett.

Waddaquodduck see Wataquadock.

Wagumcaquog Middlesex County, Mass. Natick, probably same as Maguncaquog, "place of the gift." 
Waguntaquab Pond, Nantucket County, Mass. Wampanoag, "sandy gravel."

Wagwises pastures near Forest Dale, Rutland County, Vt. Abnaki, "a fox."

Wahconah Falls and Park, Berkshire County, Mass. Mahican, "daybreak,"? or Moharok, "the old fort."?

Wahkasekhoc Penobscot County, Me. Abnaki, "at the small enclosure," or "at the place of the small pelt-frames."? ?

Wahktoohook Berkshire County, Mass. Mahican, "place of the river bend."

Wahnoosnook Worcester County, Mass. Nipmuck, "deep brook," from monoosuck.

Wainooset Hills and Stream, Worcester County, Mass. Nipmuck, "a deep brook." See Monoosuck.

Wajoses Pigeon Hill, Washington County, Me. Abnaki, "little mountain."

Wakalosen Fort Knox, Waldo County, Me. Abnaki, "rocks in a circle;" hence an enclosure, a fort.

Wakeag see Waukeag.

Wakepeke Brook, Worcester County, Mass. Nipmuck? "he pitched his tent here," or "reeds for making houses"? or "dwelling place"?

Wakogumaak Back Bay, Cumberland County, Me. Micmac, "at the end of the bay." See Wackquigut.

Wakoquet Barnstable County, Mass. Wampanoag, "house place."

Waktiompsk Middlesex County, Conn. Wangunk, "rock house," or "dwelling among the rocks," or possibly "rock cave dwelling." See Watiomsquit.

Walastegw see Wallastook.

Walintuk Stream, Penobscot County, Me. Abnaki, "cove in the brook," or "bend in the stream."

Walkamigosek Penobscot County, Me. Abnaki, "sand scooped out." This term is sometimes applied to geological "kettle holes."

Wallagrass Lake, Stream and Village, Aroostook County, Me. If this is Indian, perhaps Micmac, "good river" (easy canoe traveling); or Abnaki, "shallow, full of coves."

Wallamanticuk see Willimantic. 
Wallamanumps Falls, Hampden County, Mass. Nipmuck, "red cliffs," or "red steep rocks."

Wallamanumpscook Worcester County, Mass. Nipmuck, "at the rock standing in the red-paint place," an important boundary marker.

Wallamatogus Hill, Hancock County, Me. Abnaki, "coves in a little river."

Wallastook the St. John River, Aroostook County, Me. Micmac, "the beautiful river." Also Woolastook.

Wallempteweekek South Twin Lake, Piscataquis County, Me. Abnaki, "place of the deep round lake." Some Indians give it as, "coves surrounded by burned land." Also Wallenipteeweekek.

Walloomsac River, Bennington County, Vt. Mahican, "beautiful rocks," or "paint rocks."

Wallum Pond, Lake and Village, Providence County, R. I. and Lake, Worcester County, Mass. Nipmuck, "a dog." See Allum Pond.

Walopeconek Windham County, Conn. Nipmuck, "at the good little plantation," ? or "at the end of the plantation."?

Wamappahesett location unknown. If Natick, possibly "at the place of the field with small snares."

Wambighe now Umbagog Lake, Coös County, N. H. Abnaki, "clear lake." This name from Aubéry's I7I5 map.

Wamesit Middlesex County, Mass. Natick, "there is room for all." This was a council place of the Pawtuckets.

Wamesut Middlesex County, Mass. Natick, "place beloved of all."

Wamkeag Providence County, R. I. Narragansett, "white land," or "white place."

Wammasquid Nantucket County, Mass. Wampanoag, "at the plain."

Wampaketatekam Plymouth County, Mass. Wampanoag, "clear gushing spring," or "white sandy fountain."

Wampanic sepoot River, Berkshire County, Mass. Mahican, "at the white (clear) stream."

Wampanoag Lake, Worcester County, Mass., and Trail, in Providence and Bristol Counties, R. I. Wampanoag, "people from the east." This was a major tribe of the Plymouth-Nantucket- 
Martha's Vineyard region, important in early colonial history. Actually a confederacy of several sub-groups, they are one of the few tribes in southern New England to maintain their own identity to the present day.

Wampanucket School, Plymouth County, Mass. Wampanoag, "at the dawn place," or "at the east," also "place of white earth." Also, Wapanucket.

Wampatuck Norfolk County, Mass. and Pond in Plymouth County, Mass. Natick, from the name of the famous John Wampatuck of Braintree, who aided Pilgrims in I62I. Translated as "white river," "eastern river," or "goose."

Wampecack Creek, Bennington County, Vt. Natick? "place of white (fruit), chestnuts." (Or "brant goose place"?)

Wampee Pond, Litchfield County, Conn. Paugussett, "clear water."

Wampenum Brook, Berkshire County, Mass. Mahican, "white falls"? or "coming from the east."?

Wamphanack Avenue, Middlesex County, Conn. Wangunk, "place of the wild goose," or possibly "(look to) the place eastward."

Wamphaneeskitt Plymouth County, Mass. Wampanoag, "place where little white shells abound;" also "white meadow place."

Wamphassuck Point, New London County, Conn. Eastern Niantic, "at the eastern bog," or perhaps, "walnut trees here." Given also as "marshes" and "wet meadows." Variant, Womphasset.

Wampi-hussun-ac River, Windsor County, Vt. Natick? "clear stony place." See Pompanoosuc.

Wampnesick Providence County, R. I. Narragansett, "place of chestnut trees" (womppimish)? or "place at or in the east." ?

Wampum Norfolk County, Mass. Natick, "white stuff." This is the collective name of the white beads or "peag" used as currency. Although the purple (or "black," as they are also known), beads used in barter and in ceremonial "wampum belts" were properly called sauhock, English settlers called these wampum, too.

Wampum Swamp, Plymouth County, Mass. Natick? Wampanoag? "white stuff," white beads used for barter and ceremonial purposes. 
Wampum's Rock, Norfolk County, Mass. Natick, from an Indian family named Wampum which lived near this boulder.

Wamsutta (or Mooanum) Bristol County, Mass. Wampanoag, Wamsutta was one of Massasoit's sons, known to the English as Alexander. He died in I662. One translation of Wamsutta is, "he has a kind heart." See Wamesut.

Wanagamesswak especially in Penobscot County, Me. Abnaki, "rock fairies." These "wee people" were supposed to be friendly to the Abnaki; they made arrowheads, claystones, etc., and warned of approaching enemies, especially raiding Mohawk warriors.

Wanascohockett Plymouth County, Mass. Wampanoag, "at the end place," or "at the hill marking the plain's end."

Wanascottaquet River, Bristol County, Mass. Wampanoag, "land (or place) at the end (or source) of the tidal river."

Wanashack Brook, Litchfield County, Conn. Mahican, "at the end-place," or "at the source."

Wanashquodtinook Berkshire County, Mass. Mahican, "at the top of the mountain," or "summit place."

Wanasquatomska River, Plymouth and Bristol County, Mass. Wampanoag, "rock summit."

Wanasquatuck Bennington County, Vt. Abnaki, "head or source of the river." (Headwaters of Otter Creek.)

Wanasquatuckqut Providence County, R. I. Narragansett, "at the end of the tidal river."

Wancenquag Brook, Plymouth County, Mass. Wampanoag, "at the bend or turn." Variants, Wankinquog, Wankonquag, Wankinco, etc.

Wanchatopek Pond, Worcester County, Mass. Nipmuck, "where to get roots" (for sewing, etc.), or "roots soaked in water"? Also Wanketopic, "crooked roots place"?

Wanepoonseag Providence County, R. I. Narragansett, "place where the nets are set" (for fish? for birds?). More likely, "where the brook floods." See Wawepoonseag.

Wangam Brook and Lake, Litchfield County, Conn. Paugussett, "bend." Also Wangum.

Wangan River, Piscataquis County, Me. Abnaki, "the bend." 
Wangombog Tolland County, Conn. Nipmuck, "crooked pond." Spelled also Wangunbog. See Wongumbaug.

Wankewan Lake, Belknap County, N. H. Abnaki, "on the crooked route."

Wankinco River, Plymouth County, Mass. Wampanoag, "the bend in the stream," or "crooked rocks place"?

Wannacomet Pond, Nantucket County, Mass. Wampanoag, "at the beautiful field," ? possibly "pond field"? or "beautiful house."?

Wannametonamuc Hill, York County, Me. Abnaki, "it produces red earth," or "at the red paint hill." See Tonemy, and Wannemetonamy.

Wannamoiset Neck, Bristol County, Mass. Narragansett, "good (fishing and) resting place," or "place at the point." Also Wanomoycet.

Wannamoisett Kent County, R. I. Narragansett, "at the good fishing place."

Wannashowatuckqut Worcester County, Mass. Nipmuck, "at the fork of the river."

Wannemetoname York County, Me. Abnaki, "red paint hill."

Wannemetonomy Newport County, R. I. Narragansett, "good mountains." ? Chief Tonomy was sachem of Aquidneck in r638. See Wannemetoname.

Wannomack Ponds, Worcester County, Mass. Nipmuck, "grape country." Variants, Wanomchonck, Wannumchunk.

Wannomoiset Washington County, R. I. Narragansett, "at the good fishing place." Variants, Wanamoyset, Wanomoyset, Wannamoiset, etc.

Wannuchecomecut Neck, Washington County, R. I. Narragansett, "enclosed camping place," or "plantation at the end of the hill."

Wannuppe Island, Litchfield County, Conn. Mahican, "good water," or "good pond."

Wanomoycet see Wannamoisett.

Wanonkapooke Litchfield County, Conn. Mahican, "crooked pond," or "land at the bend of the pond."

Wanoosnuc see Monoosmoc.

Wanotimyes River, Middlesex County, Mass. Natick, "pleasant village," ? or "good land."? 
Wanpawcutt Pond, Bristol County, Mass. Narragansett, "place of flags or rushes."

Wanquanchet Plymouth County, Mass. Wampanoag, "at the bend" of the stream.

Wanshuk Brook, Providence County, R. I. Narragansett, "at the steep place." Wanskuk and Wanshuck.

Wanskuck Pond, Providence County, R. I. Eastern Niantic, "at the end-place."

Wansokett see Woonsockett.

Wantastiquet Mountain, Cheshire County, N. H. Abnaki, "at the end of the river."

Wantastiquet the West River, Windham County, Vt. Pennacook, "head or source of the river."

Wanungatuck Brook and Hill, Windham County; Conn. Mohegan, "winding river," or "at the river bend."

Wapanaki Lake, Caledonia County, Vt. Abnaki, "from the east," or "from the dawn land."

Wapanoos Point, Washington County, R. I. Eastern Niantic, "place over east,"? or "east wind blowing"?

Wapanucket see Wampanucket.

Waping Addison County, Vt. Delaware? "an opposum"? Or possibly a misspelling of Wapping, "east land" or "east place" ?

Wapoompaukset Plymouth County, Mass. Wampanoag, "waiting or resting place at the small pond."

Wapososhequash Hill, Windham County, Conn. Nipmuck? "white grasses"? or "white fruits;" that is, "chestnuts"?

Wapowage see Wepowage.

Wappanuckett Plymouth County, Mass. Wampanoag, "place of white earth." See Wampanucket.

Wappaqua Brook, Windham County, Conn. Nipmuck, "flags or cat tails."

Wappaquassett Pond, Windham County, Conn. Nipmuck, "at the place of flags or cattails."

Wappewassick Island, now Prudence Island, Newport County, R. I. Narragansett, "at the narrow straits." Variant, Wapewasick.

Wapping Road, Newport County, R. I. Narragansett, "east land," or "dawn place." See Wapping, Conn., below. 
Wapping Village, Hartford County, Conn. Sicaog? "east land," or "dawn-land;" same meaning as Abnaki.

Wapping Plymouth County, Mass. Wampanoag, "in the east" or "dawn place."

Wappinger Confederacy; a federation composed of many small Algonquian-speaking bands located chiefly in New York, but extending into Connecticut south and west of Hartford. The name means, "easterners." They joined the Stockbridge peoples following the King Philip War. The Wappinger were prominent in the manufacture of wampum.

Wappoquian's Brook, Windham County, Conn. Nipmuck, from the name of a Nipmuck Indian, possibly "the white plume." Wenepaykin, alias Sagamore George, was in Massachusetts I6I6-I684.

Wapskenigan Washington County, Me. Abnaki, "white rocks portage." Variant, Wapskehagan.

Waptook Lake, Washington County, Me. Malecite, "wild goose."

Wapwayset Providence County, R. I. Narragansett, "narrow place, forded at low water." Also, Wapwaysitt, Wepwassit, etc.

Waqua Point, Dukes County, Mass. Wampanoag, "at the end."

Waquaick Oak Bay, Washington County, Me. Abnaki? Malecite? "at the head of the bay."

Waquapaug see Weekapaug.

Waquepaug see Wecapaug.

Waquettaquage Nantucket County, Mass. Wampanoag, "pond at the end." Also Waquittaquay.

Waquoit Bay and Village, Barnstable County, Mass. Wampanoag, "at the end." Also Waquoid.

Waquompohchukoit Plymouth County, Mass. From Wampanoag, "boundary mark corner."

Waqutuquaib see Waquettaquage.

Waramanticut see Willimantic.

Waramaug Lake and State Park, Litchfield County, Conn. Mahican, "good fishing place." Variant, Warramock.

Warametonomy Hill; see Wannemetonomy.

Waronoco Hampden County, Mass. Nipmuck? "winding about." Also Woronoco, Worrinoke, etc. 
Warramanticut see Willimantic.

Warramaug a sachem of Weantinock. See Werewaug.

Warunlug see. Waentug.

Warunscopage see Maruscopag and Achetaqupag.

Waruntug Worcester County, Mass. Eastern Niantic? "a good tree."

Wasaketuwen Bristol County, Mass. Wampanoag, "torch place"?

Wasapskotock Hampden County, Mass. Nipmuck, "at the shining rock." (But possibly "flax or hemp at this river." ?)

Wasa-umkeag Sears Island, Waldo County, Me. Abnaki, "at the shining sandy beach." Also Wasumkeag and Wassamkeag.

Washakamaug Village, Middlesex County, Mass. Natick? Delaware? "fishing place for eels or lampreys."

Washakum see Weshacum.

Washaqua Hill, Dukes County, Mass. Wampanoag, "place beyond, on the other side," or "place at the end." A boundary-mark.

Washiack Hartford County, Conn. Mohegan, "at the mountain." This was one of the boundary marks of the Mohegans.

Washining Litchfield County, Conn. Mahican, "mountain country" or perhaps a corruption of Ossining, "stony land." Variant, Washee.

Washouset Point, Long Neck, Pawtuxet Cove, Providence County, R. I. Narragansett, "place of the small hill." But see Washquisset.

Washqua Outlet, Dukes County, Mass. Wampanoag, "at the end."

Washquisset Point, Providence County, R. I. Narragansett, "boundary brook."

Washucke River, Rockingham County, N. H. Abnaki, "at the hilly place."

Washukquatom Hill, Providence County, R. I. Narragansett, "summit of hill."

Washwantonowmoh Hill, Windham County, Conn. Nipmuck, "hill where testimony was taken."? Also Washwantownowmoh.

Washwantowminunk Hill, Windham County, Conn. Nipmuck, "hill where the large strawberries grow."? See Washwantonowmoh.

Waskosing Rock, Dukes County, Mass. Wampanoag? Narragansett? "whalebones." 
Wasquadomesit River, Providence County, R. I. Narragansett, "at the end of the hill,"? or "summit of the hill"?

Wasquakage Franklin County, Mass. Nipmuck? Pennacook? See Squakheag. Variants, Wussquackheag, Squakheag, etc.

Wasque Bluff and Point, Dukes County, Mass. If Wampanoag, "at the end;" if Narragansett, "whalebone," from waskeke.

Wasquonasak New London County, Conn. Mohegan, "place at the end of the rock" or "rocky point." See Obscob.

Wass Island, Washington County, Me. Abnaki, "shiny," or "white."

Wassabastegu the White River, Orange and Windsor Counties, Vt. Abnaki, "clear water stream," "white river," and "shining river."

Wassaic Stream, Litchfield County, Conn. Mahican? Delaware? "rocky land."

Wassamkihemuk Penobscot County, Me. Abnaki, "at the white sandy place." This is High Head, Bangor, Me.

Wassapacoassett Bristol County, Mass. Narragansett, "place of pine roots,"? or "rat place"?

Wassaquoick Lake, Piscataquis County, Me. Abnaki, "a clear, shining lake," possibly where fish were speared by torchlight. Also, Wassataquoick.

Wassassabskek Rapids, Penobscot County, Me. Abnaki, "at the slippery rocks."

Wassataquoick Stream, Penobscot County, Me. See Wassaquoick Lake.

Wassategwewick the East Branch of the Penobscot River, Penobscot County, Me. Abnaki, "at the place in the river where fish are speared by torchlight," or "at the bright sparkling stream."

Wassaticook Stream, Penobscot County, Me. Abnaki, "at the place of the shining, clear stream."

Wassiog Hartford County, Conn. Mohegan, "hill place," or perhaps "place or country beyond."

Wassokeag Lake, Penobscot and Somerset Counties, Me. Abnaki, "shining fishing place," or "at the whitefish place;" perhaps, "fishing by torchlight."

Wassoosumpsquehemock Island, Marshe's Island, Penobscot County, Me. Abnaki, "slippery rocks place." 
Wassquagos Pleasant River, Washington County, Me. Abnaki? Malecite? "shallow, rough," or "low, rough, overflowed."

Wassuc Hartford County, Conn. Wangunk, "between the forks" of Cold Brook and Roaring Brook, at Glastonbury. See Assawassuck.

Wassumke Wadjo White Cap Mountain, Piscataquis County, Me. Abnaki, "white sand hill."

Wassup Lake, Washington County, R. I. Narragansett, "clear stream."

Wataba Lake, Fairfield County, Conn. Mahican, "roots," the kinds of roots used for cord and for thread in sewing canoes.

Watachun Spring, Kent County, R. I. Narragansett, "on a hill" or "on a hillside."

Watagua a reedy shallow stretch in Lake Fairlee, Orange County, Vt. Abnaki, "pickerel."

Watananock Hill, Worcester County, Mass. Nipmuck, "place where the river winds around the hill," or "bend in the river at the hill."

Watanic now Nashua, Hillsboro County, N. H. Abnaki, "climbing place," or "end of the mountain."

Watannanuck Hill, Hillsboro County, N. H. Natick, "the place where we climb."

Watanock River, Worcester County, Mass. Natick, "land near the hill."

Wataqua now Nashua River, in Worcester County, Mass. and Hillsboro County, N. H. Abnaki, "a pickerel."

Wataquadock Brook, Hill and Road, Worcester County, Mass. Nipmuck, "place where we get fire-wood; branches of trees;" or perhaps "lookout place."

Watatick Pond and Mountain, Worcester County, Mass. Nipmuck, "a mountain stream," or possibly "mountain covered with trees." Also perhaps, "wigwam river," or "lookout place."

Watcha Pond, Dukes County, Mass. Wampanoag, "a hill."

Watchamoquot Village, Providence County, R. I. Narragansett, "place of the great spring"? But see Watchemoket.

Watchaug Pond, Washington County, R. I., and Brook, Hampden County, Mass. Nipmuck, "hill country." 
Watchemoket Cove, Providence County, R. I. Narragansett, "end of the fishing place." See Watchamoquot.

Watchemoquit Cove and Neck, Bristol County, Mass. Narragansett, "end of fishing place," or "end of the cove." Also, Watchymoquett, Watchimoquet, etc.

Watchemoyket see Watchamoquot.

Watchic Pond, York County, Me. Abnaki, "at the mountain." Variants, Watchig, Wachuk, Wadehuck, etc.

Watchogash Tolland County, Conn. Mohegan, "near the mountains," or "mountain country."

Watchoog Brook, Tolland County, Conn. Nipmuck, "at the hill," or "hilly country."

Watchquadnach Litchfield County, Conn. Mahican, "at the end of the mountains." Other spellings Wechquadnach, Wequagnock, etc. This was the site of a Moravian mission around I750.

Watchusesic Hill, Worcester County, Mass. Natick, "at the small hill."

Waternomee Mountain, Grafton County, N. H. Pennacook? Abnaki? "a place to climb."

Waterquechee Falls, Sullivan County, N. H. Same as Ottauquechee Falls, Vt.

Waterqueechy River, Windsor County, Vt. In this form the name is not Indian, but archaic English for "moist, damp, boggy ground." This is the same stream as the Ottauquechee River.

Watesamoonsuck Hill, Washington County, R. I. Eastern Niantic, "other side of the outlet"? or "junction of brooks."?

Watoolwan Gamock St. John Pond, Somerset County, Me. Abnaki, "good hunting from a canoe around this pond."

Wattamatogue see Wallamatogus.

Wattankshausepo an old name for Farmington River, Hartford County, Conn. Wangunk, "fast flowing stream with many turns." Also, Tunxis, Tunkseese, Tunckseapose.

Wattanumon Brook, Merrimack County, N. H. Named for a Pennacook chief who died near Concord, I632.

Wattiomsk Middlesex County, Conn. Mohegan, "at the end of the rock" or "reef's end" or "rocky point." Also Watiomsque, Wattiomsquit, etc. 
Wattoonug River, Fairfield County, Conn. Mahican, "rocky place"?

Watunkshausepo Hartford County, Conn. Same as Tunxis, q. v.

Watuppa Pond, Tolland County, Conn. Nipmuck, "roots to be used in sewing," or "where we sit and talk"?

Watuppa Pond, Bristol County, Mass. Wampanoag, "roots" to use in sewing.

Waubansconcett Middlesex County, Mass. Natick? Pennacook? "place of white rocks."

Waubeoka Lake, Fairfield County, Conn. Mahican, "a crossing place."

Waubeyaussuc New London, Conn. Eastern Niantic, "a crossing place," "a passage" or "a strait." See Weybosset, R. I.

Waubosset see Weybosset.

Wauchimoqut see Watchamoquot.

Waukeag Neck, Hancock County, Me. Abnaki, "a great knoll."

Waumbek Mountain, Coös County, N. H. Chippewa, "place of the white rock."

Waumbekket Coös and Carroll Counties, N. H. Abnaki? "at the place of the white rocks."

Waumbekketmethna Coös and Carroll Counties, N. H. Abnaki? Chippewa? "principal hills at the white rocks place;" the White Mountains.

Waumpanickseepoot Berkshire County, Mass. Mahican, "place of a white foamy waterfall in the river."

Waunashqua Nantucket County, Mass. Wampanoag, "at the end," or "summit."

Waunnakeseag possibly at one of the Casco Bay Islands, Cumberland County, Me. Abnaki, "place of the finely painted ones;" that is, "place of the mackerel."

Waupanheeskit Plymouth County, Mass. Wampanoağ, "small white falls."

Wauregan Pond and Village, Windham County, Conn. Mohegan, "a handsome thing."

Waurenaug Fairfield County, Conn. Paugussett, "good place." Waushacum see Weshacum.

Wauwaskepog see Usquebaug. 
Wauwinet Nantucket County, Mass. Wampanoag; Wauwinet was an early sachem and counselor at Nantucket in I660. The name approximates, "witness my hand," or "testify."

Wauwonoquassick Fairfield County, Conn. Siwanoy? "witness rocks," ? or "witness rock boundary place."?

Wawalona Providence County, R. I. Narragansett, said to be derived from the name of Wawaloam, the wife of Chief Miantonnomoh, prior to 1643 .

Wawashekit Providence County, R. I. Narragansett, "place of carrying burdens on backs," that is, "a portage."

Wawayontatt Plymouth County, Mass. Wampanoag, "winding creek."

Wawaytick Creek, Dukes County, Mass. Wampanoag, "winding tidal creek."

Wawecoes Brook, Litchfield County, Conn. Mahican, "meandering or twisting."? Also, Wawecoos, Wawwecous.

Wawecus Hill, New London County, Conn. Mohegan, Wauwecus (Wauweekus or Wawequa) was a brother of the famous Uncas circa I6oo. The name may mean "a swan," or "moonlight."

Wawela Park, Worcester County, Mass. Pennacook, "white ducks."?

Wawenock Knox, Lincoln and Sagadahoc Counties, Me. Abnaki, "bay country" or "inlet places." Until I73o (?) populated by Walinakiak, "people of the bay country" who moved to Canada. The last known speaker of the Walinakiak dialect died at Becancour, Québec, in IgI9. See Weeweenock.

Waweonk Creek, Kent County, R. I. Narragansett, "twisting stream," ? or "place of testimony," ? or "the bay or cove."

Wawepoonseag Providence County, R. I. Narragansett, "place where the stream overflows." (This is sometimes given as "place where birds are snared;" but such words are a description of what happened there, rather than a translation of the name.)

Wawobadenik the White Mountains, Coös, Grafton and Carroll Counties, N. H. Abnaki, "at the place of the white mountains." This name is also applied to Mt. Marcy, in the Adirondacks, New York.

Wawog Pond, New London County, Conn. Nipmuck, "crooked pond." 
Wawona Bristol County, Mass. Narragansett? "the turn."?

Waworamawak New London County, Conn. Mohegan, "where the path.turns," or "place of turning." Also, Wanwaraneanauk and Warronawarack.

\section{Wawoskepog see Usquepaug.}

Wawuttaquatuck Washington County, R. I. Eastern Niantic? Narragansett? "twisting, turning, meandering tidal stream."

Wawyachtanock Litchfield County, Conn. Mahican, "place at the bend of the river"

Wayanitoke Point Judith, Washington County, R. I. Eastern Niantic, "twisting current"? or "waves around a bend"?

Waybosset Neck; see Weybosset and Waypoiset.

Waylumkituk Penobscot County, Me. Abnaki, "place of the round sandy cove."

Waymessick Middlesex County, Mass. Natick, "winding brook," or "distant brook."

Waypoiset Narrows, Newport County, R. I. Narragansett, "at the narrow place or strait."

Wayunkeag Hill; see Wayunkeke.

Wayunkeke Hill, Providence County, R. I. Nipmuck, "at the bend," or "land at the bend."

Waywaypounshag Bristol County, Mass. Narragansett, "twisting outlet."

Wdamoganaspskak Aroostook County, Me. Abnaki, "at the place of the pipe rocks."

Wdepsek Isle aux Têtes, in the Richelieu River north of Franklin County, Vt. Abnaki, "heads." Abnaki and Mohawk warriors exhibited heads of their enemies on this island.

Weahtaqua Dukes County, Mass. Wampanoag, "end of the creek."

Weakpocoink Hill, Plymouth County, Mass. Wampanoag, "at the end of the pond," or possibly "house poles place."

Weantinock Fairfield County, Conn. Paugussett? "where the water swirls and tumbles around a hill." Variants, Weantinoque, Wiantenuck, Wyantenug, Weantinaug.

Weantucket River, New London County, Conn. Eastern Niantic, "at the winding stream." Also Weatucket.

Weataug see Weatogue. 
Weatauwanchu Mountains, Litchfield County, Conn. Mahican, "wigwam shaped (dome shaped) mountain."

Weatic Litchfield County, Conn. Mahican, "at the (village of) wigwams." Also Wiatic.

Weatogue Hartford County, Conn. Tunxis, "at the wigwams place," or "he is at home there." This Weatogue is at Simsbury. Also, Wetawk.

Weaxcashuck Tolland County, Conn. Mohegan, "at the end of the meadow or swamp." Variants, Wexcashuck, Weexhashuck, Wixhassock, etc.

Webatuck Stream, Litchfield County, Conn. Mahican, "narrow stream,"? or "brant goose"?

Webhannet River and Village, York County, Me. Perhaps Abnaki, "at the clear stream"?

Webomskat New London County, Conn. Mohegan, "narrow passages between rocks,"? or "at the white rock"? See Wasquonasak and Obscob.

Webotuck Litchfield County, Conn. Paugussett, "river in the narrow pass, or gorge."

Wecapaug Brook, New London County, Conn. Eastern Niantic, "head of the pond," or "end of the pond." Also Wequapaug.

Wecatheconnet Kent County, R. I. Narragansett, "place at the end of the enclosed field," or "house in the enclosed field."

Wechkotetuk Union River, Hancock County, Me. Abnaki, (free translation), "going upstream, the canoeist is suddenly confronted by an impassible barrier." Literally, "coming out facing river falls."

Wechquadnach see Wachquatnach.

Wechquapackat Berkshire County, Mass.? Mahican, "at the extreme point." Variants, Wickquapubon, Wichquapuchat, and Weekwapawcutt.

Wecketuckett Brook, Plymouth County, Mass. Wampanoag, "at the end of the stream" or "wading river."

Weckwannuck Brook, now Sugar Loaf Brook, Hampden County, Mass. Nipmuck, "at the end of the hill."

Wecoachett Meadow, Barnstable County, Mass. Wampanoag, "at the end of the hill," or possibly "basswood hill place."

Wecobaug see Wickabaug. 
Wecobemeas New Haven County, Conn. Quinnipiac, "basswood," or "linden" or "tying-bark fiber."

Wecopesuck a small rocky island at the end of Fisher's Island, south of Stonington, New London County, Conn. Narragansett, "small object at the end." Also, Weekapeesuck, Wickaposset, Wicopesset, etc.

Wecquaesgeek Fairfield County, Conn. Paugussett? "at the end of the march." Sometimes given as (probably incorrectly) "birch bark kettle," or "birch bark place." This tribe was an important member of the Wappinger Confederacy, and was almost totally annihilated by a Dutch party in I643 led by Capt. Underhill. Variants, Wechgaek, Wickerscreeke, and Weghquagsike.

Wecuppemee Stream, Litchfield County, Conn. Mahican, "linden or basswood inner bark" (wikopi), used for netting, string, rope, etc.

Wecups Hartford County, Conn. Tunxis, "linden or basswood"? Also Wekups.

Wedebegek Knox County, Me. Malecite? Micmac? "head of the bay," the beginning of Penobscot Bay.

Weeataug Litchfield County, Conn. Paugussett? "place of the dwelling," or "at the wigwam." This place was near Salisbury; there was a Moravian mission built here around I750. Also Wehtack, Wehtak, Wiatack, etc.

Weechagaskas Norfolk County, Mass. Natick, "place at the end of the rocks," or perhaps "rushes or flags for making mats."

Weecodnoy Nantucket County, Mass. Wampanoag, "at the end of the hill."?

Weegoschook New London County, Conn. Mohegan, "near the mountain with birches on it." Also Wegasoeguck; but see Wachaqueage.

Weekaasuck Island, Middlesex County, Mass. Wampanoag, "place of the small house, or small wigwam," or "small wigwam near the outlet." Also, Weecasuk.

Weekachommet see Wecatheconnet.

Weekapaug Point, Village, Inlet and Pond, Washington County, R. I. Narragansett, "at the end of the pond."

Weekasoak Brook, Rockingham County, N. H. Natick? Abnaki? "place of small dwelling" (near outlet?) 
Weepatuck Mountain, Litchfield, County Conn. Mahican, "at the narrow passage, or gorge."

Weepecket Dukes County, Mass. Wampanoag, possibly from Waubackuck, "white headed eagle," or "osprey."?

Weepoiset Kent County, R. I. Narragansett, "at the small ford," or "the narrow strait."

Weequakut Barnstable County, Mass. Wampanoag, "at the end."

Weequancett Neck, Plymouth County, Mass. Wampanoag, "the place of the torch-light."

Weeset Barnstable County, Mass. Wampanoag, "slippery place," or "shiny place."

Weesquobs River, Barnstable County, Mass. Wampanoag, "shining rocks." These at the river mouth? There is a hint of torch-light along the stream, perhaps spearing eels?

Weetamoe's Crossing, Bristol County, Mass. Wampanoag, "the lodge keeper," or "house keeper," or "house wife." ? At this place Weetamoe was drowned in 1676 ; see below.

Weetamoo Mountain, Grafton County, N. H. Weetamoo (or more commonly, Weetamoe) was the suncksqua, "female chief," of the Pocassets.

Weeweantitt River, Plymouth County, Mass. Wampanoag, "the crooked river."

Weeweeder Ponds, Nantucket County, Mass. From Wampanoag? "winding about."

Weeweenock Lincoln County, Me. Probably the same as Wawenock, but Weeweenock is cognate with Natick words meaning "turning, bending, meandering" probably in reference to shore line. Other spellings Wewoonoc, Wewenock, etc. See Weweantit, Weweantic, etc.

Wegquapamisk Tolland County, Conn. Nipmuck, "at the rock summit," or "at the end of the rocks" or "rocks at end place." This was a boundary-mark at the western end of the Wabaquasset country.

Weguadnach see Wachquatnach.

Weikeset Island, Middlesex County, Mass. Wampanoag, "place of the little tent or wigwam." See also Weekasoak.

Weinshauks New London County, Conn. Pequot, "great rock cliff." 
Wekapekatonnic Brook and Hill, Worcester County, Mass. Natick, "hill at the end of the divided brook"? or "end of pond near hill"? or "dwelling near the split mountain."?

Wekapekatonnuc Plymouth County, Mass. Wampanoag, "end of pond near the hill," or "hill at end of the branched stream."

Wekopee Pond, Litchfield County, Conn. Mahican, "wigwam or dwelling near pond," or "house near water." See also Wicopee.

Wekowamadensees Bird Mountain, Rutland County, Vt. Abnaki, "little mountain shaped like (a hemispherical) Indian dwelling."

Wekwabigek Little River (Utloskes), Washington County, Me. Malecite, "end of the tidal part."

Welokinbacook Lake, Androscoggin County, Me. Abnaki, "bay and cove place;" or "many curves and indentations." See also Wonunkapaugkook.

Wembemiscook Worcester County, Mass. Nipmuck, "place of chestnuts," or "place of small white fruit." Also Wombemisiscook, Wombemiscunck, Wombomesscock, etc.

Wemesuck Brook, Litchfield County, Conn. Mahican? "council place."

Wenachus Lake, Essex County, Mass. Natick, "a small bowl"? or "chimney"? or "good hill"? perhaps, "berry hill"? Also, Wennuchus.

Wenaniasoug Windham County, Conn. Mohegan? "end of (our) country;" a boundary mark.

Wenashoge Brook, Litchfield County, Conn. Mahican, "grapes country"?

Wenatuxet Stream, Plymouth County, Mass. Wampanoag, "place of the good brook." Also, Wenatukset, Winnetuxet, etc.

Wenaumet Bluffs, Barnstable County, Mass. Wampanoag, "at the good fishing place,"? or "grapes here"?

Wenekei-amaug Hartford County, Conn. Agawam, "at the end of the fishing place," or "boundary fishing place." See Congamond.

Wenekocamaug Lake, Hartford County, Conn. Tunxis, "near the boundary lake," or "fishing place at the boundary."

Wenimessett Worcester County, Mass. Nipmuck, "at the good fishing place"? or "grape vines here."? 
Wenk wida wiwie wak Hermon Pond, Penobscot County, Me. Abnaki, "where stream enters, goes down." See Edawimaniwick.

Wennanatoke Point Judith, Washington County, R. I. Eastern Niantic, "winding river."? See Weynanitoke.

Wentukset Stream, Plymouth County, Mass. Wampanoag, "at the good tidal stream."

Wepansock Hartford County, Conn. Tunxis, "narrow place," or "ravine"?

Wepaug Tolland County, Conn.? Nipmuck? "edge of the pond." Wepawaug Reservoir and River, New Haven County, Conn. Paugussett, "narrows," or "crossing-place."

Wepecket see Weepecket.

Wepoiset Bristol County, Mass. Narragansett, "at or near the little ford."

Wepoiset Providence County, R. I. Narragansett, "at the little ford, or crossing place."

Wepowage New London County, Conn. Perhaps Mohegan?"at the narrows," or "at the crossing place." Variants, Weepowaug, Wypewoke, etc.

Wequadnack Litchfield County, Conn. Mahican, "at the end of the mountain."

Wequaes Worcester County, Mass. Nipmuck, "at the end or boundary." Variant, Uquaes.

Wequagawaysuk First Falls, Eddington Bend, Penobscot County, Me. Micmac or Malecite, "head of the tide."

Wequanhausick Hampden County, Mass. Nipmuck, "at the end of the neck," or perhaps "abode of swans,"? or "at the end of the meadow"?

Wequanoc River, Plain and Island, Middlesex County, Conn. Western Niantic, "end of the hill."

Wequanunk Fairfield County, Conn. Paugussett, "stump of a tree," or perhaps "mortar for pounding corn" made out a tree stump.

Wequapaug Brook, New London County, Conn. Narragansett, "at the end of the pond." Also Wecapaug, Weakapauge, Wekopag, Wickapog, Wequapoag, etc.

Wequapaugset Kent County, R. I. Narragansett, "at the end of the small pond." 
Wequaquet Lake, Barnstable County, Mass. Wampanoag, "the shining place"? or "torchlight place"? Possibly "swampy place"?

Wequarunch New Haven County, Conn. Quinnipiac, "an upright rock at the end."

Wequash Pond, now Wigwam Pond, Belknap County, N. H. Narragansett, "a swan." A Pequot sachem of this name died at Saybrook, Conn., in I642. Also Wicwas, Wickwas.

Wequashacke Dukes County, Mass. Wampanoag, "place of brightness, as of torches."

Wequashcook New London County, Conn. Western Niantic, from the name of an Indian, Wequash-cuk, "it is probably true."? But the roots indicate "place of the swans," or "bright shining place."

Wequatuck Washington County, R. I. Eastern Niantic, "at the end of the river."

Wequatucket Cove and River, New London County, Conn. Eastern Niantic, "at the end of the tidal stream."

Wequatuxet Cove, Washington County, R. I. Narragansett, "at the end of the small stream."

\section{Wequechackomuck see Wecatheconnet.}

Wequeenuck Swamp, New London County, Conn. Mohegan, "at the end of the hill."

Wequehacke Berkshire County, Mass.? Mahican, "at the end of the territory (or tract)."

Wequepamish Windham County, Conn. Nipmuck, "place at the end of the rocks."

Wequepogue Washington County, R. I. Eastern Niantic, "end of the pond," or "head of the pond."

Wequetequock New London County, Conn. Mohegan, "place at the end of the tidal stream," or "as far as the river goes."

Wequitaquage Nantucket County, Mass. Wampanoag, "place at the end of the tidal stream," or "as far as the tidal stream goes."

Wequittayaug Hampden County, Mass. Pocumtuck, "land at the end of the cove." Variant, Wequettayag, "place at the end of the stream."

Wequobsket Cliffs, Dukes County, Mass. Wampanoag, "place at the end of the rocks." Given also as Wequobscut. 
Weraumaug Litchfield County, Conn. Mahican, "good fishing place."

Werewaug Fairfield County, Conn. Mahican, "good fishing place."

Weronke Hampden County, Mass. Nipmuck, "winding about." See Woronack, Woronoco, etc.

Wescogus Pleasant River, Washington County, Me. Abnaki, "shallow, rough, flowing over."

Wesconnaug see Wishquodiniack.

Wescunck Fairfield County, Conn. Paugussett, "a stone pounding mortar," for making corn meal.

Wescussauco Essex County, Mass. Pennacook? "end of the rocks." Also, Wessacaucon, Wessacumcon, Wissacumquan.

Wescussue Fairfield County, Conn. From the name of a Paugussett Sachem; possible meaning, "he makes earthen pots."?

Wesgustogo Cumberland County, Me. Abnaki, "at the mouth of the river."

Weshacum Worcester County, Mass. Pennacook, "surface of the sea." See Waashacum, Washakum.

Weshokastaneek see Wachocastinook.

Weskeag Creek, Knox County, Me. See Wessaweskeag.

Wesquacksaug Brook, Windham County, Conn. Nipmuck, "end place," or "source."

Wesquadomeset Sayles Hill, Providence County, R. I. Narragansett, "place at the end of the hill"? or "place of walnut trees"? (from wussoquatomiset).

Wesquage Pond, Washington County, R. I. Narragansett, "the end place;" a cove? (If Wisqussuk, the meaning would be "maker of clay pots.")

Wesquantook New Haven County, Conn. Quinnipiac, "source of the river," or possibly "as far as this place."

Wesquo Nantucket County, Mass. Wampanoag, "white, shiny stone" or "edge of cliff"? Other spellings Wesco, Wesko, etc.

Wesquobsk Cliffs, Dukes County, Mass. Wampanoag, "at the end of the cliff" or perhaps "white shiny rocks." Also found as Weskobs.

Wessagusset Beach, Norfolk County, Mass. Natick, "at the small salt water cove, or outlet," or "place at the edge of the rocks." Also Wessaguscus, Wessaquscus, etc. 
Wessappicoassett Plymouth County, Mass. Wampanoag, "edge of the resting place at the pine grove."

Wessaweskeag Knox County, Me. Abnaki, "tidal creek at the peninsula." Now Weskeag. Variant, Wessamesskek.

Wesserunsett Stream, Somerset County, Me. Abnaki, "bitter water place"? Also, Wesserunsicke and Wesseronsaëg.

Westconnaug Brook and Reservoir, Providence County, R. I. Narragansett, "place where walnut trees grow on the hill"? See Wesquadomeset.

Westenhoek Berkshire County, Mass., and Litchfield County, Conn. This is not Indian, but Dutch; it is sometimes given as Western Chuck, "west corner;" often confused with Housatonic.

Westgustoqua Barnstable County, Mass. Wampanoag, "place at the end of the river." Also given as "end of the cliff."

Westotucket Washington County, R. I. Eastern Niantic, "at the river's end," or "as far as the end of the stream."

Westquodniake see Wesquadomeset.

Wesumpsha Path, Middlesex County, Conn. Wangunk? Natick? "shining or glistening rocks."

Weswick Sagadahoc County, Me. Abnaki, "tide runs out, salt bay here," or "commonly bordered with salt-marsh growth, creek runs dry or nearly so at low tide." Also Whiskeag.

Wetauk see Weatogue.

Wetau Wadchu Brook and Mountain, Litchfield County, Conn. Mahican, "dwelling place on the mountain," or "wigwam shaped mountain." Also, Wetawanchu, Weetauwachon, Wetautauwachon, etc.

Wetchi sankasek Sunkhaze Stream, Penobscot County, Me. Abnaki, "concealing outlet."

Wethunganet Creek, Kent County, R. I. Narragansett, "house on the other side."

Wetuset see Wachusett.

Wewaskepaug see Usquebaug.

Weweantic River, Plymouth County, Mass. Wampanoag, "crooked stream," or "stream that wanders about."

Weweantit Washington County, Me. Abnaki? Natick? "winding, twisting river." 
Weweeder Creek, Nantucket County, Mass. Wampanoag, "winding about."

Wewensett Plymouth County, Mass. Wampanoag, "at the abode of horned creatures," probably deer.

Wexcodowa New London County, Conn. Pequot-Mohegan, "as far as the end of the brook." See Wexquapaug.

Wexquapaug New London County, Conn. Eastern Niantic, "as far as the pond (extends)."

Weybosset see Wapwayset.

Weynanitoke Point Judith, Washington County, R. I. Eastern Niantic, "a sweep around a high point"” or "winding river."?

Weyouchaug Windham County, Conn. Nipmuck, "as far as the mountain" or "at the end of the mountain." Also given as Weyeouchaug, Weyouchaugguck.

Whakepee Barnstable County, Mass. Wampanoag, perhaps wickopy or "tying-bark;" possibly "swamp."

Whichquopuhbau Berkshire County, Mass. This is in the southwest corner of the state. Mahican, "at the end of the flat place," or "at the end of the fork in the trail." ? Also, Wichquapuchat.

Whiskeag Creek, Sagadahoc County, Me. Abnaki, "creek runs nearly dry at low tide."

Wianno Barnstable County, Mass. Wampanoag, from the name of Chief Iyannough (or Wianno), "He who wages war."

Wianumcisses New London County, Conn. Eastern Niantic, "good little fish." Also, Wyanemesis.

Wiashquagwunsuck Tolland County, Conn. Mohegan, "good brook at the end of the meadow."

Wiatik Litchfield County, Conn. Mahican, "village of wigwams." Also Weatic, Wiatik.

Wichetseconnet see Wecatheconnet.

Wichigaskitaywick Wiscasset, Lincoln County, Me. Abnaki, "at the river outlet."

Wickabaug Pond, Worcester County, Mass. Natick, "at the head of the pond." Also, Wickabog, Wickapogue, etc.

Wickaboxet Windham County, Conn. Nipmuck, "end of the small pond," or "as far as the small pond."

Wickaboxet Pond and State Forest, Kent County, R. I. Narragansett, "at the end of the small pond." 
Wickapicket Brook, Worcester County, Mass. Nipmuck, "among the basswoods," or "where we get wickopy (tying-bark)." Possibly. from Sachem Wickobema, whose name means "basswood." But see also Wickabaug.

Wickasauke Island, Middlesex County, Mass. Natick? Pennacook? "house at the end of the outlet." Also, Wickasaukee, Wicosuck, Wehquasuck, etc.

Wickataquay Dukes County, Mass. Narragansett, "at the end of the pond," or possibly "the house at the end."

Wicketaquock see Wequatucket.

Wicketiquack see Wequatucket.

Wickwas see Wequash.

Wicopee Hill, Windham County, Vt. Abnaki, "tying bark." This is the bast from which cord is made.

Wigudi River, Aroostook County, Me.? Old Abnaki, "canoe."?

Wigwam Perhaps a score of place names in New England incorporate this term, as Wigwam Hill, Wigwam Pond, Wigwam Brook, etc. All of them derive from the Algonquian wigwam, meaning "a dwelling, habitation, or lodge." These were rounded or conical structures usually covered with bark or rush matting, or occasionally, hides. They are not the buffalohide tipi of the Plains peoples.

Wikapokotownow Worcester County, Mass. Nipmuck, "village at the end of the pond," or "hill at the end of the divided brook"? See also Wekapekatonnic.

Williguaganum Grand Lake, Washington County, Me. Abnaki? Malecite? "good lake but so shallow I have to drag my canoe."

Willimansett Hampden County, Mass. Nipmuck, "place of red earth,"? or "good berries place."?

Willimantic Reservoir and River, Tolland County, and City, Windham County, Conn. Mohegan, "good cedar swamp." Also Waramanticut, Wallamanticuk, Wewemantic, Weammantuck.

Willimantic Village, Piscataquis County, Me. Nipmuck, "good cedar swamp."

Wimpeting Litchfield County, Conn? Mahican, "ruinous heap,"? or "pile of discarded goods." ? 
Winatompic Washington County, R. I. Eastern Niantic, "place at the top of the rock," ? or "place at the end of the rocks."?

Wincheck Pond, Washington County, R. I. Nipmuck, "at the pleasant place."

Wincittico River, Shepscot River, Lincoln County, Me. Abnaki, "crooked river," or "winding river."

Winechaug Mountain, Hampden County, Mass. Nipmuck, "good mountain;" but if Minnechaug, "berry mountain."

Winegegwok Island Pond, Cheshire County, N. H. Abnaki, "abode of otters." Sometimes found as Wnegigwak.

Winichahanet at Dover Point, Strafford County, N. H. Abnaki, "current flows around this place." Also, Winnechahanett.

Winicowett Windham County, Conn. Nipmuck, "place of good pines."

Winkompaugh Brook and Village, Hancock County, Me. Abnaki? "a good enclosed pond."

Winnapauckett Plymouth County, Mass. Wampanoag, "at the good pond."

Winnapaug Pond, Washington County, R. I. Narragansett, "a good pond."

Winnapauket Kent County, R. I. Narragansett, "land or place at the good pond."

Winnatuxet Brook, Norfolk and Plymouth County, Mass. Natick, "at the good brook." Also Winnatuckset.

Winneagwamuk Bagaduce River, Hancock County, Me. Abnaki, "around the place where we pull the canoes out," or "at the portage-place."

Winneamkeak Washington County, Me. Abnaki, "at the rough, sandy bank."

Winneashimut Suffolk County, Mass. Natick, "at the good spring." (Otherwise Winnesummet. These are old names for Chelsea.)

Winneasquam Essex County, Mass. Natick, "beautiful red rock summit."

Winnebassakek near South Wolfeboro, Carroll County, N. H. Abnaki, "portage at the outlet."

Winnechahannet Rockingham County, N. H. Abnaki, "current flows around this place." 
Winnecook Village, Waldo County, Me. Abnaki, "at the portage."

Winnecott or Winnecowet River, Rockingham County, N. H. Pennacook, "where water flows out." Sometimes translated, "at the portage."

Winnecumek near Hampton Falls, Rockingham County, N. H. Abnaki, "narrow, closed-in portage." Also, Winnecumet and Winnecunnet.

Winnecunnet or Winneconnet Bristol County, Mass. Wampanoag, "at the place of the good (or beautiful) pines." ?

Winnegance East Boothbay, Lincoln County, Me. Abnaki, "the little portage." Also at Bath, Sagadahoc County, Me. Variants, Winneqansek, Winneqansege, Winniqans, Winnaganset, etc.

Winnegance Bay and Creek, Sagadahoc County, Me. Abnaki, "little portage," or "little carrying place."

Winnekeag Lake, Worcester County, Mass. Nipmuck, "good fishing place."

Winnemaug Lake, Litchfield County, Conn. Mahican, "good fishing place."

Winnemisset Brook, Worcester County, Mass. Nipmuck, "place of grape vines." Also Winnimisset.

Winnemoiset Norfolk County, Mass. Natick, "good resting place."

Winnepesaukee Lake, Belknap and Carroll Counties, N. H. This name, which has more than one hundred acceptable spellings, is Pennacook. Two of the most reasonable translations are "land around the lakes," and "land at outlets around here." Some favor Winipisiocket, "at the good lake marsh," or "good black earth around the lake." See pages 6-7.

Winnepocket Lake, Merrimack County, N. H. Abnaki, "at the portage from the pond." Another spelling is Winnepauket.

Winnepuck Fairfield County, Conn. Paugussett? "good water" or "good pond."

\section{Winnesummet see Winneashimut.}

Winnetuxet River, Bristol County, and Brook, Plymouth County, Mass. Wampanoag, "at the good little river," or "near the good brook."

Winnianebiskek Alton Bay, Belknap County, N. H. Abnaki, "the land around the lake." The late Chester A. Price's translation is "where water flows down from the narrow part of the lake." 
Winnicutt Great Bay, Rockingham County, N. H. Abnaki, "at the portage." Also, Winnicott.

Winnimisset Brook, Worcester County, Mass. Nipmuck, "near the grape vines." Another source gives "deep place or valley."

Winnipauk Village, Fairfield County, Conn. Mahican, "beautiful pond." Winnipauk was a Sachem of Norwalk in I640.

Winnipeseekett Essex County, Mass. Pennacook, "land of good water all around" ? or Natick, "place of good outlets." ?

Winnisimmet Suffolk County, Mass. Natick, "fine spring." Named for Chief Winnisimmet, Sachem of Chelsea in I625.

Winnisquam Lake, Belknap County, N. H. Abnaki, "salmon fishing around here" or "salmon fishing at lake outlet."

Winnocks Neck, Cumberland County, Me. Abnaki, "good place."

Winona Belknap County, N. H. Chippewa, "first born daughter." In Longfellow's poem, Winona was Nokomis' daughter, and the mother of Hiawatha.

Winooskeek an ancient village at the mouth of the Winooski River. Abnaki, "wild onion place."

Winooski City and River, Chittenden and Washington Counties, Vt. Abnaki, "wild onions."

Winooskitook literally the Winooski River in Vermont. Abnaki, "wild onion river."

Winotimies River, Middlesex County, Mass. Natick, "single deep place," or "solitary valley."

Winsachewett New London County, Conn. Eastern Niantic, "place of the good mountain,"? or "at the brink or edge of mountain cliff."?

Winskeag Otter Creek Point, Mt. Desert Island, Hancock County, Me. Abnaki, "long point."

Winsochook New London County, Conn. Mohegan, "at the rocky cliff"? or "at the mountain"?

Wintechog Hill, New London County, Conn. Eastern Niantic, possibly a modification of Minnechog, "berry place."

Wintoak the lower part of the Lamoille River, Chittenden County, Vt. Abnaki, "bone-marrow river"? Also Wintaak, "bad sounding."

Wintucket Cove, Dukes County, Mass. Wampanoag, "at the good tidal creek." 
Winyah Bay, Dukes County, Mass. Modified name of Winyaw Indians, a now extinct and little-known Siouan tribe of North Carolina.

Wionhege "land at the bend." See Wayunkeke.

Wionkhiege see Wayunkeke.

Wiquasseck Litchfield County, Conn. Mahican? "place at the end of the marsh."

Wisawogamak the narrows between Ferrisburg, Addison County, Vt. and Essex County, N. Y. Abnaki, "straits or narrows."

Wiscasset Village, Lincoln County, Me. Abnaki, "at the hidden outlet." See Wetchi Sankasek and Wichigaskitaywick.

Wiscogosis Lower Sabao Lake, Washington County, Me. Abnaki, "lakes strung along."

Wisconemuck Pond, Hillsboro County, N. H. Pennacook, "place of walnut trees"? or "pottery making place."?

Wishoea Pond, Plymouth County, Mass. Wampanoag, "at the end," ? or "kettle shaped." Variant, Wishoca.

Wishquagawans Windham County, Conn. Nipmuck, "mist over the end of the meadows."

Wishquodiniack Windham County, Conn. Nipmuck, "place of the walnut trees." Also, Wishquatenniog, Wussoquatak, etc.

Wisquades New London County, Conn. Mohegan, "little earthen pot."

Wissatinnewag Franklin County, Mass.? Mahican, "slippery hill"? or Nipmuck, "shining hill" ? This was an ancient village somewhere on the Connecticut River, I663.

Wiwiliamecq the dreaded "monster-of-the-water" which features in Maine and New Brunswick Indian legends. The death-thrashings of this horrible beast keeps the water roily in Boyden's Lake, Washington County, Me. Present day Indians derisively call common snails by this name.

Wiwinam River, Litchfield County, Conn. Mahican, "winding"? Or modified Paugussett, "grape." ? See Weweantic.

Wnahtookook Berkshire County, Mass. Mahican, "land at the end of the river." Sometimes translated, "great meadow near the river." Also Wnoghquetookoke.

Wnoghquetookoke Berkshire County, Mass. Mahican, "land at the end of the river (great meadow)."? 
Wobegilseebis Goose Creek, Addison County, Vt. Abnaki, literally "goose creek."

Woboostook Baker Stream, Somerset County, Me. Abnaki, "white water (or clear water) river."

Wochokieskquas Tolland County, Conn. Mohegan, "place near the mountains."

Wochsquammuguck Brook, New London County, Conn. Mohegan, "salmon fishing place."

Woepecket see Weepecket.

Wojahosen Rock Dunder, Chittenden County, Vt. Abnaki, "Forbidder's Rock." This boulder, sometimes called "Guardian Rock," figures prominently in folk lore.

Wokonocob River, Plymouth County, Mass. Wampanoag, "edge (or end) of the rocks" (or "curved ledge" ?)

Wollamansak sepe Hampden County, Mass. Nipmuck, "redearth country stream."

Wollimosset see Molligwasset.

Wolomonopaug Norfolk County, Mass. Pennacook? "red paint pond"? Also Wolomopoag.

Wolomopoag Pond, Norfolk County, Mass. Nipmuck, "beautiful pond" or "shallow pond," (perhaps "dog pond"?).

Wolopeconnet Pond, Washington County, R. I. Eastern Niantic, "shallow enclosed cove," or perhaps "fine cleared land."

Wombemando Island, Penobscot County, Me. Abnaki, "white devil." This term was applied to Maj. Robert Rogers of the Rangers, who surprised and burned St. Francis in I759.

Wombemesisacook Worcester County, Mass. Nipmuck, "place of the white fruit, or white nuts;" chestnuts. Also Wombamsicunk, Wombameescock, etc.

Womenshenick Brook, Litchfield County, Conn. Mahican, "place of steep rocks."

Wonalancet Mountain and Village, Carroll County, N. H. Pennacook, Wonalancet, "The Governor," was a great Pennacook chief who led his people to new homes in Canada during the middle I670's.

Wonasquam see Annisquam.

Wonasquatuckett Stream, Plymouth County, Mass. Wampanoag, "at the end of the tidal stream." 
Wonastitguk meadows at West River, Windham County, Vt. Abnaki, "place at the end of the river."

Wonchesick see Woonsechocksett.

Wonckompsk Brook, Hampden County, Mass. Mahican? "end of the rocks," or "rocky bend"? Also given as Wanckompss.

Wongateeg Tolland County, Conn. Mohegan, "place at the bend."

Wongatuck or Wongattuck, see Wanungatuck.

Wongonshock see Wongunshoake, below.

Wongum Middlesex County, Conn. Wangunk, "a bend."

Wongumbaug Lake, Tolland County, Conn. Mohegan, "overflowed pond," or Nipmuck, "crooked pond."

Wongun Hampshire County, Mass. Nipmuck, "the bend."

Wongunk Hartford County, Conn. Wangunk, "the bend" in the Connecticut River at Glastonbury. This gave the name to the Wangunk tribe which controlled a large area.

Wongunshoake Hartford County, Conn. Mohegan, "place at the bend." See Ungwonshackook.

Wonickcomquacke River, Plymouth County, Mass. Wampanoag, "long crooked marsh," ? or "meadow swamp place"? Another spelling, Wonocomquake.

Wonkees-ohke "fox country;" another name for King Philip.

Wonkemaug Pond, New London County, Conn. Mohegan, "fish curing place at the bend of the river."

Wonketopic see Wanchatopeck.

Wonkituck Windham County, Conn. Nipmuck, "crooked river."

Wonkkecomaug see Wonkemaug.

Wonksacoxet see Woonksechocksett.

Wonksunkamug Pond, Hartford County, Conn. Mahican? "fishing place at the bend in the stream."

Woonashquoom see Annisquam.

Wonnehockset Merrimack County, N. H. Pennacook, "at the place of the beautiful trees." Variants, Hooksett, Onnahookset.

Wonnumetonomy see Wannemetonomy.

Wonococomaug Pond, Hartford County, Conn. Nipmuck, "fish weir" ? or "fishing place"?

Wonokakeetookeese Little Otter Creek, Addison County, Vt. Abnaki, "little river abode of otters." 
Wonomenok Pond, Cheshire County, N. H. Now Monomonock Lake, q. v.

Wononke New Haven County, Conn. Quinnipiac, "hollow place;" probably one of the glacial kettle holes.

Wononkpakoonk Litchfield County, Conn.? Mahican, "an open place;" from Wunnompamukquok, or Wunnompeukonat?

Wononpacook Lake, Litchfield County, Conn. Mahican, "land at the bend of the pond."

Wononsco Lake, Litchfield County, Conn. Mahican, "place at the bend of the pond."

Wononskopomuc Lake, Litchfield County, Conn. Mahican, "rocks at the bend in the lake."

Wonunkapaugkook Lake, Litchfield County, Conn. Paugussett, "rocky point where the lake bends."

Woodtick New Haven County, Conn. Quinnipiac? "boughs, branches, or limbs," (wudtuckquash) or "piece of wood," (wudtukqun.)

Wookutcakoospa Barnstable County, Mass. Wampanoag, "at the top of the rocky cliff," ? or "rock-lined pool or well." ?

Woolamonuppoque Norfolk County, Mass. Natick, "red pond."

Woolastookwaguamok Baker Lake, Somerset County, Me. Abnaki, "near the source of the beautiful river," (the St. John's River).

Woonachasset Coasters Harbor Island, Newport County, R. I. Narragansett, "crooked little hill,"? or "at the place of separation;" that is, a boundary mark?

Woonasquatucket Park, Reservoir and River, Providence County, R. I. Narragansett, "at the head of the tidal river" (as far as the tide goes, not the source?)

Woonksechocksett Worcester County, Mass. Nipmuck, "fox country." See Chocksett. Another spelling, Wonksacoxet.

Woonsocket City, Hill and Reservoir, Providence County, R. I. Nipmuck, "place of steep descent." (Or "two brook place," from Niswesocket.)

Wopowage Worcester County, Mass. Nipmuck, "at the narrows," or "at the crossing place."

Wopowog Middlesex County, Conn. Wangunk, "a crossing place." 
Woquogonset Washington County, R. I. Narragansett, "at the end of the plain," or "as far as the place at the plain."

Woronock New Haven County, Conn. Quinnipiac, "turning place" or "winding stream"? Also Oronoke. See Woronoco.

Woronoco Hampden County, Mass. Nipmuck, "winding about." Other spellings, Woronoack, Worrinoke, Warronoco, etc.

Worromotogus Kennebec County, Me. Abnaki, "coves in brook," near Pittston. See Wallamatogus.

Worumbo Aroostook County, Me. Abnaki, "a cove or bay."

Woruntuck an ancient Massachusetts village; location uncertain. Mahican? "winding river."

Wotchaugh Washington County, R. I. Eastern Niantic, "hill place."

Wotesamoonsuck Pond, Washington County, R. I. Narragansett, "junction of brooks."

Wowoskepaug see Usquepaug.

Woxadowa see Wecapaug.

W'takantschan Berkshire County, Mass. Mahican, "great wooded mountain." This is the same as Taghkanick, q. v.

Wullamanic Hill, Worcester County, Mass. Nipmuck, "at the place of red paint."

Wune Wahjet (Indian Hill Cemetery) Middlesex County, Conn. Wangunk? Natick? "at the place of the good mountain," or "on the beautiful hill."

Wunnamuktukoogk Barnstable County, Mass. Wampanoag, "good fishing place at the river."

Wunnaqueckset Hampden County, Mass. Nipmuck, "at the end" or "end-place." (Probably a boundary mark.)

Wunnashowatuckqut Worcester County, Mass. Nipmuck, "where the river splits" or "at the river fork."

Wunnegunset Hill, New London County, Conn. Mohegan, "bowl shaped place."

Wunnompamukquock Fairfield County, Conn. Wappinger, "in the open place;" an intervale. See also, Wononkpakook.

Wunohke Norfolk County, Mass. Natick, "good land."

Wuskowhananaukit Worcester County, Mass. Nipmuck, "at the abode of pigeons," or "pigeon country." 
Wussquackheag see Squakheag.

Wussquamhegonset places below the falls on tributaries to the Penobscot River, Me. Penobscot-Abnaki, "at the place where fish can be scooped out in a net." See also Quamphegan.

Wuthommonassak River, New Haven County, Conn. Hammonassett, "at the small well"? See Hammonassett.

Wuttochoquisk Tolland County, Conn. Nipmuck, "rocky hill." Variants, Wachaqueage and Wochokeisquas.

Wuttoonug River, Fairfield County, Conn. Mahican, "rocky place."

Wyantenuc Litchfield County, Conn. Mahican, "at the parleyplace." Possibly this was the council place near Bantam.

Wyapumscut Washington County, R. I. Narragansett, "place of rushes,"? or "place at end of rocks."” Another source gives "hill (or rocky hill) at end of cove." Variant, Wyaxcumscut.

Wyasup Lake, New London County, Conn. Mohegan, "flags, or wild flax, or rushes."

Wyben Hampden County, Mass. Nipmuck, "white stuff" (possibly a fabric made from inner bark.)

Wyngaersheek Essex County, Mass. Not an Indian name, but Dutch: Wyngaer's Hoek, i. e., Wyngaer's Point.

Wyoma Lake, Essex County, Mass. Natick, "a gathering,"? or Delaware, "a flat place"?

Wyoming Essex County, Mass. and Washington County, R. I. Delaware, "the large prairie."

Wytopitlock Village, Aroostook County, Me. Abnaki, "at the place where there are alders."

\section{$-\mathbf{X}-$}

Xsebem Moosehead Lake, Piscataquis County, Me. Abnaki, "extensive water," or "big lake."

$$
-\mathbf{Y}-
$$

Yacum Hill, Hillsboro County, N. H. No satisfactory translation. If Indian, perhaps Natick, "yonder house."

Yageshon Bear Notch, Orange County, Vt. Mohawk, "among the rocks." 
Yagompoh Brook, Washington County, R. I. Narragansett, "that opening (mouth of stream) is crooked."

Yagompsh New London County, Conn. Narragansett, "on that side of the rock," or "extended rocks."

Yagunsk New London County, Conn. Eastern Niantic, "on that side of the rock" or "extended rocks."

Yankee Tuladi Brook, Aroostook County, Me. English and Malecite, "togue" or "nammycush," or "fork-tailed trout," but see Tulanic.

Yanondasa hills in Essex County, Vt. Huron, "small mountains, or foothills."

Yantic River and Village, New London County, Conn. Mohegan, "as far as the tide goes up this side of the river."

Yantuc River, New London County, Conn. Mohegan, "extended river," or "going as far as the tidal stream;" also "on that side (one side) of the stream." Other spellings Yomtack and Yontahque.

Yantuckkoyog Windham County, Conn.? Mohegan, "boundary place of the extended stream," or "source of the stream"?

Yashkwongunnuck Pond, New London County, Conn. Mohegan, "place of the extended pond or lake or fishing place." Other spellings, Yoskcowwongamuck and Yaskowunganuck, sometimes translated as "extended bends."

Yaubucks New London County, Conn. Mohegan? "on that side (or one side) of the small pond."

Yawgobby New London County, Conn. Mohegan, "extended rocks."

Yawgoo Pond, Washington County, R. I. Narragansett, "red pond"? or "fire place"? or "as far as this place" ? Variants, Yawgoag, Yawgoog, Yawgook, Yawcook.

Yawgoog Pond, Washington County, R. I. Narragansett, "one side of the pond," or perhaps "here are many lice."

Yawgunsk Brook, Washington County, R. I. Narragansett, "as far as that rock."

Yayompoh Brook, Washington County, R. I. Narragansett, "that opening (mouth of stream) is crooked." Also Yogompoh.

Yeapskasset River, York County, Me. Abnaki, "near that big rock." 
Yecompsky New London County, or Windham County, Conn.? Mohegan, "as far as those rocks." (A boundary marker.) Also Yeeompsky and Yeeomskgie.

Yennenstyaks Franklin County, Vt. Mohawk? "one breaks corn grain" or "corn seeds broken by crushing." Possibly the site of extensive corn meal grinding.

Yeushquatuck Windham County, Conn. Mohegan? or Pequot? "as far as the river extends," or "as far as the end of the river."

Yewtack New London County, Conn.? Mohegan-Pequot, "fire place." Yotaanit is given as "fire-god" by Roger Williams.

Yocum Pond, Berkshire County, Mass. Mahican, from the name of Sagamore Yokun, or "Captain Yoghum." Also Yokun.

Yokun Seat (Mountain), Berkshire County, Mass. Mahican, from the name of Chief Yokun, or Yoghum, a Stockbridge who aided Jonathan Edwards in $\mathrm{I} 788$.

Yomtack see Yantuc.

Yomtonoc Washington County, R. I. Eastern Niantic, "flood tide there." Variant, Yomtunnock.

Yontahque see Yantuc.

Yoskowwunganuck see Yashkwongunnuck.

Yotenyatarokte Lake Champlain below the Narrows, Rutland County, Vt. Mohawk, "end of the lake." (Found in older works as Caniaderi-Oit, "tail of the lake.")

Yowunck homuck Hampden County, Mass. Nipmuck, "at the other side, or end, of that field."$$
-\mathbf{Z}-
$$

Zooquageers a name applied to the Abnakis along Lake Champlain, Vt. by themselves and their relatives. Probably from Abnaki, Sokokis, "those of the southern part," or "those of the river outlet." Variant, Zooquagees. 
APPENDIX 
The following section presents a glossary of the most common root words found incorporated into various Algonquian place names. In the first column are the English equivalents, arranged alphabetically, giving as closely as is possible the sense conveyed by the Algonquian term. In the second column are those place names found in Northern New England, (NNE), which incorporate these roots, together with variant forms.

The third column, in italics, lists the root itself which has been incorporated into the preceding place name. Literal translations are included to more clearly explain the usage.

In the fourth column are those place names more commonly found in Southern New England dialects, (SNE), and the final column again gives the root term found in the preceding, as well as literal interpretations.

It is the hope of the compiler that this will enable the interested reader to make further analyses of Algonquian geographical terms, and also that it will more clearly reveal the methods whereby the Indian joined various phonemes to form such place names. 


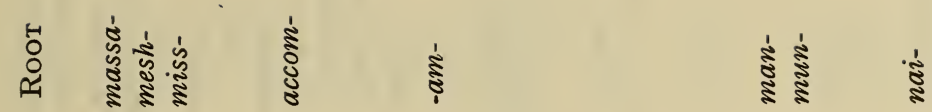

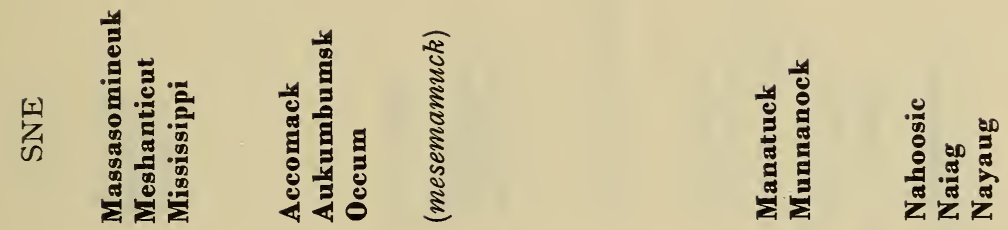

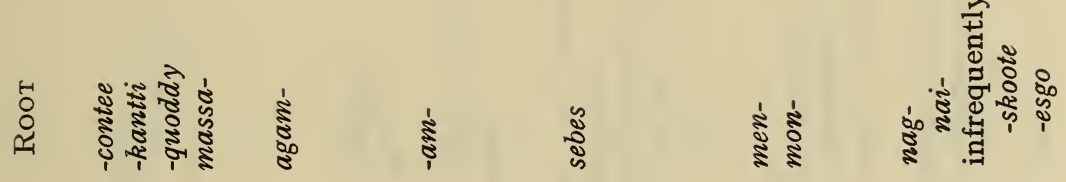

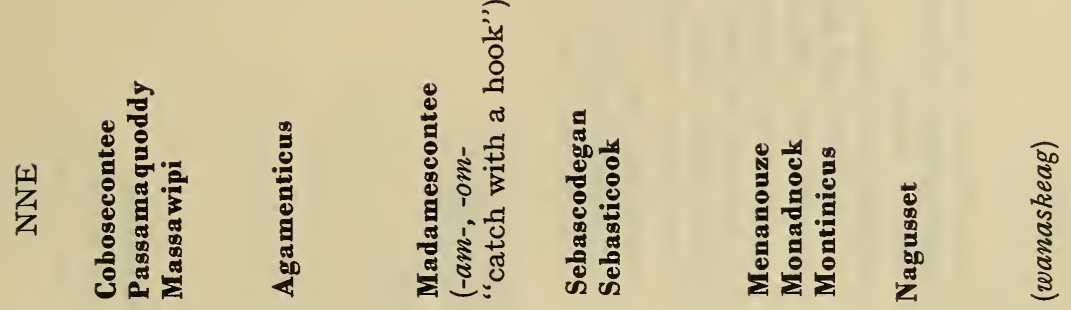

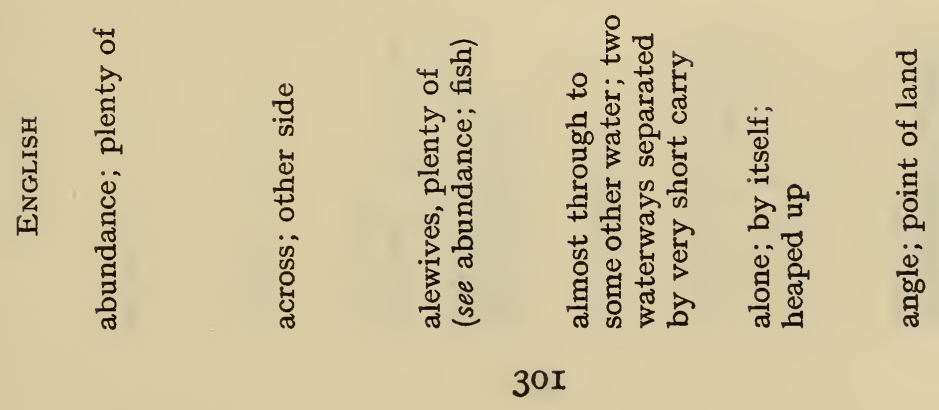


$H$
0
0

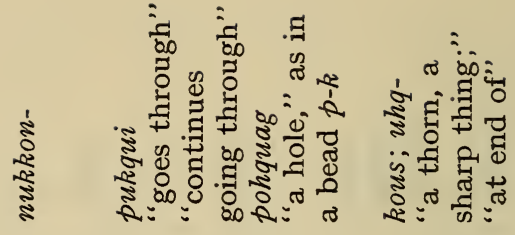

孚 苍苍范

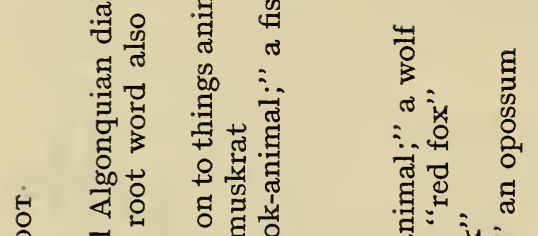

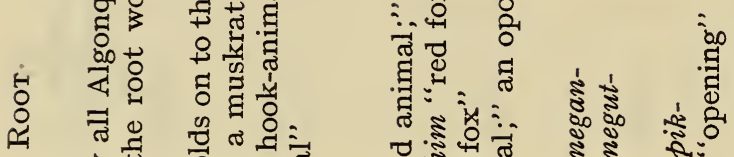

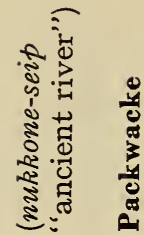

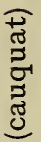

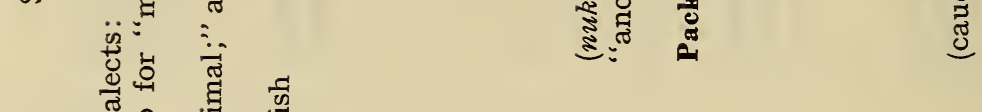

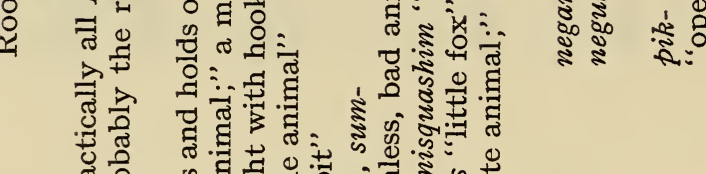

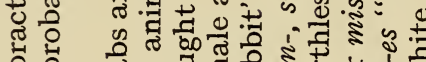
ज. गु
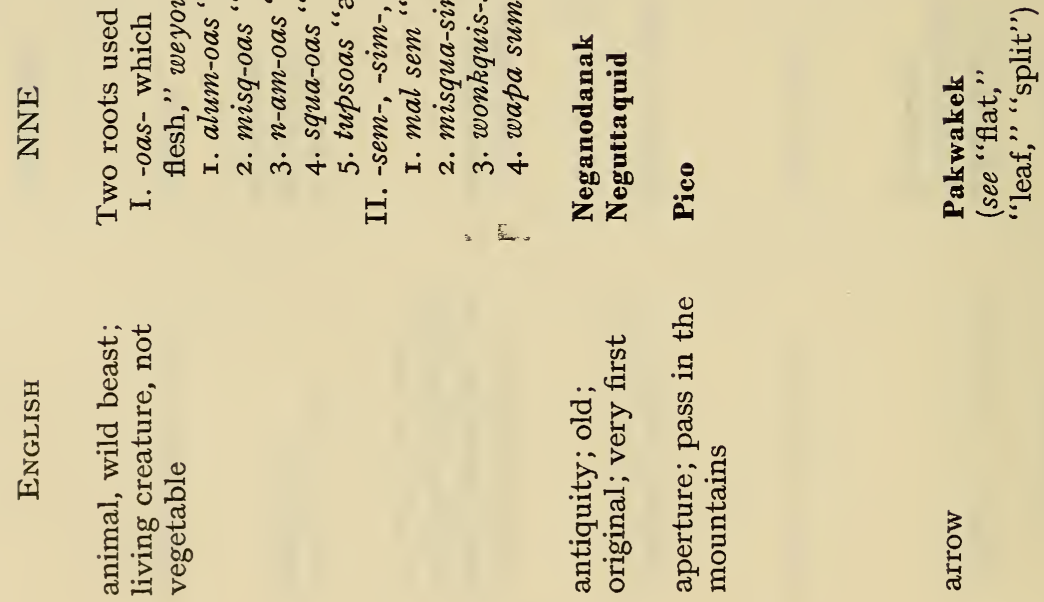

ลุ 


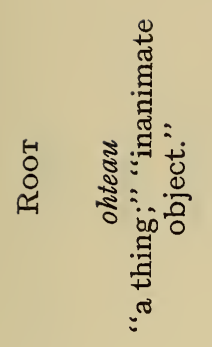

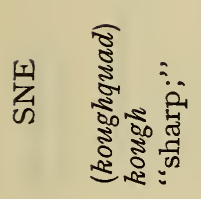
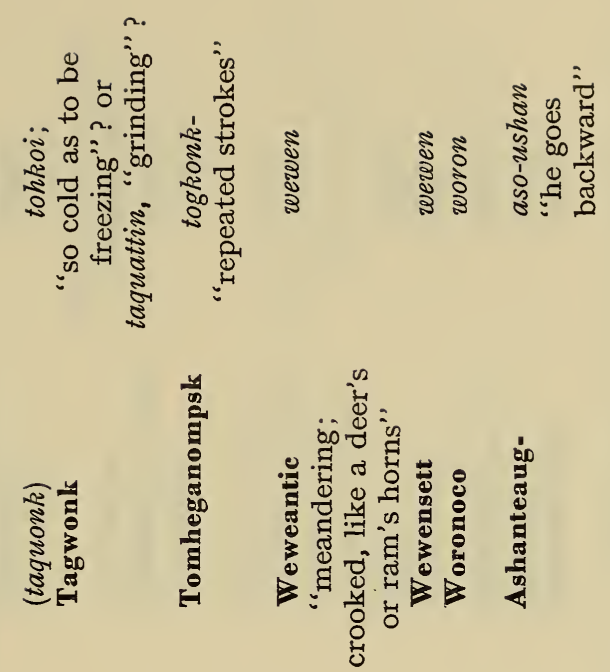

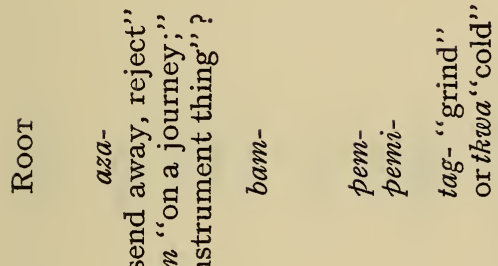

势:

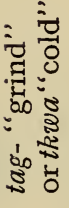

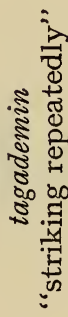

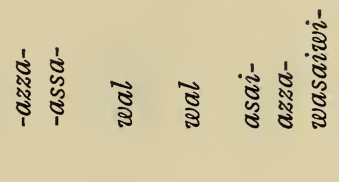

最

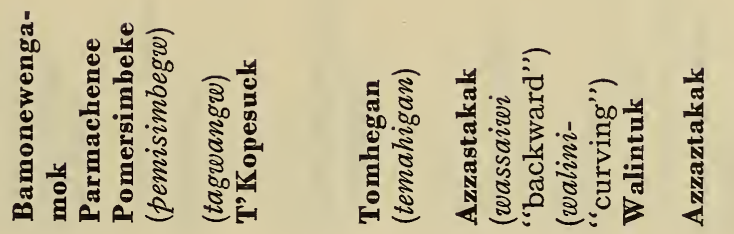

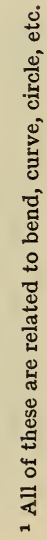

303 


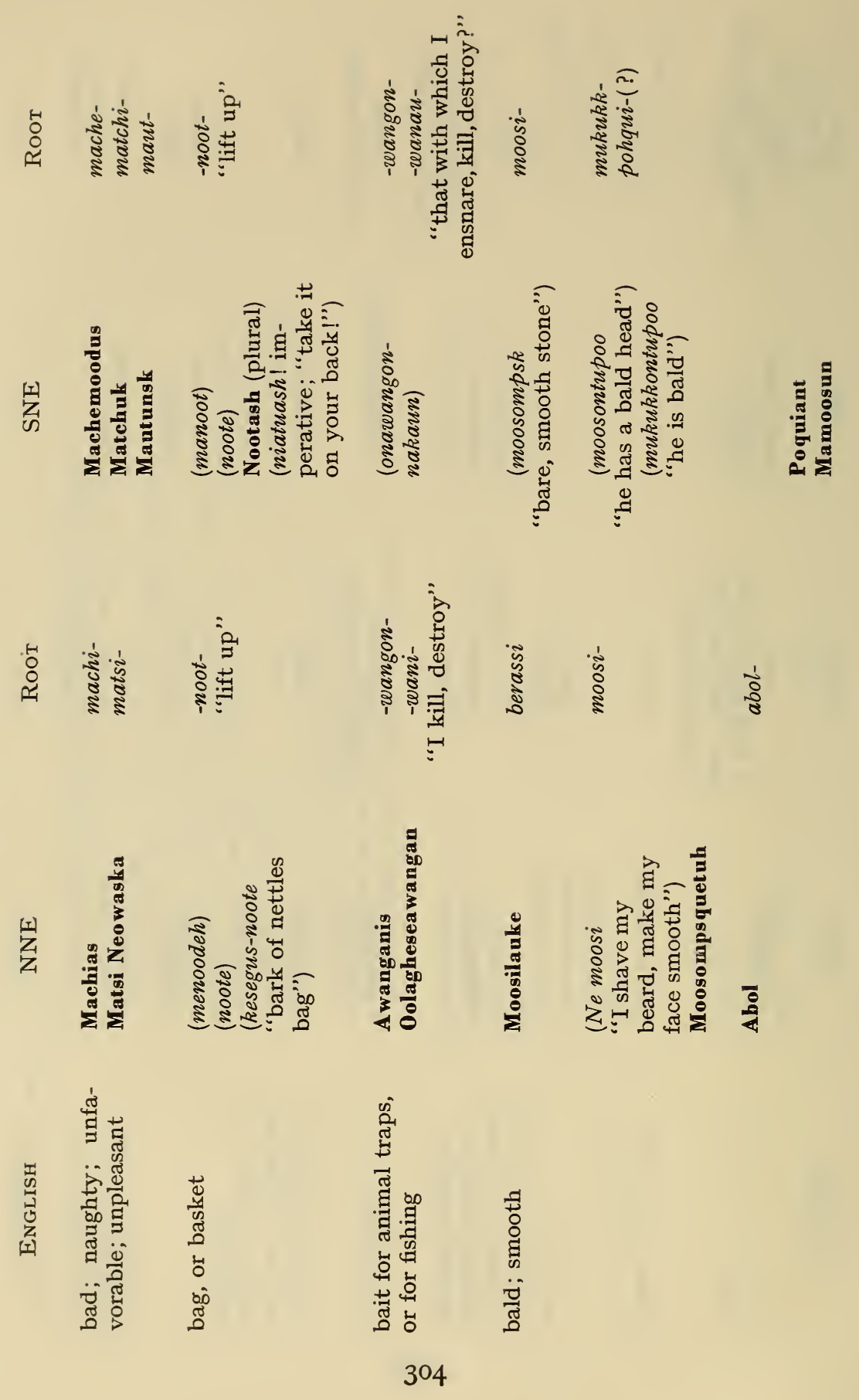




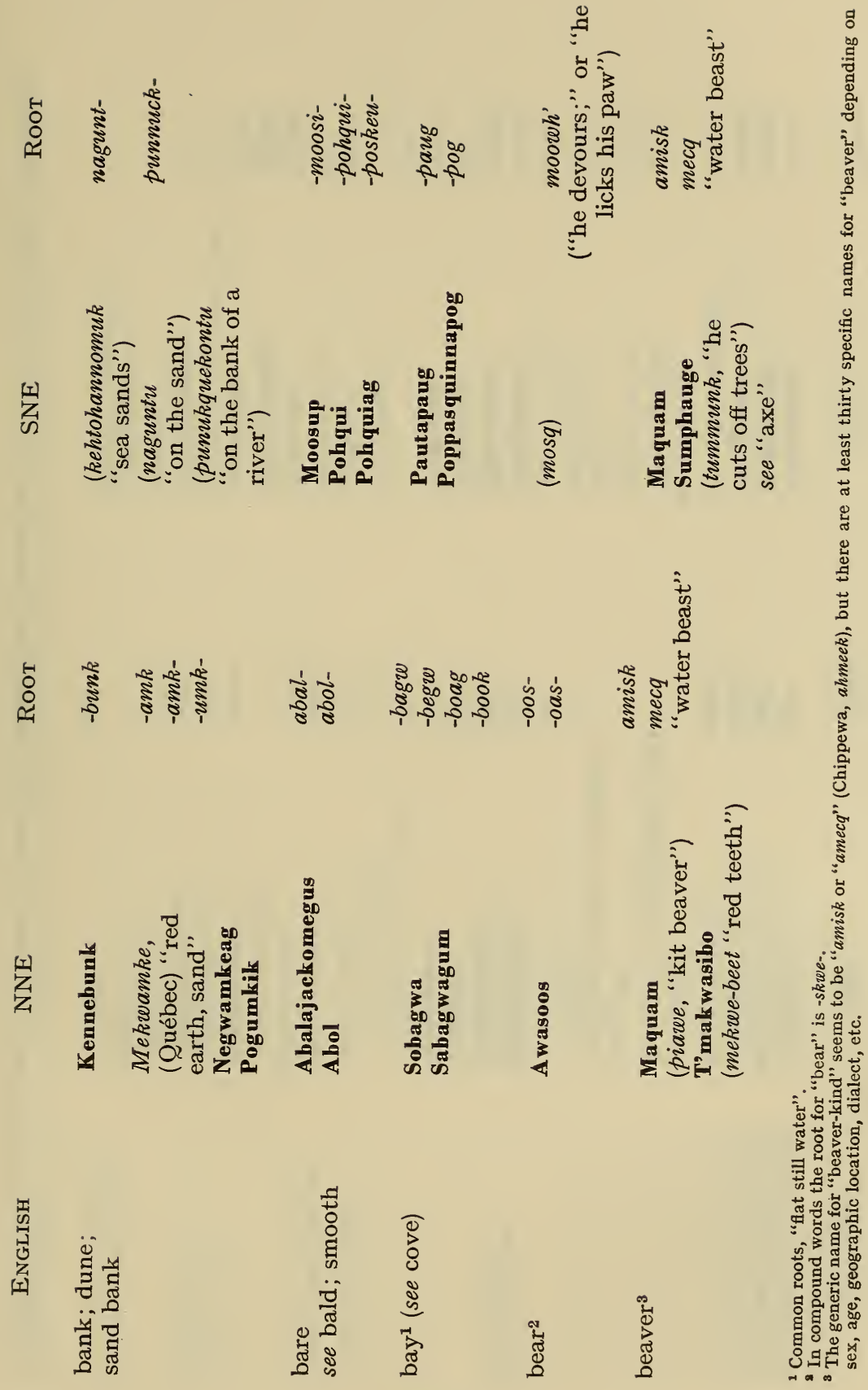


言蓄章

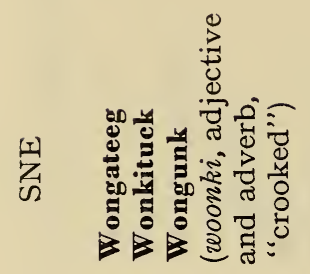

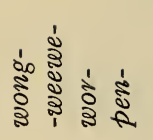

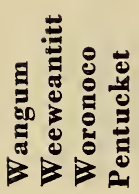

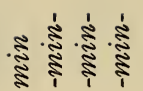

产

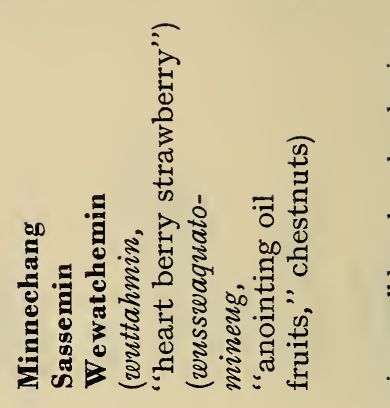

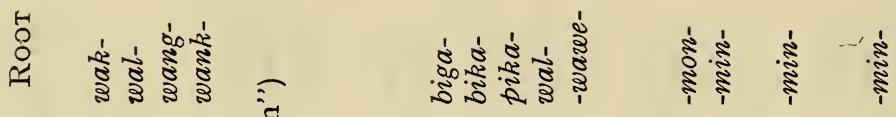

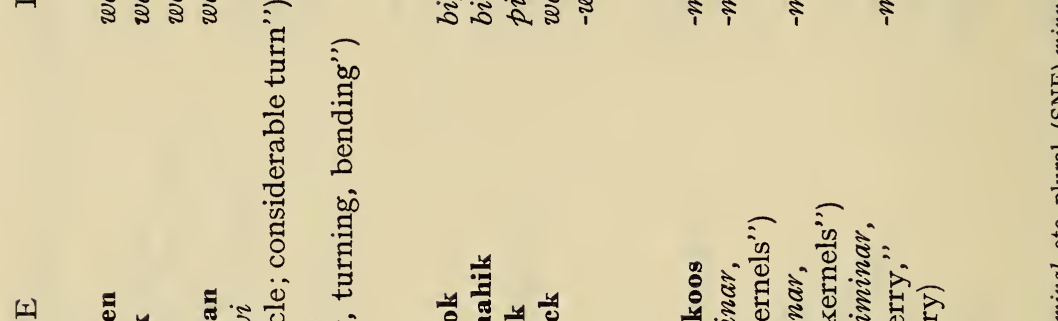

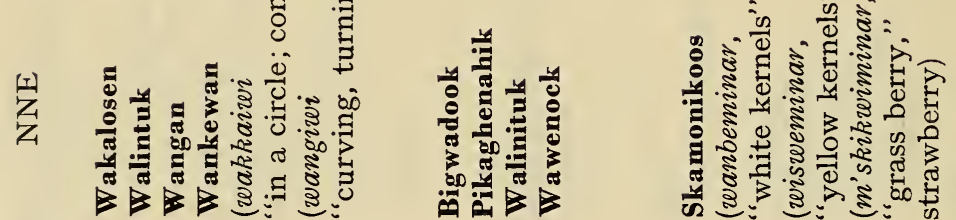
की<smiles>C1CC1C1CC1</smiles>

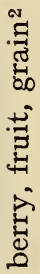

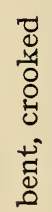

306 


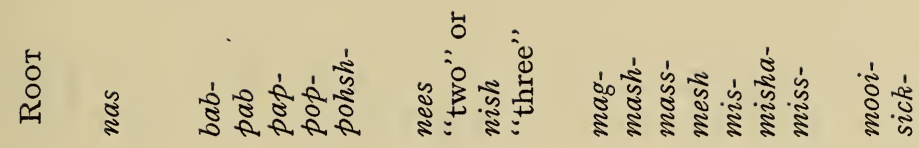

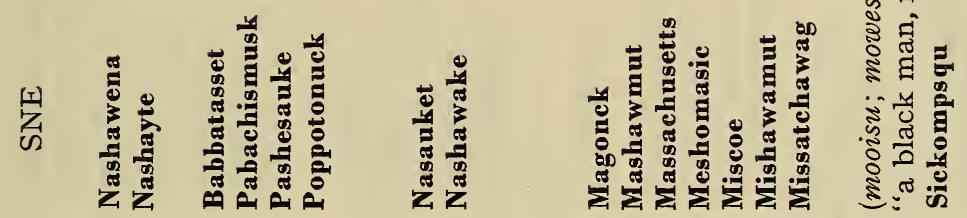

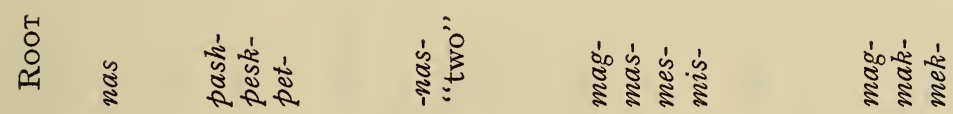

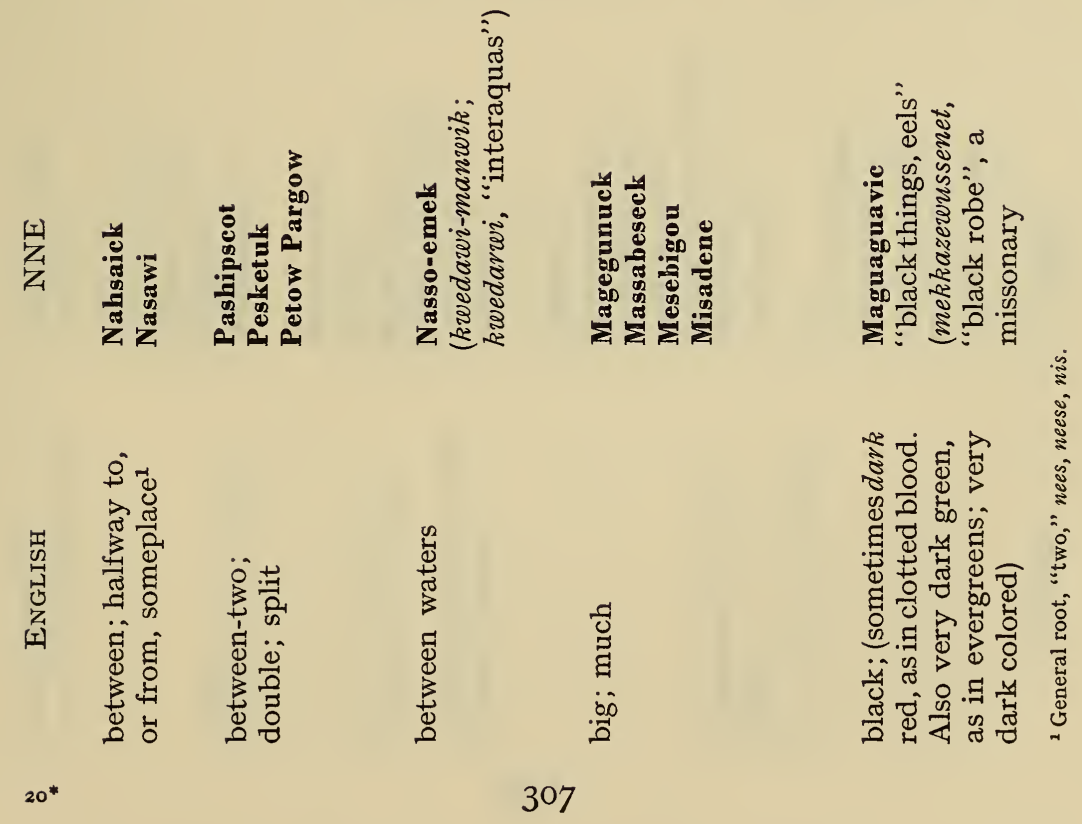


高

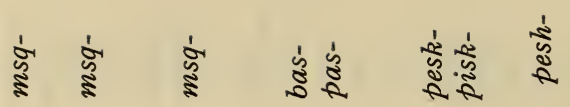

1
క్
1

㸵
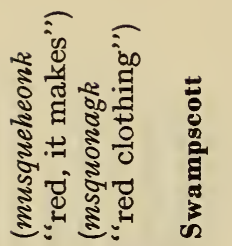

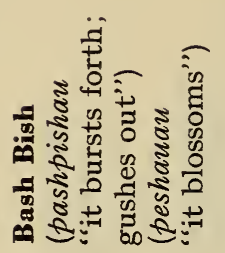

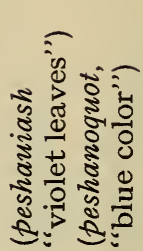

है

葍訾离

๕

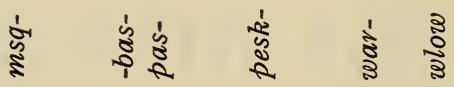

⿳亠े冖 ํำ

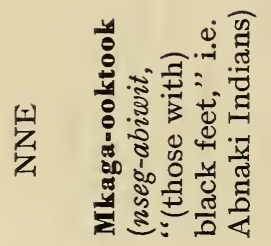
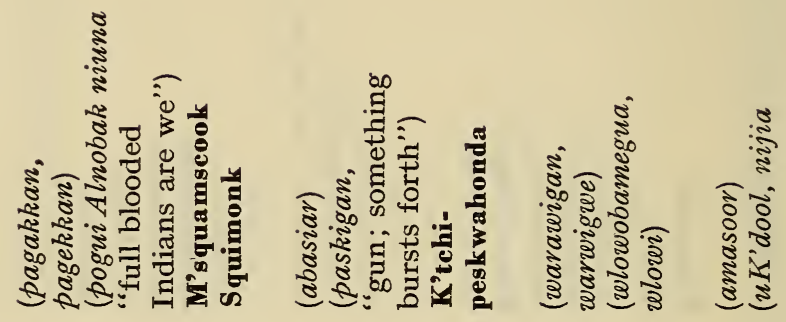

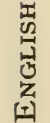
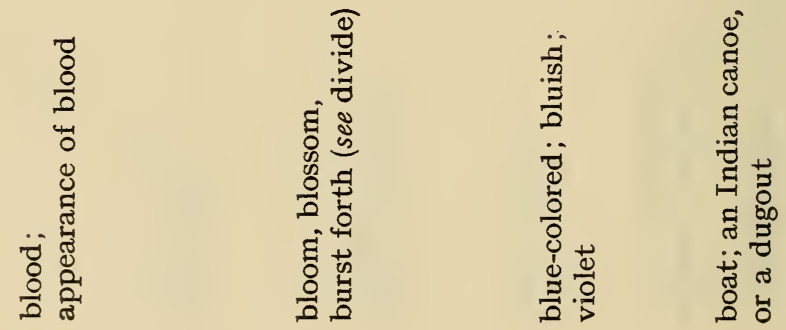

308 


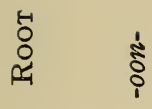

1ิ

ํํำ

ঠั่

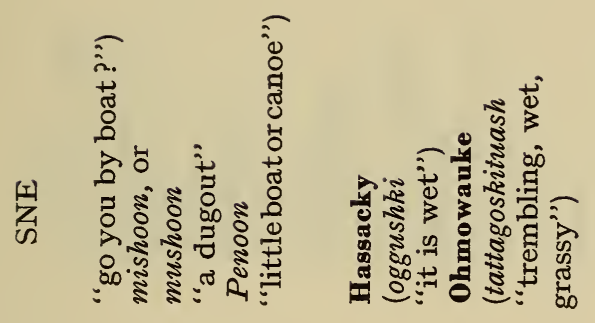

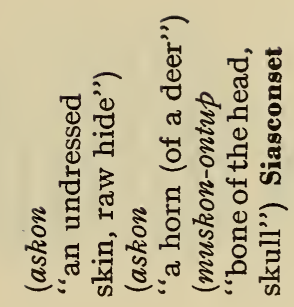

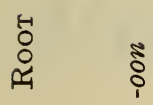

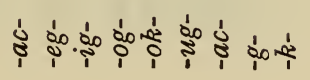

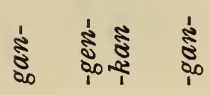

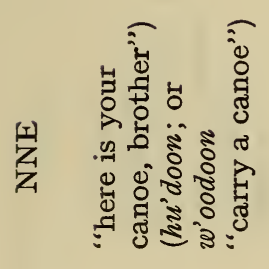

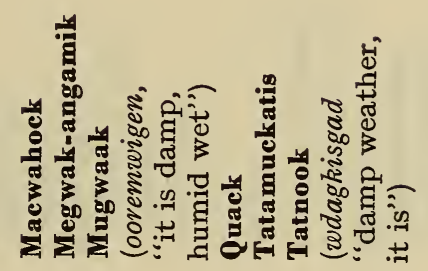

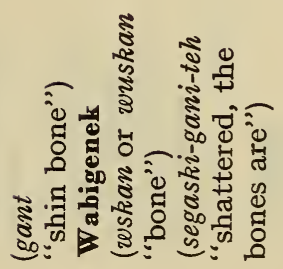

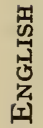

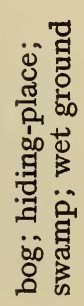

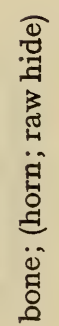




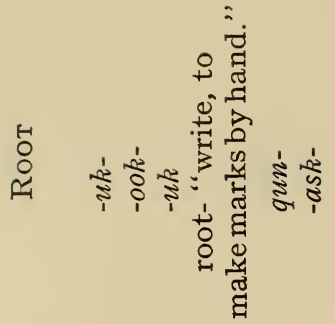

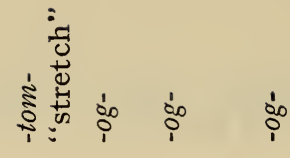

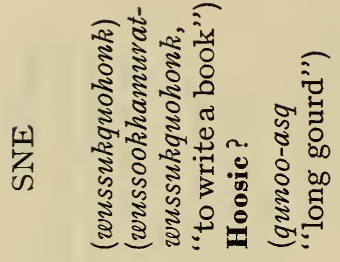

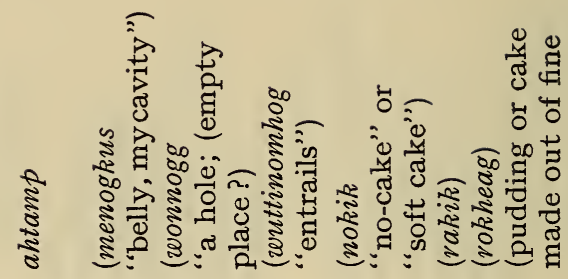

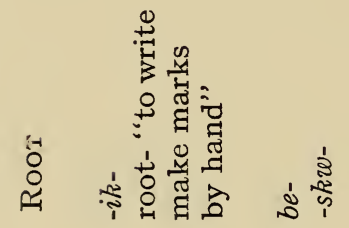

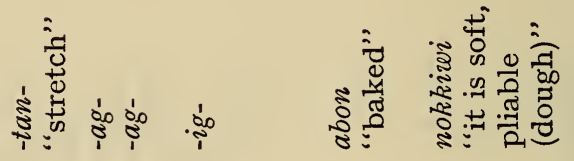

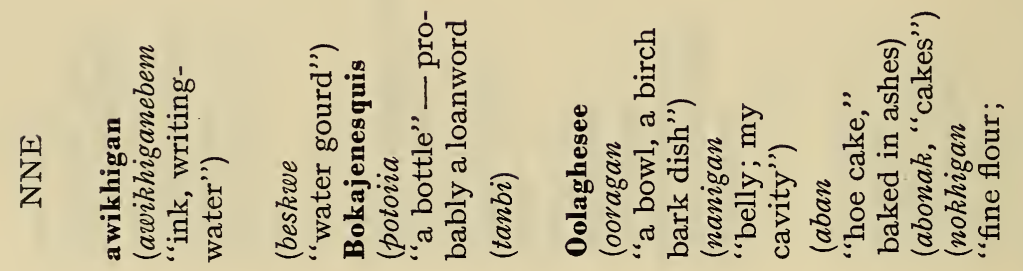

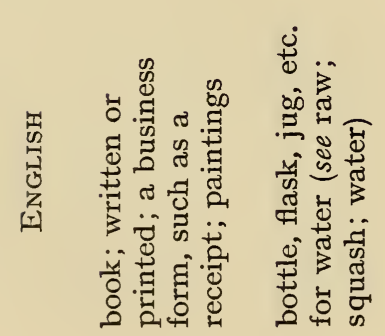

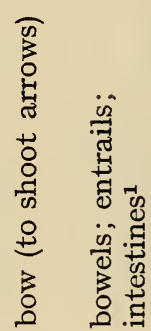

警言

.

गี

त्

嫼星

310 


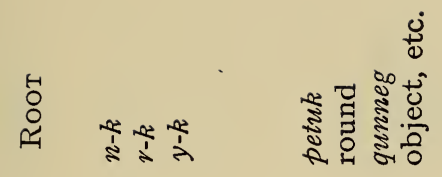

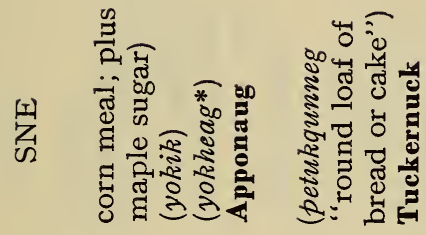

喜受

葛高

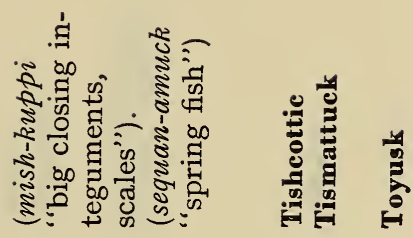

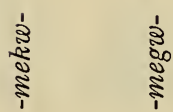

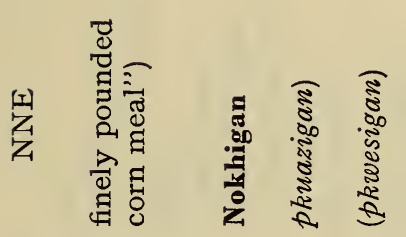

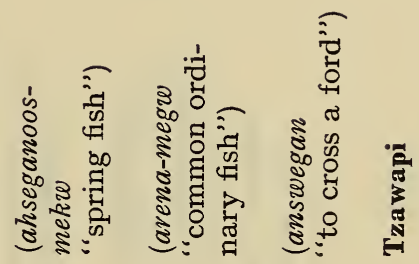

党

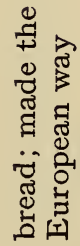

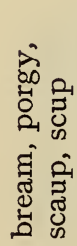

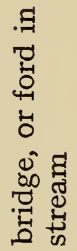




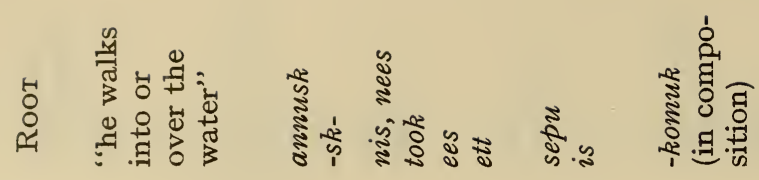

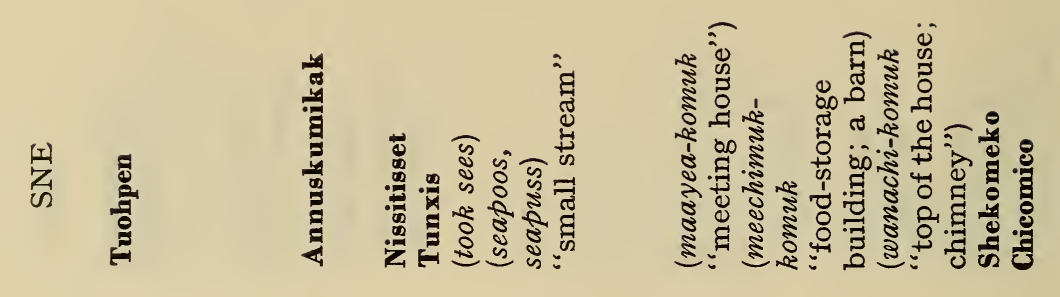

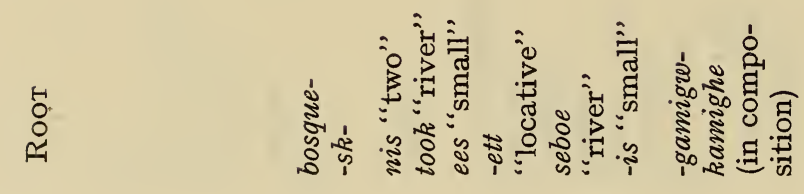
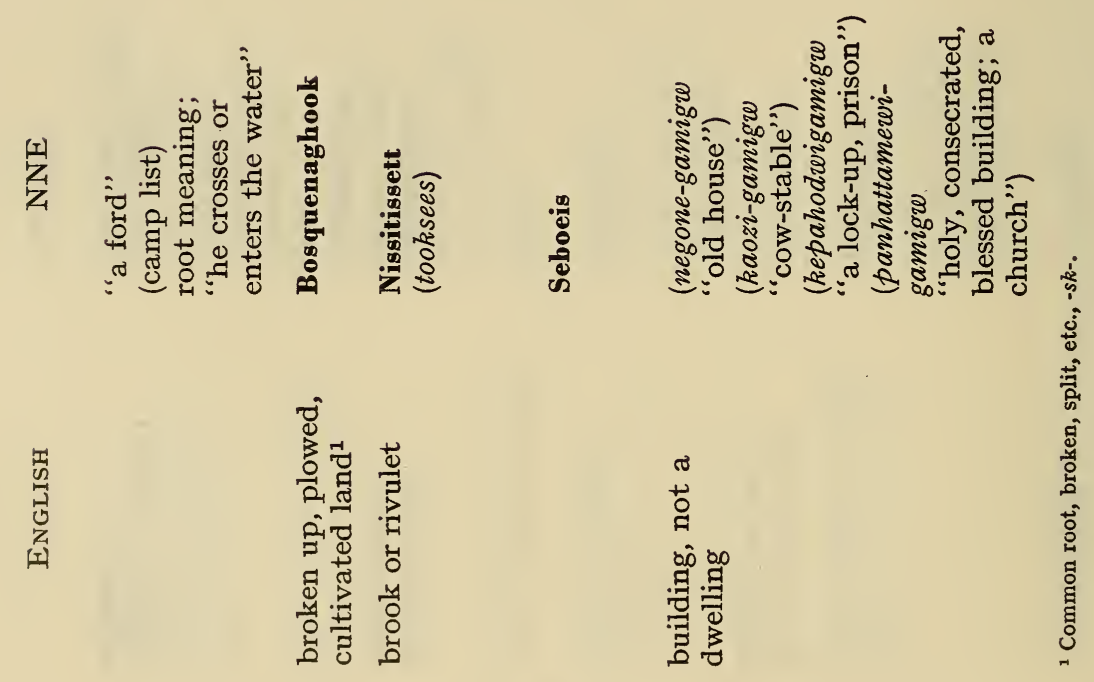
高

賈

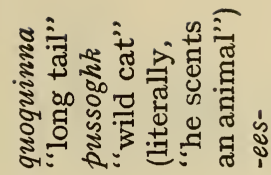

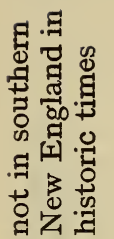

(I)
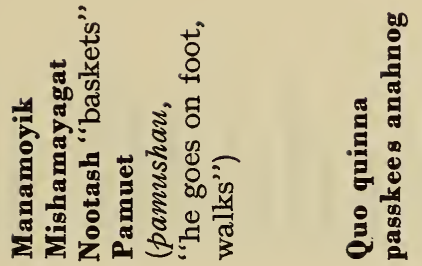

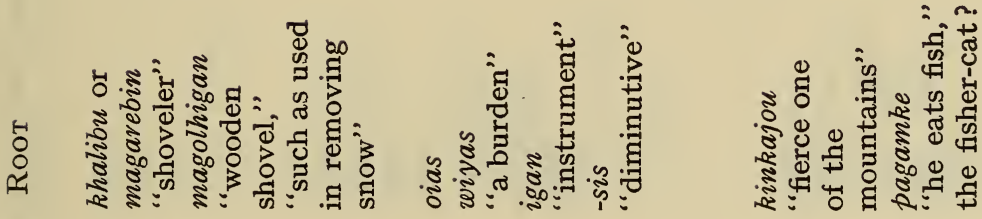

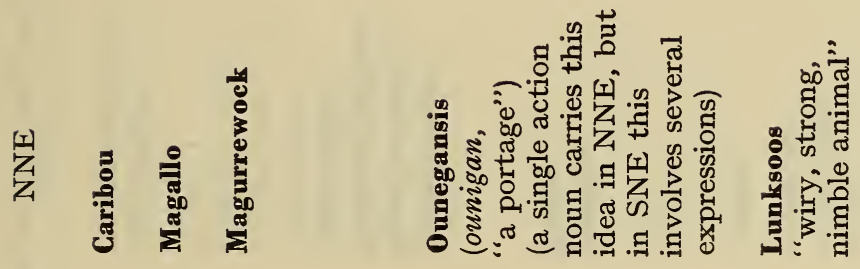

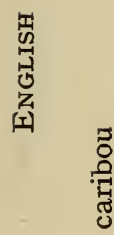
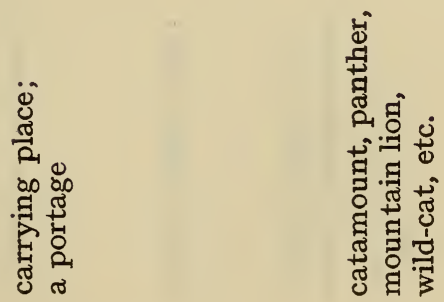

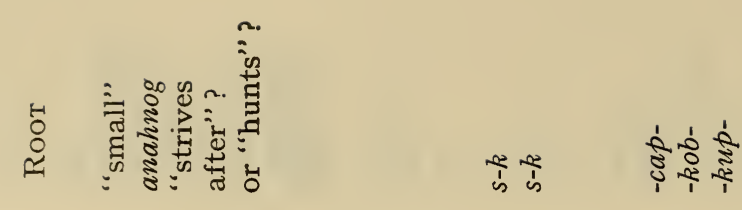

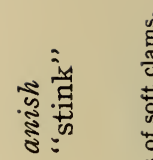

족
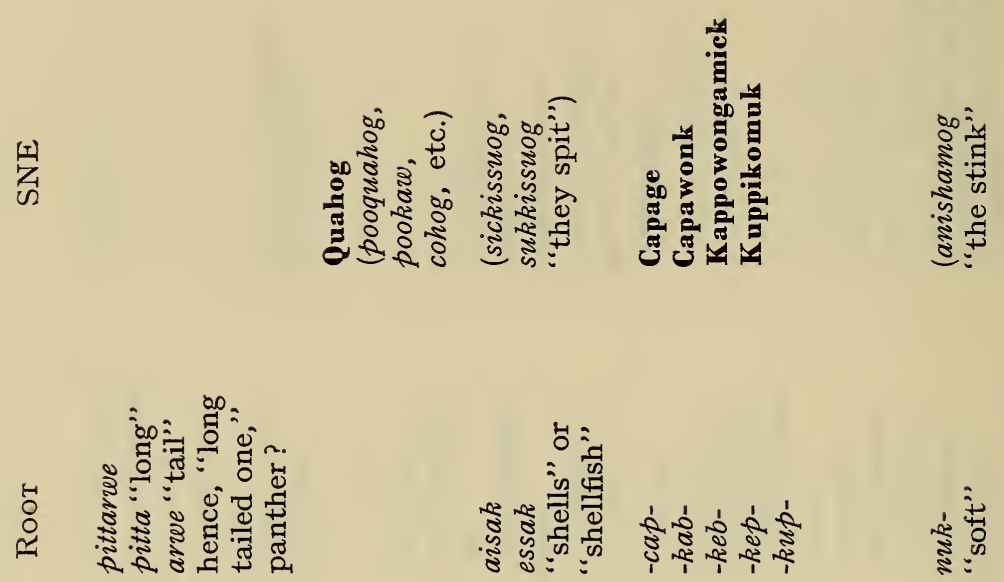

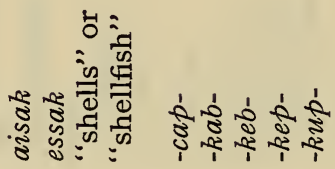

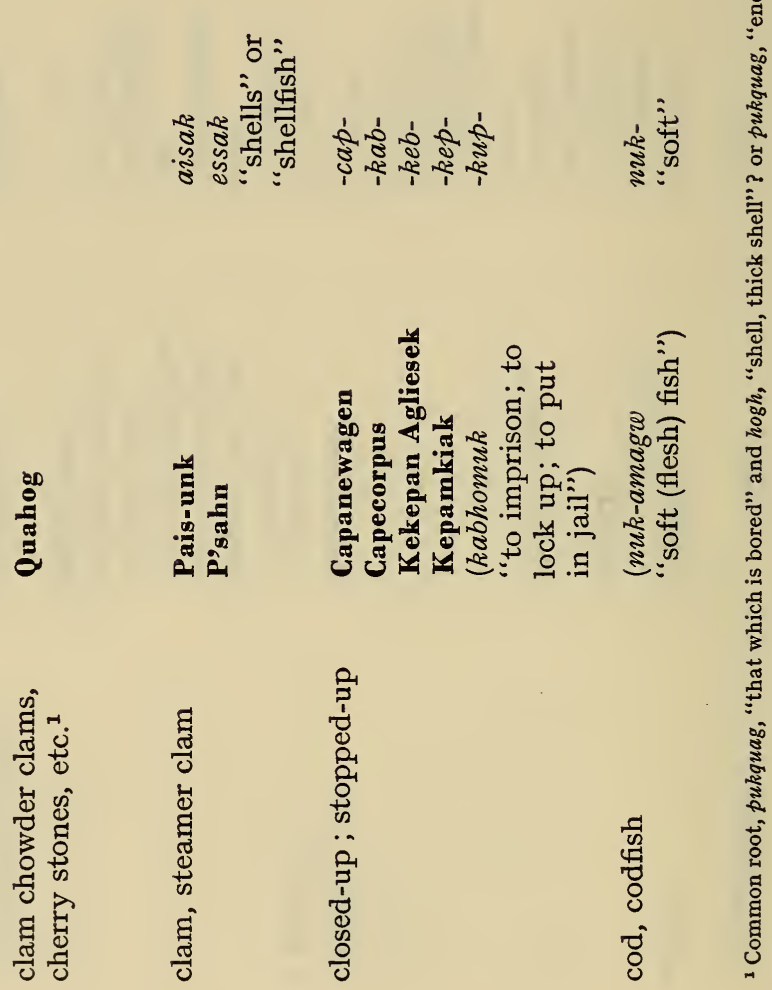

직 


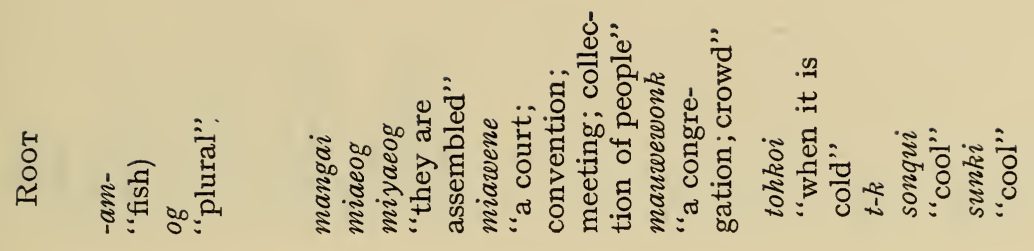

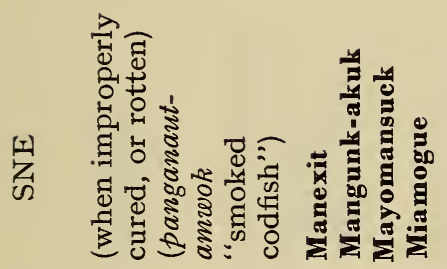

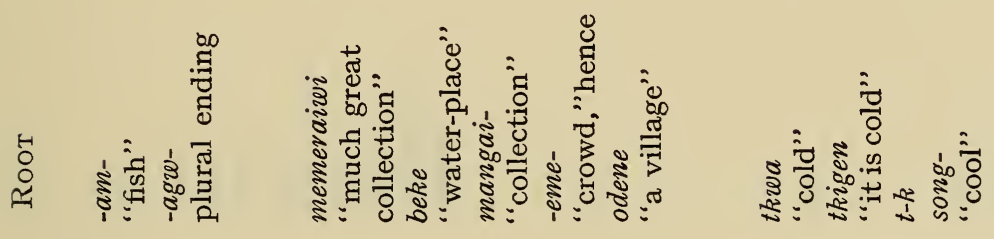

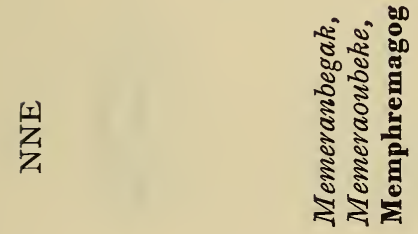
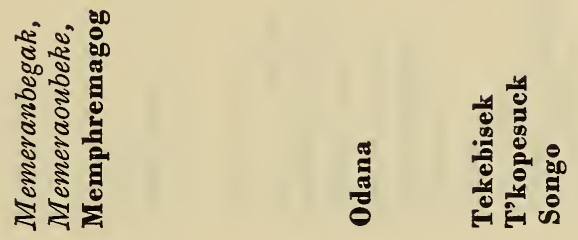

善
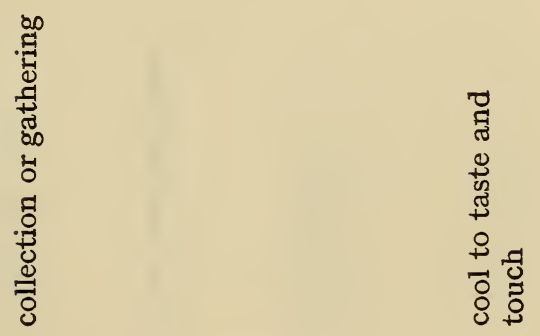


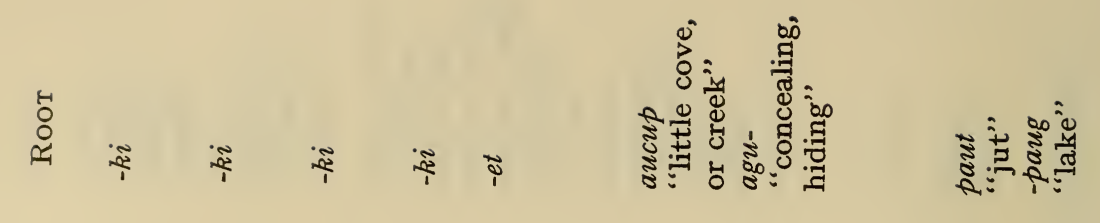

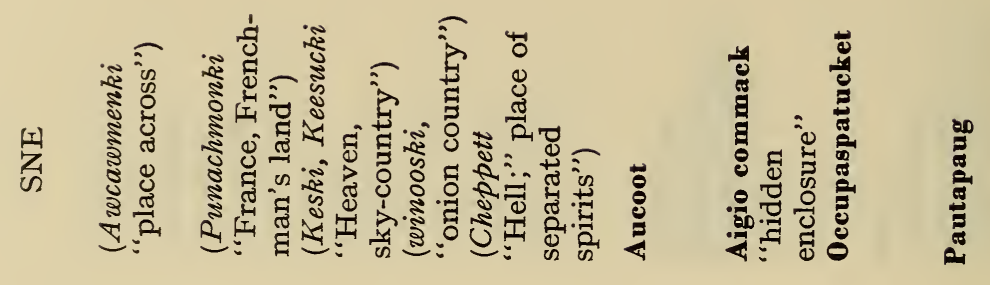

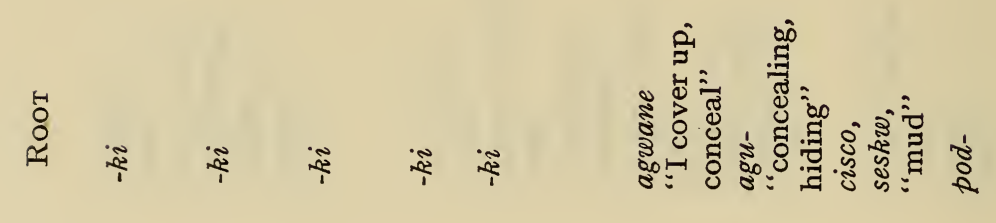

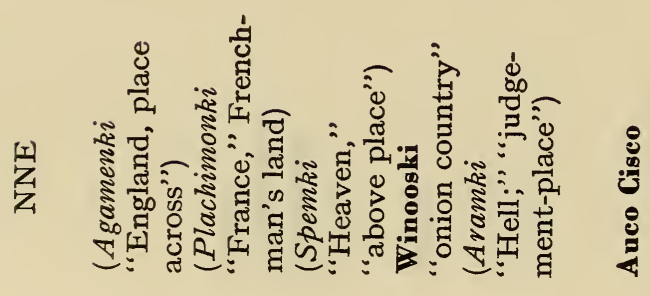
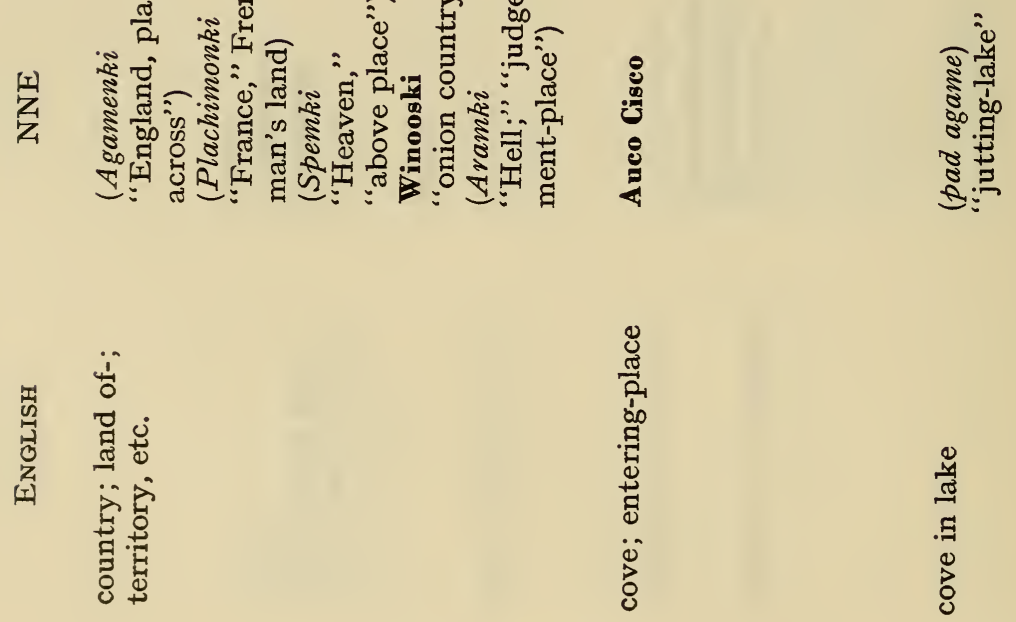


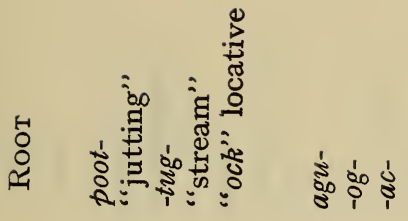

혼

舆

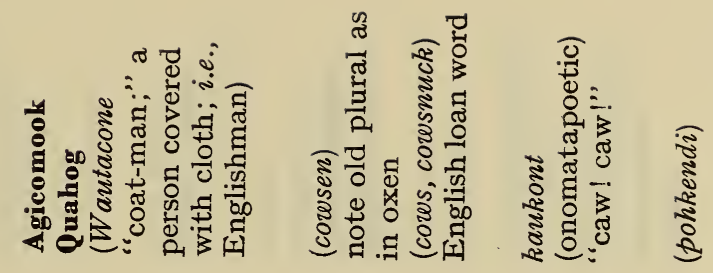

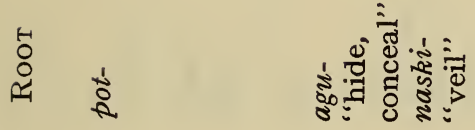

$\frac{5}{\sqrt{2}}$

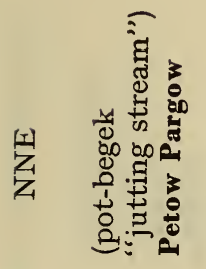

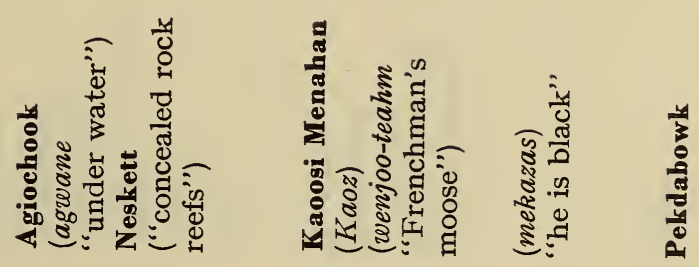

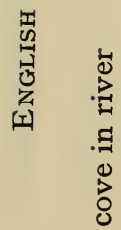

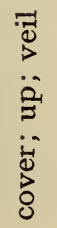

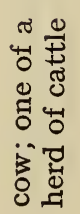

$\frac{2}{2}$

@्ञ

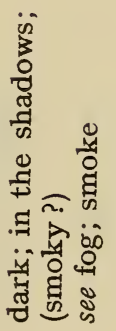




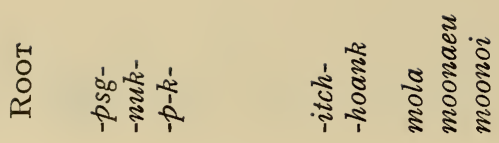

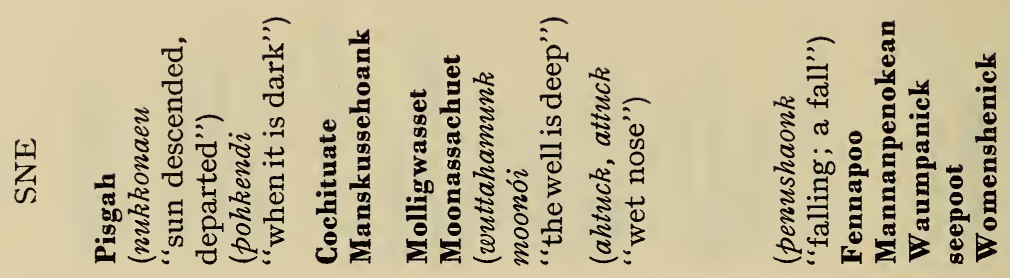

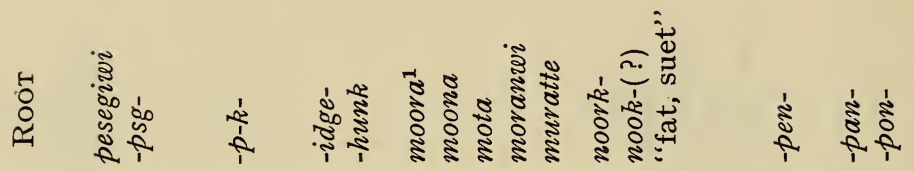

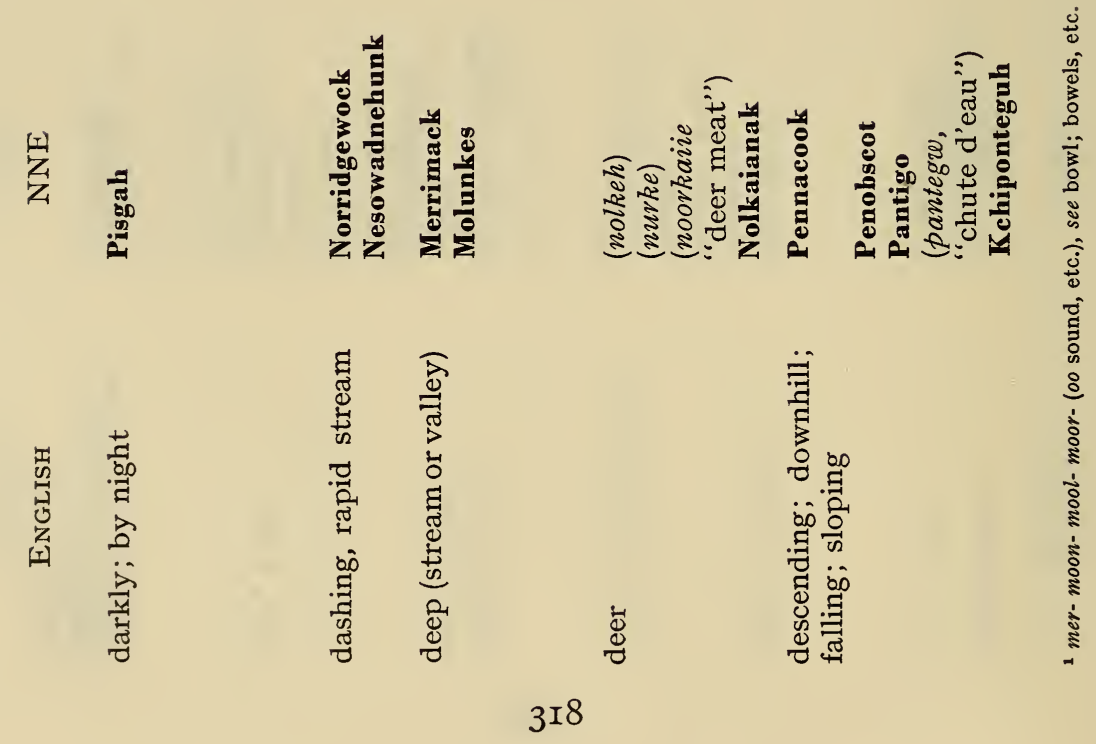




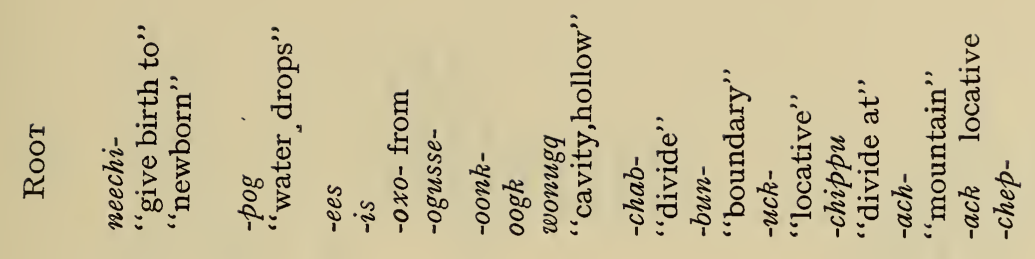

(H)

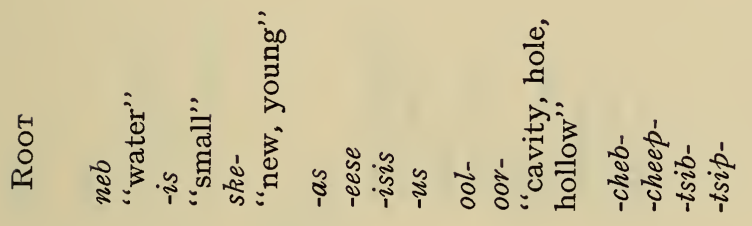

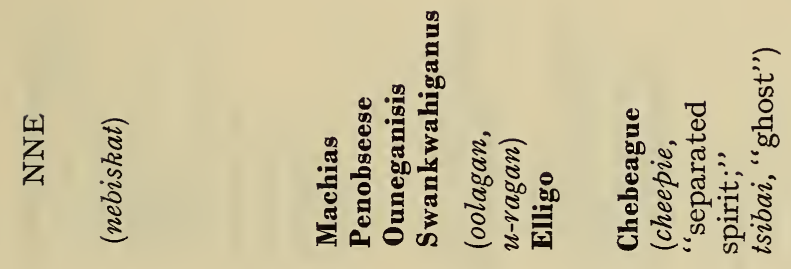

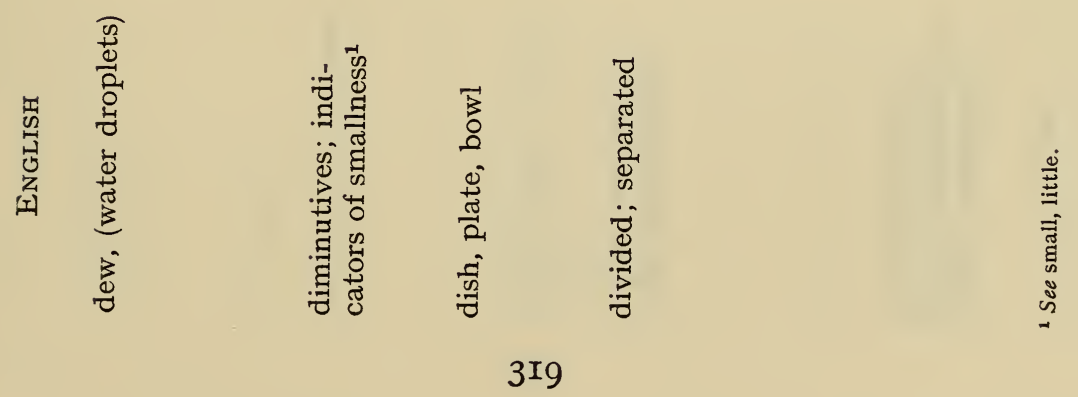




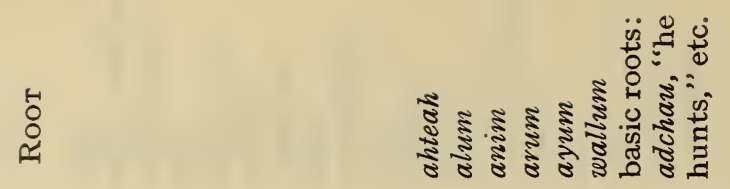

ह

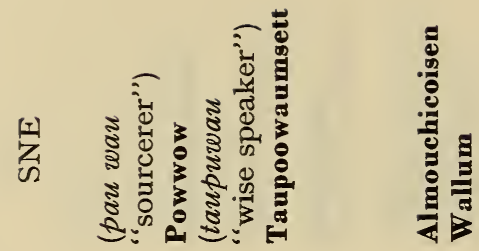

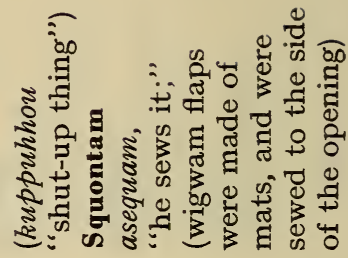

落

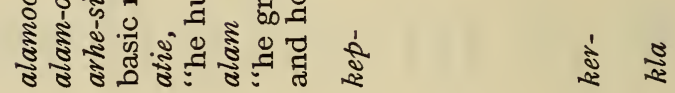

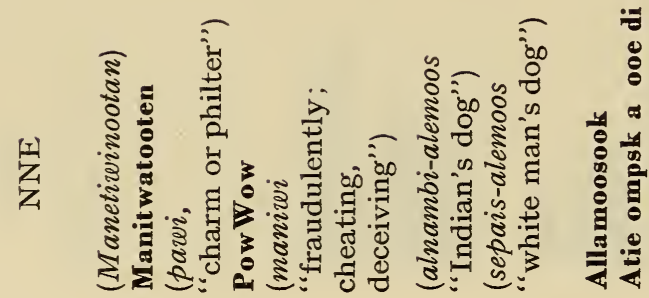
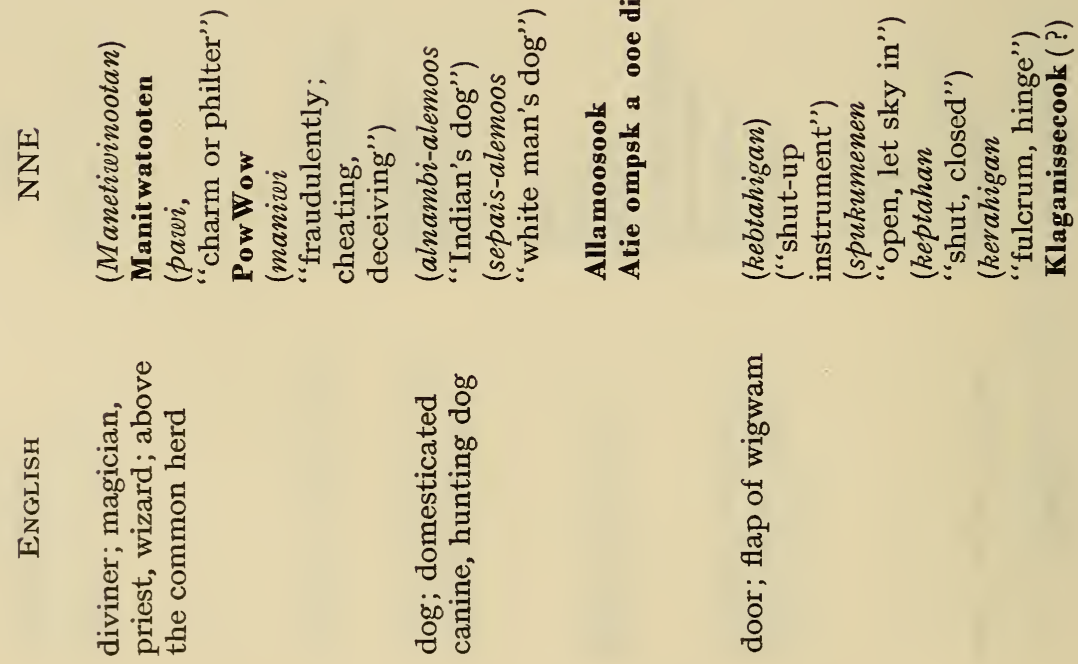

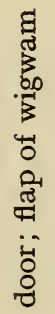

320 


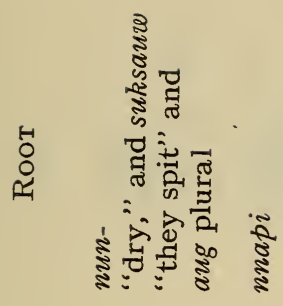

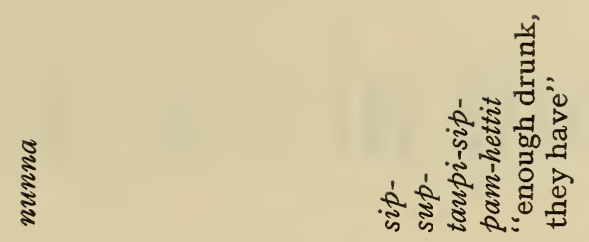

以

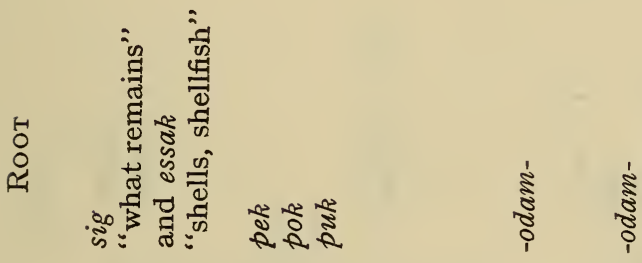

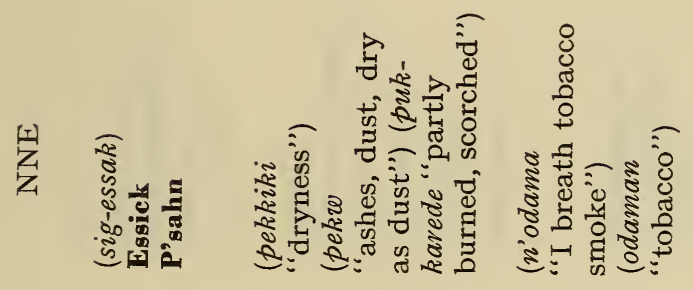

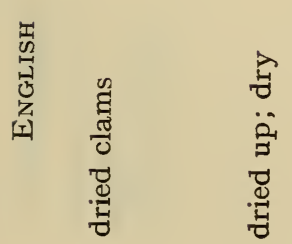

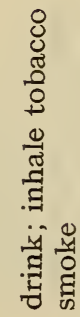

$32 \mathrm{I}$ 

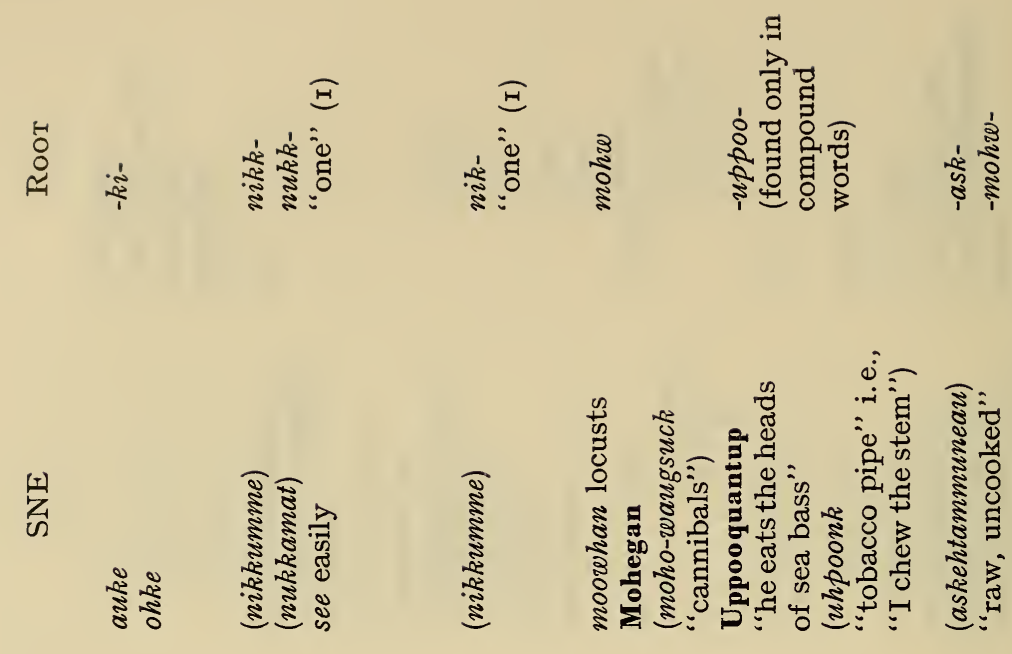

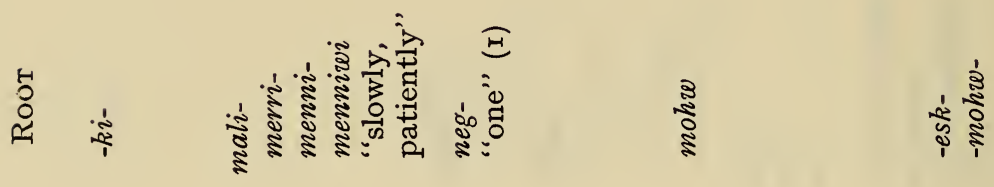
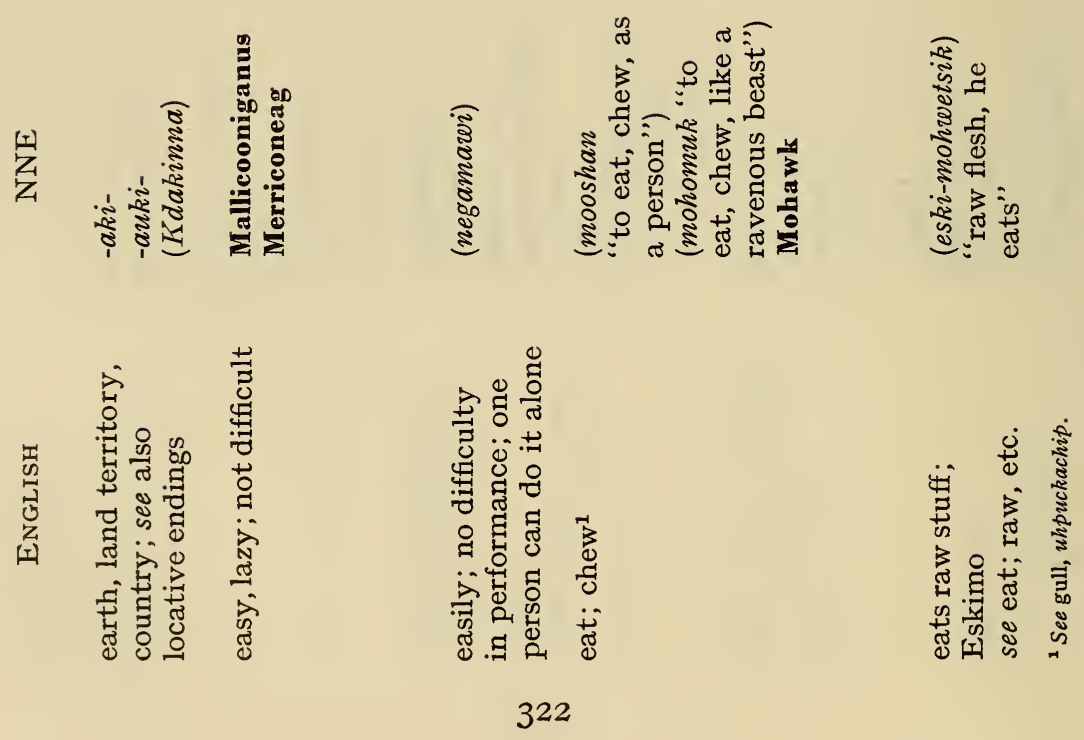
")

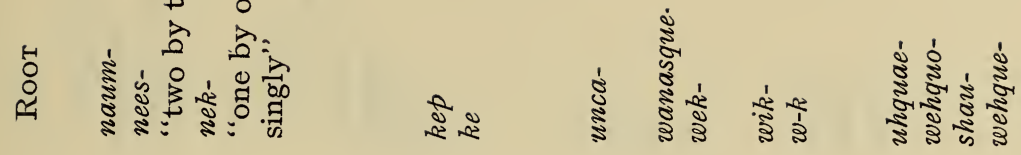

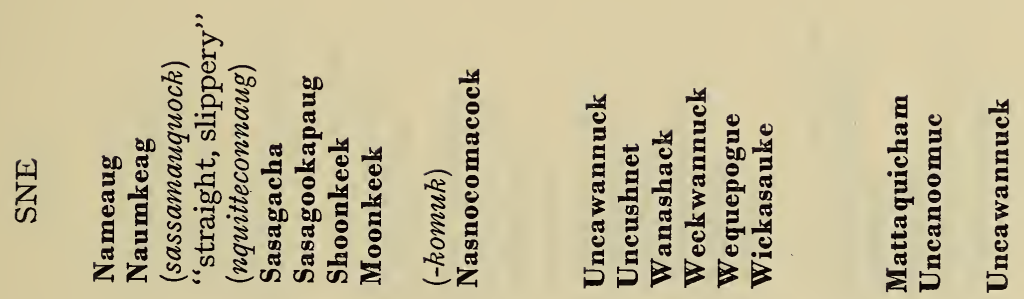

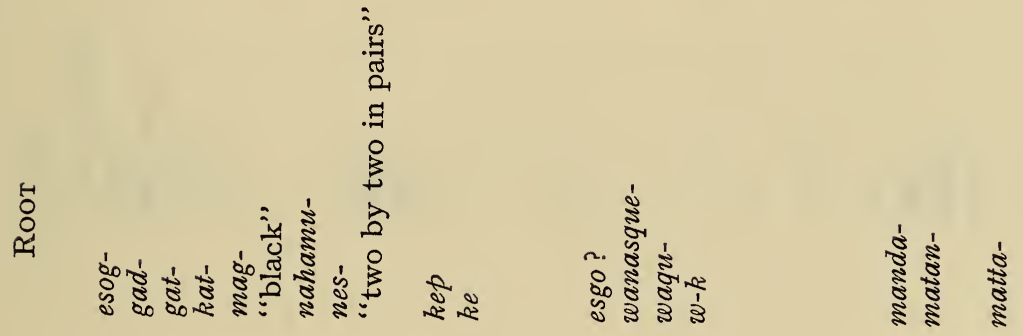

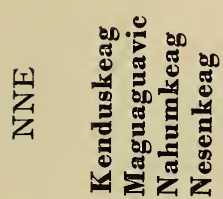

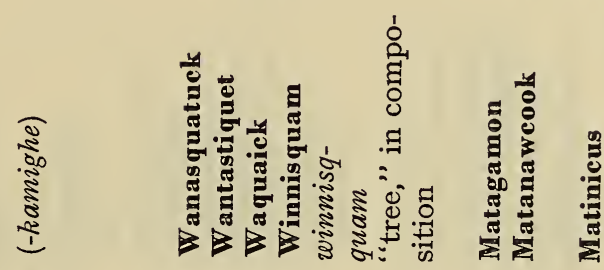

壱

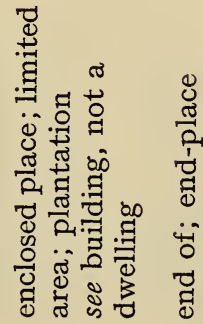

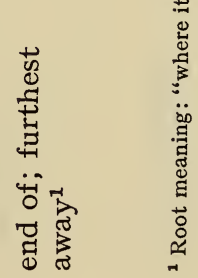

323 


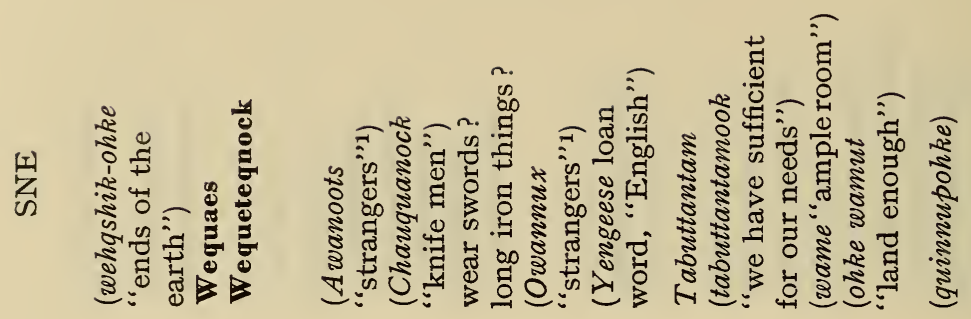

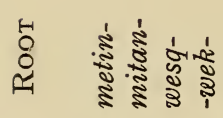

รูำ

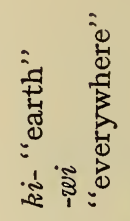

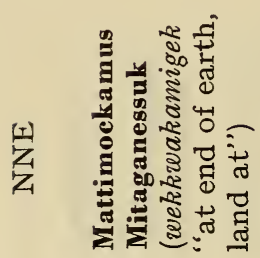

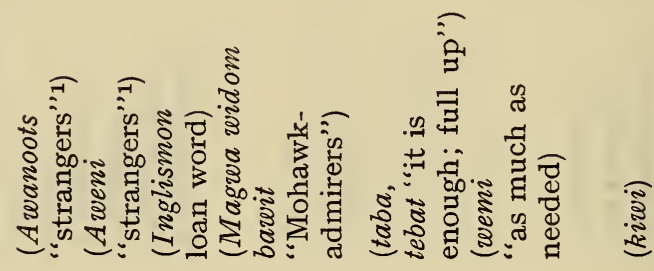

= 


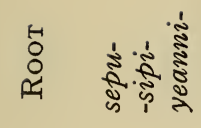

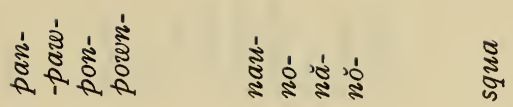

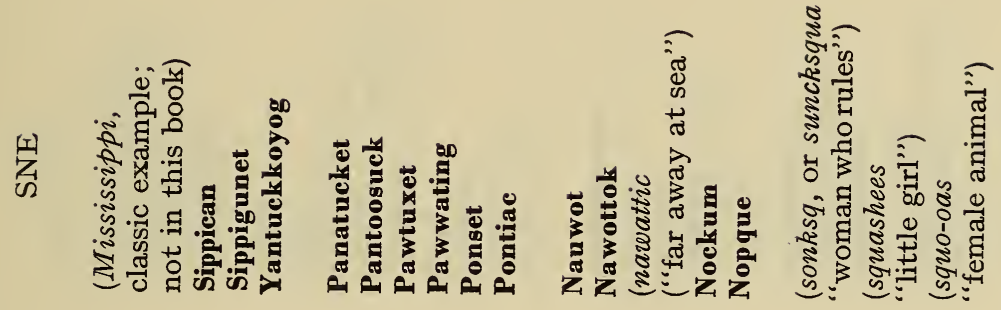

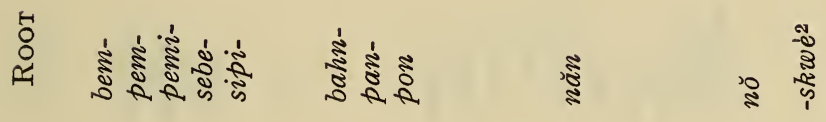

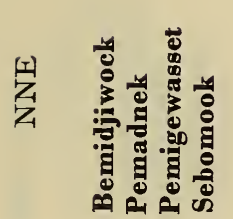

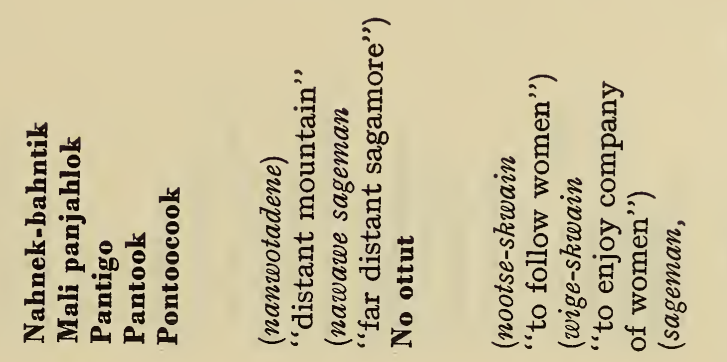

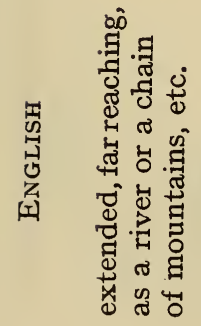

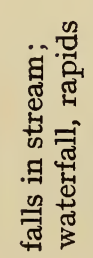

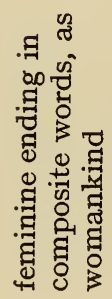

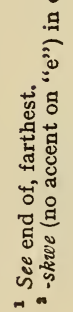




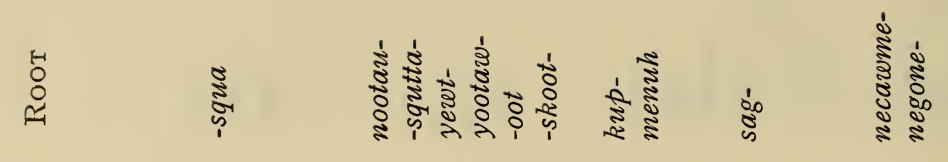

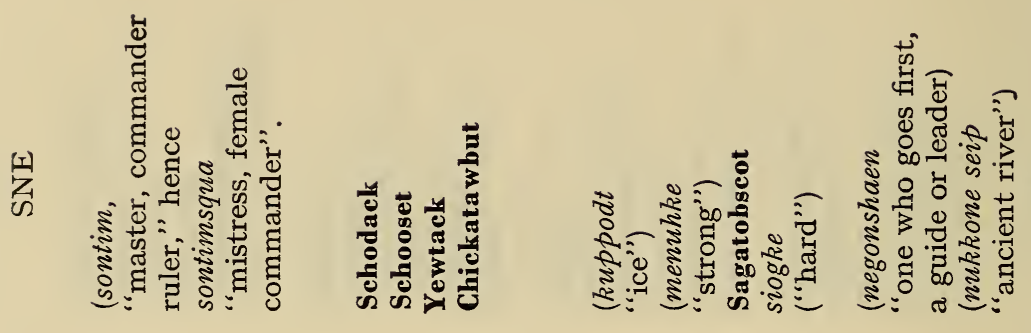

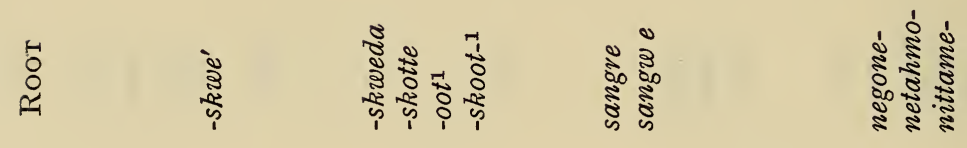

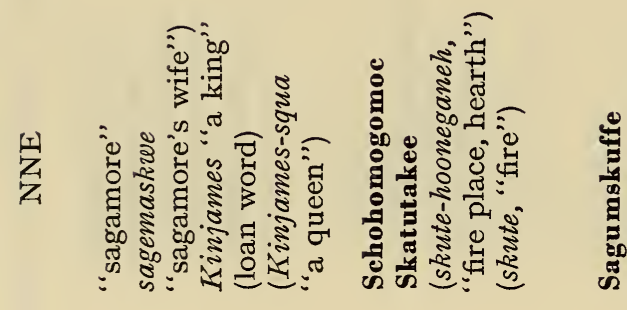

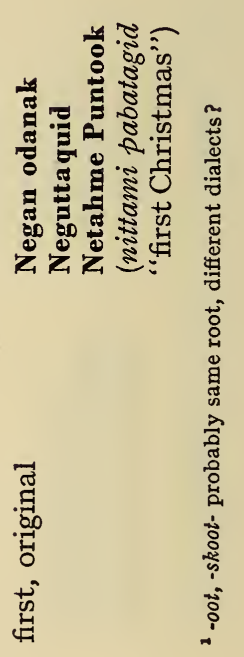

326 

高高高

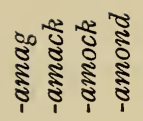
วิำ

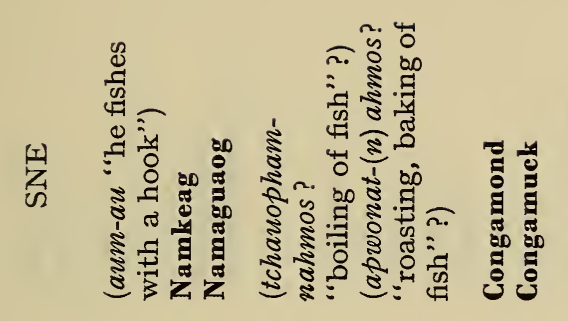

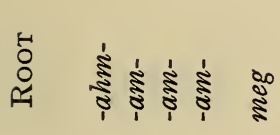

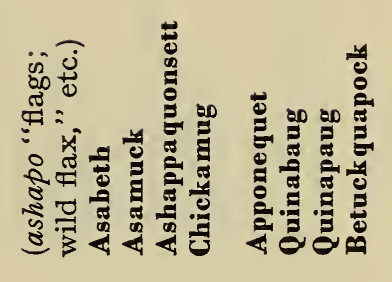

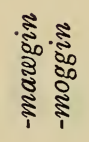

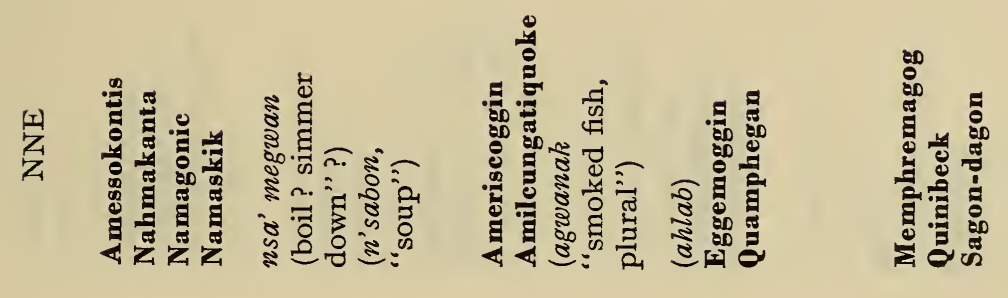

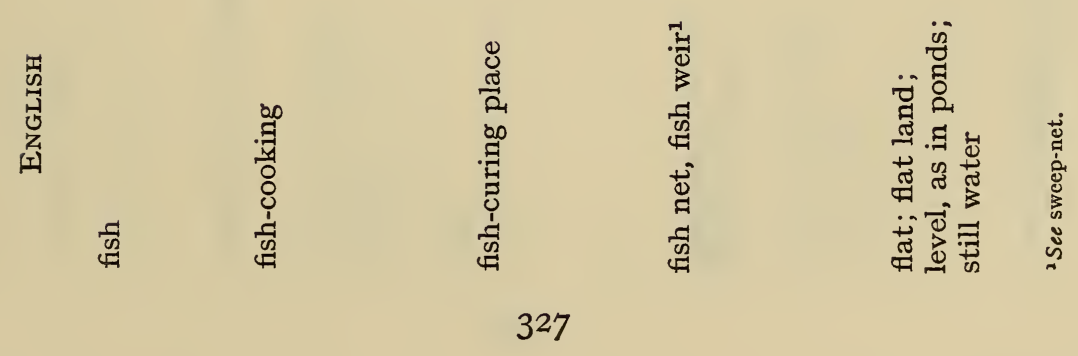




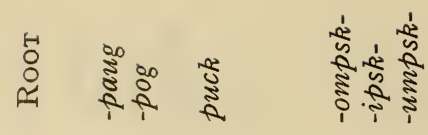
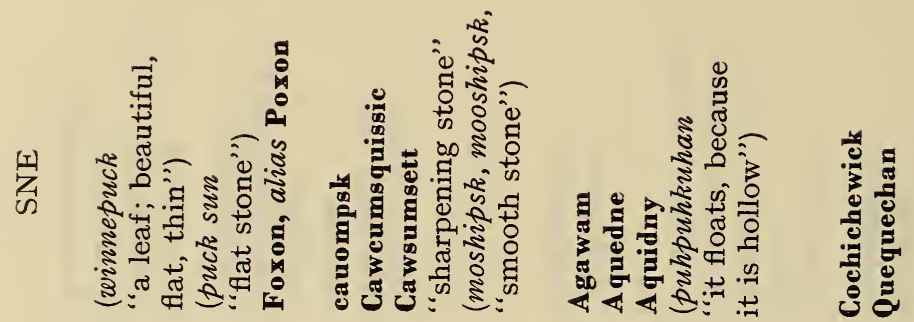

เั

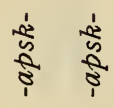

홍홍

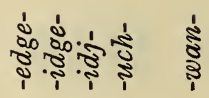

Z 다 Z
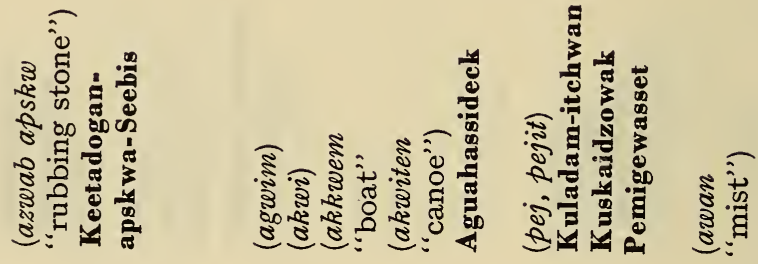

善
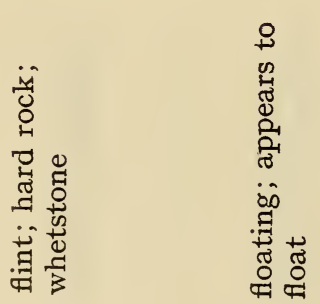

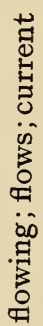

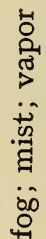


絗

妥亳

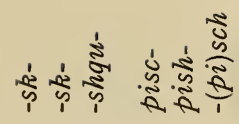

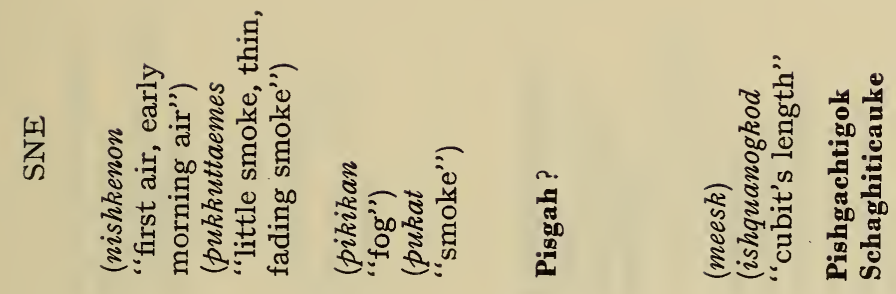

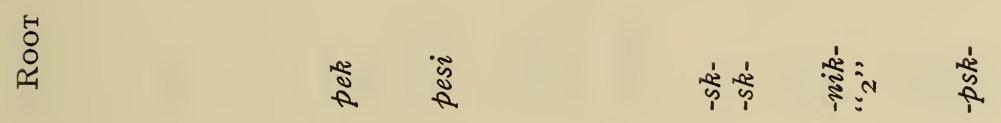

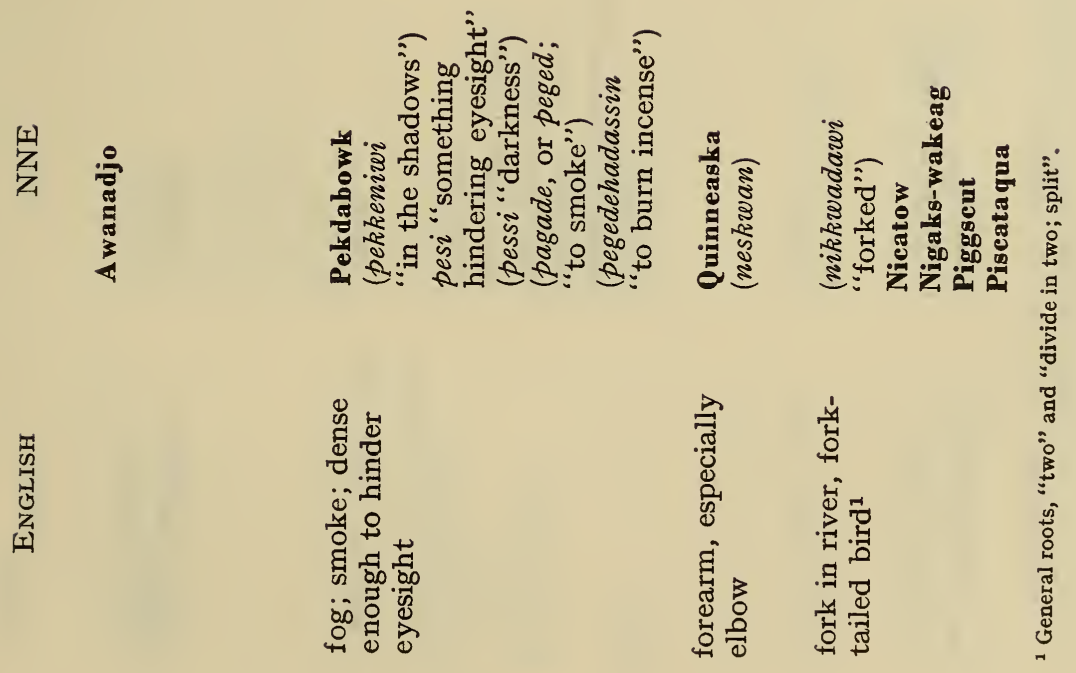




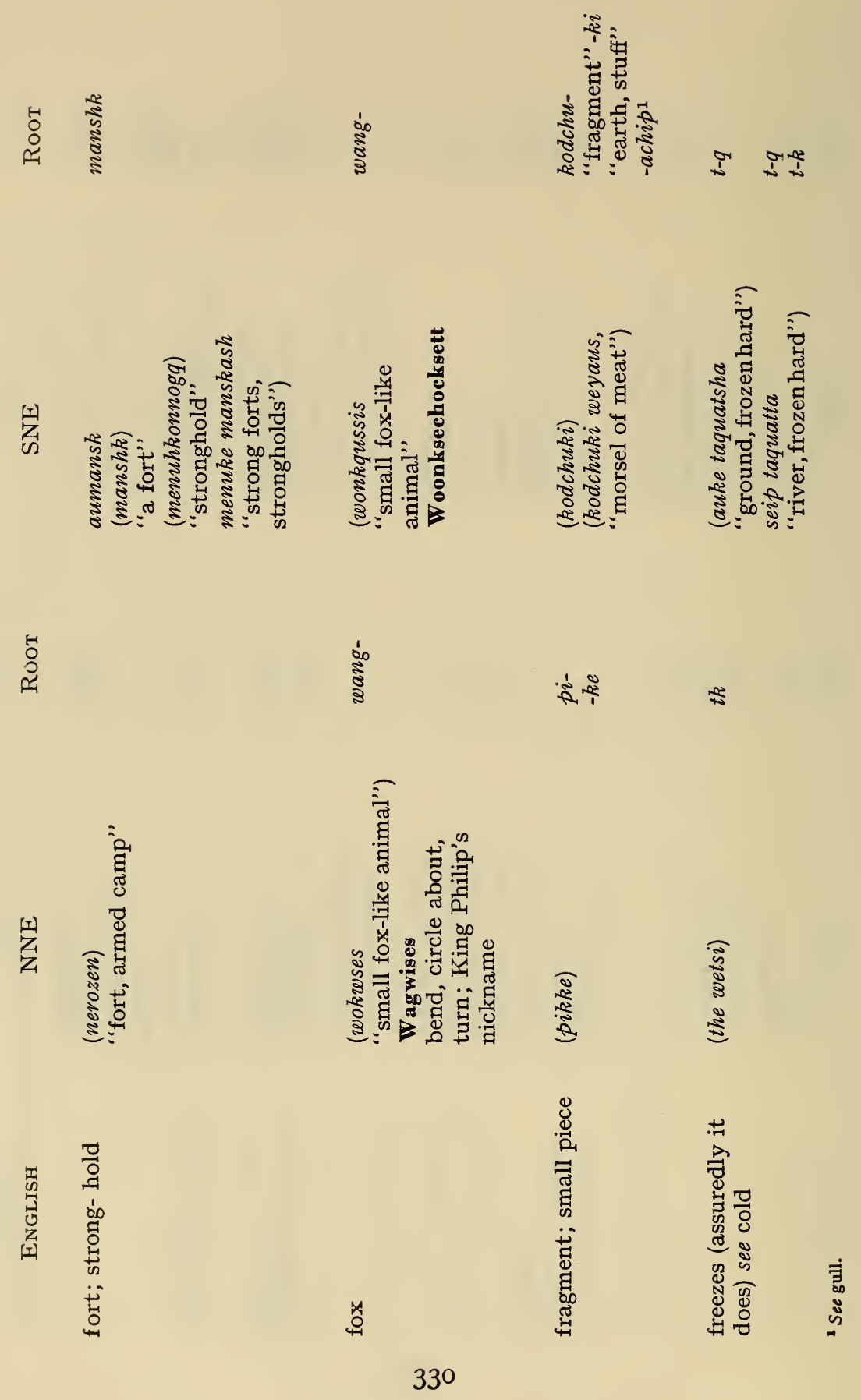


草

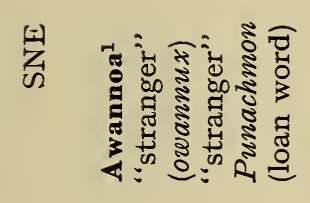

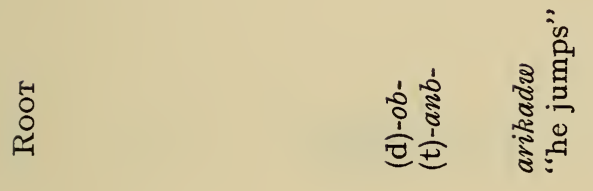

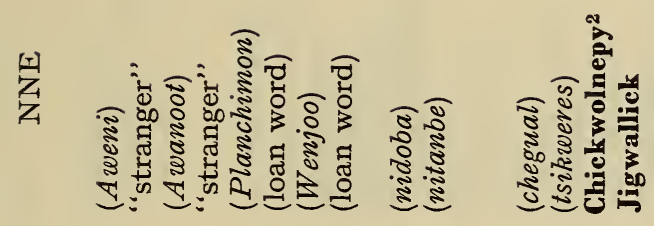

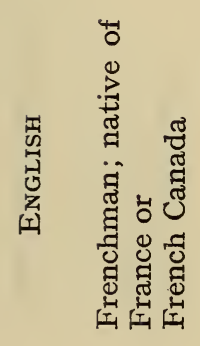

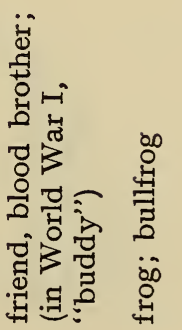

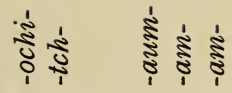

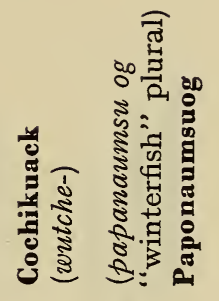

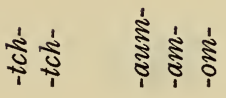

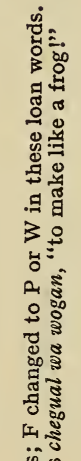

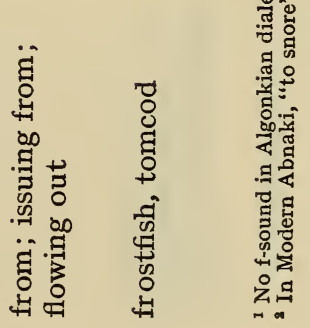



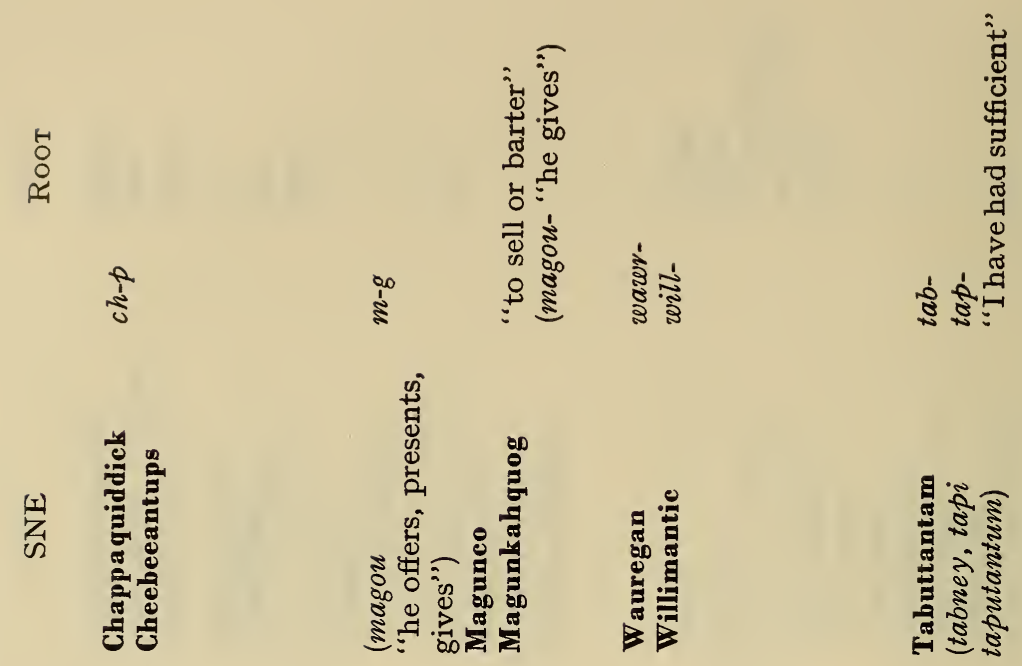

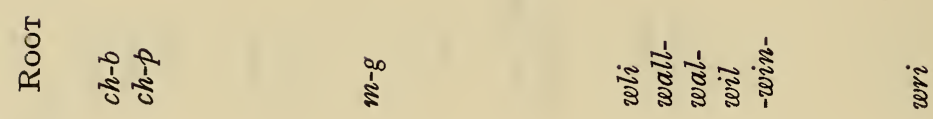

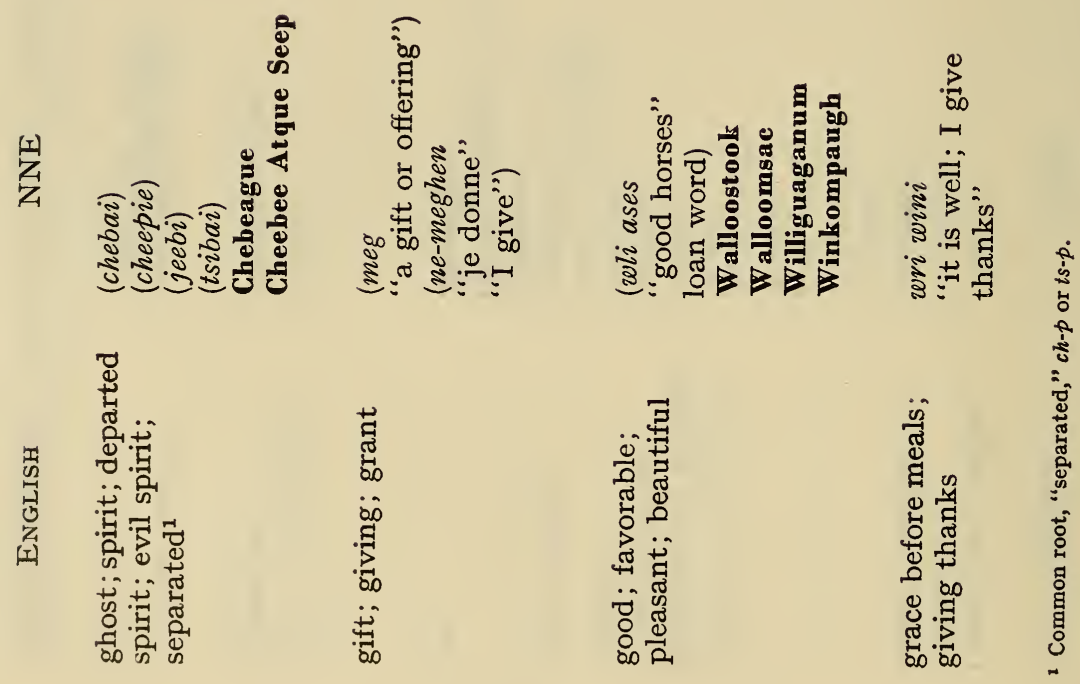


bo

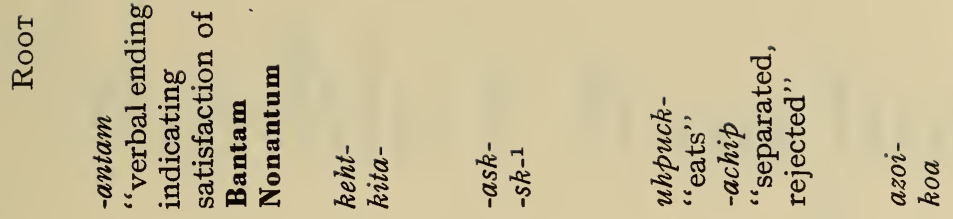

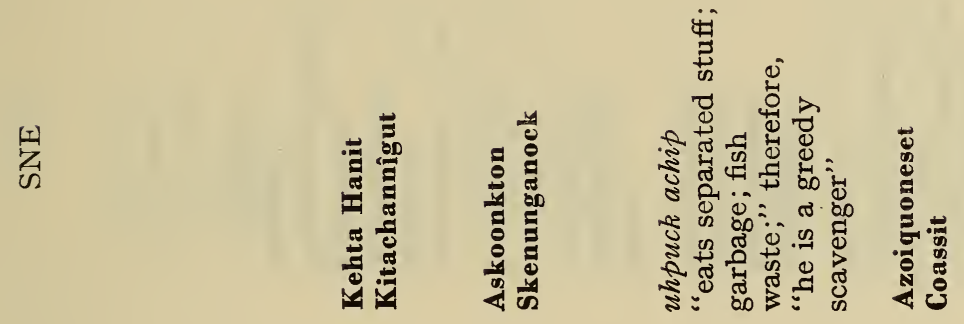

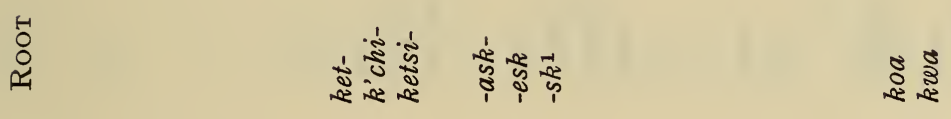

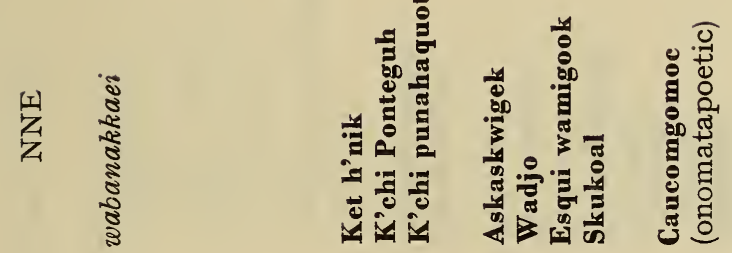

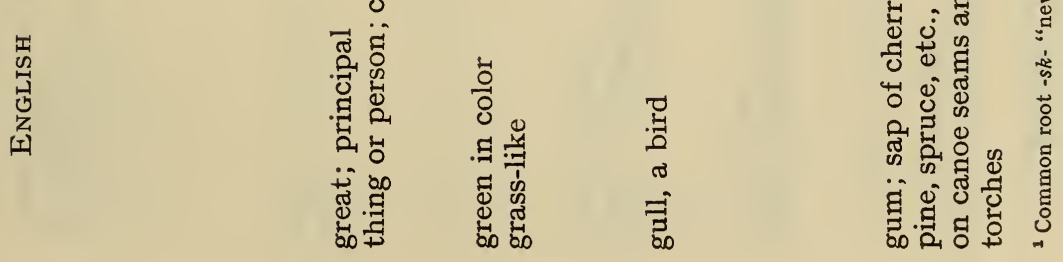


言

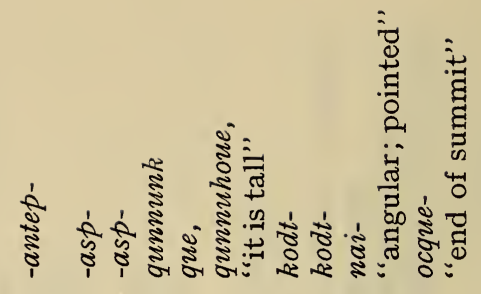

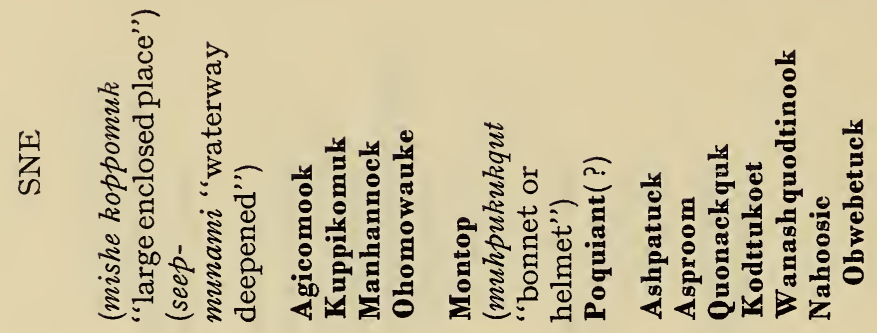

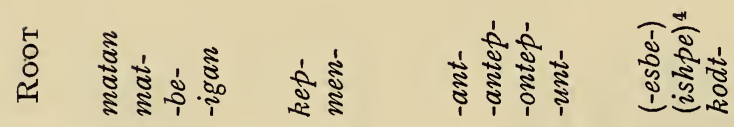

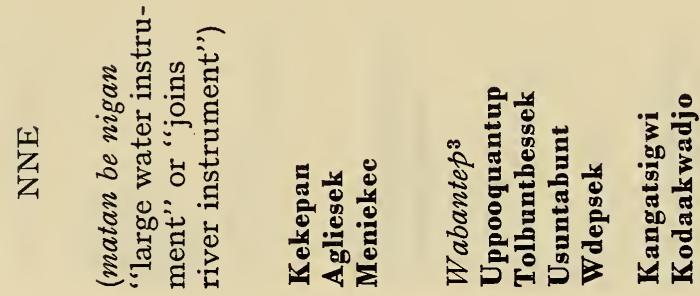

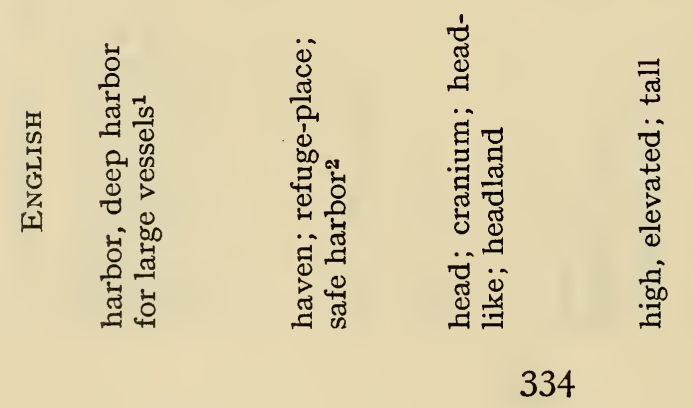

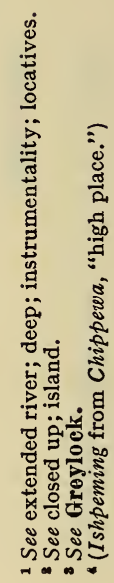




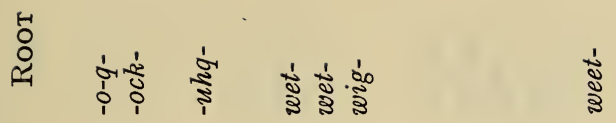

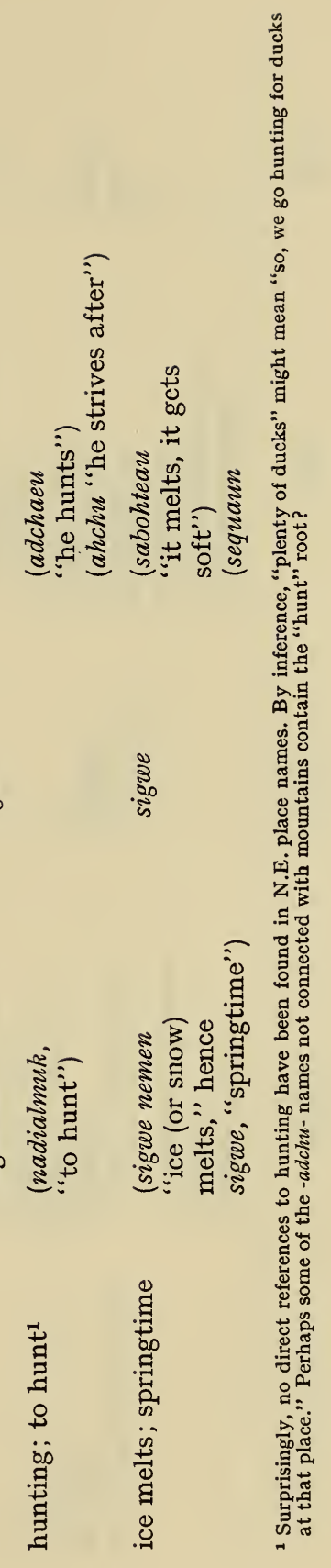


+
0
0

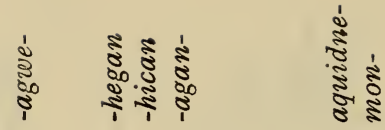

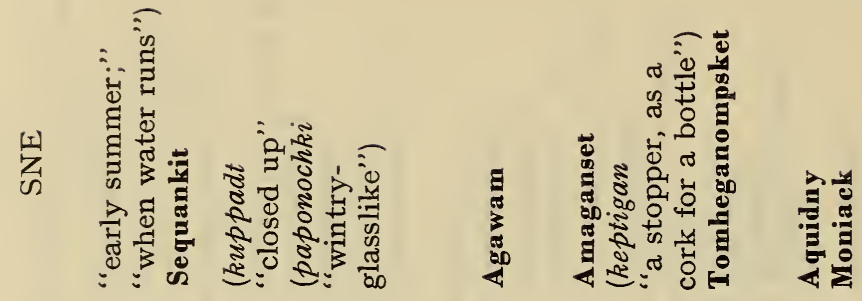

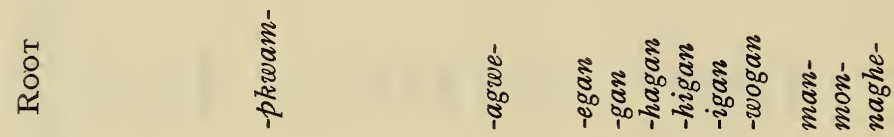

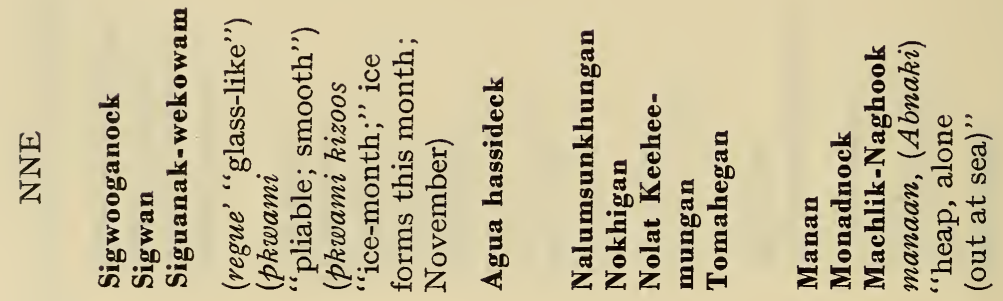

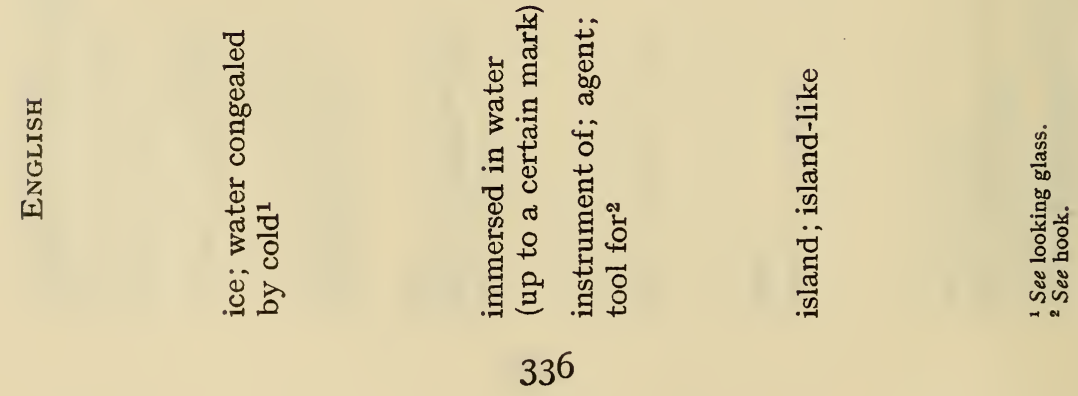




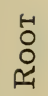

हू ई

$\frac{1}{1} \sqrt{5}$

".

㧒

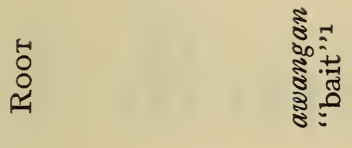

$\frac{1}{2}$

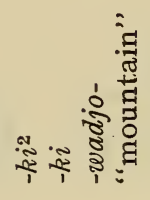

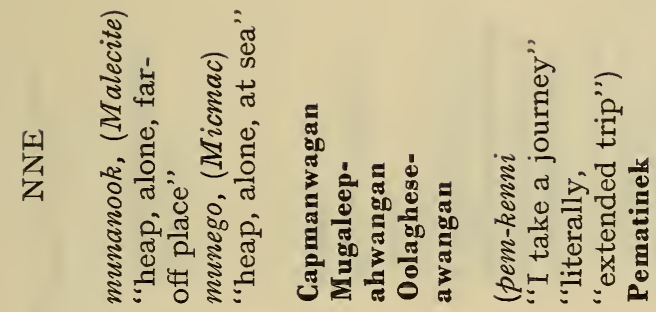

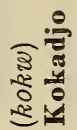

焉

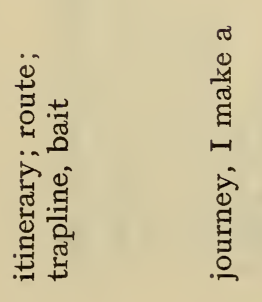

苂

華

章

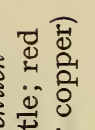

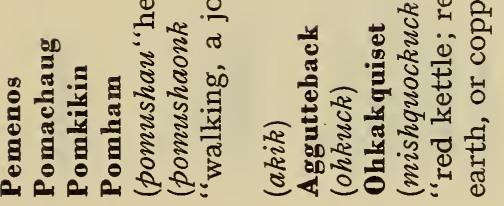

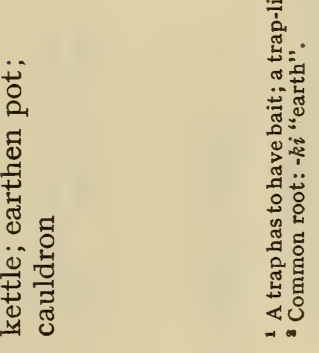




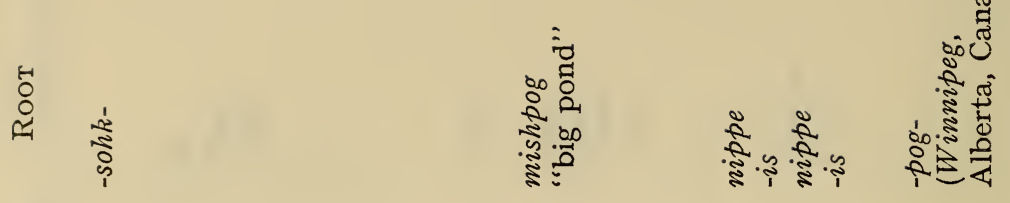

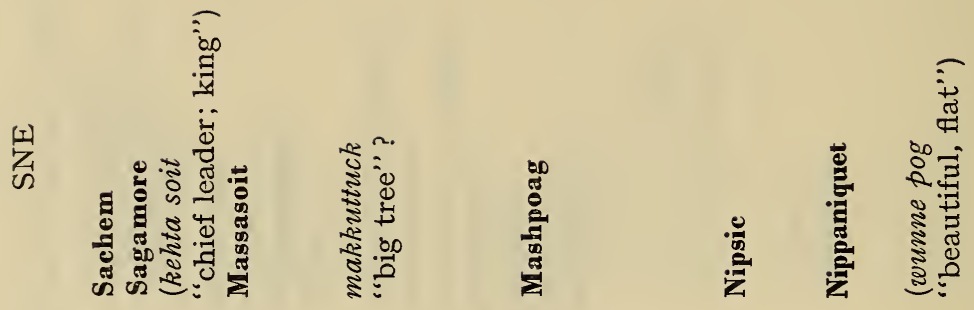<smiles>[124In]</smiles>

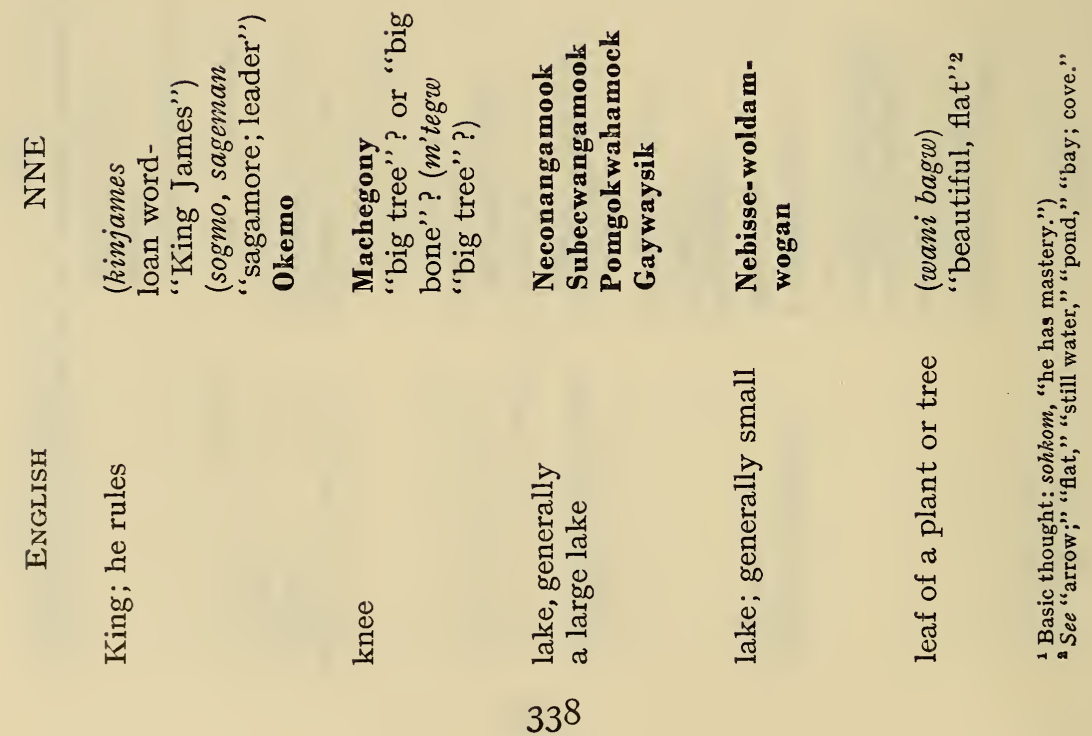




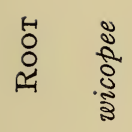

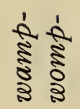

iัّ

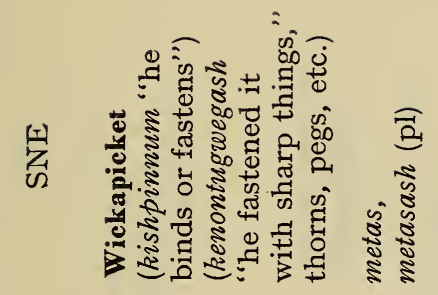

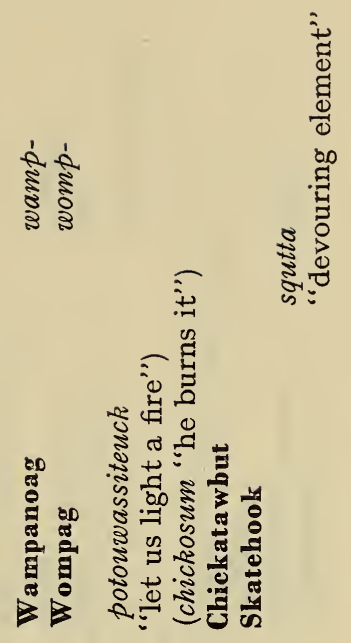

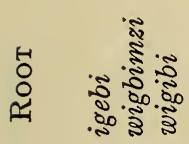

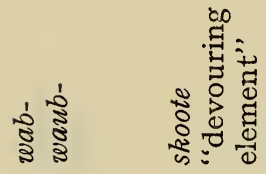

(1)

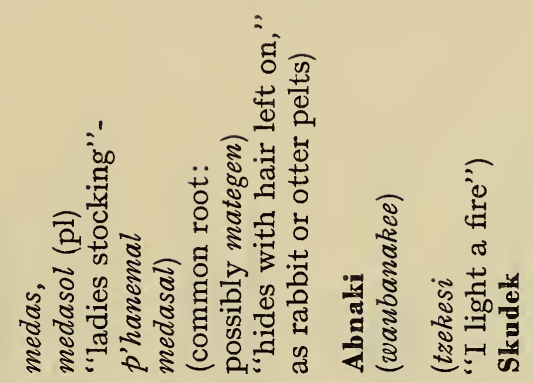

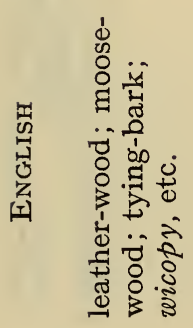

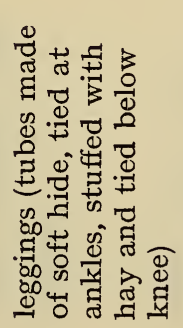

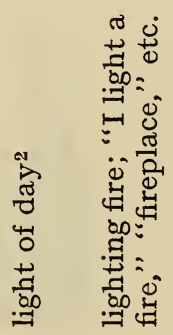


㸒

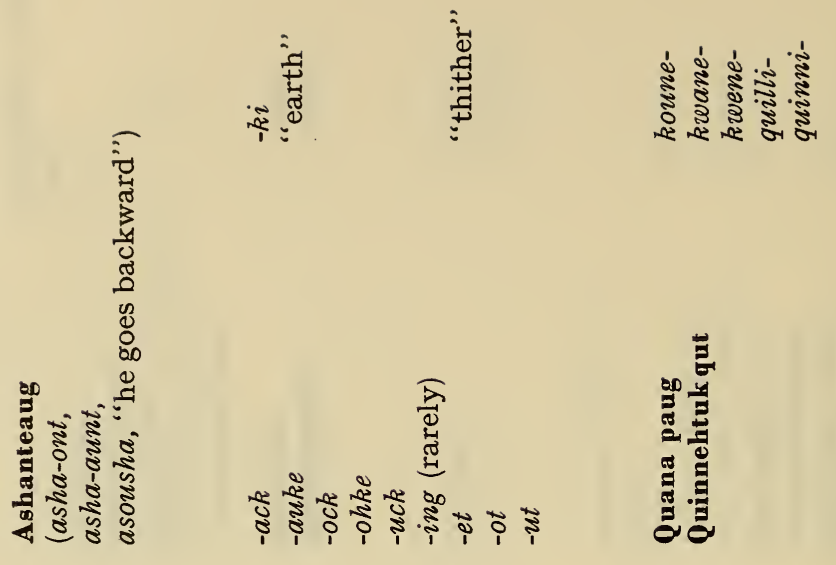

言
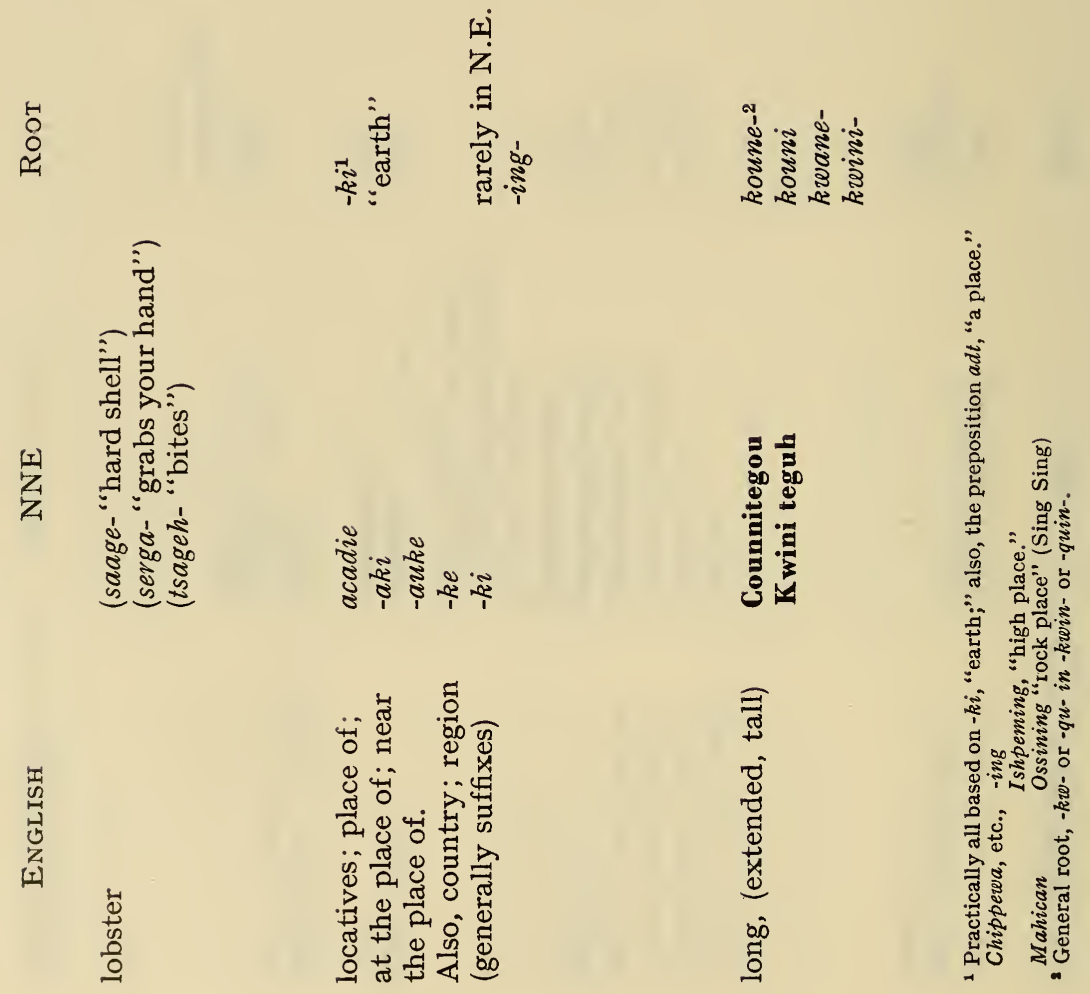
高

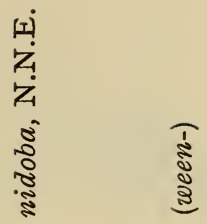

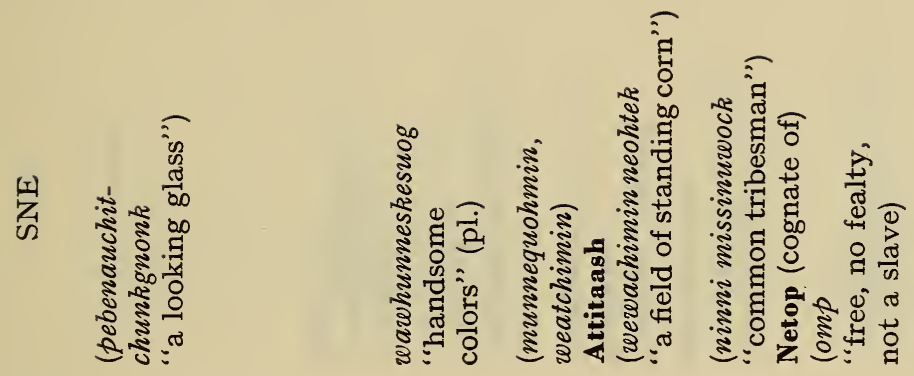

मे

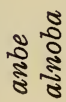

.

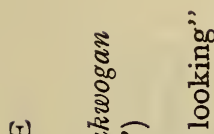

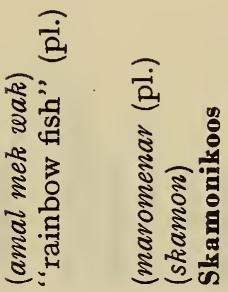

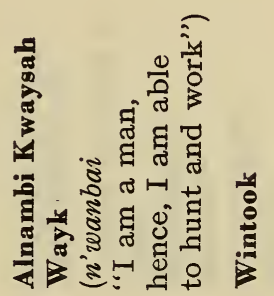

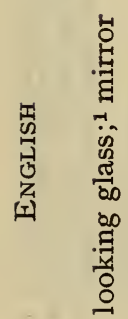

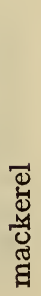

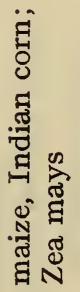

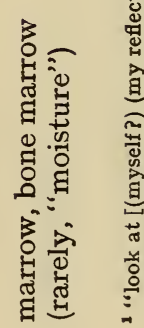

$34^{I}$ 


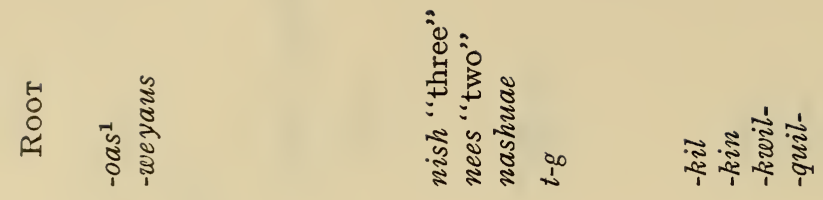

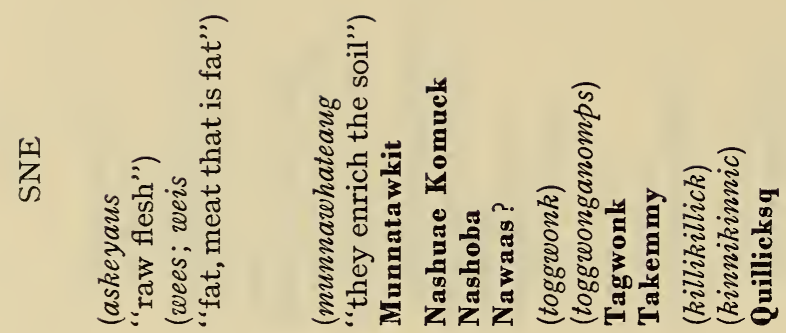

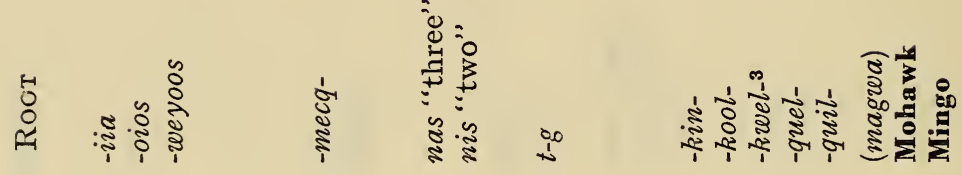

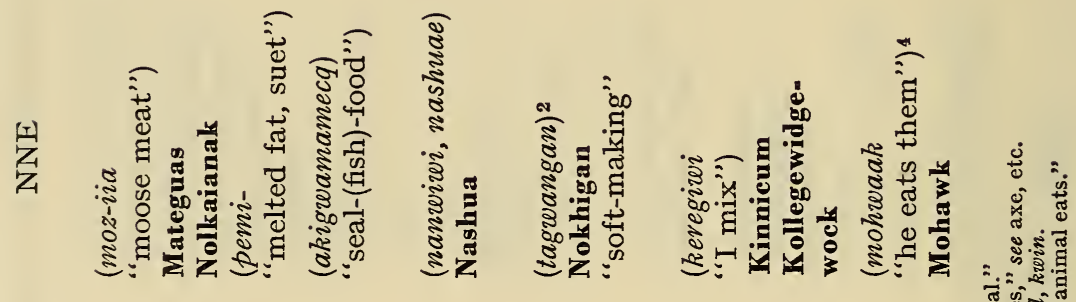

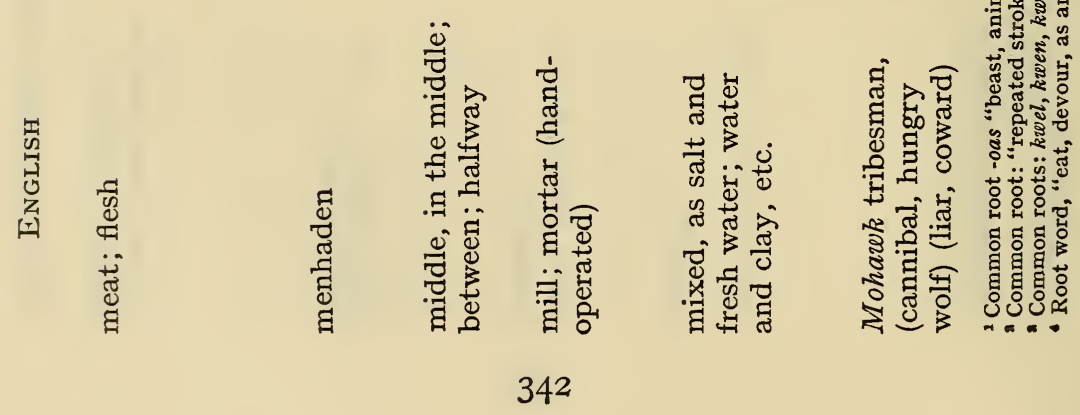



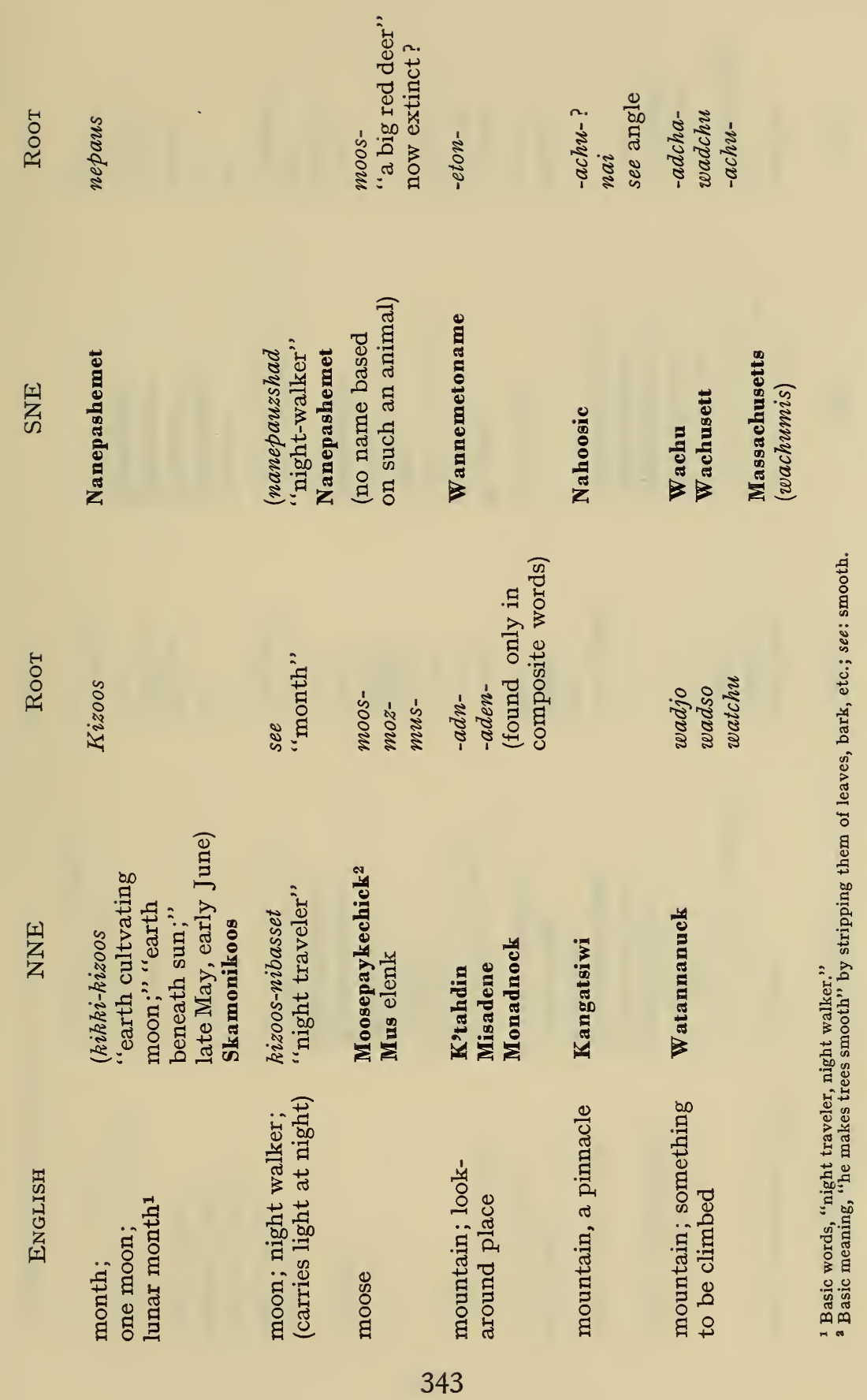


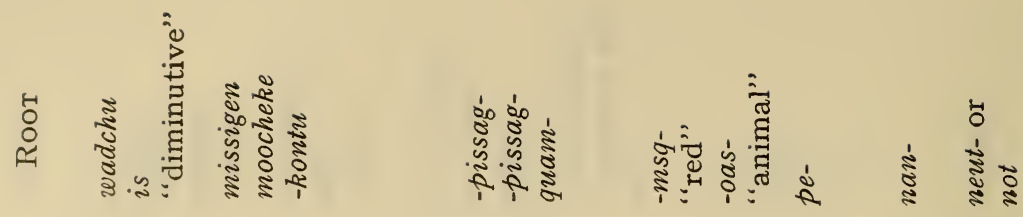

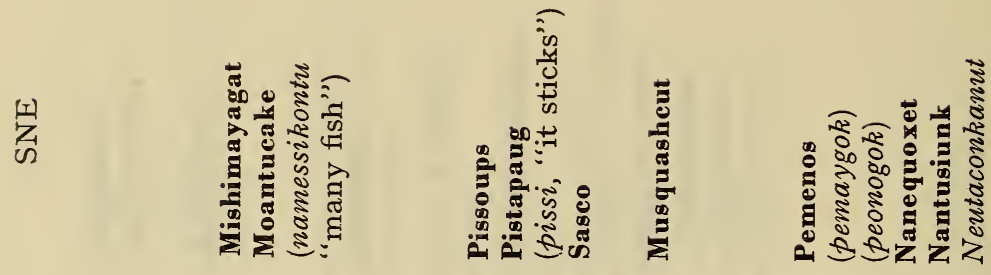

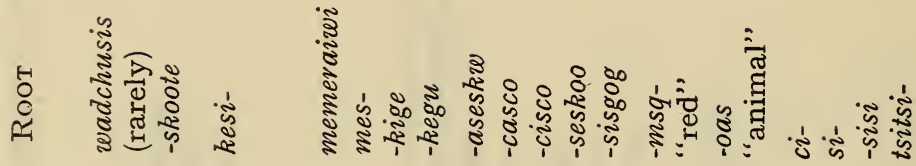

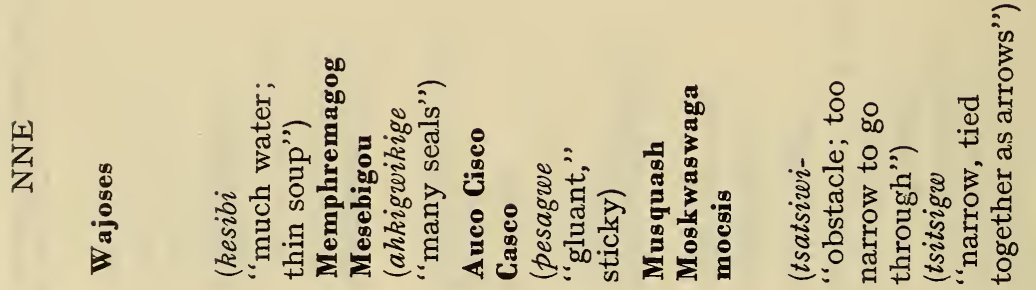

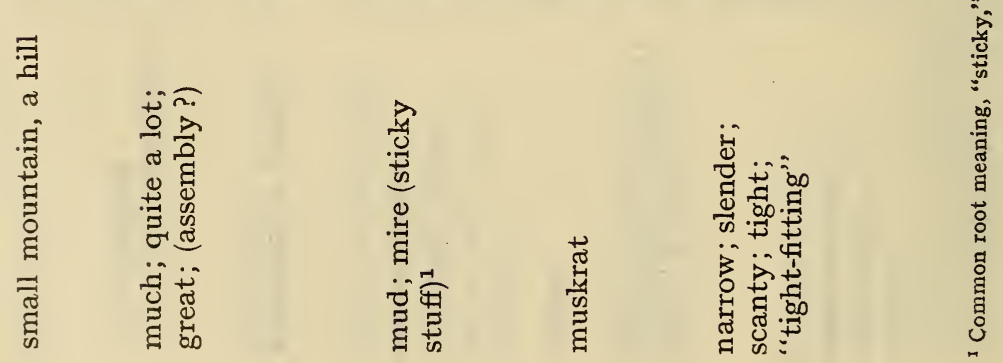




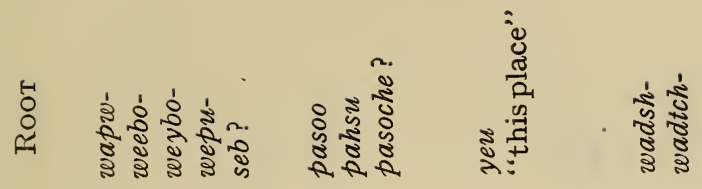

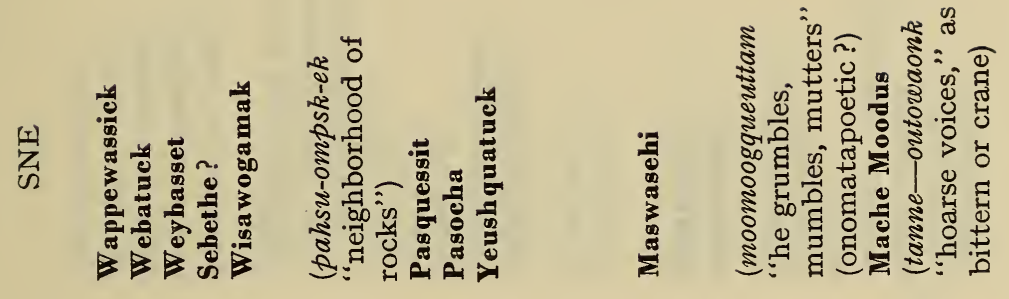

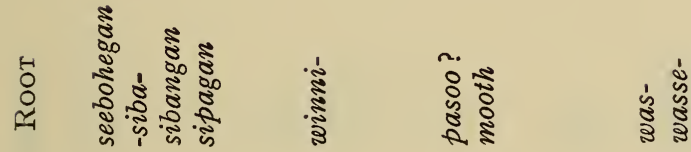

是

咅

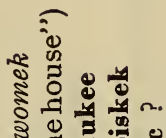

\%

)

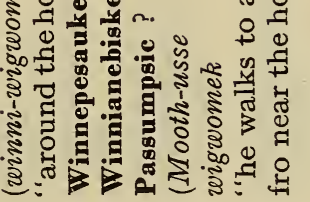

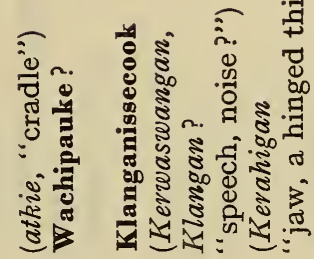

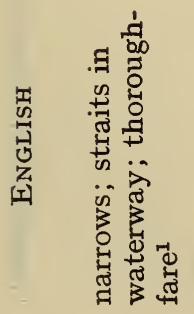

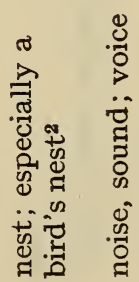

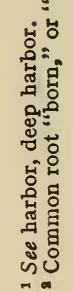




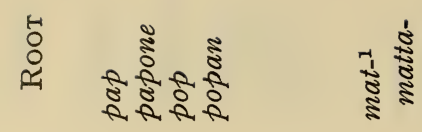

旁

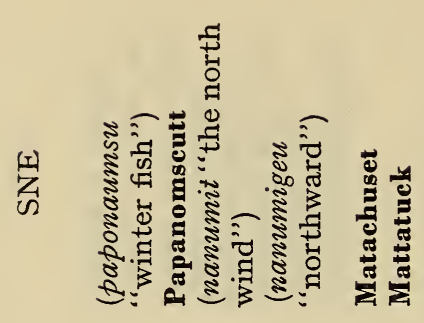

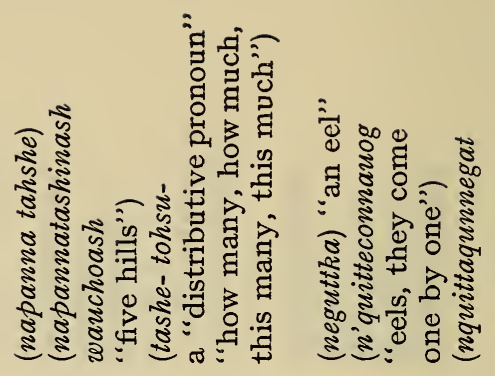

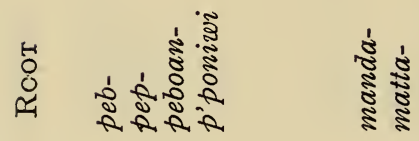

$\frac{1}{2}$

站

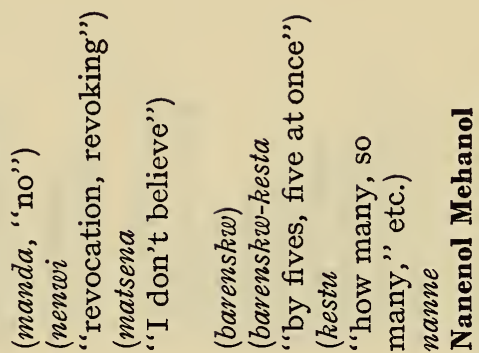

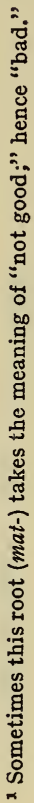

346 


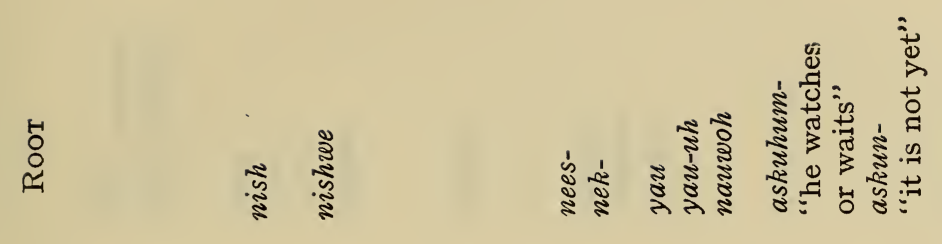

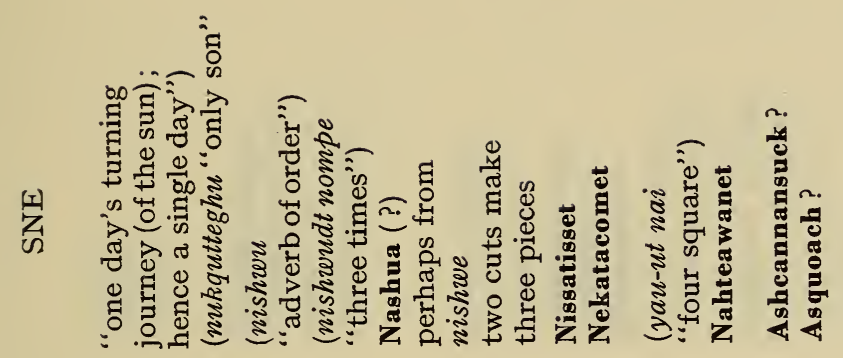

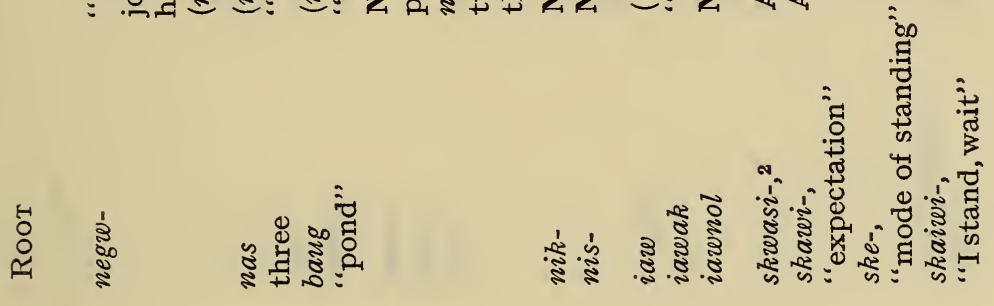

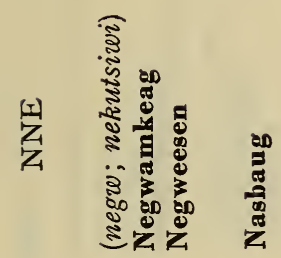

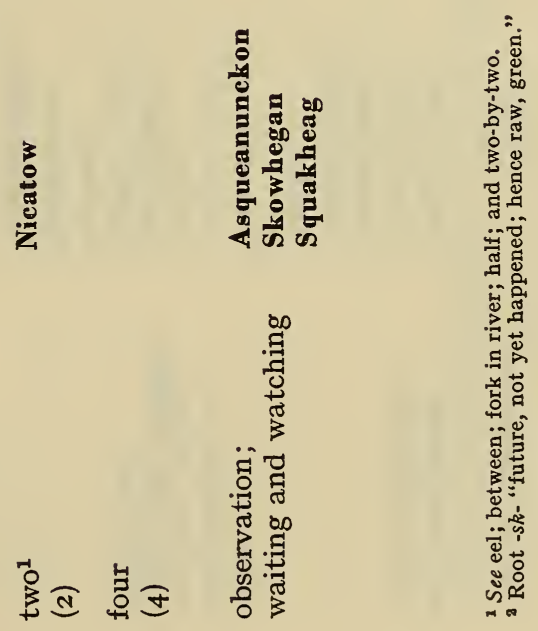

站两

茎 

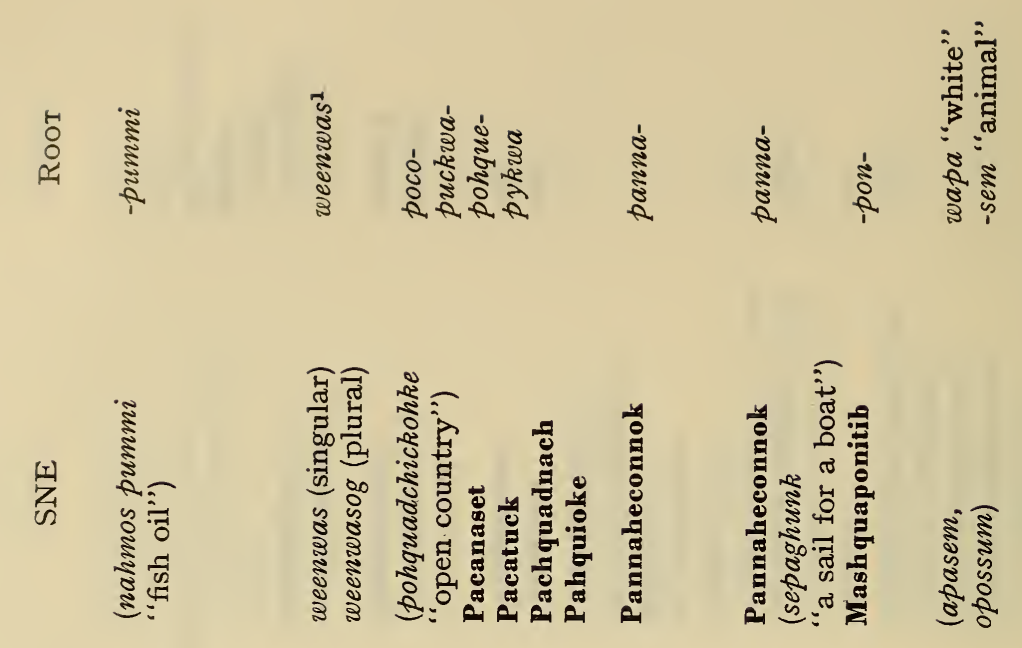

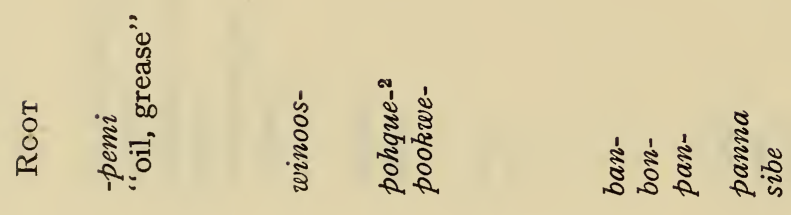

Z

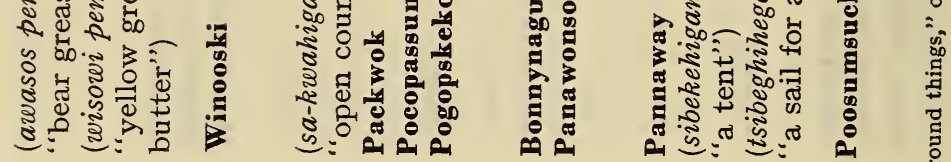

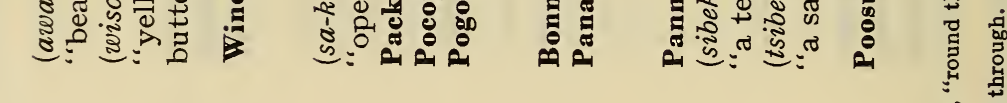

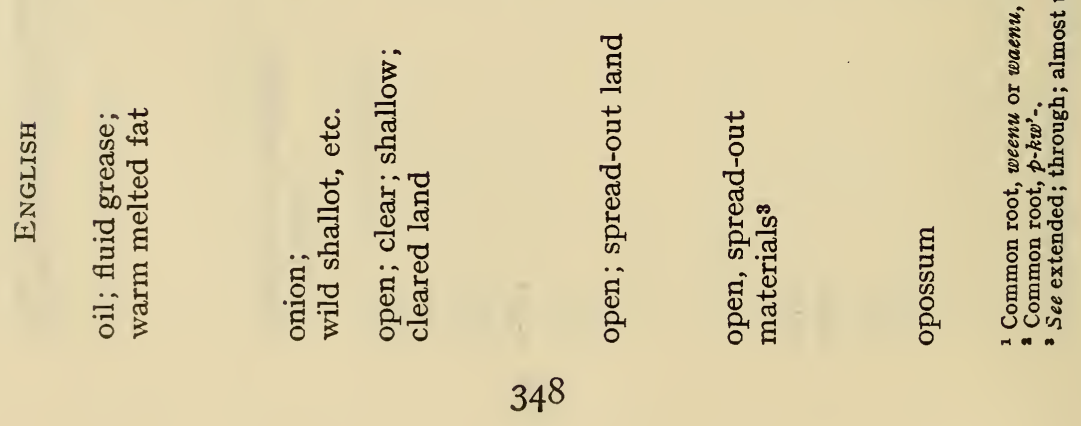




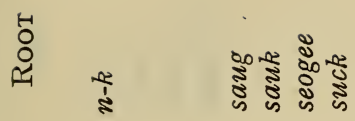

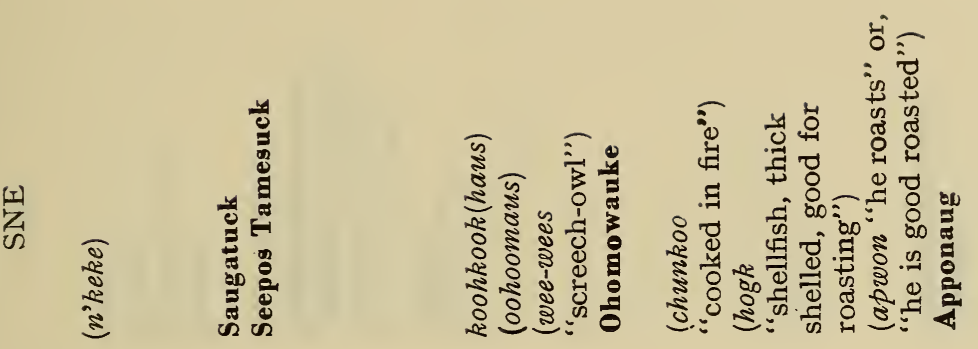

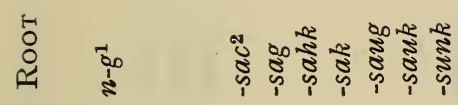

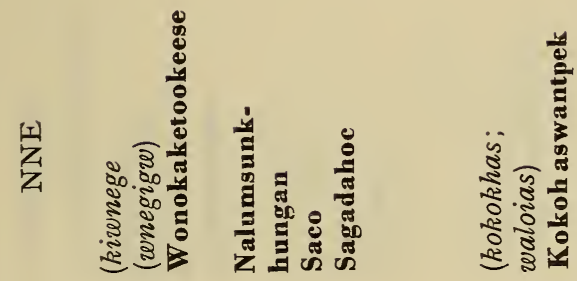

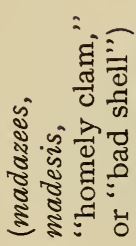

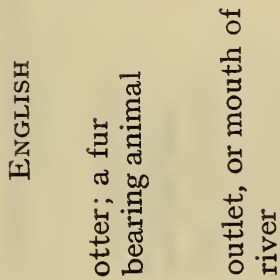
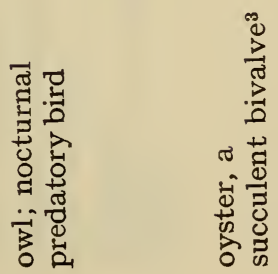


\section{苔}

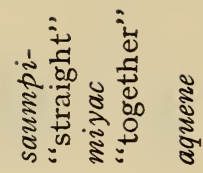

ฐั้

先

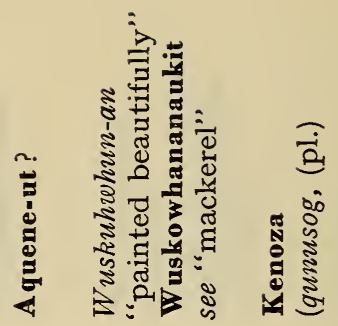

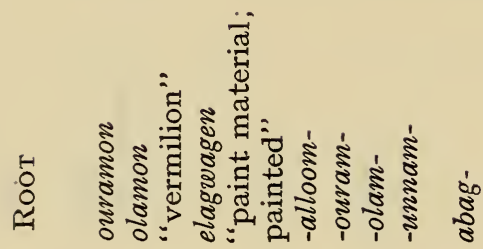

క్ర

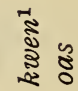

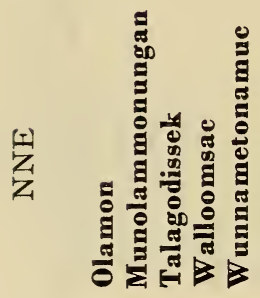

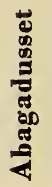

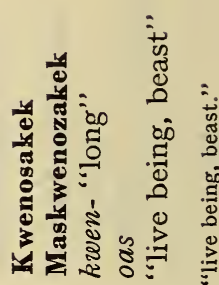

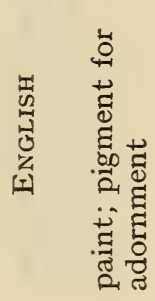

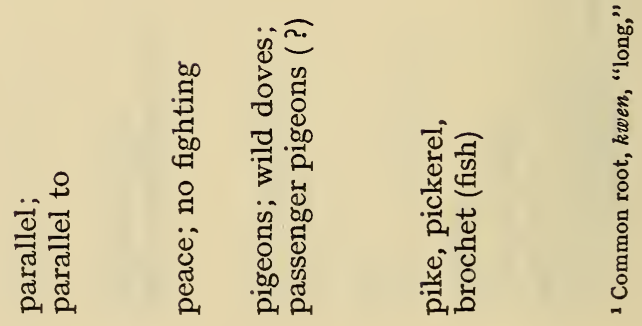




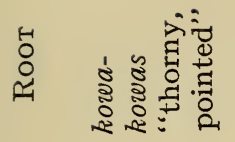

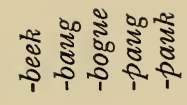

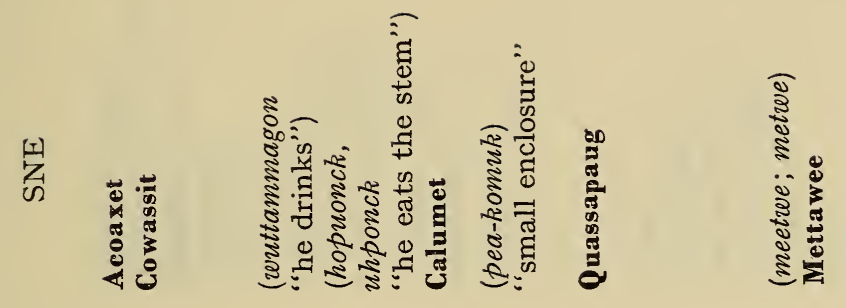

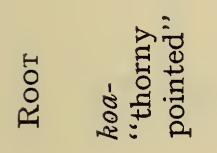

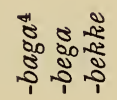

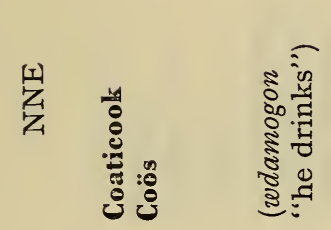

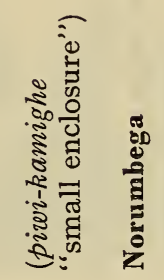

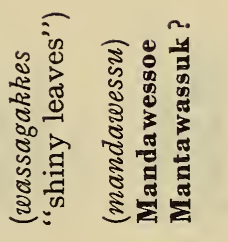

ind

童善

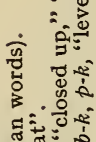

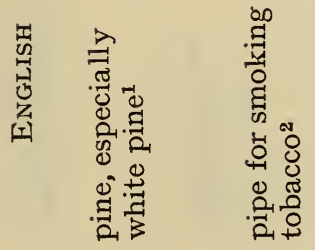

产通

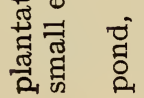

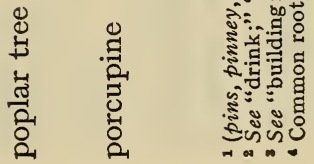

35 I 


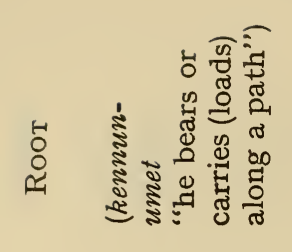

$$
\text { 畏臂 }
$$

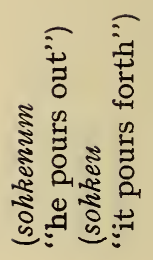

न्न
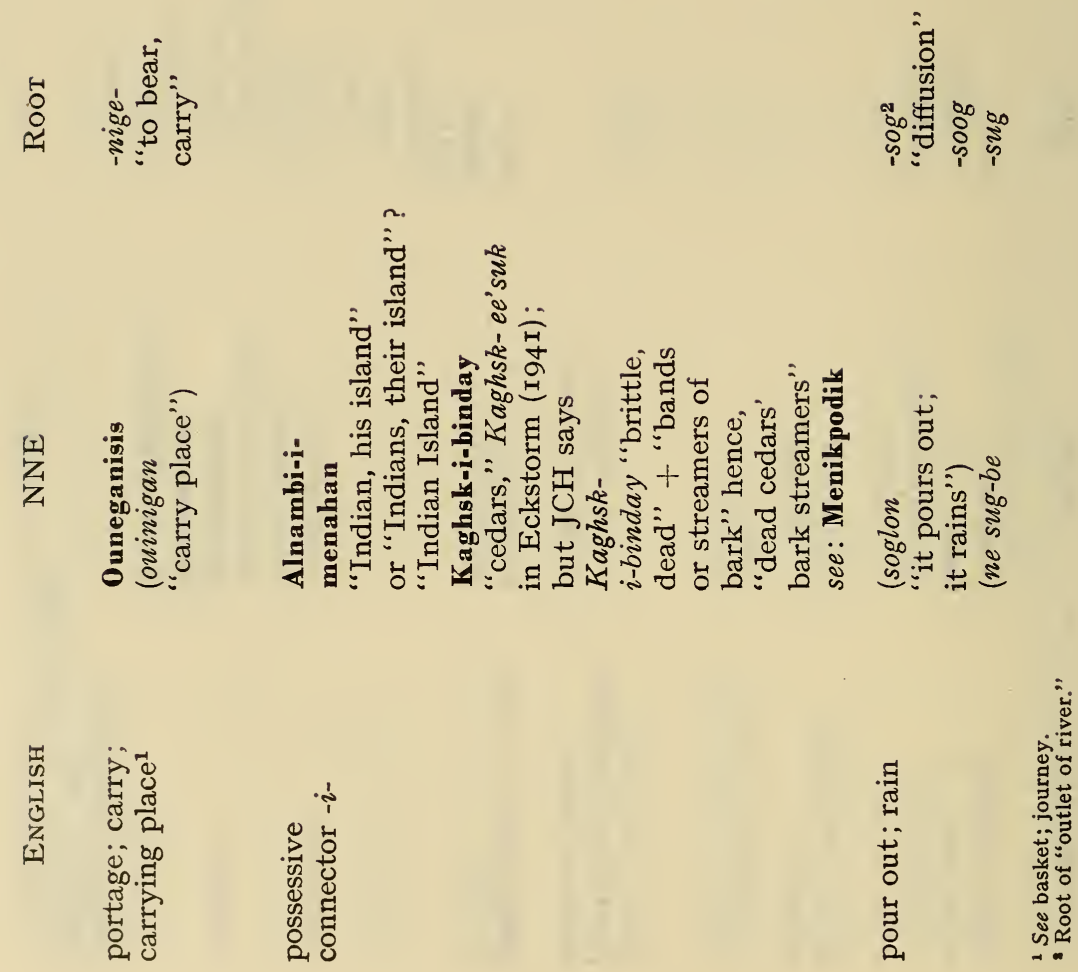
高

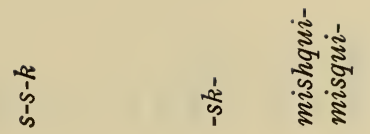

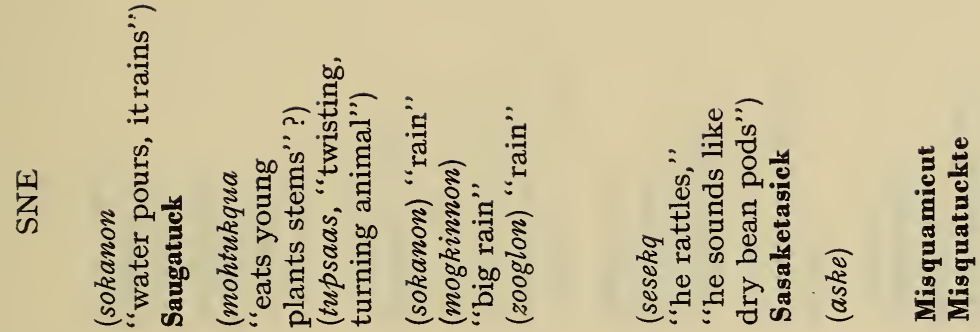

言

言

素

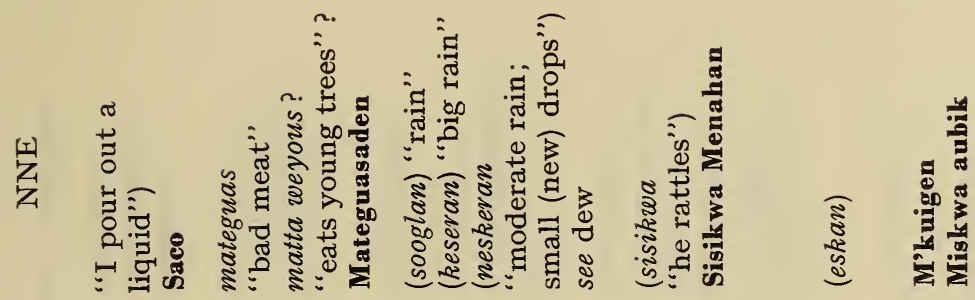

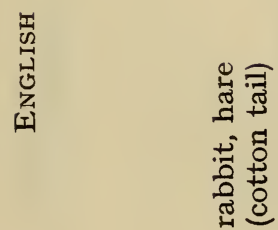

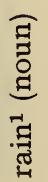

离

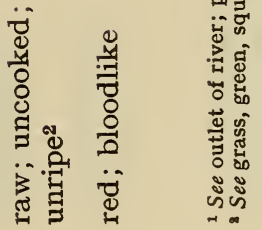




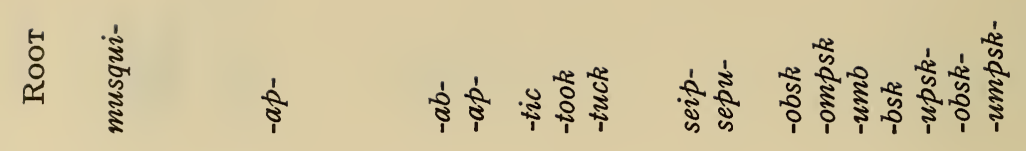

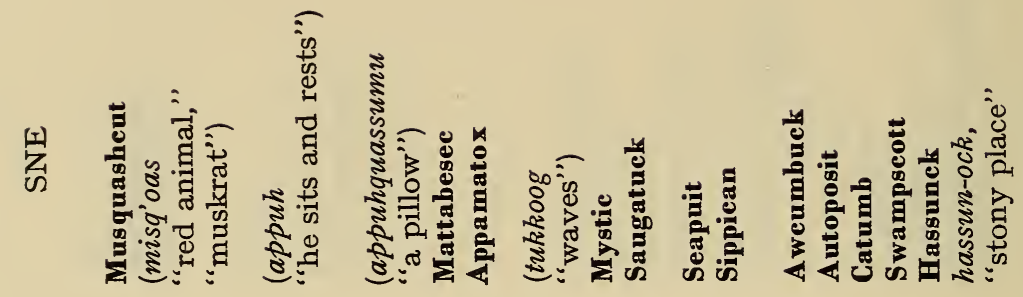

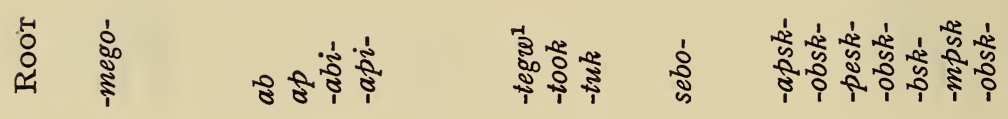

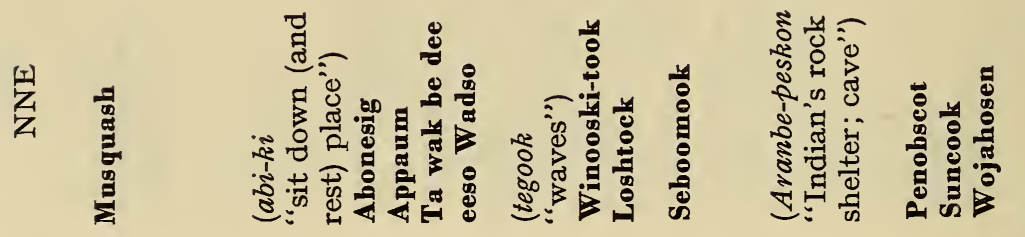

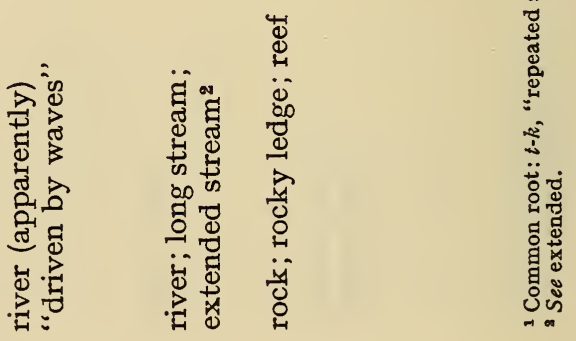

354 


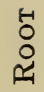

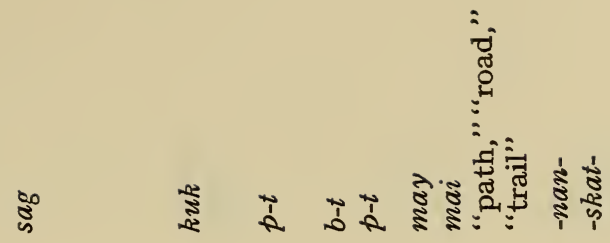

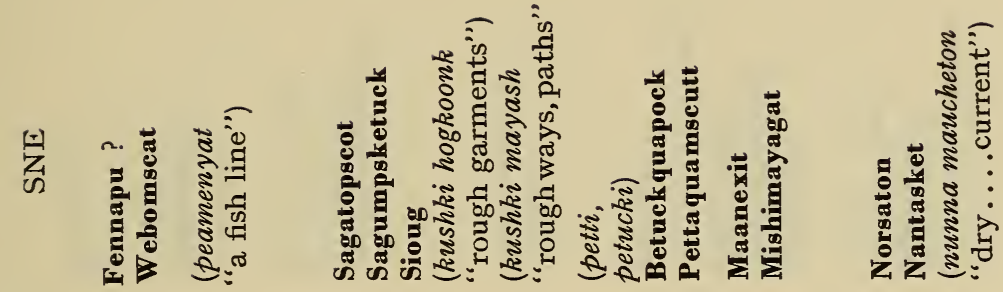

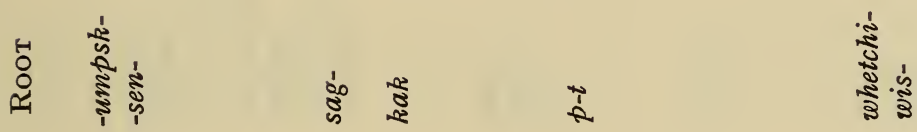

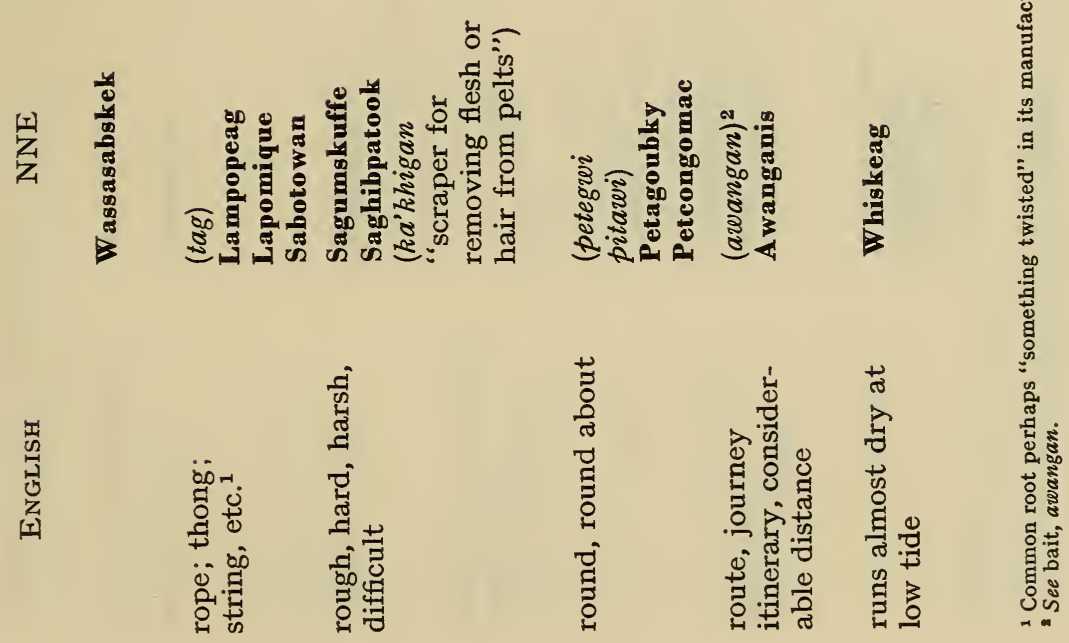

$23^{*}$ 


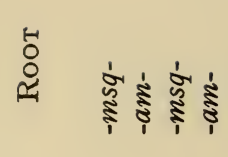

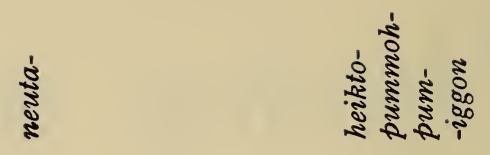

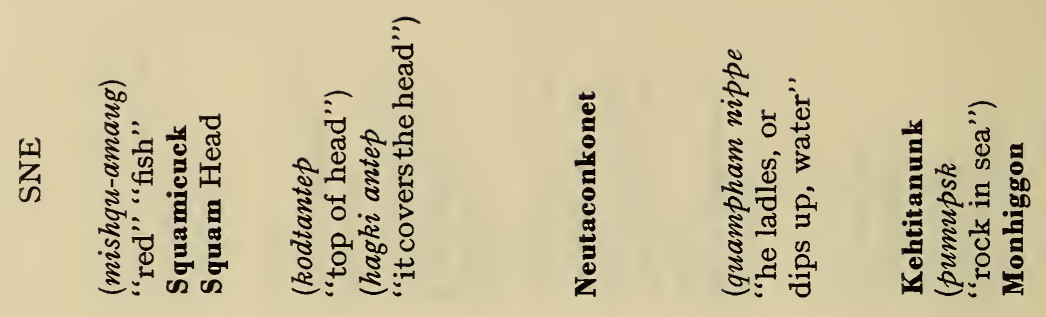

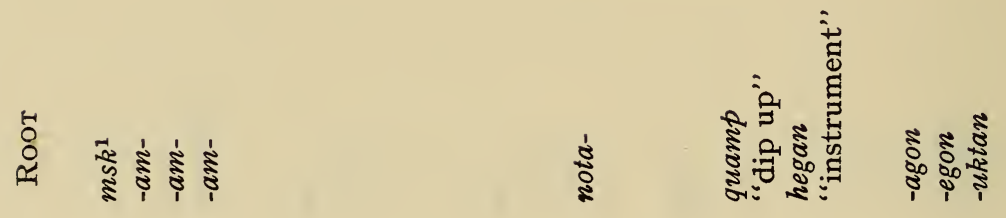

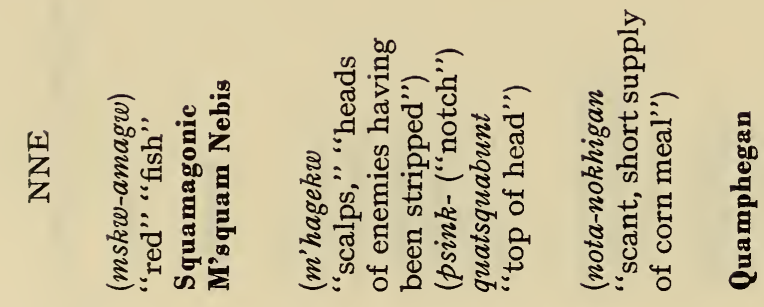

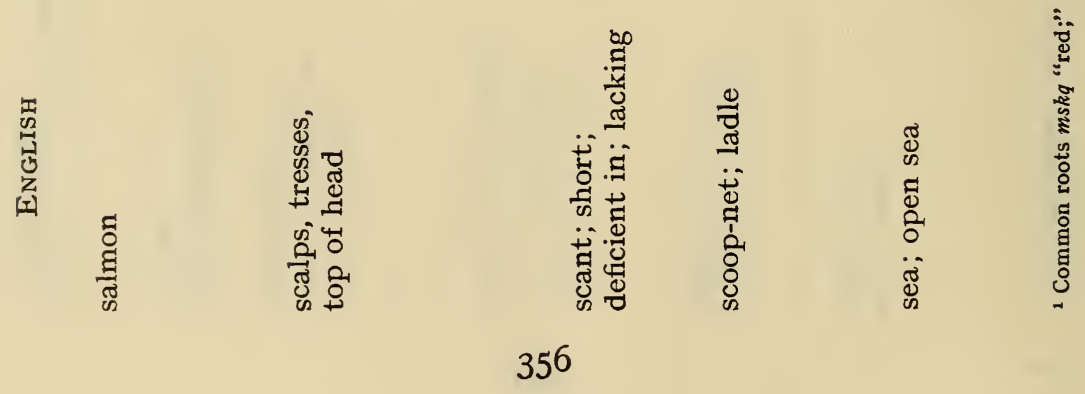




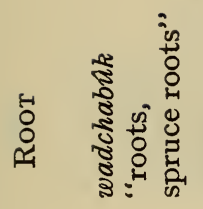

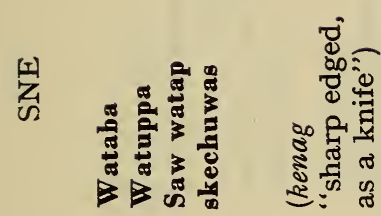

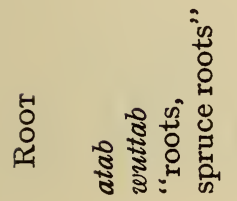

离定

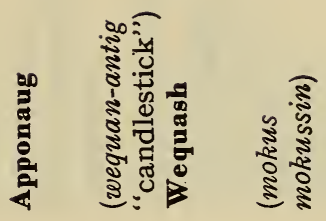

is

善

棠

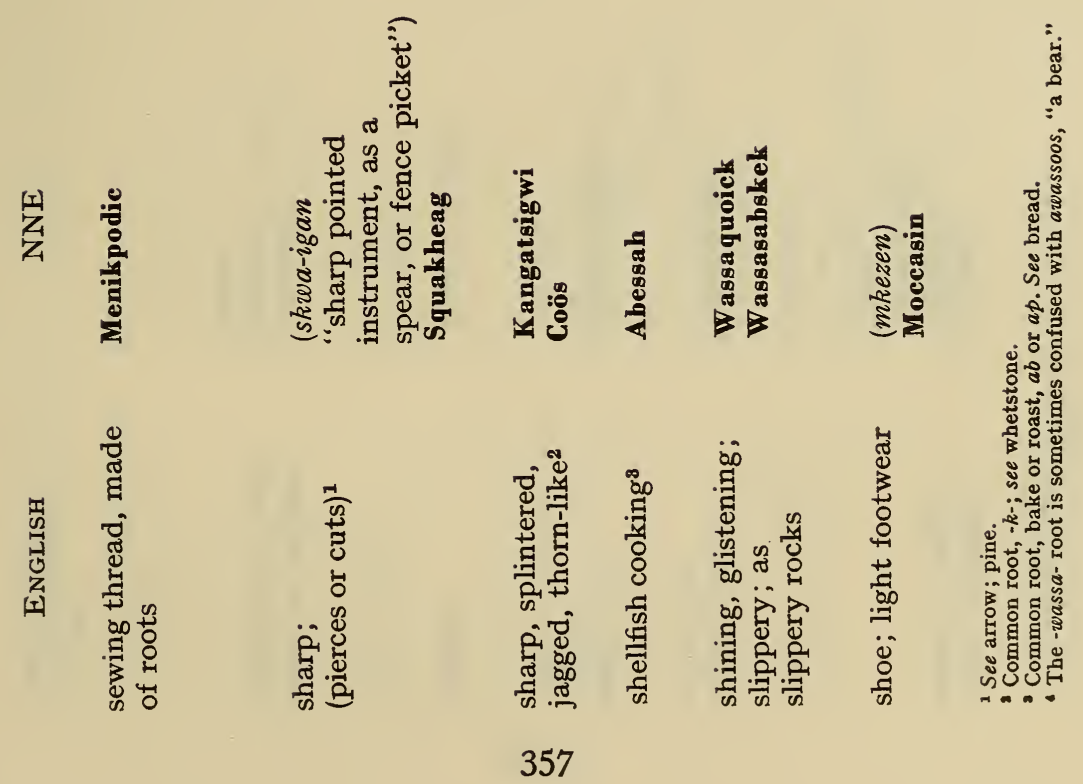




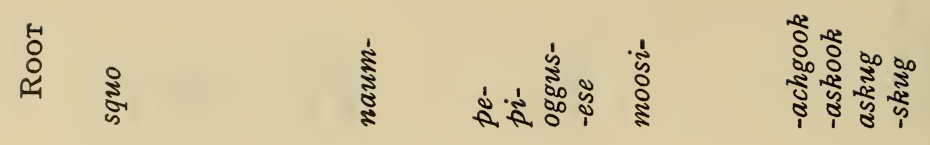

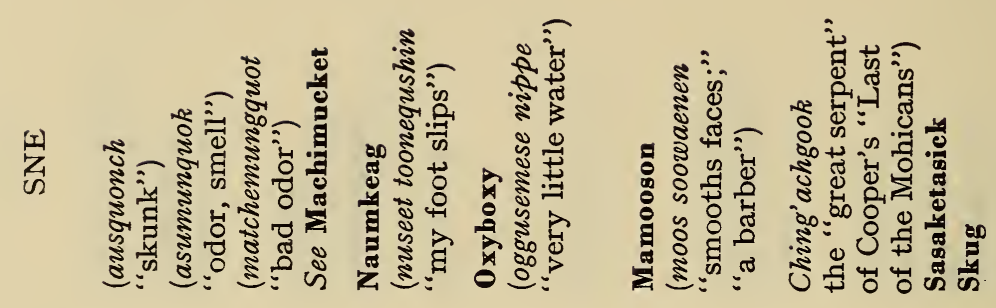

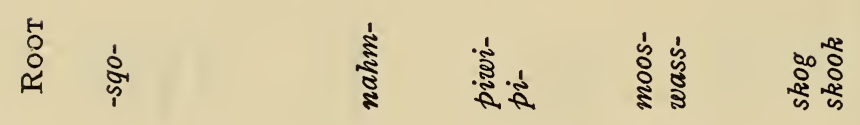

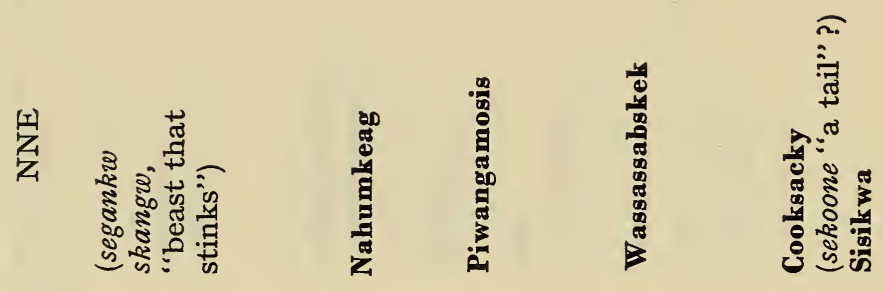

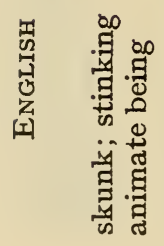

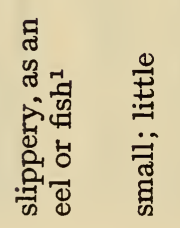

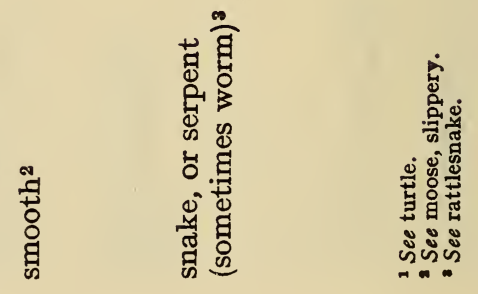




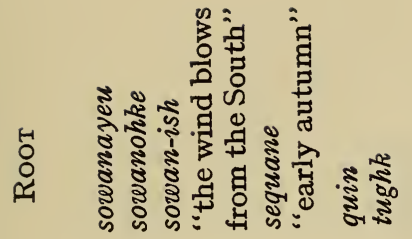

孚 莺

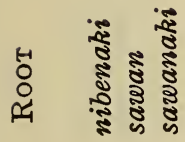

孚

敢

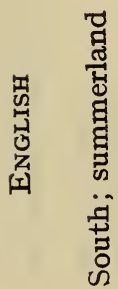

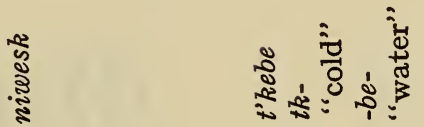

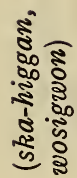

के 명

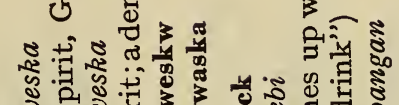

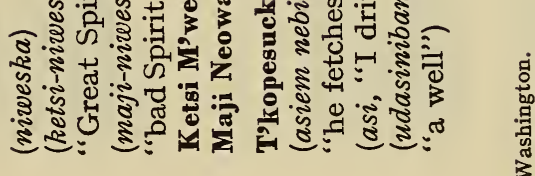

菢

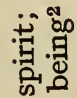

量
药

究品

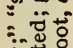

훈 


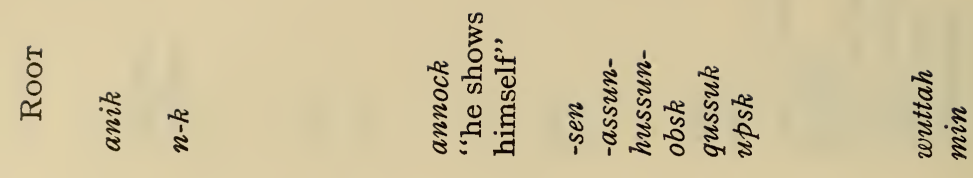

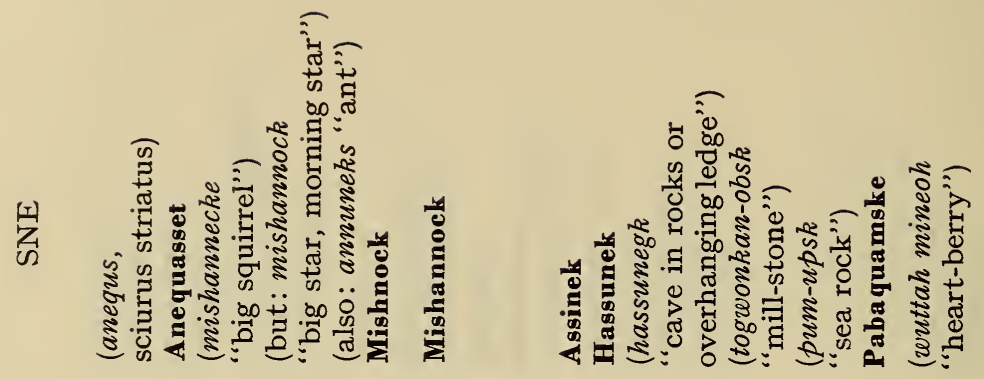

莒喜

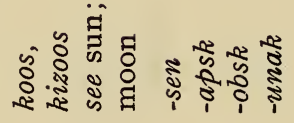

䇏战

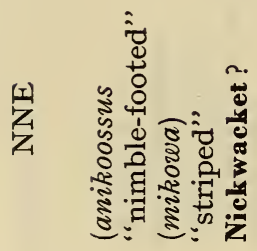

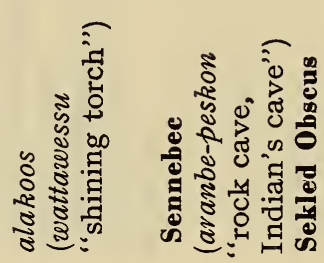

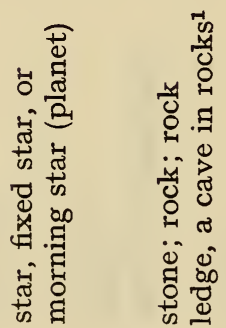

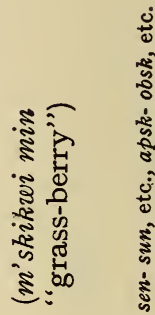

贸

360 


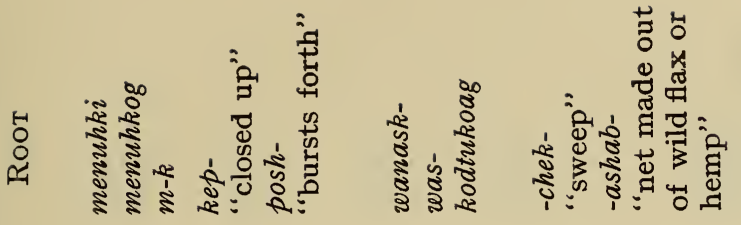

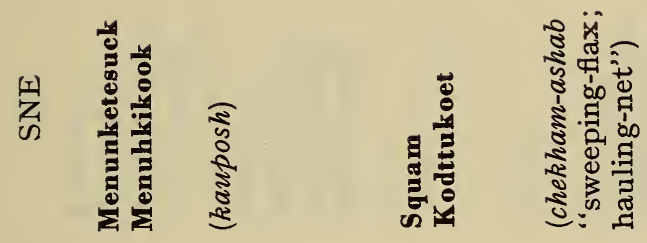

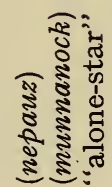

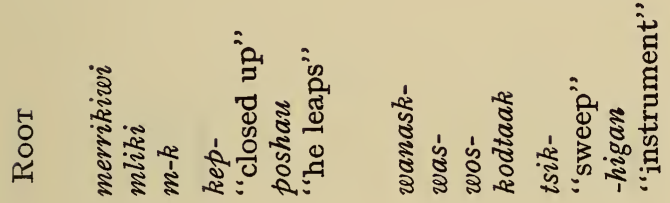

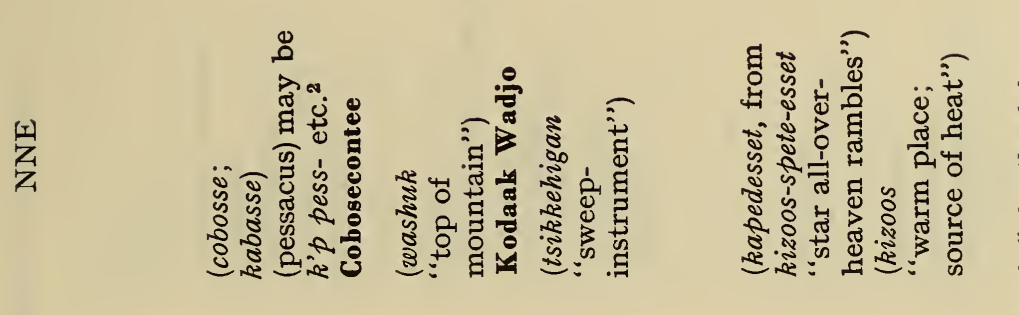

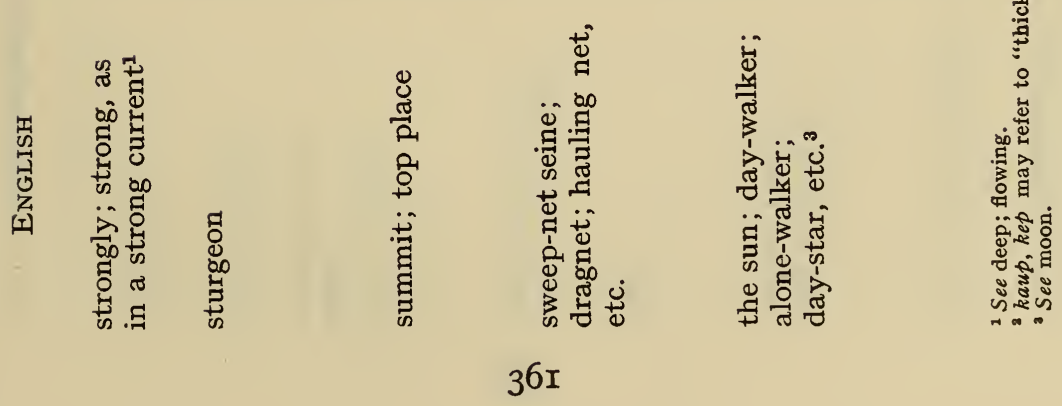



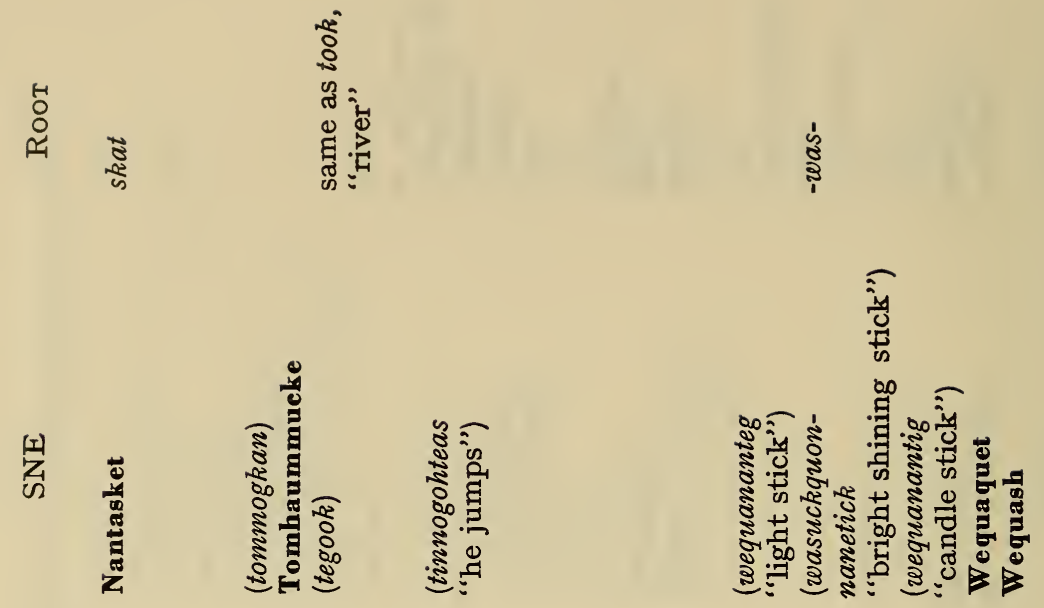

高

管

亭

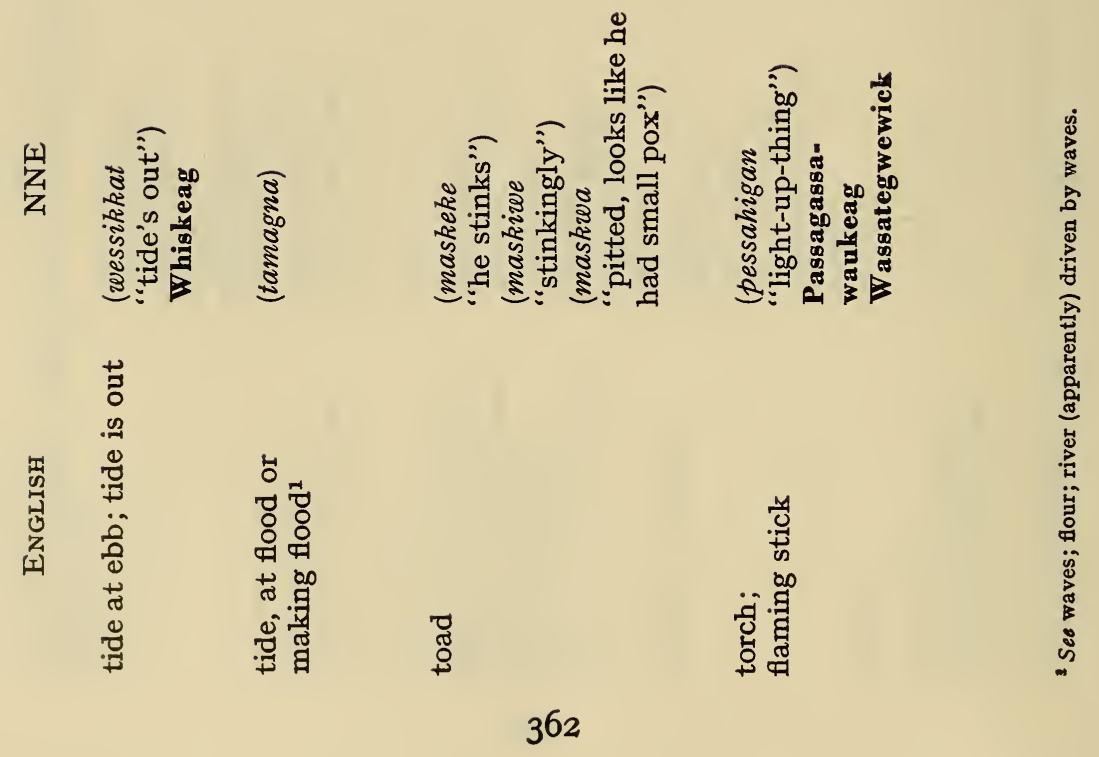




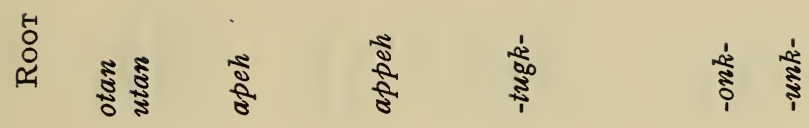

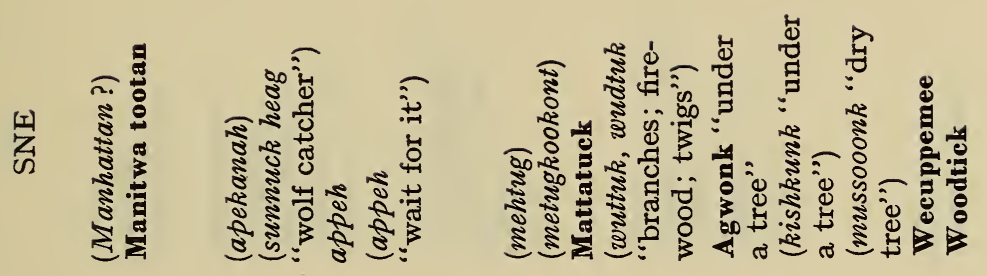

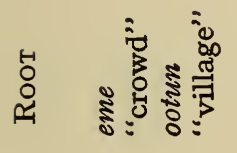

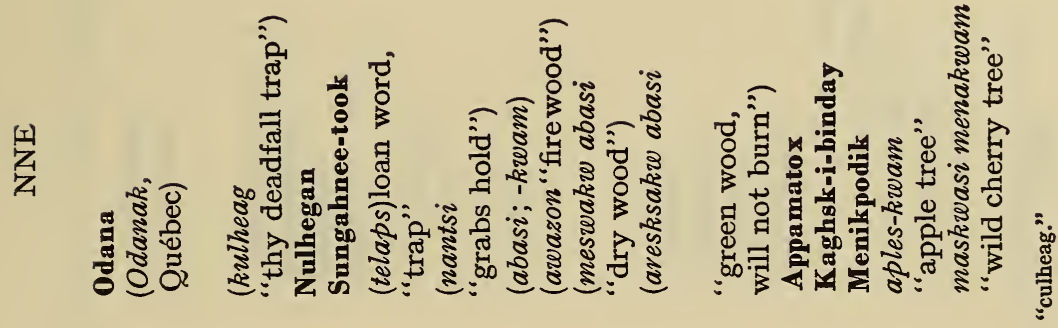

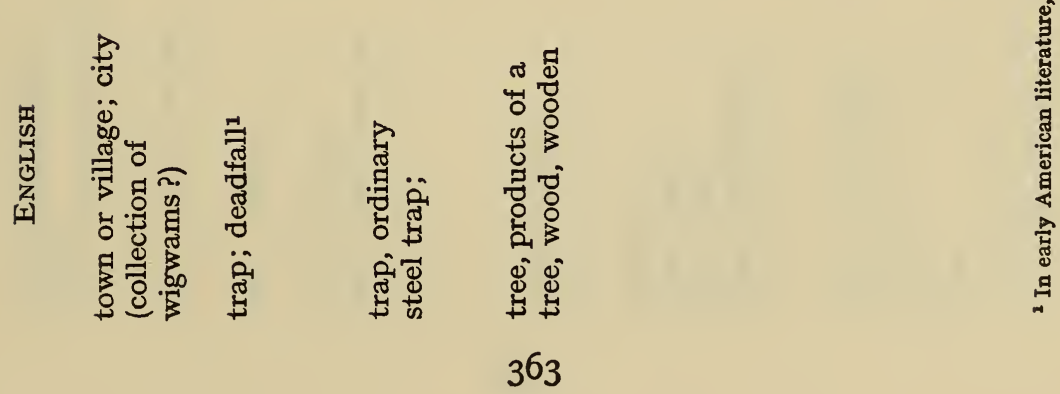




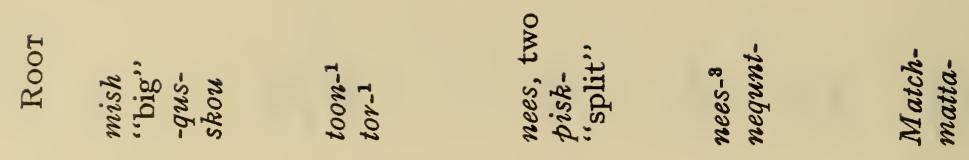

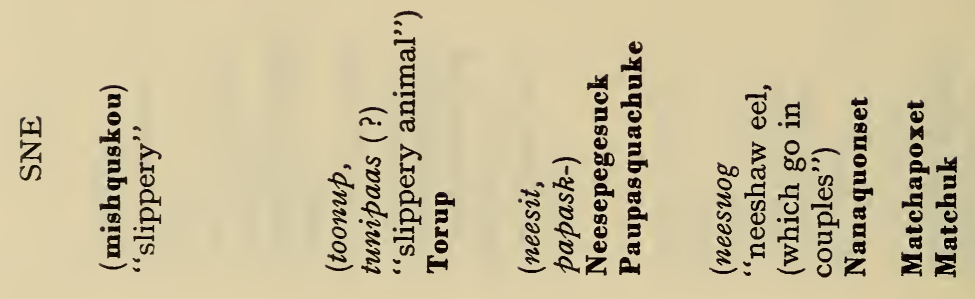

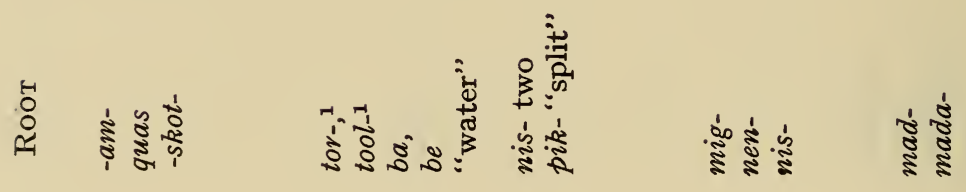
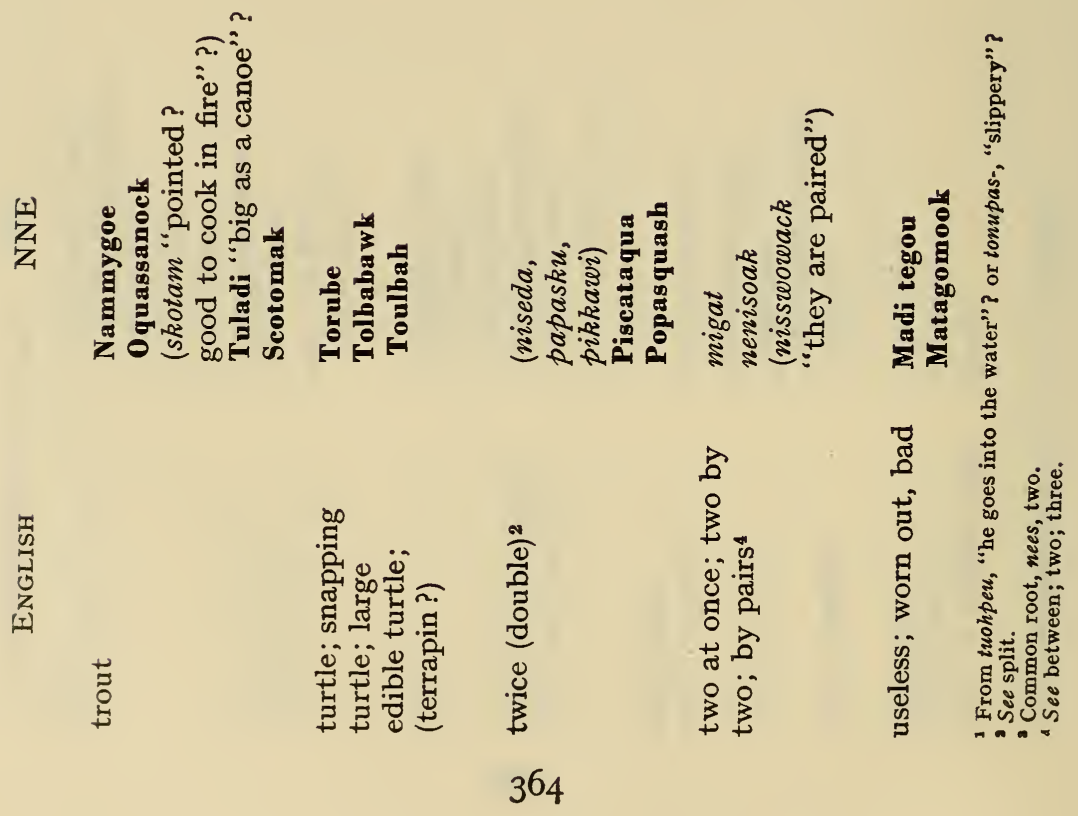


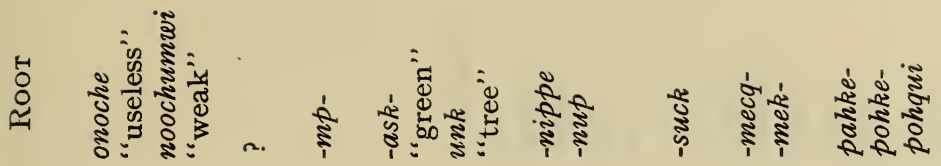

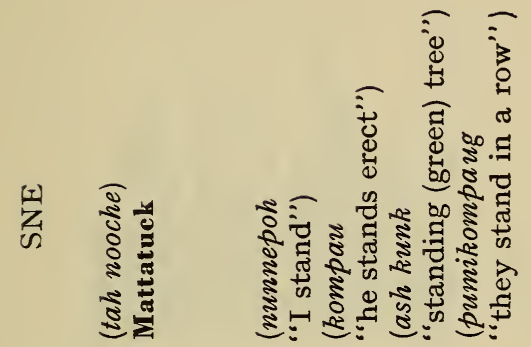

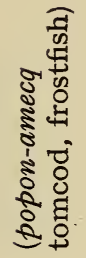

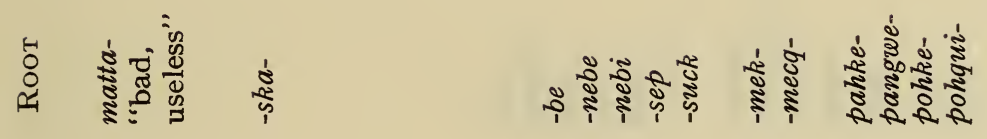

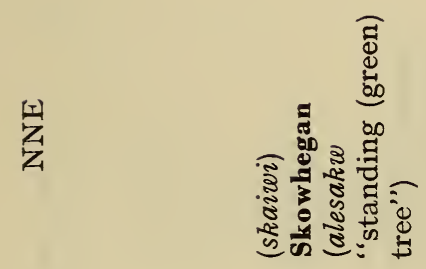

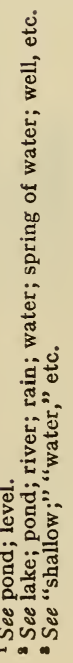

365 


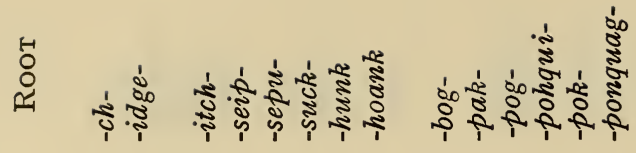

罢

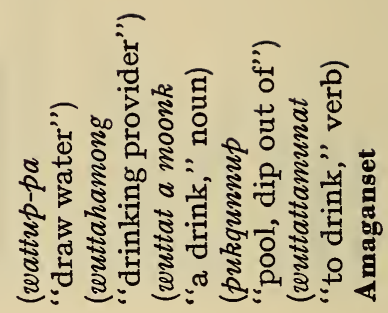

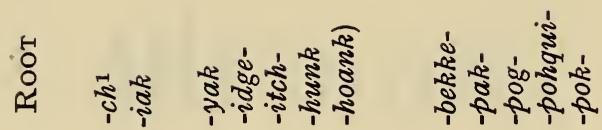

荟

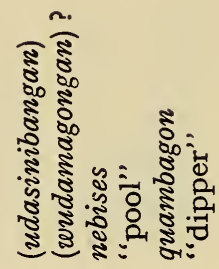

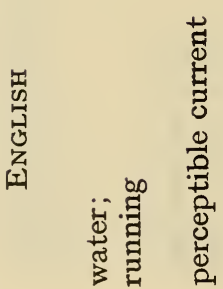

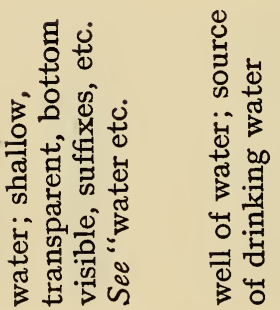

366 
言

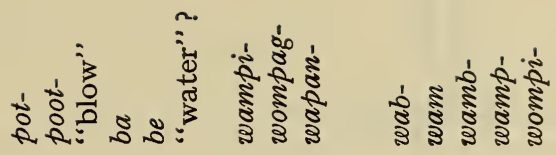

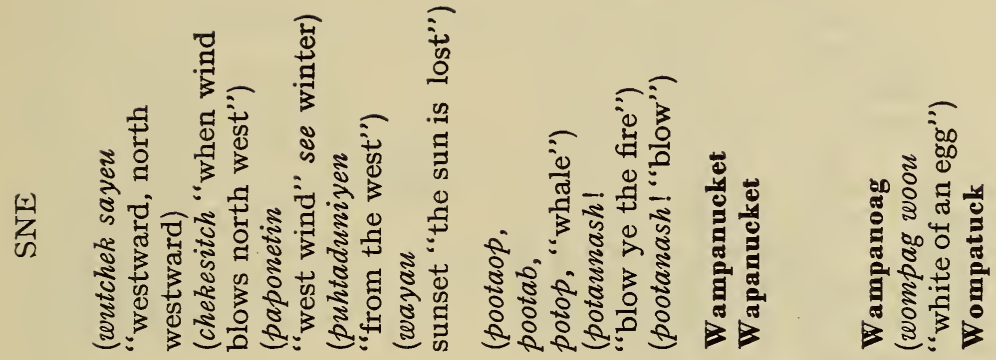

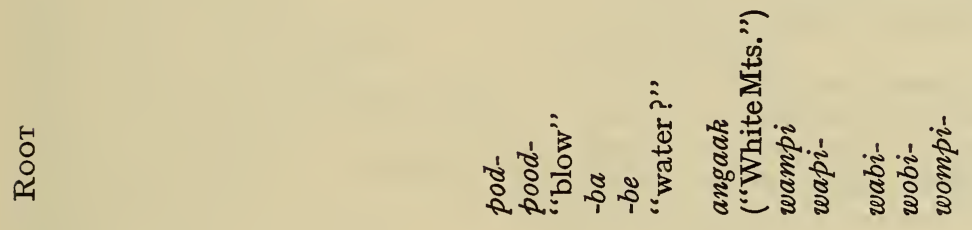

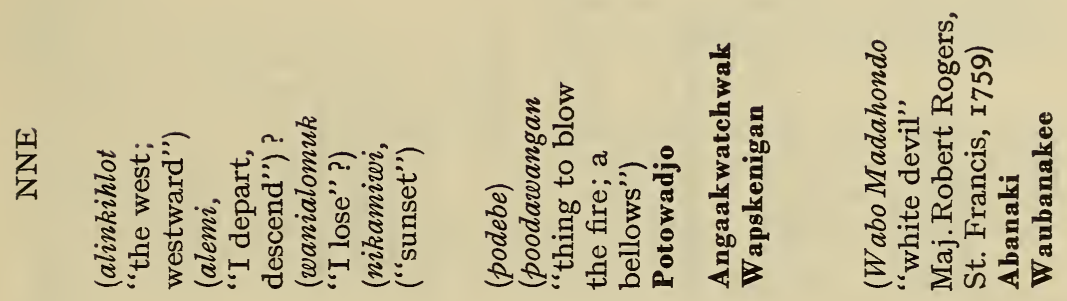

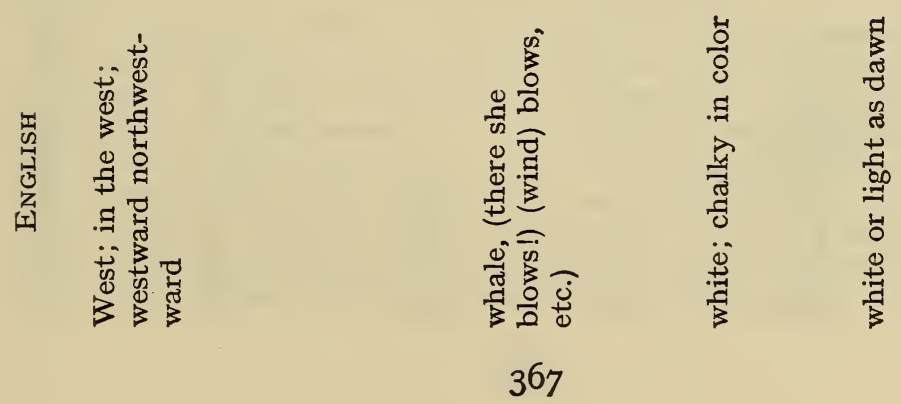




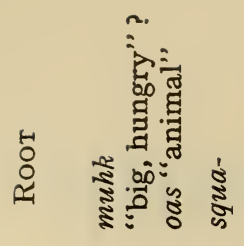

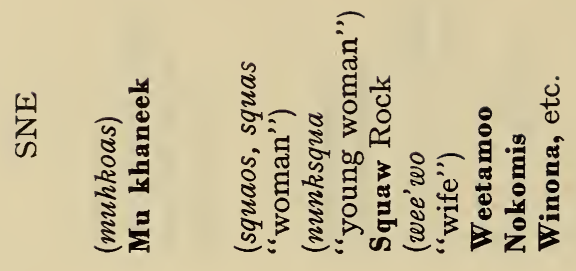

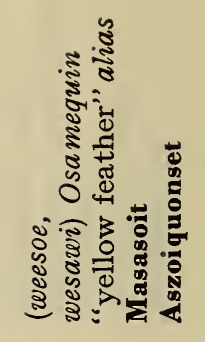

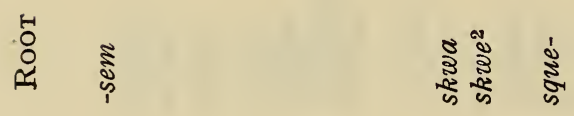

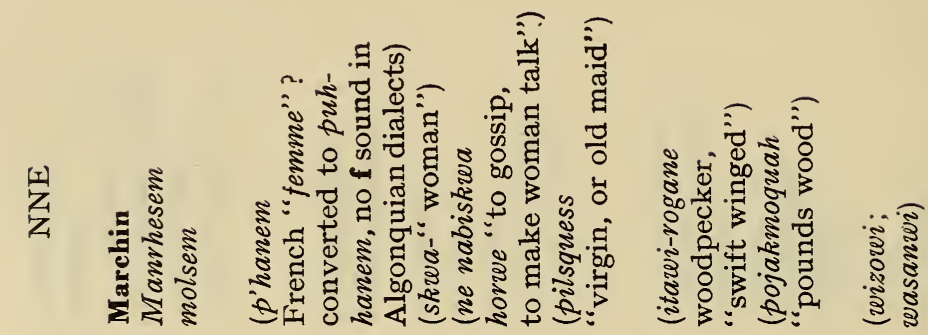
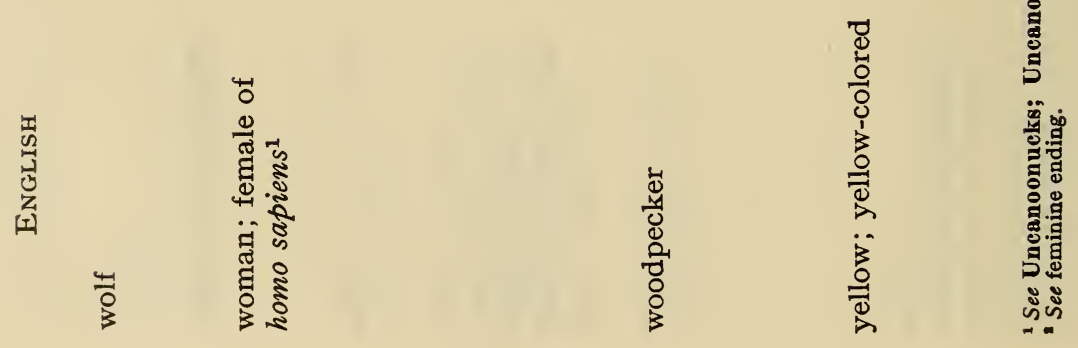


\section{PLACE NAME GLOSSARY}

Abandoned lands, Tawawag

Abnaki spring, Tekebisek, T'Kopesuck

Acorns, Anchamaunnack, Kachewalunk

Adder snakes, Iroquois

Alder place, Wytopitlock

Alewives, Ma-adameg, Madahumuck, Madamascontee, Meddybemps

Alexander, Wamsutta

Ancient beaver hunting, Koghserage

Arrow place, Pakwakek

Artificial outlet, Sahkahegan

Bald eagle, Sowangan

Bare rock, Punkups

Bark cabin stream, Allagash

Basket, Munt, Nootash

Beads, Sawacook, Sawgogue, Sheehauge, Wampum

Bear's den, Pegansic, Puckhunkonnuck, Squapan

Beautiful island, Gawenio

Beautiful river, Aroostook, Ohio, Oloostook, Wallastook, Walloomsac

Beautiful valley, Genesee

Beaver, Dawinehpeh, Gaentake, Hochelaga, Jamaica, Oselaga, Sekidawe, Shumack, Sumpauge, Sympaug, Tamonquas, T'makwa, Tomaquag; also Koghserage

Bend in stream, Walintuk, Wangan, Wankinco, Wonckompsk, Wongum, Wongunk, Wonkemaug, Woronoco

Berry mountain, Attitash, Minnechaug, Minnechoag

Big boulder, Cutchegun, Massekump

Big shoulders, Moosamuttuck

Big still water, Jimskitikuk

Bird carvings, Sepsis-edal-apskit

Bittern Kaskoi, Kaskoo, Tallawanda, Taquahunga, Tihonet

Black feather, Sassaquin, Tisquaquin
Black earth, Metewemesick, Mettawee, Saccanossett, Sassucksuck, Sicaog, Sicojoeke

Black potion, Osceola

Black Sachem, Tisquaquin

Black William Manatohqua

Blessed hereafter, Ponemah

Blood-brother, Netop

Boards, Chimenasanganum, Dekaswenkarorens, K'chi p'saganum, Menes-saganaganis, Psiscontic, Wabasgach, Wabossagock

Bog, Hassacky, Maasbaak, Macwahoc, Magawok, Maspenock, Massaco, Megwak-angamik, Narraguagus, Quack, Quag, Tatamuckatakis, Tatomuck

Boundary, Bunganut, Bungee, Canangogum, Chaubongum, Cheebee Atque, Neutaqunkanet, Notaconkanut, Pepemightughk, Topabsqua Bowl, Onagunset, Wunnegunset

Breakers, Pemategu

Breast, Tacoma, Uncanoonuc

Burnt land, Pataweekongomoc, Quawejoos, Schodack, Schohomogomoc, Scoodeag, Wallempteweekek

Canoe harbors, Agawam, Aigio commuck, Bedabek, Cataumet, Catawamkeak, Meniekec, Mishaum, Shawomet, Shawmut, Tahonwenta, Tolam

Caribou, Magallo, Magurrewock, Megurrewock

Caribou trail, Mugaleep-ahwangan

Carrying place, Deyehonwakwatha, Mannamoiset, Mishimayagat, Manamoyik Neekounegan, Ouneganisis, Pewagon, Unaganek, Winnecook, Winnegance

Carvings on rock, Assonet, Hoosic, Hoosic whisic Humolatski hegon, Sepsis-edal-apskit 
Catamount, Lunksoos, Quoquinna

Cats, Poohookapaug, Poohpoosaug

Cemetery, Bosquenaghook, Ponemah, Wune Wahjet

Chestnuts, Wampnesick, Wombemesisacook

Chief's house, Pometacomet, Sachimo Comaco

Chipmunk, Anequasset, Anqueet

Circler, Uncas, Wonkees-ohke

Clams, Essick, Poquahaug, Quahog

Codfish, Pacamkik

Cold spring, Takebi-suk, T'Kopesuck

Cold stream, Ammadamast, Sankaty, Scituate

Cornfield, Nutskamongan, Tawawag

Corn grinding, Nokhigan, Samp Mortar, Taghommay, Tagwahogan, Tagwonk, Takemmy, Takwangan, Tomhollisick, Yennenstyaks

Corn harvest, Scammon, Sconunganuc, Skamonikoos

Cowardly Spirit's rock, Regiochne, Rodsio Canyatare, Rogeo Rottsiichni

Cranberry, Omquemenikeag, Sasagacha, Sassamon, Sassaquin

Crane, Kaskoi, Tallawanda, Taquahunga, Tihonet

"Crouching Panther," Tecumseh

Curing fish, Ahmelagognetercook, Ameriscoggin, Amessecantee, Ammacongan, A moskeag, Arrockaumecook, Atgatogwisas, Comphegan Congamond, Skipmaug, Wussquamhegonset,

Dancing, Alnobaganock, Nodawangak, Pauchauog, Pomagusset, Pompanuck, Popumossett

Dawn land, Abenaki, Abnaki, Wampanoag, Wapanaki, Wapping

Dead water, Jimskitikuk, Skaki, Skitticook

Deep, Merrimac, Mollywooket, Molunkes, Mona, Monoosuck, Moonassachuet, Temiscouata

Deer, Askenonten, Nohlkai, Nolkaianak, Ohskenonton

Defender, Uncas
Destroyers, Pequod, Pequot, Pequt toog

Devil's Den, Madahondo apskwa

Dirty mountain, Quagachusque, Quaweejoos

Divided, see Split

Dog, Almouchicoisen, Alum, Atie

Double hill, Papasquash, Paupasquachuke, Popsquatchet

Double pond, Petow Pargow, Pocotopaug

Double stream, Nissitisset, Pampaskeshanke

Down-hill, Penacook, Penecoög

Drowned land, Maasbaak, Onderiguegon

Duck, Colicum, Darangowa, Kwikwemes, Quacumquasset, Sabagwilha, Shecoway, Sip, Sipsaconta, Umcolcus, Umcolquis, Ungowaumsock, Wawela

Eagle, Millimagassett, Sennemahgesso, Sowangan, Swango, Weepecket

Eels, Conomo, Cushankamaug, Kenduskeag, Kochisuk, Konomoc, Maguaguavic, Mettuckquashapock, Naamkeak, Nahumkeag, Naumkeag, Nequamwicke, Nesenkeag; Nesopack, Onshaukamaug, Sassawich, Skunkamug, Tabusintac, Taubakonommock, Unshemamuck Eggs, Owweonhungganuck

Englishmen, Scammon, Swank wahiganus

Extended current, Pemigewasset

Extended fog, Panawansot

Extended land, Pemaquid

Extended mountains, Pemadnek, Pemadinisikek

Extended rocks, Seremobscus

Extending water, Pannaway

Extinct seals, Hahkik Watpuk

Falls in river, Bashbish, Mali Panjahlok, Nahnek-bahntik, Netahme Puntook, Panatucket, Pantigo, Pantook, Pantukket, Pat-higget, Patuxet, Pawtucket, Sahnghib pahntook 
Feather, Kutshamakin, Massasoit, Osamequin, Sassaquin, Tiasquam Tisquaquin, Wappoquian

Fertilizer, Koomasabunkawitt, Menada, Munnatawkit, Namaskeese, Namassakeeset

"Finely painted," Waunnakeseag

Fire, Bomoseen, Cabassauk, Chackacust, Cheekheek, Chequaquet, Chickatawbut, Edjida Waskodek, Mahackemo, M'skutook, Schodack, Schohomogomoc, Schoodic, Yewtack

First-born daughter, Winona

Fish, Amaseconti, Amoskeag, Canoza, Chamcook, Cobossecontee, Katamaug, Keekomkwak, Kenosha, Kwenosakek, Madahumuck, M'squamscook, Mameeg, Naacook, Nahmakanta, Namaascottic, Namaskik, Nemdamassuagum, Numdemosees, Obumkeag, Oquossoc, Pacamkik, Papanomscutt, Pokahganeh, Quinshepaug, Sickenames, Skutarza, Squam, Squamicuk, Squeteague, Togue, Tuladi, Uppooquantup, Wataqua

Fish line, Pemunneaht

Flags, Abaquage, Ayasup, Mundoouscoo, Upaquoag, Wappaquasset, Wyasup

Food cache, Muttoneguis, Tsabakwa Foods, Anitaash, Apwonnah, Attebemeuck, Attitash, Minnechoag, Owweonhungganuck, Essick, Nokhigan, Paupock, Quahog, Samp, Sippenak, Sipsaconta, Succotash, Torube, Uppooquantup, Wampnesick, Wenaumet, Wapososhequash Winnemisset, Wintechog, Wintoak, Wish quodiniack, Wombemesisacook. See also Acorns, garden stuff, ducks, eels, fish, etc.

Foothills, Penacook, Penagou

Fording place, Astimoost, Aswaguscawadic, Kedumcook, Ozwazogehsuck, Pomkikin, Poniken, Taascamessick, Tauskounk, Tismattuck, Tist, Toskaunk, Toskibunke, Towantic, Toyusk, Tueskennck- inck, Tuscomanset, Tuskheganuck, Tzawapa, Wapwayset, Weepoiset, Weybosset

Forest, Mashantackack, Mashantucket, Mashentuck, Mattatig, Mattatuck, Shantituck, Tachannike, Taconic, Tokenake, Tug, Watatick, Woodtick

Fork in stream, Anackatuseck, Nicatow, Nichmug, Nickwacket, Nikisipik, Piscatacook, Piskaquoag, Scatacook, Schaghaticauke Schaticook Fork-tailed terns, Nigakws-wakeag Fountain, Ashimuet, Astimoost, Keekamuit, Tohke commumwachak, T'Kopesuck, Toikiming, Wampaketatekam

Fox, A'wumps, Muchaquema, Wagwises, Wonkees-ohke it

Fox country, Chocksett, Ocsechoxit, Shockolog, Woonksechocksett

Frog, Chickwolnepy, Jigwallick

Frost fish, Papanomseutt, Popponesset

Games, Pompanuck

Garden stuff, Agutteback, Ashquoash, Caskak, Easkissey, Squannacook, Squannahonk

Gathering place, Maanexit, Miamogue, Wamesit

Geological terms, Monadnock

Glacial kame, Adowaukeag, Douaquet, Waukeag

Glooscap stories, Kineo, Kokadjo, Madagamus, Moos-i-katchick, Moosepayechick, Oolaghesee, Osquoon, Sabotowan

Gluey, Squeteague

Good, Handsome, Wauregan

Goose, Hinkum, Honkamonk, Sakonnet, Seekonk, Wampatuck, Waptook, Wobegilseebis

Graphite, Cajoot, Metewemesick

Grassy, Askaskwigek Wadjo, Menasquassicook, Meskeekwagamasic, Missisquoi, Moz Mushkeg, Muskagwah, Muskapasset, Muskeget, Muskehtu-auk, Musquetohauke

Great Commander, Massasoit 
Great Spirit, Ketsi M'weskw, Manitoo

Great Swamp Fight, Quawawehunk, Sasco

Great trail, Mishimayagat

Gulls, Caucomgomoc, Kaouasaki

Hardwoods, Kamouraska, MachlichNagook, Pemmaquan, Pemsquamkutook

Hell, Chaboken, Chebacco, Chepinoxet, Shabokin

Hiawatha's mother, Winona

Hidden outlet, Sunkaze, Wiscasset

High, Acomes, Acqunkoke, Adowaukeag, Aspetuck, Asproom, Attawaugan, Cocumcussuc, Cos Cob, Cotackta Gungy Wamps, Hoosic, Kodttukoet, Kongscut, Konikey, Kunckquachu, Mahoosuc, Quinuqui

Hill, Penacook, Wajoses, Washouset, Washucke, Wassiog, Watchaug, Watchoog, Watchusesic, Wotchaugh

Hollow, Pogotossuc, Sologismoodik, Wenachus

Hooded seals, Hahkik, Quaco

Hook, Harco monco, Hoccanum, Hockanum, Hocquan, Mamacock, Mashoquen

House, Annawoncoate, Asnacancomic, Assameek q, Chicomico Hassunek, Macomet, Mogewetu, Natick, Nukkekummees, Odana, Pometacomet, Quinsnikit, Sachimma Comock, Shekomeko, Wakoquet, Weatic, Weatogue, Wekopee, Wiatik, Wigwam Wickasauke

Indian Joe, Sozap

Indian Molly, Mali bowk

Indian Preacher, Occum

Infant, Papoose

Insect, Alkarmus, Jabish, Kermes, K'mokadich, Okemo, Occooch

Island, see names containing syllables quid-; man-; mon- -naghe

Joint, Machegony, Quinneaska Junction, see branch; split
Kettle, Augutteback, Chee Coke, Cocasset, Cuppan augunit, Hoosac, Keeck, Keoka, Kochekok, Kok adjeweem, Kokadjo, Mangunk akuk, Nanacoicus, Nonacoicus, Ohkakquiset, Oukote, Walkamigosek, Wesquage, Wisconemuck King Philip, Chocksett, Metacom, Pacanoket, Pokanoket, Pometacom, Sowamsett, Squakheag, Wampanoag, Wonkees-ohke, Woonksechocksett

Knee, Chegony, Machegony, Magegunuck, Mashoquen

\section{Lady Rebecca, Pocahantas}

Lakes strung out, Amgopilasig, Pomersimbeke, Oulakese, Wiscogosis

Lamprey eels, Konomoc, Taubakonnommock

Lands, abandoned, Tawawag. See burnt lands

Largest N. E. boulder, Cutchegun

"Lazy portage," Mallicooniganus

Leatherwood, Menikpodik, Wecuppemee, Whakepee, Wickapicket, Wicopee

Lighthouse, Pessakenew Agenek

"Little God," Montowese

Little portage, Winnegance

Lobsters, Ashanteaug, Masenunckquock

Lookout, Manatuck, Manetic, Matianuck, Matomy, Matunuck, Monadnock, Skowhegan, Squakheag, Tyontkathotha

\section{Mackerel, Waunnakeseag}

Magical rushes, Mundoo-uscoo

Mahican, Muhhekaneew

"Mahican's River," Manigana-ticauoit

Maples, Pemmaquan

Mats, Abaquakea, Ahapaconsett, Uppanash Konameset, Wabaquasset, Wabquisset, Wappaquassett Medicine man, Paw Woh, Ponus, Pow Wow

Micmac, Tarratine

"Moon Chief," Nanepashemet 
Moose, Moosabek, Moz Mushkeg, Original

Moravian missions, Peantam, Shekomeko, Watchquadnach, Weeataug, Wequadnack

Mountain, see names with: wadwatch, waj-, -adn-; also names of larger mountains.

Mud, Auco Cisco, Casco, Chuquisak, Kebumkewis, Macwahoc, Passanoke, Pishatipaug, Podunk, Quillicksq, Sasco, Sicaog, Sisquisic, Susquesong

Muskellunge, Maskwenozakek

Muskrat, Kahus weskeete, Moskwaswagamok, Musquash

Muskrat lodges, Donwagayon, Odonwagayon

Nammycush, Nammygoe, Togue, Tuladi

Narrows, Piamikin, Wappewassick, Wapwayset, Waubeyaussuc, Webotuck, Weepatuck, Weepoiset, Wepawaug, Wepowage, Weybosset, Wopowage

Narrow trail, Pemenos, Pokamquoh

"No-cake," Nokhigan

Oak, Puckcommeagon

Ocean, see Atlantic

Opposite points, Teckyadough, Umsaskis

Otter, Dawinehneh, Sekidawe, Winegegwok, Wonokakeetookeese

Otter slide, Aguahassideck

Outlet sandbar, Sakadamkiak

Oxbow, Azzastakak, Pattagussis, Petagoubky, Weeweantitt, Woronoco

Oysters, Apponaug, Apwonnah

Paint, Metewemesick, Olamon, Talagodissek, Wannametonamuc, Wullamanic

Papoose-bear, Passaconaway

Parallel, Abagamok, Abahos

Path, Minnewokun, Sogkunate

Pestilence, Mashepagonoke

Pickerel, Kenosha, Kenoza, Quinshepaug, Watagua
Pigeons, Wuskowhananaukit

Pine, Coassit, Coaticook, Cohoes, Coös, Koessek

Pinnacle, Mahoosuc

Pipes, Calumet, Megantig, Uhuponckash, Wdamoganaspskak

Plateau, Pachquinacook

Point, Adonniate, Naiag, Naquag, Narragansett, Nauseag, Nayatt, Nayaug, Nayumpsk, Nehantic, Niantic, Nyac, Schodack, Schoodic, Scoodik

Portage, see Carrying place

Prairie, Wyoming

"Praying Towns," Chabanakongkomuk, Coatue, Cowate, Hassanamesit, Magaenak, Magunhukquok, Magunkahquog, Manitwa tootan, Nashamoiess, Nashoba, Natick, Nemasket, Okommakemsit, Pakemit, Panatucket, Pequimmet, Ponkapog, Quanatusset, Titicut, Wamesit, Wamesut

Quail, Paupock, Popoquash

Quarry, Tomheganompsket

Rabbit, Madagascar, Mateguasaden Rapids, Cocheco, Cochichewick, Cochituate, Kassanum, Kawapskitchwak, Kuladam-itchwan, Metichawon, Nallahoodus, Narantsoak, Nesowadnehunk, Newichwannock, Norridgewock, Orantsoak, Ottauquechee, Pejipscot, Pemigewasset, Pennechuck, Pennichuk, Queachik, Quequecham, Skitchewaug, Skookum Chuck, Sobscook, Tunkahoosen, Tunxis, Wattanks= hausepo

Ravine, Molechunkemunk, Molnichwock, Molunkes, Packwacke

Red paint, Mun-olammon ungun, Olamon, Oolamongamook, Tonomy, Wallamanumpscook, Wannametonamuc, Wolomonopaug, Wullamanic

Rejoicing, Nonantum

Resting place, Abonesig, Appamatox, Apponaug, Mattabesec, Mattapan, Mattapoisett 
Robin, Opechee

Rock, bare, Punkups

Rock carving, Assonet, Humolatskihegon, Sepsis-edal-apskit

Rock circle, Wakalosen

Rock fairies, Wanagamesswak

Rocking stone, Tataesset, Tattahassun

Root thread, Wanchatopek, Watuppa

Rotten corn, Anitaash

Round, Betuckquapock, Pataconkset, Pattaquattic, Puttacawmaumshcuck, Puttuck qumpscut

Round clam, Quahog

Round loaf, Tuckernuck

Salmon, Gonic, M'squam Nebis, Quamscook, Squam, (Head), Squamagonic, Squamok wisseeboo, Winnis quam

Sand bar, Bamedumpkok, Capawack, Gunas quamecook, Hammonassett, Katawamkeag, Kebec, Kebumkewis, Kennebunk, Kepamkiak, Kwetahwamkituk, Mashawmut, Mattawamkituk, Mattawamkeag, Medumcook, Mekwamkesk, Memkeeswe, Meniekec, Merriconeag, Mishawum, Nolangamoik, Nunnakomac, Onset, Pamedomcook, Pautonk, Pemadumcook, Petekamkes, Pogamqua, Pogumkik, Puggamugga, Quotonset, Shawmut, Tolam, Waylumkituck

Sausage casing, Oolagheese, Oulakese

Scoop net, Atgatogwisas, Comphegan, Quaiombog, Quamphegan, Wussquamhegonset

Sea, Kehtah hanit, Sobagwa, Tahanock; see Atlantic

Sea bass heads, Uppooquantup

Sea gulls, Caucomgomoc, Kaouasaki

Sea shore trail, Quinnipiac, Mishimayagat, Sacunyte

Seed corn, Menomee

Selected tree, Pepemightughk

Separated, Chaboken, Chappaquiddick, Chepinoxet, Chipchug, Chi- ponaug, Chippachaug, Chippuachack, Tsabakwa

Shaking meadow, Hassacky, Tatamuckatakis, Tatnock, Tatomuck, Totomak

Shark-shaped, Sekled Obscus, Sisladobsis

Sharp bend, Kananghet'ne, Quinneaska, Mashoquen

Shell beads, Sawgogue, Solikuk, Sowanoxet, Succanesset, Wampum

Shell people, Siwanog, Siwanoy

Shining, Passagas-sa-waukeag, Pennessewassee, Wabassus, Wasa-umkeag, Wass, Wassaquoick, Wassokeag, Weesquobs, Wequashacke, Wicwas

Short carry, Mallicooniganus, Ooniganissek

Shortest route, Sebasticook

Shoulder, Moosamuttuck

Skull, Cheebeeantups

Slender trout, Oquasanock

Sloping ledge, Sawad Apskek

Small bend, Pewonganuck

Small fork, Nicatowis

Smoking mixture, Kinnicum

Snake, Sasagook, Scook, Scucurra, Sisikwa, Skug; also Iroquois

Snake dance, Skogogwaganock

Snowshoe tracks, Madagamus, Mosemadage

Soothsayer, Moharmet's, M'teoulin

Split, Nicatowis, Pabaquamske, Paghmaigah, Pashesauke, Pashipscot, Pauqununch, Peskebegat, Peskeompscut, Pesketuk, Piscataqua, Pocotopaug, Scaticook, Topabskwa, Wunnashowatuck qut; see Branch

Spring (of water), Ashimuet, Nippoo, Nipsic, Scamscammuck, Shamuet, Shawkemo, Shenipsit, Shimmoah, Shumuit, Squog Kane Kaneek, Takebi-suk, Tashmuit, Teikiming, Tekebisek, T'kopesuck, Winneashimut, Winnisimmet

Spring (of year), Seeconnesset, Segunesit, Sigwanawock, Singrawac Squirrel, Anequasset, Mishanneke 
Steam baths, Kussompskauk, Pattaquasset, Pattaquonset, Paukyowohog, Pesuponek, Pissapogue, Pissepunk

Steamer clam, Essick, P'sahn, Saquish. See Bar Harbor, Mount Desert

Strange light, Pennessewassee

Sturgeon, Cobosseecontee, Kobossee, Passawaukeag

Sucker fish, Keekomkwak, Nemdamassuagum

Summit, Annisquam, Hoosac, Kodaak, Kodttukoet, Kongscut, Mahoosuc, Qununckwattehu, Wanashquodtinook, Wanasquatomska, Washukquatom, Waunashqua, Wawobadenik, see High

Suncksqua, Potucko's, Quaiapen, Weetamoo

Sunken land, Quabacook

Supernatural, Gallowa, Ketsi M'weskw, Kinkajou, Lunksoos, Madahondo, Mundoo, Wiwiliamecq

Swamp, Hassacky, Ohomowauke, Quack, Quag, Quakish

Swan, Wawecus, Wequash

Sweat lodges, see steam baths.

Tail of lake, Yotenyatarokte

Teardrop, Peegwon

Thankfulness, Tabuttantam

There she blows! Potowadjo

Thunderstorm, Nimpanick Hickanuh, Patant atonet

Tomcod, Papanomscutt, Popponesset

Top, Ongeachonta

Torchlight spearing, Passagas-sawaukeag, Wabassus, Wassaquoick

Trading place, Assawompset, Paudowaumset, Pautonk, Potowomut

Trail, Awanganis, Manomet, Mishimayagat, Popumossett, Subecwangamook

Trap, Appaum, Chickamug, Eggemoggin, Kenduskeag, Naperhan, Nulhegan, Mousam, Sungahnee took

Tree stump, Wequanunk

Trembling, Hassacky, Kekamooch- aug, Quaquoountuck, Quawawehunk, Tatamuckatakis, Tatnock, Tatomuck, Tatuppequaog

Trout, Acquessuc, Musquinepash, Namaaskotic, Namagonic, Nammygoe, Oquassanock, Scotomak, Skuthazis, Togue, Tuladi

Turning place, Chepachague, Chepachewag, Pachaug, Pachet, Packa= chaug, Paskhommuck, Patchogue, Quinnipiac, Waworamawak

Turtle, Namokanok, Ohnowarake, Tolbuntbessek, Torube, Torup. Tunipus

Two-by-two, Nesenkeag

Tying bark, Menikpodik, Wecobemeas, Wecuppemee, Wecups, Whakepee, Wickapicket, Wicopee, Wyben

Upright stone, Nepasooeneg

Virginia Princess, Pocahantas

Waiting place, Appaum, Apponaug, Skaki, Skowhegan, Squakheag

Wampum, see shell beads

Wampum belt, Machemux

Weakfish, Squeteague

Wee people, Mikumwessak, Muggs, Mukyaweesug, Nutskamongan, Wanagamesswak

Weeping rocks, Nebissee-woldam-wogan

Wells, Amaganset, Ashimuet, Hammonasett, Machaquamaganset, Meshomasic, Nipsic, Onkoskopsug, Shenipsit, Shickasheen, T'Kopesuck

Wet head, Usuntabunt

Whalebone, Siasconset, Waskosing, Wasque

Whiskey Pond, Usquepaug

White boards, Wabasgach

White bone, Siasconset, Wabigenek, Waskosing, Wasque

White captive, Pewampskine

White falls, Wabeno bahntuck

White plume, Wappoquian's 
White sand, Wassamkihemuk

White stone, Wapskenigan, Wesquo, Wesquobsk

Whortleberries, Attitash

Widening stream, Pannaway, Pocasset, Pocowset, Powakasik

Wigwam place, Weatogue, Wiatik

Winding stream, Wawayontatt, Weantinock, Weweantic, Weweantit, Weynanitoke, Woronoco

Winter fish, Papanomscutt, Popponesset

Wintry place, Keewaydin, Koghserage, Peboamauk

Wolf, Marchin

"Wolf People," Mohanhegumewog, Mohawk, Moheganick

Woman's mountain, P'ahnmoiwadjo

Woodchuck, Acquasimink

Woodpecker, Pongokwahemook, Rockabema

Yellow plume, Osamequin, Massasoit

Younger sister, Sepunamus 


\section{CAMP NAMES}

Abasandogganal. Modern Abnaki, the Northern lights or Aurora borealis.

Achsinik. Mahican, at the big rock.

Acquasimink. Mahican, abode of the woodchuck.

Adchaukoma. Natick, hunting lodge.

Agwonk. Natick, under a tree.

Akinagan. Abnaki, a cradle.

Akwatain. Abnaki, source of the river.

Alnobaganock. Abnaki, dancing place.

Anaquesuonk. Natick, a joint (such as an elbow).

Angawi. Abnaki, hidden.

Annumwussukuppe. Natick, willow tree.

Anockqus. Natick, a star.

Anwohsinook! Natick, rest ye!

Appehmehtig. Natick, resting tree, or bower.

Appin. Natick, place for sitting; place for sleeping; bed; place for resting.

Aquene. Narragansett, peace; or, a truce.

Aquene-ut. Narragansett, peace place; or, truce place.

Arami. Abnaki, delight; joy.

Ashim. Natick, a spring of water.

Ashkoshquhkontu. Natick, in green pastures.

Askuhquame-ut. Natick, a greenwood tree; or, under the greenwood tree.

Askuwhetae-komuk. Natick, watch-tower.

Assemi. Modern Abnaki, indulge in a bath.

Assunekoaz. Natick, thorn bush; briar patch; refuge place.

Atki. Abnaki, nest or cradle.

Attitash. Narragansett, huckleberries.

Auwépin. Narragansett, calm; the wind ceases; calm of the evening.

Awikhigan-gamigwe. Modern Abnaki, writing room.

Bessanswi-zambon. Modern Abnaki, I heat soup.

Chepewissin. Natick, northeast wind.

Chepiokomuk. Natick, house of ghosts; hell; haunted place. 
Chickabi. Natick, swift water.

Chikkup. Natick, red cedars; a cedar.

Chippa puock. Narragansett, they are apart; they form a group by themselves.

Gernamen. Abnaki, hand-holding.

Gwanso. Abnaki, I stay overnight.

Hassunegk. Natick, a cave; a cavern; a rock shelter.

Hopuonck. Narragansett, a (tobacco) pipe.

Huan. Abnaki, I walk in the snow. (I ramble)

Itsin. Abnaki. I tear flesh. (I wolf down my food)

Kahagon. Iroquois, in the forest.

Kahyonhes. Moharek, long stream.

Kandadiwi. Modern Abnaki, a feast.

Kanosera. Iroquois, temporary shelter.

Kanti. Modern Abnaki, plenty, or abundance, e.g.

kemak-kanti. plenty of lice or gnats.

namagwe-kanti. plenty of fish.

norke-kanti. plenty of deer

sipsi-kanti. plenty of birds (ducks)

Kaski. Modern Abnaki, deep.

tegwe-kaski. river, deep.

nebes-kaski. pond or lake, deep.

Kateganiwi. Modern Abnaki, by night, nocturnally.

Katinon Kwat. Huron, heart's desire.

Kchi Alakwas. Modern Abnaki, morning star; evening star; great star.

Kchi Pontegwa. Modern Abnaki, a grand rapid; the principal rapid.

Keda Kukusis. Modern Abnaki, thy little kettle.

Keektawhank. Mahican, rapid stream; dashing current.

Kehchippam. Natick, on the shore.

Kehtahannupog. Natick, waters of sea.

Kenn! Modern Abnaki, Thanks! Thank you!

Kerahigan. Modern Abnaki, log trap, deadfall.

Kespatte. Modern Abnaki, dry.

Kestaubeunk. Mohican, principal camping place.

Ketasoot. Natick, a king. 
Ketsessaiwi. Abnaki, near the fire.

Kiga bait. Modern Abnaki, a bachelor (man).

Kiga eskwait. Modern Abnaki, a bachelor girl; an "Old Maid."

Kikki-Kizos. Abnaki, land of the sun; month of May.

Kishkituckock. Mahican, by the riverside; on the banks of the stream.

Kisos. Modern Abnaki, sun or moon.

Kitompanisha. Natick, break of day.

Kodtompskoag. Natick, summit of a cliff; top of the rock.

Kodttukoe. Natick, summit (of a mountain or hill).

Kojkehooponat. Natick, to be drunk; state of being drunk.

Kuppokoma. Natick, a grove; enclosed place; place of refuge; hiding place.

Kussamp kussum. Natick, a furnace or oven; literally, "he makes rocks hot."

Kussitchuan. Natick, rapid stream.

Kutsah'arigan. Modern Abnaki, stew.

Kutsa-harigan-kanti. Modern Abnaki, stew-a-plenty.

Kutshamunat. Natick, lightning.

Kwadarwi. Abnaki, between waters.

Kwask watsi. Modern Abnaki, extreme cold.

Machemotae. Natick, lasting; enduring; long-wearing; dependable. Magoshketomp. Natick, a huge man; a giant.

Maguagon. Modern Abnaki, a rainbow.

Mahanepes. Mahican, slow water; sluggish stream.

Mahshagquodt. Natick, (time or place or condition of) famine.

Maiyaéog. Natick, they assemble.

Mamada-ki. Modern Abnaki, barren land.

Mamankwe. Modern Abnaki, chew ye! (eat!)

Maneskammen. Modern Abnaki, a flocking together.

Manoonsket. Natick, place of clay.

Manshk. Natick, a fort; a place of defense.

Manusqussedash. Narragansett, beans.

Maskwamozi. Modern Abnaki, a birch tree.

Massatigk. Natick, great tree.

Matanbenigan. Abnaki, a harbor.

Matsi Nepi. Abnaki, bad water (undrinkable).

Mattanaukonash. Narragansett, mats to sleep on; (and by inference) sleeping-bags.

Mattapsh yoteg! Narragansett, sit by the fire! 
Mautabon. Natick, dawn; morning; daylight.

May-yea-komuk. Narragansett, meeting house; gathering place; wayside-inn.

Meechumee-komuk. Natick, food-house; pantry; (or by inference) grub tent; dining hall.

Meitch! Narragansett, eat thou! or, come on, eat!

Mekwamke. Abnaki, red earth.

Menahan. Modern Abnaki, an island.

Meskwaka. Abnaki, always green; ever green; spruce tree.

Messubisuk. Abnaki, place of the large pond.

Michemohteau. Natick, it endures forever.

Mickuck askeete. Narragansett, a meadow.

Migat. Modern Abnaki, two at once.

Mishadchu. Natick, a great mountain.

Mishannock. Natick, great star; morning star.

Mishoadtue. Natick, precious; of great price.

Mishoon. Natick, canoe.

Mishquammauquock. Narragansett, red fish; salmon.

Mishquawtuck. Narragansett, red wood; cedar.

Mishquockuk. Narragansett, a red copper kettle; (or sometimes) a red earthen pot.

Mishqushkou. Natick, a trout.

Mogke koma. Natick, a great house.

Mohtompan. Natick, it is day.

Monunks. Natick, ash-tree.

Moonooe nippeash. Natick, deep waters.

Moosompsquetuh. Natick, upon the smooth, weathered rocks.

M'skikwiminar. Abnaki, strawberry; grass-berry.

M'skwooteh. Abnaki, field; burned-over-land.

Munnanock. Natick, the moon; wonderful star.

Muscoota. Mahican, grassy land; meadow; pasture.

Nadialoi-ki. Modern Abnaki, hunting ground.

Nagontu. Natick, in the sand; among the dunes.

Nahoosik. Natick, a pinnacle.

Nanacheestawack. Mahican, fort; place of safety.

Nanwat. Abnaki, far away.

Nanwiwi. Abnaki, in the middle.

Napachamach. Mahican, fish weir; place of the fish trap.

Naskade. Abnaki, a bottomless pit.

Nebes. Modern Abnaki, a lake; at the lake. 
Nebessis. Modern Abnaki, a pond; a small pond.

Necawnauqun. Narragansett, the old barn.

Necawnaqunash (plural). Narragansett, the old barns.

Neckick. Narrägansett, (to) my house.

Nedak kikkem. Modern Abnaki, my garden.

Nedakki. Modern Abnaki, my estate, property, land.

Neimpauog. Narragansett, thunder.

Ne ketemenesk. Modern Abnaki, I am lazy.

Nepanon. Natick, a shower of rain.

Nepauz-pashpishau. Narragansett, (it is) sunrise.

Netop. Natick, my friend; blood-brother.

Niben. Modern Abnaki, summer time.

Niben-aki. Modern Abnaki, land of summer time; south-land.

Nikamiwi. Abnaki, sunset; sunset lodge.

Nipauz. Narragansett, the sun; place in the sun.

Nittauke. Narragansett, my land.

Nodawangak. Abnaki, dancing-place.

Nompakou. Natick, a jewel; a treasure.

Nootau. Narragansett, a fire.

Notamiset. Natick, at the oak tree.

Nunksqua. Natick, a girl.

Nunnakoma. Natick, dry land; the shore.

N'wedderra. Modern Abnaki, to get out of doors.

Ohkuk. Narragansett, a kettle.

Omaen. Natick, fisherman.

Omaenuog (plural). Natick, fishermen.

Onahgesona. Iroquois, silver birches.

Onkouchtae mehtugquash. Natick, shady trees; shady grove.

Onkouohtae. Natick, shade; shadow.

Onkuppe. Natick, strong drink.

Onontakah. Iroquois, hillside.

Oonouhkoi. Natick, a valley; low land.

Otahnagon. Iroquois, in the pine woods.

Ouwan. Natick, the mist.

0'wdesis. Modern Abnaki, a path; a trail.

Panjahlok. Modern Abnaki, a cascade; a waterfall.

Pantucoog. Abnaki, place at the falls in the river.

Paponetin. Natick, the west wind. 
Paskamontamoonk-komak. Natick, starvation manor; where-you-starve plantation.

Pauwasq. Natick, a sorceress; a witch (female).

Pauwaw. Natick, a sorcerer; a witch (male).

Pawtuck. Narragansett, a water fall.

Pebon-ki. Modern Abnaki, wintry land.

Peeksq. Natick, a night-hawk.

Peeyaush, netop! Narragansett, come hither, friend!

Pekkeniwi. Abnaki, in the shadows.

Petukunok. Hammonassett, at the round rock.

Pili Kisos. Modern Abnaki, new moon.

Pipegiwi. Abnaki, stormy water.

Pisowa-kamigwa. Modern Abnaki, the wilderness.

Piwi. Abnaki, small; pee-wee.

Pogwas. Modern Abnaki, moon.

Pohkuhtemis. Natick, a white oak.

Pohkuk-misqui. Natick, red oak.

Pompanuck. Natick, a place for playing games; game room; rumpus room.

Pontegwa. Modern Abnaki, a rapid.

Powwaw. Narragansett, a conjurer; sorcerer; wizard.

Puppinashimwog. Natick, wild beasts.

P'wadagameh. Abnaki, cove in lake.

P'wat-begek. Abnaki, cove or bay in a river.

Pwatsobagwa. Abnaki, cove in the sea; bay on the sea cost.

Quannacut. Natick, the rainbow.

Quequecumauog. Narragansett, ducks.

Qunnunkque-komuk. Natick, a high tower.

Sachem. Natick, a king.

Sachemaoug (plural). Natick, kings.

Sachimo comaco. Natick, chief's house.

Sagamore. Natick, a king; a chief.

Sagamore-connuck. Natick, a chief's house.

Sakkade. Abnaki, flat; level.

Sasaunck pamuck. Narragansett, sassafrass tree.

Say-kwahigan. Abnaki, (by inference), hunting ground; open country.

Sekidawe. Abnaki, beaver dam.

Senojiwi. Modern Abnaki, the shore; a bank (of a stream). 
Sepoese. Narragansett, little river; a brook.

Sibo. Modern Abnaki, a river.

Sibosis. Modern Abnaki, a brook; a stream; a small river.

Siguan. Modern Abnaki, springtime.

Siguanak-wekowam. Modern Abnaki, vernal lodge.

Sipsi konta. Abnaki, birds a-plenty.

Skassen. Abnaki, the northwest wind.

Skwahigan. Abnaki, waiting place; tryst.

Sobagua. Modern Abnaki, the ocean.

Sohkohsuonk. Natick, victory.

Sohsumwae. Natick, shining.

Sonkippog. Natick, cold water.

Sonkisq. Natick, a queen.

Sowan-aki. Modern Abnaki, south-land.

Sowanishin. Natick, south wind.

Sowanohke. Natick, the south-land.

Spemki. Modern Abnaki, heaven; paradise.

Spiwi. Abnaki, on high; upwards.

Squashes. Narragansett, a little girl.

Sunnadin. Natick, north wind.

Swish cuttow wauog. Narragansett, literally, "wigwam with three fires;" the special name for the Belt of Orion constellation.

Taatabiwi. Modern Abnaki, happily.

Tabuttantamoonk. Natick, thanksgiving.

Taguahogan. Modern Abnaki, mill.

Taguahogan-sebo. Modern Abnaki, mill stream.

Taguogo. Modern Abnaki, autumn.

Tahonwenta. Mohawk, canoe-launching place.

Takekom. Narragansett, a spring of water; a fountain.

Tapantam. Natick, it satisfies.

Tapapimin. Natick, room enough for all.

Taushkaubik-au. Chippewa, a split rock.

Tegwa. Modern Abnaki, a river.

Tekasmwadi. Abnaki, bathing place.

Tese Ramsen. Abnaki, the wind blows.

Tkassem wagan. Modern Abnaki, bath.

T'kebi. Modern Abnaki, spring water; fountain; springing fountain.

Tomhannock. Natick, flooded; brimming.

Tsibai. Abnaki, ghost. 
Tuohkomuk. Natick, a forest.

Tzawapi. Abnaki, a crossing-place; ford in a stream.

Tzekesi. Modern Abnaki, I light a fire.

Ukquanoequon. Natick, a rainbow.

Uppohquos. Natick, a tent; an awning; a covering.

U-ragan. Modern Abnaki, bark dishes, plates.

Wad amiwi. Modern Abnaki, baggage.

Wadchmeshash (plural). Natick or Narragansett, small mountains; hills.

Wadchumes. Natick or Narragansett, small mountain; hill.

Wadtch. Natick, a bird's nest.

Wanaskwinigan. Abnaki, the end of the trail; journey's end.

Wanaskwiwi. Abnaki, at the highest point; summit; at the furthest place.

Wasaiwi. Abnaki, at the top.

Waskwaha. Iroquois, camp.

Wassenema. Modern Abnaki, give light, illumine.

Wattawesswa. Abnaki, a star; evening star.

Wawwhunnekesuog (plural). Narragansett, they have handsome colors; they are finely painted. Refers to "mackerel."

Wayaawi. Natick, sunset.

Wayont. Natick, sunset.

Wegenagat. Abnaki, the length of a night; overnight; an overnight stop.

Weetimoquet. Narragansett, it has a sweet odor (as, new mown hay, fresh baked corn bread, etc.)

Weetimung quot. Natick, a perfume; a sweet, pleasant odor (as in a glade with wild honeysuckle.)

Wekinea uquat. Narragansett, fair weather.

Wekkwakamigek. Abnaki, land at end of the earth; trail's end?

Wenauwetu. Natick, well housed; sturdy home.

Wenomin. Narragansett, grape.

Wenomineash (plural). Narragansett, grapes.

Wequanunkq. Natick, stump of a tree; wooden mortar for grinding corn.

Wequarran-woddish. Natick, eagle-nest.

Wequashim. Narragansett, moonlight; a swan; (name of an early chief).

Wesattimis. Natick, a red oak. 
Wessikkat. Abnaki, ebb tide.

Wetapwauwwas! Narragansett, sit ye down and talk with us; squat and chat.

Wetomp. Natick, a favorite; a dear friend.

Wetompaog (plural). Natick, favorites; dear friends.

Wetu. Narragansett, a house.

Wetu-hahanuonk. Natick, house of laughter; (and by inference) house of merriment.

Wetuomemese. Narragansett, a little house.

Wetu-wayont. Natick, house (of the) sunset; sunset cottage.

Wili-ki. Modern Abnaki, fertile land.

Windigo. Abnaki? ruler of winter region; mighty, dreaded giant.

Wirini. Modern Abnaki, give thanks, "it is well."

Wisawogamak. Modern Abnaki, at the straits or narrows.

Wiwibiwi. Abnaki, alone; solitary; off by itself.

Wohsumoonk. Natick, shining forth.

Wompatucket. Natick, abode of the gray goose.

Wompiskuk. Natick, osprey; great white bird; eagle.

Wonogq. Natick, a hole; a hollow.

W'ski wigwam. Abnaki, a new house.

W'tseka-aban. Abnaki, morning breaks.

Wudtuckquanash! Narragansett, lay wood on the fire!

Wunnegin! Natick, welcome!

Wunnegin! cowish! Narragansett, welcome! Sleep here!

Wunneta wetu. Natick, happiness manor.

Wunni komuk. Natick, happiness manor; great happy home.

Wunohke. Natick, good ground.

Wusapinuk. Natick, edge or bank of river.

Wuske. Natick, new.

Wussaumpatamoonck. Narragansett, a prospect; a view.

Wussock hosick. Natick, writing-house; office.

Wussooquatomis. Pequot, a walnut tree.

Wussoquatominash (plural). Pequot, walnut trees.

Wuttahamununk moonoi. Natick, the well is deep.

Wuttamasim! Narragansett, give me tobacco!

Wuttaminneoh. Narragansett, strawberry.

Wuttaminneash (plural). Narragansett, strawberries.

Wuttogki. Natick, it is wet (deer's nose).

Yotahkoda. Iroquois, at the end of the trail.

Yau-ut nashin. Natick, it is four-square; it is on the square. 


\section{BIBLIOGRAPHY}

Aubéry, Joseph, Rev.

I7I5 Abnaki Dictionary. Manuscript in the archives of Nicolet College, Québec. Copied in I89I by Very Rev. M. C. O'Brien;

BALLARD, EDWARD manuscript in Maine Historical Library, Portland. 49opp.

I87I "Geographical Names on the Coast of Maine." Report of the Superintendent of the Coast Survey, I868. Appendix No. I4. Washington: G. P. O. pp. 243-259

BANKS, ChaRLES EDWARD

I9I I History of Martha's Vineyard. Vol. I. Boston: G. H. Dean. $535 \mathrm{pp}$.

BARAga, Frédéric, Rev.

I878 A Dictionary of the Otchipwe Language, Explained in English. Part I, English-Otchipwe. Montréal: Beauchemin and Valois. $301 \mathrm{pp}$.

Barbeau, C. Marius

I9I5 "Huron and Wyandot Mythology." Anthropological Series: Memoir 8o, No. II. Canadian Department of Mines Series, Geological Survey. Ottawa: Canadian G. P. O. $43^{8}$ pp.

BARRATT, JOSEPH, Dr. (editor)

I85I The Indian of New England and the North-eastern Provinces, etc. Middletown: Charles H. Pelton, $24 \mathrm{pp}$. [This was based upon information obtained by Barratt from Nicola Tenesles, an Indian informant].

Barrows, Charles $\mathrm{H}$.

I9I6 Meaning of Indian Local Names, etc. A Historical Address Delivered May 26, to the Citizens of Springfield, Mass. Connecticut Valley Historical Society. Ioo pp.

Beauchamp, William M., Rev.

1907 "Aboriginal Place Names in New York." New York State Department of Education; Museum Bulletin No. I08. Albany.

BoAs, Franz $333 \mathrm{pp}$.

I9II; "Handbook of American Indian Languages." Bureau of Amer-

1922 ican Ethnology, Bulletin 40. Washington: Smithsonian Institution. 2 parts.

Boyd, Stephen G.

I885 Indian Local Names With Their Interpretation. York, Penn: privately printed, $70 \mathrm{pp}$.

Brinton, Daniel Garrison and Rev. Albert Anthony Seqaqkind

I888 A Lenâpé-English Dictionary. Philadelphia: Historical Society of Pennsylvania. $236 \mathrm{pp}$.

CABot, William Brooks

I920 I 7 th and I8th Century Vocabularies of the Penobscot, Passamaquoddy, Massachusett, etc. Unpublished, typed copies in Bangor, Maine, Public Library. Approximately I5o pp. 
Cady, John Hutchins

1936 Rhode Island Boundaries I636-1936, State of Rhode Island and Providence Plantations. Providence: Tercentary Commission. I $22 \mathrm{pp}$.

Charland, Thomas-Marie, Rev.

1942 Histoire de Saint-François-de-lac. Ottawa: Collège Dominicain. $34^{\circ} \mathrm{pp}$.

Charlevoix, Pierre François Xavier de

I76I Journal of a Voyage to North America. London: R \& J Dodsley. (2 Volumes) $382 \mathrm{pp}$.

COFFIN, PaUL

I856 "Missionary Journeys in Maine, I796-180o." Collections of COTTON, JosIAH Maine Historical Society, Vol. IV. Portland. 405 pp.

I829 Vocabulary of Massachusetts (or Natick) Indian Language. Cambridge: E. W. Metcalf. II 3 pp.

Cotton, JosiaH

I830 "Natick Word List." Collections of Massachusetts Historical Society, 3rd Series, Vol. 8. Boston: II3 pp.

COOKe, Charles ANgus

I956 Five Thousand Iroquoian Proper Names. Unpublished typescript. Copies in the Library of the National Museum, Ottawa, and at the American Philosophical Society, Philadelphia. About 500 pages.

CROCKETT, WALTER

I92I History of Vermont, Vol. I. New York: Century Historical Co. $546 \mathrm{pp}$.

CUOQ, JEAN-ANDRÉ, Rev.

I886 Lexique de la Langue Algonquine. Montréal: J. Chapleau et fils. $446 \mathrm{pp}$.

DAY, GORDON M.

I96I "The Name Contocook." International Journal of American Linguistics, Vol. XXVII, No. 2, pp. I68-I7I.

DAY, GoRdon M.

I959 "Note on St. Francis Nomenclature." International Journal

DEAN, LEON of American Linguistics, Vol. XXV, No. 4, pp. 272-273.

I9I4 "Champlain's Famous Rock." Vermonter Magazine, Vol. XIXI. p. 29.

DEFOREST, JOHN W.

I85I History of the Indians of Connecticut, from the Earliest Known Period to I850. Hartford: William James Hamersley. 509 pp.

Douglas-Lithgow, Robert A.

1909 Dictionary of American Indian Place and Proper Names in New England. Salem: Salem Press. 400 pp.

DOUglas-Lithgow, RoberT A.

I9I4 Nantucket, a History. New York: Putnam's. Pp. I7-34.

Dunlap, A. R. and C. A. Weslager.

I950 Indian Place Names in Delaware. Wilmington: The Archaeological Society of Delaware. 6I pp. 
Eckstorm, Fannie HaRdy

I94I "Indian Place Names of the Penobscot Valley and the Maine Coast." University of Maine Studies in History and Government, No. 55. Orono: Reprinted at the University Press, I960. $272 \mathrm{pp}$.

ECKSTORM, FANNIE HARDY

1938 "Prefixes, Suffixes, Roots and Derivatives in Maine Place Names." Unpublished; typed copy in Bangor, Maine, Public Library. 52 pp.

EDWARDS, JONATHAN JR.

I823 "Observations on the Mohegan (Connecticut) Language." Edited by John Pickering. Massachusetts Historical Society Collections, and Series, Vol. X, pp. 8I-I6o.

ELIOT, JoHN

I666 The Indian Grammar Begun. Cambridge: Marmaduke Johnson. $66 \mathrm{pp}$.

GaHAN, LaURENCE $\mathrm{K}$.

I960 "Methods of Translating Indian Place Names." Bulletin of the Massachusetts Archaeological Society, Vol. 21, Nos. 3 and 4, pp. 46-47.

GAHAN, LAURENCE K.

I96I "Gleanings from the Indian Languages." Bulletin of the Massachusetts Archaeological Society, Vol. 22, Nos. 3 and 4, pp. 65-67.

Ganong, William Francis

I905; "Monographs of Place-Nomenclature, Cartography, Historic

1912- Sites, Boundaries and Settlement Origins of the Provinces of

I918 New Brunswick." Contributions to the History of Nerw Brunswick. Ottawa: The Royal Society of Canada. $600 \mathrm{pp}$.

Gatschet, Albert Samuel

I897 "Indian Place Names in Maine." Washington: National Geographic Magazine Vol. XXXX. Continued in Eastport Sentinel, VIII, Sept. I5, I897. Copies in Maine State Library, Augusta.

Gatschet, Albert Samuel

I887- Vocabularies, Treaties, etc. Unpublished notes (probably

I900 taken at Old Town, Maine) on deposit at Smithsonian Institution, Washington.

Greenleaf, Moses

I904 "Indian Place Names on the Penobscot and St. John Rivers in Maine." In Moses Greenleaf, Maine's First Mapmaker. Bangor: DeBurian Club, privately printed, I2 pp.

Hodge, Frederick WeBb (editor)

1907; "Handbook of American Indians North of Mexico." Bureau

r9I0 of American Ethnology, Bulletin 30. Washington: Smithsonian

Holmer, NiLs M. Institution. 2 Volumes.

I948 "Indian Place Names in North America." Essays and Studies on American Language and Literature, No. VII. Cambridge: Harvard University Press., 44 pp. 
HORSFORD, EBEN NORTON

I886 The Indian Names of Boston, and Their Meaning. Cambridge: John Wilson, \& Son. 28 pp.

Hubbard, Lucius LeE

I884 Woods and Lakes of Maine. Boston: James R. Osgood and Co. Appendix I, Place Names, pp. I9I-2I4; Cross index (in English) to Indian names in the preceding list, pp. 215-2I6.

Huden, John C.

I955 "Indian Place Names in Vermont." Vermont History, Vol. XXII, No. 3, July I955, pp. I9I-203; Vol. XXV, No. I, pp. 66-8o. HUDEN, JOHN C. Reprinted privately in 1957 at Burlington: Lane Press, $32 \mathrm{pp}$.

I959 "Champlain Maps, I 542-I 792." Vermont History, Vol. XXVII, No. I, pp. 34-40; Vol. XXVII, No. 2, pp. 85-87; Vol. XXVII, No. 3, pp. 190-193. Also Burlington: Lane Press, privately printed. $20 \mathrm{pp}$.

Hutchinson, Viola L.

I945 The Origin of New Jersey Place Names. Trenton: New Jersey Public Library Commission. 33 pp.

JONES, WILLIAM

I904 "Some Principles of Algonkian Word-Formation." American Anthropologist, n. s., Volume VI, No. 3, pp. 369-4II.

Kelton, Dwight H., Captain

I888 Indian Names of Places Near the Great Lakes. Detroit: Kelton. $55 \mathrm{pp}$.

KenNicuti, Lincoln N.

I909 Indian Names of Places in Plymouth, Middleborough, Lakeville and Carver. Worcester: Commonwealth Press. $50 \mathrm{pp}$.

KenNicutt, Lincoln N.

I9I I Indian Names of Places in Worcester County, Massachusetts. Worcester: Commonwealth Press. 42 pp.

Lacombe, Albert, Rev.

I874 Dictionnaire et grammaire de la langue des Cris. Montréal: Beauchemin \& Valois. 709 pp.

LAURENT, JosEPH (alias Lolo, SozAP)

I884 New Familiar Abenakis and English Dialogues. Québec: Léger Brousseau. $230 \mathrm{pp}$.

LAURENT, STEPHEN

I960 "New Hampshire's First Language." Concord: The New Hampshive Archaeologist, No. Io. pp. 5-6.

LAURENT, STEPHEN

I955, "The Abenakis, Aborigines of Vermont." Vermont History, I956 Vol. XXIII, No. 4, pp. 286-295; Vol. XXIV, No. I, pp. 3-II.

LONGFELLOW, HENRY WADSWORTH

I883 Poems. Boston: Houghton, Mifflin and Company. The Song of Hiawatha, pp. I4I-I89. (Vocabulary of Indian Names and their translations, pp. 482-483).

LONGFELLOW, HENRY WADSWORTH

1856 The Song of Hiawatha. Boston: Ticknor and Fields. 316 pp.; Vocabulary of Indian words and their translations, pp. 3I4-3I6. 
LAYNG, THEODORE E.

I956 Sixteenth Century Maps Relating to Canada. Ottawa: Public Archives of Canada. Published by the Queen's Printer. 238 pp.

LOUNSBURY, FLOYD G.

I960 "Iroquoian Place Names in the Champlain Valley." Champlain Basin Past-Present-Future; Report of the New York-Vermont Interstate Commission on the Lake Champlain Basin. Albany: New York State Legislative Document (1960) No. 9, pp. 2I-66.

McAleEr, George, Dr.

I906 A Study in the Etymology of the Indian Place Name Missisquoi. Worcester: Blanchard Press. 137 pp.

Masta, Henry Lorne

I932 Abenaki Indian Legends, Grammar and Place-Names. Victoriaville; Québec: La Voix des Bois Francs. I Io pp.

Maurault, J. A., Abbé

I866 Histoire des Abenakis, depuis I605 jusqu'd nos jours. Sorel: Imprimé à l'atelier typographique de la "Gazette de Sorel." $632 \mathrm{pp}$.

MayHew, EXPERIENCE, Rev.

I885 "Letter of Experience Mayhew, I722, on the Indian Language." Boston: New England Historical and Genealogical Register, Volume 39, pp. ro-r 7 .

MenCKen, Henry Louis

1948 The American Language, Supplement II. New York: A. A. Knopf. 328 pp.

Nichols, Frances S. (compiler)

I954 "Index to Schoolcraft's 'Indian Tribes of the United States'." Bureau of American Ethnology, Bulletin I52. Washington: Smithsonian Institution. $257 \mathrm{pp}$.

Nudénans, JEAN Baptiste

I760 Radicum Wabanakkcerum Sylva. A manuscript Abnaki-Latin Dictionary at Nicolet College, Québec. A r934 typescript copy is in the Bangor, Maine, Public Library. 108 pp.

O'Brien, Michael Charles, Rev.

I887 "A Grammatical Sketch of The Abnaki Noun." Portland: Maine Historical Society Collections, IX. pp. 259-294.

O'Brien, Michael Charles, Rev.

I870- Notebooks and Memoranda collected while Father O'Brien

1890 was working with Maine Indians. In Maine Historical Society Library, Portland.

PaINe, George W.

1902 Abnaki Vocabulary. Unpublished manuscript on deposit in Smithsonian Institution, Washington.

Parsons, Usher, Dr.

r86r Indian Names of Places in Rhode-Island. Providence: Knowles, Anthony and Company. 54 pp.

Pike, Judith Johnstone

I953 A Study of Place Names in Middletown, Connecticut. Unpublished M. A. Thesis, Trinity College (Hartford) I23 Pp. 
Piper, Winthrop W.

I949 Place-Names of New Hampshire. Physical Features from Origin to 1857 . Unpublished M. A. thesis, Columbia University. $15^{8} \mathrm{pp}$.

Pilling, James Constantine

I89I "Bibliography of the Algonquian Languages." Bureau of Ethnology, Bulletin I3. Washington: Smithsoinan Institution. 6I 4 Pp.

Pownall, Thomas

r959A topographical Description of the Dominions of the United States of America. (Edited by Lois Malkaern). Pittsburgh: University

Price, Chester B. of Pittsburgh Press. 235 pp. (Original manuscript, 1784).

I958 "Historical Indian Trails in New Hampshire." New Hampshive Archaeologist, Number 8. Concord: pp. 2-r3, plus map.

Prince, J. Dyneley and Frank G. Speck

1903 "The Modern Pequots and Their Language." American Anthropologist, n. s., Volume 5, No. 2, pp. I93-2I2.

Prince, J. Dyneley and Frank G. Speck

I904 "Glossary of Mohegan-Pequot Language." American Anthropologist, n. s., Volume 6, No.I, pp. 18-45.

Purchas, Samuel

I906 Purchas His Pilgrims, or Hakluytas Posthumus. Volume VIII. Glasgow; James Maclehose and Sons. pp. 358-359.

Quasuk, Paumatuck

I824 "Will of Paumatuck Quasuk, Father of Squaw Betty: I673." Massachusetts Historical Society Proceedings, Vol. 2. Boston: pp. 289-290.

Rand, Silas Tertius, Rev.

I875 A First Reading Book in the Micmac Language. Halifax: Nova Scotia Printing Co. $126 \mathrm{pp}$.

Rand, Silas Tertius, Rev.

1902 Rand's Micmac Dictionary. Charlotte Town: (P. E. I.) Patriot Publishing Co. (An earlier edition, 1888. Halifax: Nova Scotia Printing Co.)

Rasles, Sébastian, Rev.

I724 "A Dictionary of the Abnaki Language in North America." Cambridge: E. W. Metcalf and Co. (I832). Edited by John Pickering, in American Academy of Arts and Sciences, Memoirs, n. s., Volume I, pp. 375-565.

Rider, Sidney S.

I904 The Lands of Rhode Island as They were Known to Canounicus and Miantunnomu When Roger Williams Came in I636. Providence: Sidney S. Rider. $40 \mathrm{pp}$.

Ruttenber, Edward Manning

I906 "Footprints of the Red Man." (Indian Geographical Names in Hudson Valley, Mohawk Valley and on the Delaware) Albany: Proceedings of the New York State Historical Assoc., Vol. VI. $242 \mathrm{pp}$.

Schoolcraft, Henry R.

I853 Historical and Statistical Information Respecting the History, Condition and Prospects of the Indian Tribes of the United 
States. Volume I. pp. 284-299. "Massachusetts Indians"; Volume II, pp. 353-508, "Grammatical Structure of the Algonquin Language, and Vocabularies," Philadelphia: Lippincott, Grambo and Co. 6 Volumes.

Sealock, Richard B. and Pauline A. Seely

I949 Bibliography of Place Name Literature. Chicago: American Library Association. 33I pp.

Seely, Pauline Augusta

1955 "Place Name Literature in the United States and Canada." Journal of American Name Society, Volume III, No. 2, 20 pp.

SHERWIN, REIDERT

1940- The Viking and the Red Man: Old Norse Origin of Algonkian

I953 Language. New York: Funk. 7 Volumes.

Sleeper, Myron O.

I949 "Indian Place Names in New England." Massachusetts Ar. chaeological Society Bulletin. July, pp. 89-93.

Speck, Frank G.

1928 "Native Tribes and Dialects of Connecticut," and "A MoheganPequot Dictionary," in Forty-third Annual Report of the Bureau of American Ethnology. Washington: Smithsonian Institution.

SPECK, Frank G. pp. 203-287.

I915 "The Eastern Algonkian Wabanaki Confederacy." American Anthropologist, Vol. I7, pp. 492-508.

SPECK, Frank G.

I940 Penobscot Man. Philadelphia: University of Pennsylvania Press. 325 pp.

Spiess, Mathias

I933 "Indians of Connecticut." Hartford: Tercentenary Bulletin, $X X I X$ Committee on Historical Publications. $33 \mathrm{pp}$.

Stewart, G. R.

1945 Names on the Land. New York: Random House. 4I8 pp.

STRACHEY, William

I955 "The Original Strachey Vocabulary of the Virginia Indian Language." Edited by John P. Harrington. Bureau of American Ethnology, Bulletin I57, Anthropological Papers, No. 46. Washington: Smithsonian Institution. pp. 189-202.

SWANTON, JOHN R.

1952 "The Indian Tribes of North America." Bureau of American Ethnology, Bulletin I45. Washington: Smithsonian Institution. $726 \mathrm{pp}$.

TANTAQUidgeon, GLadys

I928 "Mohegan Medicinal Practises, Weather Lore and Superstition." Bureau of American Ethnology, 43rd Annual Report.

Washington: Smithsonian Institution, pp. 264-279.

Thoreau, Henry David

I864 The Maine Woods. Boston: Ticknor and Fields. 328 pp.

TOOKER, WILliam Wallace

I 895 "Louisquisset - Letter on Name, dated November I7, r894." Providence: Booknotes, Volume XII, No. 8, pp. 85-86. 
Tooker, William Wallace

I901 Algonquian Series. Io Volumes. New York: Harper Press.

TOOKER, William Wallace

r904 "Algonquian Names of Some Mountains and Hills." Boston: Journal of American Folk Lore, Volume XVII, pp. I7I-I79.

TOOKER, WILliam WALLACE

I904 "Remarks on the Names Massasoit, Ousamequin, and Packanoket." Book review in American Anthropologist, n. s., Volume VI, No. 4, pp. 547-548.

TOOKER, WilliaM WALLACE

I9I I Indian Place Names on Long Island, and Islands Adjacent. New York: G. P. Putnam's Sons. 3I4 pp.

TRUMBUll, James Hammond

I870 "The Composition of Indian Geographic Names." Hartford: Connecticut Historical Society Collections, Volume 2. pp. 3-5I.

TRUMBUll, James HAMmond

I88I Indian Names of Places etc., in and on the Borders of Connecticut. Hartford: Case, Lockwood and Brainerd. 93 pp.

TRUmbull, James Hammond

1903 "Natick Dictionary." Bureau of American Ethnology, Bulletin 25. Washington: Smithsonian Institution. $349 \mathrm{pp}$.

Wallis, Wilson D. and Ruth Sawtell Wallis

I955 The Micmac Indians of Eastern Canada. Minneapolis: University of Minnesota Press. pp. 72-94.

Wallis, Wilson D. and Ruth Sawtell Wallis

1957 "The Malecite Indians of New Brunswick." National Museum of Canada, Anthropological Series No. 40, Bulletin 148. Ottawa: G. P. O. 54 Pp.

WATKIns, Edwin ARTHUR, Rev.

$1865 A$ Dictionary of the Cree Language, as Spoken by the Indians of the Hudson's Bay Company's Territories. London: Society for Promoting Christian Knowledge. $460 \mathrm{pp}$.

WiLliams, ROGER

I643 A Key Into the Language of America. London: Gregory Dexter. 224 pp. Reprinted, 1827, Collections Rhode Island Historical Society, Vol. I, pp. 17-163.

WRIGHT, HARRY ANDREW

I939 "Some Vagaries in Connecticut Valley Indian Place Names." New England Quarterly. September, pp. 535-544.

Writers Program, Massachusetts

I94I Origin of Place Names in Massachusetts. New York: Harian Press. 55 pp.

Wzokhilain, Peter Paul (alias Osunkhirine)

1830 Wobonaki Kimzowi Awikhigan. (Abnaki Religious Writings, also Spelling, Reading, Vocabularies, etc.) Boston: Crocker and Brewster. $90 \mathrm{pp}$.

Zeisberger, DAVid, Rev.

r887 Zeisberger's Indian Dictionary. Cambridge: John Wilson \& Son. 236 pp. 


\section{INDEX}

Abington, Mass., Manamookeagin

Addison, Me., Wassquagos

Addison, Vt., Chemaun-Nayaug, Kanionra

Agawam, Mass., Cottinackeesh, Naggawoomcom, Pauhunganuck, Usquaiok

Alburg, Vt., Algonkine, Ga non wa ro hare, Wdepsek

Alder Brook, Me., Peskedopikek

Alexander's Lake, Conn., Mashipaug

Alfred, Me., Bunganut

Allis Park, Vt., Ahwassoos Aden

Alton Bay, N. H., Quannipi, Winnianebiskek

Amesbury, Mass., Attitash

Amston, Conn., Ashowat

Andover, Conn., Watchogash

Andover, Mass., Quayachick

Androscoggin River, Me., Nagusset, Pescedona

Ansonia, Conn., Pacoquarocke

Ant Hills Island, Me., Emikus wassissek

Apple Tree Point, Vt., Darongowa

Argyle Boom Island, Me., K'noonaghek

Arlington, Vt., Kansas, M'kuigen, Nebraska, Ondawa, Tyetilegogtakook

Arrowsic Island, Me., Ke Kepan Agliesek, Manaskek, Roswic

Ashaway, R. I., Ashawaug

Ashburnham, Mass., Watatick

Ashby, Mass., Neesepegesuck, Nemoset, Squannacook

Ashford, Conn., Acquebatuck, Assawaug

Ashland, Mass., Magunhukquok, Magunkahquog

Athol, Mass., Pahquioque, Pequiog

Atlantic, Kehta Hanit, Sobagwa
Augusta, Me., Cushnoc, Negwamkeag Ayers Rips, Me., Nalumsunk-hungan

Bagaduce River, Me., Winneagwamuk

Baker Lake, Me., Woolastookwaguamok

Baker River, N. H., Abocadneticook, Asquam Chumakee

Baker Stream, Me., Woboostook

Baker's Island, Mass., Fennapoo

Bald Hill Cove, Me., Edali teh

Bald Hill Mountain, Me., Eskweskwewadjo

Bangor, Me., Paamtegwitook, Pemtegwatook

Bantam, Conn., Peatam, Wyantenuc

Bar Harbor, Me., Abessah, Ahbasak, Manesaydik, Pais-unk, P'sahn. See Mount Desert.

Barnet, Vt., Scotomak

Barnett, Mass., Kuppowonkunok

Barnstable, Mass., Canaumet, Cottonchusett, Cummaquid, Mattakesett, Pawpoesit, Saughtuckquett, Sawaquatock, Skunkamug, Waquoit

Barrington, R. I., Maskituash, Nahett, Nockum

Barter Island, Me., Moniecook

Bartlett, N. H., Pequawkett

Bartlett Peak, N. H., Attilah

Bath, Me., Towessek, Weswick, Whiskeag, Winnegance

Batten Kill, Vt., Ondawa, Saratogue, Tyetilegogtakook

Beach Pond, R. I., Pawcomet

Beacon Falls, Conn., Capage

Bearcamp River, N. H., Awassokik

Bear Island, N. H., Awasaswi Menahan

Bear Notch, Vt., Owassoosen, Yages= hon 
Beaver Pond, N. H., Shoneeto

Beaver Tail Point, R. I., Qummunagat

Bedford, N. H., Massasecum

Belchertown, Mass., Paquonk quamaug, Paskesickquopoh

Belfast, Me., Paquatanee, Passagassa-waukeag

Bellows Falls, Vt., Chee Coke, K'chi Ponteguh, Kitchee Ponteguh, Niben

Bennington, Vt., Askaskwigek Wadjo, Moosompsquetuh, Sancoik, Taascamessick, Walloomsac

Berkshire Mountains, Mass., etc., Tachannike, Taghkannuc

Berlin, Mass., Kequassagansett

Bernardston, Mass., Coassit, Koessek, Nallahamcongan, Natanas

Berwick, Me., Neguttaquid, Osabeg

Bethel, Conn., Unkawa, Waubeoka Beverly, Mass., Mingo

Big Lake, Me., Amilesk, Chimenasanganum, Ellitegway gamek, Menes-saganaganis

Big Ledge Carry, Me., Katopskonegan

Billerica, Mass., Popossego quohock egge

Birch Stream, Me., Pemsquamkutook

Black Duck Stream, Me., Kwikwimes-witicook

Black Island, Me., M'kazanikutmenahan

Blackman Stream, Me., Madamiscomtis

Blackmore Pond, R. I., Pesaumkamesquesit

Black Pond, Me., Meskeekwagamasic

Black River, Lincoln County, Me., Abonegog

Black River, Orleans County, Vt., Elligo-sigo

Black River, Penobscot tributary, Me., Kahgognamock

Black River, Windsor County, Vt., Alskachewack, Kaskactchawack, M'kazawitego
Black River, Lower Kennebec, Me., Sasanoa

Blackstone River, Mass., Kittituck, Kuttutuck, Mutighticoss, Neet mock, Sneechteconnet, Tittituck, Wunnashowatuckqut

Block Island, R. I., Manisses, Sachem

Bloody Point, N. H., Squamscott

Bloomfield, Vt., Mozias, Nebisonbik, Skahogan

Blue Hill, Me., Awanadjo

Blue Hill Tide Falls, Me., Kuladam. itchwan

Bonnabeg Pond, Me., Squimonk

Boom Islands, Me., Menehanis-cook

Boston, Mass., Accomonticus, Amaganset, Hoanantum, Mashawmut, Shaw mut

Boston Neck, R. I., Namkook

Bowditch Ledge, Mass., Fennapoo

Boyden's Lake, Me., Nahsaick, Nesayik

Bradford, Vt., Kikon, Maasbaak, Madagascar, Madahondo apskwa, Mahsunquamassec, Nasawi, Nokhigan

Braintree, Mass., Cochessett, Mananticut, Minotoquid, Wampatuck

Brandon, Vt., Neshobe, Wagwises

Brattleboro, Vt., Keetadoganapskwa Seebis

Brewer, Me., Aguncia, Kaghsk-ibinday, Pesut amesset, Sedunkehunk

Bridgeport, Conn., Miamogue, Pequonnock

Bridgewater, Mass., Nameunkquasset, Nunkatusset

Brighton, Vt., Oswegachie

Brimfield, Mass., Ashquoash, Quiuabaag, Wullamanic

Bristol, R. I., Chisawannock, Papasquash

Brookfield, Mass., Masquabamisk, Massaquockumnie, Matchuk, Musquebaug, Nacommuck, Nanantomqua, Neesepegesuck, Pataug, Podunk, Quabaconk, Wullamanic

Broken Island, Me., Boskquenuguk 
Brooklyn, Conn., Tatnic

Brooksville, Me., Winneagwamuk

Brunswick, Me., Pescedona, Pogamqua, Puggamugga, Seogogguanegabo

Brunswick, Vt., Nebisonbik, Nolkaianak

Bucksport, Me., T'kopesuck

Bullock's Cove, R. I., Wannamoiset

Burden's Pond, Me., Puscommatas

Burlington, Vt., Darongowa, Miskwa-aubik, Nonnowigil Menahol, Otstenrowanen, Paliten, Regiochne, Skanentgraksenge, Winooskeek

Burrilville, R. I., Matomy

Buzzards Bay, Mass., Poughkeeste

Byram River, Conn., Armonek, Cockamong, Comonck

Cabot, Vt., Mali bowk, Mali panjahlok

Cambridge, Mass., Anmoughcawgen

Camden, Me., Mada-Kamigosek, Megunticook, Negunticook

Camel's Hump Mountain, Vt., Dyonondakren, Ta wak be dee eeso Wadso

Canaan, Vt., Namaaskotic, Yanondasa

Canterbury, Conn., Wanungatuck

Canterbury, N. H., Plausawa, Sabbatus

Cape Elizabeth, Me., Poodik, Purpoodik, Quack

Cape Neddick, Me., Quaco

Cape Porpoise River, Me., Mousam

Cape Rosier, Me., Edali chichi

Caribou Lake, Piscataquis County, Me., Mahnekebahntic, Nahnekbahntic

Casco Bay, Me., Manan, Marchin

Castine, Me., Bagaduce, Edali, Mosemadage, Pentagoet

Castleton, Vt., Wekowamadensees

Cathance Lake, Me., Posseps Cangamock

Centerville, Mass., Wequaquet

Central Connecticut, Nawaas, Nowashe
Chamberlain Lake, Me., Apmoojenegamook, Baamchee nungamook

Charles Island, Conn., Poquahaug

Charles River, Mass., Ashunaiunk, Cowate, Messatsoosec, Quinnebequi, Shannock

Charlestown, Mass., Accomonticus, Mashamugget, Mashawmut, etc. See Boston.

Charlestown, R. I., Bapetanshant, Chemunganock, Machaquamaganset

Charlotte, Vt., Adonniate, Aswahdenick, Mateguasaden, Ohnowarake, Pawonbowk, Tommy Squat. ter, Topabskwa

Charlton, Mass., Quassaponikin

Chatham, Mass., Manamoyik, Manamoquack, Matchapoxet

Chebeague, Me., Jebucto

Chelmsford, Mass., Neesepegesuck, Tadmuck, Waymessick

Chelsea, Mass., Naamkeak, Winneashimut, Winnisimmet, see Boston

Chepachet, R. I., Matomy, Squammicott

Chester, Conn., Pattaquonk, Sepunamus

Chesterfield, N. H., Wantastiquet

Chibanook Lake, Me., Saponac

Chicopee, Mass., Chequapee, Chikabi

Chilmark, Mass., Nashuae-Komuk, Nimpanick Hickanuh

Chimney Point, Vt., Chemaun Nayaug, Kanionra, Teckyadoughinyariga

Chimon Island, Conn., Mamachimins

Chittenden, Vt., Nickwacket

Choke Cherry Island, Me., Attebemeuck

Churchill Lake, Me., Allagask wigamook

Clifton Hills, Me., Pemadinisikek

Clyde River, Vt., Misquiquaungameck

Coasters Harbor Island, Me., Woonachasset

Cohasset, Mass., Musquashcut

Colchester, Conn., Cassacubque, Cheebeeantups 
Colchester Point, Vt., Agenyateonnia,

Concord, Mass., Annursnack, Musketaquid, Nasawtuck, Punkatesset

Concord, N. H., Wattanumon

Concord, Vt., Skahogan

Connecticut River, Counnitegou, Guonitogou, Kwini teguh, Maniganatica-uoit, Quinnehtukqut, Quintikoock, Quonektacut, Passquishunk, Sicojocke, Sokoquois

Cononicus' Boulder, R. I., Neotaconkonitt

Corinth, Vt., Oquassanock

Corinth-West Fairlee, Vt., Yageshon

Corliss Cove, R. I., Saxafrax

Cos Cob, Conn., Cassacubque

Cousin's River, Me., Sisquisic, Susquesong, Sysquisset

Coventry, R. I., Machepaconapunsuck, Scatacook

Cowesett, R. I., Metaubscot

Cow Island, Me., Kaoosi Menahan

Cranston, R. I., Maushapogue, Pataconkset, Papaquinapaug,

Crawford, Lake, Me., Gaywaysick

Crooked Island, Me., Bakun Gunahik, Pikaghenahik

Cross Island, Me., Sibegehanuck

Cross Lake, Me., Bamonewengamok

Cuttyhunk Island, Mass., Cappiquat, Kehtitanunk, Poo Cuttahunk Anow

Daggett Pond, Me., Moskwaswagamocsis

Damariscotta, Me., Kananghetne, Madamascontee, Tamescot

Damariscotta River, Me., Appowick, Pedaugbiouk

Damariscove Island, Me., Aguahega

Dana, Mass., Neeseponsonet, Pottapaug

Danbury, Conn., Pahquioke, Tankiteke, Unkawa

Danville, Vt., Sozap Nebees

Dartmouth, Mass., Akusenag, Assameek q, Coquitt, Cushenag, Mackatoy, Nukkekummees, Pascomanset

Dedham, Mass., Pomham, Powisset, Pumham, Tist
Deerfield, Mass., Paucomptucke, Pocomtakuke, Pocumtuc

Deer Island, Me. Nohlkai manahan Dennis, Mass., Nobscusset

Denny's River, Me., Ket h'nik

Derby, Conn., Squantuck, Weantinock

Devils' Head, Me., see D'Orville's

Devil's Island, R. I., Chepinoxet

Dighton Rock, Assonet

Dobsy Lake, Me., Deekeewenskek, Sisladobsis

Dochet Island, Me., Mutanagwes

Dorchester, Mass., Kutshamakin, Megansett, Menchoiset, Shawmut

Dorset, Vt., Netop, Wanasquatuck

D'Orville's Head, Me., Quagachusque, Quaweejoos

Dover, N. H., Chebeague, Cocheco, Jebucto, Newichwannock, Panway, Scatuate, Winichahanet

Dracut, Mass., Augamtocook

Dudley, Mass., Kekamoochaug, Kekamowadchaug, Quitemaug, Tohke commumwachak

Dummerston, Vt., Skamonikoos, Wicopee

Dunstable, Mass., Mashepagocke, Mashepagonoke

Dutch Island, R. I., Aquednesset, Quatenus

Dyer's Neck, Me., Kemboes Kisek, Nasso-emek

Eagle Lake, Me., Pongokwahemook East Boothbay, Me., Winnegance

Eastern River, Me., Mundoo-uscoo took

Eastford, Conn., Abaquage

East Greenwich, R. I., Pootowoomet

Eastham, Mass., Naamcoyicke, Ocinamunt, Potenumacut

Easthampton, Mass., Aspowunk, Assatayag, Minhan, Nashawannuck, Nayasset, Pasacomuk, Petowwag

East Hartford, Conn., Namareck, Upaquoag

East Haven, Conn., Mioonkh-tuk

East Limington, Me., Watchic

East Lyme, Conn., Mamacock 
Easton, Mass., Hockamock

East Pepperell, Mass., Babbatasset

Eastport, Me., Cobscook, Muselenk

Eddington, Me., Mantawassuk, Mitaganessuk

Edgartown, Mass., Kamesset, Nunnipaug. see Martha's Vineyard

Eel Brook, N. H., Nesenkeag

Elbow Lake, Me., Numachinagamook, Numtsceenaganawis

Elizabeth Islands, Mass., Onkatomka, Pacamkik, Peschameeset

Ellsworth, Me., Wechkotetuk

Equinox Mountain, Vt., Ekwanok

Essex, Conn., Pataquasek

Etna Pond, Me., Nawlombages

Exeter, R. I., Bassoqutogaug, Hakewamepinke, M'squamscook, Shankhassick, Shawunkhassick

Fairfield, Conn., Chickons, Weantinock

Fairhaven, Vt., Cooksacky

Fairlee, Vt., Sennemahgesso

Fall River, Mass., Copicut, Quequecham, Watuppa

Falls Point, Me., Adowaukeskeag, Douaquet, Waukeag

Falmouth, Me., Scittery gusset, Skaki

Falmouth, Mass., Ashimuet, Shamuet, Succanesset

Farmington, Conn., Tunxis, Wepansock

Farmington, Me., Amaseconti, Amessagunticook, Amessecantee

Farmington River, Conn., Cheesechankamuck, Pacomtock, Pautonk, Tunxis, Watunkshausepo

Feeding Hills, Mass., Noycoy

Fenton River, Conn., Nahwesetuck

Ferdinand, Vt., Seneca

Ferrisburg, Vt., Dawinehneh, Donwagayon, Jigwallick, Pekontuk, Sungahnee took, Wisawogamak, Wobegilseebis

Field's Pond, Me., Piwan gamosis

First Eddington Falls, Me., Wequagawaysuk

Fisherman's Island, Me., Epituse

Fisher's Island, N. Y., Munnatawkit
Five Islands Falls, Me., Kessalogesso Modik, Sologismoodik

Forest Dale, Vt., Wagwises

Fort Knox, Me., K'tchi-peskwahonda, Wakalosen

Fort Point, Me., Aguahassideck, Negas

Fort Trumbull, Conn., Mamacock

Foster, R. I., Connaug, Mashentuck

Foxboro, Mass., Cocasset

Fox Island Narrows, Me., Hahkik Watpuk, Sahbahesset

Fox Island, R. I., Azoiquoneset, Nanaquonset, Nanequoxet, Sonanoxet, Sowanoxet

Framingham, Mass., Nobscot, Penobscot

Franklin, N. H., Nikisipik

Freese Island, Me., Nolat keeheemungan

French Island, Me., Keenaht Nassick, Mada-waniganook

Fryeburg, Me., Pigwacket

Gales Ferry, Conn., Awcumbuck

Gardiner's Lake, Conn., Mashipaug

Gardiner's Lake, Me., Nemdamassuagum

Gardner, Mass., Quag, Watatick

Garner's Neck, R. I., Mattapoysett

Gates Pond, Mass., Kequassagansett

Georgetown, Me., Sagassett

Georgia, Vt., Sekidawe

Gilford, N. H., Menunquatucke, Norwottock

Glastonbury, Conn., Assawassuck, Kongscut, Mabautuantucksuck

Glocester, R. I., Paquantuck, Shinskatuck

Glover, Vt., Nagwadog, Pekdabowk

Goat Islands, R. I., Nantusiunk

Goffstown, N. H., Uncanoomuc, Uncanoonucks

Goose Creek, Vt., Wobegilseebis

Goshen, Conn., Massapoag

Gould's Island, R. I., Aguspemokick, Aquopimokuk

Grafton, Mass., Hassanamesit

Granby, Conn., Congamuck 
Granby, Mass., Pitchawamache, Quaquoontuck, Suchow

Granby, Vt., Moceasin

Grand Isle, Vt., Gawenio, K'chenamenahan, Kahwehniyo, Oselaga, Ohskenonton

Grand Lake, Me., Cheputneticook, Chilnucook, Matagamook, Willi. guaganum

Grand Landing Place, Me., Catawamkeak, K'tahguantek

Grand Manan Channel, Manahnook

"Grand Marais," Onderiguegon

Great Barrington, Mass., Maswasehi

Great East Lake, Me., Newichwanimak

Greenfield, N. H., Sebosenec

Green Island, Me., Manaskoos

Greenland, N. H., Winnicutt

Greenwich, Conn., Asamuck, Sioascauk

Griswold, Conn., Chabunnuck

Groton, Conn., Gungy Wamps, Poquiant, Shenecosset

Groton, Mass., Abaquakea, Massapoag, Petapauket, Waubansconcett

Gull Island, N. Y., Nayantacawnick

Haddam, Conn., Cossonowock

Hadley, Mass., Chusick, Coassit, Hoccanum

Hadley's Lake, Me., Sabagwagum, Subecwangamook

Hale Brook, Me., M'squamagweseebo

Hamilton, Mass., Aspowunk

Hampden, Me., Edali weekek, Sawad Apskek, Sewad apskak, Tolbuntbessek

Hampton River, N. H., Winnecumek, Winnecunnet

Hancock, Me., Adowaukeag

Hancock, Vt., Texas

Hanover, Mass., Assinipi

Hardwick, Mass., Magus, Sasagookapaug, Wombemesisacook

Hardwood Island, Me., MachlichNagook

Harpswell, Me., Merriconeag, Quohoag, Sebascodegan
Harrington Lake, Me., Pokumkesawangamoksis

Harrison's Island, Me., Epuk unikek

Harrow Pond, Me., Megwakangamosis

Hartford, Conn., Appaquag, Nawaas, Saukiog, Sequassen, Sicaog

Hartland, Vt., Waterquechee

Harvard, Mass., Chaboken, Shabokin

Harwich, Mass., Monamesset, Satuit, Sawaquatock, Sawkatucket

Hatfield, Mass., Capawonk, Cappowongamuck, Chickons Cattones, Mincomonk, Wonckompsk, Yowunck homuck

Hathaway Point, Vt., Sabagwilha

Haverhill, Mass., Contoocook, Pentucket

Haydenville, Mass., Unquamonk

Haymock Lake, Me., Nahma jimski congomoc

Hermon Pond, Me., Edawi maniwik, Kwedawi-manwik, Wenk wida wiwie wak

Heron Island, Vt., Kaskoi Menahan Herring Pond, Mass., Commquessakumkanet

High Head, Me., Wassamkihemuk

Hinsdale, N. H., Squakheag

Hobart Stream, Me., Acrupsak

Hockanum, Conn., Noeutuck

Hockomock Point, Me,. Hobbomocka

Hog Island, R. I., Chisawannock

Holden, Mass., Hassunek

Holeb Pond, Me., Pascongamoc

Holland, Mass., Kesiog, Massapoag

Holland Pond, Vt., Massawipi

Holliston, Mass., Boggistow

Hoosac River, Vt., Atgatogwisas, Kaskekouke, Schaticook

Hopkinton, Mass., Kattenanit,' Magunquog, Magunhukquok, Magunkahquog

Hopkinton, R. I., Mamaquag, Moshowunganuck, Neshunganset, Wotesamoonsuck

Hop River, Conn., Sagumumpsketuck

Housatonic River, Conn., and Mass., Ousatunick 
Hubbardston, Mass., Canestow

Hughesdale, R. I., Wanepoonseag

Humphrey's Pond, Me., Monnebassa

Indian Hill Cemetery, Middletown, Conn., Wune Wahjet

Indian Island, Old Town, Me., Alnambi-i-menahan

Indian Point, Bucksport, Me., Alnambi kwaysah wayk, $T^{\prime}$ Kopesuck

Indian Pond, Kennebec Co., Me., Sebahticook, Seebaticook

Ipswich, Mass., Agawam

Island Pond, Vt., Menanbawk

Isle au Haut, Me., Solikuk

Isle la Motte, Vt., Gawenidakhe, Tgawistaniyonteh

Islesboro, Me., Kaskoo naguk, Pitow baygook

Isles of Shoals, N. H., Montinicus, Mootinoo

Israel's River, N. H., Sigwooganock, Singrawac, Soucook

Jacques' Island, Me., Sachtalen

Jamaica, Vt., Shatterack, Skamonikoos

Jewett City, Conn., Pautuxet

Jockey Cap Mountain, Me., Pequawkett

Joe-Mary Lakes, Me., Melanpswangamoc

Joe's Pond, Vt., Sozap Nebees

John Compound's, Conn., Compounce

Johnson Brook, Me., Mantawassuk Johnston, R. I., Absolonomiscut, Assapumsic

Jonesport, Me., Esqui wamigook, Moosepayechick, Seguin

Jug Island, Me., Bokajenesquis

Kendall Head, Me., Wabigenek

Kent, Conn., Pateook, Scaticook, Schaghaticauke

Kidderville, N. H., Mohawk

Killingly, Conn., Aspinook, Massapoag

King Philip, Metacom, Metacomet, Pometacomet, Wonkees-Ohke
Kingston, N. H., Massapaug

Kingston, R. I., Cajoot, Namcook, Shamcook, Shewatucquese, Shippaquonset

Kittery Point, Me., Amiciskeag

Knightsville, R. I., Mashantackack

Lake Bomoseen, Vt., Maskeekohwogam

Lake Champlain, Vt., Andiarocte, Aniadarawonte, Caniaderi Guarunte, Canuxsawhory, Kanondoro, Kanyatatakwaronte, Onderiguegon, Oneadalote, Onyatalot, Petonbowk, Petow Bowk, Petow Pargow, Rodsio Canyatare, Skanentgraksenge, Skanetoghrowa, Teckyadoughinyariga, Ticonderoga, Tieronderaquegon, Wisawogamak, Yotenyatarokte

Lake Chauncey, Mass., Naggawoomcom

Lake Fairlee, Vt., Kejigigilhasis, Quinibeck, Tolbabawk, Watagua Lake Sunapee, N. H., Oquasskikonaquam

Lakeville, Mass., Pocksha

Lambert's Cove, Mass., Konikey

Lamoille River, Vt., Kwenosakek, Wintoak

Lamprey River (Lamper Eel Stream), N. H., Piscasset

Lancaster, Mass., Chesquonopog, Hosokey, Penacook

Lancaster, N. H., Sowniganock

Lanesboro, Mass., Shonkamonke, Shoonkeek Moonkeek

Lebanon, Conn., Poquechanneeg

Ledyard, Conn., Acomekes, Cuppacomuck, Maushantuxet, Ohomowauke

Leicester, Mass., Mossonachud, Towtaid, Wekapekatonnic

Lenox, Mass., Perquanapa qua, Squog Kane Kaneek

Levant, Me., Tchebatigosak

Lewey's Lake, Me., Ellitegway gamek

Lewis Creek, Vt., Jigwallick, Sungahnee took 
Lewiston, Me., Amitgon pontook

Lilly Bay, Me., Potobek

Lincoln, Me., Pematinek

Lincoln, N. H., Kancamagus

Lincoln, R. I., Amataconet, Quinsnikit

Lisbon, Conn., Showatucket

Litchfield, N. H., Naticook, Nesenkeag

Little Bell's Place, Me., Swank wahiganus

Little Black River, Me., M'kaga-ook took

Little Compton, R. I., Coaxet, Nukkekummees, Pachet, Tunipus

Little Dochet Island, Me., Muttonegwenish

Little Falls Stream, Me., Ehkapsak

Little Hemlock Island, Me., K'seuski-naghassik

Little Otter Creek, Vt., Wonokakeetookeese

Little River, Me., Wekwabigek

Little Round Lake, Me., Pataquongamis, Piwan gamosis

Little St. Croix River, Me., Maguaguavic

Littleton, Mass., Magunco, Nashoba

Lobster Lake, Me., Peskebegat

Lone Tree Hill, Vt., Nagwadog

Long Island, Penobscot County, Me., Cheemahn

Long Island, Waldo County, Me., Pitow baygook

Longmeadow, Mass., Asnuntuck, Masacksicke, Pecowsic, Quillicks q, Scantic, Watchaug

Long Neck, R. I., Washouset

Long Reach, Me., Kwaykway-nahmak

Lonsdale, R. I., Wawepoonseag

Loon Lake, Me., Kwanok sagamik

Lowell, Mass., Massick, Naamkeak, Pantookaëg, Pantoocoög, Wickasauke

Lower Dobsy Lake, Me., Sisladobsis Lower Sabao Lake, Me., Wiscogosis Lower Saco River, Me., Sockhigones Lower Waterford, Vt., Muskagwah Lubec, Me., Kepamkiak, Unaganek
Ludlow, Mass., Minnechoag, Winechaug

Lunenburg, Mass., Catacoonamug, Makamacheckamucks, Massapoag, Uncachewalunk

Lunenburg, Vt., Tug

Lyme, Conn., Mamachoag

Lyme, N. H., Ordonakis

Madison, Conn., Tuxisshoag

Maine Coast, Apistama, Neskett

Malletts Bay, Vt., Ouisnouski

Mallison Falls, Me., Nagwamqueeg

Manchester, Mass., Mascanomo

Manchester, N. H., Cohoes, Namaskik, Naumkeag, Squog

Manchester, Vt., Ekwanok

Manomet, Mass., Aptuxet, Scook, Scusset

Manomet River, Mass., Pimsepoese

Mansfield, Conn., Nawbesetuc

Marblehead, Mass., Massabequash, Nanepashemet

Marion, Mass., Acoont, Aucoot, Metukpogkottomis, Pawhikchatt, Pitchoohutt, Seipican

Mark Island, Me., Kaskoo-naguk

Marlboro, Conn., Ungwonshackook

Marlboro, Mass., Ockoogangansett, Okommakemsit, Teightaquid

Marshes Island, Me., Wassoosumpsquehemock

Marshfield, Mass., Massasaugatukut

Marsh River, Me., Kwikwimes-witicook

Martha's Vineyard, Mass., Capawack, Cape Higgon, Capoag

Mason's Island, Conn., Chippachaug Matinicus Island, Me., Emmetinic, Menasquassicook

Medway, Me., Nicatow, Patagumkis, Petekamkes

Megantic Pond, Me., Damisokantic Mendon, Mass., Miscoe, Nippsatchuk, Quisset, Tattamasket, Tuttomnest Meredith, N. H., Aswaguscawadic, M'squam Chumaki

Merrimack River, Mass., and N. H., Cabassauk, Kaskaashadi, Mommock 
Merrymeeting Bay, Me., Abagadusset, Chisapeak, Kebec, Nassouac, Quabacook

Mianus, Conn., Mayannoes

Middleboro, Mass., Keticut, Muttock, Nadhuset

Middlebury, Conn., Meshapock

Middleton, Mass., Annasnappet

Middletown, Conn., (Indian Hill

Cemetery) Awannoa, Coschow

Middletown, Conn., Mattabesec

Milford, Conn., Owenoke, Poquahaug, Woronock

Milford, Mass., Magomiscock, Maspenock

Milford, Me., Saquaische, Sunkaze

Milford, N. H., Wisconemuck

Millbury, Mass., Packachoog

Miller's River, Mass., Baquag, Pappacontucksquash

Millis, Mass., Boggistow

Mill River, Mass., Pewonganuck

Mill River, Me., Cowesiseck

Milton, Mass., Hoosicwhisic, Uncataquisset

Milton, Vt., Kwenosakek, Wintoak

Mirror Lake, N. H., Aroosabaug

Moat Mountain, N. H., Atie ompsk a ooe di

Mohawk Brook, Mass., Nepasooenegg

Mohegan, Conn., Mangunk akuk, Mohanhegumewog, Muckhaneek, Muggs

Molly's Pond, Vt., Mali bowk

Monhegan, Me., Mananis

Montville, Conn., Cochikuack

Monument Brook, (on the MaineNew Brunswick Boundary), Cheebee Atque Seep

Monument Mountain, Mass., Mauswaseekhi

Monument Pond, Mass., Kitteaumut Moosabec Reach, Me., K'mokadich

Moose River, Me., Kweu-euk tonoonk hegan, Sahkabehaluk

Moosehead Lake, Me., Mozodup Nebes, Tomahegan, Xsebem

Mopang Lake, Me., Apskikek, Escutnagen

Morgan, Vt., Namagonic
Moutonboro, N. H., Chenayok, K'chi Nayok

Mt. Agamenticus, Me., Sasanow

Mt. Anthony, Vt:, Askaskwigek Wadjo

Mt. Ascutney, Vt., Askachewack, Askutegnik

Mount Desert Island, Me., Pais-unk, Pemadnek, Pemategu, Pemetic, P'sahn, see Bar Harbor

Mt. Mansfield, Vt., Dyonondakren, Mozodepowadso

Mt. Paxton, Mass., Asnebumsket

Mt. Philo, Vt., Aswahdenic, Mateguasaden, Tyontkathotha

Mt. Toby, Mass., Kunckquachu, Qununkwattchu

Mt. Tom, Mass., Paskhommuck

Mt. Waldo, Me., Kinabsk atnek

Mt. Washington, N. H., Agiochook, Kodaakwadjo, Maji Neowaska

Muck Pond, Me., Pockwockamus

Mud Pond, Me., Megwak-angamik, Pamgockamock

Muscongus Island, Me., Amobscot, Seremobscus

Muskeget Island, Mass., Kotget

Namokanok Island, Me., K'tolbeh

Nashua, N. H., Pennichuck, Watanic, Wataqua

New Bedford, Mass., Nequechoke, Potumska, Quequecham

New Braintree, Mass., Menemesseck, Wenimessett, Winnemisset

Newbury, Mass., Quascacunquen, Wescussauco

Newbury, N. H., Oquasskikonaquam

Newbury, Vt., Coös, Coösuck, Musquash, Sozap

Newburyport, Mass., Moniack

Newcastle, Me., Nasso-emek, Pedcoke gowake

Newcastle, N. H., Pannaway

New Found Lake, N. H., Passaguanik

New Haven, Conn., Mautunsq, Quillipiac, Mishimayagat

New London, Conn., Aukumbumsk, A wcumbuck, Catumb, Mameeg, Nameaug 
Newmarket, N. H., Washucke

New Marlboro, Mass., Umpachene

New Milford, Conn., Kenunck Pacooke, Metichawon

Newport Pond, Me., Sagon-dagon

Newport, R. I., Nonequit, Tittituck

Newport, Vt., Misquiquaungameck

New Sharon, Me., Amaseconti

Newton. Mass., Ammocangen, Winotimies

Nichols Rock, Me., Sobscook

No Man's Land Island, Mass., Cappoquidnet, Tequenoman

Noroton, Conn., Norsaton

Norridgewock, Me., Narantsoak

Northampton, Mass., Pewonganuck, Wequittayaug

North Branch, Marsh River, Me., Catamawawa

Northeast Harbor, Me., Asticou

Northern N. Y. and Vt., Koghserage

Northfield, Mass., Coassit, Coasuck

North Kingston, R. I., Matatucket, Shewatucket, Wannuchecomecut

North Lake, Skiff Pond, Me., Nigakws-wakeag

North Schoodic Lake, Me., Omquemenikeag

North Smithfield, R. I., Nippsatchuk, Quatoncanit

North Stonington, Conn., Assekonk, Chickamug, Cosattuck, Cuppan augunit Maumsuck

Norwalk, Conn., Mahackemo, Nauwot, Norwauke, Prosewamenos

Norwottock, Mass., Masquomp

Notched Head, Me., Psinkskilhigani-ontop

Oak Bay, Me., Waquiack

Oak Bluffs, Mass., Assanotucket, Hummock, Quinnamuck see Martha's Vineyard

Olamon, Me., Waylumkituk

Old Town, Me., M'skutook, Negan odanak, Neekounegan, Pannawambskek, Panoumke, Tegoak

Oliverian Stream, N. H., Umpammonoosuc

Orleans, Vt., Keekomkwak
Orson Island, Me., Asnela, K'chi mugwock

Otter Creek, Vt., Dawinehneh, Kahyonhes, Neshobe, Pekontuk, Netahme Puntook

Owl's Head, R. I., Ohomowauke

Owl's Head, Me., Kokohas wantepek

Owl's Place, Conn., and R. I., Ohomowauke

Oxford, Mass., Augutteback, Sacarappa

Palmer, Mass., Pattaquattic

Palmertown, Conn., Mashantackack

Patience Islands, R. I., Chibacoweda

Pawlet, Vt., Mettawee

Pea Cove, Me., Kwetahwamkituk

Peebles' Neck, R. I., Papanomscutt

Pelham, Mass., Jabish

Pembroke, Me., Pemtaquiauktook

Pembroke, Mass., Namaskeese

Pepperel, Mass., Nissitisset

Perry, Me., Sebeskiak, Wajoses, Wiwiliamecq

Perry Island, R. I., Chisawannock

Peterborough, N. H., Minniwawa

Petersham, Mass., Nichewaug

Petit Manan, Me., Menanouze

Pickpocket Falls, N. H., Pakwakek

Piermont, N. H., Moz Mushkeg

Pigeon Hill, Me., Wajoses

Pittsfield, Mass., Ouschankamaug, Washakamaug

Pittston, Me., Nahumkeag, Nehumkeag, Worromotogus

Pleasant Lake, Me., Maklic-ongomoc

Pleasant Point, Me., Seebyik

Pleasant River, Me., Mun-olammon ungan, Wescogus

Plymouth, Mass., Caskak Chesquash, Chipascutt, Gashee, Hobbomoc

Point Judith, R. I., Mattoonuc, Nayanticot, Nianticut

Pokomoonshine, Lake, Me., Menikpodik

Pomfret, Conn., Newichawannock, Tamonquas, Uhquanchaug, Wappoquian

Portland, Conn., Maromas 
Portland, Me., Acocisco, Auco Cisco, Machegony

Portsmouth, N. H., Sagamore

Portsmouth, R. I., Pocasset

Potter Hill, R. I., Chippachooag

Pownal, Vt., Nebissee-woldam-wogan, Schaticook

Preston, Conn., Checapseaddock

Princeton, Mass., Wanchatopek

Princeton, Me., Memada-Kamioguk, Wapskenigan

Priestly Lake, Me., Awanganis

Prospect, Me., Penopsquacook

Prospect Ferry, Me., Edali quh

Providence, R. I., Chaubatik, Moosehausic, Pattukkett, Pomham

Prudence Island, R. I., Wappewassick

Putney, Vt., Kawassentekwa, K'chi Muskegw

Quoddy Village, Me., Sebayek

Ragged Island, Me., Raggertask

Ragged Lake, Me., Menhaneekek, Nikanagamak

Ragmuff Stream, Me., Pataweektook

Rainbow Lake, Me., Mahnagwanegwa Sebem

Randall's Pond, R. I., Topamisspauge

Randolph, Mass., Cochessett

Rattlesnake Hill, Conn., Somersic

Rattlesnake Island, N. H., Sisikwa Menahan

Rattlesnake Mountain, Mass., Manskussehoank

Rattlesnake Peak, Mass., Deowcook

Raynham, Mass., Cohanit, Nippahonset

Red Hill, Vt., M'kuigen

Redrock Point, Vt., Miskwa-aubik

Rehoboth, Mass., Annawon, Chachapacasset, Mamwhauge

Rhode Island, R. I., Aquednessick, Aquidneck, Aquidnic

Richmond, N. H., Nipmuck

Richmond, R. I., Ashunaiunk, Conob, Ohomowauke, Shawnuck

Ridgefield, Conn., Asproom, Candatowa, Ramapoo
Ripogenous Lake, Me., Nolan gamoik Roach Pond, Me., Kok adjeweem gwasebem

Robbinston, Me., Mekwamkesk, Quinnisk-wouk-ook, Qunnoskwamkook, Wabasgach, Wabossagock

Rochester, N. H., Schohomogomoc, Squamagonic

Rochester, Vt., Michigan, Texas

Rock Point, Vt., Mistasenee, Negweesen, Otstenrowanen

Rockland, Me., Bedabek, Catawamkeak, Katawamkeag, Chickawaukee, Madam Bettox

Rockwood, Me., Tomhegan

Roily Lake, Me., Nesayik, Wiwiliamecq

Roque Bluffs, Me., Humolatski hegon

Rose Island, R. I., Conockonquit

Round Pond, Me., Petegwamgamek

Royall's River, Me., Swegustagoe

Rumford Falls, Me., Pennecook

Russell Mountains, Me., Tonquewack

Rutland, Mass., Pascatiguage, Pomagusset, Quaquananawich

Ryegate, Vt., Scotomak

Saco, Me., Chouacoet, Massagika, Sakadamkiak, Sawacotuck, Shawacotoc, Sockhigones, Sowhigginock

St. Albans, Vt., Kanyagwaronton, Popasquash, Sabagwilha

St. Anne's Shrine, Vt., Tgawistaniyonteh

St. George's Island, Me., Meniekec

St. George Peninsula, Me., Konesanouskek

St. George River, Me., Saquid, Segocket

Salem, Conn., Pagwonk, Paugwonk Salem, Mass., Nameaug, Namkeag, Nanepashemet, Naumkeag

Salisbury, Conn., Kisnop, Pannaheconnok, Weatic, Wiatic

Salisbury, Vt., Keewaydin, Moosalamoo

Salmon Falls, N. H., Newichwannock, Seminenal 
Sandwich, Mass., Aquednet, Ashimuet, Katomuckett, Massassomineuk, Skauton, Pimsepoese, Pumpisset

Sandy River, Me., Penobsquisumquisebou

Sawyer's Mountain, N. H., Cunney Saybrook, Conn., Pashebesauke

Sayles Hill, R. I., Wesquadomesset

Scalp Rock, Me., Psinkwandissek

Scarboro, Me., Owascoag

Seal Head, Me., Hahkik watpuk

Seal Rock, Me., Minnecopscook

Sears Island, Me., Awassawamkeak Searsmont, Me., Quontabacook

Sedgwick, Me., Sepsis-edal-apskit

Seponac Lake, Me., Chibanook

Seymour, Conn., Scucurra

Seymour Lake, Vt., Namagonic

Shad Island, Me., Sataylan

Shadow Lake, Vt., Pekdabowk

Shad Pond, Me., Nalaseemagamobsis

Shaftsbury, Vt., Tomhannock

Shallow Lake, Me., Moskwaswagamok

Sharon, Conn., Guna, Wachquatnach, Wequadnack

Sharon, Mass., Wolomonopaug

Sharon, Vt., Pantigo

Sheepscott, Me., Aponeg, Cowsigan, Nekrangen, Pashipscot

Sheffield, Mass., Mannanpenokean

Shelburne, Vt., Quinneaska

Shoreham, Vt., Kanondora, Teckya. dough Nigarige

Silver Lake, Me., Oolamonogamook

Simsbury, Conn., Massaco

Sipp Bay, Me., K'chi punahaquot

Skiff Pond (North Lake) Me., Nigakws-wakeag

Smith Brook, Me., Messatoocus

Smithfield, R. I., Toskaunk, Tueskennckinck

Socatean Stream, Me., Mesakketesagewick

Sock's Island, Me., Sachtalen

Somers, Conn., Missatchawag

Somersworth, N. H., Pisgatoek, Quamphegan

Soper Brook, Me., Peskebskitegwek Sugar River, N. H., Askutegnik
South Berwick, Me., Newichawannock

Southbridge, Mass., Cohasset

South Corinth, Vt., Oquassanock

South Kingston, R. I., Ohomowauke, Pesquamscot, Quawawehunk

South Orrington, Me., Walintuk

Southport Me., Nekrangen

South Windsor, Conn., Nowashe

South Wolfeboro, N. H., Winnebassakek

Spencer Pond, Me., Kok adjeweem gwasebemis

Springfield, Mass., Masacksicke, Paca tuck, Pecowsic, Wequanhausick

Springfield, Vt., Poosumsuck, Skitchewaug

Spruce Island, Me., Esqui wamigook

Squam Lake, N. H., M'squam Nebis

Squaw Mountain, Me., P'ahnmoiwadjo

Stamford, Conn., Mahackemo, Totomak, Umpewage

Sterling, Mass., Chocksett, Moantukcake, Tahanto

Stetson Pond, Me., Nexaongermek

Stewartstown, N. H., Metallak

Stillwater Lake, Me., Seeogamook

Stockbridge, Mass., Mahkeenac,

\section{Wnahtookook}

Stockton, Me., Essick, Ooniganissek Stoddard, N. H., Quinibaak, Winegegwok

Stonington, Conn., Asupsuck, Aushpook, Canonchet

Stony Creek, Conn, Agiocomook, Aigio commuck

Stoughton, Mass., Pakemit, Pekenut, Pequimmet

Stow, Mass., Pompositicut

Stratford, Conn., Cupheag, Nayumps, Pisqueheege

Sturbridge, Mass., Poohookapaug, Quinebaug

Sudbury, Mass., Magaenak

Sugar Island, Me., Sugalmanahan

Sugar Loaf Brook, Mass., Weckwannuck 
Sunderland, Mass., Saw watep skechuwas

Sunkhaze Stream, Me., Wetchi sankasek

Sutton, Mass., Manchaug

Swansea, Mass., Keekamuit, Wannamoiset, Wepoiset

Swanton, Vt., Maskwenozakek, Missisiasuk

Tauton, Mass,. Cohannet, Masstucksett, Nesquabinanset, Seewamuck

Telosimis Lake, Me., Pataquongamis

Telos Lake, Me., Sahkahegan

Thames River, Conn., Pequod, Pequot, Puccatannock, Sepos Tamesuck

The Gulf, Me., Mahkonlahgok

The Hook, Me., Kedumcook

The Narrows, R. I., Weybosset

The Notch, Mass., Norwottock

The Notched Head, Me., Psinkskihigan-i-ontop

The Weirs, N. H., Aquadoctan

Thomaston, Me., Saw quid, Squitcomegek, Tahanock, Wessaweskeag

Thompson, Conn., Maanexit, Mananexit, Manexit

Thompson's Point, Vt., Ohnowarake, Tommy Squatter

Thoroughfare Brook, Me., Sahbimskitegwek

Three Heads Mountain, Me., Nesuntabunt

Tisbury, Mass., Ohkonkumme. See Martha's Vineyard

Tiverton, R. I., Pocasset, Puncatest, Quamquit

Tolland, Conn., Nipmuck

Topsfield, Maine, Simquish

Topsham, Me., Psazeske, Sawacook

Townshend, Vt., Kamouraska

Treat's Falls, Me., Bemidjiwok, Pemjeedgewock

Treat Island, Me., K'chi penabsq

Treat Webster Island, Me., Talagodissek

Truro, Mass., Meeshawn

Turner Brook, Me., Ahsedakwasic

Turner's Falls, Mass., Peskeompscut
Twin Islands, Me., Tagwesi Menahol Twin Lakes, Conn., Pannaheconnok, Wallempteweekek

Union River, Me., Sennebec, Wechkotetuk

Uxbridge, Mass., Chockalog, Wacuntug, Watchusesic

Veazie, Me., Wabeno bahntuck

Vergennes, Vt., Netahme Puntook, Pecontook

Vernon, Vt., Koessek, Odana, Squakheag

Verona Island, Me., Nalagwem Menahan

Victory, Vt., Mozias

Voluntown, Conn., Monhungunuck, Walopeconek

Waits River, Vt., Mahsunquammasec

Waldoboro, Me., Penobseese

Wallis Pond, Vt., Namaaskotic

Wallum Lake, R. I., A'wumps

Walpole, N. H., K'chi Ponteguh

Ware, Mass., Wembemiscook

Wareham, Mass., Cromesit, Misquitucket, Wawayontatt, Weweantic

Warehouse Point, Conn., Nameroke

Ware River, Mass., Menameset, Misquatucket

Warner, N. H., Schodac

Warren, Mass., Naltaug

Warren, R. I., Nahett, Pacanoket, Pokanoket, Scamscammuck, Sowampsett, Toskaunk

Warwick, R. I., Aquitamoset, Mishawomut, Pomham

Waterbury, Conn., Achetaqupag

Waterford, Conn., Oswegatehie

Watertown, Mass., Pitchgussett, Winnemaug

Waterville, Me., Keta Kouan, Negwamkeag, Taconnet

Webster Stream, Me., Madunkehunk, Nemadunkehunk

Wellfleet, Mass., Nampshaket, Punonakanit

Wells, Me., Nampscoscoske, Neguntequit, Webhannet 
West Arlington, Vt., Annisquassi- White Mountains, N. H., Anniscoke

Westboro, Mass., Hobbomoc, Naggawoomcom

Westbrook, Conn., Pachaug

Westbrook, Me., Sacarappa

Westerly, R. I., Achagomiconset, Acontaug, Ascomacut, Mamaniskak

Westfield, Mass., Pachasuck, Pawtuxet, Poyasuck,

Westford, Mass., Kissacook, Nabnasset, Tadmuck

West Greenwich, R. I., Mishnock, Tippecansett, Wequapaugset

West Haven, Vt., Gashekwaneh, Tieronderaquegon

West Lake, Me., Abagamok

Westmore, Vt., Pisgah

Westport, Conn., Hassacky

Westport, Mass., Gotomska

West Quoddy, Me., Pessakenew Agenek

West River, Vt., Wanasquatuck, Wantastiquet

West Rock, New Haven, Conn., Mautunsq

West Windsor Locks, Conn., Unshemamuck

Wethersfield, Conn., Pyquiag, Sicaog, Sowheage

Weymouth, Mass., Weechagaskas

Whale Mountain, Me., Potowadjo

Whately, Mass., Capawong, Weckwannuck

Whetstone Brook, Vt., Keetadoganapskwa Seebis

Whetstone Falls, Me., Popokumuk wodchussu

Whiskey Pond, R. I., Usquepaug

White Cap Mountain, Me., Wassum. ke Wadjo quam, Attilah, Cowissewaschook, Kodaak Wadjo, Misadene, Wabademsolduwak Wajowuk

White Oak Pond, Me., Pawnook

White River, Me., Woboostook

White River, Vt., Cascadnac, Madi tegou, Pantigo, Wassabastegu

White Rock Portage, Me., Wapskenigan

White Squaw Island, Me., Kahnonahjik

Whiting River, Me., Keght Niganish

Wickford, R. I., Kesickamuck, Namquoxet, Opuitowaxet

Williams River, Mass., Taupagoh

Willimantic, Conn., Mamaquag, Nahwesetuck

Winchendon, Mass., Penkese

Winchester, Mass., Aberjona

Windham, Vt., Kamouraska

Windham, N. H., Gaentake

Windsor, Conn., Chesechankamauk, Cushankamaug

Winthrop, Me., Maroonscook

Wolcott, Vt., Wapanaki

Wolves Rocks, Me., Adogenadesk

Woodstock, Conn., Segunesit, Senexet, Pompwanganug

Woodstock, Vt., Ohio, Pogue

Woolwich, Me., Nasket

Woonsocket, R. I., Niswosaket

Worcester, Mass., Packachaug, Sagatobscot

Worden's Pond, R. I., Aquebapaug

Wrentham, Mass., Wolomonopaug

Wright's Island, Conn., Manhannock

Yarmouth, Me., Wesgustogo

Yarmouth, Mass., Acqueet, Hocquan

York, Me., Yeapskasset 



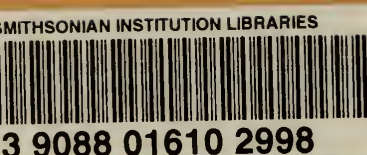

